\title{
DEVELOPING A THEORY OF IMPLEMENTATION FOR BETTER CHRONIC HEALTHCARE MANAGEMENT: A COGNITIVE MAPPING AND SYSTEM DYNAMICS APPROACH
}

BY

DAVID REES

A thesis

submitted to the Victoria University of Wellington in fulfilment of the requirements for the degree of Doctor of Philosophy

Victoria University of Wellington

2013 


\begin{abstract}
While chronic disease is viewed by some as the 'healthcare challenge of this century', and academics and practitioners around the world extol the virtues of chronic care management programmes, we are still a long way from fully specifying the causal connections that are needed to design and implement them successfully. Whilst the factors that are important in such systems of care are well articulated in the literature, it is less clear what the relationships between them are, and it is unclear how those factors can be implemented in a way that retains the integrity of the system they are a part of. The result is that despite strong clinical and management support, progress in implementing such programmes is slow.
\end{abstract}

The goals of this research are therefore to:

- develop a better understanding of the system of causality underpinning the key factors known to be important in implementing new models of chronic health care management,

- understand how context influences this system, and

- use the answers to the above questions to provide a model of implementation that can inform both theory and practice

The research uses in-depth interviews with seven clinical, management and policy leaders within the New Zealand health system to develop a 'theory of implementation' that is described using System Dynamics. The research uses the cognitive mapping method to elicit the key concepts in the 'expert' theories by analysing both the individual maps and a composite map developed by combining data from all seven interviews. The cognitive maps are then used to inform the development of a causal loop diagram that depicts the key causal connections that are seen to be important in implementing such programmes and provides the basis for a simulation model.

The findings from this research fall into two groups. The first group are findings that relate directly to the challenge of implementing programmes to improve care for people with chronic conditions. Within this group are findings that emphasise the importance of clinicians' self-efficacy, the paradox that striving to implement best practice may, in 
some contexts, decrease performance and the acknowledgement that implementation will always be a 'local affair'. The second group of findings relate to the process of implementation research. The world of implementation is a world of multiple, interacting variables that change over time and this research provides an approach, combining qualitative and quantitative data, that can be used in other contexts where the interest is in understanding how innovative ideas are implemented in practice.

The research has therefore some implications for the practice of implementing new health innovations in primary care and provides a set of heuristics to inform such endeavours. The research also describes an approach for those who want to conduct research into the complex world of practice, by exploring the dynamics of many interacting factors, rather than isolating individual factors from each other and the context within which they exist. 


\section{Acknowledgements}

Undertaking $\mathrm{PhD}$ study is an arduous task. Undertaking $\mathrm{PhD}$ study part-time, well into one's fifties, could be considered foolish and there have been many times over the last few years when I contemplated the foolishness of the task. On more than one occasion I made the decision to quit. My supervisors however, encouraged me and interspersed their academic critique with enough confidence-giving feedback to keep me going. Bob Cavana and Jackie Cumming stuck with me throughout this long journey, acting as coaches and guides, both challenging and supporting, and ensuring all the time that I was heading in the right direction with enough momentum to keep the wheels turning. I am forever grateful for their wisdom and encouragement throughout this research.

I also want to thank the health experts, who gave up time to share their wealth of knowledge about health, and the challenges of trying to implement programmes to improve care for people with long-term conditions. It is their expertise that sits at the centre of this work, and without their willingness to share their expertise with me, this research would not have gone very far.

I am also grateful for the support that my work colleagues provided, especially over the last 18 months, when I was often absent, as much in mind as in body, as I tried to get the research completed. Their cries of, 'now go and get the bloody thing finished' as we broke for our Christmas break echoed in my ears, and some of the energy required for the long hours over the last few weeks has come from the fear of heading back into the office in 2013 to face their wrath if it was not finished.

Undertaking the $\mathrm{PhD}$ has in many ways been an attempt to complete 'unfinished business'. I first enrolled in a $\mathrm{PhD}$ in the UK in 1986 but a young baby always made it difficult, and a second, following in her footsteps, put paid to any hopes that it was going to be a realistic option. My children, now very much grown up and tackling their own challenges, have always encouraged 'dad' to get on and get it done, and their continual enquires about, "how's the $\mathrm{PhD}$ coming along Dad" have ensured that it was never too far away from my attention. 
It is my wonderful partner Jacqueline, however, who has had to live with my highs and lows on a daily basis. Never far away, she had the knack of knowing when to tell me to, "get my act together and get stuck into it", or provide the gentle encouragement and support needed to sit down once again and write. 


\section{Contents}

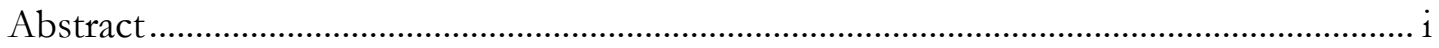

Acknowledgements ........................................................................................................ iii

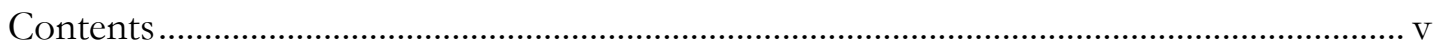

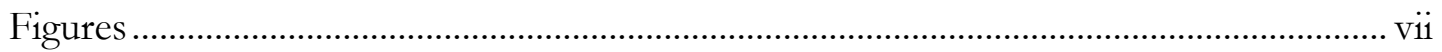

Tables ………………………………………………………………………………………

Glossary of Terms and Abbreviations............................................................................

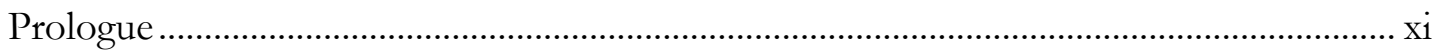

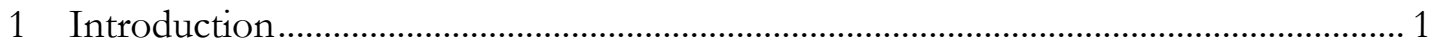

1.1 The Burden of Chronic Conditions .........................................................................................

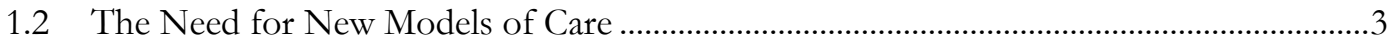

1.3 From Models of Practice to Models in Practice.....................................................................5

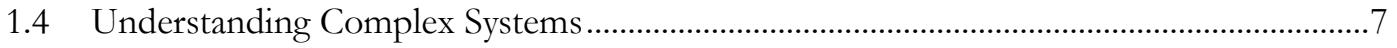

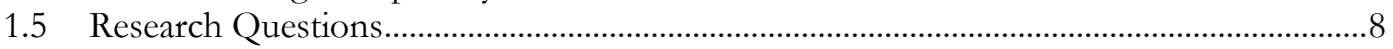

1.6 Summary ………………………………………………………………………....

1.7 Outline of Thesis.............................................................................................................................

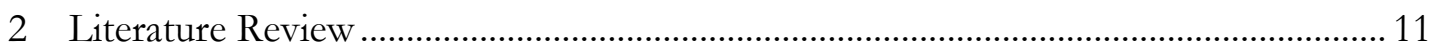

2.1 Meeting the Challenge of Chronic Disease ........................................................................... 11

2.2 Frameworks for an Effective Response …………………………………………………1 16

2.3 Limitations of Current Frameworks .................................................................................... 23

2.4 Understanding Interactions: Taking a Systems Approach................................................ 25

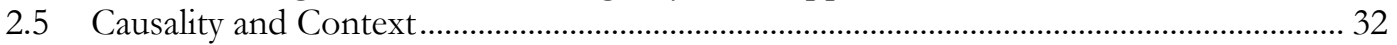

2.6 Applications to Healthcare …………………………………………………………………... 35

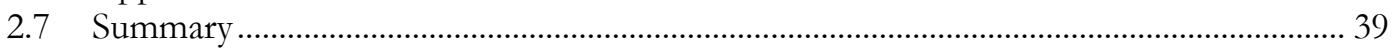

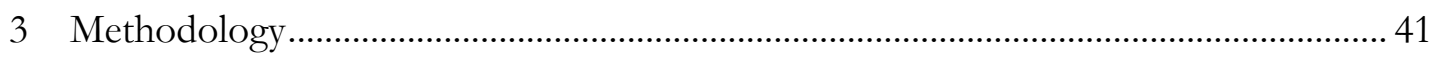

3.1 What Informs the Approach to This Research ............................................................... 42

3.2 Implementation Research ...................................................................................................... 46

3.3 The World of Research and the World of Practice ……………………………………..... 49

3.4 Implementation as a Design Challenge ................................................................................. 51

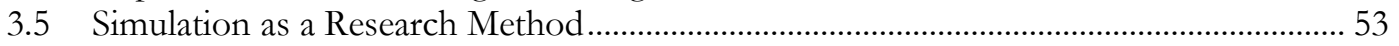

3.6 The Research Method …………………………………………………………………………... 56

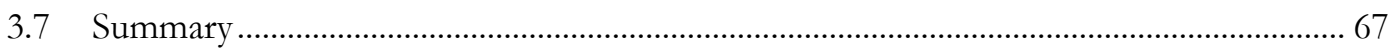

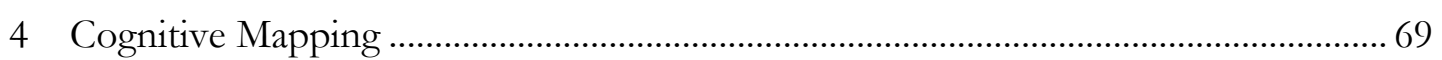

4.1 Moving Beyond Lists: the Argument for a Systems Approach ........................................... 69

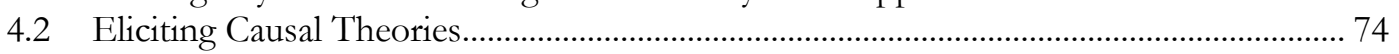

4.3 Analysis of Cognitive Maps .................................................................................................. 79

4.4 Thematic Analysis Of Composite Map …………………………………………………….... 86

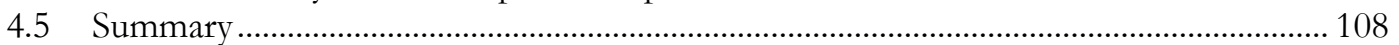

5 Causal Loop Modelling …………………………………………………………………... 109

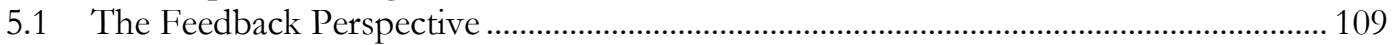

5.2 The Model of Implementation................................................................................................. 114

5.3 Summary of 'Expert' Theory...................................................................................... 130

5.4 Policy Recommendations.................................................................................................. 135

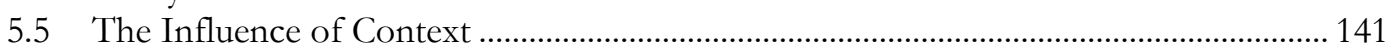

5.6 What Makes a Good Conceptual Model? ......................................................................... 142 


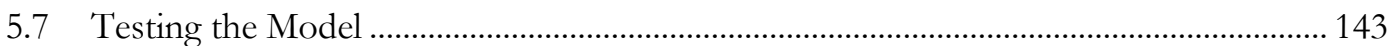

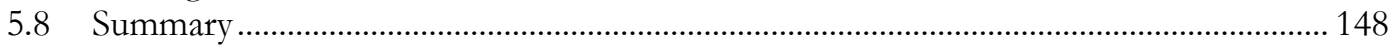

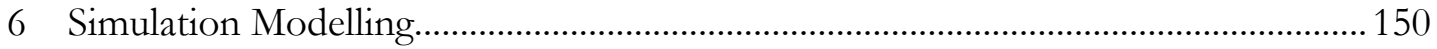

6.1 Core Components of the Theory of Implementation......................................................... 151

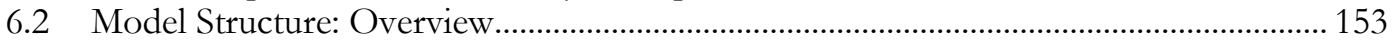

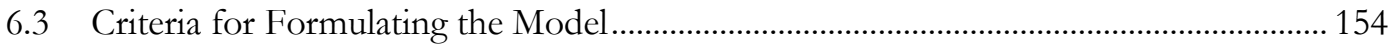

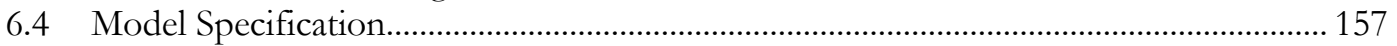

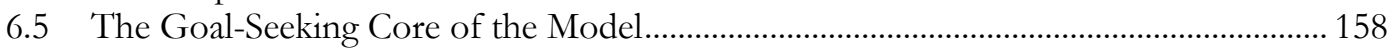

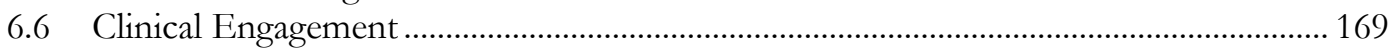

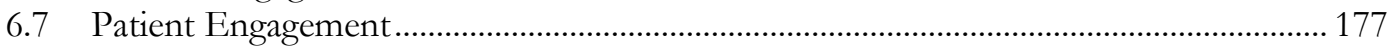

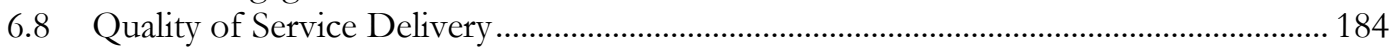

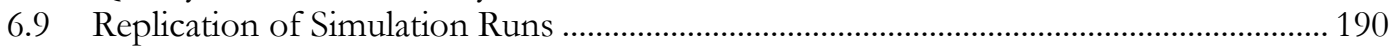

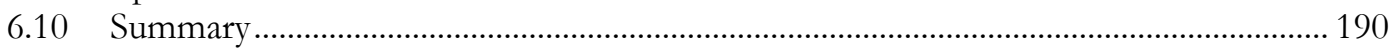

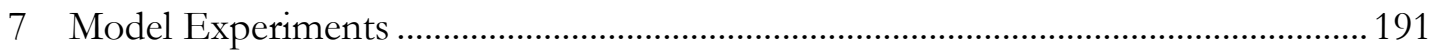

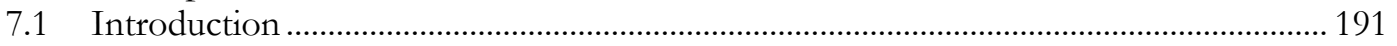

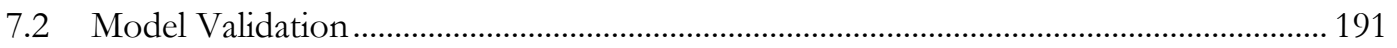

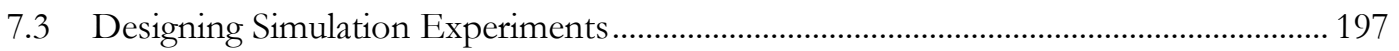

7.4 Structure for the Simulation Experiments ....................................................................... 198

7.5 Exploring the Behaviour of Different Primary Care Practices ....................................... 199

7.6 Establishing the Base Case ............................................................................................... 204

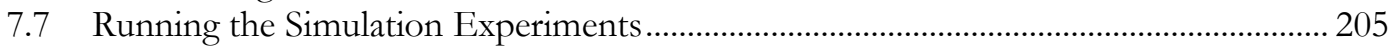

7.8 Improving the Quality of Care by Setting Higher Goals ................................................. 206

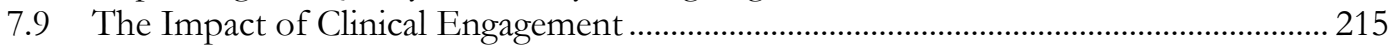

7.10 Options for a Resource Constrained Practice- Patient Engagement............................. 220

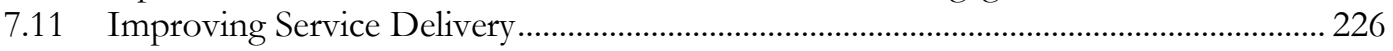

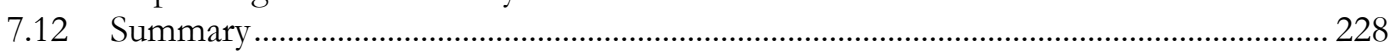

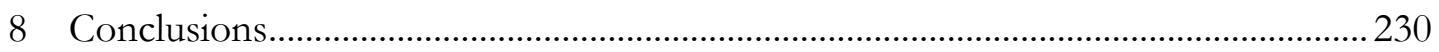

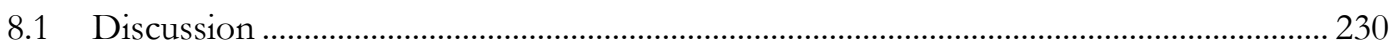

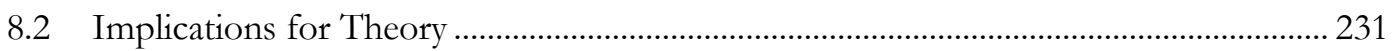

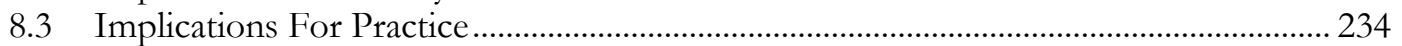

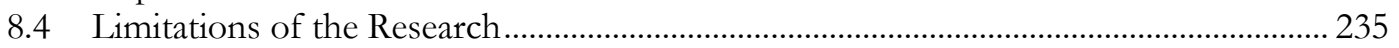

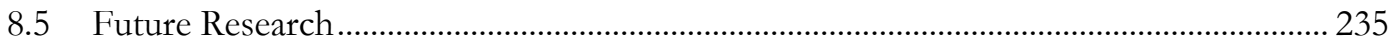

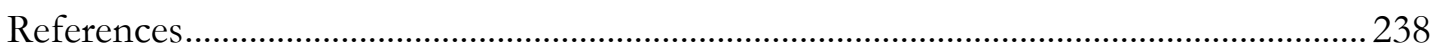

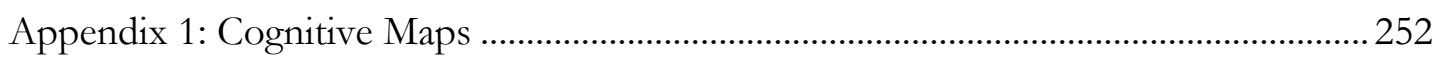

Appendix 2: Material Used to Feedback Model to Health Experts Interviewed............ 260

Appendix 3: Sensitivity Tests ......................................................................................... 272

Appendix 4: Model Documentation................................................................................. 277 


\section{Figures}

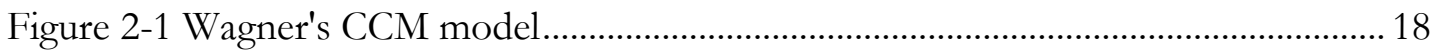

Figure 2-2 Sustainability of Primary Care Programmes ...................................................... 22

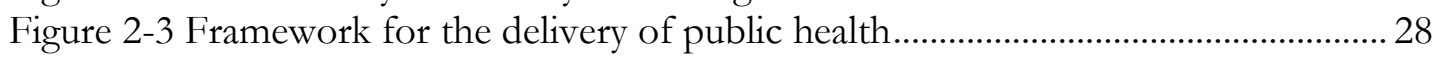

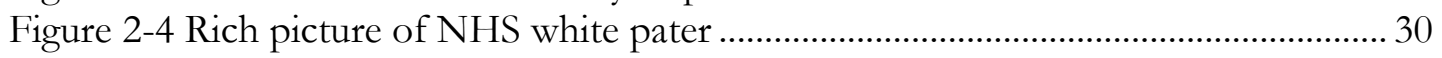

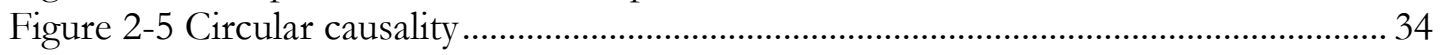

Figure 2-6 'System Dynamics' and 'Health' articles 1998-2012 _.......................................... 35

Figure 3-1 The interactive process of management theory and simulation modelling ... 55

Figure 3-2 Overview of the research method ...................................................................... 58

Figure 3-3 Illustrating the links between data and clinical engagement .............................63 63

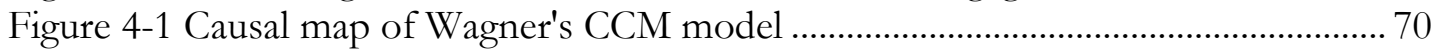

Figure 4-2 Cognitive Mapping Example: 1st interview with interviewee \#1 .................... 77

Figure 4-3 Cognitive Map Example: 2nd interview with interviewee \#1 ........................... 78

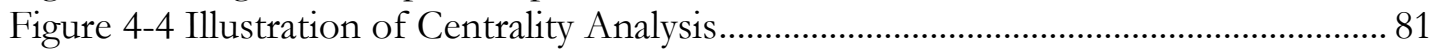

Figure 4-5 Causes and conditions related to the engagement of providers ........................ 82

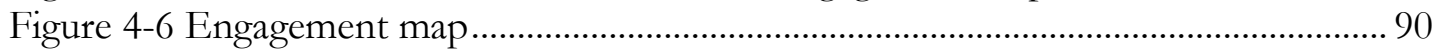

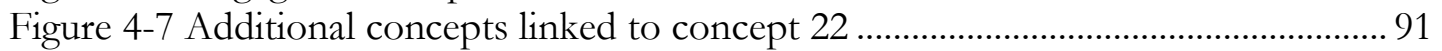

Figure 4-8 Performance feedback map .......................................................................... 94

Figure 4-9 Concept 181 shown with connections outside the performance feedback

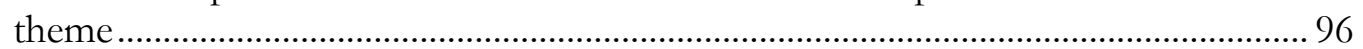

Figure 4-10 Provider performance map …………….......................................................... 99

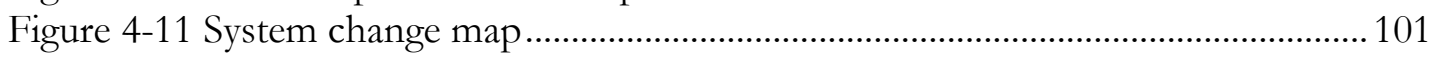

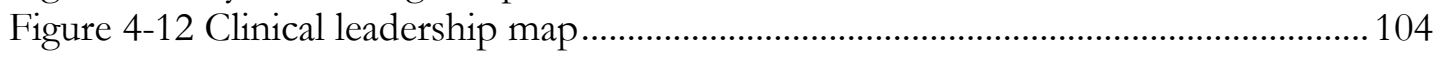

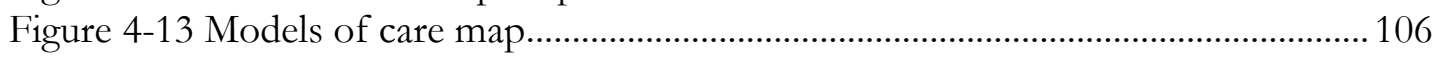

Figure 5-1 Managing the tension between chronic and acute care ................................... 111

Figure 5-2 Impact of clinical engagement on the effective management of chronic

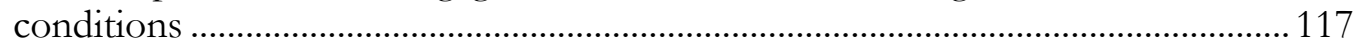

Figure 5-3 Closing the loop between engagement and action ........................................... 117

Figure 5-4 Impact of performance feedback on clinical engagement (R1) .......................120

Figure 5-5 Impact of performance data on quality of effort (R2) .................................... 121

Figure 5-6 Impact of performance data on quality of processes (R3) ............................... 121

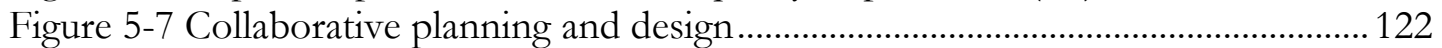

Figure 5-8 Patient engagement and self-management (R4)............................................ 124

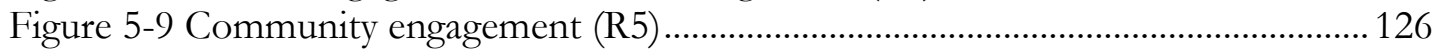

Figure 5-10 Resource demand (B1) and self-efficacy (R6) .............................................. 128

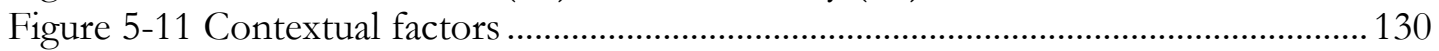

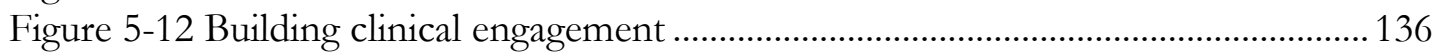

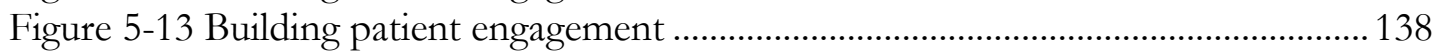

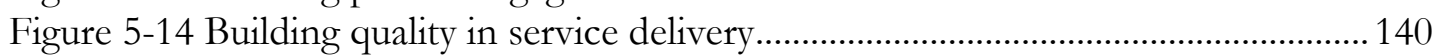

Figure 6-1: Published papers "Management" AND "Theory" AND "Simulation" ...... 150

Figure 6-2 A causal theory of implementation.................................................................... 152

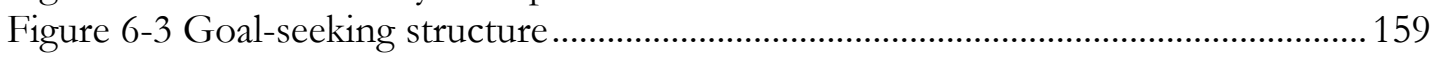

Figure 6-4 Impact of improvement activities on process quality ...................................... 161

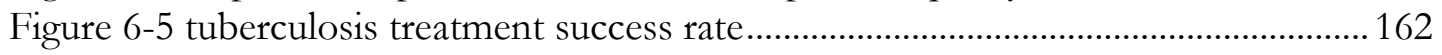

Figure 6-6 Effective management of chronic conditions .................................................... 164

Figure 6-7 Incorporating loss of quality and limits upon improvement capacity ........... 166

Figure 6-8 Effective management of chronic conditions: impact of capacity................ 168 
Figure 6-9 Clinical engagement and the development of the implicit goals 170

Figure 6-10 Effective management of chronic conditions: impact of clinical

engagement

Figure 6-11 Impact of clinical engagement upon the weight given to the stated goal.. 172

Figure 6-12 Timeframes and self-efficacy.....

Figure 6-13 Impact of self-efficacy upon the effective management of chronic conditions

Figure 6-14 Impact of self-efficacy on the improvement effort....................................176

Figure 6-15 Patient engagement and its impact upon self care ........................................ 179

Figure 6-16 Impact of patient engagement on self care .................................................... 181

Figure 6-17 Impact of experience on visits by self care patients...................................... 182

Figure 6-18 Impact of engaged patients on the effective management of chronic

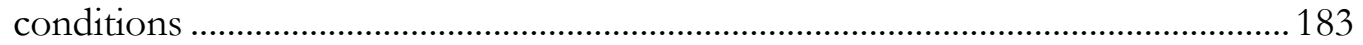

Figure 6-19 Quality of service delivery ........................................................................... 184

Figure 6-20 Effect of improvement activities on process quality ….................................185

Figure 6-21 Effect of process quality on quality of effort ............................................... 185

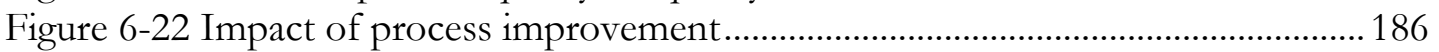

Figure 6-23 Time to perceive change in performance …................................................187

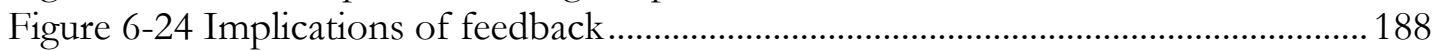

Figure 7-1 Validation testing: impact of clinical engagement on weight given to stated goal.

Figure 7-2 Testing the impact of clinical engagement on the weight given to the stated goal.

Figure 7-3 Policy experiment: adjusting goals .................................................................. 207

Figure 7-4 Policy experiment: striving for best practice …………….................................. 209

Figure 7-5 Policy experiment: impact of resources ........................................................ 211

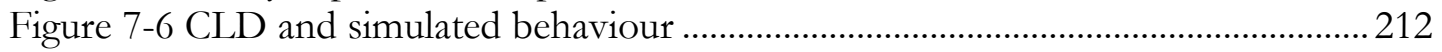

Figure 7-7 Policy experiment: matching goals to performance ………………..................214

Figure 7-8 Policy experiment: changing initial levels of clinical engagement .................. 217

Figure 7-9 Policy experiment: clinical engagement and self-efficacy .................................218

Figure 7-10 Policy experiment: lower levels of clinical engagement................................219

Figure 7-11 Policy experiment: changing levels of patient engagement activities........... 222

Figure 7-12 Policy experiment: impact of changing investment in patient engagement

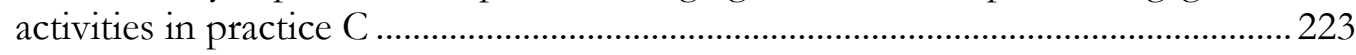

Figure 7-13 Policy experiment: impact of high resource demands in practice C............224

Figure 7-14 Policy experiment: setting targets based on local capabilities in practice $\mathrm{C}$

Figure 7-15 Model structure: impact of service delivery.................................................226

Figure 7-16 Policy experiment: impact of process improvement activities ..................... 227 


\section{Tables}

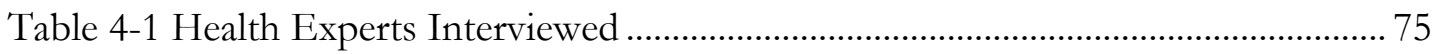

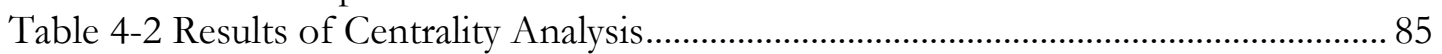

Table 4-3 Key Themes Arising Out of Centrality Analysis ................................................... 86

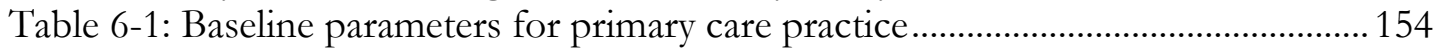

Table 6-2 Key parameters in the baseline run...................................................................... 163

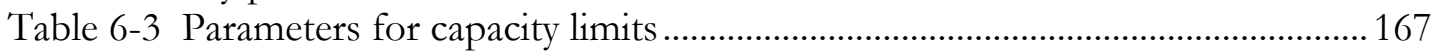

Table 6-4 Key Parameters for Patient Engagement ............................................................ 179

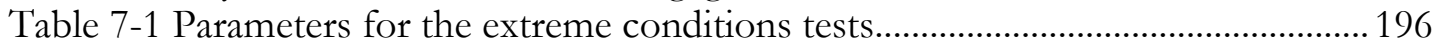

Table 7-2 difference between simulation and laboratory experiments ............................... 197

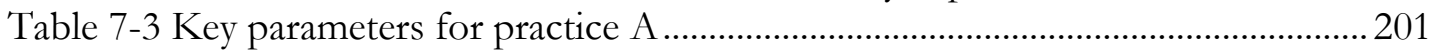

Table 7-4 Key parameters for practice B ...........................................................................2202

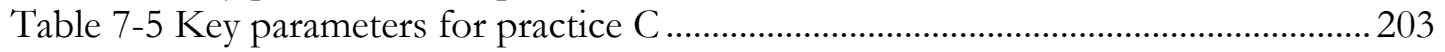

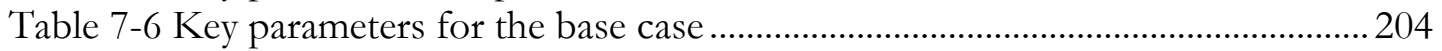

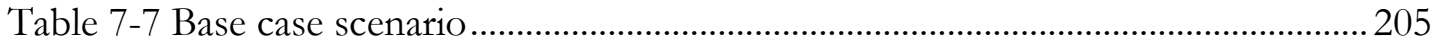

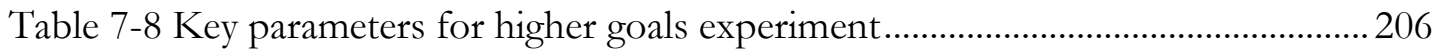

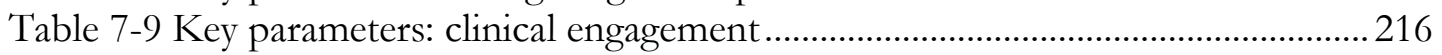

Table 7-10 Key parameters: resource constrained practice .................................................221 


\section{Glossary of Terms and Abbreviations}

\section{CCM}

Concept

Construct

Chronic conditions

Chronic disease

CLD

CVD

DHB

GP

Long-term

conditions

Factors

Practice Teams

Self-care

Self-management

Theme

Variable
Chronic Care Management. A programme designed to integrate key aspects of care for people with chronic conditions

Refers to the individual ideas in the cognitive maps developed from the initial interviews.

Refers to the 'higher-order' ideas that were 'constructed' from the analysis of the cognitive maps. For example 'clinical engagement' was one of the key constructs that arose out of studying the concepts within the cognitive maps

These are conditions that are ongoing, long-lasting and have an affect on the physical, social, psychological and economic aspects of a person's life.

see 'chronic conditions'

Causal Loop Diagram developed from the interviews to describe the casual links between the key ideas and developed into the initial theory of implementation

Cardiovascular disease. These are amongst the most prevalent group of diseases, and principally refer to cardiac disease, vascular diseases of the brain, such s stroke, and kidney and peripheral arterial disease.

District Health Board. DHBs are organisations, established by the New Zealand Public Health and Disability Act 2000, to mange heath services in their District.

General Practitioner. The term used to describe the primary care physician in New Zealand

see 'chronic conditions'

The term 'factors' is used when referring to 'parts' of the larger system. It is used when discussing, for example, factors identified in the literature as being important in implementation and when discussing parts of the system outside of their specific use in the models developed in this research.

Refers to the clinical team of doctors, nurses and allied health professionals that together make up the primary care practice

Self-care is central to the effective management of long-term conditions and refers to the patient's ability to take an active part in their care programme. It is a major factor in increasing levels of adherence to treatments being recommended by the patient's doctor. see 'self-care'.

The term 'theme' is used to describe groupings of concepts generated in the concept mapping phase.

Individual elements within the CLD are referred to as variables. 


\section{Prologue}

In May 1940, a policeman scratched himself while pruning his roses. This seemingly innocuous event led to staphylococcal septicaemia, an infection that soon had him in hospital and on his deathbed. This was not an uncommon occurrence at the time as there was little available to treat infections that became septic. However, while he lay on his bed a team of researchers at Oxford University, building on earlier work by Alexander Fleming, who first described the effects of penicillin, had just inoculated eight mice with fatal streptococci doses. All died except the four that had been given penicillin. Eager to try it on a patient, the team of researchers saw the dying policeman as the perfect opportunity and although there was very little of the drug, as production techniques had not yet been developed, they injected him with the penicillin and within four days he had improved remarkably. The power of antibiotics, as they became known, was no longer in doubt and by 1943 penicillin was in mass-production in both the United States and Great Britain. So successful was this drive for production that, by DDay in June 1944, there was enough penicillin for unlimited treatment of thousands of allied servicemen (Porter, 1997).

This discovery of the powerful benefits of penicillin was to revolutionise medicine, and medical research, with the result that new drugs quickly appeared, making major inroads into once very common fatal diseases. These drugs proved successful against diseases such as smallpox, measles, mumps, typhoid fever, rubella, diphtheria, tetanus, yellow fever, whooping cough, and poliomyelitis, as well as bacterial conditions, some viral infections and numerous disorders (Porter, 1997). Many of the drugs still used today are derivatives of those first discovered in the 1940s and 1950s (Fanu, 1999), and continue to be central tools of modern medicine. This explosion in pharmaceutical knowledge however, all came too late for the policeman; he died. Not able to produce enough penicillin, the researchers were unable to continue his treatment.

For many decades the diagnostic skills of doctors were far more advanced than their ability to treat, and their "pharmacopoeia was a bag of blanks" (Porter, 1997). However, by the end of World War II, doctors finally had a range of effective treatments that they could dispense, and this was to have a major impact upon how medicine was practised. 
But, as doctors' increased their ability to dispense drugs that actually worked, they began to lose that special bond that had existed for many years between the doctor and their patient. More and more of the interactions between doctors and their patients took place in the doctors' surgery rather than in the home of the patient. They had a new bag of pharmacological tricks and were increasingly confident of their ability to use it, with the result that the psychological significance and benefits of the relationship that developed between family doctors and their patients were being forgotten. The prescription, and the benefits it now held, became a way of avoiding a more timeconsuming analysis and treatment. It provided the mechanism for cutting short the consultation (Porter, 1997).

The irony in all this is that within a few short decades of getting powerful drugs to give to their patients, the disease landscape began to change. As a result of better living conditions, better nutrition and better therapies, many of the diseases that killed people prior to World War II started to disappear. In their place, from the mid-1970s onward, came a new crop of diseases. These were modern 'lifestyle' diseases: lung cancer, heart disease, stroke, diabetes and chronic degenerative diseases such as dementia. The drugs that gave doctors such powerful means of curing infectious disease were not so effective against these newer arrivals, and doctors were increasingly being faced with diseases that, once again, were not so amenable to the clinical toolkit.

In less than 50 years the 'miracle cures' were no longer that miraculous, and doctors were again being faced with the reality that their influence on the health of their patients had severe limitations. The diseases of the twenty-first century, such as heart disease, diabetes and cancer are largely incurable. Their worst effects can be controlled. The symptoms associated with them can be managed. People who have them can live long, active and fulfilling lives. However one cannot be 'cured' of diabetes or heart disease. Furthermore, to control the worst effects of the conditions, to effectively manage their symptoms, requires a response that goes beyond the confines of the doctor-patient relationship. The patient, their family and the community within which they live all have a role to play, and need to become a part of the team that delivers an effective response to chronic disease. Medicine, and the toolkit that comes with it, is still enormously powerful, but the task it is now faced with cannot be met by medicine alone. 
An effective response to the major diseases affecting people in the twenty-first century has to be system-wide. Understanding the dynamics of this response, within the system of primary care in New Zealand, is the focus of this thesis. 


\section{Introduction}

\section{The Changing Landscape of Health}

\subsection{The Burden of Chronic Conditions ${ }^{1}$}

The World Health Organisation refers to chronic conditions as the "healthcare challenge of this century', highlighting the enormous personal and social costs of chronic conditions (World Health Organisation, 2005). Even though 'advanced' countries have made progress in some areas, reducing the death rates from heart disease, for example, by around 50 per cent over the last fifty years, other chronic conditions, such as diabetes, have risen dramatically so that chronic diseases currently account for approximately 60 per cent of the world's disease burden (World Health Organisation, 2005). Cardiovascular disease (CVD) accounts for 10 per cent of the global disease burden. The social costs of this burden are horrific. Not only do chronic conditions contribute to an early death, with over 45 per cent of chronic disease deaths occurring prematurely, but they also lead to to disabilities, often lasting for decades of a person's life. As a result of improved nutrition, sanitation and the success in fighting infectious disease, many people are now living longer, and in New Zealand mortality rates have been on a continual decline since the 1950s. However, while people are living longer, the consequence is that many are now living long enough to develop chronic diseases. People may be living longer, but for many it is a life of suffering.

But the burden of chronic disease does not fall evenly. 80 per cent of chronic disease deaths occur in low and middle-income countries and there is a direct link between chronic disease and poverty; both connected in a vicious reinforcing cycle (World Health Organisation, 2005). People who are poor have increased exposure to the risk factors $^{2}$ associated with chronic disease and have less access to health services. Poor

\footnotetext{
${ }^{1}$ In health practice, and in the health literature the terms chronic disease, chronic conditions and longterm condition are synonymous.

2 This thesis is concerned with 'whole systems' and their 'parts'. As it will be combining information from a number of disciplines, each with its own terminology, consistency of language is difficult, especially for terms describing the parts of a system. When, in chapter 4, I develop the key themes for a theory of implementation, using cognitive mapping, I will stick to the terminology of the discipline and refer to each idea as a concept. When this is developed into a qualitative model each element in that model will be referred to as a variable. I will use the word 'factor' when referring to key 'system parts' in the broader
} 
people suffering from chronic disease find it even harder to break out of the poverty cycle with an increasing amount of their meagre earnings being spent on the treatment and care of their disease.

While we might not consider ourselves to be a poor country, the story is the same in New Zealand; the poorest amongst us suffer most from chronic diseases. It is the poor, Māori, and Pacific peoples who carry the greatest burden of chronic disease in New Zealand. Māori over 35 are three times more likely to die of cardiovascular disease (CVD) than non- Māori. Māori have higher rates of diabetes, are more likely to be hospitalised and die earlier. Māori are disproportionately represented in the lower income groups and consequently disproportionately affected by chronic conditions (Ellison-Loschmann, King, \& Pearce, 2004; Gentles et al., 2006). The same overall story applies to Pacific peoples. They die younger, have greater incidence of a range of chronic diseases and are poorer than the general population (National Health Committee, 2007). The story in New Zealand is a microcosm of the story around the world; chronic disease is rising to epidemic proportions and those who suffer most are the poor; those with the least resources to cope.

On top of the enormous personal costs, there are also major financial costs. In New Zealand, while there is a need to develop much better data on the overall costs of chronic conditions, data on specific chronic conditions are alarming (Jaine, 2009; National Health Committee, 2007). As documented by the National Health Committee (2007) the direct costs of strokes are estimated to be around $\$ 150$ million a year; the total financial costs of arthritis are estimated to be $\$ 2.35$ billion; asthma costs the country over $\$ 800$ million per year, and patients with diabetes generate hospital costs that are around 2.5 times greater than someone without diabetes. In terms of overall financial impact we do know that chronic conditions in New Zealand are the leading cause of hospitalisations, use 70 per cent of health funds, and account for 80 per cent of all deaths (National Health Committee, 2007).

literature, and when speaking about implementation in a general sense, outside any specific reference to the models used in this research. When I am referring to larger parts of a system, which may involve multiple factors I will use the terms 'component'. 


\subsection{The Need for New Models of Care}

But, while more and more of our time and dollars are spent grappling with chronic conditions, much of our health system has been built up to respond to acute care. The health system that has evolved since World War II has focused on acute care, treated by a complex mix of new surgical and pharmaceutical tools. This has contributed to undermining the development of any meaningful relationships between the doctor and their patients and the 15 minute visit to the General Practitioner (GP) (Bindman, Forrest, Britt, Crampton, \& Majeed, 2007), that usually ends with the sound of the pharmaceutical script being ripped off the pad, is now the norm. In 2012 however, this sound has been replaced by the 'click' of the return key on the computer.

Furthermore, it is the patient who usually initiates the visit to the doctor and the doctor responds to what is presented to them. This model is only 50 years old but it is now an 'old model of care' and no longer meets the requirements of people with chronic conditions, who need help to understand and manage their conditions; help that cannot effectively be dispensed within the 15-minute time slot of the GP consultation. The interaction between patients and those who deliver health services to them needs to be re-emphasised, and the way we design and fund those services needs to support those relationships. The world of acute disease has been replaced by the world of chronic conditions, and the skills and attitudes that once were central to that special relationship between doctors and patients are now, once again, being asked to take centre stage.

This rise in chronic conditions is having a significant impact, not just on patients, but also on the pattern of health delivery, forcing health professionals to look beyond their own discipline to obtain a broader picture of the needs of chronic patients. Diabetes is a typical example of this change. As pointed out by Homer et al., the approach to diabetes has changed considerably over the last few decades (Hirsch \& Homer, 2004b; Homer, Hirsch, \& Milstein, 2007). During the 1970's the focus was on educating people with diabetes and health professionals in the proper care of people with diabetes with the aim of reducing diabetes-related complications. During the 1980's there was increasing emphasis on screening and in the 1990's, the focus shifted to the intensive control of blood sugar levels. Over the last few years the focus has begun to shift to population- 
level initiatives in which the focus goes beyond the health sector to involve social and community agencies as well as the patient and their family (Homer et al., 2005).

The nature of this change requires everyone in the health sector to acquire new knowledge and skills as well as new ways of working. These new ways of working require partnerships across the sector; partnerships that go beyond the normal peer relationships within health disciplines; to partnerships with other disciplines, organisations, communities, and the people who have chronic conditions as well as their families/whānau (National Health Committee, 2007). It is also a change requiring the design and implementation of new healthcare programmes better suited to the changing needs of people with chronic conditions. As difficult as these challenges are, however, healthcare professionals are helped by the fact we know a lot about what an effective healthcare response to chronic conditions looks like.

In the New Zealand context, the author has been involved in modelling diabetes to support the design of a programme in South Auckland; 'Let's Beat Diabetes'. The scope of this programme involved social and community agencies operating beyond the health sector and, within the health sector, involved health professionals from health prevention and promotion as well as secondary specialists, such as renal physicians, treating patients with end-stage renal failure. This 'system-wide' approach is increasingly recognised as being necessary if the challenges presented by the rising prevalence of chronic conditions are going to be met (Singh, 2008). Internationally, there has over the last 15 years, been a number of models of care that have been developed in response to this rise in chronic conditions (Singh \& Ham, 2008). Motivated by the continuing rise of chronic illness and the resulting challenges it is placing on our health services, there has been extensive research into what constitutes an effective service response (Singh \& Ham, 2008). This research has been successful in developing a much better understanding of the key factors required to deliver effective care for people with chronic conditions and models, such as the Chronic Care Model (CCM) developed by Wagner (Wagner, Austin, \& von Korff, 1996), are used extensively to guide programme structure. And, while this research has given us a much richer understanding of what factors need to be incorporated into an effective response to chronic illness, more recent research has begun to tease out what key factors are important in implementing 
such a response. (Connolly et al., 2010; Singh, 2008). With all this research it is clear that health professionals now have a good idea of what is required if we are to improve our response to this growing issue (Wells \& Jackson, 2011).

However, despite knowing the essential factors for an effective healthcare response to these chronic conditions (Connolly et al., 2010; Groves \& Wagner, 2005; Martin \& Sturmberg, 2008; Nolte \& McKee, 2008; Rea et al., 2007), despite the evidence supporting the efficacy of these factors (Singh, 2005; Wagner et al., 2001), and despite the fact that over the last 10 years there have been a number of major programmes developed to help bring about these changes (National Health Committee, 2007; Rea et al., 2007) the orientation in both primary and secondary care is still strongly weighted towards acute care (Connolly et al., 2011). Less than 25 per cent of patients who suffer from chronic conditions receive care that could be considered consistent with best practice (Schoen et al., 2011). In New Zealand, for example, a survey by the New Zealand Branch of the Thoracic Society of Australia and New Zealand found that less than 50 per cent of District Health Boards (DHBs) had implemented any aspects of the long-term condition programme for chronic obstructive pulmonary disorder (COPD), an increasingly prevalent chronic condition (Connolly et al., 2011).

This is concerning given that chronic conditions are, as discussed earlier, the leading cause of mortality, morbidity and inequitable health outcomes in New Zealand.

Furthermore, chronic illness is projected to continue rising, and is the major contributor to an expected doubling of demand for health services over the next 10 years (Gorman, 2010). As a consequence we need to get much better at understanding the dynamics of implementing new healthcare innovations. We need to get beyond describing models of practice and get better at developing such models in practice.

\subsection{From Models of Practice to Models in Practice}

However, putting these models into practice is difficult, as the world of healthcare implementation is complex (Institute of Medicine., 2001). One of the most commonly used frameworks to support this shift is the Chronic Care Management model (CCM), developed by Edward Wagner (1996), which was designed to integrate key evidence- 
based factors important to improved care (A. Coleman, Checkland, Harrison, \& Hiroeh, 2010). Martin and Sturmberg (2008) in describing the characteristics of the CCM model note that it is a programme that requires major health system redesign and major social engineering in its implementation. Furthermore, to be successful it "...requires an alignment of all system components with the patient's health experience" (Martin \& Sturmberg, 2008, p. 573).

The challenge of implementing new health models designed to tackle chronic conditions is therefore a difficult one. It is made more difficult by the fact that the implementation literature is short on the level of detail needed to understand what is actually involved in successfully implementing a new health innovation, such as a programme to improve care for people with chronic conditions. So, while we know a lot about what constitutes effective care for people with chronic conditions, the gap between theory and practice reflects the limited evidence on implementation (Proctor et al., 2009). Trisha Greenhalgh and her colleagues (Greenhalgh, Robert, Bate, MacFarlane, \& Kyriakidou, 2005), in their very influential work on innovation in health service organisation make that point that within the innovation literature the issue of implementation is the least well developed. Klein and Sorra are more blunt in their assessment, concluding that the “implementation literature offers, unfortunately little guidance" (Klein \& Sorra, 1996, p. 1059).

Some research simply notes that at the stage when a new innovation begins to be implemented, "it is inevitable that other factors within the organization become involved at this point” (Dewett, Whittier, \& Williams, 2007, p. 12). These 'other factors' are not described and the issues involved in implementing innovations remain a mystery.

In other research, some of these factors are at least noted. For example, Klein and Knight (Klein \& Knight, 2005), identify infrastructure as a key aspect of implementation, but the 'body of variables' that make up this construct are not investigated, making it very difficult for a practitioner wanting to understand how to adjust infrastructure to make it supportive of implementation, or a researcher to explore what aspects of infrastructure help or hinder the innovation of any new practice. 
To make it even more complex, Klein and her colleagues (Klein \& Knight, 2005) argue that 'ideal' studies should look at implementation 'over time'. This is echoed by Dewett, who notes that his own model of implementation is a 'snapshot' and the "... role of time must be more fully examined" (Dewett et al., 2007, p. 21).

Finally, CCM and other programmes designed to improve care for people with chronic conditions are highly context dependent (A. Coleman et al., 2010). The characteristics of the organisation that is trying to implement the new innovation and the population they are trying to serve has a significant impact upon how it is implemented and the effect of that implementation (Hovmand \& Gillespie, 2006).

\subsection{Understanding Complex Systems}

The challenge of implementation therefore involves dealing with a number of factors that interact and influence each other over time, the pattern of which is highly context dependent (Martin \& Sturmberg, 2008). This creates very special practical and research challenges and, as discussed above, the innovation implementation literature has not progressed far in addressing this complexity.

As a consequence, whilst the new models of healthcare delivery are clear about many of the changes required, and we know many of the factors that are important in implementing them, the research to understand the causal mechanisms by which they interact and influence each other is largely untouched. Furthermore, the research community is not confident that it has the tools to conduct research in this context (Dewett et al., 2007; Greenhalgh et al., 2005).

To address this situation we need to acknowledge that implementing programmes to improve care for people with chronic conditions means grappling with multiple, interacting factors that are context dependent. In short, we have to confront the dynamics of complex systems and use tools that are designed to work in that context.

Qualitative and quantitative systems modelling are approaches that are being increasingly used to explore complex issues in health care (Homer \& Milstein, 2004;

Wolstenhome, Monk, McKelvie, \& Arnold, 2007) and other settings (Cavana \& Clifford, 
2006; Repenning, 2002). It is an approach that is especially suited to research environments that involve a complex web of interacting variables that change over time. In such systems each of the individual processes may be well known and understood. For example, it has been well known for many years that clinical leadership is an important factor in the success of new health initiatives (Ham, 2003). However, the outcomes of the interactions between an individual process, such as clinical leadership, and other processes known to be important are less obvious, especially over time. It is because of this capability that systems modelling sits at the centre of the research approach used in this research to help unravel the complexity of implementing new health innovations. Specifically, this research will use two systems-based modelling methods; cognitive mapping (Eden, 1988) and system dynamics (SD) (Forrester, 1961)

\subsection{Research Questions}

As noted above, much has been done to help increase understanding of what is involved in the process of implementing new health innovations. However, there are still significant questions that remain unanswered, and can only be answered by taking a 'whole-system' perspective that explores the key factors involved in a way that allows their contextual, interacting nature to be explored. This thesis adds to the research that has been done on implementing healthcare innovations by using qualitative and quantitative systems modelling techniques (i.e. cognitive mapping and SD) to examine three research questions that evolve out of the concerns noted above.

- What is the system of causality underpinning the key factors known to be important in the implementation of new models of chronic health care management?

- How does context influence this system?

- Can the answers to the first two questions provide a model of implementation that informs both theory and practice? 


\subsection{Summary}

The continuing rise in chronic conditions is challenging healthcare systems to find new ways of delivering healthcare to people whose needs cannot be met in the 10-15 minute appointment typical of current interactions between patients and their doctors. While new models of healthcare delivery, such as the CCM model, are increasingly being presented as providing effective alternatives, implementing them is difficult and our knowledge of how to do this is much less than our knowledge of the models themselves. This is, in part, because the world of implementation is difficult, for practitioners and researchers alike. To make progress it is going to require grappling with the nature of complex systems, especially the fact that they involve multiple factors interacting and influencing each other over time.

To try and understand the dynamics of implementation, in the context of models of care designed to improve care for people with chronic conditions, this research addresses three key equations:

- What is the system of causality underpinning the key factors known to be important in the implementation of new models of chronic health care management?

- How does context influence this system?

- Can the answers to the first two questions provide a model of implementation that informs both theory and practice?

\subsection{Outline of Thesis}

This thesis will describe the development of a conceptual model (using cognitive mapping) and a causal model (using causal loop diagrams) that shows how key factors, important in implementing of improved chronic care management, interact and influence each other. The causal model also provides the basis for developing a simulation model that explores how these factors interact over time and in different contexts. In addition, the thesis will demonstrate a method of conducting research that uses the experience of 'experts' to understand the complexity of implementation 
practice to inform the development of implementation theory. The remainder of the thesis is as follows:

Chapter 2 provides a review of the literature relating to the three domains covered in this thesis: i) the management of chronic conditions, ii) the implementation of innovative health programmes to improve care for people with chronic conditions, and iii) the use of a systems perspective to shed light on the complexity of implementation. Chapter 3 will describe the research design. Chapter 4 describes the conceptual model that provides the framework for the development of key themes central to implementing chronic care management programmes. This results in a qualitative model describing the key casual relationships within and across these themes, which is described in chapter 5, and a dynamic model, which explores the impact of these relationships over time for a 'virtual primary care practice', which is described in chapter 6. These results are then presented and discussed in chapter 7 . The thesis concludes, in chapter 8 with a discussion of the contribution made by this research and possible avenues for further research to build upon the work documented here. 


\section{Literature Review Health Service Challenges in the New Landscape}

As described in chapter 1 , the continuing rise in chronic conditions is requiring major changes in the way care is delivered. The pharmacological and surgical toolkit, while still important, is not sufficient to tackle the challenges posed by chronic conditions, which require a broader, 'whole-system' approach (Martin \& Sturmberg, 2008).

This chapter will explore current understandings of this challenge, drawing on the health literature of chronic disease, and the literature on innovation and the challenges of implementation. The review of the chronic disease literature will focus less on the actual burden of disease and more on the challenges it poses for health delivery systems and the models of care that have been developed in response to those challenges. The aim will be to describe the nature of the changes that are required if the challenges are to be met successfully. The review of the innovation literature will focus on the implementation of new health innovations and what it tells us about implementing new health programmes for people with chronic conditions.

This chapter will also explore the literature on complex systems as it pertains to health, focusing on the tools and methods that can help increase understanding of the dynamics involved in implementing new health innovations for people with chronic conditions.

\subsection{Meeting the Challenge of Chronic Disease}

The systemic nature of chronic disease discussed in chapter 1 brings with it a number of major challenges for the delivery of healthcare services. There is a need to co-ordinate the large number of people and services involved in providing care for people with chronic conditions. Primary and community care have a very special role to play and more and more services are being moved to primary and community settings.

Furthermore, the care has to be delivered, not by a clinician acting independently, but by a transdisciplinary team comprising health professionals as well as community agencies, the patients and their families. Chronic disease does, in fact, require a substantial rethink 
about how healthcare is organised and delivered, and who actually delivers it. Key components of this rethink are discussed in the following sections

\subsubsection{Co-ordination of Delivery Systems}

The fact that most people with chronic conditions suffer from more than one such condition means that the health system needs much better mechanisms to communicate and co-ordinate care. Someone with diabetes, for example might interact with a range of secondary specialists; a diabetologist, a renal physician and a cardiologist. They are likely to visit their primary care practice on a regular basis and see their GP as well as primary nursing staff. They may have their feet checked by a podiatrist and their eyes checked by an ophthalmologist. They may also be part of a community support group and attend weekly exercise classes for people with diabetes. The limited ability to even know about, let alone co-ordinate, the range of people interacting with the patient is becoming increasingly troublesome and increasingly entering the political and public arena. A highly publicised death at Wellington hospital, which prompted a scathing report by the New Zealand Health and Disability Commissioner, highlights a number of these challenges (Health \& Disability Commissioner, 2007).

The report of the Health and Disability Commissioner criticized the hospital in question for poor care planning. What the report did not explore in depth is the fact that the patient, referred to as Mr. A, had multiple co-morbidities, including mental health issues, and no-one had the overall responsibility to manage and/or co-ordinate the care required for him. He came into the hospital for a specific procedure, but all the other conditions he suffered from would have had a bearing on that procedure, conditions which the hospital staff may have only had a partial knowledge of. The health system simply does not have the structures in place to support co-ordinated services across the continuum of care needed to meet the needs of people with chronic conditions.

While the country reacted to the tragic case of Mr. A, tragedies, such as this, are very rarely the stuff of headlines; they take place over years not days. Chronic conditions last for a substantial period of time, wax and wane in terms of their severity and typically cannot be cured. A chronic condition is enduring and is not simply a series of unconnected complaints. Furthermore, unlike the sudden onset of most acute 
conditions, chronic conditions develop slowly over time. People often have the condition long before they are aware of it, or before it is actually diagnosed. Chronic conditions have multiple causes and can emerge long after interactions with the causal factor (Kane, Priester, \& Totten, 2005). What was ignored in the Commissioner's report is why Mr. A was admitted to hospital in the first place. How well was his asthma, and any other conditions associated with it, managed prior to his admission? How important was this pre-admission management to the events that followed? Did the hospital staff have any knowledge of the broader health issues associated with Mr. A? Did they have a report from his GP in front of them? Was his GP even aware of his broader health issues?

Mr. A is typical of many patients in the current health system, in that his admission to hospital was precipitated by a long-standing chronic condition, not an isolated acute episode with a sudden onset which can be cured by swift and expert intervention in a hospital. He would have had interactions with many clinicians in community, primary and secondary care, many of whom would have been unaware of each others' role. He may have also had interactions with alternative therapy providers, who are even less likely to part of any cohesive management team for Mr. A. The simple fact is that people with long-standing chronic conditions will interact with many people from many disciplines during their life. Many people, trained and untrained, will provide care and treatment of different sorts.

\subsubsection{From Hospitals to Communities}

Within the domain of public health it is recognised that any effective response to chronic conditions needs to acknowledge that an individual's health goes well beyond his or her physical and biological characteristics and is influenced by the broader social, cultural and economic context within which the individual lives. These 'social determinants of health' are well recognised and documented (Marmot \& Wilkinson, 2006). It is also recognised that these social determinants are interdependent, linked together to provide the context within which individual and population health is determined. 
Because of this recognition, there is a strong impetus to move the focus of the health sector away from hospitals to the community (Sibbald, McDonald, \& Roland, 2007). In New Zealand this movement has and continues to be led from the top:

"The new government in 1999 had made health one of its key campaign issues and it moved quickly to bring a stronger population health and community orientation into policy. This found its expression in the Public Health and Disability Act 2000...”" (Barnett \& Barnett, 2005, p. 188)

... and taken up by District Health Boards (DHBs) across the country:

"Whilst we will always strive to ensure excellent hospital services are available for people who are sick or injured, we are increasingly shifting our focus to support people to keep healthy, and access services earlier and in community settings” (Counties Manukau District Health Board, 2006, p. 3)

The current Government in New Zealand, elected in 2008, has put a great deal of emphasis on cutting costs and increasing the efficiency of hospitals. Despite this however, their key health policy statement - 'Better Sooner, More Convenient' - focuses on increasing co-ordination across the sector and moving more and more services into primary and community care.

"Better, Sooner, More Convenient Primary Health Care is the Government's initiative to deliver a more personalised primary health care system that provides services closer to home and makes Kiwis healthier. Primary health care has a part to play in helping reduce acute demand pressure on hospitals by better managing chronic conditions and proactively supporting high need populations.” (Ministry of Health, 2011).

But making these shifts is not easy. A recent review of the literature indicates that shifting services to the community is a plausible strategy for improving access. This can be achieved by, for example, having access to diagnostics through primary care, rather than having to get specialist referrals, or being able to have minor surgery in the primary care practices (Sibbald et al., 2007). There are risks however, in terms of reduced quality and increased cost (Sibbald et al., 2007). This is an important consideration given that 
one of the major drivers is the concern that patients with chronic conditions are not getting good quality care (McGlynn, Asch, Adams, \& Keesey, 2003) and that there is an overall concern about the continuing rise in healthcare costs (Gorman, 2010). This is supported by an empirical research that looked at the consequences of shifting the balance of care from the hospital to community settings (K. Taylor, Dangerfield, \& Le Grand, 2005). One of their conclusions was that shifting care to community settings stimulated demand, which is one factor that would contribute to rising costs.

Despite these risks, however, in New Zealand, many health initiatives are, by their design, heavily focused on the community, whilst acknowledging the multiple stakeholders and complex relationships within and beyond the health sector. They are operating in an environment where there is no clear, coherent and agreed model of what that broader, community-centred health system looks like. From a design point of view, how should it be structured; how should it be funded and how should it be staffed? In the current world most patients with chronic conditions not only receive their care in primary and community settings, but require ongoing care for conditions for which there is no cure. This ongoing care requires interaction with a wide range of health providers, many of whom will not know each other and work within different paradigms. At this point in time, except in isolated cases, no-one is responsible for this co-ordination; no-one is responsible for ensuring that the relationships between the providers is working well and there is no infrastructure to enable that to occur.

\subsubsection{From Individual Clinicians to Transdisciplinary Teams}

The need to co-ordinate care and the push to place more and more of that care into primary and community settings means that more and more of that care has to be provided by teams rather than individuals. As noted above, one of the major challenges that the rise in chronic conditions and the shift from hospital to community poses is the need for a number of people to be involved. Chronic conditions necessitate a perspective broader than the hospital, a perspective that incorporates a wider range of involvement in a patient's care. Chronic conditions, by their very nature, impact not just their physical health but their economic, social, cultural and spiritual health as well. The doctor alone, working with his or her patient, cannot solve the problem of chronic conditions; nor can it be solved by the planner, the nurse, the accountant or any single 
health discipline. To work effectively in this environment, health professionals need to work together with a wide range of people. These people come from a range of professions and disciplines; many are volunteers and many have no formal training in any aspect of healthcare. Despite this, all have a role to play.

Leonard Syme highlights the challenges that this creates for healthcare professionals when he states, with his tongue firmly in his cheek:

"While we in public health know the importance of involving community partners in our programs, we also know how difficult it is to do. The challenge of involving the community is especially difficult if one has been trained, as I have been trained, to be an arrogant, elitist prima donna. I am the "expert," after all, and I help people by sharing my expertise” (Syme, 2004, p. 1)

It is because of this mix of people involved that I use the term 'transdisciplinary' rather than 'multidisciplinary' as what is called for is not just co-operation across the professional disciplines but co-operation that transcends professional boundaries and in the words of one research team involves "joint problem-solving among science, technology and society" (D. Thompson, Edelsberg, Colditz, Bird, \& Oster, 1999).

There have been a number of attempts to develop responses that take account of these challenges, and the following section describes the Chronic Care Management (CCM) model, developed by Edward Wagner and colleagues (Wagner et al., 1996)

\subsection{Frameworks for an Effective Response}

In designing responses to the challenges noted above, one of the most influential writers has been Edward Wagner, from the McColl Institute for Health Care Innovation (Bodenheimer, Wagner, \& Grumbach, 2002a; Wagner et al., 1996). His Chronic Care Management model has provided the inspiration for a number of programmes providing care for people with chronic conditions in New Zealand (Wellingham, Tracey, Rea, \& Gribben, 2003), and is central to both the research and practice of providing care for people with long term conditions. The CCM model has also been adopted by the World Health Organisation (Epping-Jordan, Pruitt, Bengoa, \& Wagner, 2004). 
The CCM model describes six key factors for the effective care of chronic disease; self management support, delivery system design, decision support, clinical information system, health care organisation and community (Wagner et al., 1996). These come together into a model that focuses on changing the nature of the relationship between the primary care doctor and the patient. It is this relationship that, according to Wagner, is central to bringing about better care and better outcomes for chronic patients. The CCM model does therefore address one of the central concerns described in chapter 1 ; focusing attention on the importance of the relationship between the doctor and their patient. Whilst this is placed, appropriately, in a broader clinical and community context, it acknowledges its centrality and thus reaffirms the importance of the therapeutic relationship which has, for the last fifty years, been undermined by the power of medical technology.

The CCM model has attempted to identify the specific practice and system changes needed to improve the care of patients with chronic conditions. The original model focused very much on the primary care practice and how they delivered care (Wagner et al., 1996). It looked at the use of explicit guidelines, the reorganization of primary care practices, patient education, use of expert systems and the use of information to support patient follow up. This initial model was criticized for not indicating the mechanisms through which the system enhancements would result in improved processes of care and improved health outcomes (Wagner, Davis, Schaefer, von Korff, \& Austin, 1999). The changes that resulted from this feedback led to version 2 of the model, shown in figure 2-1 below. It is the version that has had a significant impact on chronic care programmes developed in New Zealand.

The big shift in this second version of the CCM model is that it viewed the health system, not as an isolated system with all it needed to tackle health problems, but as part of a larger community. Within this model, the effective provision of services for people with chronic conditions required that the health system be closely linked with the necessary resources available within the local community. With the development of this model, the boundaries between health and social policy becomes blurred (Milstein, 2008). The effective management of chronic conditions was no longer simply a matter of the provision of health services. It required a close link between health services and 
the broader community within which these services sat (Wagner et al., 1999). The role of the health system was to provide the leadership, incentives and resources to help primary care practices change. Together these two foundations supported the, “... development of both informed, activated patients and prepared, proactive professional practice teams"(Wagner et al., 1999, p. 58). It is this interaction that assures the service delivery mechanisms that deliver improved outcomes.

\section{The Chronic Care Model}

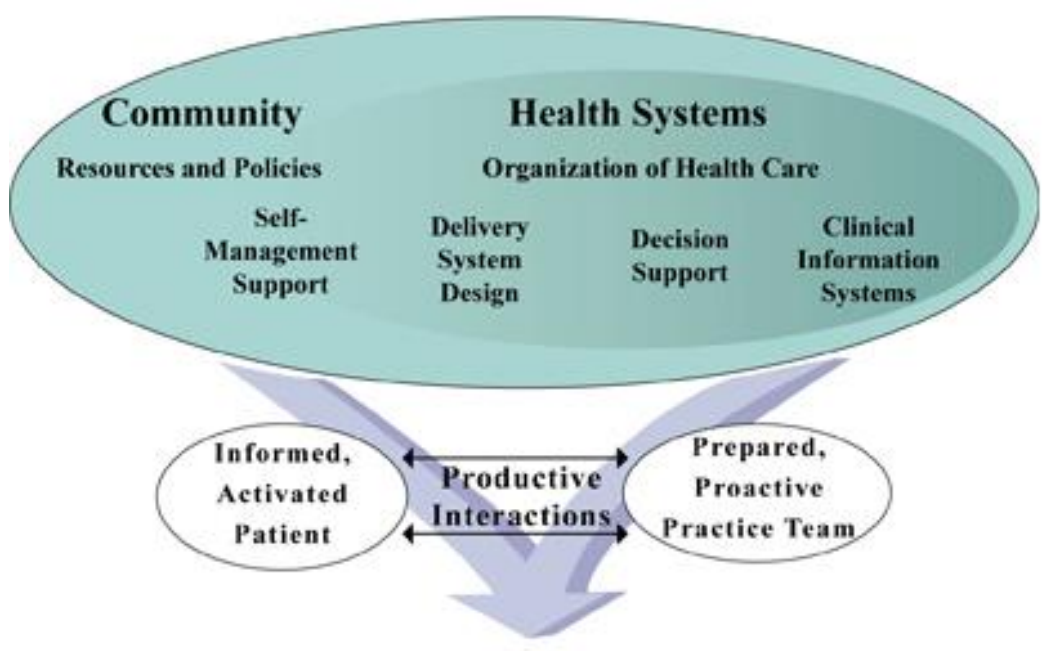

Improved Outcomes

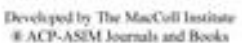

Figure 2-1 Wagner's CCM model

(Source: Wagner, E. H., et al., (1999). Figure 2.)

In describing these productive interactions, the new version of the model clearly articulated a mechanism by which system enhancements would deliver improved outcomes. While it may not have answered all the questions, it does point to the issues noted above: the need to co-ordinate care, the importance of linking with the community, and to deliver care through teams rather than individuals.

Since the CCM model was first formulated, a number of other factors have been added to the original thinking; cultural competence, patient safety, care coordination, case management, and health promotion (Rea et al., 2007). Most developed countries now draw on this model for the development of chronic care policies (Singh, 2005). 
Much has been learned from the work of Wagner and others and we have a very good understanding of the building blocks of an effective programme of chronic care management. However as a review of chronic care clearly states:

"Despite the growing body of clinical knowledge about the care and management of chronic conditions and the range of proven building blocks for reconfiguring the system, there is still no consensus on how to combine these tools into coherent and effective chronic illness care” (Kane et al., 2005, p. 215) [my emphasis].

This view is also shared by Wagner himself. In a review of CCM programmes, he concluded by saying that, "None of the organizations has achieved full implementation of the chronic care model...” (Bodenheimer, Wagner, \& Grumbach, 2002b). A study of nominated 'best practice' programmes found that none had made the system changes necessary to fully implement the model (Wagner et al., 1999). Some aspects were in fact common. For example, 82 per cent had implemented some form of case management. 60 per cent used explicit practice guidelines. Despite the fact that the model explicitly described the importance of close links between the health practices and their local communities, less than half of the nominated programmes had developed community linkages. In New Zealand, attempts to implement best practice are well advanced. Despite this however we remain, as most of the world does, with partially implemented programmes struggling to fulfil the intent of their designers (Connolly et al., 2011).

In many ways this is not surprising, in that the model calls for a major redesign of the health delivery system, internally through process change and externally through a critique of what constitutes the boundary of concern. To be successful each practice not only has to reconsider what they do, but also reconsider what they do it with. The CCM model does not specify how it is to be implemented, and each organisation has to find its own way of translating the framework provided by the model into its own local context (K. Coleman, Austin, Brach, \& Wagner, 2009).

The problem, in part, is that Wagner's CCM model is a conceptual framework only. It provides no specific steps or methods to implement it. Whilst being thoughtfully grounded in the best evidence available, it is still little more that a checklist. 
Any organisation that wants to implement the CCM model must, therefore, figure out what parts are relevant for its particular situation and how they come together as a whole. In discussing one organisation attempting to implement the CCM programme, Hroscikoski noted that:

"As a conceptual framework, the CCM model was useful for thinking about the types of care processes needing to be addressed. It was at best a vague guide to change, however, there were no specifics about the actual care process changes to be made and no description of the change process needed to achieve them" (Hroscikoski et al., 2006, p. 324).

The result is that there are no complete examples of an implemented CCM programme in the literature (Solberg et al., 2006), or in practice (Rea et al., 2007). We simply do not know what are the best and most important changes required in the care delivery system, nor do we know what the most effective change process for implementing programmes for the care of people with chronic conditions is. In addition, in New Zealand at least, we have not been effective in transferring learning from the implementation efforts that have taken place.

"Despite anecdotes of many chronic care management and integrated care projects around New Zealand, there is no formal process to collect and share relevant learning within (but especially between) District Health Boards (DHBs)" (Rea et al., 2007, p. 1).

Furthermore, those that have been implemented in New Zealand only have a tenuous hold, with questions being raised about their clinical and financial viability. As pressure is put on DHBs to 'balance their books', programmes are being forced to justify their investments with returns in terms of reduced use of health services. Whether or not they will be sustainable over the long term is a moot point, despite the fact that they are seen as the major initiative designed to respond to the growth in chronic conditions.

Whether driven by the desire for improved care of people with chronic conditions, or by the desire to control rising healthcare costs, programmes to prevent and manage chronic conditions are seen as an essential part of any future healthcare system.

Knowing how to improve their sustainability is crucial. 
In addition to models of care, such as CCM, there are also models that provide frameworks to ensure the sustainability of such models (Sarriot, Winch, Ryan, Bowie, et al., 2004). Some would say that the debate about sustainability is premature. The focus needs to be on setting them up; getting them successfully implemented and then issues of sustainability can be thought through. However, as pointed out by Sibthorpe et al., (2005) and Sarriot et al., (2004), the lack of understanding about what makes health innovations sustainable, impacts on their ability to be established successfully. Thus research about the successful design and implementation of programmes for the prevention and management of chronic conditions must understand the dynamics of sustainability if we are to avoid short-lived programmes that do little to address the challenges raised in section 2.1 .

There is however a significant gap in the literature here. Trisha Greenhalgh and her colleagues (Greenhalgh et al., 2005), undertook a survey of innovations in health care. This was a major investigation into healthcare innovations, however, as pointed out by Sibthorpe and her colleagues, the authors of that review, “...found so few studies addressing sustainability that they did not include it in their journal article based on the review" (Sibthorpe et al., 2005). The question that Greenhalgh and her colleagues were exploring was how to spread and sustain health service innovations. However, the literature on the sustainability of health service innovations was so sparse that they did not include it in the article that preceded the publication of the full findings (Greenhalgh, Robert, MacFarlane, Bate, \& Kyriakidou, 2004). In the full research they noted that they found only two references to the term 'sustainability', in over 1000 sources. This is in part due to confusion over its meaning and its limited use in the literature. However, it is also because 'sustainability' assumes that the innovation has been implemented and the research about implementation of innovations is also, "complex and relatively sparse" (Greenhalgh et al., 2004, p. 610)

There is, however, a close relationship between the key factors of Wagner's CCM model for chronic care and models of sustainability of primary health care programmes. Essentially Wagner's model has three main components, broken down into six distinct factors. These are the nature of the health service delivered (supporting and encouraging self-management), the characteristics of the organisation that delivers it (the 
design of the delivery system, the decision support tools, the clinical information systems and overall health care organisation), and the community context within which it sits. Sarriot and his colleagues (Sarriot, Winch, Ryan, Bowie, et al., 2004) put forward a framework to assess the sustainability of primary healthcare programmes. This framework has three key dimensions; health and health services, organisation and community. Health and health services are further broken down into approach, quality and outcomes. Organisation is further broken down into capacity and viability and Community is further broken down into competence and capacity, and the local ecological, human, economic, political and policy environment. A graphical representation of their framework is shown in figure 2-2 below:

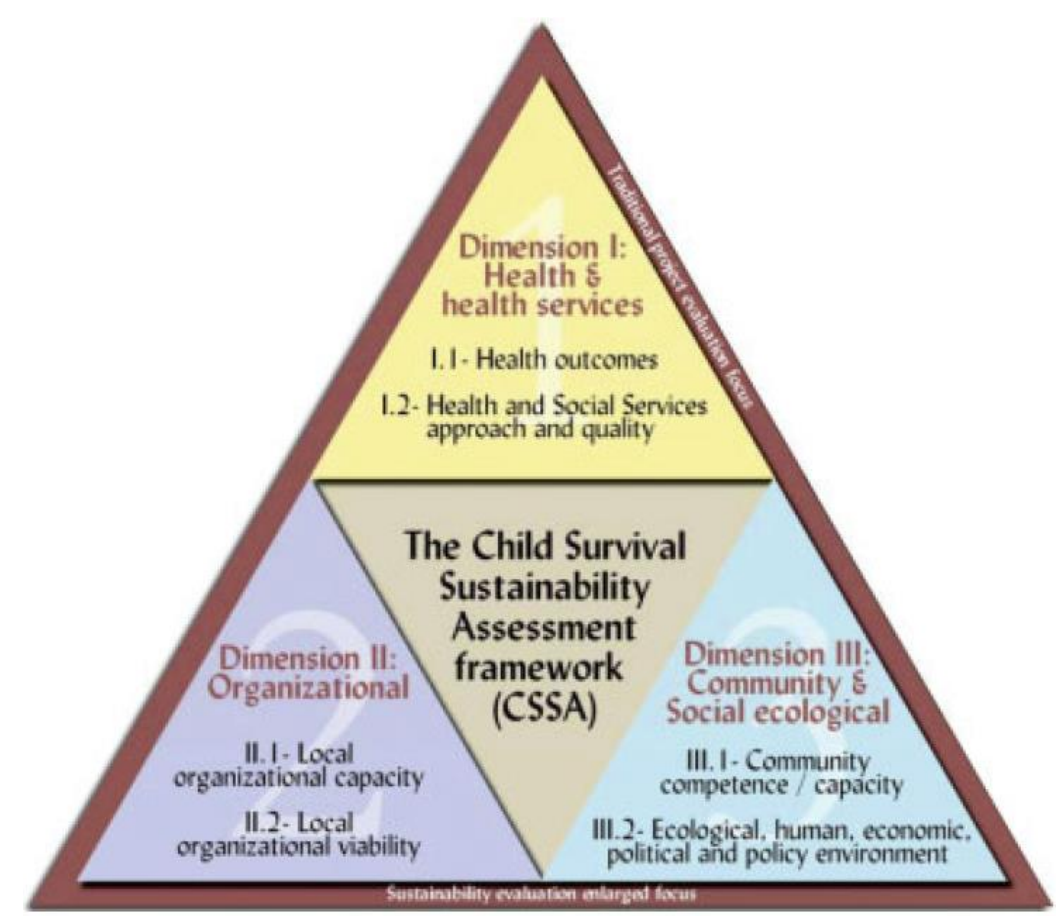

Figure 2-2 Sustainability of Primary Care Programmes

(Source: Sarriot, et al., (2004). Figure 2.)

The differences here are not of substance but of emphasis. Given Wagner's background, as a practicing clinician, his model gives most emphasis to the nature of health service delivery. Sarriot, on the other hand has a strong public health focus, especially on child health programmes in third world counties. It is not surprising therefore that he gives a 
great deal more emphasis to the nature of 'community' and the context within which the programme sits.

Together they provide a framework to explore not just what is required to design and implement effective chronic care programmes but how to design them so that they last.

Specifically, the CCM model describes the factors that need to be included in any effective programme for preventing and managing chronic conditions. Sarriot's work provides a framework to assess whether or not any particular programme is likely to be sustainable in a given context. This assessment framework looks at i) the health services delivered and their outcomes, ii) the organisation of those health services and whether or not they build local capability and iii) the community context within which the programme sits.

In summary therefore, we have a lot of information about what is needed in any programme to improve care for people with chronic conditions and what is required to sustain those programmes over time. That is, we know the pieces of the puzzle, but not yet how to put the pieces together.

\subsection{Limitations of Current Frameworks}

One reason it is so difficult to put the pieces of the puzzle together is that the current models being used to design and implement chronic care programmes are static descriptions of key component parts. In addition they tend to focus on a single condition, such as obesity, or a single intervention, such as a lifestyle or screening programme. There is very little research that explores the reality of chronic disease i.e. interactions between multiple conditions and multiple interventions. For example, in the area of health promotion Ziglio et al., make the point that,

"Despite an apparent widespread acceptance of a socio-ecological model of health amongst people working in health promotion, most health promotion activity has continued to be issue based or else has focused on only one determinant at a time. Rhetoric has largely failed to become reality" (Ziglio, Hagard, \& Griffiths, 2000, p. 145). 
Whilst these 'single condition' studies are important and relevant, those who suffer most in terms of poor health generally have more than one health condition. For example, as well as being obese or overweight they may suffer from a heart condition, have diabetes or may suffer from bouts of depression. It is also possible that they may have poor living conditions in terms of housing, employment, resource access and community support networks. All of these factors interact to create poor health. Furthermore, the interaction between these factors will affect the success of any single intervention.

For example, in one patient group suffering from end-stage renal failure it was found that everyone suffered from at least one other co-morbidity (Australia and New Zealand Dialysis and Transplant Registry, May 2006). Within that patient group, of those receiving renal dialysis in 2004/2005, 84 per cent were suffering from hypertension severe enough to require treatment; 39 per cent suffered from type II diabetes; 36 per cent were suffering from coronary heart disease; 20 per cent were suffering from peripheral vascular disease; 14 per cent had chronic lung disease and 12 per cent suffered from cerebro vascular disease. Calling them renal patients hides the truth of a more complex set of health problems. A review of the literature however reveals that there is very little research that explores these multiple interactions (Vogeli et al., 2007). Where research has taken place, it is noted that those who do have multiple conditions use a wider range of health services, making the co-ordination of care, noted above, even more difficult (Robert Wood Johnson Foundation, 2004). Other research also notes that those with multiple chronic conditions find it harder to engage in selfmanagement, one of the key goals of CCM (Hunt, Townsend, \& Wyke, 2003). Overall what little research there is on multiple co-morbidities tells us that the challenges associated with chronic conditions are increased when multiple conditions are present (Anderson, 2003). As Vogel et al., sum up their findings:

"The small amount we do know suggests that specific chronic conditions combine and impact health costs in unpredictable ways, and that specific combinations have particularly large impacts on health or costs of care" (Vogeli et al., 2007, p. 394).

However, little help can be gained from this research in terms of implementing responses to these challenges, as it tells us little about the interaction of these multiple 
chronic conditions with the broader social, cultural, environmental and economic context within which they sit. One notable exception to the is the 'Syndemics Prevention Network' at the Centres for Disease Control (http://www.cdc.gov/syndemics/index.htm) which has been set up to specifically explore this complexity. Such research is crucial if we are to better understand the implementation issues involved in chronic care programmes.

A key feature of the literature on chronic disease is that the key factors of an effective response have been well identified and common themes are emerging. Evidence is also showing that at least some of the interventions are producing better outcomes (Singh, 2005). It is also recognised that the system has to change to meet the rise in chronic conditions. However when it comes down to designing and implementing specific programmes for specific patient populations, much remains unknown. The world in which such chronic care models as CCM exist is one of multiple interactions. The models themselves however, despite describing the key factors, do not describe in any detail, the nature of the interactions between these factors.

\subsection{Understanding Interactions: Taking a Systems Approach}

As noted above, interventions based on the CCM model have repeatedly been shown to improve care for people with chronic conditions:

"In an effort to reduce mortality and morbidity, programmes of chronic disease management have evolved with the aim of achieving formalised, population-wide implementation of components of the chronic care model developed by Wagner et al. Results of rigorous evaluations of such programmes suggest improved survival and/or disease control with reductions in hospitalizations and adverse clinical events.” (Scott, 2008, p. 427)

Other models have complemented the CCM in a number of ways. The Kaiser Permanente 'Triangle' focuses on integrating services and removing barriers between primary and secondary care (Feachem, Neelam, \& White, 2002). The Evercare model focuses on those patients who are at highest risk of hospitalisation (Singh \& Ham, 2008). The World Health Organisation worked with Edward Wagner to extend the scope of 
the model to take a global perspective and focus more on the role of community and policy in improving chronic care (Epping-Jordan et al., 2004; Nuno, Coleman, Bengoa, \& Sauto, 2012). However, despite its influence, and despite the fact that it acknowledges the systemic nature of the challenge and describes factors in that system, it does not describe the nature of the interactions between those factors or how they may evolve over time. This may, in part, be a consequence of the tendency of researchers to ignore the systemic basis, which informed the initial development of the model. For example, in an influential paper the description of the model is introduced with the question, "What are these 6 pillars of the chronic care edifice" (Bodenheimer, Wagner, et al., 2002a). This metaphor has unfortunately stuck (Connolly et al., 2010) and most research focuses on the individual pillars and not the system that ties them together. And, despite acknowledging the systemic nature of the issues it is trying to address the CCM model falls short of providing insight into that system.

"With rare exceptions the overall system dynamics have not been directly addressed; the focus has been on policy initiatives rather than on the context within which policy is developed, and on countless explanatory variables that do not fit together coherently to allow for explanation rather than description" (McCubbin \& Cohen, 1999, p. 59).

Therefore, despite the fact that the CCM model and others like it, seem to cover the factors that are needed by programmes for managing long-term conditions they are still little more than checklists. While arguing for the importance of the factors in their models they offer little to help someone who wants to implement them.

Each programme focusing on chronic conditions is implemented in a specific context. This context has a population with distinct characteristics, thus requiring different responses from the health provider. It also has a different set of resources. For example, it is clear that in areas of high social deprivation the disease burden is greater and the resources available are usually less. The resources required to follow up patients who are poor, and often transient, are much greater than those who are well off living in stable home conditions. The particular mix of population characteristics and resources has an enormous influence on the design and implementation of programmes for preventing and managing chronic conditions. These contextual factors are not considered in either 
Wagner's or Sarriot's models. However, when one is implementing such programmes it is precisely these factors that need to be considered. How they influence the design and implementation of such programmes has not been considered and is a large gap in our understanding of how to make chronic care programmes successful.

This is a design problem. "Every design problem begins with an effort to achieve fitness between two entities: the form in question and its context" (Alexander, 1964). The real focus then, in a design task, is not just the form alone, in this case the programme for preventing and managing chronic conditions, but the 'whole system', comprising the form and its context. In the example of programmes targeting chronic conditions the key aspects of context are population characteristics and resource mix. The former determines the service requirements of the design, the latter determine the boundaries of feasibility, so that the design process becomes the engineering of locally functional solutions (Joseph, 2004, p. 235). A large urban centre has a much bigger and richer set of resources to draw upon than a small rural centre. While they may both serve similar populations in terms of health needs, it is likely that a successful response will look quite different in the two contexts.

So whilst there is recognition that health is embedded in the broader social, cultural, environmental and economic context, there remains a gap between the conceptual understanding of health as a dynamic phenomenon and the tools that are used to plan and evaluate health policy and health practice, especially in the context of the rising prevalence of chronic conditions. For example, the Ministry of Health's, now disbanded, Healthy Eating Health Action (HEHA) strategy made the point that health is affected by a dynamic interplay of intrapersonal, socio-cultural, policy and physical environment factors (Ministry of Health, 2007). However, despite this recognition, the HEHA strategy did not attempt to provide formal analysis of those links in any of their documents or models. As Homer and Milstein point out,

"Most formal models in the field simply have not been made to adhere to the basic properties of ecological systems [... and as a consequence they are...] unable to capture the causal feedback that makes health problems resistant to change." (Homer \& Milstein, 2004, p. 2). 
The term 'ecological approach' is being used increasingly to describe programmes that look at the patient in their broader social and cultural context. It accepts that the determinants of disease go beyond the individual and are found in the broader environment. The term is often used concurrently with the phrase 'social determinants of disease'. They both refer to the same idea of health being determined by issues beyond the health sector. However, even models that explicitly state that they are taking an ecological approach fail to meet the most basic requirements of an ecological system. Take, for example, a paper describing what the authors called, "A framework for the delivery of public health: an ecological approach” (Nurse \& Edmondson-Jones, 2007). In the paper, the authors describe an ecological perspective as, "the science and relationships between organisms and their environment" ( $p$ 557). They describe the key factors of an ecosystem, being part of a cyclical system; each part influencing the other. They describe their framework as, “...relating the interaction of the multiple components" ( $p$ 557). What they provide, shown in figure 2-3, is a picture of a 'Greek Temple' with the various factors of concern to public health included in its structure and environment.

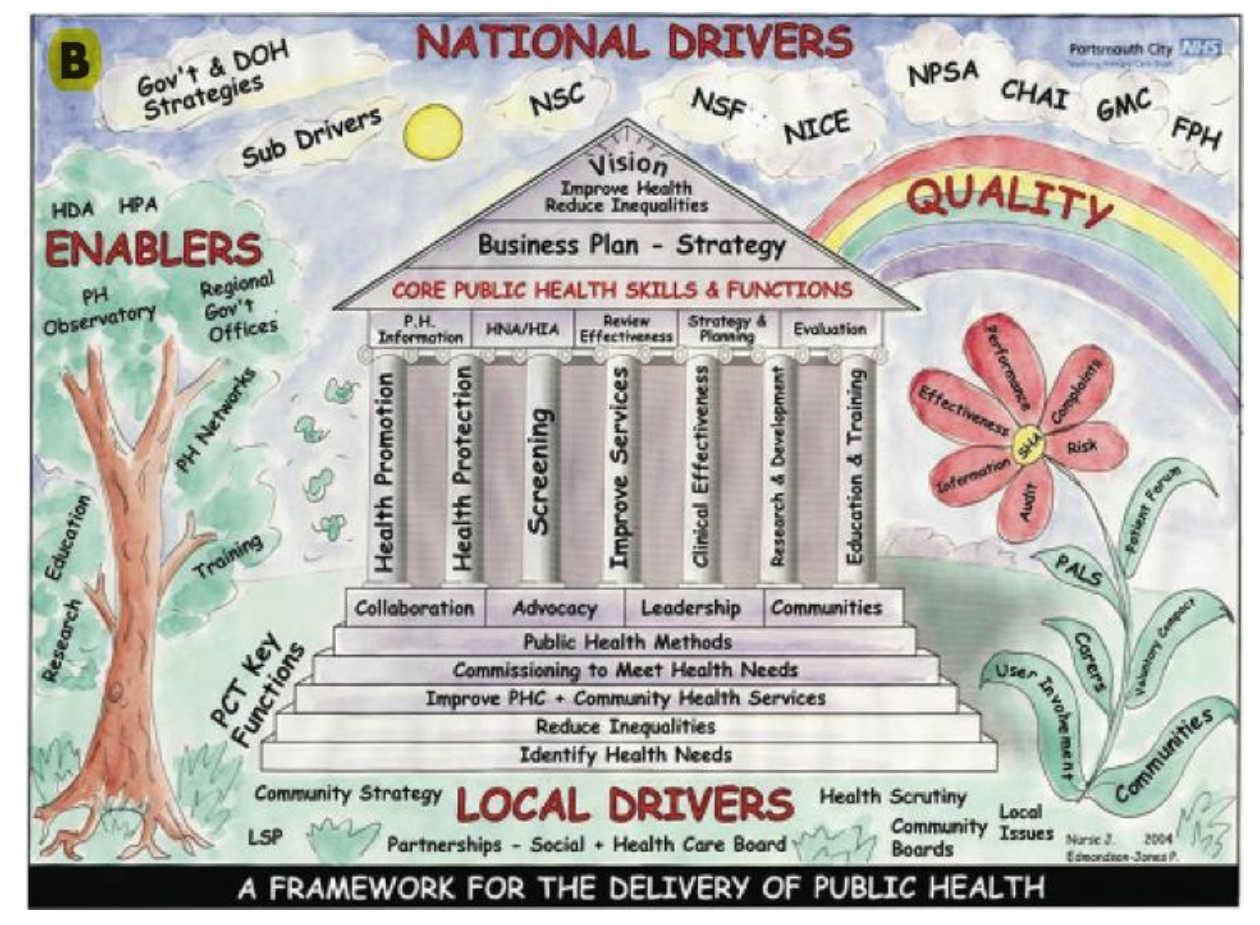

Figure 2-3 Framework for the delivery of public health (Source: Nurse, J., et al., (2007). Figure 2.) 
Whilst such a picture can be a useful aid to remembering important factors there is nothing in the framework that provides any insight into how those factors relate to each other and it certainly does not describe, what they refer to as, the "clear pathways between components". What is the nature of those relationships, what factors impact upon them, and how do they evolve over time? It is a static picture of important 'bits'. It is not a dynamic description of an ecosystem providing insight into how that ecosystem performs and evolves over time. It is typical of the 'models' used within health to inform the planning and implementation of health policy and practice. They use pictures to give the impression of being 'ecological' or 'systemic'. In the end however they are little more that pictorial checklists. Now, checklists are very useful and I am not arguing against their value. However, if one is purporting to portray a system, or to take an ecological approach, because the nature of the problem demands it, then to conform to the most basic requirements of an ecological approach the model must put as much focus on the relationships between the factors as on the factors themselves. Most models do not meet that requirement.

Systems approaches have a potential role to play here. One such example is Peter Checkland's 'rich picture' (shown in figure 2-4) of the information system implications of major reforms in the UK's National Health System as outlined in a White Paper (HMSO. 1997). 'Rich Pictures are one of the tools that make up 'Soft Systems Methodology' and serve to portray the, “...complexity of multiple interacting relationships" (Peter Checkland, 2000, p. S22). 


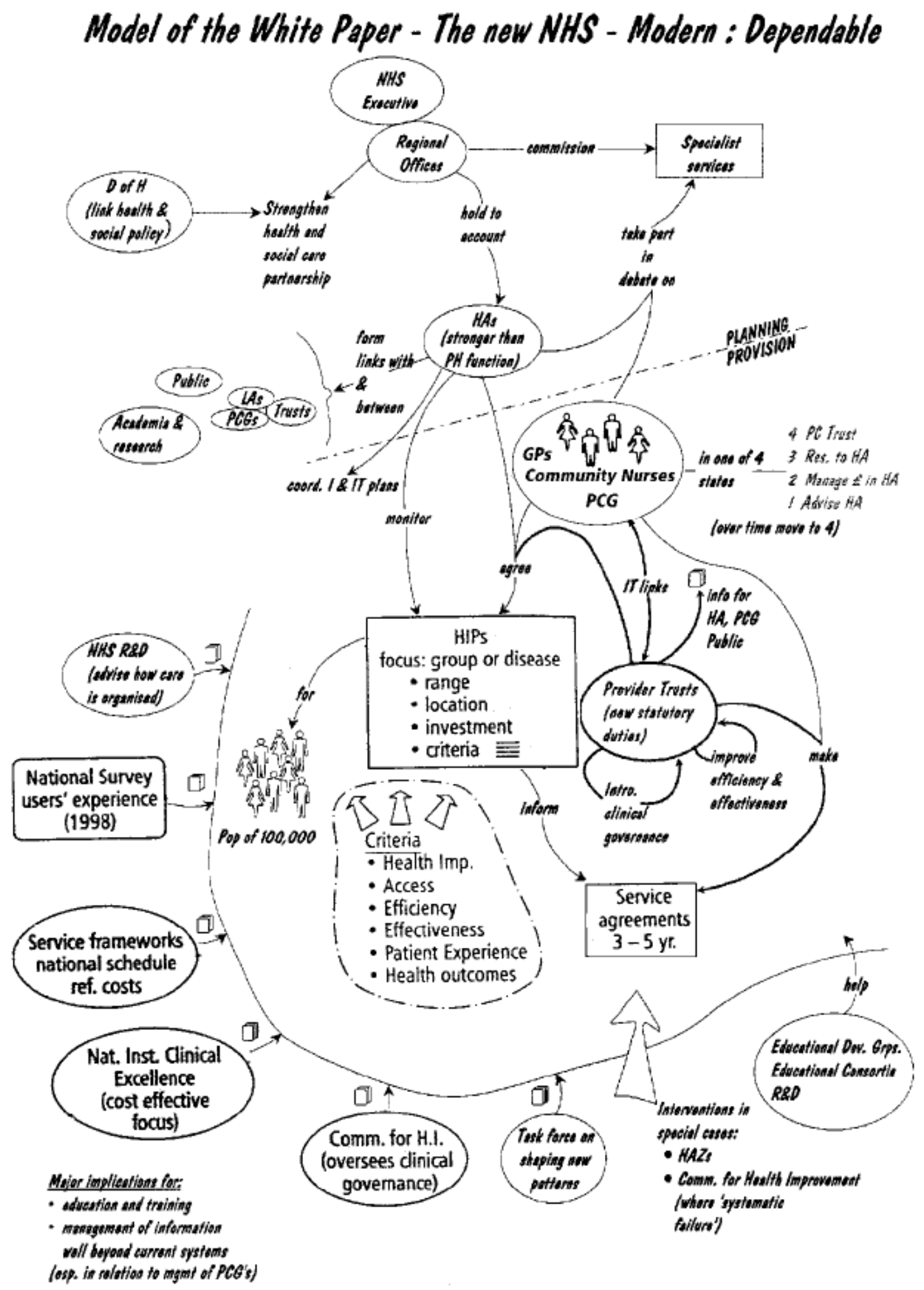

Figure 2-4 Rich picture of NHS white pater

(Taken from: Checkland, P. (2000). Soft Systems Methodology: A Thirty Year

Retrospective. Systems Research and Behavioural Science, 17, S25.) 
While it is still qualitative and visual, in contrast to the picture presented by Nurse and Johnston, Checkland's 'rich picture' puts a great deal more emphasis on the relationships between the factors portrayed. It still has the 'informal' characteristics of a hand-drawn object, but now we are informed that, for example, the 'HIPS' in the central rectangle inform the 'Service Agreements'. Both are important factors in the White Paper and we now have some understanding of how they interact with each other. Checkland's approach helps to tease out the relationships that occur in systems we consider 'complex'. This ability to highlight the relationships that contribute to that characteristic we call complexity is important in understanding care for people with chronic conditions, as pointed out by Rea et al.,:

"It is clear both from experience and from the literature that apparently-small differences in programmes may have a major impact on uptake and effectiveness, even when comparing projects that are all based on best-practice guidelines and designed specifically to enhance patient care. Health systems are recognised as classic examples of 'complex systems'....” (Rea et al., 2007).

Checkland's work, an example of a systemic approach, provides some insight into how the complexity of systems can be portrayed, and stands in contrast to most research and practice that has, instead, focused on telling the story of the key factors, providing little insight into how those factors interact. In this thesis, however, I want to go beyond a qualitative and visual description and, in addition, explore the quantitative nature of the interactions and how they impact each other, especially as they evolve over time. Because of this interest, the core systems approach that will be used in this research is SD (Forrester, 1961), which has an increasing body of literature in health applications.

The SD method has relevance in two key areas. Firstly it provides a process and a set of tools from mapping the causal relationships between the key factors in models such as those proposed by Wagner (1996) and Sarriot (2004). SD helps us unravel the complex web of relationships that exists to uncover those that have the most impact upon the programme. Secondly SD can help us understand the local context that affects health status and the capacity of any community to respond. 
The primary goal of this research is, therefore, to build upon the conceptual models of people like Wagner and Serriot, to create a model of effective and sustainable chronic care programmes that provides insight into the dynamic nature of the system under consideration. A key aspect of this goal is to explore the causal dynamics involved in the interactions between factors known to be important in chronic care and how context affects that causality.

\subsection{Causality and Context}

The above discussion of the CCM model was focused on highlighting the fact that while it provides a consistent and coherent description of the factors that define good care for people with long-term conditions, it does not provide insight into how those core factors interact together, nor how they interact with the context, of which they are a part. However, there is now a significant body of empirical research that supports the validity of the claims being made by the proponents of CCM (K. Coleman et al., 2009; Singh, 2005). Whilst there are debates about which specific factors of the models have the most effect, and whether or not the outcomes are worth the investment needed to bring them about, there is little doubt that effective chronic care management does improve patient outcomes (Dennis et al., 2008; Nolte \& McKee, 2008; Zwar et al., 2006).

However, the causal theories that underpin these descriptions focus on linear relationships between two or more variables. For example, the causal relationship that is argued to exist between 'self-management education' and clinical outcomes: "Evidence from controlled clinical trials suggest that...programs teaching selfmanagement skills are more effective than information-only patient education in improving clinical outcomes" (Bodenheimer, Lorig, Holman, \& Grumbach, 2002, p. 2469). 'Self-management education' and 'clinical outcomes' are two variables, extracted from the complex web of context and relationships, treated as though they were the same regardless of the context within which they occur. Much research, and certainly clinical trials, take this form; $Y=f(X)$. The research focus is on establishing that the values of $Y$ can be determined, given the values of $X$, independently of any other features of the contexts in which both $\mathrm{X}$ and $\mathrm{Y}$ occur. As a result there is considerable research on key factors in the models, such as self-management education. Context, 
where it is acknowledged, is usually treated as 'noise' affecting the relationship between two variables, that are presumed to be causally related, regardless of the context within which they exist (Hohmann \& Shear, 2002).

However, people always act in specific contexts and if the understanding and explanation that arises out of such research is to be translated into action then additional requirements have to be met. These additional requirements can be provided if one adopts a theory of causality based on design (Argyris, 1996). Designs specify the actions to be taken to achieve intended consequences. At the core of such designs therefore is a notion of causality, which can be stated as, "...given such and such conditions if A then B" (Cavana \& Mares, 2004). This concept of causality puts description and explanation at the service of action i.e. the theory requires that the conditions specified in the causal links are specified and the mechanisms by which they are created described. Description and explanation are still central but the theory of causality that now underpins it acknowledges and requires a deeper understanding of context - the conditions that determine the characteristics of 'if A then B'. If those conditions do not exist then the causal relationship between $\mathrm{A}$ and $\mathrm{B}$ may no longer exist. Context is no longer noise but a central part of the causal theory. This is important in developing 'actionable knowledge'; knowledge that informs 'how' rather than just 'what'.

"Knowledge produced by empirical research can have external validity, which means it is relevant to the everyday world. Actionable knowledge is that knowledge required to implement the external validity (relevance) in that world" (Argyris, 1996, p. 390).

$\mathrm{SD}$ is an approach that is well suited to developing actionable knowledge. SD practitioners think in terms of feedback loops and accumulations to account for observed behaviours (Forrester, 1961; Richardson, 1991; Senge, 1990). The notion of causality flowing in one direction, as described, for example by the equation $Y=f(X)$ is replaced by chains of reciprocal, causal relationships among variables, linked together in a structure of 'mutual causality' (Dent, 2003). Thus while these chains of causality can be expressed as $Y=f(X), X=f(Z)$ and $Z=f(Y)$, they often do not run in straight lines but form a web of closed relationships, as shown in figure 2-5. It is this web of relationships 
that become the important unit of analysis; becoming a higher conceptual unit than the variables that make it up.

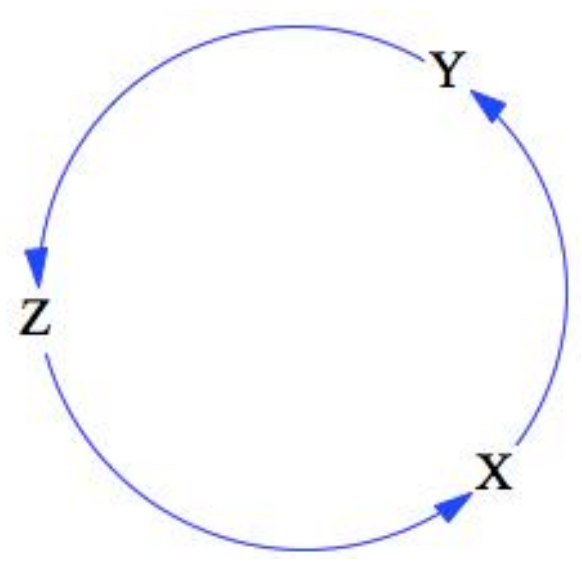

Figure 2-5 Circular causality

When the feedback approach of SD is combined with notions of designing something that does not yet exist, it makes very little sense to say X causes $\mathrm{Y}$, because it only does so in the context of the causal loop, and because it is a loop, $\mathrm{Y}$ also has an effect on $\mathrm{Z}$ which affects $\mathrm{X}$. Take away that web of causality and there is no basis for the causal relationship between $X$ and $Y$. The causal statement that $Y=f(X)$ has no relevance on its own; cause and effect do not exist outside of the context within which they sit. In the feedback loop the proposition 'If $\mathrm{Z}$ then $\mathrm{X}$ ' is only true in the context of $\mathrm{Y}$. Causal propositions, in a design-focused approach are always of the form "In situation S, to achieve consequence, C do A" (Argyris, 1996; Argyris, Putnam, \& McLain Smith, 1985)

Furthermore, within these webs of circular relationships, SD also acknowledges that things accumulate; knowledge increases, hospital beds get filled and people develop chronic conditions. These accumulations are the 'conditions' central to design causality. For example, given 'high levels of trust between a patient and their health provider' (a condition) then if there is a 'self-management education programme' implemented (A) then the 'patient is likely to benefit' (B). If the condition does not exist then the causal relationships may no longer hold. Therefore, from a design orientation, it is important 
to understand the conditions that support, for example, the beneficial relationship between self-management education and clinical outcomes. Understanding what these conditions are and what nurtures them is an important requirement if one is to successfully implement the knowledge obtained from empirical research.

\subsection{Applications to Healthcare}

In this section I look at the application of SD to issues in healthcare. Specifically I want to highlight the increasing range of applications that reflect an increasing interest in the relevance of the approach to providing insight into complex health issues. As shown below in figure 2-6, there has been a considerable growth in the number of papers on SD and healthcare. Using the search query "system dynamics modelling" OR "system dynamics modeling" AND "healthcare" the "hits" on Google Scholar grew from 8 in 2002 to 110 in 2012.

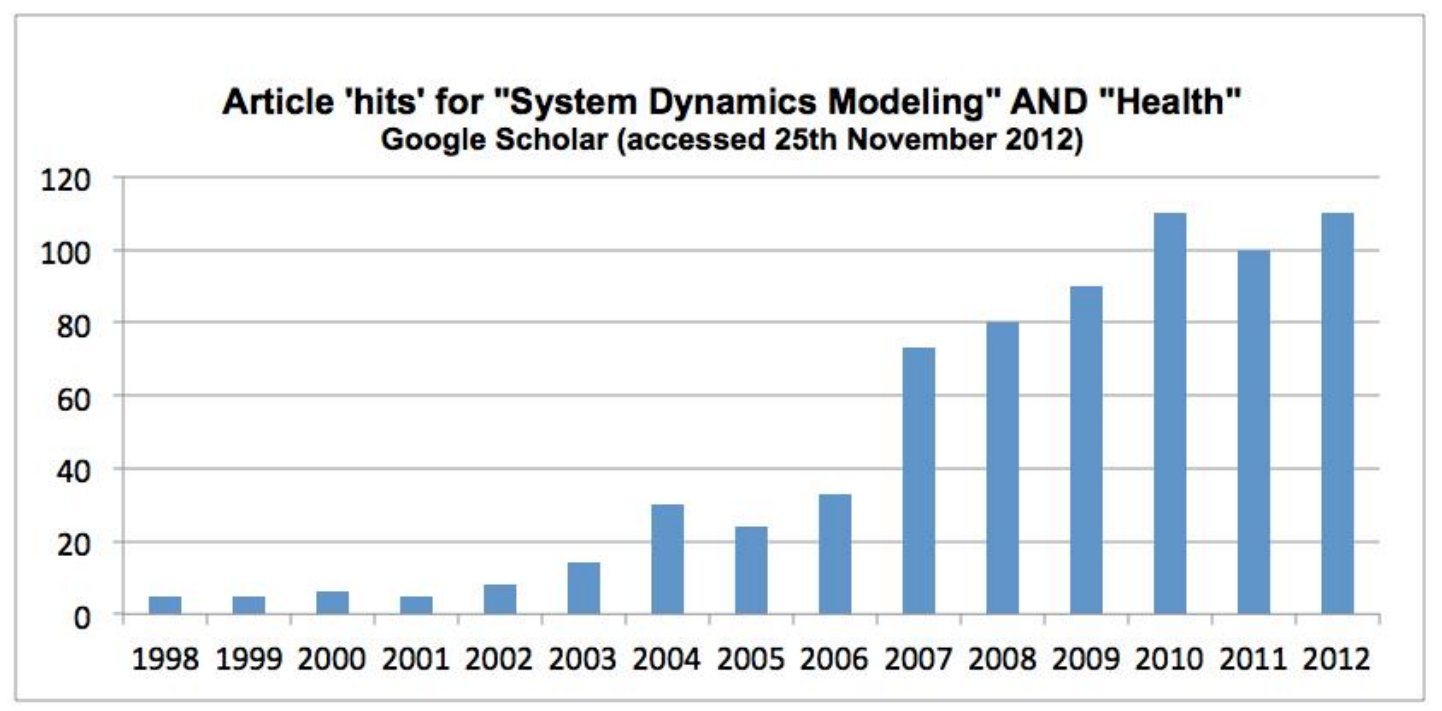

Figure 2-6 'System Dynamics' and 'Health' articles 1998-2012

Furthermore, issues within the health sector have been well represented within the International System Dynamics Society and in their Journal, the 'System Dynamics Review' (SDR). The first article focusing on health issues appeared in 1993 in volume 9 of the SDR (Homer, 1993) and since then have grown in number. Following that first article on the prevalence of cocaine, articles appeared in 1997 on mental health (Huz, 
Anderson, Richardson, \& Boothroyd, 1997) and drugs (Coyle \& Alexander, 1997), and in 1999 on epidemics (Ritchie-Dunham \& Galvan, 1999). In 1999 there was also a special issue dedicated to health issues focusing on waiting lists (Gonzalez-Busto \& Garcia, 1999; van Ackere \& Smith, 1999b), patients flow within hospitals (Wolstenhome, 1999), AIDS, (Dangerfield \& Roberts, 1999) health policy (Royston, Dost, Townshend, \& Turner, 1999), integrated care (Hirsch \& Immediato, 1999) and quality of health services (Cavana, Davies, Robson, \& Wilson, 1999). Since that time health articles have appeared most years in the journal; in 2000 there was an article on antibiotic resistance (Homer et al., 2000), in 2001 AIDS was the focus (Dangerfield, Fang, \& Roberts, 2001), in 2002 there was an article on obesity (Abdel-Hamid, 2002) and in 2004 there was an article on chronic disease (Homer, Hirsch, Minniti, \& Pierson, 2004b) and in 2006 tobacco control (Cavana \& Clifford, 2006). In 2007 articles have appeared on health innovation (Bayer, Barlow, \& Curry, 2007) and chronic care (Homer et al., 2007). In 2008 the Jay Forrester Award, the supreme award in the field, was awarded to Kimberly Thompson for a paper on the global management of poliomyelitis (K. M. Thompson \& Tebbens, 2008). In 2009 the boundaries between health and social policy overlapped in a paper on domestic violence (Hovmand, Ford, Flom, \& Kyriakakis, 2009), and 2010 saw a paper published on addiction to online gaming (Park \& Ahn, 2010). In addition, the Society has an active special interest group focusing on health policy.

In the United States there is a growing body of literature that uses the SD method to explore aspects of chronic disease. These include Hirsch and Homer (2004b), Homer, Hirsch, Minniti and Pierson (2004a), Homer and Jones et al., (2005) and Homer and Milstein (2008).

Hirsch and Homer's (2004b) paper focused on the additional workload that new chronic care programmes imposed upon providers and describes two models that look at the interplay between chronic care programmes and the capacity of the delivery system. The paper by Homer, et al., (2004a) describes the use of SD modelling to support the planning of a programme for diabetes and heart disease, while the paper by Homer and Jones et al., (2005) focuses more on aspects of policy, specifically in terms of evaluating intervention strategies. 
In addition, the same group of researchers have explored the dynamics of broader system change within the context of chronic conditions. For example, see Homer and Hirsch et al., (2007). In this paper the authors build on their previous work to develop a generic model of chronic illness in the U.S. population. The model is used to help explain the pattern of disease and interventions as well as demonstrating the impact of moving 'upstream' by addressing known risk factors to prevent the onset of the illness.

In the United Kingdom, there is also a growing body of research and practice based on the application of SD to complex health issues. Some have focused on the overall benefit of the approach for tackling health issues (Wolstenhome, McKelvie, Monk, Todd, \& Brady, 2008). Others have focused on specific conditions such as mental health (Smith, Wolstenhome, \& Repper, 2005) and AIDS (Dangerfield et al., 2001). The paper by Smith et al., (2005) describes a case involving the application of SD modelling in a Mental Health Trust and the challenges of applying what is learnt from the modelling process in practice. Ann van Ackere's work focused on hospital waiting lists for elective surgery (van Ackere \& Smith, 1999a) and David Lane's work focused on patient flow through emergency departments (Lane, Monefeldt, \& Rosenhead, 2000) while Kathryn Taylor and colleagues focused on shifting care to community settings ( $K$. Taylor et al., 2005), highlighting that the desire to improve the provision of services by making them closer to home can be undermined by the increased demand that has been stimulated by the shift.

These last two papers highlight a phenomena that is often identified in SD modelling efforts; what Forrester (1975b) calls the counterintuitive nature of social systems, the tendency of systems to produce results that were unexpected and sometimes, 'counterintuitive'. One of the reasons for this, described by Forrester (1975b) is the conflict that arises between the short-term and long-term consequences of interventions. The future is rarely a simple extrapolation of the present.

In New Zealand, the conflict between short and long-term perspectives was the focus of papers describing the design of chronic care programmes in South Auckland (Rees \& Orr-Walker, 2006), the pattern of renal disease (Rees, Naden, \& Field, 2008), and cardiovascular disease (Kenealy et al., 2012). Each of these papers reflects work that focused on trying to help managers, policy makers and clinicians understand the 
differing consequences of short and long-term perspectives. Work by Cavana and colleagues (Cavana \& Clifford, 2006; Cavana \& Tobias, 2009; Tobias, Cavana, \& Bloomfield, 2010) focused on policy options for tobacco control.

As well as these researchers specifically using SD modelling to support their work in health, there are also people outside of SD who are either using, or calling for the use of systemic approaches to understand complex health issues. David Kernick highlights the complex nature of health and calls for approaches that see health as a complex adaptive system (Kernick, 2002). McCubbin in his work on mental health systems states that; "Problems are acknowledged to be system-wide, yet few writers have used a method of analysis appropriate for systemic problems." (McCubbin \& Cohen, 1999). Bobby Milstein has developed an approach that explores the dynamics between social and health policies in the context of the multiple morbidities that often afflict patients suffering from chronic conditions (Milstein, 2008). Mooy has explored the use of computer models to support health impact assessments (Mooy \& Gunning-Schepers, 2001). In New Zealand, Rea talks of health as being a classic example of a 'complex system' (Rea et al., 2007).

One body of work that is very relevant to this research is the SD modelling work undertaken through the auspices of the Centers for Disease Control and Prevention. This work has included a number of projects focusing on specific disease conditions such as diabetes (Milstein et al., 2007), obesity (Homer, Milstein, Dietz, Buchner, \& Majestic, 2006) and cardiovascular disease (Homer et al., 2008) as well as projects focusing on the dynamics of health system change (Hirsch, Homer, McDonnell, \& Milstein, 2005). The models developed in this extensive body of work go beyond the static models described earlier and use SD modelling techniques to explore the consequences, over time, of the many and varied connections involved in the CCM model. For example, the work of Gary Hirsch and Jack Homer has shown the impact of capacity constraints on the successful implementation of programmes for the care of people with chronic conditions (Hirsch \& Homer, 2004a). By the use of SD modelling techniques, they directly addressed one aspect of the context discussed above. Specifically, they showed that implementing a chronic care programme without understanding its impact upon the chronic care nurses, who are central to the 
programme's success, can limit the programme's impact and, in severe cases, threaten the programme's viability. Chronic care nurses are a crucial resource in helping patients with chronic conditions develop a degree of control over their illness. If this resource is not developed and supported, those nurses involved in the programme become overwhelmed. Furthermore, if the resource is inadequate a referral backlog of patients develops. These patients continue to add to the overall demand and can swamp the system spilling over and affecting patients' commitment to self-control, thus further adding to patient load while decreasing positive outcomes. It is a good example of research highlighting specific aspects of context that can affect the implementation success of any programme.

\subsection{Summary}

The challenges posed by the rise in chronic conditions requires better co-ordination between different parts of the healthcare system, shifting more services into the community and away from hospitals and more reliance on transdisciplinary teams rather than individual clinicians. While models such as Wagner's CCM model (Wagner et al., 1996) and Sarriot's model for assessing the sustainability of primary healthcare programmes (Sarriot, Winch, Ryan, Bowie, et al., 2004) describe the key factors required for an effective response, they provide little insight into how to implement them. In this chapter I have tried to show that while we have a great deal of knowledge about the key factors required for an effective response to the increasing burden of chronic disease, the models that describe these factors fall short in some key areas. Specifically they fail to illustrate the fact that these factors interact with each other over time and that the nature and impact of those interactions are highly context dependent.

The challenge of implementation is a design challenge, one that seeks to achieve fitness between two entities: the programme and its context (Alexander, 1964). I have argued that in order to explore this context-dependent world of multiple interactions it is important to have tools that enable one to explore and describe complex patterns of causality. As much of the literature points out, there are many factors that can affect the success or otherwise of any given programme, and if research on implementation is to go beyond the exploration of single factors, or even discrete lists of factors, new tools 
will be required. While there are a number of systems approaches, this research uses SD as it provides both a process and a set of tools to explore causal interactions between multiple factors within given contexts. Furthermore, it is an approach that has been applied to a number of complex health problems.

In the next chapter I will describe a research design that aims to explore the dynamics involved in implementing chronic care programmes in a manner that overcome some of the limitations described above. 


\section{Methodology \\ Designing Research to Unravel Complex Systems}

As described in chapter 2, the successful implementation of chronic care programmes involves a complex interplay between the community, the healthcare system, the provider organisation and the patient and their families. (Bodenheimer, Wagner, et al., 2002a). Models, such as CCM, emphasize the importance of key factors but the underlying causal mechanisms that enable them to work effectively together are left unstated. We learn, in the CCM model for example, that linkages between the provider organisation and community resources are crucial, but we do not learn about the causal mechanisms that develop and sustain those linkages. We learn that, "If an organization's goals and leaders do not view chronic care as a priority, innovation will not take place", but we do not learn about the key causal factors that prioritise chronic care in the organisation's goals and leadership (Bodenheimer, Wagner, et al., 2002a).

If we are to become more effective in implementing new health innovations for chronic conditions, we must become more knowledgeable about the causal mechanisms at play. As Anjali Sastry (1997) puts it, when discussing theories about organisational change, "...too often, the casual structures of the theories are not fully specified and that theoretical frameworks and empirical results are not well integrated"(Sastry, 1997, p. 237), and because, “... action is central to theories of organisational change, a causal modelling approach suitable for capturing dynamics is needed"(Sastry, 1997, p. 237).

This chapter describes the 'causal modelling approach' used in this thesis. It begins by describing the epistemological framework that informs the approach. It then summarises the research challenges presented by health innovations such as CCM and describes how these challenges are addressed in the innovation literature. Following that, the chapter describes simulation modelling, why it has a place within research into health innovations and why, despite a growing interest in it as a research method, its use in management and organisational research still lags behind its use in other social and physical sciences. The chapter finishes with a detailed description of the specific 
research method used in this thesis, which is built around systems thinking and simulation modelling and aims to address the challenges outlined.

\subsection{What Informs the Approach to This Research}

This research is informed by two major 'worldviews'. The first is constructivism; the second is system science. These, provide the framework of ideas that inform the research, and determine what constitutes 'knowledge' about the situations being researched. The reason for trying to makes this framework of ideas explicit is that, "such a declared framework...allows those interested in the research and its outcomes to recover the process by which the results were obtained. Hence they can see how these arose and decide how believable they are" (P Checkland \& Holwell, 1998).

\subsubsection{Constructivism}

The first worldview that influences this research is the belief that human beings continually construct and re-construct, through dialogue and action, the world in which they live (Watzlawick, 1984). This perspective leads to an 'interpretive' approach to research, that begins from the assumption that people experience the same physical and social world in different ways (Cavana, Delahaye, \& Sekaran, 2001, p. 91). If one begins from this perspective, that people are continually constructing their understanding of the worlds within which they live, then research becomes an '...organised discovery of how human agents make sense of their perceived worlds, and how those perceptions change over time...." (P Checkland \& Holwell, 1998, p. 22).

Interpretive research involves, therefore, working alongside those people who are the subjects of the research, and doing so in their context. This is required as the, “...generation of meaning is always social, arising in and out of interaction with a human community" (Creswell, 2009, p. 9).

However, it is important to realise that those involved in the research do not necessarily see that they are constructing their own world; for them, the world may be an 'objective reality' rather than an 'invented' one. 
"In other words, what is supposedly found is an invention whose inventor is unaware of his act of invention, who considers that it is something that exists independently of him; the invention then becomes the basis of his world view and actions" (Watzlawick, 1984, p. 10).

Research that operates from this perspective has therefore, to not only 'interpret' meaning in a way that is explicit and falsifiable, but also help participants see that their meanings are constructed and unique to them. Only then can they be open to collaborative critique and the possibility of other meanings being considered. This is crucial if the participants in this research are to become involved in creating new meanings that go beyond their own individual perspectives and see the outputs that emerge as valid and useable.

The scientific legitimacy for this interpretive approach is often attributed to Kurt Lewin. Working in the decades before, during and after the Second World War, Lewin was concerned with the interplay between science, democracy and education (Argyris et al., 1985, pp. 7-8). He was also concerned, from a research point of view, to point out that it was possible to research, with scientific rigour, much more than simply the directly observable world. While this may be taken for granted today, in the 1930s and 1940s this was not the case. Reviewing an earlier study looking at group processes, which were a major area of interest to him, he reported in 1943 that:

"Observing the interrelation of a group of individuals, it was possible to collect reliable data about such items as who moved his arm, turned his head, or moved from one place to another. However, no reliable data could be obtained about friendliness or unfriendliness or many other social characteristics of behaviour. The study seemed to lead to the unfortunate conclusions that what can be observed reliably is socially meaningless and what is socially meaningful cannot be observed reliably" (Lewin, 1997, p. 279).

Lewin devoted much of his research to prove this conclusion wrong, and in doing so became seen the 'father' of social psychology and of action research (Argyris et al., 1985). 


\subsubsection{Systems Science}

The other reason for referring to Lewin is that he was also very influential in advocating an approach to social research that is consistent with the second major perspective that influences this research; systems science.

In a paper, looking at the impact of education on the national psychology of the United States and Germany, first published in 1936, Lewin made the following points:

"One has to face the educational situation with all its social and cultural implications as one concrete dynamic whole. One will have to understand the dynamic interrelations between the various parts and properties of the situation in which, and as part of which, the child is living....If one wishes to understand the interrelation between the parts and properties of a situation" (Lewin, 1997, p. 24).

Here, Lewin succinctly describes the key elements of a systems approach that is concerned with trying to understand and intervene in the world; an understanding of interrelationships and an understanding of those interrelationships in a specific context. As an aside, it is interesting to note that the GP in New Zealand's health system is often referred to as a 'gatekeeper' in that he/she often controls access to secondary services. This concept was developed by Lewin as part of his Field Theory in Social Science, to describe a key role that affects things, "Entering or not entering a channel and moving from one section of a channel to another" (Lewin, 1997). Policy makers concerned with modifying and improving the gatekeeping role of GPs could do far worse than to read Lewin's work. Despite being written over 50 years ago it still has much to offer.

The systems sciences are a loosely affiliated group of disciplines that are held together by a worldview that emphasises the interconnected nature of the world and the importance of understanding the interplay between sets of connected systems and the contexts within which they exist. Some of the known disciplines within this field are general systems theory (Bertalanffy, 1969), soft systems methodology (Peter Checkland, 1993), cybernetics (Beer, 1994), system dynamics (Forrester, 1961) and complexity theory (Holland, 1995). 
Despite their differences in emphasis, and methods used, the different 'schools' within the field of systems science all attempt to develop understanding through analysis of the interactions between elements within a system. These interactions have their own characteristics, which can be analysed, and which can provide understanding of how systems develop and change over time. A significant consequence of this perspective is that it forces researchers to look inside the system for points of influence, rather than at external factors, that is, a systems perspective looks for an 'endogenous' explanation.

“'The word 'endogenous' means 'arising from within.' An endogenous theory generates the dynamics of a system through the interaction of the variables and agents represented in the model.” (Sterman, 2000, p 95).

In contrast, an 'exogenous' (arising from without) explanation looks at variables outside the system of concern. For example, in the context of chronic conditions, it is acknowledged that an ageing population presents many problems as, on average, older people place greater demands upon the health system. A number of research projects have been undertaken to understand the impact of this ageing population For example, see Garrett and Martini (2007). Garrett and Martini's study tried to estimate the impact of the ageing population on medical costs in the United States. They concluded that, despite limitations in their model, they have provided a starting point for more precise estimates of the impact of ageing on costs (Garrett \& Martini, 2007, p. 59). What is ignored in all of that work however, is the possible response of the system to these changing demands. The paper limits its interest to the exogenous variables associated with an ageing population.

In contrast, a systemic perspective focuses on the endogenous variables, to understand how the system responds to this growth in the ageing population, and how those responses affect the extent of its impact. That is, how much of the impact is embedded in the characteristics of the ageing population, and how much is embedded in the design and response of the systems that are affected by it?

For those who take a systemic perspective, when looking at chronic conditions, the ageing population is an exogenous variable. However, the rise in costs and the growth in residential care, for example, are a result not just of an ageing population, but also of the 
way we have designed the health system to respond to it. From a systemic perspective, the responses to the ageing population are endogenous to the system and therefore able to be influenced by it. For example, the choices we have made as a society to undervalue the work of family members, who care for the older members of their family, has contributed to a major growth in residential care. In other countries different responses have occurred with different results. The systems perspective argues that it is in the design of our response that we should look for causes of increased demand on health services, not simply the fact that people are living longer.

Our ability to 'see systems', and our role in designing them, are therefore, crucial determinants of how the world evolves, and it is this dual focus on 'constructivism' and 'systems', that provides the lens through which the research is framed, and by which the methods are chosen.

\subsection{Implementation Research}

As described in chapter 2 , the rise of chronic conditions is presenting many challenges for the health sector. No longer can health professionals simply focus on the physical condition of the person they are seeing. While research on the success of CCM programmes, “...is based on discrete and successful interventions related to specific chronic diseases, ... its purview is in fact far reaching into multiple dimensions beyond disease" (Martin \& Sturmberg, 2008, p573). As a consequence, regardless of the diagnosis, treatment for those with chronic conditions requires input and support from the clinicians' colleagues, the patients' families and broader support networks, as well as the patients themselves (Dubois, Singh and Jiwani, 2008). Furthermore, if the patient's conditions are advanced and/or complex they are likely to be seeing a range of health professionals, whose knowledge of each other's involvement may be marginal, and in some cases non-existent. In short, health provision no longer revolves around the exclusive relationship between a clinician and their patient. Instead there is a network of multiple relationships, which interact to help and sometimes hinder the provision of optimal care. Thus, the system of health care has changed, and many players within it have different understandings of the relationships and their meanings. 
In this context, the world of the health professional is far more complex and best practice, as exemplified by health care models such as CCM, requires significant changes on the part of individual providers and their organisations. This has led to many attempts to rethink our approach to healthcare provision and CCM is just one example of an innovative health practice that is driven by evidence, focused on improving care, and cognizant of the challenges that the shift in focus away from 'cure' to 'care' creates for health providers. CCM has been the subject of detailed research, with the result that most of the factors associated with improved and impaired performance are well known (Singh \& Ham, 2008). However, while the factors may be well documented, understanding the causal mechanisms by which they work, in practice, is still largely untouched. As pointed out by Tricia Greenhalgh and her colleagues, implementation is the least understood area in the innovation literature and "...empirical studies on implementing and maintaining innovations in service organisations had been undertaken from a pragmatic rather than an academic perspective... [and]... implicitly or explicitly assumed simple causal relationships between variables..." (Greenhalgh et al., 2005, p. 227) As a consequence, whilst models of care such as the CCM model are clear about many of the changes required in the individual and organisational attributes, little research has been undertaken on the implementation of such programmes and little is known about how to successfully implement those changes.

Chapter 2 highlighted the fact that programmes for the care of people with chronic conditions are complex, often requiring significant changes to clinical behaviours as well as organisational structures and processes. This is because chronic conditions are generally not curable in the sense that a medical intervention can make the condition 'go away'. Instead, the curative model, when applied to chronic conditions, delivers benefits that, at best, "... buy a little time, and which are easily nullified by external countervailing factors" (Porter, 1997). Furthermore, while the major advances in healthcare have meant that infectious diseases have been significantly reduced, chronic conditions have risen to take their place, and the extra years of life that have been granted by the successes in defeating infectious disease have left a hollow victory, in which our longer lives have translated into “...more time to be ill.” (Porter, 1997). 
Research within this context is problematic. While it may be possible to distil out individual processes the challenge is, given the nature of chronic conditions, to understand how the interactions between the many different processes, and people, evolve over time. This is the domain of innovation implementation; how individuals and organisations implement new practice within their organisations. The problem is that the literature on innovation implementation is scarce. Innovation literature focuses instead on adoption and diffusion, leaving the complex world of implementation largely unexplored. As one writer put it "innovation implementation is often treated like a black box" (Dewett et al., 2007). This is reflected in the gap between what we know about effective care and what is actually delivered to patients (Proctor et al., 2009).

Throughout the innovation literature, issues of implementation are often referred to but rarely investigated. It should, however, be of interest to researchers given that the benefits of an innovation only accrue after they have been implemented, and research indicates that the success of implementing innovations is very low (Damschroder et al., 2009). For example, within the health sector, research on the implementation of handhygiene practices (crucial to reducing infection rates in hospitals) compliance to agreed protocols was consistently below 50 per cent (Achterberg, Schoonhoven, \& Grol, 2008). Furthermore, The Lancet, a leading medical journal, reported studies that suggest between 30-40 per cent of patients do not receive treatment that accords with best practice and, even worse, around 20-30 per cent receive care that is not needed or is potentially harmful (Grol \& Grimshaw, 2003). In the United States one study found that only 55 per cent of patients received recommended care (McGlynn et al., 2003). And, as discussed in chapter 2 the success in implementing chronic care programmes is highly variable.

The rest of this chapter explores the challenges of conducting research within this context. Firstly, it highlights the fact that the world of practice is a world of multiple, interacting variables that change over time. Secondly, it explores the difficulty that traditional research paradigms have in conducting research under these conditions. Thirdly, it explores the issues raised by the fact that implementation is a design challenge, and that research into this challenge cannot be satisfied by simply understanding the world as it is, but must be able to explore that world as it could be, and as practitioners 
are trying to make it be. Finally, it describes an alternative approach to research combining systems thinking simulation approaches - that is well suited to this context.

\subsection{The World of Research and the World of Practice}

As described in chapter 2, chronic care programmes require major redesign in the way care is delivered and major shifts in the relationships between care providers and their patients. This is a very complex environment in which to undertake research and hints as to why so little research has been done in this area can be seen in some of the comments of the innovations researchers themselves. For example, Todd Dewett, describing the influential work of Teresa Amabile on organisational innovation, points out that when it came to discussing implementation all that she could say was, that "it is inevitable that other factors within the organization become involved at this point" (Dewett et al., 2007). Amabile goes no further though; these 'other factors' are not described and the issues of implementing innovations remain a mystery. In other cases some of these factors are at least noted. For example, Klein and Knight (2005) identify infrastructure as a key aspect of implementation, but the 'body of variables' that make up this construct are not investigated, making it very difficult for a practitioner wanting to understand how to adjust infrastructure to make it supportive of implementation, or a researcher to explore what aspects of infrastructure help or hinder the innovation of any new practice.

One paper aiming to address this gap (Damschroder et al., 2009), develops a 'Consolidated Framework for Implementation Research' (CFIR) that provides an overarching typology to promote implementation theory about what works across multiple contexts. However, the focus of this work is, like most other research in the implementation field, on identifying key factors. Their CFIR has five major 'domains' within which are 37 'constructs'. While providing a comprehensive typology of important factors, it does not address one of the major concerns being raised here; namely the interactions between these factors. While describing the factors that are believed to influence the success of implementation programmes it, “...does not specify the interactions between those constructs". (Damschroder et al., 2009, p. 3) This is an odd omission given that they start the paper with the assertion that; 
"Many interventions found to be effective in health services research studies fail to translate into meaningful patient care outcomes across multiple contexts."

(Damschroder et al., 2009, p. 1)

How one can meaningfully explore how implementation programmes work across multiple contexts without exploring the interactions between key constructs is unclear. This paper is typical of implementation research, working within a paradigm that focuses on generating a list of factors (independent variables) that are considered to have the biggest impact upon the issues of concern (the dependent variable). Furthermore, these independent variables are considered to have some causal connection to the dependent variable. The nature of those causal relationships is not discussed however. In fact the word 'cause' does not appear in the paper.

Furthermore, as soon as you look at implementation as involving multiple interactions you bring in the idea of time, and because of that Klein and Knight (2005) argue that 'ideal' studies should look at implementation 'over time'. This is echoed by Dewett et al., who note that their own model of implementation is a 'snapshot' and the "...role of time must be more fully examined" (Dewett et al., 2007, p. 21).

The world of implementation requires an understanding of the causal mechanisms at play and there is very little research that incorporates well-specified causal mechanisms. Instead, the focus is on the development of 'factor theories' (Downs \& Mohr, 1976), that try to identify the important 'independent variables' that are believed to increase or decrease the successful implementation of an innovation (Dewett et al., 2007; Klein \& Knight, 2005).

So, we are left with a view that extensive system redesign and social engineering is required but, to-date, research provides us with little more than a list of factors that are important. How those factors interact and evolve over time is still largely sitting inside Dewett's 'black box'.

What we have then is a body of literature that often points to the importance of implementation but says little about the causal mechanisms that influence it. Even where the research acknowledges the complexity of multiple interacting variables it 
tends to assume, “... simple relationships between variables... and has failed to take due account of contingent and contextual issues" (Greenhalgh et al., 2005, p. 227).

Furthermore, in quoting the work of L. Potvin, Greenhalgh states that,

"In an important theoretical article, Potvin argues that because of the highly complex nature and relentlessly contextual nature of dissemination programmes they should be treated as a 'special case' in research." (Greenhalgh et al., 2005, p. 228)

So what type of research would help address these limitations? The next section describes qualitative and quantitative research as the main paradigms used to describe and design research approaches in this field. It then argues for a 'design research' approach, in which simulation plays a major role.

\subsection{Implementation as a Design Challenge}

Implementation is concerned with changing the current state. It is about creating something and in that sense it is a design task. As a consequence research into implementation is concerned with more than just understanding the world as it currently exists and moves from the realm of both the sciences and the humanities and enters the world of design. In Herbert Simon's terminology design is the 'science of the artificial'. "Everyone designs who devises courses of action aimed at changing existing situations into preferred ones." (Simon, 1996, p. 111). The objects under study are artificial in the sense that they are the result of human endeavour, 'moulded by goals or purposes' and rather than conforming to universal laws are highly contingent upon their environment. Design then is a distinct approach to research that integrates different theoretical domains into a specific domain of practice and application with the purpose of creating something that does not yet exist. Research into design is therefore research about the processes that bring about that which does not yet exist; not just the world 'as is' but the world as it 'could be'. It also conforms to the pragmatist worldview being concerned more with what works than with what is true, but is not simply an 'application', as it is concerned with developing theories in the form of 'design propositions' (Akkermans \& 
Romme, 2003) that can inform 'experiments' beyond the specific case example and be tested.

The design approach is not new to management and it was once at the centre of management research. Frederick Taylor (F. W. Taylor, 1911) was an engineer whose designs for manufacturing processes, initially only published in engineering journals, became known as scientific management (Barley \& Kunda, 1992). Scientific management has had a major impact upon management theory and practice, focusing on improving managerial control and co-ordination, especially in relation to development of systems for cost accounting, production control and wage payment plans (Romme, 2003). As described by Romme (Romme, 2003) design methods were also central to the work of eminent management theorists such as Emery and Trist (Emery \& Trist, 1972) in their work on sociotechnical systems and Jaques (Jaques, 1962) in his work on human relations. More recently, Chris Argyris and Donald Schön have applied design methodology in an extensive body of work on organisational learning (Argyris, 2004; Argyris \& Schon, 1974).

Design is an integrative discipline that pulls its theory and its practice from a number of domains. Technology and engineering are the domains of practice for product designers who pull their theories from the natural sciences. The artist, sitting firmly within the creative and applied arts, is informed in their use of paints, inks and other materials by the natural sciences and in the compositions they create by the humanities and liberal arts. Those concerned with designing organisations to fulfil particular purposes sit within the human professions and services drawing their knowledge heavily from the social and behavioural sciences.

Design research also has a particular view of causality. The most dominant form of causality in contemporary research sits within the paradigm of 'factor theories' discussed earlier, focusing on understanding the most important factors regardless of the local context. 'Design causality' (Argyris, 1996) however is context dependent. Furthermore, the design perspective sees human beings as designers, taking action to change existing situations into preferred ones (Simon, 1996), and interventions as 'human experiments' that have the goal of creating new worlds (Argyris, 1993). 
So, if design is about creating that which does not yet exist, and the patterns of causality within those designs are highly context dependent, then tools that enable one to work with 'virtual worlds', with different patterns of causality, within different contexts, maybe of value. Simulation is one of these tools.

\subsection{Simulation as a Research Method}

The world of implementation is then a world of interactions between multiple variables over time, that transcend any specific discipline and which have a concern with evolution over time. Furthermore it is a world of design, in which people are trying to create something new; concerned not just with 'what is', but also with what 'could be. It is these very characteristics of the complex world of implementation that make simulation a valuable research method. As Harrison and Carroll (Harrison \& Carroll, 2006, p. 35) describe:

"Computer simulations seem to us especially helpful in studying the behavior of complex systems, or systems composed of multiple interdependent processes. In such systems, each of the individual processes may be simple and straightforward, and each may well be understood from previous research or at least well supported theoretically. But the outcomes of the interactions of the processes may be far from obvious, especially over time. Simulation enables the systematic examination of the simultaneous operation of these processes in a specified theoretical model over time."

While the use of simulation in organisational research has a long history going back to Jay Forrester's work on 'Industrial Dynamics' (Forrester, 1961) and Cyert and March's work simulating organisational behaviour (Cyert \& March, 1963) it is largely ignored in textbooks on research design, which, as noted above, tend to approach the subject from a perspective based on qualitative and quantitative methods, either on their own or brought together as 'mixed methods'. As described by David Morgan (Morgan, 2007, p. 48);

"For the past two decades, much of the discussion in social science research methods has focused on the distinction between Qualitative Research and 
Quantitative Research....and the current alternative approach, which—depending on the language you prefer-either combines, integrates, or mixes qualitative and quantitative methods."

Quantitative research has largely been deductive in nature, developing theories that are then tested on the basis of empirical research. This approach, common in the physical sciences, is problematic in the social sciences because of the complex and stochastic nature of social processes, often making the mathematical techniques used in the physical sciences unworkable. This has led to the development of theories based on simplifying assumptions such as 'perfect rationality' (Aharoni, Tihanyi, \& Connelly, 2011), not because the assumptions reflect reality but because, simplifying assumptions are useful for deriving consequences (Harrison, Carroll, \& Carley, 2007). This difficulty has contributed to the growth of qualitative research in the social sciences, which up until the early 1980s was not treated with the same degree of 'respect' as quantitative methods (Morgan, 2007). In contrast to quantitative methods, qualitative methods have relied largely on inductive methods, developing theories about the relationships between variables from the analysis of data. A major problem however is the availability of data. Often the variables are unobservable (Godfrey \& Hill, 1995) or difficult to measure (Anantatmula \& Kanungo, 2006), a situation made even more difficult if one is concerned with implementation, which involves change over time. As pointed out earlier, 'time' is a factor largely ignored in implementation research.

Simulation methods can be used to overcome these constraints on both the deductive and inductive approach. The mathematical difficulty in deriving solutions within complex systems is overcome by using numerical methods and multiple simulation runs. The data issues involved in inductive research can also be partially overcome by the fact that the simulation generates its own 'virtual data'. The very nature of simulation methods combines both inductive and deductive reasoning. The building of a model is a process of theory building, using deductive reasoning to make explicit theories about the relationships between variables. By making mental models explicit the model captures the 'theories' of those involved in building it. A model is therefore a theoretical statement. Furthermore, these theoretical statements in the model are design propositions, describing a new design that is likely to evolve if the assumptions in the 
model hold true. The experiments using the model then become tests of the theory, providing data to confirm, disconfirm or refine the original theory using an inductive process.

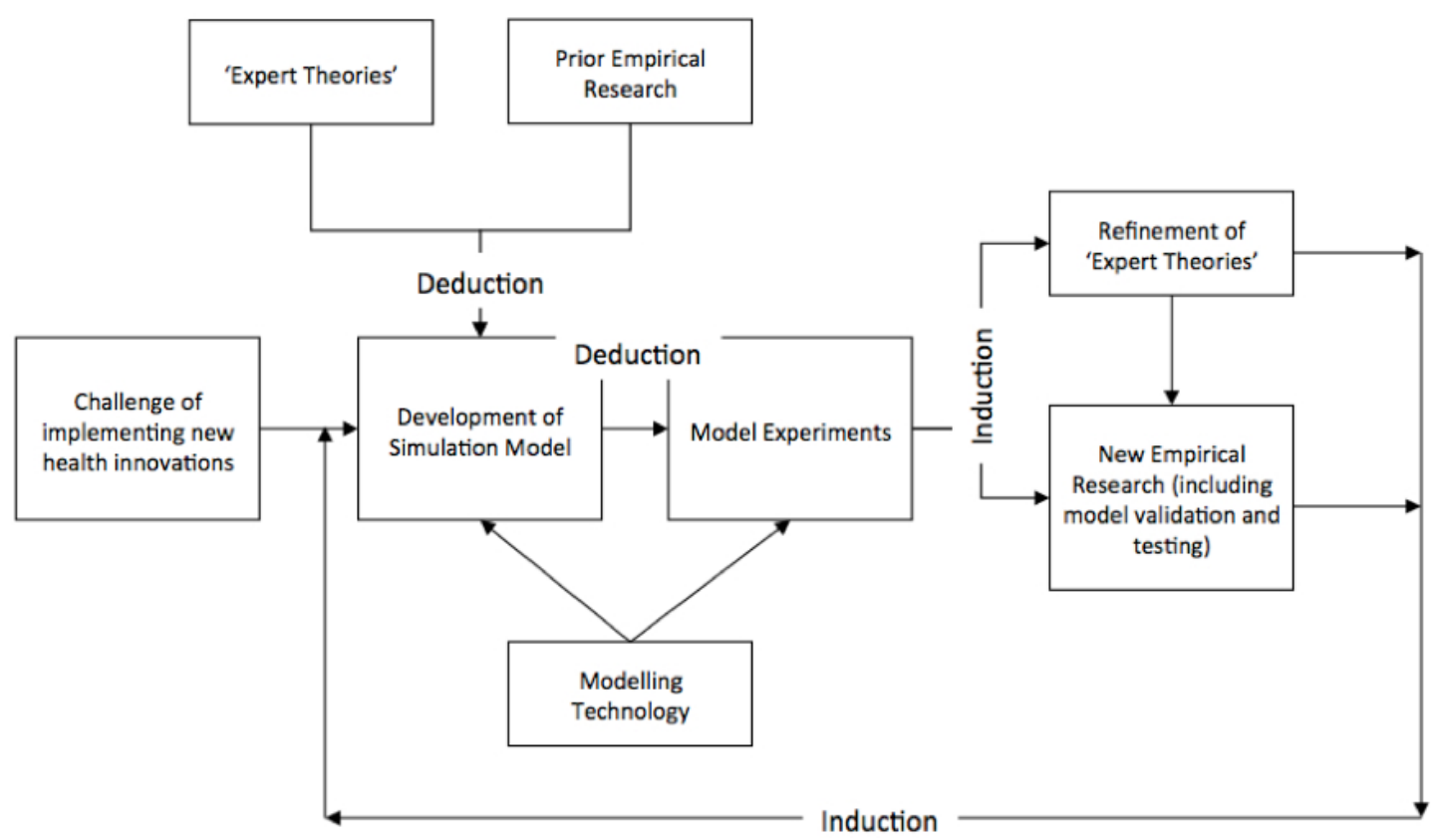

Figure 3-1 The interactive process of management theory and simulation modelling Based on (Harrison et al., 2007, p. 1240)

This deductive-inductive cycle is shown in figure 3-1. The diagram emphasises the link between complex problems, such as implementing new health innovations, and simulation modelling as a theory development process; in this case building upon and refining the 'theories of successful implementation' provided by experts working within the New Zealand health sector. In addition, the model construction is informed, by previous empirical research, which provides further information to illuminate the 'expert theories' and assist in the model construction. Experiments run with the use of this model then develop and refine the original 'expert theories' and also direct new empirical research, including research undertaken to validate and test the model. The results from the theory development and the empirical research feed back into the modelling process. Model construction is also linked to the computational technology, which provides the means to build and run the models. Simulation, because it uses deductive and inductive approaches, combining both quantitative and qualitative data has been described as, “... a third way of doing science” (Harrison \& Carroll, 2006, p. 27). 
As described above, the challenge in undertaking research in complex systems such as CCM is that the success or failure of any implementation is dependant upon a set of complex interactions between the, "... attributes of the innovation, the behaviour of individual adopters, the nature of communication and influence, and various structural and sociological features" (Greenhalgh et al., 2005). The ability to generalise from one context to another then becomes difficult, if not impossible and a simulation model provides a 'virtual world' (Schon, 1983) which enables general principles to be explored in specific contexts.

\subsection{The Research Method}

The research method used in this thesis is based on the work of Maani and Cavana (2000). This method utilises five distinct phases, each of which provides an increasing understanding of the system which cumulatively adds to the value and power of the research. This method is embodied within the discipline of SD.

While $\mathrm{SD}$ is an approach to research that combines both qualitative and quantitative data it generally approaches issues of data from a constructivist view of the world. As the founder of the field, Jay Forrester noted:

“...vast amounts of information exist in the minds of those participating in the particular social system. To ignore this information is to cut off our greatest source from which we may learn, but to accept everything which is said at face value would be an equal mistake" (Forrester, 1968, p. 612).

Furthermore, in relation to the use of both qualitative and quantitative information Forrester states:

"Perceptive observation, searching discussions with persons making the decisions, study of already existing data, and the examination of specific examples of decisions and actions will all illuminate the principal factors that influence decisions" (Forrester, 1961, p. 103) .

This position is put even more strongly by Donella Meadows (1980), when she states, speaking of SD modellers, that: 
"They would regard a series of conversations with mothers about their children to be as useful a source of information as a twenty-year time series on fertility data" (D. Meadows, 1980, p. 51).

In $\mathrm{SD}$, this process of exploring meaning in these conversations, of understanding how the person constructs their world, is explored through the concept of 'mental models'. Senge describes mental models as the “...deeply ingrained assumptions, generalizations, or even pictures or images that influence how we understand the world and how we take actions" (Senge, 1990, p. 8). From a modelling perspective an individual's mental models incorporate their, "... beliefs about the causes and effects that describe how a system operates, along with the boundary of the model... and the time horizon we consider relevant" (Sterman, 2000, p. 16).

$\mathrm{SD}$ is an approach grounded in the systems sciences and since its earliest beginnings, understands that the world and therefore the models we produce within it, are 'constructed'. It is important, therefore, to build into the modelling process, steps to understand the meanings that any particular construction of reality includes.

These steps in the research method are shown in the following diagram (Figure 3-2). 


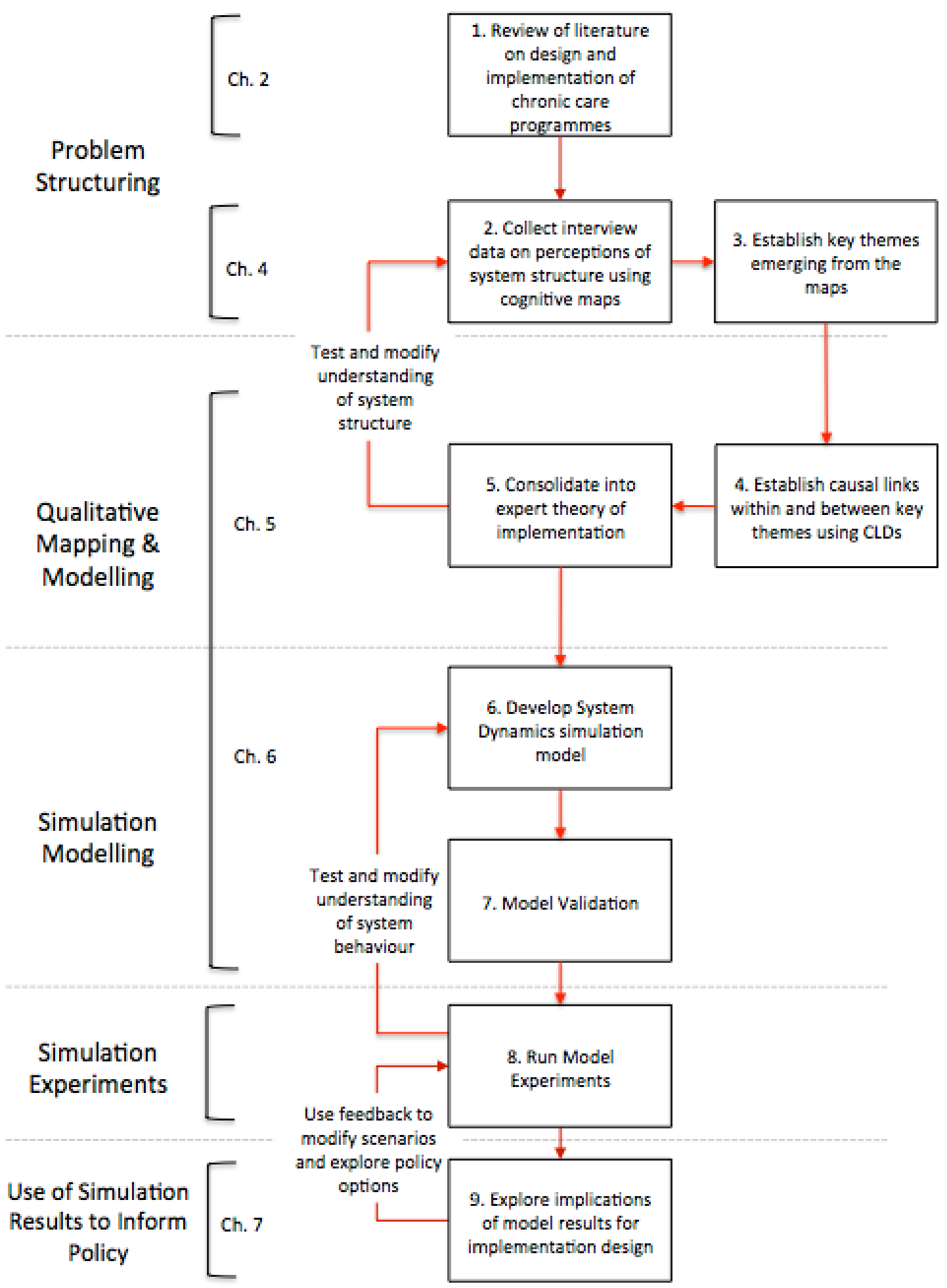

Figure 3-2 Overview of the research method 
3.6.1 Phase 1: Problem Structuring

"In this phase, the situation or issue at hand is defined and the scope and boundaries of the study are identified" (Maani \& Cavana, 2000, p. 17).

A characteristic of complex problems is that there can be many interpretations of what the problem actually is and many proposed solutions, based on these different interpretations. The problem-structuring phase is designed to define the scope and boundaries of the study; to understand the different interpretations; and to understand the nature of the world within which those interpretations are being made.

“...the most demanding and troubling task in formative decision situations is to decide what the problem is. There are too many factors; many of the relationships between them are unclear or in dispute; the most important do not reduce naturally to quantified form; different stakeholders have different priorities" (Rosenhead \& Mingers, 2001, p. 1).

This initial phase explores, using input from a number of health experts working within New Zealand, what the 'problem of implementation' is when discussing chronic care programmes.

Steps 1 to 3, shown in figure 3-2, focus on problem structuring; gathering data on the issues of concern, developing an understanding of the system structure and establishing key themes. This initial problem structuring will be developed though an in-depth review of the literature (step 1) and interviews with experts in the field (steps 2 and 3). The interviews will be undertaken with health experts, who have extensive experience and expertise in the design and delivery of programmes for people with chronic conditions. The results of these interviews will be consolidated into a series of structural representations using the cognitive mapping technique (Eden, 1988).

The problem-structuring phase is crucially important as it is in this stage that the scope is set and the key issues defined. The issue of concern in this thesis is complex and therefore choices have to be made about what to model. This initial conceptualisation 
phase is about ensuring some rigour and validity in choosing what to model and to avoid the real danger that the part of a situation to be modelled is chosen accidentally or by what others, outside of the context, consider important. (Eden, 1994). To overcome this, the problem-structuring phase will utilise the cognitive mapping method (Eden, 1988, 1992; Eden, Ackermann, \& Cropper, 1992). Cognitive maps, developed during steps 2 and 3 in the research method provide a qualitative understanding of how each individual sees the 'implementation problem' and what is important to them in that problem, i.e. how they construct meaning.

The cognitive mapping technique focuses on understanding the causal relationships between key concepts that are understood to be important and provides both a description of the issues and an understanding of what can and cannot be done about them. It does this by placing each concept within the context of what it explains (consequences) and explains it (causes) (Eden, 1994). The cognitive map begins the process of theory development, describing the theories held by those interviewed on the challenge of successfully implementing chronic care programmes.

This mapping technique will be used for each individual interview. Each map will then be integrated into an overall conceptualisation of the system structure based on the common themes arising out of the interviews. These maps therefore will help inform the understanding of the structural factors involved in implementing health innovations, specifically programmes to improve care for people with chronic conditions.

\subsubsection{Phase 2: Qualitative Mapping}

"During this phase, conceptual models of the problem, known as causal loop diagrams (CLDs) will be created.” (Maani \& Cavana, 2000, p. 17).

One of the characteristics of a systems paradigm, and central to the SD method, is the importance given to changes over time. Most of the key factors that comprise the most important variables involved in implementing chronic care programmes will be embedded in the cognitive maps developed in phase 1, as they will describe key outcomes that people see as important and the key factors involved in delivering them. 
Phase 2, therefore, begins by exploring these key outcomes and their drivers to tease out, in more detail, the causal links between them.

As pointed out in chapter 2, the current models are largely 'check lists' of the factors that are important in programmes designed to improve the care for people with chronic conditions. What, for example, actually constitutes an 'informed and activated patient', a concept central to Wagner's CCM model, is hard to discern from the literature, yet this concept is central to most models. The cognitive mapping process will uncover some of the structural detail that sits behind this concept; and understanding the factors that are involved in supporting and/or hindering its development and the consequences that follow.

From the perspective of programmes designed to improve the care for people with chronic conditions, understanding how things evolve over time has a special importance. The changes, financial and clinical, that emerge from successful programmes for the care of people with chronic conditions take many years to appear. These timescales are longer than the time many people stay with any particular funder (insurance company or government purchasing authority) and longer than the period within which the politicians who fund healthcare spending are wanting to see the results of their expenditure (Nolte \& McKee, 2008). As Nolte and McKee point out there are many pressures on funding organisations and they often force these agencies:

“...to take a short-term view and focus on providing more acute care to deal with current demand rather than investing in chronic conditions management with its likely longer term benefits. There may well be wider political reasons for so doing (e.g. reducing waiting times for elective surgery rather than investing in chronic conditions management programmes) as governments are held to account for their achievements over a relatively short time cycle" (Nolte \& McKee, 2008, p. 203).

Developing an understanding of the linkages between outcomes and the causal factors involved provides a series of 'structure-behaviour pairs', which link descriptions of the system with the behaviours that they are perceived to have generated. 
These are then brought together into an overall conceptualisation of the system (step 5 in the research method). This conceptualisation constitutes a dynamic hypothesis of the 'structure' of relationships that is causing the issue of concern. This is achieved by using 'Causal Loop Diagrams' (CLDs).

CLDs are a tool for describing the casual links between variables. They provide a visual picture of the pattern of causality that is believed to generate the behaviour. CLDs provide, therefore, a 'theory-of-action' (Argyris et al., 1985): a theory that describes the pattern of causality that produces the consequences described in the behaviours.

Because CLDs make this theory explicit it becomes testable, and a key part of this phase will be to test the theories embedded within the CLDs with those who have been involved in the interviews. They will also be compared with the literature, highlighting any similarities and differences.

To illustrate the use of CLDs, the following example (figure 3-3) is provided, based on an interview with a senior clinical advisor at the Ministry of Health. A key part of their argument was that effective implementation of chronic care programmes requires high levels of engagement with providers. Furthermore, they argued that this could only occur if there was good use made of performance data that clearly showed the gap between current performance and the performance needed to make the programme successful. Within this argument there are two key loops; loop 1 focusing on the link between data and provider engagement (R1) and loop 2, the link between provider engagement and the ability to improve the quality of that data (R2). At this stage this is simply one person's 'theory' of what is required to implement such programmes. It is included however to illustrate the use of CLDs within this research. 


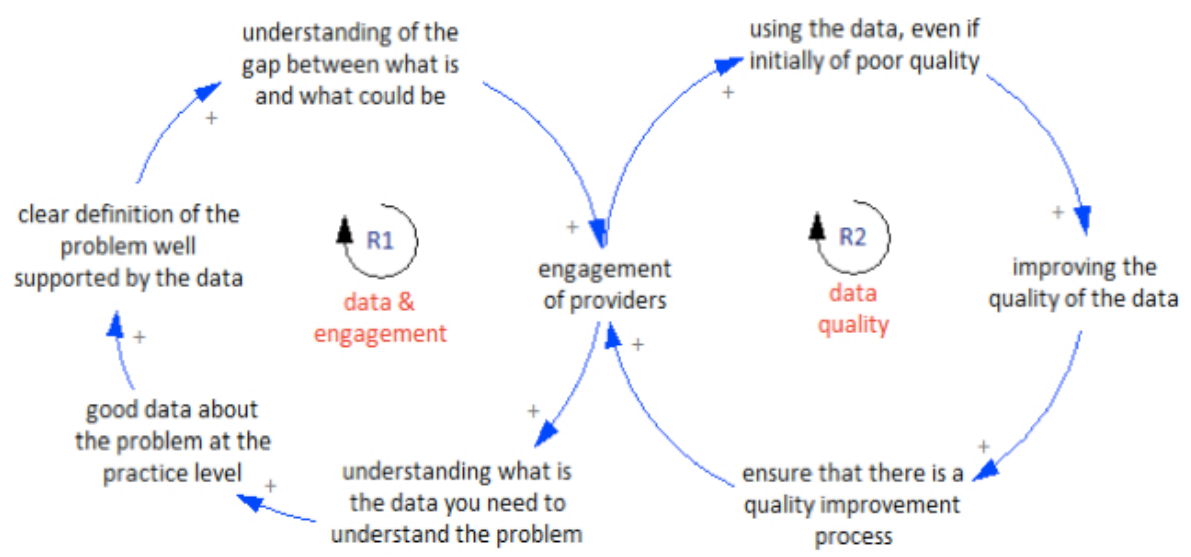

Figure 3-3 Illustrating the links between data and clinical engagement

\subsubsection{Phase 3: Simulation Modelling}

"Although causal loop modelling is very powerful...there are a number of advantages to be gained from developing a computer simulation model to investigate more deeply the dynamic issues that are of concern to management." (Maani \& Cavana, 2000, p. 56).

While CLDs are useful for describing the causal links between key variables, they fall short as a tool upon which policy can be developed. While explicitly representing the causal links described by the health experts, the behaviour of the system cannot be confidently inferred, as CLDs say nothing about the strength of those links, or the size of the effects they cause. CLDs also ignore the 'accumulations' in the system; that is those factors that you can see, feel, count at any given time, that build up and decay over time. For example, the number of patients visiting a primary care practice, the level of social deprivation in a community, or the number of GPs in a practice. These accumulations are explicitly captured in the 'stock-flow' language of SD. Because of this, the qualitative CLD model will be developed into a SD simulation model (step 6 of the research method). The development of the simulation model not only provides a more robust description of the system, but allows data to be utilised that then enables 'what if scenarios to be run, thus testing some of the initial hypotheses that the CLDs generate.

The simulation model, therefore, provides a tool to help structure the information obtained from the interviews, and the literature, to provide a better understanding of the variables and connections that influence the effectiveness and sustainability of the 
programmes. Simulation has been shown to be very valuable in helping people understand the consequences of actions over time (Akkermans \& Romme, 2003; Hirsch, Levine, \& Miller, 2007; Senge, 1990; Sterman, 1994) and as such provides a powerful tool to help illuminate the issues in designing programmes that are effective and sustainable. The simulation model is the formalisation of the theories about effective implementation, espoused by the health experts interviewed in phase 1 .

As pointed out by Robert Axelrod (Axelrod, 2003, p. 6), the goal of simulation modelling is to, “...enrich our understanding of fundamental processes that may appear in a variety of applications." As such, simulation is primarily a descriptive research tool. In this research the fundamental processes that are the focus of the modelling effort are the interactions between the factors seen to be important in implementing chronic care programmes. It does this by:

- developing operational definitions of the key factors of the programmes,

- exploring the interactions amongst these factors, and

- developing a model of an effective and sustainable programme that captures the key factors and their interactions.

The purpose of the simulation model is, therefore, to provide a tool that will help those involved in designing, implementing and evaluating programmes for the care of people with chronic conditions.

\subsubsection{Phase 4: Simulation Experiments}

"In this phase, various policies and strategies are postulated and tested. Here 'policy' refers to changes to a single internal variable such as hiring, quality, or prices. Strategy is the combination of a set of policies and as such deals with internal or controllable changes. When these strategies are tested under varying external conditions, this is referred to as scenario modelling" (Maani \& Cavana, 2000, p18).

Prior to using the model to run a range of experiments (step 8 in the research method) it is important to validate the model (step 7). Model validation is a process of assessing whether or not the model is suitable for the purpose it has been built for (Forrester, 
1961). That is, does the model have sufficient 'soundness and usefulness' (Forrester \& Senge, 1980, p. 210) for it to be used confidently. The specifics of this process will be discussed in chapter 6 . These model experiments will be used to explore the effects of different contextual factors on the behaviour of key factors identified in the model conceptualisation stages, as being important to the successful design and implementation of programmes for the care of people with chronic conditions.

The analysis phase focuses on developing scenarios and policy options that can be used to guide the implementation of CCM programmes and similar health innovations targeting people with chronic conditions.

\subsubsection{Phase 5: Use of Simulation Results to Inform Implementation Design}

"Once simulation models have been developed, they can be enhanced by extending them into a microworld. Microworlds (also known as management flight simulators) provide an interactive and user-friendly interface for managers to experiment with the model.” (Maani \& Cavana, 2000, p. 19).

If the models, and the model development process, used in this research are to inform both policy and practice they need to contribute to what Chris Argyris (Argyris, 1993) calls 'actionable knowledge'. They do this in a number of ways. First, as described above SD modelling adopts a perspective of 'design causality'. That is, the model explains i) how the observed structure and the consequential behaviours arose in the first place; ii) why some changes will not have the effects desired; and iii) introduces new suggestions of how new patterns of behaviour can be generated. But, to be useful, no matter how convincing the arguments, the knowledge has to be actionable. For this to be achieved, "...it must be possible to derive from them the actual behaviour required for effectiveness" (Argyris, 1993). In the concern to make knowledge actionable, Argyris is focusing on the same issue that Forrester described in 'Industrial Dynamics'.

"If management is the process of converting information into action, then it is clear that management success depends primarily on what information is chosen 
and how the conversion is executed. The difference between a good manager and a poor manager lies at this point" (Forrester, 1961, p. 93).

Helping make explicit this link between knowledge and action is at the heart of learning and implementation and SD modelling can play a significant role as it provides a visual portrayal of the explicit 'rules' that govern decision-making. As Forrester points out;

"The formulation of a model is based on an explicit statement of the policy (or rules) that govern the making of decisions.... The decision making process consists of three parts: the formulation of a set of concepts indicating the conditions that are desired, the observation of what appears to be the actual conditions, and the generation of corrective action to bring apparent conditions towards desired conditions" (Forrester, 1961, p. 93).

This bears a very close resemblance to Argyris's concept of 'theories of action'. Argyris and his colleague, Donald Schön, have spent over 30 years undertaking research in this field and make the point that all theories of action have the same form: "in situation S, if you want to achieve consequence C, do A" (Argyris \& Schon, 1974). This is essentially the same as Forrester's description of decision-making above.

What a SD model does is to make this theory transparent, and therefore testable. In the modelling process the current situation is modelled through the use of a 'stock', also known as a 'state variable'. This could be something as tangible as the number of people waiting for a surgical procedure or as intangible as the level of support for a particular programme. Decision making - taking action - is therefore precipitated by seeing a gap between the current state, number waiting for the procedure, or level of support for the programme, and the desired state, size of waiting list being sought, or level of support desired. The implementation of that decision is affected by the size of the gap and the effort and/or time associated with closing it. By modelling this dynamic explicitly, SD helps support productive reasoning, in that it, “(1) produces valid and validated knowledge, (2) creates informed choices, and (3) makes personal reasoning transparent in order for the claims to be tested robustly" (Argyris, 2004). 
However, while it is true to say that a SD model can support this by ensuring that the 'theories' that underpinned decisions are transparent and therefore testable, that will only occur if supported by the modelling process.

SD models can be complex artefacts that people can find difficulty interacting with. They have a strange visual language and are governed by differential calculus. That language alone is enough to put many people off. However, SD can be said to support improved public discourse. In the preface to Marjan van den Belt's (2004) book on mediated modelling, Thomas Dietz made the following comment;

"In this volume, van den Belt deploys one of the most broadly integrative tools in the sciences - system dynamics - in the service of public discourse. Her discussion of mediated modeling shows that it can be both robust and subtle. It acknowledges the tentative and partial character of all systems models. It is thoughtful about the process of interaction with a model and, more important, the process by which discourse leads to changes in the perspectives of participants" (Van Den Belt, 2004, p. xv).

The key point here is that the potential of SD models to support learning and change is dependent on the process by which the model is built. The steps outlined in this phase are therefore, important throughout the five phases of this research method.

Throughout the process, from phase 1 onwards the model will be open, explicit and testable. By ensuring this, it makes it more likely that the model that is finally developed will be accepted as a valid representation, and as a tool that can be used to explore alternative representations of future possibilities.

\subsection{Summary}

Understanding what is involved in implementing new innovations in healthcare delivery requires an understanding of the causal mechanisms at play. However, the literature on implementing innovations is sparse and what exists is usually anecdotal, using simplistic causal assumptions (Greenhalgh et al., 2005) and ignoring the impact of context and time. 
This research proposes a design orientation as understanding implementation requires an understanding of the world as it 'could be' and not just the world 'as is'. A design orientation also sees causality as being context dependent, and as such addresses one of the major limitations in much of the research on innovations and implementation. However, conducting research into worlds that do not yet exist is a difficult proposition and simulation methods provide a useful approach. Simulation applies both deductive and inductive approaches, using both qualitative and quantitative data in a manner that Harrison and Carroll (2007) describe as a 'third way of doing science'.

The chapter concluded with a description of the research method used in this research, combining SD simulation modelling with cognitive mapping and qualitative SD modelling using CLDs. 


\section{Cognitive Mapping}

Development of Key Themes

While chronic disease is viewed by some as the 'healthcare challenge of this century' (World Health Organisation, 2005) and academics and practitioners around the world extol the virtues of chronic care management (Rea et al., 2007), we are still a long way from understanding how to design and implement the system that will deliver the care that so many say is necessary, if the worst fears about the future 'burden of chronic conditions' are to be avoided. Whilst the factors needed in such systems are well articulated (Bodenheimer, Wagner, et al., 2002a; Wagner et al., 2001), the causal structures of the theories are not fully specified and it is unclear how those factors can be implemented in a way that retains the integrity of the system they are a part of.

The prime purpose of this research is to stand back from the theories about chronic care management and elicit the 'theories of implementation' as espoused by seven experts who are active at a senior level within the New Zealand Health sector. What do they say about programmes designed to improve care for people with chronic conditions and importantly what are the issues that need to be addressed if they are to be implemented successfully? The aim is to develop an understanding of some of the key factors that a theory of implementation would need to encompass. It is important therefore that this research elicits causal theories from the interviewees: their argument for what needs to occur if such programmes are to be implemented successfully.

\subsection{Moving Beyond Lists: the Argument for a Systems Approach}

To develop a causal theory of implementation, this research has to go beyond describing a list of factors that are considered important. The reason for this is that such lists, while claiming to describe the universe they purport to represent, are unable to do so in a way that makes the list useful; because the nature of lists aims at a description of discrete factors that, in reality, are not discrete. For example, a recent comprehensive study of chronic conditions within New Zealand, (Connolly et al., 2010) proposed a list of 10 'action areas'. These action areas are described as 'dimensions critical to effective 
chronic conditions management" (Connolly et al., 2010, p. 3). Action area 8 'delivery system design', for example, focuses on effective design of such programmes. This, the authors state, is based on Wagner's model of chronic care management (CCM).

Wagner's model however goes beyond action area 8 and encompasses a number of the other action areas described in the report; action areas 3, 5, 6 and 9. In Wagner's model, 'Delivery System Design' is part of a system of connected parts, not an isolated pillar. A causal map of Wagner's model, which highlights the linkages between each of the 'pillars' (shown as yellow boxes) in the CCM model, is shown in figure 4-1 below. It was developed from a paper describing the CCM model (Bodenheimer, Wagner, et al., 2002a).

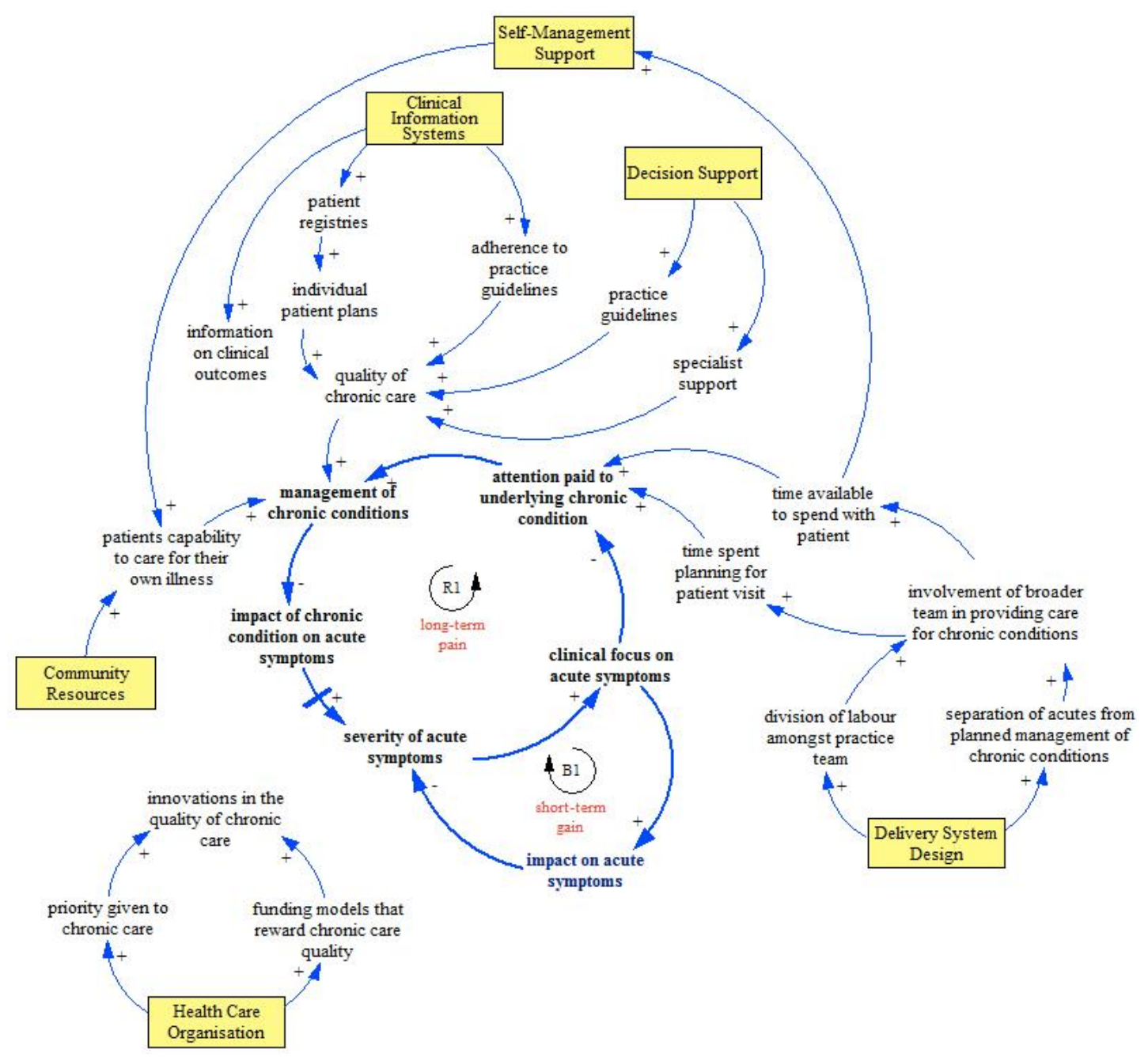

Figure 4-1 Causal map of Wagner's CCM model

(Based on Bodenheimer, 2002a) 
It is a complicated map and, even though many of the linkages are not well-defined, it clearly shows that in developing the CCM model, Wagner and his colleagues had a sense of a model that was made up of connected parts. Connolly's research however chose to ignore those connections, discussing each as a discrete area of focus.

As the map shows, within Wagner's model, self-management is dependent upon 'delivery system design' and it makes no sense to talk of improving self-management in the absence of delivery systems that can support it. Wagner and his colleagues argue strongly that the 'delivery system design' has to take place so that time is freed up, enabling the clinical team to spend the time with the patients that is necessary to provide credible and adequate support for self-management. Ignoring the links between key constructs, such as 'delivery system design' and 'self-management support', undermines the integrity of Wagner's original thinking and ignores the research, upon which the model was built.

What the map also shows is that the causal theory is far from complete; there is nothing, for example, that describes the link between 'health care organisation' which supports 'innovations in the quality of chronic care' and the other 5 factors in the CCM model. Furthermore, while each of the 6 factors within the model is described, there is nothing, with the exception of the link between 'delivery system design' and 'self management support' that describes the causal links that will ensure its implementation.

So, while the research by Connolly and his colleagues highlights many important factors known to be important in the care of people with chronic conditions, by placing little attention on the links between those factors it undermines the ability, of anyone who wishes to, to successfully implement any of the factors on the list itself. By ignoring these interdependencies, lists are unable to move beyond description to explanation, which is a requirement of any information that is meant to inform practice (Argyris, 1996). It is unclear, in a list, how one is to bring about the consequences one is striving for. For that to occur the information provided:

“...should inform the users not only what is likely to happen under the specified conditions but how to create the conditions and actions in the first place. Otherwise the generalization is not actionable. For example, there is much 
research in the empirical literature on the relevance of trust in managing. However, there is little attention paid to informing the reader on how to create trust" (Argyris, 1996, p. 392)

Lists therefore, do not possess any information about design causality; “...the specifications of actions to be taken (often in a specified sequence) to achieve the intended consequence" (Argyris, 1996, p. 396). Lists also ignore context. In a manner consistent with much in the sciences, lists assume a "...scheme of isolable units acting in one-way causality" (Bertalanffy, 1969, p. 45). They assume that somehow there is a direct and isolatable causal link between, for example, 'adherence to clinical guidelines' (action area 3 in Connolly's research) and improved care for people with chronic conditions. While it could be argued that clinical guidelines are necessary, they are not sufficient and they will only deliver improved care if they are combined with other necessary conditions that enable them to be taken up and integrated into the way care is delivered. As shown in the causal map of the CCM model (figure 4-1), clinical guidelines affect the quality of chronic care. However, they will only improve the management of chronic conditions if there is a redesign of the clinical practice so that it supports the reallocation of resources needed to pay attention to the underlying chronic conditions, rather than simply focusing on the more immediate acute symptoms presented by the patient. Clinical guidelines are, along with every other action area on Connolly's list, what American philosopher E. A. Singer refers to as 'producers'. As described by Ackoff (Ackoff, 1981), Singer differentiated between a 'producer-product' relationship and a 'cause-effect' relationship and;

"...the view of the universe revealed by viewing it in terms of producer-product is quite different from the view yielded by viewing in terms of cause-effect. Because a producer is only necessary and not sufficient for its product, it cannot provide a complete explanation of it. There are always other necessary conditions, co-producers of its product.... These other necessary conditions taken collectively constitute the...environment" (Ackoff, 1981, p. 21).

It should be noted at this point, that this distinction can create some issues with language and shifting from discussing 'cause and effect' relationships to 'producer and product' relationships can overly complicate the writing. It is an important idea 
however and Singer's description of the relationships between 'producer' and 'product' has much in common with design causality discussed in chapter 3. Therefore, although the terminology of cause and effect will be used throughout this thesis, it will be used recognising the key ideas Singer was describing when he talked of 'producers' and 'products'. That is, the perspective taken in this thesis is that there are very few 'effects' that result from single 'causes', context nearly always has a part to play and, for a 'cause' to bring about a given 'effect' there will be additional, necessary, conditions that will determine whether or not it occurs. So, for example, if 'self management' is a 'producer', what are the additional conditions and co-producers required to bring about the desired outcome of clinical improvement for the person with the condition, that need managing? As Ackoff points out, “...the use of the producer-product relationship requires the environment to explain everything whereas use of cause-effect requires the environment to explain nothing." (Ackoff, 1981, p. 21).

It is interesting to note that this idea is at least 2,500 years old, being a central teaching of Buddhism. An important concept in Buddhist teaching is 'dependent co-arising' (paticca samuppäda), which states that everything in this world has 'arisen' from previous causes AND conditions. Both have been central to bringing us to where we are today, and both will create the future. Future consequences are the results of current causes and conditions (Macy, 1991).

So, lists have a number of shortcomings that make their use problematic if the concern is to take action. First, they create confusion in that items on a list that is concerned with action are rarely discrete; often overlapping and sometimes encompassing others. Second, by ignoring the links between them lists assume a single one-way model of causality that does not exist in the real world. Finally, any item in such a list is a 'producer', a necessary but not sufficient causal factor in bringing about the desired 'product' and therefore it is unlikely that any list item could be implemented in the absence of the necessary contextual or environmental factors. 


\subsection{Eliciting Causal Theories}

To respond to this criticism of action lists, this thesis aims to develop an initial 'theory of design' that as well as describing key factors affecting the implementation of chronic care programmes also provides insight into the causal relationships between the factors.

As chronic disease has become increasingly prevalent and initiatives to tackle it have increased there have been numerous efforts to evaluate their effectiveness, both internationally (Singh, 2005) and in New Zealand (Connolly et al., 2010). Some evaluations have involved a comprehensive review of the literature (Singh, 2005), others have focused on descriptions of specific initiatives (Wagner et al., 1999), while others have focused on surveys of current practices (Connolly et al., 2010) to develop an understanding of what is happening in such programmes and how effective they are.

Because the concern here is to develop a deeper understanding of causality, this thesis focuses on developing an in-depth understanding of the views of seven people who are actively involved, at a senior level, in the design and implementation of chronic care initiatives. The seven people interviewed are all involved at a national and regional level and four are also practicing clinicians, who combine their clinical practice with involvement in policy at both national and regional levels. The question that formed the basis of the interview was; "What are the key issues that you consider to be important in the implementation of chronic care programmes?" The seven people interviewed were:

\begin{tabular}{lll}
\hline 01 & Primary care clinician & $\begin{array}{l}\text { Interviewee \#1 is a General Practitioner, who has also spent many } \\
\text { years at national and regional level acting in management and policy } \\
\text { roles. }\end{array}$ \\
\hline $02 \quad$ Primary care clinician & $\begin{array}{l}\text { Interviewee \#2 is a General Practitioner, who combines a continuing } \\
\text { clinical practice, with senior advisory roles at national and regional } \\
\text { level. }\end{array}$ \\
\hline $03 \quad$ Senior health manager & $\begin{array}{l}\text { Interviewee \#3 has held senior health management positions at } \\
\text { national and regional levels for over 20 years. }\end{array}$ \\
\hline $04 \quad$ Secondary care clinician & $\begin{array}{l}\text { Interviewee \#4 is a senior, well-respected secondary physician who also } \\
\text { has an active role in policy and implementation at national and regional } \\
\text { levels. }\end{array}$ \\
\hline $05 \quad$ Senior health planner & $\begin{array}{l}\text { Interviewee \#5 has many years experience in planning primary care } \\
\text { programmes for the Ministry of Health, DHBs and NGOs. }\end{array}$ \\
\hline
\end{tabular}




\begin{tabular}{lll}
\hline 06 Senior health IT planner & $\begin{array}{l}\text { Interviewee \#6 has spent many years managing and developing the IT } \\
\text { systems to support primary-secondary integration. }\end{array}$ \\
\hline $07 \quad$ Primary care clinician $\quad \begin{array}{l}\text { Interviewee \#7 is a practicing General Practitioner who spent many } \\
\text { years in a policy role, providing leadership within the Ministry of } \\
\text { Health. }\end{array}$ \\
\hline
\end{tabular}

Table 4-1 Health Experts Interviewed

To overcome the limitations inherent in lists, described above, this research attempts to develop a more holistic view, by trying to gain a picture of the whole, and its emergent properties, rather than focusing on discrete parts. That is, it tries to elicit the 'necessary conditions' needed for any given factor to have the effect it purports to have. The key steps in the approach are:

i. developing individual cognitive maps that reflect the thinking of key experts in the field.

ii. analysing individual maps to elicit key themes,

iii. combining the cognitive maps into one composite map to cluster the constructs within each theme,

iv. using the cognitive maps of each theme to gain a richer understanding of what each theme comprises, and

v. using the thematic maps as the basis for creating an overall theory of implementation.

This chapter describes the outputs of steps i) to iv). Chapter 5 describes the outputs of steps v).

\subsubsection{Cognitive Mapping}

Cognitive mapping, as developed by Eden (1988), is a visual mapping technique used to elicit peoples' description of a situation and/or issue; why it is the way they see it and why it is important to them. The interview process, using cognitive mapping, teases out the key ideas - termed concepts - related to the interview focus and through the use of unidirectional arrows depicts the line of argument. Thus meaning “...is not deduced from a semantic analysis but rather from the context of the concept - what it explains 
(consequences) and what explains it" (Eden, 1994). Cognitive maps, therefore, make explicit the additional conditions needed for the 'producer' to deliver the 'product'. Cognitive maps also have an additional advantage in that by laying out the interviewee's responses in the form of a visual map the interpretation of meaning is made explicit, enabling it to be tested and therefore changed.

To ensure that the interpretation of what was said in the initial interviews reflected what the interviewee was in fact trying to say, all people were interviewed twice. In the second interview, the cognitive map developed in the first interview, was tested and refined. In all cases, the second interview led to further additions to the map, concepts they thought were not covered, or not covered in enough detail. It was rare to have any of the concepts in the first version deleted. In most cases the second interview provided the opportunity for a richer, more detailed discussion of key ideas.

For example, figure 4-2 shows the cognitive map that emerged from the first interview with one of the participants. Figure 4-3 shows the cognitive map that emerged after the second interview with the same participant; the most significant change being the development of a line of argument around the engagement of patients. This extra line of argument is shown on the right-hand side of the map. Other changes included slight changes to wording to better reflect what the interviewee was trying to describe.

In all there were 7 cognitive maps developed and each one was refined in a second meeting with the interviewee. These are shown in appendix 1. 


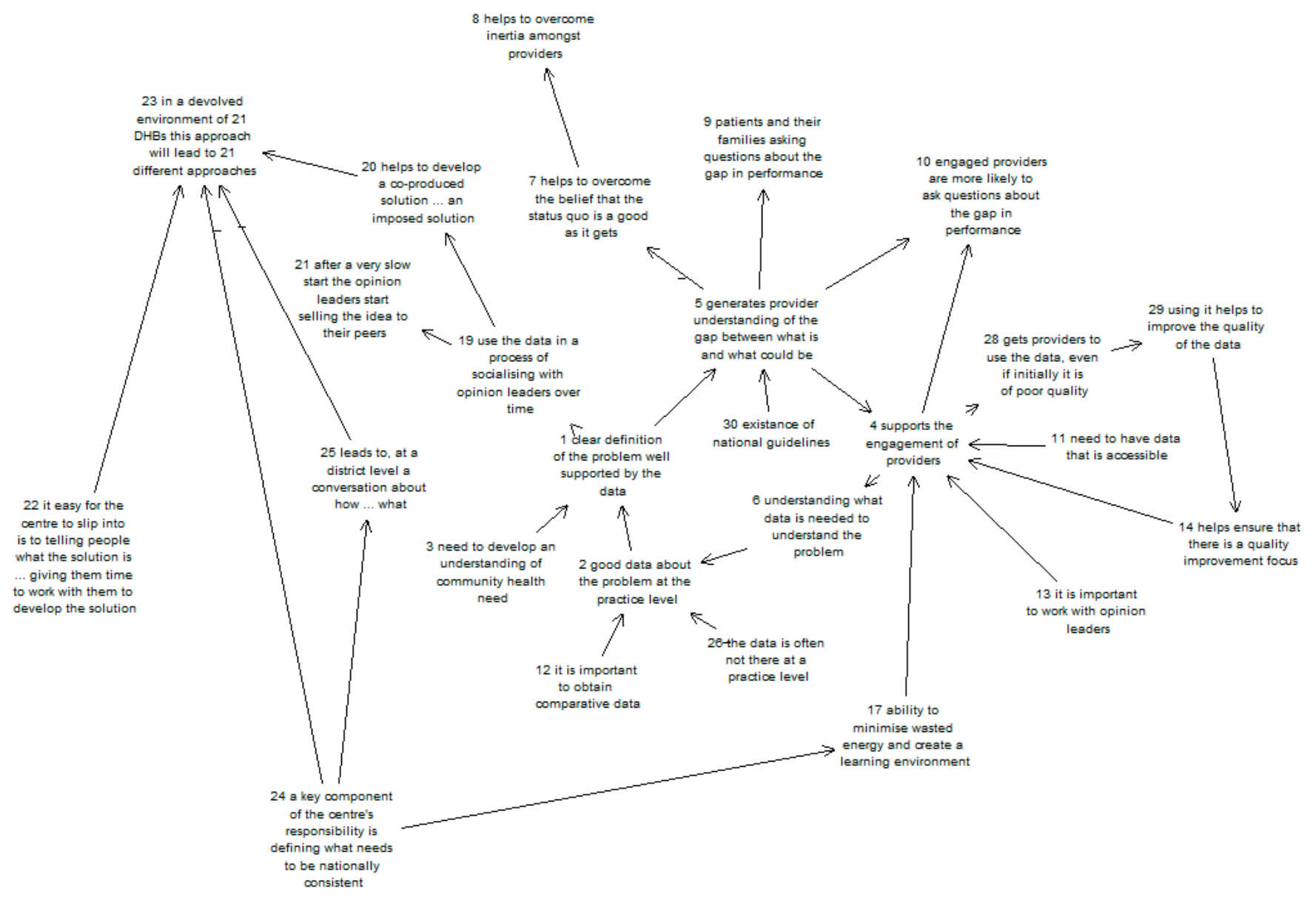

Figure 4-2 Cognitive Mapping Example: 1st interview with interviewee \#1 


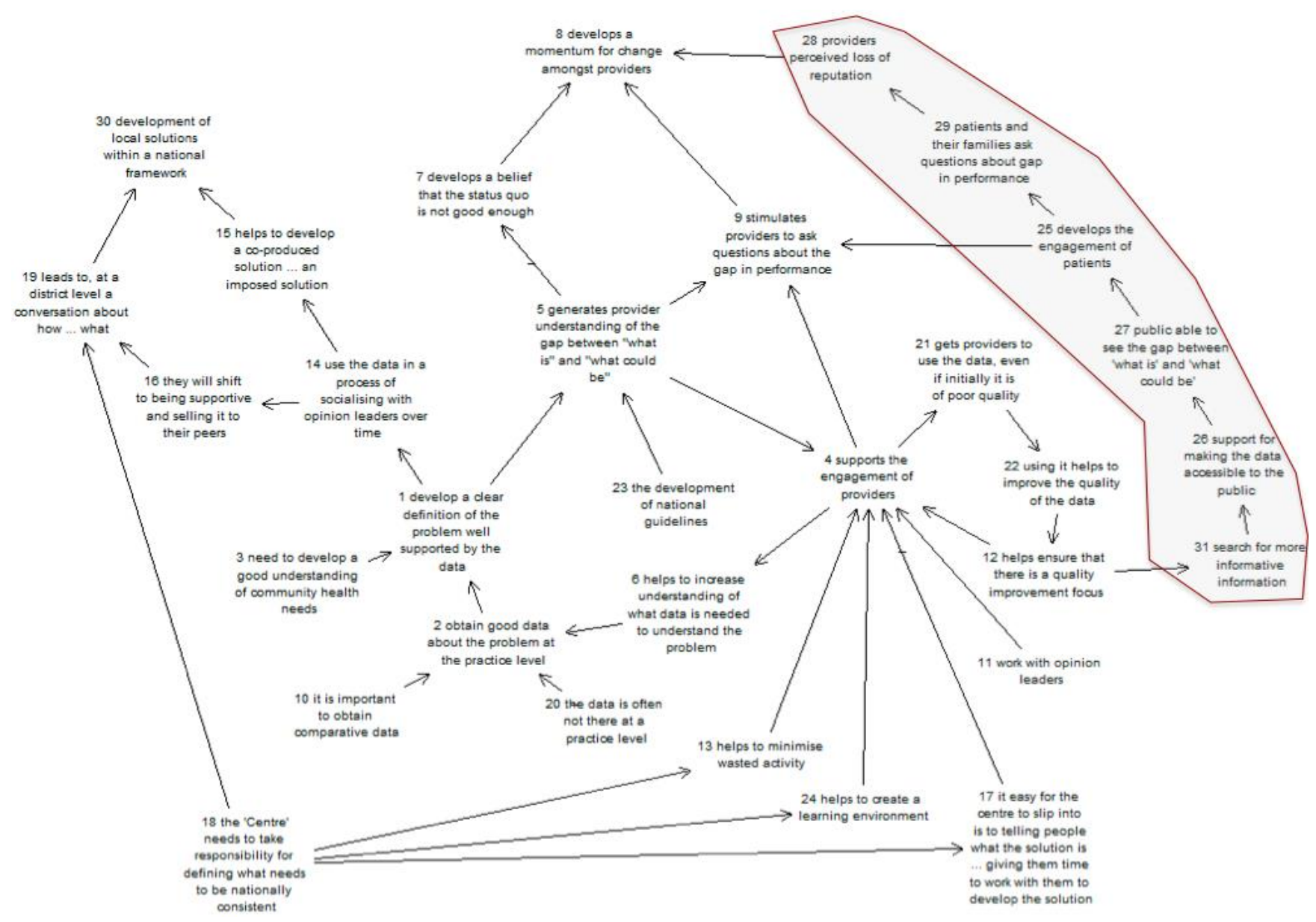

Figure 4-3 Cognitive Map Example: 2nd interview with interviewee \#1 


\subsection{Analysis of Cognitive Maps}

Cognitive maps are causal maps. That is, they provide a graphical representation of the causal links between concepts, in this case, concepts developed during the interviews process. Analysing such maps has generally focused on attempting to explore the complexity of the map by either counting the number of 'nodes' or concepts within it or, by counting the links between them (Eden et al., 1992). The number of concepts is assumed to reflect the complexity of the map. However, counting the number of concepts is fraught with problems as the number of concepts elicited during an interview is more likely to reflect the length of the interview and the skills of the interviewer than any complexity in the ideas discussed (Eden et al., 1992). A common form of analysis of the links is known as 'domain analysis' (Eden et al., 1992). It is referred to as a 'domain' analysis because the method counts the number of in and out arrows from each, that is within its immediate domain. This approach pulls out those concepts that have a higher level of complexity, as evidenced by the density of its linkages. However, by simply focusing on the immediate links this analysis ignores the wider context. To overcome this, a centrality analysis (Eden et al., 1992) is used and is the analytic technique used to explore the key themes within the maps.

\subsubsection{Centrality Analysis}

The cognitive map shown in figure 4-2, as well as those developed in the other 6 interviews, were all inputted into 'Decision Explorer', a software tool developed by Colin Eden to display and analyse cognitive maps ${ }^{3}$. Individual maps ranged in size from 25 to 53 concepts. However, as noted above, the number of concepts in any map reflects more the length of the interview and the skills of the interviewer than it does of any complexity of ideas expressed by the person being interviewed (Eden et al., 1992). So little, if anything, can be inferred from the difference in number of concepts in each interview. Of more importance are the links between them.

The analysis of those links was undertaken using a centrality analysis. Centrality analysis highlights how central a concept is and, “...indicates the richness of meaning of each

\footnotetext{
${ }^{3}$ Detailed information about the software can be obtained from the Decision Explorer website, http://www.banxia.com/dexplore/
} 
particular concept" (Eden et al., 1992, p. 313), by calculating the number of in-arrows (causes) and out-arrows (consequences) from each concept. This is an important analysis as it pulls out from the large number of connected concepts those that are central to the ideas being presented by the interviewee. Using the software to do the analysis avoids preconceptions of the interviewer to determine what is, and is not important to the interviewee. What is important are those ideas that are densely connected, affecting and being affected by a large number of other ideas put forward during the interview process.

To ensure that the wider context of the concept is taken into account successive layers, or domains, are considered, that is, not just the concepts to which it is immediately linked, but also those that are further removed. As Eden et al., (1992) point out, "Intuitively, it seems sensible to give each successive layer of concepts a diminishing weight - a distance decay function" (Eden et al., 1992, p. 313).

Those that are further removed are given a diminishing weight i.e. those that are directly connected to the concept are given a weight of 1 . Those that link into them, i.e. level two are given a score of $1 / 2$. Those that link into them, i.e. level three, are given a score of $1 / 3$. This is illustrated in figure $4-4$ below with an extract from one of the cognitive maps. 


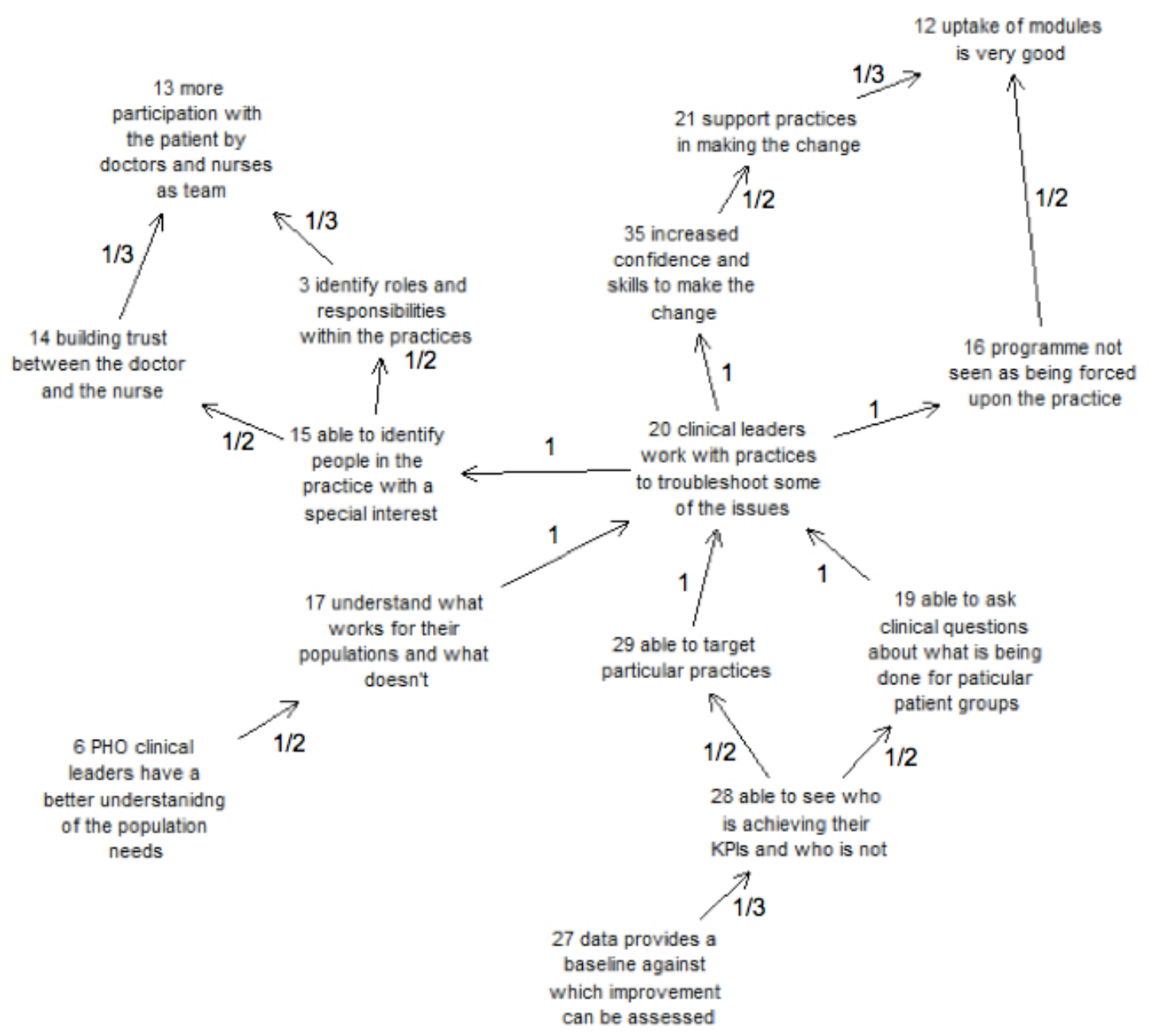

Figure 4-4 Illustration of Centrality Analysis

In this example, the central concept is linked to 14 other concepts. Using the scores noted on the map the score given to this concept is (in metric) 10.83. It is therefore described has having a score of 11 from 14 concepts. It should be noted that this is a simplified extract, to illustrate how the scores are calculated. To avoid making the diagram too complicated, the example does not show all the links present. In describing the scores, the numbers are also rounded to the nearest whole number.

Centrality analysis isolates core concepts and provides a method for developing a summary, or overview of the total map, highlighting the concepts that have a significant importance for the interviewee. For example, in the domain analysis conducted on the map shown in figure 4-5, the concept that had the highest centrality score and thus was 
seen to be the most important for the interviewee was; 'supports the engagement of providers' (concept 4).

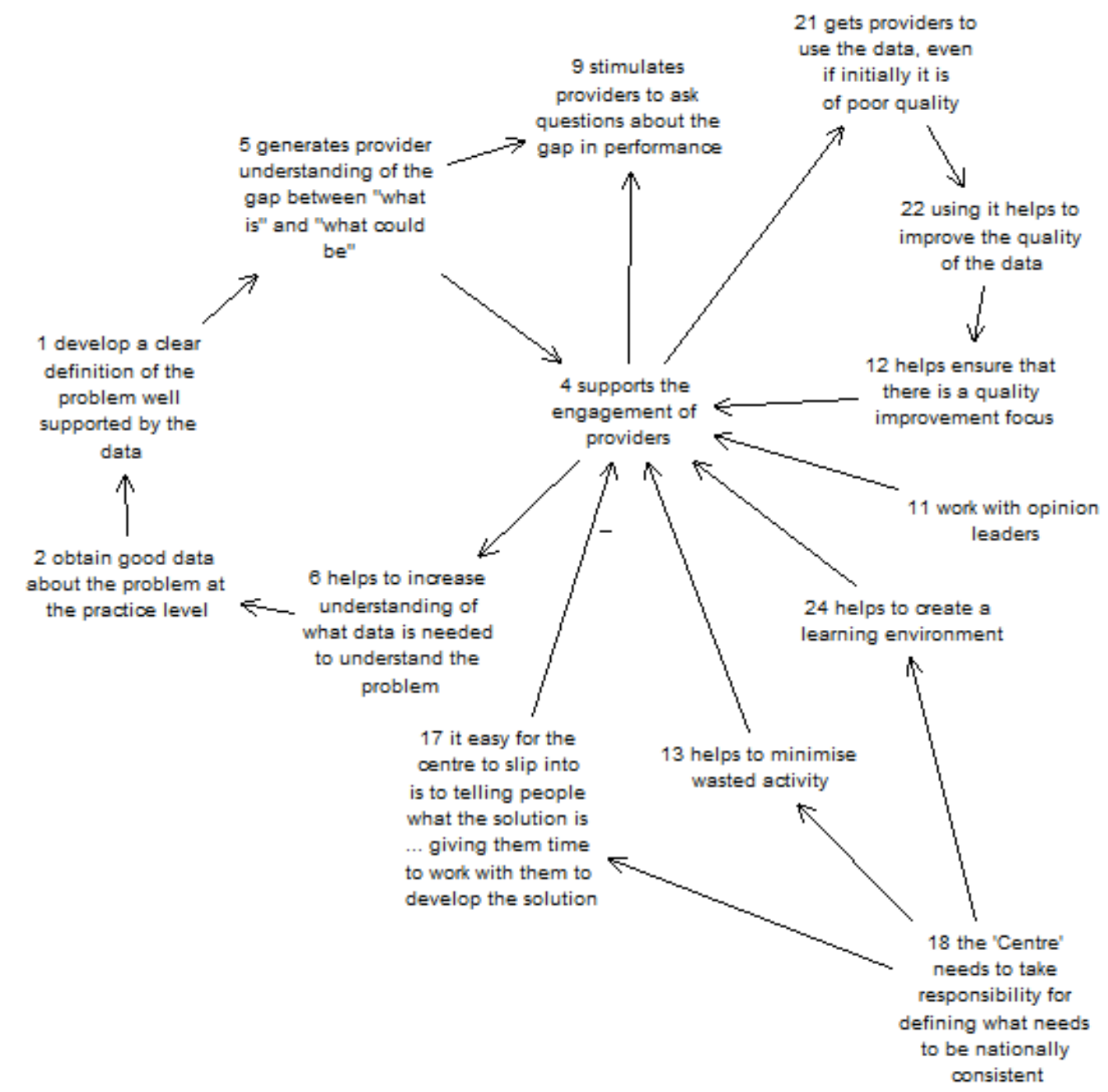

Figure 4-5 Causes and conditions related to the engagement of providers

When shown within the context of the map, concept 4, 'supports the engagement of providers' takes on a much richer meaning. The 'necessary conditions' needed to bring about that engagement are shown - concepts with arrows leading into concept 4 . That is, they have an influence in bringing it about. In addition, the consequences of developing that engagement are shown at the end of the arrows leading out of concept 4. 
Shown in the context of the map it becomes clearer why this concept is considered important by the interviewee, and what is required if the intent associated with it is to occur. As the map shows, the engagement of providers is considered important by the person interviewed because it is a causal factor in increasing understanding of what data is needed to understand the problem (concept 6), supporting the use of data, even when it is of poor quality (concept 21) and stimulating providers to question performance gaps (concept 9). To develop that engagement the interviewee considers it important to have a quality improvement focus (concept 12), minimise wasted activity (concept 13), develop a learning environment (concept 24), give people time to work closely together in developing the solution (concept 17), work with opinion leaders (concept 11) and develop provider understanding of what is and what could be (concept 5). In addition, there is also an important feedback loop at play in which the engagement of providers, promotes the use of data (concept 21) which enhances the quality of data available (concept 22) which in turn helps ensure a quality improvement focus (concept 12) that supports the further engagement of providers.

Exploring a map in this way reveals what the interviewee considers important and what their line of argument is. It does provide a 'list' of key items but also uncovers the context within which they sit; how they link to other items and the meaning it has for the interviewee. The use of cognitive maps begins to describe the causal theories of the interviewee, not just the factors considered important.

In this case, the list item, 'provider engagement', is seen as a key factor in moving from the 'as is' situation to a situation in which programmes for the care of people with long term conditions are being designed and implemented effectively. It is also linked very closely to data and changed behaviour amongst front-line service providers. In moving from a simple item on a list to a concept embedded in a rich web of context, the analysis provides an initial sense of what an effective theory of implementation will need to contain.

Each of the interviewees had a centrality analysis conducted on their individual maps to ascertain those concepts that had a central position in their thinking. To develop some idea of what was most important to the interviewees, the top five most central concepts for each person were extracted, and a thematic analysis conducted on the resulting 35 
concepts. The results are shown below in table 4-2. The scoring on the right hand side shows the number of other concepts the central concept is connected to and the score itself reflects the distance of each of those concepts from the central concept as described at the beginning of this section. So, for example, a centrality score of ' 15 from 26 concepts' for the concept, 'develops the engagement of providers' means that the concept, 'develops the engagement of providers', is linked to 26 other concepts and adding up the scores, of all the linked concepts down to level three, using the method described above, provides a score of 15 .

\begin{tabular}{|c|c|c|}
\hline & Central Concepts & Centrality Score \\
\hline \multirow[t]{5}{*}{ Interviewee 1} & Develops the engagement of providers & 15 from 26 concepts \\
\hline & $\begin{array}{l}\text { Generates provider understanding of the gap between } \\
\text { what is and what should be }\end{array}$ & 14 from 17 concepts \\
\hline & $\begin{array}{l}\text { Stimulates providers to ask questions about the gap in } \\
\text { performance }\end{array}$ & 12 from 24 concepts \\
\hline & $\begin{array}{l}\text { Develops a clear definition of the problem well } \\
\text { supported by the data }\end{array}$ & 11 from 23 concepts \\
\hline & $\begin{array}{l}\text { Helps to increase understanding of what is needed to } \\
\text { understand the problem }\end{array}$ & 10 from 22 concepts \\
\hline \multirow[t]{5}{*}{ Interviewee 2} & $\begin{array}{l}\text { Support practices to do the right things around the } \\
\text { evidence }\end{array}$ & 15 from 26 concepts \\
\hline & $\begin{array}{l}\text { Have data on key process measures where we know } \\
\text { those processes lead to clinical outcomes }\end{array}$ & 12 from 24 concepts \\
\hline & $\begin{array}{l}\text { Collect data to let us know whether or not we are } \\
\text { doing better }\end{array}$ & 11 from 23 concepts \\
\hline & $\begin{array}{l}\text { Pay more attention to getting the patient engaged and } \\
\text { activated }\end{array}$ & 11 from 21 concepts \\
\hline & Practices able to use data to see how they compare & 10 from 21 concepts \\
\hline \multirow[t]{5}{*}{ Interviewee 3} & $\begin{array}{l}\text { There are now a wider range of stakeholders, } \\
\text { including community and consumers }\end{array}$ & 7 from 13 concepts \\
\hline & The problem definition often shifts over time & 7 from 13 concepts \\
\hline & Engage people in the conversation & 7 from 11 concepts \\
\hline & Develop team-based care in a primary setting & 6 from 13 concepts \\
\hline & $\begin{array}{l}\text { Develop a consensus that we would want to work } \\
\text { together }\end{array}$ & 6 from 11 concepts \\
\hline \multirow[t]{2}{*}{ Interviewee 4} & Define your units of community & 13 from 22 concepts \\
\hline & Budget holding & 13 from 22 concepts \\
\hline
\end{tabular}


The community would hold all the budget

It is a community problem, therefore it has to be a community solution

Establish clinical governance for health and provision

\begin{tabular}{|c|c|c|}
\hline & Establish clinical governance for health and provision & 9 from 20 concepts \\
\hline \multirow[t]{5}{*}{ Interviewee 5} & Improve the provider, patient relationship & 18 from 31 concepts \\
\hline & We need multiple things to happen...one lever & 17 from 31 concepts \\
\hline & Change driven by the provider & 13 from 30 concepts \\
\hline & Change driven by the patient & 13 from 30 concepts \\
\hline & Effective management of LTCs may buy time & 13 from 26 concepts \\
\hline \multirow[t]{5}{*}{ Interviewee 6} & $\begin{array}{l}\text { Clinical leaders work with practices to troubleshoot } \\
\text { some of the issues }\end{array}$ & 15 from 29 concepts \\
\hline & Increased confidence and skills to make the change & 13 from 27 concepts \\
\hline & Able to target particular practices & 11 from 23 concepts \\
\hline & $\begin{array}{l}\text { Develop strong partnership between } \mathrm{DHB} \text { and } \mathrm{PHO} \\
\text { clinical leaders }\end{array}$ & 10 from 19 concepts \\
\hline & Programme not seen as being forced upon the practice & 10 from 23 concepts \\
\hline \multirow[t]{5}{*}{ Interviewee 7} & Attention is diverted away from the important stuff & 14 from 26 concepts \\
\hline & Develop a coherent model of care & 12 from 23 concepts \\
\hline & $\begin{array}{l}\text { The Ministry of Health needs to highlight priorities } \\
\text { that are not implemented }\end{array}$ & 11 from 21 concepts \\
\hline & $\begin{array}{l}\text { We need to focus less on services, such as wellness } \\
\text { checks, that are not delivering much value }\end{array}$ & 10 from 25 concepts \\
\hline & $\begin{array}{l}\text { Provide evidence that the process of change will } \\
\text { deliver outcomes }\end{array}$ & 10 from 21 concepts \\
\hline
\end{tabular}

12 from 26 concepts

10 from 17 concepts

from 20 concepts

18 from 31 concepts

31 concepts

15 from 29 concepts

.


There is no rationale, other than the need to focus this research, for picking the top five concepts from each interview, as opposed to the top six, or any other number of concepts. A check was done to see if any significant change in themes would occur if a greater number of concepts were included from each interviewee. To do this, a further centrality analysis was done to include the top seven concepts for each person, giving a total of 49 concepts in all. When this analysis was done, no new themes emerged. In deciding how many of the concepts to include in the thematic analysis a balance had to be struck between a focus on the concepts of most importance to the interviewees, and obtaining a rich understanding of all concepts. Looking firstly at the five most important, and then the seven most important, was an attempt to keep a degree of focus on what was most important, without narrowing the focus too much and ignoring concepts considered important by the interviewees.

The themes and their scoring under the two options are shown below:

\begin{tabular}{lll}
\hline Theme & Scoring of top 5 & Scoring of top 7 \\
\hline Performance Feedback & 6 & 8 \\
Engagement & 5 & 7 \\
Provider Performance & 5 & 7 \\
System Change & 5 & 6 \\
Clinical Leadership & 4 & 6 \\
Models of Care & 3 & 6 \\
\hline
\end{tabular}

Table 4-3 Key Themes Arising Out of Centrality Analysis

Having obtained the key themes from the initial interviews, the next step was to combine the data into an overall model that captured the concepts and their connections across all seven interviews. It was then possible to develop thematic maps, that describe the key concepts and their connections within each theme.

\subsection{Thematic Analysis Of Composite Map}

A major benefit of utilising the 'Decision Explorer' software is that it makes it possible to manage large amounts of qualitative data in a structured way. To do this the first step 
was to combine all the individual maps into one overall composite map, containing all the concepts and their links into one group map. This produced a map with 270 distinct concepts.

The second step was to go through each of the 270 concepts and code them into one or more of the six themes that emerged out of the analysis of the individual interviews. Maps were then created for each of the themes and each map was reviewed to merge concepts, where their meaning was the same. This resulted in the total number of distinct concepts reducing to 199 .

These thematic maps then became the component parts from which a system dynamics model was built to explore how, for example, the theme of engagement could be developed over time and how changes in the levels of engagement could affect the other five themes, and how together they could improve the care for people with chronic conditions.

The following section discusses these themes with the subsequent modelling discussed in the following chapter.

\subsubsection{Theme 1 Engagement}

Coding the concepts within the combined model resulted in 30 distinct concepts focusing on the theme of engagement. These are shown below:

1 initiative less likely to be seen as just another programme

13 more participation with the patient by doctors and nurses as team

14 building trust between the doctor and the nurse

22 makes sure everyone is on the same page

34 Develop strong partnership between DHB \& PHO clinical leaders

38 improved provider, patient relationship

72 used to treat patients' as pawns as well, simply telling them what to do

73 treat patients with sense of respect and mana, working for ... done to

77 changes the nature of the conversations between patient and provider to focus on joint problem solving

90 it is a community problem therefore it has to be a community solution

95 develop community support for patient self-management

101 develop common employment contracts and home base

102 people feel part of a team

108 provide space and time for community input

117 often have to pull together people to work together who are all working under different employment contracts and different employers 
119 the lack of a shared budget means it disintegrates at the slightest change

133 define the problem in a way that people can relate to

138 develop an understanding of the environment for clinicians to believe that, "I can only solve this by working with you"

145 engagement is the key issue

151 pay more attention to getting the patient engaged and activated so that they can do more on their own

153 patients become aware that they are in a different sort of programme that involves a 'contract'

154 patients adhere better to the treatment recommendations medication, diet etc.

156 need to be explicit in 'signing up patient' to the deal involved in CCM

158 programme rules can be a barrier to clinician engagement

168 work with key groups to come up with solutions ... bringing in solutions from somewhere else

184 develops the engagement of clinicians and other providers

188 develops a momentum for change amongst providers

189 stimulates providers to ask questions about the gap in performance

206 support making the data accessible to the public

207 public able to see the gap between 'what is' and 'what could be'

This is the numbers on the left-hand side are used by the Decision Explorer software to identify each concept. Furthermore, the use of "..." in the concept i.e. 'working for...done to' is used in the software as shorthand for the phrase 'rather than'. So, the phrase would read 'working for rather than done to'. It is often used to clarify the concept by comparing it with its opposite.

The centrality analysis conducted on all 30 concepts, within the engagement theme, resulted in concept 22, "makes sure everyone is on the same page", being the most central. The second and third most central concepts however highlight that this theme is focusing on the relationships between providers and patients, who are the key groups that need to be 'on the same page'. The second most central concept was concept 184, "develops the engagement of clinicians and other providers". The third was concept 38, "improved provider patient relationship". The centrality analysis thus highlighted the two key clusters, i.e. concepts linked together, within the engagement theme. These were the engagement of clinicians and the engagement of patients and the communities within which they lived. Together they interact to change the nature of the relationships between provider and patient. This is consistent with the original formulation of the CCM model by Wagner and highlights that fact that it is the relationship, rather than specific characteristics of clinicians or patients that is the crucial element. 
It should be noted that the centrality analysis incorporates all of the 199 concepts in the composite model. So, when the software undertakes the centrality analysis on the 30 concepts in the engagement map it includes concepts outside of that group in calculating the centrality score. The following figures show the engagement map. Figure 4-6 shows the map of all the concepts categorised within the engagement theme. Concept 22 is clearly not that central when only the concepts categorised within the engagement theme are shown. However when the concepts outside of the 'engagement theme' (shown in larger grey font), which are linked to concept 22 are shown (figure 47), its centrality becomes more apparent. This highlights the connectivity across the key themes and emphasises the importance of understanding the relationships between factors and not just the factors themselves. This is common in the thematic maps that follow. Those concepts with high centrality scores often do not 'seem' to be central when one looks at the thematic maps. This is because the density of their connections, as shown in figure 4-7, is often driven by concepts outside of the central theme, further emphasising a central idea in this thesis that 'factors' identified as important are in reality embedded in a rich context of meaning, and teasing out this context is important for understanding the dynamics of implementation. 


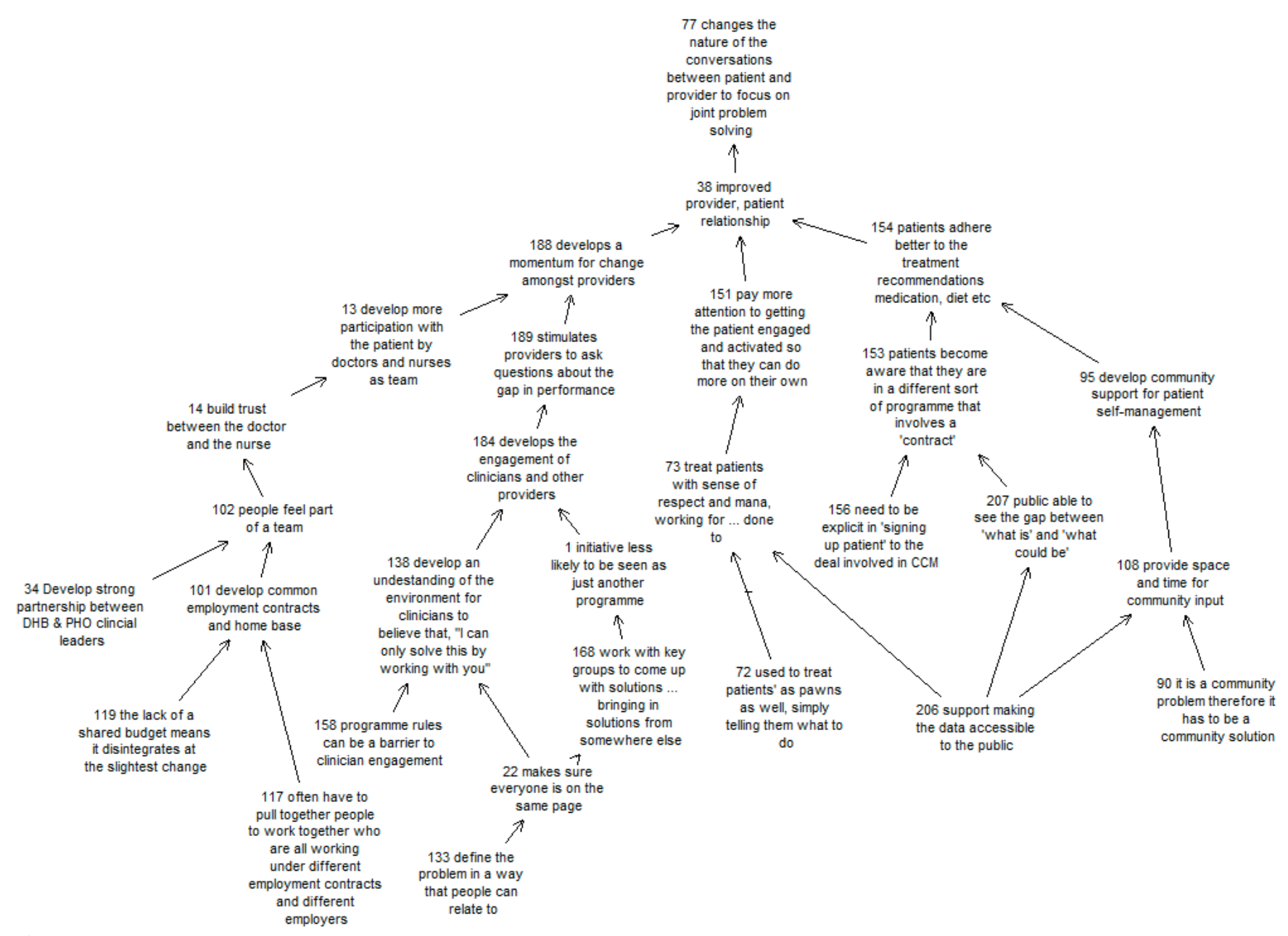

Figure 4-6 Engagement map 


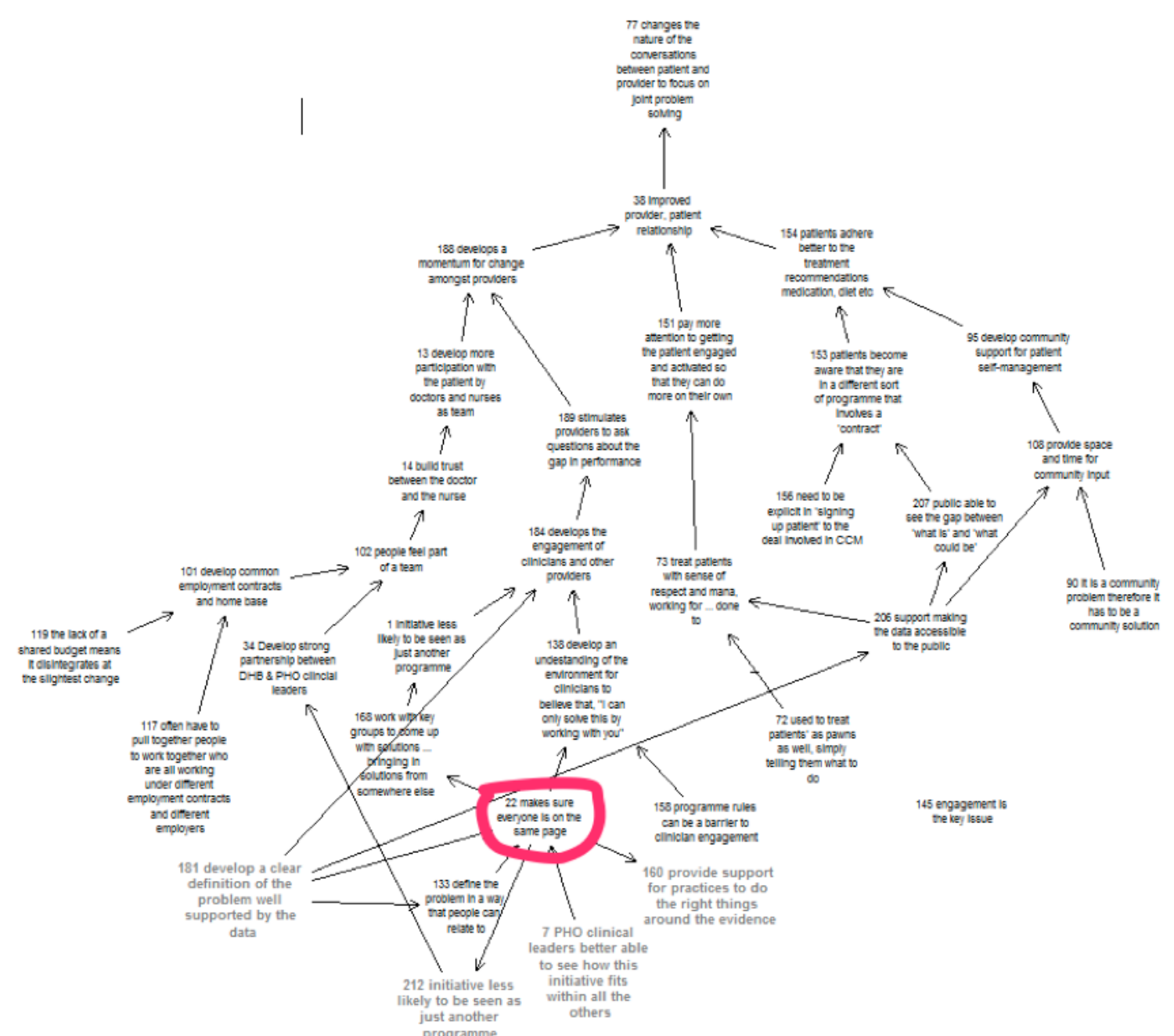

Figure 4-7 Additional concepts linked to concept 22 
A closer look at the map, shown in figure 4-7, shows if 'every one is on the same page' then it will be easier to develop solutions internally, rather than bringing them in from the outside (concept 168), which, in turn, will help clinicians see it as more than 'just another programme' (concept 1), further developing their engagement (concept 184). This then develops a more critical view of gaps in performance (concept 189) that, in turn, helps to develop a momentum for change (concept 188). It is this momentum that contributes to improving provider-patient relationships (concept 38). However, as highlighted in Wagner's original model, and in the centrality analysis noted above, providers are only one half of the therapeutic relationship and the theme of engagement also pinpoints patient engagement as a key element. Once treated and respected as 'partners' (concept 73), patients are more likely to take on more responsibility for their own care (concept 151) and, if they have some support (concept 95) as well as being aware of the nature of the programme itself (concept 153) are more likely to adhere to treatment recommendations (concept 154).

The main ideas highlighted in the engagement map are therefore the importance of engaging clinicians and patients and finding ways to plan and design the programmes in a collaborative manner, whether that is collaboration between planners and clinicians, between clinical groups, or between clinicians and patients. The important consequence of this engagement is an improved relationship between the provider and their patients, resulting in joint efforts and accountabilities to address the key concerns.

\subsubsection{Theme 2: Performance Feedback}

The theme of performance feedback contained 32 distinct concepts:

6 PHO clinical leaders have a better understanding of the population needs 17 develop understanding of what works for practice populations and what doesn't

19 able to ask clinical questions about what is being done for particular patient groups

26 obtaining comparative data helps us understand the practice population

27 data provides a baseline against which improvement can be assessed

28 able to see who is achieving their KPIs and who is not

29 able to target particular practices

33 data can then be used to provide more informed conversations about what the patient needs

39 provides ability to demonstrate that things are getting better or worse in our communities

133 define the problem in a way that people can relate to 
134 the problem often looks different from different perspectives

143 enables practices to know how what they do compares with others

147 ability to respond to what the information is telling us

159 there are some bottom line things - the evidence is clear that this is what needs to happen

165 capture data on key process measures where we know those processes lead to clinical outcomes

166 get the feedback loops working so that the data is interrogated

167 reinforces what the value of the data is

179 enables discussions about the real issues eg why patients are not taking their pills

181 develop a clear definition of the problem well supported by the data

183 good understanding of community health need

186 helps to increase understanding of what data is needed to understand the problem

202 helps improve the quality of the data

220 able to ask clinical questions about what is being done for paticular patient groups

241 provide the information to those who are trying to improve outcomes?

244 pragmatic difficulty is that with the information available it is hard to see if we are improving

247 better understanding of what is needed to make an impact?

248 help us understand the structure, processes and outcomes needed to bring about the change

255 evidence for improvements in LTC takes a long time to accumulate

256 because of time lag in LTC the focus is often on the easiest thing to fix

257 attention is diverted away from the important stuff

258 what may make things worse in the short term delivers benefits in the long-term

2267 make diagnostics available to patients

The map containing these concepts is shown below in figure 4-8. 


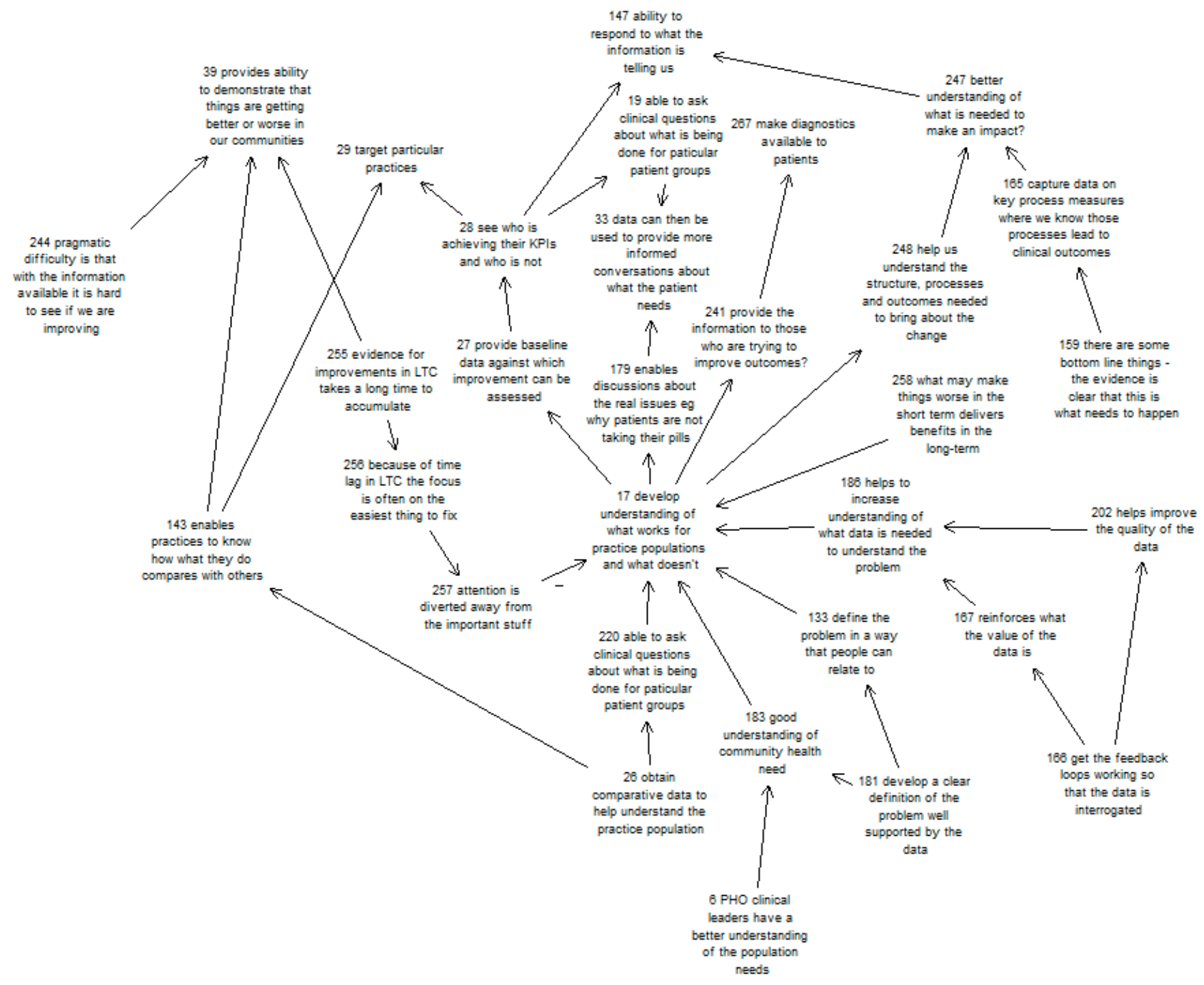

Figure 4-8 Performance feedback map 
The centrality analysis of the performance feedback map highlighted the importance of information to inform practices about their performance and to develop an understanding of what works and what does not. The most central concept was concept 181, "develop a clear definition of the problem well supported by the data". The second most central concept was concept 147, "ability to respond to what the information is telling us". The third most central concept, concept 202, focused on the quality of the data itself, "helps improve the quality of the data". The clear focus here is on quality data being linked to the problem being addressed and, importantly, having the ability to respond to what the data is saying.

To reinforce the point that the centrality score incorporates concepts outside of the central theme, the following map includes the key connections to concept 181, "develop a clear definition of the problem well supported by the data" which had the highest centrality score in the performance feedback map yet did not 'seem' to be that central by a simple visual check. As the following map figure 4-9 shows, many of its connections were with concepts not categorised within the theme of performance feedback. 


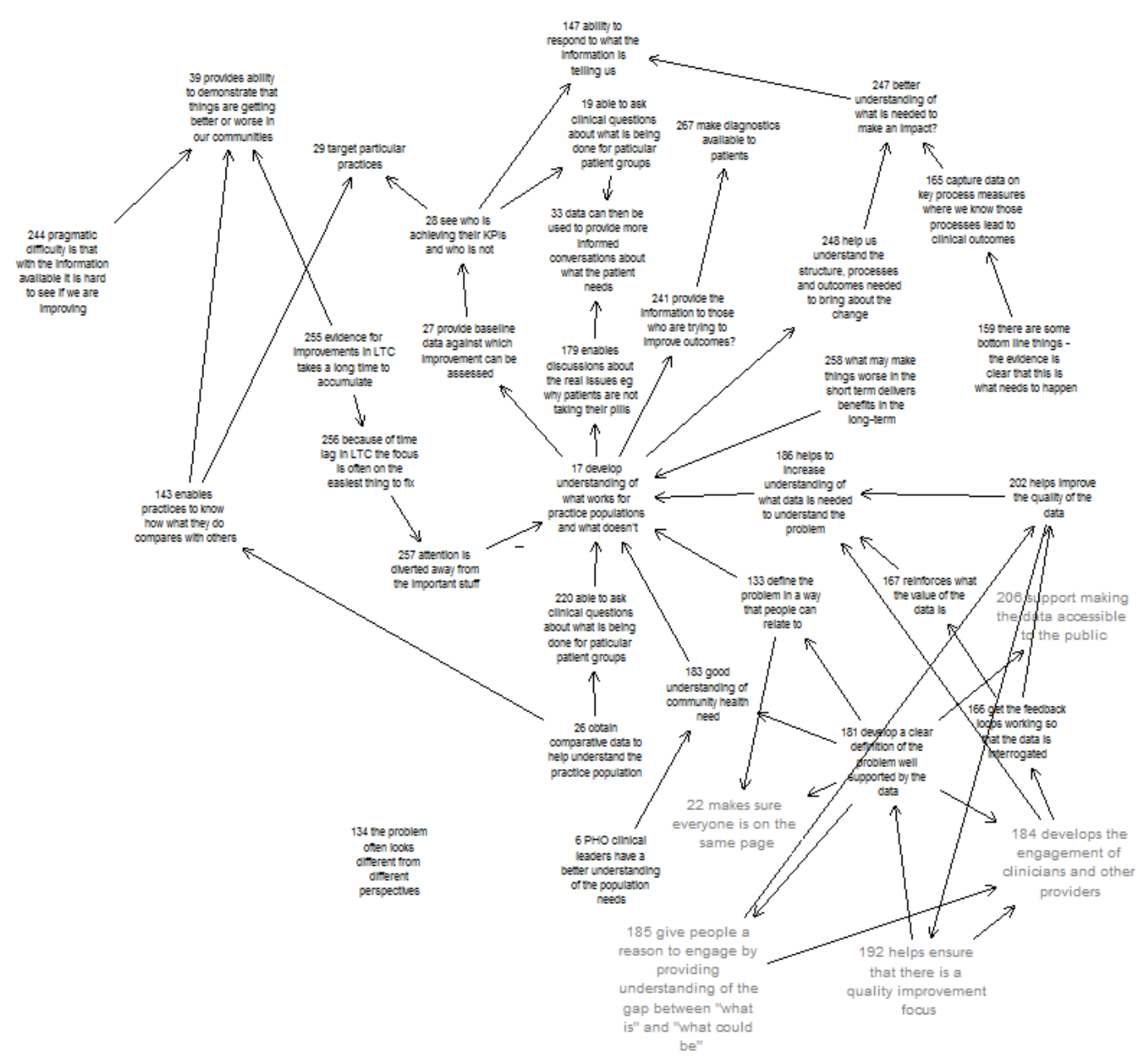

Figure 4-9 Concept 181 shown with connections outside the performance feedback theme 
This theme is, therefore, very much about ensuring practices are provided with data that confronts their performance, highlighting how well, or not, they are doing to deliver improved health outcomes for patients with chronic conditions. A consequence is that the, "data provides a baseline against which improvement can be assessed", concept 27. In addition, this theme also emphasises information that provides insight into what process actually work and which do not. For example, concept 165 emphasises how important it is to "capture data on key process measures where we know those processes lead to clinical outcomes".

Thus the theme of performance feedback focuses on the collection and use of quality data and is closely linked to theme one, in that feedback of performance data, as noted in the discussion on engagement, is seen as a major element in developing clinical engagement and driving improved performance.

\subsubsection{Theme 3: Provider Performance}

The provider performance theme contained 34 concepts:

2 there is a lot of programme fatigue in the practices

3 identify roles and responsibilities within the practices

5 develop implementation plans at a practice level

8 every practice will have different issues to deal with

12 uptake of modules is very good

23 make sure there are adequate people on the ground to support practices

25 programme becomes 'business as usual'

30 there are competing priorities and resource constraints

35 increased confidence and skills to make the change

51 give providers exposure to different ways of working

52 provide good decision support tools

54 provide 'best practice' bulletins

58 need to get change at that front-line practice

59 there are many and varied providers

61 put majority of focus on the provider

62 provider personalised feedback on how they are doing with their patients

63 forums where they can discuss the feedback

64 give providers a sense that they are being rewarded for good practice

124 develop better primary care

148 there are huge training issues to get the prepared proactive team

149 training to cover clinical skills

150 training to cover self management skills

155 not getting the outcomes we would expect to see 
160 provide support for practices to do the right things around the evidence

175 provide clinical resource to facilitate across the practices

176 dramatically improved outcomes

177 close the treatment gap so that the right thing is happening

188 develops a momentum for change amongst providers

192 helps ensure that there is a quality improvement focus

193 helps to minimise wasted activity

208 providers perceived loss of reputation

243 small improvements each year could reverse worst to the best

245 learn to discriminate within the small range of improvements to learn quickly

261 it is harder to put up with short term pain for long-term impact

The map for this theme is shown in figure 4-10. The centrality analysis revealed that the key ideas about provider performance focused on support for practices to make the changes. The most central concept was concept 160, "provide support for practices to do the right thing around the evidence". The second was concept 35, "increased confidence and skills to make the change" and the third was concept 177 "close the treatment gap so that the right thing is happening".

Thus, the provider performance theme describes the focus of performance, namely the need to close the 'treatment gap' but also highlights that to achieve this, providers are going to need support to do so. In fact 18 of the 34 concepts within the provider performance theme focused on either the support that providers would need, or the challenges that providers face in bringing about the improvements. Concept 30, for example, noted that "there are competing priorities and resource constraints", while concept 148 identified that there are "huge training issues to get the prepared proactive team". Developing this performance therefore requires i) training, for example concept 149, "training to cover clinical skills", ii) information, for example concept 52, "provide good decision support tools", and iii) resources, for example concept 23, "make sure there are adequate people on the ground to support practices". 


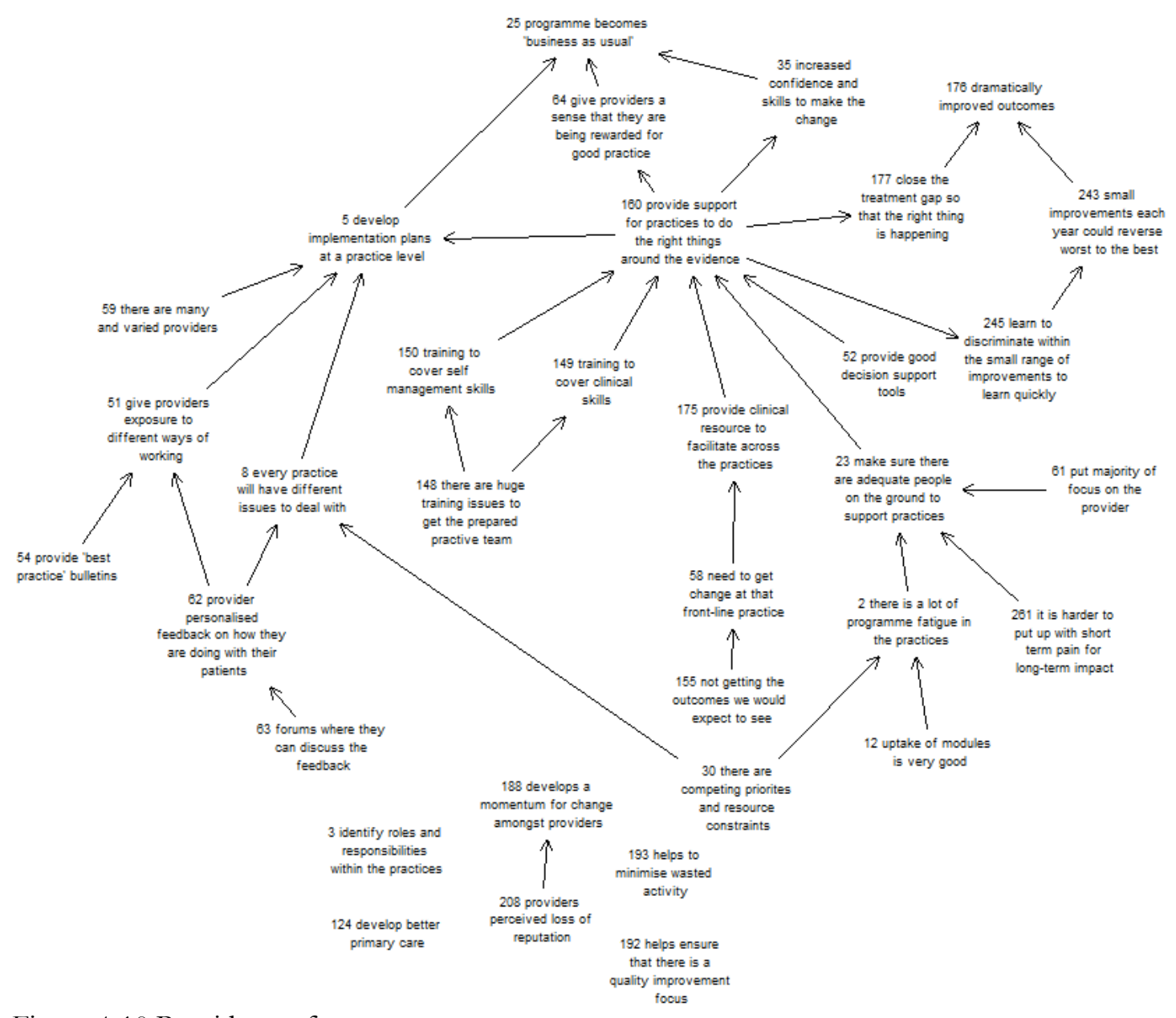

Figure 4-10 Provider performance map 


\subsubsection{Theme 4: System Change}

The system change theme contained 30 distinct concepts:

40 we need multiple things to happen ... one lever

57 changes are often driven by ideology hence the focus on structure

58 need to get change at that front-line practice

61 put majority of focus on the provider

64 give providers a sense that they are being rewarded for good practice

80 formalised structure e.g. computer recalls

85 more we focus on secondary to primary shift the more we will overload primary care

86 primary care will suffer the tyranny of the urgent

87 don't believe it actually frees up a bed

98 budget holding

101 develop common employment contracts and home base

104 integration ... primary and secondary divided

105 shared services

106 GP property holding

110 deciding the size of the village is a business decision

111 the units of behaviour change need to be smaller

112 to form your units you look at geography and demographics

113 define your units of community

115 the community would hold all the budget

116 the old concepts of primary and secondary care are irrelevant for LTCs

117 often have to pull together people to work together who are all working under different employment contracts and different employers

118 strong capital investment in the current system can impede change

119 the lack of a shared budget means it disintegrates at the slightest change

128 \$ in primary care is no longer a motivation

129 change is no longer about entrepreneurial zeal

130 focus on whole system change

131 in LBD for example the GP is no longer in the lead

$132 \mathrm{MoH}$ sees the NZ health system as isolated islands of business

139 strongly held view that hospitals can't keep growing

170 flexible IT that makes the right thing the easiest thing to do

The map of system change, shown in figure 4-11, is less coherent than the others, having a number of seemingly discrete concepts, making it difficult to tease out the focus of ideas within the overall theme. However, the centrality analysis did highlight an interesting pattern. The most central concept, which had a significantly higher score that the others, was concept 40, 'we need multiple things to happen rather than use one lever'. Following that were a number of initiatives each of which could be considered 


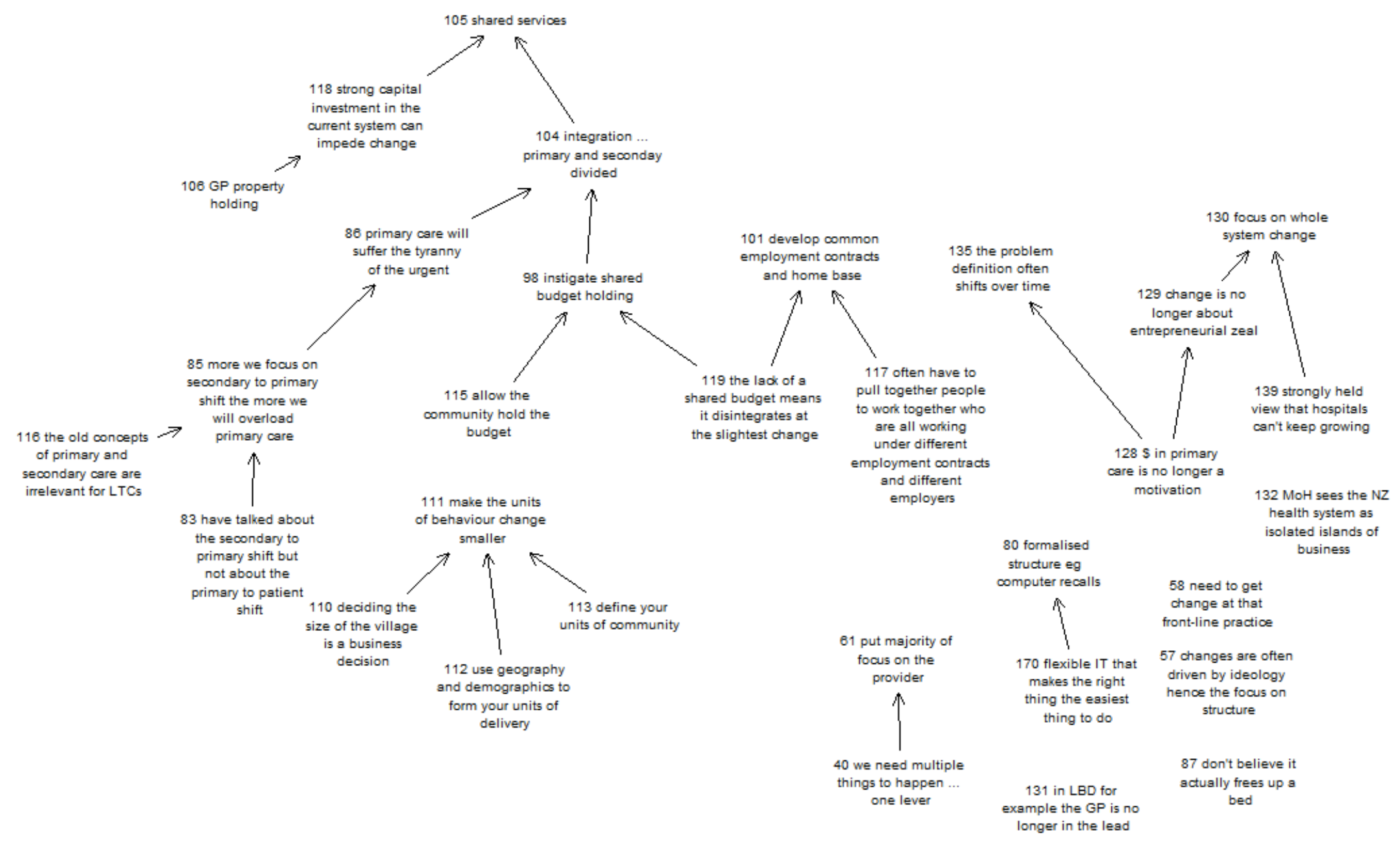

Figure 4-11 System change map 
one of the 'multiple things'. For example, concept 170 talked of the need for 'flexible IT'. Concept 80 asked for more formalised structures such as computer recalls. Concept 113, required a better definition of the 'units of community' and concept 105 called from more 'shared services'. Thus, while there were not a lot of linked concepts the message was strongly that there is no one thing that will bring success and any approach has to be multi-pronged. In many ways it is this theme that argues for the systemic approach that is being developed in this thesis. Making the changes necessary for practices to effectively deliver programmes for people with chronic conditions will require a mix of initiatives. Understanding these initiatives and how they interact with each other over time is a central focus of this thesis.

\subsubsection{Theme 5: Clinical Leadership}

While clinical leadership was stated by as important by the interviewees the detailed coding resulted in only 9 concepts. These were:

$6 \quad$ PHO clinical leaders have a better understanding of the population needs

$7 \quad$ PHO clinical leaders better able to see how this initiative fits within all the others

11 clinical leaders able to connect into secondary care and other areas of support for practices

34 develop strong partnership between DHB \& PHO clinical leaders

76 there is not always strong clinical leadership in the PHOs

100 establish clinical governance for health provision

142 create clinical leaders

174 identify internal champions who are able to get the resources required to run the programme

221 ensure clinical leaders work with practices to troubleshoot some of the issues

The map for clinical leadership is shown below in figure 4-12. The most central concept within this theme focused on what was wanted of clinical leaders, namely that they worked, "with the practices to troubleshoot some of the issues" (concept 221). The second most central concept (concept 100), focused on how such leaders were to be developed, through the establishment of 'clinical governance'. The third most central (concept 142), simply asserted the need to, "create clinical leaders". 
The small number of concepts may indicate that clinical leadership is considered axiomatic. It has to be there and little more needs to be said. So, whilst clinical leadership is considered important, with only nine concepts, the interviews shed very little light on what it involved and what was required to develop it. Exploring this theme, within the broader context of the themes discussed here may be a useful avenue for future research. 


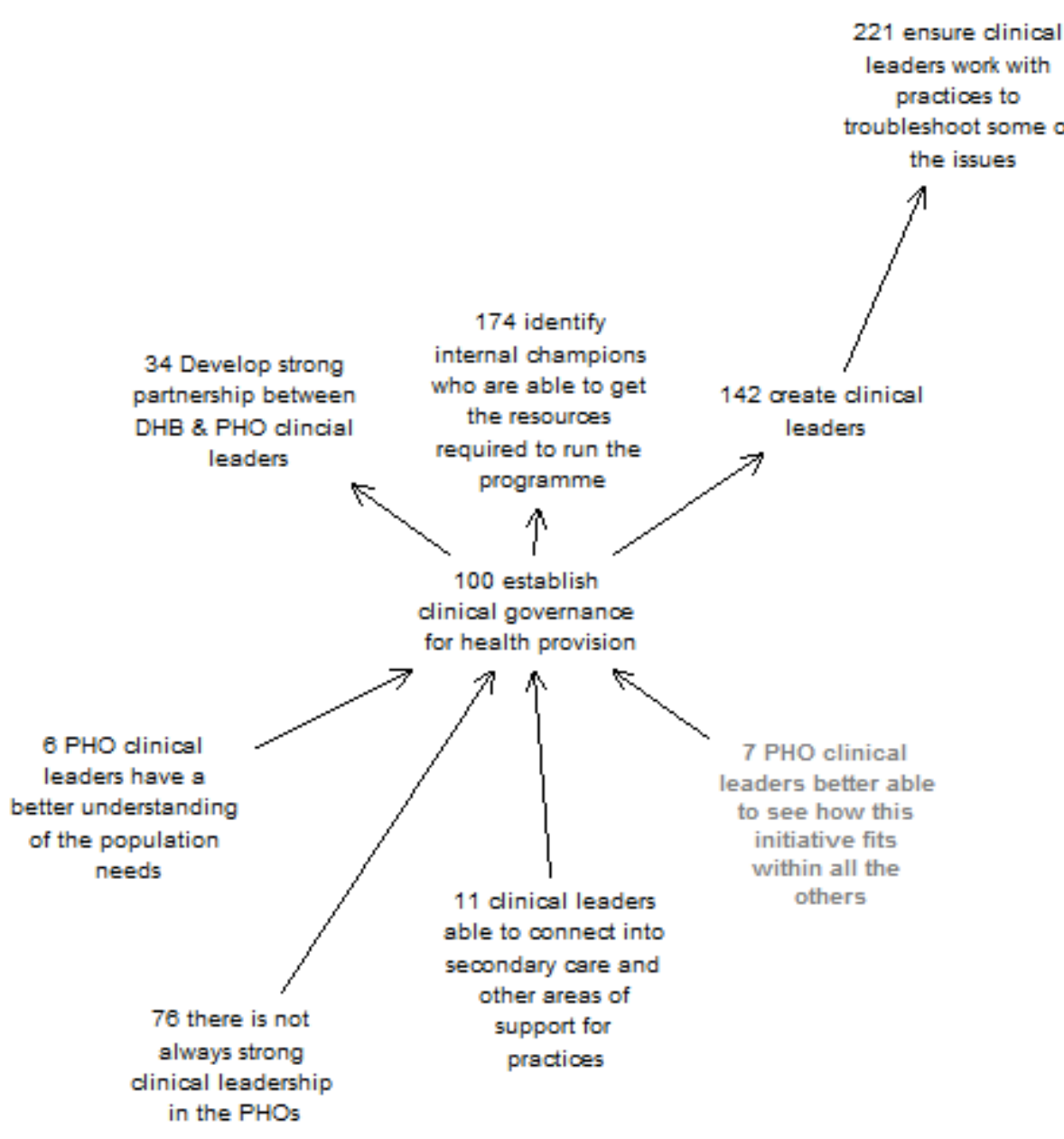

Figure 4-12 Clinical leadership map 
4.4.6 Theme 6: Models of Care

Models of Care contained 33 distinct concepts. These are:

17 develop understanding of what works for practice populations and what doesn't

24 leads to frustration and it is not too long before people throw up their hands - it is too hard, I don't want any of this

25 programme becomes 'business as usual'

44 the beauty of LTCs is that you have time, time to experiment

45 development of self management

47 If case managers simply help patients navigate a complex system it's simply a sticking plaster

48 use case managers to simplify the system through clever design

49 not convinced that you have a big impact on acute demand

80 formalised structure eg computer recalls

82 in terms of sustainability self care is the only option

83 have talked about the secondary to primary shift but not about the primary to patient shift

85 more we focus on secondary to primary shift the more we will overload primary care

86 primary care will suffer the tyranny of the urgent

94 long term conditions are about human behaviour and social justice ... the modern hospital which is about 'widgits' and an industrial process

96 long term conditions require a different attitude on the part of providers

97 long term conditions require different training as social director ... widget producer

111 make the units of behaviour change smaller

128 \$ in primary care is no longer a motivation

131 in LBD for example the GP is no longer in the lead

140 develop team-based care in a primary setting

146 important to have a structured programme

151 pay more attention to getting the patient engaged and activated so that they can do more on their own

159 there are some bottom line things - the evidence is clear that this is what needs to happen

240 models, such as Wagner, make intuitive sense but how do they deliver improved outcomes

248 help us understand the structure, processes and outcomes needed to bring about the change

250 do we have any evidence that we do need a model of care

251 develop a coherent model of care

252 describe which people will deliver the services

263 we have to devolve care into primary and community care

264 we have to devolve primary care into families and communities

265 devolving care means self-care rather than GPs etc

266 need to start treating individuals and whānau as part of the workforce

269 we need to focus less on services, such as wellness checks, that are not delivering much value

The Models of Care map is shown below in figure 4-13. 


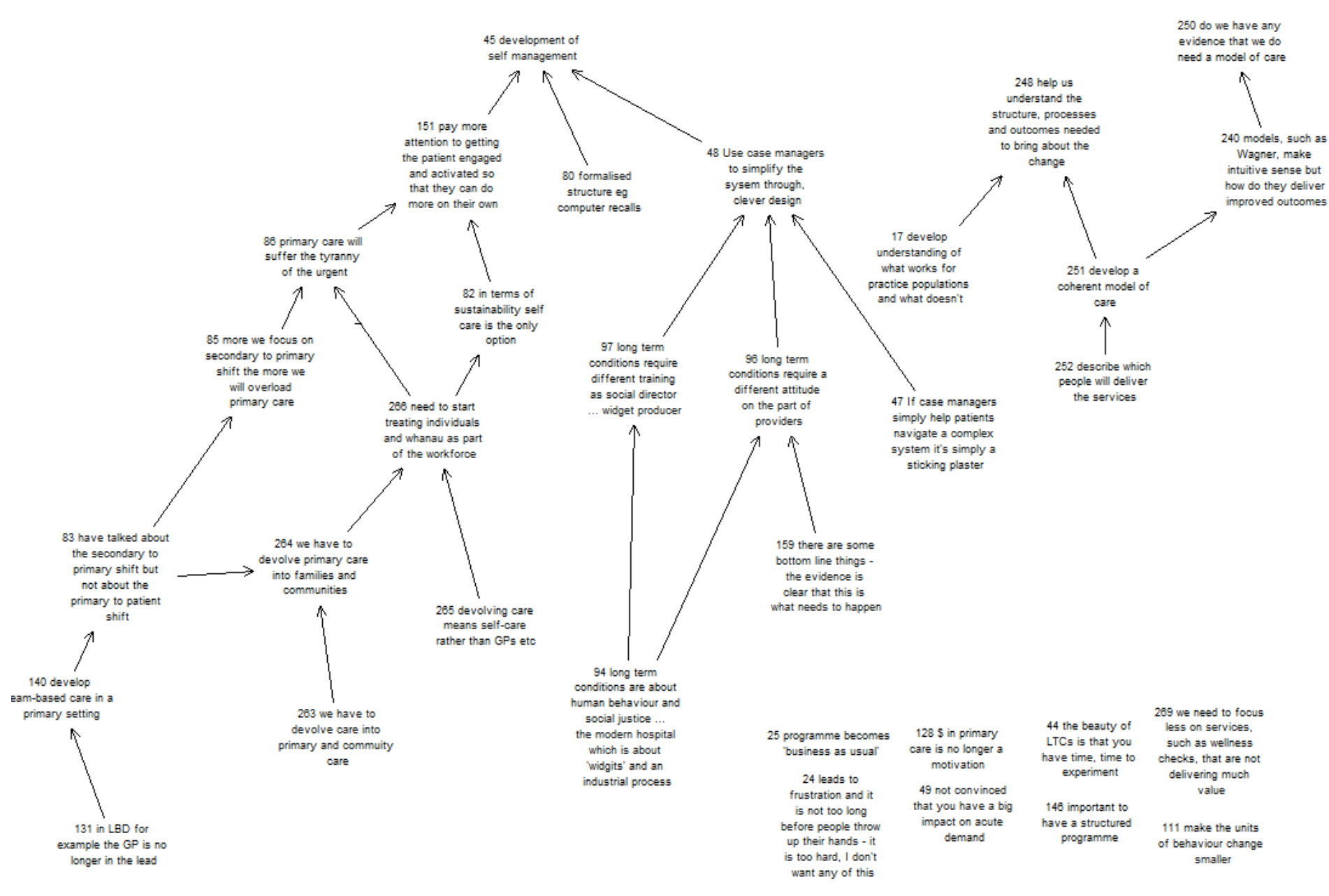

Figure 4-13 Models of care map 
The third most central concept resulting from the centrality analysis is the simple assertion that there is a need to, "develop a coherent model of care" (concept 251). The first and second most central concepts however reveal key characteristics of this coherent model, namely that it is based on an "understanding of what works for practice populations and what doesn't" (concept 17) and that it must, "pay more attention to getting the patient engaged and activated so that they can do more on their own" (concept 151). These two concepts also sit within the themes of 'performance feedback' and 'engagement'.

Looking closely at the concepts within this theme it emerges that 11 of the concepts relate to the behaviours of both providers and patients, both in terms of what is needed on the part of providers, for example concept 96, "long term conditions require a different attitude on the part of providers" and what is needed on the part of patients, for example concept 45 , "development of self-management". 


\subsection{Summary}

What this chapter has tried to do is describe how the thinking of seven health experts in the field of planning and implementing chronic care models in New Zealand was used to develop an understanding of the key concepts that are central to a theory of implementing chronic care programmes. The interviews with the health experts, using the cognitive mapping approach, and the subsequent development and analysis of the maps that emerged from those interviews, highlighted six themes that point to important ideas about implementing new health programmes designed to improve care for people with chronic conditions. These themes, which are not about the programmes themselves, but what is required if such programmes are to be implemented are: performance feedback, engagement, provider performance, system change, clinical leadership and models of care. As has been discussed earlier, while chronic care programmes such as the CCM model of Edward Wagner (1996) describe in detail the factors required in any programme designed to provide good chronic care, little is known about how to implement these programmes successfully. In this chapter I have begun to elicit what some of the key factors involved in effective implementation are, and how they connect to and influence each other. While these factors overlap with other research looking at care for people with chronic conditions, such as the work of Connolly and his colleagues (Connolly et al., 2010), the use of Cognitive Mapping has helped to tease out the web of interconnected factors that give them a much more context-rich meaning.

To provide a useful theory of implementation, however, it will be necessary, on the one hand, to stand back from the detail of 199 distinct concepts, and focus on the most central concepts within each theme described above, and on the other hand, establish more clearly the causal connections that exist within and between them.

The next chapter focuses on exploring these interactions in more detail. Using the insights of the health experts and the relevant literature, chapter 5 builds a coherent theory of implementation. 


\section{Causal Loop Modelling Developing A Theory of Implementation in Primary Care}

Chapter 4 described the problem structuring phase within the research method outlined in chapter 3 , in which seven health experts were interviewed using the cognitive mapping technique to tease out their understanding of the key issues involved in implementing new models of care for chronic conditions. The results of these interviews were then mapped into a number of key themes. The next step in formalising the theory of implementation, covered in this chapter, is to relate the key variables together, within and across these themes. The result of this work is a set of interlinked feedback loops that represent the processes involved in developing effective management of chronic conditions. This chapter therefore describes the development and evaluation of a theory of implementation. Unlike many of the theories of implementation discussed in chapter 2 , the theory described in this chapter makes explicit the causal connections and how differing contexts influence those connections.

The first section of this chapter describes the feedback perspective used to formulate the model and the second section describes the development of the model itself. Section 3 of this chapter describes the process used to develop confidence in the model. Chapter 6 then takes it one step further by developing a fully quantified simulation model, the results of which are discussed in chapter 7.

In relation to the research methods outlined in chapter 3 , this chapter covers phase 2 : qualitative mapping. However, before describing the development of the model, the next section describes the feedback perspective that underpins it.

\subsection{The Feedback Perspective}

The feedback perspective, which underpins the modelling approach used in this thesis, provides an approach to understanding implementation that focuses on the causal mechanisms at play. As pointed out in chapters 2 and 3, much of the implementation literature in general, and the literature on CCM more specifically, focuses on identifying 
important factors such as the funding mechanisms and rules (Wagner et al., 2001), visionary clinical leaders (Bodenheimer, Wagner, et al., 2002b), support for patient self management (M. T. Coleman \& Newton, 2005) and proper management of healthcare structures and processes (Rea et al., 2007). What these studies do not do, to the same extent, is explore the interactions between these factors and how they influence each other over time. As pointed out by Hovmand and Gillespie (2008), most studies of implementing new evidence-based practices focus on describing the factors, such as clinical leadership, that increase or decrease implementation success but stop short of describing the causal mechanisms that link clinical leadership that the success rate of implementation endeavours.

What the feedback perspective does is enable the researcher to stand back from the specific details of these individual factors to describe the dynamic interdependencies at play and which ultimately govern the implementation process. To illustrate its application I develop below a qualitative feedback model, also referred to as Causal Loop Diagrams (CLDs) (Maani \& Cavana, 2000; Wolstenhome, 1990), that describes the key tension in providing care for people with chronic conditions that the CCM model is trying to address. This will help, not only to introduce the reader to the specific method being used to develop the qualitative model, but also put the model itself into the context of the challenge of effectively managing chronic conditions.

5.1.1 The CCM Model: a Feedback Perspective

As discussed in chapter 2, the CCM model, developed by Edward Wagner (Wagner et al., 1996), is an attempt to shift primary care away from a focus on acute episodes of care to an ongoing, proactive management of chronic conditions (Bodenheimer, Wagner, et al., 2002a). That is, it aims to shift the focus of clinical interventions from the immediate, acute symptoms being presented by patients, to the underlying chronic conditions that often cause, exacerbate and prolong the symptoms being presented. What the CCM model is trying to bring about is a shift away from the 'tyranny of the urgent'. The core dynamic involved in this is shown in figure 5-1, based on the work of Wagner and Bodenheimer. For example see Bodenheimer et al., (2002a). 
Although it is possible to start the 'story' of CCM embedded in figure 5-1 at any point, the description below starts with the patient.

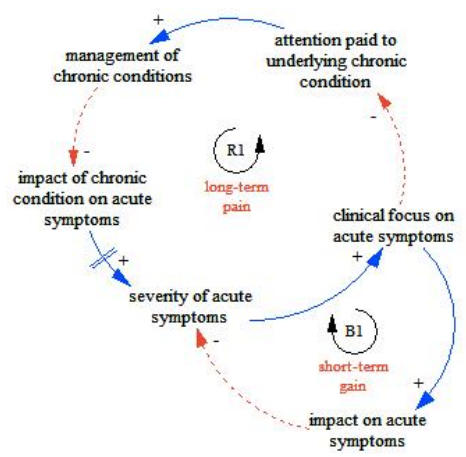

Figure 5-1 Managing the tension between chronic and acute care

An important concept in this model is the link between symptoms and the clinical response to them; in this case the severity of those symptons driving an increasing clinical focus on those symptoms. In the model this is shown by the arrow from 'severity of acute symptoms' to 'clinical focus on acute symptoms'. What this link is saying is that as the 'severity of acute symptoms' increases (or decreases), the 'clinical focus on acute symptoms' also increases (or decreases). This is a positive link, denoted by the ' + ' sign at the arrowhead. This shows that the more severe the presenting symptoms, the more focus the clinician is going to put on them. With positive causal links more of one leads to more of the other and, conversely, less of one leads to less of the other. Following the loop around clockwise (B1), the greater the focus on acute symptoms, the greater the impact on those symptoms, thereby reducing their severity. The link between impact on acute symptoms' and 'severity of acute symptoms' is a negative link and denoted by the dashed line and the '-' sign at the arrowhead. With negative causal links more of one leads to less of the other and conversely, less of one leads to more of the other. Following the story around the other loop (R1), the greater the 'clinical focus on acute symptoms', the less 'attention paid to underlying chronic condition' leading to less effective 'management of the chronic condition', which then increases the impact of chronic conditions on acute symptoms'. Over time, indicated by the delay mark - two parallel lines across the arrow - this leads to further development 
of acute symptoms, driving a further clinical response to them. When these causal links loop back to influence the starting variable a feedback loop has been created.

There are two feedback, which capture the behaviour generated by the set of causal links, in this system. Starting from any variable the behaviour of the loop is established by tracing through the effects of each link until a circuit is completed. If the net effect is to counteract the initial change, the loop is balancing, also referred to as negative, and is denoted by a 'B' in the diagram. For example, an increasing 'clinical focus on acute symptoms', leads to an increasing 'impact upon acute symptoms'. This in turn decreases the severity of the acute symptoms', which loops back and decreases the 'clinical focus on acute symptoms'. Thus, an initial increase in the 'focus on acute symptoms', through a set of linked causal connections, feeds back to bring about a decrease in this focus, by decreasing the severity of symptoms being presented by patients. Simply put, this means that if the clinician treats the symptom, the patient no longer needs to come back to the practice requiring treatment for that symptom. This then is a balancing, or negative, feedback loop in which an initial change in one direction is counteracted upon to bring the system back into balance. To describe this dynamic I have called the feedback loop 'short-term gain'. There is no doubt that by focusing on the symptom, the patient will, assuming the efficacy of the treatment, be relieved, at least in the short-term, of that symptom.

If the net effect is to reinforce an initial change in the chosen variable, the loop is reinforcing, sometimes referred to as a positive feedback loop, and is denoted by ' $R$ ' in the diagram. So, for example, the increased 'clinical focus on acute symptoms' also leads to less 'attention paid to the underlying chronic conditions', leading to less effective 'management of the chronic condition'. Over time, this leads to increased 'severity of acute symptoms', leading to even more 'clinical focus on acute symptoms'. Thus, a greater focus on acute symptoms leads to a dynamic that drives an increasing focus on acute symptoms over time. As a result the short-term gain of responding to symptoms leads to the 'long-term pain' of their re-occurrence, hence the name 'long-term pain' given to this feedback loop. In this loop the focus on the acute symptoms actually increases the severity of the symptoms over time. 
Thus, within this dynamic there are two opposing forces which impact upon the 'severity of acute symptoms' and the 'clinical focus on acute symptoms' designed to address them. To provide a simple illustration, treating a toothache with Panadol, or other similar pain relief, is an example of focusing on the acute symptom to relieve its severity. That is fine, and it will reduce the severity of the presenting symptom, but if that continues without addressing the underlying 'chronic condition', which may, for example, be gum disease, the toothache returns, often more painfully and with more severe consequences. Many 'off-the-shelf pharmaceutical treatments, such as pain relief and cold remedies, follow this pattern by treating the presenting symptom, but doing nothing to address the underlying causes. For some conditions, such as the common cold, this is fine, but for others, such as the long-term conditions which are the focus of this research, unless they are complemented with interventions targeting the cause they can contribute to behaviours, on the part of the patient and the clinician, that, over the long term, make the condition much worse.

This dynamic interplay between 'short-term gain' and 'long-term pain' is at the heart of the implementation challenge. The two feedback loops are linked by a common resource - clinicians and patients - and in reality are far more complex than shown in this model, with more variables, interacting over time, shifting the balance between the two feedback loops. For example, even in primary care practices with the commitment and resources to improve care for those with long-term conditions, the seasonal rise in the number of patients with cold and flu symptoms often takes up so much resource that little else can be done other than respond to the immediate needs of acute patients.

When seen from this feedback perspective, the purpose of chronic care management is to shift the balance between these two feedback loops so that the management of chronic conditions can, over time, reduce the clinical focus on acute symptoms and thereby create a self-reinforcing pattern of behaviour that reduces the severity of symptoms experienced by patients over time. Much of the writing and research on chronic care management is about the factors that can bring about this shift (for an example see Epping-Jordan et al., (2004)). The model, developed below, based on the views of the health experts described in chapter 4 , is an attempt to develop a coherent 
theory of what is involved in bringing this shift about by focusing on the causal mechanisms that are at play.

\subsection{The Model of Implementation}

To develop this model the question that was addressed in the interviews with health experts was about the issues and challenges involved in implementing 'effective management of chronic conditions'. What the previous chapter described was the key themes that emerged out of those interviews. This chapter takes the next step and interrogates the perspectives of the health experts in more detail to ascertain their theories about how to bring about more effective management of chronic conditions. I will do this by working through each of the themes and tease out their causal patterns in more detail to develop a causal model that captures their theory about effective implementation of programmes for chronic care management.

The key themes that make up the model, and the concepts within them that were considered most important by the interviewees are:

\begin{tabular}{ll}
\hline Theme & Concepts Considered Most Important \\
\hline Engagement & $\begin{array}{l}\text { makes sure everyone is on the same page } \\
\text { develops the engagement of clinicians } \\
\text { improved provider patients relationship }\end{array}$ \\
\hline Performance Feedback & $\begin{array}{l}\text { develop a clear definition of the problem well supported by the } \\
\text { data } \\
\text { respond to what the information is telling us } \\
\text { improve the quality of the data }\end{array}$ \\
\hline $\begin{array}{l}\text { Provider Performance } \\
\text { evidence } \\
\text { increase confidence and skills to make the change } \\
\text { close the treatment gap so that the right thing is happening }\end{array}$ \\
$\begin{array}{l}\text { ensure clinical leaders work with the practices to troubleshoot } \\
\text { some of the issues } \\
\text { establish clinical governance for health provision } \\
\text { create clinical leaders }\end{array}$ \\
\hline $\begin{array}{l}\text { develop a coherent model of care } \\
\text { develop understanding what works for the practice population }\end{array}$ \\
\hline
\end{tabular}


and what doesn't

pay more attention to getting the patient engaged and activated so that they can do more on their own

The theme 'System Change', discussed in chapter 4, is not included in this model. The reason is that, as pointed out in chapter 4, the map of system change was not coherent, being made up of a number of seemingly discrete concepts. The key message within the theme was that there was no one thing that would bring success and an effective implementation programme has to be multi-pronged. However, while not incorporated directly, the nature of the model that is being built in this chapter will highlight what those 'multi-pronged' initiatives need to be.

Of the six themes that emerged out of the initial interview five are used to develop the model. A further element, context, is added to explore how some of the key contextual concepts raised by the interviewees, such as the level of resources available to primary care, affects the overall dynamics.

The following sections build up the model step by step, based on the interview data described in chapter 4. Throughout the development of the model, the literature is also incorporated to 'tease out' some of the details that were not clear from the interview data.

\subsubsection{Clinical Engagement}

A central theme raised by the health experts during the interviews was engagement. Engagement, as used by the health experts is broader than clinical engagement, referring to the engagement of clinicians, patients and the broader community within which the patient lives. This section focuses on clinical engagement.

In the Engagement cognitive map discussed in Chapter 4, both clinical and patient engagement came together with the concept 'improved provider patient relationship'. In the minds of the health experts, clinical engagement was seen to be an important element in any successful implementation of chronic care programmes. 
This is not a surprising perspective, in that it is the clinicians who have to deliver the programme and, as highly trained professionals, they are unlikely to invest in learning new knowledge and skills, let alone change the way they practice, unless it is an initiative that they are committed to. It is a simple assertion: that clinical engagement is needed if you expect clinical staff to make the effort to implement the programme. This is not a unique or unusual perspective and it has been repeated in a number of documents looking at the implementation of new health practices (for example see Bradley, et al., (Bradley et al., 2004)).

In New Zealand, the report by Connolly et al., (Connolly et al., 2010) for example, talks about leadership and collaboration as being two, out of three, higher order factors, amongst the 12 they identified in their research as being important in a chronic care management model. Chris Ham, a leading health researcher argues that improving the experience for patients relies on "changing the day-to-day decisions of doctors, nurses and other staff" and making these changes is "best achieved through clinical engagement" (Ham, 2003). Bowns and McNulty (1999) found, in their evaluation of a major change programme in the Leicester Royal Infirmary in the United Kingdom that, "significant change in clinical domains cannot be achieved without the co-operation and support of clinicians” (Bowns \& McNulty, 1999, p. 69). Trisha Greenhalgh's meta review of innovation in health services (Greenhalgh et al., 2004), found it difficult to separate implementation research from the broader domain of organisational change. In that literature, however, she found evidence to support the assertion that the involvement and engagement of leaders enhanced the opportunity for success in implementing new innovations (Gustafson et al., 2003). This built on the earlier work of people like Rosabeth Kanter (1984) who argued that leaders will be more supportive of the change if they are kept involved and informed.

Other research takes the need for clinical engagement as being axiomatic and focuses on the factors needed to develop it. Ruston and Tavabie (2010) focus on the role of leadership in developing clinical engagement. Hockenberry et al., (Hockenberry, Walden, \& Brown, 2007) focus on factors involved in developing an "environment for evidencebased practice" (EBP), of which clinical engagement is central. Therefore at the centre 
of the model is the clear link between engagement, action and improvements in care. This is shown below in figure 5-2.

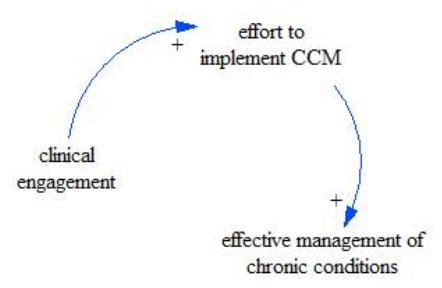

Figure 5-2 Impact of clinical engagement on the effective management of chronic conditions

Outside of health, other researchers have focused on what is involved in developing the engagement, or commitment, of people to a task. The work of Locke et al., (Locke, Latham, \& Erez, 1988) focuses on 'goal commitment' and what is required to develop it. Like the other writers noted above, they take as axiomatic the view that commitment is a precursor to action, and focus instead on what is required to develop it. One important factor is the success that action brings, feeding back and reinforcing the commitment that underpinned the original action. Locke et al's work literally closes the circle and provides a mechanism by which commitment or, to use the term of the health experts, engagement, is developed over time. In Locke's et al's work, they found that goals, in this research the effective management of long-term conditions, affect action, and preceding action was commitment. Thus, one has to have a degree of commitment, or engagement with a goal, before people will take action to achieve it.

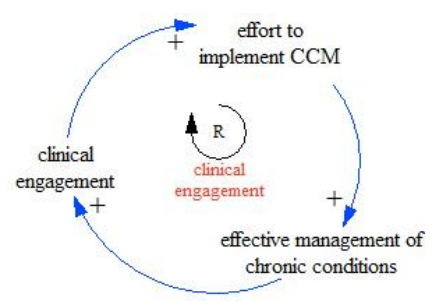

Figure 5-3 Closing the loop between engagement and action

The key variables involved in developing this engagement over time are captured in figure 5-3, which shows commitment (clinical engagement) preceding the action (effort to implement CCM) that delivers results (effective management of chronic conditions). 
The positive polarity of the linkage indicates that an increase (decrease) in clinical engagement leads to an increase (decrease) in efforts to implement CCM. Similarly an increase (decrease) in effort leads to an increase (decrease) in effective management of chronic conditions. That in turn feeds back to further influence clinical engagement. As the effective management of chronic conditions increases (decreases) so does clinical engagement. In its structure this is identical to one of the feedback loops described by Repenning (2002) in his model exploring the dynamics of innovation implementation. The key difference is that Repenning's work focuses on the commitment to 'using' an innovation. In this context an equal emphasis has to be given to the 'development' of that innovation in a particular practice, modifying it as necessary to meet the specific requirements of their population and their provider resource.

This feedback loop begins to tease out the structure behind the experts' view about the importance of clinical engagement and also points to the importance of performance feedback. This is covered in more detail below.

\subsubsection{Performance Feedback}

Because CCM is not a 'widget' which can simply be applied unchanged in any situation, but a complex set of processes and behaviours that are developed over time, this issue of feedback is crucial and one that was highlighted by the health experts. One of the three most important concepts for the health experts in regards to performance feedback was concept 147 , the 'ability to respond to what the information is telling us' 'Us' in this case, being those charged with implementing the programme.

They pointed out that there is a key intervening variable that needs to be in place if improvements in the management of chronic conditions are to feedback and support continuing clinical engagement. This variable concerns the use of performance data being fed back to clinicians. Simply put, clinicians need to know the effect of their actions upon their specific patients and the broader population that the practice is serving. The most important concept for them within the performance feedback theme was concept 181, 'develop a clear definition of the problem, well supported by the data' which is linked in the composite map to concept 184, 'develops the engagement of 
clinicians and other providers'. Thus the health experts see a clear causal link between performance feedback and clinical engagement.

Effective management of chronic conditions requires the use of data to understand both the population and the impact upon the population's health by the health provider. A key part of what defines effective chronic care management is the production of information that helps in the delivery of proactive management of the patient and their conditions(s). For example, what impact does a self-management programme have upon the levels of HbA1c (blood glucose), an important indicator of effective management of diabetes? Effective management of chronic conditions does produce data about the population and the impact that the programme is having on that population; it is an intrinsic aspect of what makes such a programme effective, and effective management of chronic conditions cannot take place without it (Kane et al., 2005). That data, if fed back to clinicians, supports their further engagement to either close the gap, if performance is not as good as expected, or improve performance further if performance is good.

This idea that performance feedback supports engagement is consistent with the literature on goal setting and motivation. The work of Locke and Latham (2002) highlights the importance of goals, in this example implementing the CCM programme, and feedback in motivating people towards high performance and performance improvement. As Locke and Latham (2002) point out, for the goals to be effective in driving engagement and action over time, people need feedback that reveals their progress in relation to their goals. While having a goal can be a motivator for engagement, "...the combination of goals plus feedback is more effective than goals alone" (Locke \& Latham, 2002, p. 708). In addition, the work of Ilies and Judge (Ilies \& Judge, 2005), building on the earlier work of Locke and Latham, shows how performance feedback affects how people feel about their performance and the goal they are striving to achieve. As they point out, “...feedback influences affect, which, in turn, influences subsequent goals" (Ilies \& Judge, 2005, p. 463). Figure 5-4 enhances the initial feedback loop, shown in figure 5-3, by incorporating feedback on performance data into the clinical engagement feedback loop (R1). 


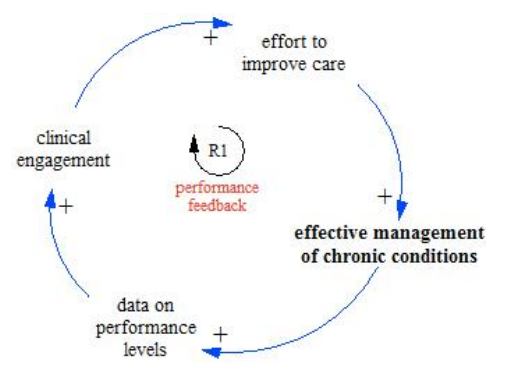

Figure 5-4 Impact of performance feedback on clinical engagement (R1)

A further point raised by the health experts however is that performance feedback is not only a good motivator to support clinical engagement, it is also a crucial element in helping the clinical team assess whether or not their efforts are making a difference. For example, in the Models of Care theme, the second most important concept was concept 17, 'understanding what works for the practice population and what doesn't.' Thus performance feedback not only supports ongoing clinical engagement, it also improves the impact of the effort. Effective management of chronic conditions is brought about, therefore, not only by more effort on the part of the practice team, but also by more effective effort, based on the feedback of performance data emerging from their programmes. In the composite map, concept 17 had a direct link to a number of concepts that focused on improving the effectiveness of what the practice does. These were concepts:

27 provide baseline data against which improvement can be assessed

179 enable discussions about the real issues e.g. why patients are not taking their pills

240 provide the information to those who are trying to improve outcomes

248 help understand the structure, processes and outcomes needed to bring about the change

This highlights another aspect of feedback that drives motivation and performance improvement; the ability of the feedback to provide some insight into the effects of the efforts currently being made on the population that they are serving. This additional reinforcing feedback loop (R2) is shown in figure 5-5. 


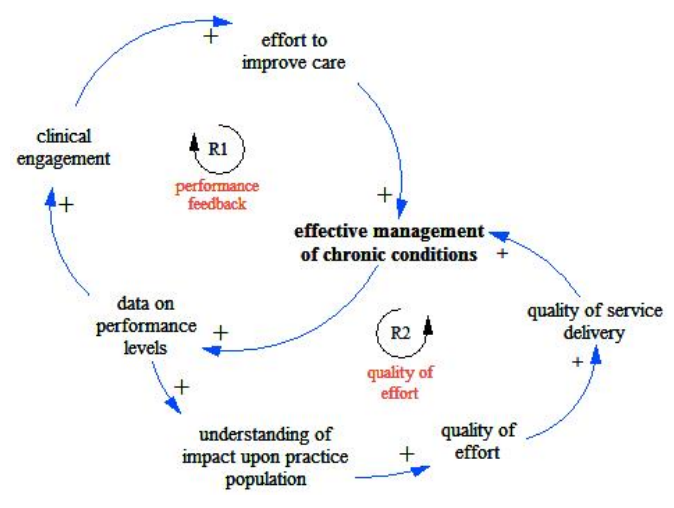

Figure 5-5 Impact of performance data on quality of effort (R2)

In addition, these linked concepts also highlight that performance feedback not only helps to increase understanding of the patients but also, as evidenced by concept 248 , the processes that deliver those outcomes.

Thus good performance feedback data also informs the practice about how their own internal processes affect clinical outcomes and what can be done to improve them. This additional loop (R3) is shown below in figure 5-6.

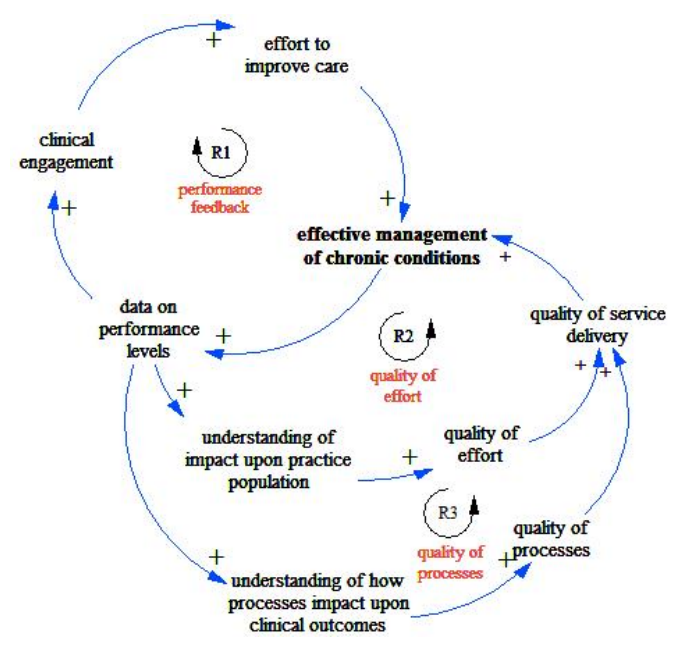

Figure 5-6 Impact of performance data on quality of processes (R3)

With the addition of this new loop, feedback is not only increasing clinical engagement and the effort that results from it, but also the quality of that effort and the quality of the process involved in delivering it. Feedback thus becomes a key aspect of learning, 
enabling clinicians to target their efforts in areas that are more likely to deliver effective outcomes. This is also consistent with the literature. Feedback, is a central concept in both SD and Cybernetics (Forrester, 1994; Richardson, 1991; Sterman, 1989) and is a key mechanism to support learning (Forrester, 1994; Sterman, 1989).

Other work (Erez \& Zidon, 1984) shows that efforts to improve performance is not so much linked to how easy or difficult that task is, or whether or not the feedback is telling one that the performance gap is closing but whether or not the goal one is striving for is one that is accepted and believed in. This highlights the other aspect of clinical engagement that the health experts emphasized; their involvement in the design of the programme and the goals it is striving to achieve. This additional element is included in figure 5-7.

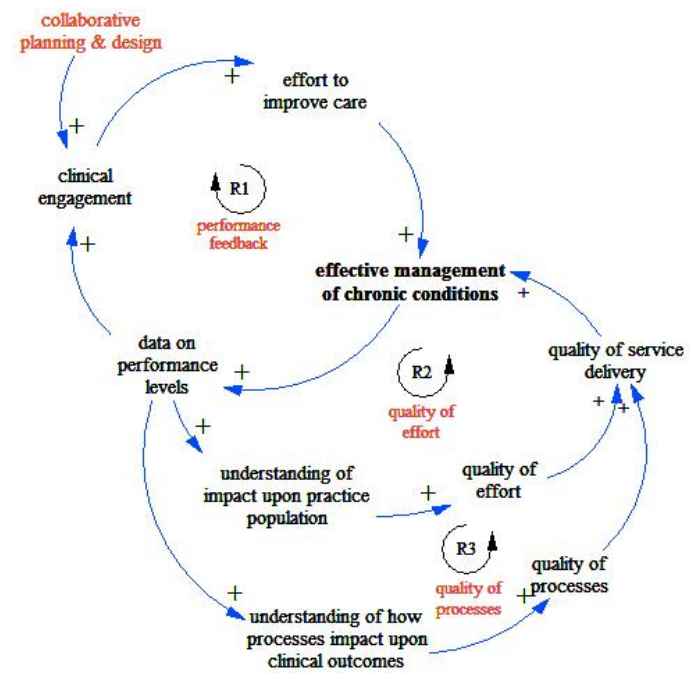

Figure 5-7 Collaborative planning and design

The literature on this particular aspect of the 'expert theory' is not straightforward. Locke and Latham (2002) have found in a series of studies that the performance of people with participatively set versus assigned goals do not differ a great deal. Erez (1984) however reached the opposite conclusion. In looking at the discrepant results Locke and Latham found that, "... an assigned goal is as effective as one set participatively provided that the purpose or rationale for the goals is given" (Locke \& Latham, 2002, p. 708). That is, collaborative planning is less important if the purpose 
for the programme and the goals being set for it are accepted. Where there is some disagreement, either about the programme or about the specific goals being set, then it does become important. Also of interest is the finding that, “...employees who were allowed to participate in setting goals set higher goals and had higher performance than those who were assigned goals" (Locke \& Latham, 2002, p. 709).

Ensuring that clinicians are involved in the planning and design of the programme seems to be an idea, put forward by the experts, that should be an important element of any implementation initiative.

At this point the theory is beginning to provide a description of the links between clinical engagement, improved performance and the important role played by performance feedback. The theory so far describes the link between engagement, effort and performance in a way that is consistent with the research on goal motivation and performance. Furthermore, it shows how data on performance, fed back to the practice team, can further enhance clinical engagement and also improve the quality of effort by providing better information about what does and does not work for the patients enrolled with any given primary care practice. The ability of this feedback, often through the use of decision support systems, to improve clinical performance is well supported in the literature (Garg et al., 2005). Finally, clinical engagement can be given the 'kickstart' it needs by ensuring that the practice team are involved in the initial planning and design of the programme. The support in the literature for this last point is however ambiguous.

\subsubsection{Patient Engagement}

As pointed out above, from the perspective of the health experts, engagement involves more than just clinical engagement. Within the engagement theme, the third most important concept was concept 38, 'improved provider patient relationships'. Within the Models of Care theme, the third most important concept was also focused on patients; concept 151, 'pay more attention to getting the patient engaged and activated so they can do more on their own'. 
Patient engagement is important within the CCM model as it is one half of the performance goal being sought; what the health experts referred to as 'improved provider patient relationships' (concept 38) and which is described in the CCM model as “...productive interactions between practice team and patients” (Wagner et al., 2001, p. 68). The incorporation of patient engagement (R4) into the model is shown below in figure $5-8$.

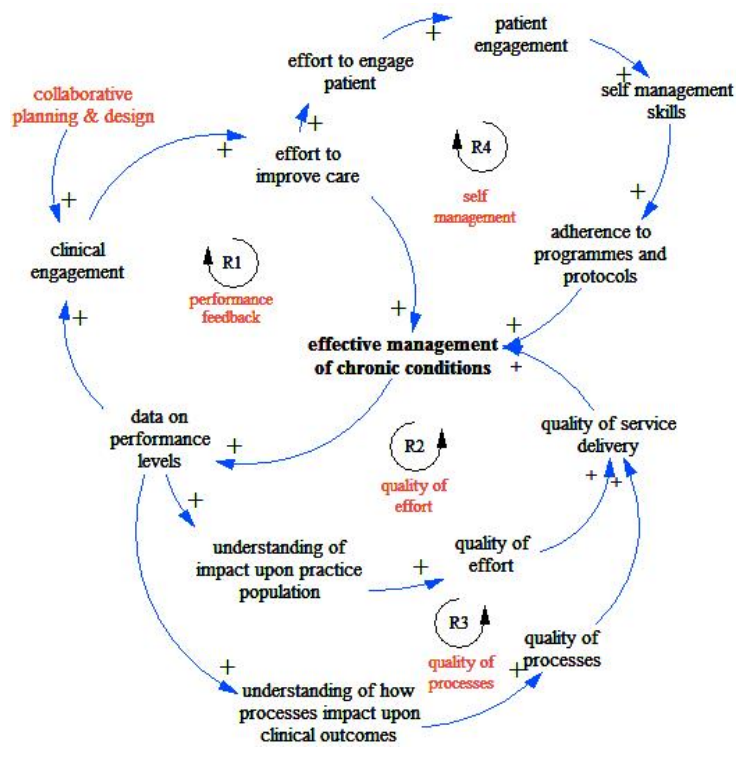

Figure 5-8 Patient engagement and self-management (R4)

In this loop, the efforts made by engaged clinicians are targeted towards engaging the patient so that they develop the self-management skills needed to adhere to the programme and protocols associated with the treatment they are receiving. Jordan and her colleagues (Jordan, Briggs, Brand, \& Osborne, 2008) also point out that strategies for patient engagement need to be integrated into the overall service design and not seen as a peripheral task outside of core health care activities. Only with this level of integration will the necessary uptake of patients and health professionals take place. National policies are now being put in place in some countries to support this idea, not just because of the ethical issues involved in supporting patients to share in the decision making about their care (Elwyn et al., 2010), but also because such efforts can improve patient-engagement (D. Stacey et al., 2012). In New Zealand, the Guidelines Group 
(New Zealand Guidelines Group, 2011) conducted research to better understand what strategies are most effective in developing this engagement.

Patient engagement is seen as a key causal link, which is important in helping patients adhere better to programmes and protocols associated with their condition. As pointed out by the health experts, developing a greater level of patient engagement helps ensure that 'patients adhere better to the treatment recommendations' (concept 154), an argument that is supported by the literature (Joosten et al., 2008). This may include ensuring that the proper medication is taken at the appropriate time and in the right amounts. It may also involve following a specific dietary or exercise programme. Herein lies one of the major challenges of chronic conditions, and one that requires a major change in clinical behaviours and delivery practices. Within the CCM model, the patient is no longer a passive recipient of clinical decisions but an active participant in deciding the treatment programmes. The patient is a central part of the multidisciplinary team (MDT). Under the acute model, treatment is short, often played out over minutes, days or weeks. As a consequence there is little urgency to develop patient self-management skills and the patient remains a passive recipient of clinical judgements. Under a chronic care model, the clinician has to provide room for the patient to become more actively involved in their care, a behavioural shift that many, who have been trained and rewarded for their skills in responding to acute symptoms, have difficulty making.

\subsubsection{Community Engagement}

Patients also do not exist in isolation. They are embedded in close family/ whānau and community networks, and research by Rosenthal et al., (Rosenthal et al., 2006) has shown that regardless of how well the patient is engaged, they often need support to continue with high levels of adherence over time. This was acknowledged by the health experts, who saw that patient engagement would need strong community supports in place, if it was to translate into ongoing adherence to programmes and protocols. This was reflected in comments like 'provide space and time for community input' (concept 108), and 'develop community support for patient self-management' (concept 95). 
This community involvement was also linked to performance data. The health experts argued that for the community to be involved they needed more information, not just clinical information about specific conditions, but about the health of their community and how it was changing over time. Thus, they argued, implementation initiatives should 'support making the data accessible to the public' (concept 206), which would enable the 'public able to see the gap between 'what is' and 'what could be" (concept 207).

Central to the viewpoints of the health experts is that health is grounded in the community, and as a consequence, the community has to be involved in responding to health concerns. This is supported in the literature (Epping-Jordan et al., 2004; Hoddinott, Britten, \& Pill, 2010) and one of the main ways that the health system can support this involvement is through making information more accessible. Community support is closely linked to improved adherence. This community dynamic is included in figure 5-9.

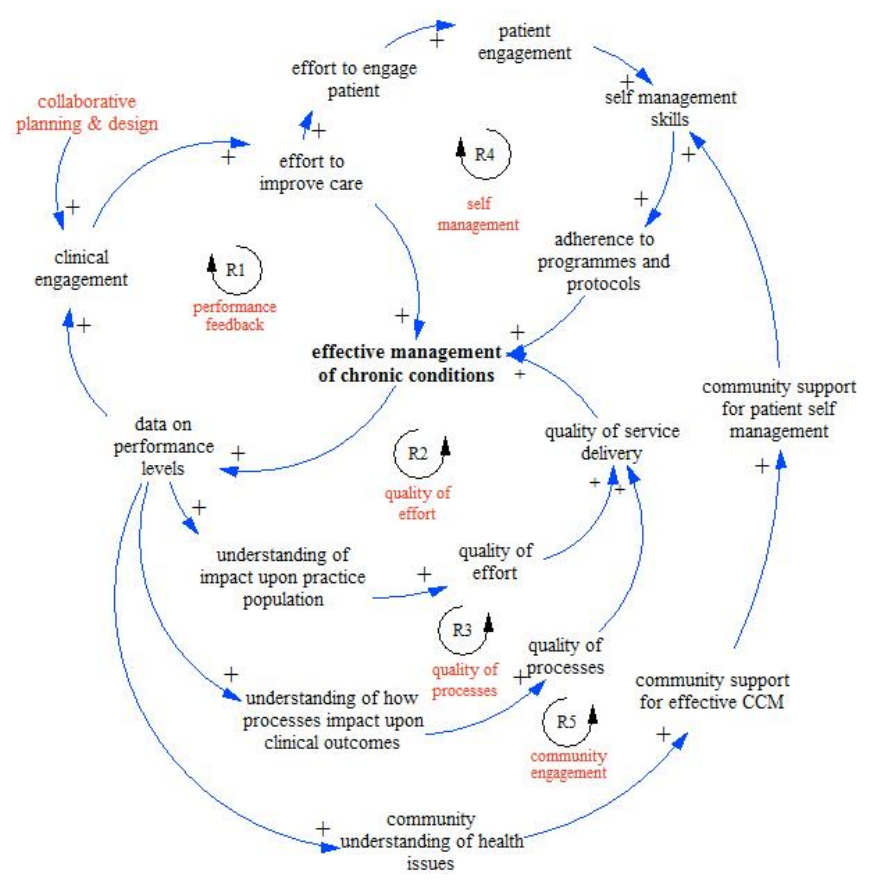

Figure 5-9 Community engagement (R5) 


\subsubsection{Support for Practices to Make the Change}

So far, the theory has focused on the dynamics that drive the change; engagement of clinicians, patients and the communities within which they live, and the importance of performance feedback to support ongoing engagement, understanding of clinical outcomes and the effectiveness of internal processes. Furthermore, the theory argues that if clinical engagement is to be developed it needs to be built upon early involvement through processes of collaborative planning and design.

The health experts also noted however that practices would need support to make the changes required to successfully manage chronic conditions. The most important concept within the theme of 'Provider Performance' was concept 160, 'provide support for the practices to do the right thing around the evidence'. The second and third most important concepts described the consequences of that support, namely 'close the treatment gap so that the right thing is happening' (concept 177) and 'increased confidence and skills to make it happen' (concept 35). A look at the map of the 'Provider Performance' theme shows that the health experts identified three key ways of providing this support. The first was through training; 'training to cover selfmanagement skills' (concept 150), and 'training to cover clinical skills' (concept 149). The second was through the provision of additional resources; 'provide clinical resource to facilitate across the practices' (concept 175) and 'make sure there are adequate people on the ground to support practices' (concept 23). The third was through the provision of decision support tools; 'provide good decision support tools' (concept 23),

Effective management of chronic conditions is resource intensive and it is often being implemented in a context where the practice is already burdened with high levels of acute workload, and as a consequence, many clinicians, even when they support the idea, do not feel they have the resources needed to make the change. This is supported by recent research indicating that, especially in small practices, “...some type of external financial incentive and quality improvement support may be essential for widespread practice change" (K. Coleman et al., 2009, p. 82).

The health experts are presenting two central ideas here. The first is that chronic care management places increasing demands upon a scarce resource. The second is that this 
impacts upon the clinicians' belief that change is possible: their self-efficacy. These two additional feedback loops are shown in figure 5-10.

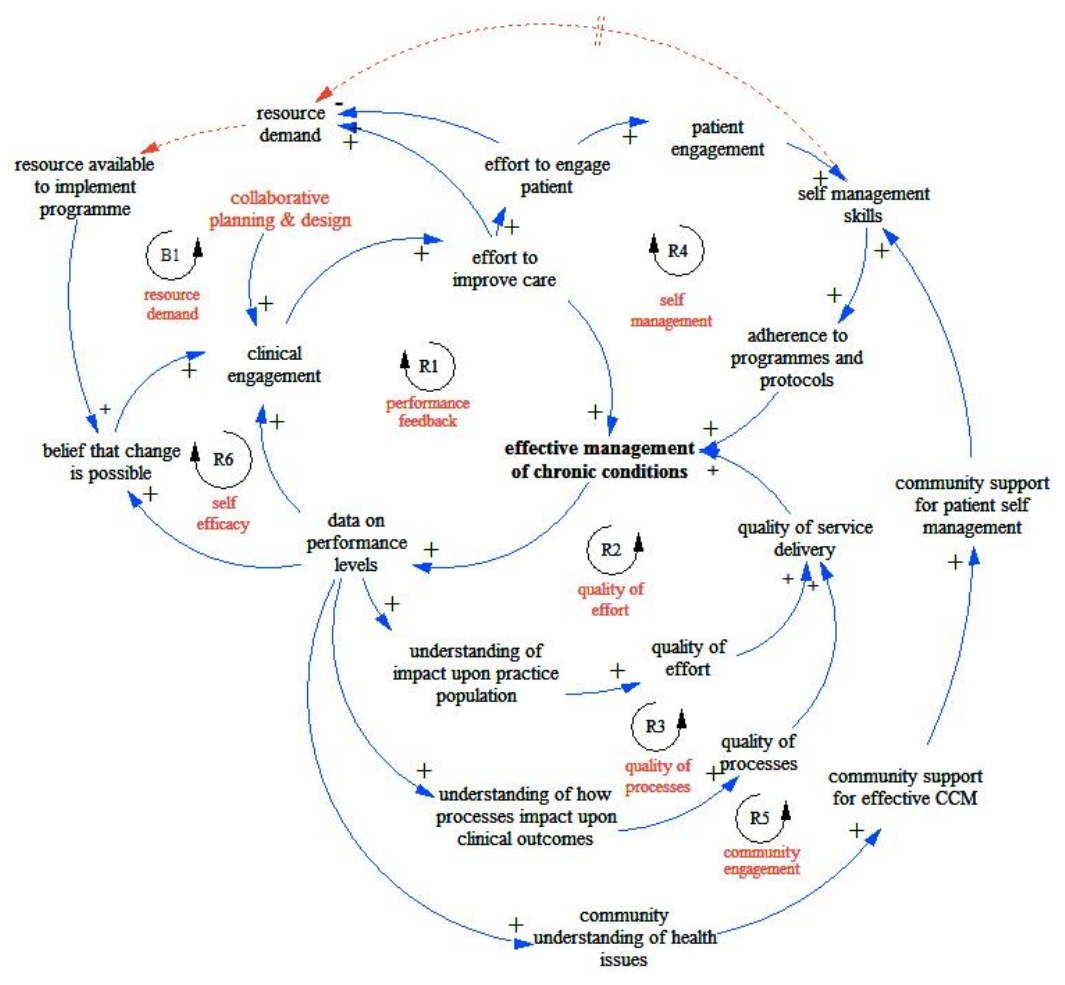

Figure 5-10 Resource demand (B1) and self-efficacy (R6)

This 'belief that change is possible', referred to as self-efficacy in the literature (Bandura \& Cervone, 1983), is central to developing clinical engagement and for ensuring that collaborative planning and design does in fact contribute to increased clinical engagement. Support for this idea has been found in the literature on implementing evidence-based practice in primary care (Turner, Nicholson, \& Sanders, 2011) and in nursing performance (Lee \& Ko, 2009). In research looking at the implementation of a parenting and family support programme, Turner and Nicholson found that higher selfefficacy was positively associated with successful implementation. The work by Lee and Ko (2009) looked at self-efficacy from the group perspective finding that 'collective selfefficacy' was a significant factor affecting nursing performance. So, what this tells us is that even where the practice team, collectively and individually have the skills to implement a programme, their self-efficacy, or belief that they can deliver the 
programme successfully, has a major role to play in their involvement in implementing new evidence-based practices such as CCM. Self-efficacy it seems plays a moderating role. When there is low self-efficacy, involvement in collaborative planning and design has little impact upon actual performance. However, when it is high the impact is different:

"When goals are self-set, people with high self-efficacy set higher goals than do people with lower self-efficacy. They also are more committed to assigned goals, find and use better task strategies to attain the goals, and respond more positively to negative feedback than do people with low self-efficacy" (Locke \& Latham, 2002, p. 706).

Thus support is needed to help develop a sense that change is possible and that the support has to address the resource demand. This has been advocated by the health experts in a number of ways such as training and capability development as well as increased use of information technology and decision support tools (Garg et al., 2005).

The important issue here is not about whether or not the practice has enough resources to make the required changes, but whether or not they believe that is the case. No doubt the two are related, but the important element, in terms of the theory being developed, is the belief held by the practice team that they have the resources, whether they be time, knowledge, skills and/or money, to actually change their model of care.

\subsubsection{Contextual Factors}

All behaviour is influenced by context and all theories are influenced by the context within which they operate. The theory of implementation described in the model above unfolds in different ways in different contexts. For example, the 'baseline workload' has an impact upon resource demand and therefore the impact of the extra effort required to implement the new programme. Developing patient engagement is influenced by the nature of the population, specifically the 'patients' level of knowledge, skills and confidence' within the resident population being served. This will be harder if the practice team do have a good level of 'clinical knowledge, skills and confidence relevant to CCM'. Furthermore the level and quality of effort described in the model will be 
harder to develop and maintain without 'structures to support clinical governance of the CCM programme' and finally the 'strength of community resources' will have an impact upon the patients' ability to develop the self-management skill needed for them to become active participants in the treatment programme. These contextual factors are incorporated into the model as shown below:

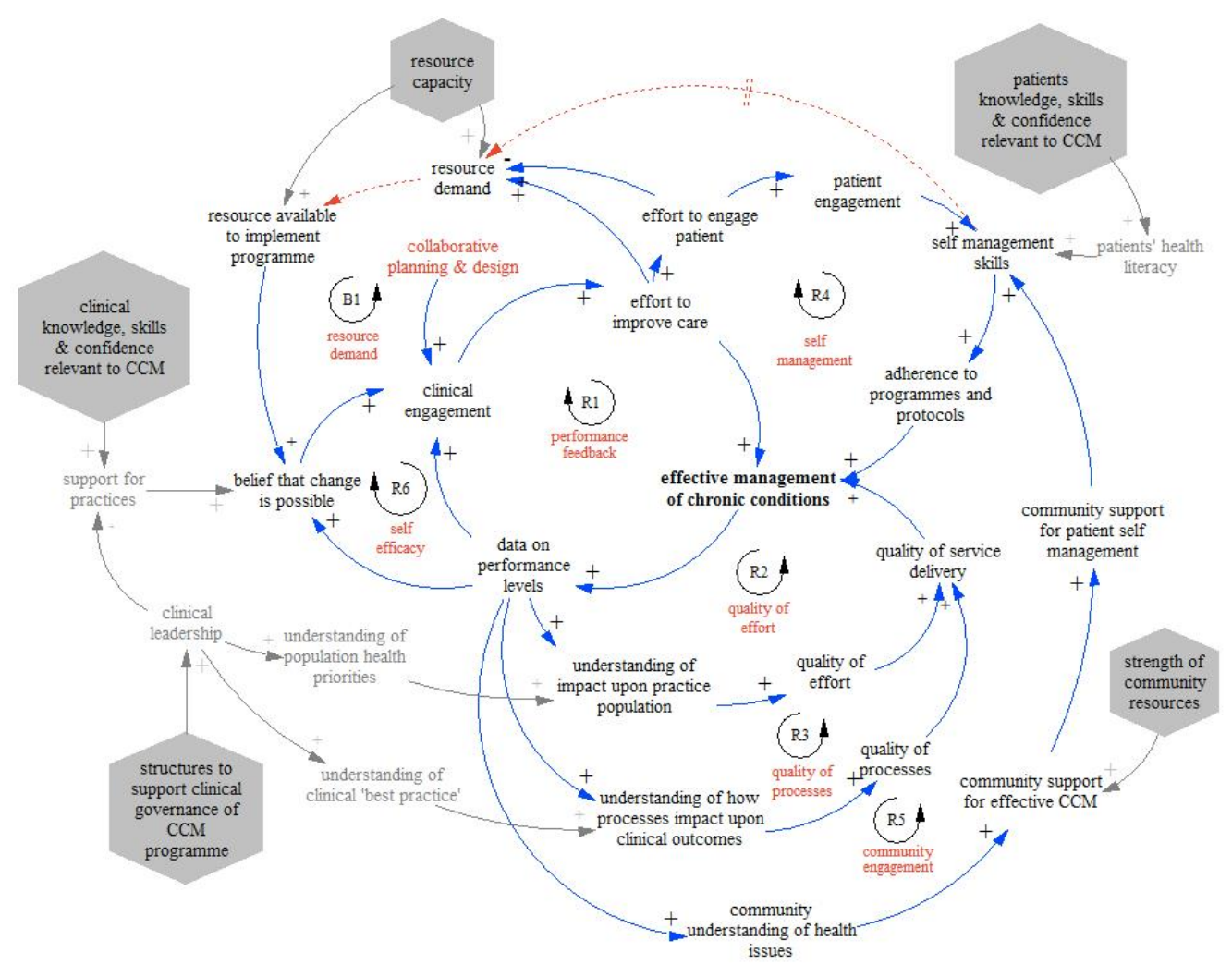

Figure 5-11 Contextual factors

\subsection{Summary of 'Expert' Theory}

The theoretical framework depicted above, focuses on how a primary care practice responds when faced with the challenge of implementing a new health programme designed to improve care for people with chronic conditions. It was based on interviews with health experts, the literature and organisational theory and focuses on the relationships between the capabilities within the primary care practice, its patient population, and key service delivery processes. As a consequence the emphasis is on the 
strategic design issues rather than day-to-day tactical questions such as whether or not, for example, to appoint a change co-ordinator, or acquire a new diagnostic instrument.

This section summarises the theory that has been developed. The purpose is to synthesise the detailed CLD shown in figure 5-11 and describe the key dynamics involved. Each loop has been developed from the interviews with the health experts and each specific link has been supported with the relevant literature. However, while each of the variables within the theory may have been mentioned elsewhere, they have not before been described explicitly as part of a dynamic system. In that sense the theory itself is new. The model does not focus on individual factors, but describes how these factors are combined into a coherent set of feedback processes that describe the dynamics involved in an implementation effort.

Within the model there are three key drivers of the effective management of chronic conditions; the effort to improve care by engaged clinicians, improved adherence to programmes and protocols by patients with self-management skills and quality service delivery processes. These are discussed, in turn, below.

\subsubsection{Summary: Efforts to Improve Care by Engaged Clinicians}

Consistent with the original writing of Wagner (Wagner et al., 1996) the health experts consider that at the heart of the effective implementation of CCM is an engaged, confident and supported clinical team interacting with knowledgeable and motivated patients living in communities that understand the requirements and responsibilities associated with self-management. Clinical engagement is required if efforts are to be made to improve care. Without that engagement little if any change will occur and ensuring they are involved in the planning and design process will ensure that at least at the start, clinicians will have a level of buy-in to the changes being proposed.

However, efforts to change requires resources and the balance between resource demand and resource availability is central to maintaining clinical support and, specifically their belief that the changes required to improve care can in fact be implemented within the resources available. This is a balance that has to be maintained if the implementation is to be successful, as it is a key driver of self-efficacy; the belief 
amongst the clinical team that the change is in fact possible (Lee \& Ko, 2009). This is a key negative feedback loop (B1) constraining the development of clinical engagement. Regardless of how 'engaged' clinicians are, how positive they are about the effects of good CCM programmes, if they do not believe that the change is possible, especially given the demands of their current workload, then they will not make the effort to implement it. Improving care for people with chronic conditions is a resource intensive endeavour and if the clinical team do not believe the resources are there to enable the changes to be made, then regardless of the positive support for the idea it is unlikely to be implemented.

An additional factor affecting clinical engagement is the feedback that clinicians get. Knowing the impact of their efforts is a key driver of continued engagement and effort. This is not just 'good' feedback informing the clinical team of how well they are doing. The same impact is created with 'bad' feedback telling the clinical team that their efforts are not working. In the former case, buoyed by their efforts, they become even more engaged. In the latter case, not wanting to fail their patients and/or be seen as poor performers by their peers they continue to make efforts to improve care. Clear, unambiguous feedback is an important element in helping people to perform well (Bakken, 2008).

Thus, in line with other writings (Ham, 2003; Ruston \& Tavabie, 2010) the theoretical framework highlights the importance of clinical engagement. The model's contribution is to show the mechanisms by which clinical engagement has its effect and the mechanisms that develop and support it over time (R1).

\subsubsection{Summary: Adherence to Treatment Programmes by Patients with Self-Management Skills}

Clinical engagement is central to improving care but the engagement of patients is crucial if that care is to deliver improved outcomes over time. The ability for even the most engaged and competent clinical team to generate sustained improvement without patient engagement is minimal. Patient engagement is therefore, the other crucial part of an interdependent dynamic (R4). On the one hand, effective implementation requires the efforts of an engaged clinical team to practice according to the best evidence 
concerning long-term care and to engage the patient and their whānau/community in that. On the other hand the patient and their whānau/community has to accept their role in developing increased capabilities in self-management.

The effectiveness of this clinician - patient relationship depends, at least initially, on the efforts of the practice team. It is the effort of the practice team that not only establishes and improves the effective management of chronic conditions, but also provides the initial impetus to the development of patient and community engagement, which is the pre-requisite for self-management. Thus, the clinical team has to take responsibility for establishing and developing the programme and the patient has to, over time, take responsibility for their part in this contract, self-management. However, effort alone is not enough and this particular dynamic, like clinical engagement itself, is enhanced with the inclusion of information about the effects of the programme upon patients and the patient population. This is important as it not only provides the clinical team with the information needed to assess the impact of their efforts but also to provide the patients with knowledge about the conditions they are trying to manage, the effect of the clinical programme and their response to that upon that condition. It is the feedback loop (R4) that provides the 'connecting glue' between clinical and patient engagement.

However, as noted above developing this relationship is just another demand upon scarce resources and one that may not deliver immediate gains. This is simply because patient engagement is aiming to change patient behaviour and behaviour change can be difficult to bring about (New Zealand Guidelines Group, 2011). The nature of longterm conditions however is that the behaviour of patients, whether that be in terms of lifestyle or adherence behaviours, is central to improved health. Clinical interventions alone are limited. Thus, on the one hand patients who are not engaged and do not, due to lack of skills and/or knowledge, take an active part in the therapeutic relationships limit how much improvement can be made. On the other hand however, a fully engaged patient, actively self-managing, not only contributes to greatly enhanced outcomes but also decreases the resource burden upon the practice.

Finally, the family and community context within which the patient lives, has a significant impact upon their engagement and the clinical practice, largely through the 
provision of health knowledge, has a role to play in helping to develop that supportive context (R5).

\subsubsection{Summary: Quality of Service Delivery}

Supporting the key dynamics involved in the therapeutic relationship are the delivery processes, by which the patient receives their care and the clinical team learn more about their population and best practice care for that population (R2) and (R3). These processes ensure firstly, that clinical and patient efforts are based upon an 'understanding of clinical best practice', thereby improving the quality of processes (R3) and secondly, that the clinical team bases their programme on a good understanding of population health priorities', thereby ensuring 'quality of effort' (R2). Both of these are important. Together, they help ensure that the efforts applied by engaged clinicians is supported by high 'quality of service delivery'.

The third key process is the one aimed at increasing community understanding of health issues (R5). While the clinical team only has a limited ability to influence the capability of the community and families to support patients with long-term conditions, one thing they can do is increase their understanding of health issues.

\subsubsection{Summary: Balancing the Networks of Relationships}

While the description above has focused on three key dynamics within the whole, a key aspect of the model is that it is describing a network of relationships. It cannot be understood by adopting a reductionist stance and assuming that one can, for example, focus on developing clinical engagement without understanding the interplay of factors that affect it or how clinical engagement affects, and is affected by, patient engagement and the delivery processes. The theory describes a pattern of organisation (Dent, 2003), characterised by mutually interacting feedback loops, and the contribution of this theoretical framework is to highlight that the impact of known factors, such as clinical engagement, depends upon this organisation.

This organisation however has temporal as well as spatial characteristics. While the temporal aspects will be covered in more detail in the description of the simulation model, there is one important aspect that deserves to be highlighted here. Efforts, 
regardless of where they are targeted, soak up resource and immediately affect the balance between resource demand and resource availability. However, the impact of those efforts is spread out over time and especially those efforts designed to enhance the family and community support for the patient may not deliver benefits for months and possibly years. This emphasises again the importance of providing the clinical team with information on their performance. Knowing the impact of their efforts is a key factor in maintaining clinical engagement, even when there is resource pressure, and choosing metrics that can be expected to change over a shorter period enhances the gain that such feedback delivers.

This section has attempted to provide a summary of the key dynamics driving successful implementation as captured in the theoretical framework shown in figure 5.11. The following section looks at the policy recommendations that emerge from this description.

\subsection{Policy Recommendations}

The model has a number of implications for implementing programmes to improve the care of people with long-term conditions. These relate to three core parts of the model described above: the efforts to improve care by engaged clinicians, the adherence to treatment programmes by patients with self-management skills and by the quality of service delivery processes.

\subsubsection{Policy Recommendations: Clinical Engagement}

Clinical engagement is central to successfully implementing programmes to improve the management of long-term conditions and the theory highlights three areas that have to be managed carefully if clinical engagement is to be developed and maintained. The first is that clinicians need to be involved in the detailed planning and design of the programme. If they are not then, despite being supportive of the core idea, they will be less engaged and therefore make less effort to make the changes necessary. The second area is feedback. Without feedback about performance, clinicians are not able to assess whether or not the efforts they are making are in fact improving clinical outcomes for patients. Instead, their experience is limited to their understanding of their specific 
patients, often based on anecdotal feedback. Many of these patients have conditions, such as diabetes, where it is hard to see progress and the clinicians' individual experiences can hide overall progress with the enrolled population. The third area relates to the ratio of available resources to resource demand. As the additional resource demand required by the new programme gets close to, and possibly exceeds resource capacity, then the clinicians' belief that it is going to be possible to make the required changes - self-efficacy - will decline, reducing the level of clinical engagement. Unless this is offset by the other two factors, early and ongoing involvement on the planning and design of the programme and ongoing feedback about performance, it maybe necessary to provide additional support and/or additional resources to offset the lowering expectations that change may in fact be possible. This is shown in the model extract below:

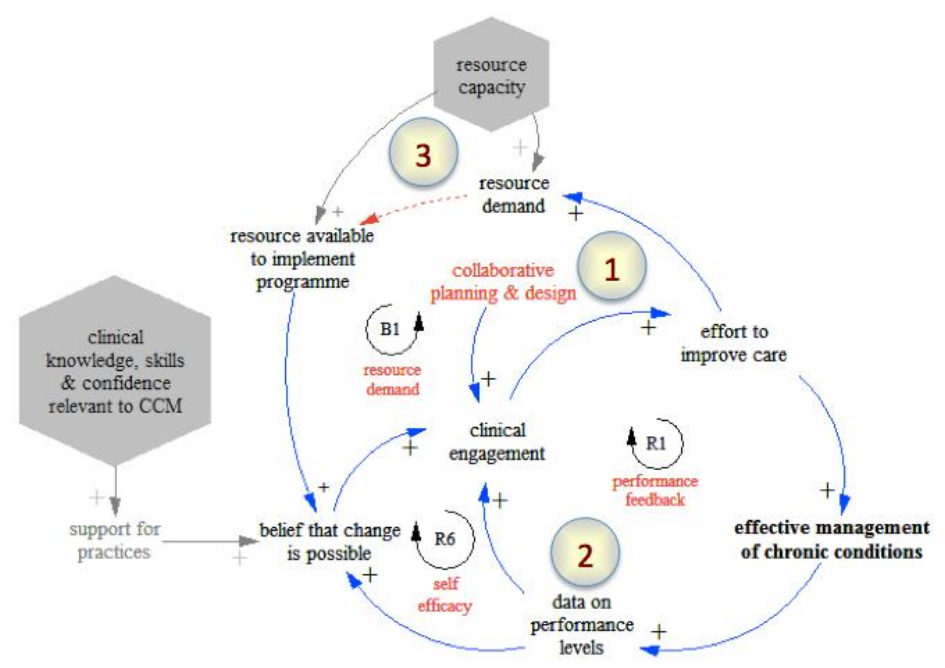

Figure 5-12 Building clinical engagement

In summary, the theory emphasizes three policy recommendations to build and maintain clinical engagement in practices. They are;

- ensure clinicians are involved in the planning and design of the programme. This should be at the outset of planning and on an ongoing basis,

- provide ongoing feedback that enables clinicians to see the impact of their efforts on patient outcomes, and 
- carefully manage the balance between resource demand and resource availability.

The consequence of these policy recommendations not being followed is that, except in practices that are 'resource rich', self-efficacy will decline and additional resource will be required to develop and support the clinical engagement that is central to successful implementation.

\subsubsection{Policy Recommendations: Patient Engagement}

Patient engagement is also crucial. Most importantly this is because it is their level of knowledge, skills and confidence (health literacy) that will set both the opportunities and constraints upon the level of change that is possible. Secondly, as they live with their conditions 24 hours a day, 7 days a week, patients have a lot of knowledge that is different, but just as important, as the clinical knowledge and can inform the treatment programme.

There are three main areas that affect this engagement. The first is the effort made by the clinical team to engage the patient so as to shift the nature of the 'therapeutic relationship' from the 'doctor as provider' and 'patient as receiver' to one of 'joint problem-solvers'. Even 'simple' interventions such as the use of medicines relies on the patient both understanding why they are required and how they need to be taken, as well as their willingness and ability to keep on taking the medicine(s) even when their symptoms seem to be under control and they 'feel better'. This recommendation immediately highlights the systemic nature of implementation. As efforts made to engage patients are intensified, it increases the overall resource demand. This means that there are now two pressures on that resource and the balance between them cannot be established ahead of time. Where the tensions lie, whether it is better to push hard on the patient engagement or on implementing the core of the programme depends on context. Without that contextual understanding it is impossible to put forward 'generic' recommendations about what should or should not be focused on. Thus arguing that successful implementation will come from engaged clinicians is an unhelpful truism that ignores many levels of complexity. 


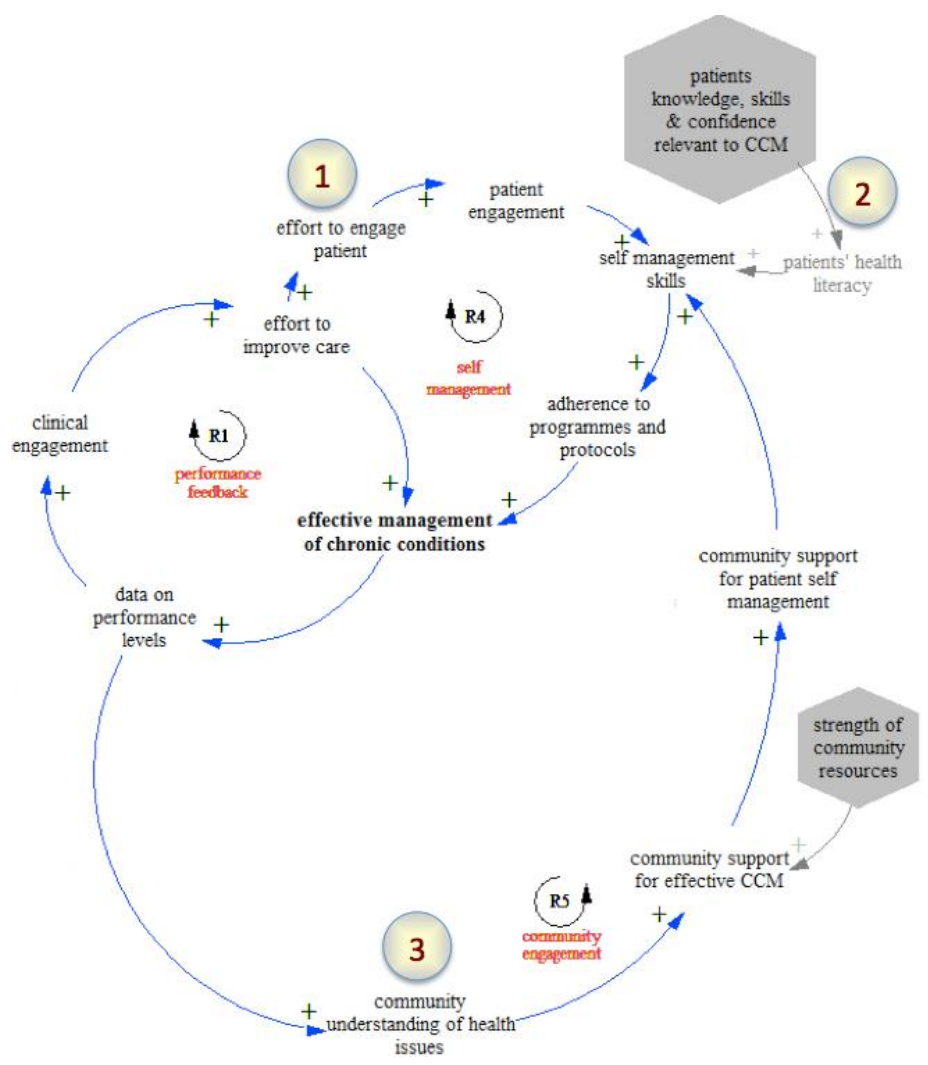

Figure 5-13 Building patient engagement

The second policy recommendation focuses on efforts to increase the health literacy of the patient by helping them gain further knowledge, skills and/or confidence in relation to the management of their long-term conditions(s). As the model points out this literacy underpins self-management, and no matter how much effort is put into engaging the patient by the primary care team, unless levels of health literacy are built up, self management skills and the consequential improvement in adherence to treatment programmes and protocols will be limited (Jordan et al., 2008).

The third policy recommendation focuses on the levels of support available to the patient within their family, whānau and broader community. It is well-documented that patients in supportive families and communities adhere better to treatment programmes and protocols, becoming better managers of their own condition(s). While the primary care practice may have limited capacity to directly support the building of these 
resources, they are well placed to increase community understanding of health issues and this understanding can be an important stimuli to greater development within the community. In summary, the policy recommendations designed to build patient engagement are:

- ensure that clinicians within the team understand the importance of the relationship between clinician and patient and the need to move it towards one of joint problem-solving. There may be training and support required for this to happen effectively;

- invest time and resource in developing the health literacy of patients as any limits here will make it difficult for the patient to make the changes that may have been agreed in the consultation with members of the practice team, and

- create links within the community so that the patients will have a support base broader than that able to be provided by the practice team.

The consequences of these policy recommendation not being followed is that the expectation for improved health will continue to be placed on the clinicians who have to spend increasing amounts of time with sicker and sicker patients. This goes back to the core dynamic underlying Wagner's original work and discussed in chapter 2. Success in managing long-term conditions requires a shift of resource from the short-term and/or immediate symptoms being presented to the longer term underlying condition. The model highlights this by saying that all practices have to balance their response to the immediate presenting symptoms with the need to increase patient responsibility for their own health. Thus, as soon as the clinician has provided an effective response to the immediate need and, at least provided some temporary relief and/or improvement, efforts have to be made to engage the patient in the care programme if they are to avoid an ongoing dynamic of responding to the acute and severe symptomatic episodes common with people whose condition is poorly managed.

\subsubsection{Policy Recommendations: Delivery Processes}

The third key component in the model is the quality of service delivery, which underpins both clinical and patient engagement. A key part of this quality is firstly, to ensure that the practice team understands the health priorities for their population so 
that the scarce resource are focused on patients with the greatest needs and secondly, to see how specific processes impact upon clinical outcomes.

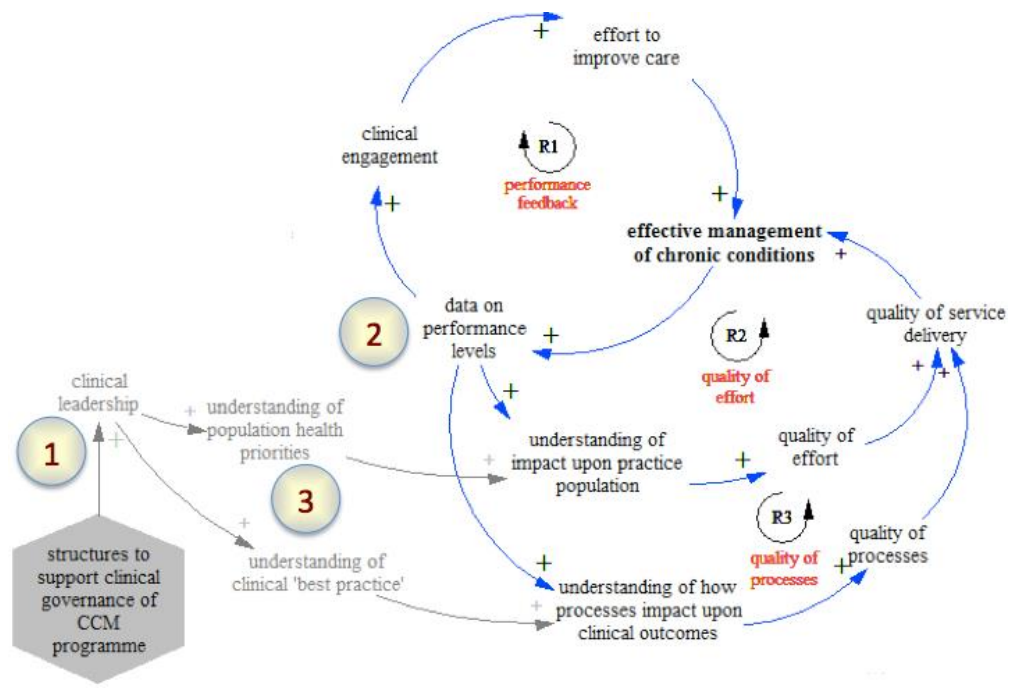

Figure 5-14 Building quality in service delivery

Performance feedback and clinical leadership are central for this to occur. As mentioned above, without feedback about performance, clinicians are not able to assess whether or not the efforts they are making are in fact improving clinical outcomes for patients. Furthermore, without clinical leadership they may not be able to make best use of this feedback. Clinical leaders are able to see the bigger picture, helping front-line clinicians see beyond their own specific patients and practices. The consequence is that clinical leaders can help ensure that effort is directed to the most important areas of health need within the patient population enrolled with the practice. They are also generally better informed about best practice, helping ensure that internal processes and practices support the clinical outcomes being sought. The policy recommendations that result from this are:

- ensure that clinical leaders are supported and 'recognised'. In many case these clinicians are seen as leaders by their peers because of their own interests and efforts, and not because of a formal position. In these cases it is important that they are recognised and supported; 
- ensure that the performance feedback loops are working so that clinicians are aware of the impact of their efforts and can see a link between the changes being implemented and the impacts they have on i) the practice population and ii) upon specific patients; and

- utilise the knowledge and skills of the clinical leaders to help focus efforts on segments of the population with high needs and where changes to primary care can make a positive difference. Also, use clinical leaders to champion and develop, where required, practice processes that are aligned with 'best practice' and where there are clear evidential links between the process and clinical outcomes.

The key consequence of these policy recommendations not being followed is that of less focused effort, which in turn will decrease the quality of the services delivered, decreases the effectiveness of the programme, undermine engagement and ultimately limit success in implementation. Poor processes mean that more resource is applied and less outcomes are achieved, and in a resource-constrained practice, the consequences are less engagement, less effort and ultimately less success.

\subsection{The Influence of Context}

Not everything described in the above policy recommendations can be implemented at the same time, nor will they have the same level of importance and priority within each practice. The key determinants of timing and priority will be context.

For example, in a practice that has patients with higher levels of personal and community-based resources, more effort can be put onto the clinical side of the doctorpatient relationship, focusing much more on the first and third sets of policy recommendations. Practices operating in communities where patients have very limited health literacy and where community resources are minimal, will have to invest much more in engaging patients. This extra workload may have to be balanced by narrowing the focus of the programme to, for example, one condition such as diabetes or maybe on a subset of the enrolled population, for example, patients with high risk of complications. Unless this is done, there would be a danger of resource demands rising 
to a level where clinicians no longer feel it is possible to implement, thereby undermining clinical engagement which will in turn undermine efforts to develop patient engagement.

What this highlights is that the implementation of new health practices often requires changes to resource flows, processes and sometimes values (Hovmand \& Gillespie, 2008). When the change disrupts current patterns, it may in fact decrease overall performance and the assumption that the implementation of 'best practice' improves performance is not always the case (Hovmand \& Gillespie, 2008).

Success is more likely if practices take account of the contextual factors and use that to design both the focus and scope of the implementation. Assuming a resource constrained environment, then balance will always be required and assessment of the context, using the five contextual factors in the model, can help establish where the priorities are, where the resource constraints are and what would be an optimal mix and timing for the implementation programme.

\subsection{What Makes a Good Conceptual Model?}

This chapter has described, in detail, the development of a model to describe a theory of implementation, based on the initial interviews with the health experts and the themes that emerged from them. The model building process also utilised the relevant literature to provide further explanation and support for the concepts discussed by the experts. The literature was especially useful in helping clarify relationships, for example, the link between clinical engagement and action. This was clarified in the work by Locke and Latham (2002) which helped make the causal link between engagement and improved care much more explicit. So, while the experts were consistent in their view that clinical engagement was important in improving care it was the literature that provided the additional information that showed how this engagement has been shown to lead to improved care. i.e. that engagement, or commitment as it is described in the goal setting literature, preceded action and it was action that delivered the improved care. The literature thus provided support for the concepts put forward by the experts and helped provide more detail about the causal links between them. What the model added was a 
plausible and coherent link between the concepts so that they were no longer seen as a set of discrete factors, but as part of a coherent set of interdependent processes. The result is a model that captures a theory of implementation in a primary care setting.

However, while each concept within the model finds support in the literature, the 'whole' is a new contribution and at this point it is not clear whether or not that contribution has much value. Does it, for example, provide an improvement on the mental models that those who work in the field already have in their head, and which they would describe if asked? In asking the question this way I am also pointing to the criteria that needs to be used in assessing the quality of the model. Models of social systems cannot be assessed against some 'imaginary perfection’ (Forrester, 1968), but against the mental and descriptive models that are currently used and which provide the basis of 'advice' in designing and implementing care for people with long-term conditions. But how will we know if the model described in this chapter is better than the mental models which people would otherwise use? It is if it provides some insight into the dynamics involved in designing and implementing such programmes that our current mental models do not.

The question that has to be asked, therefore, if confidence is to be built in the model, is to what extent do those involved in implementing new health programmes for the care of people with long-term conditions feel that the model actually provides them with some insights into the design and implementation processes? How that question was asked, and the answers that were received, is explained in the following sections.

\subsection{Testing the Model}

To test the model I have chosen to use the criteria described by Schwaninger and Grösser (2008) whose work on SD as a process of theory building provided one of the motivations for this research. Wanting a more complete and operational set of criteria they used Patterson's eight criteria for evaluating theory (Patterson, 1986), supplemented with definitions by Holton and Lowe (2007). The result is a set of ten 
criteria, which can be described in clear concrete terms and against which the quality of the model can be assessed.

Schwaninger and Grosser's (2008) work focuses on quantitative models and as a consequence two of the criteria are not applicable to a qualitative model. These are refutability and reliability. Refutability refers to the ability to test the underlying structural and behavioural assumptions of the model. Reliability refers to a model that is free of measurement errors and the results of any test, using the model, would remain constant under identical conditions. As quantitative models have an explicit set of mathematical equations it is possible for these to be tested, but as the model described above is purely qualitative, such tests are not possible.

Leaving out these two criteria left eight of those described by Schwaninger and Grösser still applicable, and it is these that were used to test the model. These are described below:

\subsubsection{Criteria for Testing Model Quality}

Importance: A model can only be assessed in relation to its purpose and by those for whom it has been developed. In this case the target audience of the model are health professionals involved in the design and implementation of programmes to improve care for people with long-term conditions. Understanding how important such a model is to them is a key test of quality.

Clarity: The model describes a set of variables and how they relate to each other. For example, patient engagement is important, as it is a precursor to improved selfmanagement skills, which in turn improve adherence to programmes and treatment protocols thus improving the management of their condition. Furthermore, patient selfmanagement will depend on i) how much effort the clinical team put into engaging the patient, ii) the current level of knowledge skills and confidence the patient has and iii) the level of community resources and support. One of the challenges in developing such a model is to ensure that the variables and the relationships between them are clear.

Parsimony and Simplicity: The model, in trying to capture the key dynamics involved in implementing new health programmes, has 20 variables connected with 34 distinct 
relationships, driving seven key feedback loops which are all affected by five different factors of context. It is complex. A challenge in trying to develop models that reflect useful aspects of the real world is in making choices about what to include and what to leave out, aiming for a parsimonious balance between simplicity and complexity.

Comprehensiveness: Although all models are a simplification of the real world they need to be broad enough to cover the substantive issues of interest. This model focuses on the primary care practice and assumes that there is general support for the idea of improving care for people with long-term conditions. Convincing people that improving such care is important is not within the boundaries of the model, although convincing them that the particular approach being recommended is worthwhile and/or feasible is. If the model is comprehensive it should allow structured discussions about the key issues facing a primary care practice that wants to implement a programme to improve care for people with long-term conditions.

Operationality: Operationality refers to how well the model includes variables that have a counterpart in the real world. To be operational, the model must be concrete enough so that the variables could be described, measured and tested in the real world of practice.

Validity: Validity refers to how well the model provides an accurate picture of the real world. Does the model imply the behaviours you would expect to see in the real world? For example, if clinicians became overloaded and resource demand exceeded resource supply would, as predicted in the PMI, clinicians start doubting that the implementation could in fact succeed and reduce their efforts to implement the programme.

Usefulness: Usefulness refers to whether or not the model provides insights into the reality of implementing new programmes for the care of people with long-term conditions. Could it, for example, illuminate discussions about the issues facing practices with differing levels of resource demand and capability, or differing levels of knowledge and/or capability within the clinical team and/or patient group? Could it, for example, be helpful in guiding practice discussions about what would be required to successfully implement a new programme within their practice? Could it, for example, 
be used to evaluate an implementation programme and assess what is contributing to current levels of performance? The extent to which it can is an indicator of usefulness.

Practicality: Practicality refers to whether or not the model provides a useful conceptual framework for practices trying to implement new programmes for the care of people with long-term conditions. A practical theory provides decision makers with a practically relevant framework of the variables that are essential for policy and management of an implementation programme.

The following section describes the results of the feedback sessions with the health experts used to assess the quality of the model.

5.7.2 Testing Model Quality: Interviews with the Health Experts

There are two fundamentally different approaches that can be taken when building models of complex systems. One approach is to develop models that are able to mimic behaviour. Econometric models are a good example of this. The focus of these models is on reproducing behaviour seen in the 'real world' with little interest in replicating the casual structure that drives the behaviour. (Bossel, 2007, p. 19). The other approach is the one taken here, in which the focus is on trying to represent system structure, to better understand what is causing the observed behaviour. The model developed in this chapter is therefore a model of system structure, not of system behaviour. Building the model has focused on understanding the parts of the system, how they are connected and how they influence each other. In testing the model therefore, the focus was on whether or not the structure, as described in this chapter, provides any new insights into the world of implementation in primary care.

To test this, the model was presented back to the health experts originally interviewed, to get their feedback, using the criteria noted above to structure the conversation. The material used in the feedback sessions is shown in appendix 2. Of the seven initially interviewed, five were able to be contacted and all were willing to provide further feedback.

In all cases the importance of the topic was still high and the experts commented that trying to capture the key dynamics was still an important and useful endeavour. In terms 
of clarity all of the experts interviewed found the depiction of the variables and the relationships between them to be clear. While the model was parsimonious, two of the experts did argue for it to be more comprehensive. One argued that the initial engagement was heavily influenced by the culture of the practice and the model would be enhanced if that aspect had been developed further. Culture in this context was described as the curiosity amongst clinicians to explore better ways of doing this and to understand how well, or poorly, they were performing. A second expert felt that performance data and feedback was so influenced by information technology that the model erred on the side of simplicity. Although making it more complex, including information technology into the model would make it more complete. In terms of operationality, two of the experts interviewed started a discussion about how the model could be used to help design implementation processes. They suggested developing checklists, aligned to key variables, to assess individual practices and, as a consequence, be better able to design effective implementation processes that took account of the specific practice characteristics. One wanted a copy of the model to share with an internal project team, as it provided him with insights into some difficulties they were having with a programme to improve the uptake of cardiovascular disease (CVD) risk screening. A second, currently operating as a workstream leader for a region-wide change programme in primary care, invited me to facilitate a working session to discuss the model and its implications for their programme. A third, senior academic and clinician immediately following the feedback sessions emailed a number of senior managers within the DHB to set up a meeting to discuss the model. It was felt to be of relevance to work currently being done developing a set of Key Performance Iindcators (KPIs) for primary care in the region.

While this is a narrow sample, and the analysis is purely qualitative, it is clear that, in terms of the above criteria of model quality, the model, as viewed by a number of health experts:

- is tackling an important subject;

- is clear and easy to follow;

- has achieved a reasonable balance between simplicity and complexity, although two of those interviewed felt that the model would be more 
comprehensive with the addition of information about culture and

information technology;

- is operational in that clear connections to real issues could be made;

- has a degree of validity in that it provided insights into current challenges, with all those interviewed being able to point to aspects of the model that connected to current issues they were facing; and

- is practical in that three of those interviewed found that the model provided some insights to issues they were facing and increased understanding of the causal mechanism underpinning them. All three also asked for copies of the model.

\subsection{Summary}

In this chapter I described the feedback perspective and showed how it can be applied to models such as CCM. I then applied this perspective to each of the themes, discussed in chapter 4. Using the themes elicited from the initial interviews with the seven health experts, and the relevant literature, a coherent model of implementation was developed and described using a CLD. The model goes beyond describing a list of key factors, showing the patterns of interactions between the key concepts in each of the themes and the key elements of context. In addition, the development of the CLD resulted in the emergence of three higher-order constructs that linked the more detailed concepts together. These constructs were, clinical engagement, patient engagement and delivery processes and were used to frame a set of recommendations that were developed on the basis of the implementation theory, described in the CLD.

The chapter concluded with a description of what makes a good conceptual model, using the schema described by Schwaninger and Grosser (Schwaninger \& Grosser, 2008) and how this was tested in the involvement of and feedback to the health experts interviewed in the development of the model.

Chapter 6 takes another step by converting the qualitative model described in the CLD into a SD simulation model. Chapter 7 then uses the simulation model to run a series of experiments, to explore the size and impact of the causal connections to see how the 
structure described in this chapter influences performance over time under a range of scenarios. 


\section{Simulation Modelling Exploring the Dynamics of a Theory of Implementation}

As mentioned in chapter 2 , much of the research on implementation has focused on identifying key factors that influence the successful implementation of new models of care for people with chronic conditions. My interest in this research has been to examine the processes occurring within a primary care practice that affect the implementation of new care practices and to explore how these processes could evolve over time. Chapter 5 put forward a theory of implementation, based on interviews with leading experts working in the New Zealand health sector, that described some of these processes and how they interact. However, as it is a purely qualitative model, it is unable to provide insight into the strength and timing of the interactions, nor how they are likely to evolve over time. That is the purpose of the simulation model described in this chapter.

As discussed in chapter 3, the use of simulation models to support theory development is a small but growing aspect of research in the social sciences and in management. As shown below in figure 6.1 a search, in Google Scholar using the terms 'Management', 'Theory' and 'Simulation' shows an increasing number of published papers, since 2000, using simulation methods to explore and develop theory in the management field.

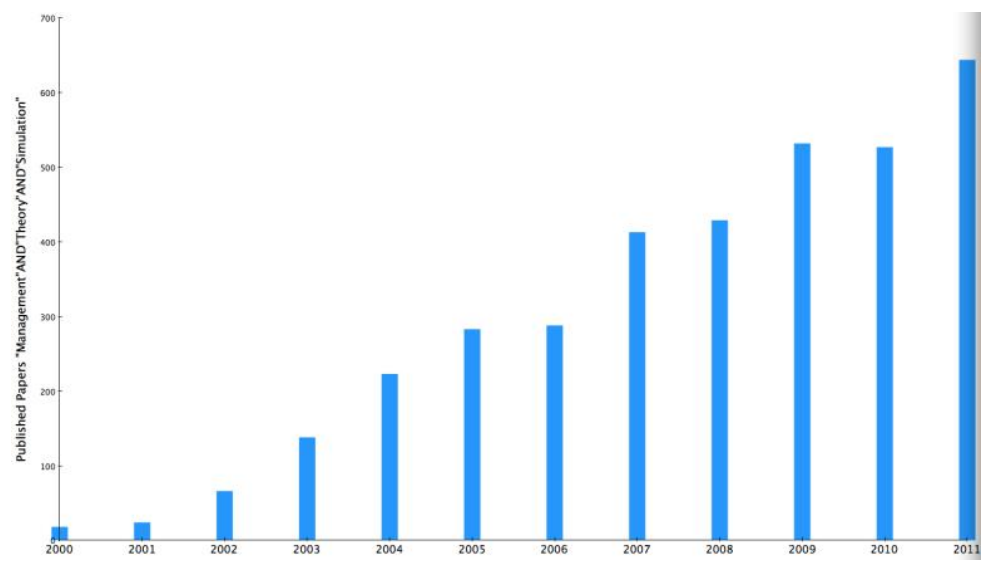

Figure 6-1: Published papers "Management" AND "Theory" AND "Simulation" 
While translating the rich descriptions provided by the health experts in the interviews into a qualitative model and then into a simulation model does result in some loss of depth, it does have three corresponding benefits that make the development of a simulation model worthwhile. Firstly, as pointed out by Repenning (2002), developing a simulation model enforces an internal consistency in the theory being modelled, ensuring that the behaviour it purports to explain can in fact be generated by the assumptions that underlie it. Developing a simulation model does, if nothing else, show, whether or not the theory is a possible explanation of the behaviour being explored. Secondly, a simulation model allows a researcher to explore the multidimensional nature of implementation, discussed in chapter 4, and go beyond the tendency in the literature to focus on single variables. Thirdly, a simulation model provides an experimental laboratory in which the implications of the theory's assumptions can be explored.

In this chapter, the qualitative model developed in chapter 5 is translated into a SD model. Analysis of the model, which will be undertaken in chapter 7, will explore the insights, as well as the limitations and constraints of the theory. This chapter will focus on describing, in detail, the major equations used to formulate the model. Appendix 4 contains the full list of equations and functions.

\subsection{Core Components of the Theory of Implementation}

The CLD model, shown in its complete form in figure 5.11, and reproduced below in figure 6.2, describes the complex dynamics involved in making the changes required to shift the focus of primary care practices away from the immediate demands of acute care and more towards managing the underlying causes of the presenting symptoms when working with people with chronic conditions. It provides a comprehensive theory of what is required to manage the tensions inherent in balancing the 'short-term gain' and 'long-term pain', which was discussed in chapter 5 and shown in figure 5:1. Within the model there are three key constructs. The first is 'clinical engagement', which is the engine of change delivering improvements in the management of long-term conditions. The model describes three feedback loops that determine how clinical engagement develops over time; 'performance feedback' (R1), 'resource demand' (B1), 'self-efficacy' (R6), as well as the initial and ongoing importance of 'collaborative planning and design'. 
The second key construct is 'patient engagement' which is required if significant improvements in care are to be made. The model describes two feedback loops here; the first being the efforts made by the practice staff to engage patients and help develop improved 'self-management' (R4), and the second is the development of a 'supportive community' (R5). The third key construct is the 'quality of service delivery' driven by two key feedback loops. The first is 'quality of effort' (R2), based on an improved understanding of the enrolled population, and the second is 'quality of processes' (R3), based on a better understanding of what services actually affect clinical outcomes.

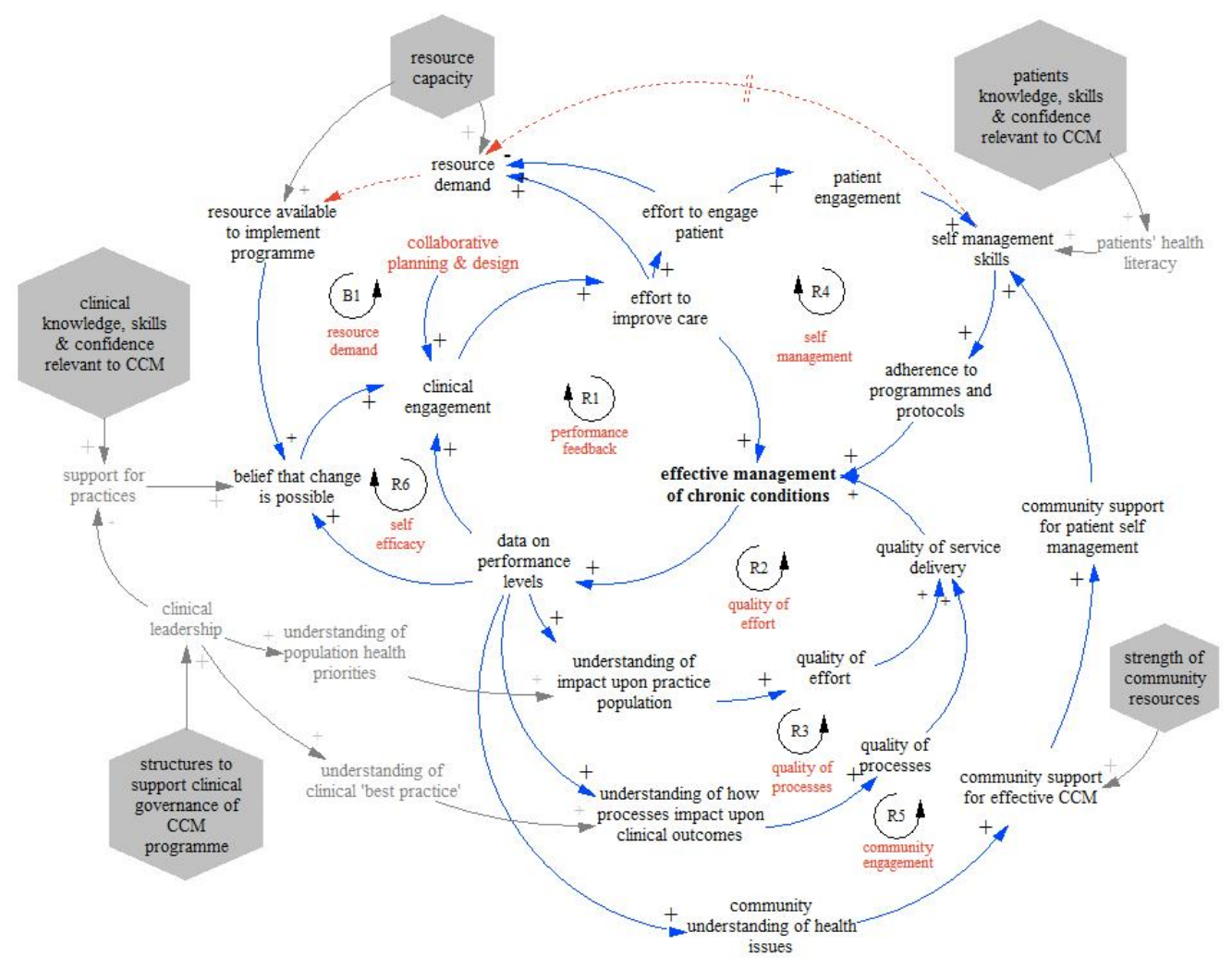

Figure 6-2 A causal theory of implementation

The theory, described in the model, postulates therefore, that the management of longterm conditions will be heavily influenced by how well or poorly the primary care practice manages the seven feedback loops described above and shown in figure 6-2. The purpose of the simulation model is to explore how this dynamic unfolds over time and to gain some insights into the interactions between these seven feedback loops. In doing so, it is hoped that it may help formulate guidelines for primary care practices 
engaging in the challenge of developing new models of care to meet the growing prevalence of long-term conditions in their patient population.

\subsection{Model Structure: Overview}

The unit of analysis for the simulation is a primary care practice. It is assumed that the practice engages in the full range of primary care including the treatment of long-term conditions. Thus their patients are broken into i) acute patients who appear for a specific acute condition, ii) patients with long-term conditions who appear when their symptoms are such that they seek medical treatment, and iii) patients with long-term conditions who are taking an active role in managing their conditions. While this is not an empirical model, the data used to set the initial conditions for the primary care practice are taken from what is known about the size of practices working within the Auckland region and the size of their enrolled populations. In addition, data has been taken from the New Zealand College of General Practitioners (Fretter \& Pande, 2006) to ensure that the parameters used in the model fit within known ranges of New Zealand primary health care practices. The patient groups are broken up into those who use primary care services largely for acute conditions, those who have long-term conditions and use their GP primarily for symptom control and those who have longterm conditions and have developed self-care capabilities. The baseline conditions used in the initial model for the size of the practice and the number of patients are shown below in table 6-1. The simulation model runs for 60 months (five years). 
Table 6-1: Baseline parameters for primary care practice

(Source of data: taken from what is known about primary care practices in Auckland and workforce and practice data from the New Zealand College of General Practitioners (Fretter \& Pande, 2006)).

Furthermore, the model assumes that the primary care practice has many of the infrastructural and technological requirements for good long-term care management and as a result does not need major investment in new capital equipment such as suitable premises or improved information technology. As described in chapter 2, good information and decision support tools are an important element for effective care of people with long-term conditions. However, improvement in the management of longterm conditions is, in this model, explored as a behavioural rather than as a technical issue. Therefore, while information is important in the model it is treated as an element of process feedback and not of information technology investment. Furthermore, as this model is not concerned with differentiating between types of conditions, such as diabetes or heart disease, effective performance is considered in relation to the overall goal of improving care for people with long-term conditions and does not address clinical differences between these conditions.

In line with the theory outlined in chapter 5 , there are three main constructs modelled; clinical engagement, patient engagement and quality of service delivery.

\subsection{Criteria for Formulating the Model}

To explain how the simulation model is built, I begin by describing the criteria used for building the simulation model, using an approach adapted from Anjali Sastry, (Sastry, 
1997). These criteria provide guidelines for specifying the model and standards against which model formulations and model performance can be judged. The following section will describe the specific formulations in detail.

6.3.1 The Search for Endogenous Explanations

A key feature of SD modelling is that it seeks to find explanations for behaviour within the interactions between variables in the system of interest. These are referred to as endogenous explanations. The word 'endogenous' comes from the Greek and means 'arising from within'. This is in contrast to exogenous explanations that focus on changes that 'arise from without'. The modelling builds this endogenous explanation by specifying how the theory is structured and the rules of interaction (the decision rules in the system). Thus, the qualitative 'expert' theory was studied to identify the key endogenous and exogenous determinants of improvement in the care for people with long-term conditions. Endogenous processes serve an important role in that they provide insight into how the behaviour of the model is generated by the variables and relationships within it and the model described in chapter 5 describes the pattern of interaction between these endogenous variables. Exogenous variables are important in that they provide key factors of context within which the dynamics described in the theory are played out. The exogenous variables, depicted inside hexagons that were described in the qualitative model (figure 6-2) were the:

- level of clinical knowledge, skills and confidence relevant to CCM held by the team charged with the implementation,

- level of patients' knowledge, skills and confidence relevant to CCM,

- structures to support clinical governance of CCM programmes,

- strength of community resources, and

- resource capacity.

Each of these is an important element of context and a prime purpose of the simulation is to explore how they impact the dynamics of implementation. 


\subsubsection{Grounding in Observed Behaviour and Evidence in the Literature}

It is important that the model formulations are grounded in what we already know about such relationships. As pointed out in chapter 3, much is known about individual aspects of implementation and what this model adds is the ability to explore how these individual aspects relate to each other, and how the relationships between them affect development over time. It is important therefore that in formulating these individual relationships best use is made of available evidence. For example, a key relationship described in the theory is the link between self-efficacy and clinical engagement. In the qualitative model, all that is asserted is that an increase in self-efficacy increases clinical engagement. To develop the simulation model the nature of that relationship has to be specified, that is describe the direction and size of the 'increase' that self-efficacy has upon clinical engagement. This and other key relationships are discussed in the model description section.

\subsubsection{The Model Must be Testable}

The third criterion is that the model must allow predictions of behaviour within the 'expert' theory to be compared with model outputs. Furthermore, where there is empirical evidence related to aspects of the theory it must be able to be compared against model outputs. Statements made by the health experts often describe behavioural patterns, which provide hypotheses against which the model can be judged. For example, much was made of the importance of providing feedback to clinicians about how well, or poorly, their efforts were in improving care. This feedback was seen as a key ingredient in building and maintaining clinical engagement and that this engagement was a key ingredient in sustaining the effort required to make the changes necessary for improved care. This is a hypothesis about how performance feedback improves care for people with long-term conditions and has to be built into the model in a way that allows it be to be tested against the statements of the health experts. How these tests have been conducted to assess the validity of the model is discussed in chapter 7.

It is important to note that the model has no 'pre-specified' behaviour built into it that determines practice performance in terms of effectively managing long-term conditions. 
The patterns of behaviour are built up from separate statements about individual relationships. The overall behaviour is a result of the interactions between these individual relationships.

\subsubsection{Model Boundaries}

It is also important to be clear about the model boundaries, as any model has to be a simplification of the real world. For example, in the original interviews, one of the interviewees commented on the need for the Ministry of Health to provide some consistency in terms of what was expected from primary care. While this may be an important issue, the boundaries of the model have been set around the primary care practice, and material that related to concepts not within the control of the practice have been excluded from the model. Like all boundary decisions, this decision was made on the basis of model purpose. In this case the purpose of the model is to explore the dynamics of implementing new health practices within a primary care practice. As a consequence, concepts emerging out of the initial interviews that were outside the control and/or influence of the practice itself were not included. While the model, as will be discussed in chapter 7, does have implications for Government policy concerning primary care, specific policies have not been included in the model itself. This research is interested in implementation, and is focusing on implementation of new health practice within the primary care practice, which, in the New Zealand context, is the main vehicle for delivering primary care services in relation to long-term chronic conditions.

\subsection{Model Specification}

In this section the key variables and constants used in the simulation are described. Supporting information for the specifications are also provided, where possible, to show that the formulations used are consistent with the 'expert theory' and with what is already known about the relationship. In addition, examples of model behaviour are shown. The purpose in this chapter is primarily to illustrate the model structure and the connections between key variables in the model. More detailed analysis of model outputs will be provided in chapter 7 . The model is also built up in stages, based around 
the three core constructs noted above; clinical engagement, patient engagement and quality of service delivery.

However, before showing how those constructs are modelled, it is important to clarify the key 'output variable', namely the 'effective management of chronic conditions'. The whole purpose of implementing new health practices is to improve the quality of care, which is described in the model as the 'effective management of chronic conditions'. This provides the focus for assessing the range of inputs; it is the level of this variable that indicates the success, or failure, of any intervention mix. The 'effective management of chronic conditions' sits at the centre of the model (figure 6-2).

\subsection{The Goal-Seeking Core of the Model}

At the centre of the expert theory of implementation described in the previous chapter is the desire to achieve a goal, in this context, the 'effective management of chronic conditions'. Before building the model that illustrates that theory it is important to develop the core 'goal-seeking' structure around which the details of the theory can be built. The initial interviews were conducted around one key question, "What do you consider to be the key issues that need to be addressed in implementing programmes for people with chronic conditions?" To enable the issues raised by the interviewees to be explored, a core 'goal-seeking' structure was developed as the focus, around which the key concepts in the model described in chapter 5 could be built.

\subsubsection{Core Goal-Seeking Structure}

The basic structure of the goal-seeking model has been well documented in the SD literature. For example see (Barlas \& Yasarcan, 2008; Morecroft, 2007; Sterman, 2000). As described by Forrester (1975a), this basic structure has four key components; i) a goal, ii) an observed state of the system's performance, iii) the difference between this observed state and the goal, and iv), an action designed to close the gap between the two. In this model, shown in figure 6-3, there is a single fixed goal goal for quality of

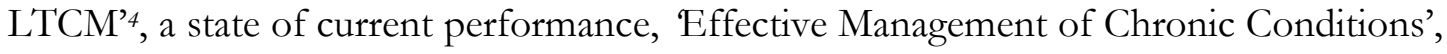
the difference between the current state and the goal, 'desired improvement in quality'

\footnotetext{
${ }^{4}$ Within the model LTCM is a short-hand for 'long-term condition management'
} 
and an action, 'change in quality of LTCM'. This structure also incorporates the time it takes to make that change, 'time to adjust quality of LTCM'.

While this configuration is simplistic, and will later be extensively modified to reflect the theory expounded by the health experts, it does provide a basic goal-seeking structure.

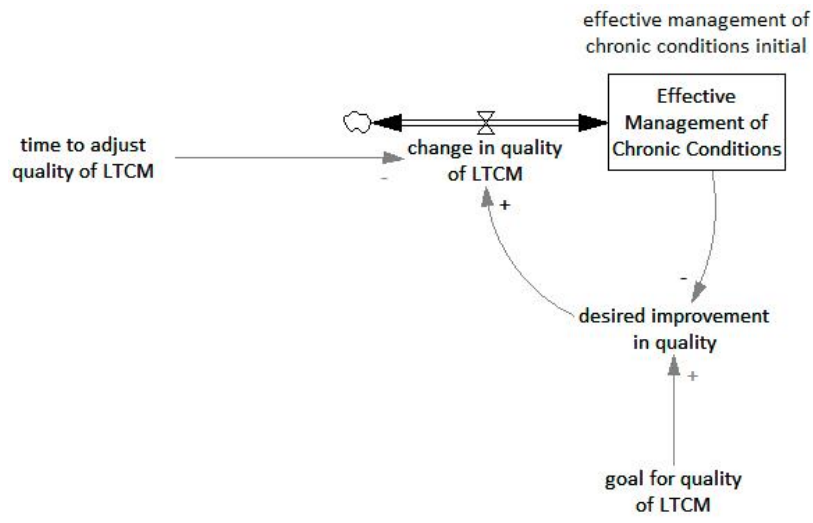

Figure 6-3 Goal-seeking structure

In this basic model, 'desired improvement in quality' is a function of the gap between current performance, 'Effective Management of Chronic Conditions' and the goal, 'goal for quality of LTCM'. The time taken to close that gap is determined by 'time to adjust quality of LTCM'. It is formulated as:

desired improvement in quality $=(($ implicit goal for quality of LTCM-Effective Management of Chronic Conditions)+(decrease in quality of LTCM care*time to adjust quality of LTCM))/time to adjust quality of LTCM

and

change in quality of LTCM $=(M I N($ desired improvement in quality, effect of improvement effort $) *(1+$ pct self care patients $* 5)$

Thus, the desired improvement in quality, over any specific time period, is affected by the gap between the current level of quality, the goal that has been set, and the time it takes to adjust quality. This desired improvement then drives the change in quality over time. The actual quality of long-term condition management is a function of the change in quality of LTCM' - that is the 'Effective Management of Chronic Conditions' accumulates all the changes in the quality of LTCM over time. 
This simple structure provides a core skeleton around which the rest of the model is built. However, before developing the model, it is important, given that this model is based on qualitative rather than quantitative data, to discuss the issue of parameter choice.

\subsubsection{Choosing Parameters}

As pointed out by Alan Graham (1980) most parameters in SD models, "are estimated on the basis of descriptive information obtained from participants in the system being modelled" (Graham, 1980, p. 144). It is because of this ability to work with descriptive information that the SD modelling approach is being used to explore the implementation of new health initiatives. As described in chapter 3 it is extremely difficult to capture and analyse data from multiple, interacting variables that change over time and in some cases the most important variables are difficult to measure (e.g. the impact of self-efficacy upon clinical engagement). This problem is compounded by the need to obtain comparable measures across a range of clinical categories and, in the case of this research, across a period of years. While the use of computer simulation does, to an extent, overcome these issues, the choice of parameters is still an important part of the model building process.

While the theory expounded by the experts does not provide empirical data, it does provide detailed qualitative descriptions that can be represented formally in a model. For example, within the theory expounded by the health experts there is the assertion that time spent on process improvement will increase process quality. This, in turn, will contribute to improving the overall quality of long-term conditions management. To model this, a quantitative relationship has to be set up between time spent on improvement activities and the level of process quality. The following graph, figure 6-4, shows such a relationship in which the qualitative assertion is given a quantitative specification. 


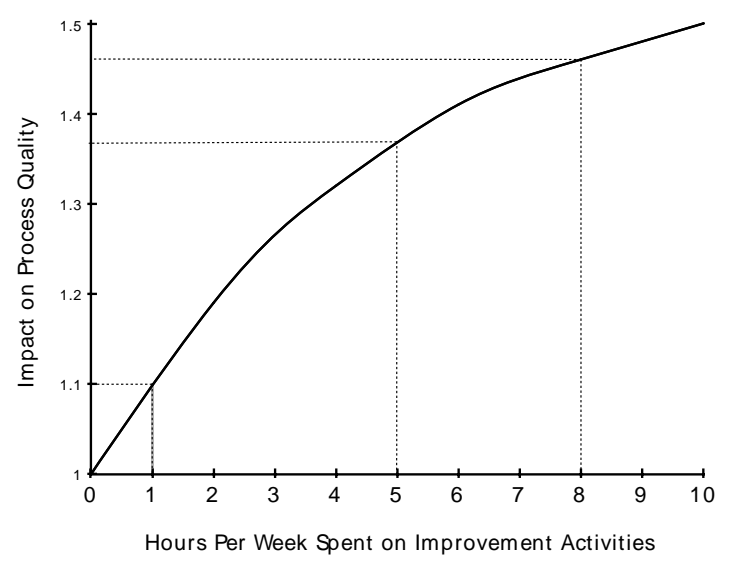

Figure 6-4 Impact of improvement activities on process quality

In this case, as more hours per week are spent on process improvement, quality improves. It is not a linear relationship however, with increasing hours providing less incremental improvement in quality. One hour per week provides a 10 per cent improvement in quality, five hours per week provides a 37 per cent improvement in quality and eight hours per week provides an increase in quality of 46 per cent.

This type of relationship between variables is common in many situations, in which a change in one variable produces a change in another, but at a decreasing rate. The following graph, figure 6.5 , shows how the successful treatment rate of tuberculosis in New Zealand has increased during the last decade. Although still improving, the large increase from 2001 to 2002 has declined over the years and now seems to be levelling off at somewhere between 70 and 80 per cent (figure 6-5). Thus, each year passing provides a smaller and smaller increment of improvement. This is a common pattern, where behaviour is striving for some goal, in this case a 100 per cent success rate in the treatment of tuberculosis. As that goal gets closer to being achieved, the incremental steps of improvement become smaller. 


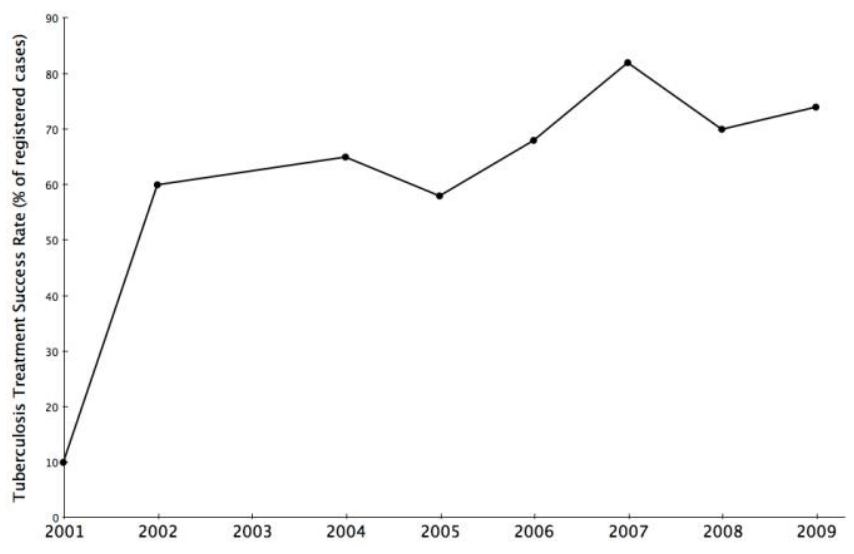

Figure 6-5 tuberculosis treatment success rate

(World Bank Indicators - New Zealand. http://www.tradingeconomics.com/newzealand/tuberculosis-treatment-success-rate-per cent-of-registered-cases-wb-data.html Accessed July 2012)

Providing a quantitative specification of the qualitative assertions is a key part of the modelling effort and each will be explained in the description of the model specification below, along with the rationale for the choice made. As a general rule, I have, in choosing parameters, endeavoured to ensure that they are clearly linked to real-world phenomena and have a basis in the empirical literature. In addition, I have developed formulations that yield operating points either between zero and 100, where 100 represents 100 per cent of what is possible or desirable or between zero and one, where 1 represents 100 per cent of what is possible or desirable. Choosing these parameters allows me to explore changes that can be formulated in language that has meaning at a practical level. For example, increasing the level of patient engagement by 20 percentage points. Even if there is no data available it still makes sense, in a very tangible way, to talk about increasing, or decreasing a variable such as the level of patient engagement by 20 percentage points or even thinking of 100 per cent engagement. While developing 100 per cent engagement of patients is unlikely to occur in practice, setting parameters between 0 and 1 or 0 and 100 does allow the impact of such an event to be tested.

It is important to point out here that, in providing a quantitative specification, the model is not claiming the degree of specificity implied in the use of numbers. So, 20 per cent improvement for example, is simply a quantitative specification of the qualitative concept of 'significant improvement'. It has no more precision, and simply 
reflects the fact that computers need numbers to run. Similarly, 100 per cent engagement does not imply that there is an explicit measure that one can use to assess 100 percent. It is simply a quantitative representation of the highest level of engagement you could imagine in that specific context.

Finally, in the analysis of the model outputs, which will be described in detail in chapter 7 , these numerical representations will be varied to explore how sensitive key model outputs are to changes in these numbers. That is, to explore the implications of shifts in specific inputs from, for example, 'little' to 'lots'.

\subsubsection{Baseline Run}

In the initial run the 'goal for quality of LTCM' has a parameter value of 80, being 80 per cent of best practice. That is, in the baseline run it is assumed that the primary care practice is seeking to achieve a goal that is close to, but not quite, 'best practice'. This reflects the reality within many primary care practices where clinicians believe that, due to a number of factors, while they can improve their performance, best practice is not achievable across the practice with all conditions and all patients. This and other parameters will be changed in later runs. In the baseline run the key parameters are:

\begin{tabular}{lll}
\hline Model Variable & Unit of Measure & Parameter \\
\hline effective management of chronic conditions & Quality & 20 \\
goal for quality of LTCM & Quality & 80 \\
time to adjust quality of LTCM & Month & 6 \\
\hline
\end{tabular}

Table 6-2 Key parameters in the baseline run

The figure of 20 for 'Effective Management of Chronic Conditions' was chosen for the baseline as it reflects the fact that that most primary care practices only apply proactive programmes for the care of people with chronic conditions to specific conditions, such as diabetes. Such programmes are very rarely spread across the range of conditions presented by the enrolled population. Thus, even if their management of diabetes is good, as it represents only a fraction of the relevant conditions, their overall quality is not likely to be above 20 per cent of best practice. Further support for a starting point of 20 is provided by a recent unpublished report of the percentage of diabetes patients 
in the South Auckland region who have met the clinical conditions for control. The report provided data on all diagnosed diabetes patients within Counties Manukau, a large region in the southern suburbs of Auckland, broken down by specific practice and locality. The clinical criteria used to assess control was HBA1c $<8$, SBP $<135$, LDL $<2.5$ and those patients meeting those criteria can be said to be receiving good quality care that enables them to effectively manage their condition. The average across all primary care practices within the South Auckland region was 19 per cent. If care for people with diabetes is a reasonable indicator of overall success in managing the longterm conditions of patients then a starting point of around 20 per cent is appropriate.

The behaviour of this initial model is a classic negative feedback loop which acts to bring the system in line with its desired state, in this case the stated goal for quality of LTCM'. This behaviour is shown below in figure 6-6.

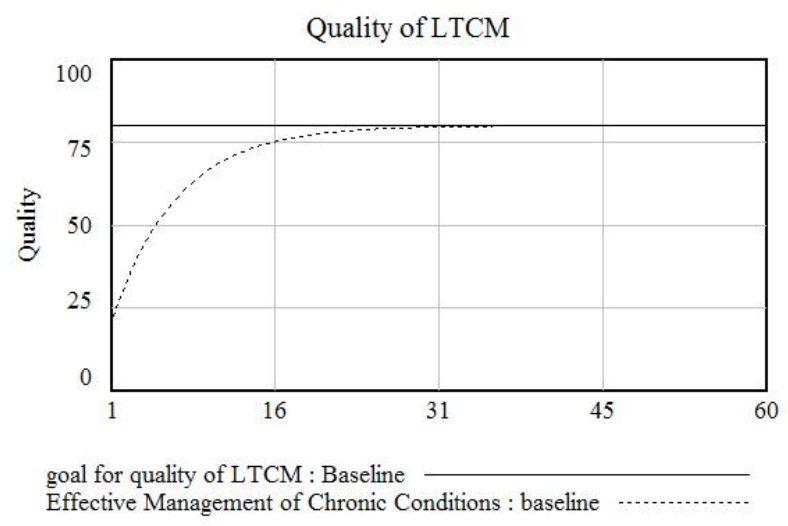

Figure 6-6 Effective management of chronic conditions

In this simple model the goal will always be reached, modified only by the stated goal, the starting position and the time it takes to adjust quality.

This model, and all subsequent variations, runs for 60 months (5 years), with a time-step of 0.25 . I have chosen the simulation time period as one month as it will allow me to interpret parameter values in terms of the effect they have on a monthly basis. Given the focus of the research, a time period of less than one month is not likely to have much impact, while time periods of a quarter, or a year, may hide many interesting changes that take place over a shorter time period. 
6.5.4 Introducing Capacity Limits and Baseline Performance ${ }^{5}$

Before beginning the description of how the key factors in the implementation theory are modelled, the goal-seeking core of the model needs to be expanded to include some limits on the ability to improve the quality of care. One of these limits is the resource available to commit to the task. As one interviewee described it, "there are competing priorities and resource constraints". This is incorporated in the model as a 'maximum improvement capacity'. This variable reflects both the number of clinicians in the practice plus their level of knowledge, skills and confidence in working with long-term conditions. It places a limit upon how much improvement is in fact possible. In addition, it is assumed that unless there is an ongoing effort to improve, or at least maintain, a level of performance it will drop to what is the accepted norm, or baseline performance, within the relative peer group; 'baseline performance of LTCM in primary care'. If programmes to improve care for people with long-term conditions are not that well developed within the peer group then, unless efforts are made to improve the level of performance within the practice it will drop to a level comparable with that group. This is based on the fact that chronic care management is not the norm within primary care practice, which is still dominated by an acute model of care (Rea et al., 2007). As a consequence, unless efforts are made to develop and maintain improvements within the practice, the acute model will dominate and the quality of LTCM will decrease to what is considered the norm within the peer group. These additional variables in the model are shown below in figure 6-7.

\footnotetext{
${ }_{5}^{5}$ All the simulation runs described in this chapter, from this point forward, can be replicated in the model. To do this all that is required is that the relevant .cin file is loaded. The .cin files replicate all the input variables used in the simulation run and are provided in the model folder. A table is presented at the end of this chapter describing each simulation run and the relevant .cin file.
} 


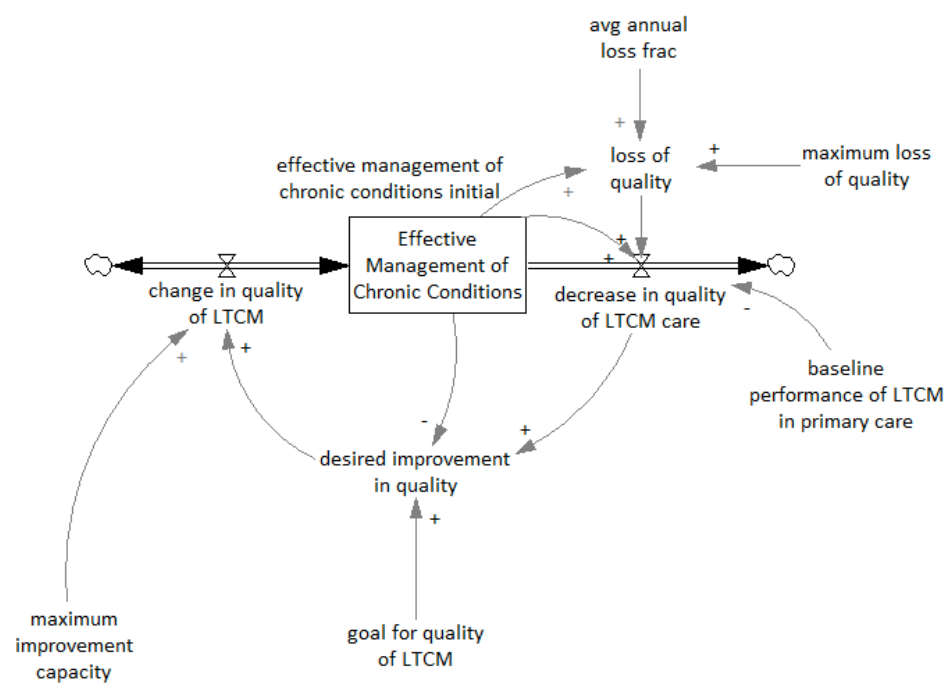

Figure 6-7 Incorporating loss of quality and limits upon improvement capacity

In this version of the model the 'desired improvement in quality' now has to take into account the ongoing 'decrease in quality of LTCM'. This is formulated as:

(goal for quality of LTCM-Effective Management of Chronic Conditions)+decrease in quality of LTCM

In addition the 'change in quality of LTCM' now has to take into account the limits imposed by the 'maximum improvement capacity'. Thus the 'change in quality of LTCM' in figure 6.7 is the minimum of the desired improvement and the capacity to improve. This is formulated as:

MIN(maximum improvement capacity/12,desired improvement in quality)

The 'maximum improvement capacity' is divided by 12 to take into account that the model is being run in months and the parameters are based on 'maximum capacity per year'.

The key additional parameters in this model are shown below in table 6-3: 


\begin{tabular}{lll}
\hline Model Variable & Unit of Measure & Parameter \\
\hline maximum improvement capacity & Effort & 30 \\
average annual loss fraction & $1 /$ Month & 0.25 \\
baseline performance of LTCM in primary care & Quality & 20 \\
\hline
\end{tabular}

Table 6-3 Parameters for capacity limits

The loss of quality in LTCM, 'decrease in quality of LTCM', is modelled as a function of a loss fraction 'avg annual loss frac' and a baseline performance, 'baseline performance of LTCM in primary care'. The baseline represents what is the norm in comparable practices, and it is the level to which the practice will fall if no efforts are made to improve and/or maintain the quality of their management of chronic conditions. The 'avg annual loss frac' of 25 means that if no effort is made to develop and/or maintain capability in LTCM the practice would lose 25 per cent of its capability per year, until it reached the baseline level. This is altered in future runs of the model as it is affected by such factors as the turnover of clinicians within the practice. 'Baseline performance of LTCM in primary care' is given a value of 20 to indicate an average level of performance within comparative practices. As described above, in the baseline case the practice is already operating at this norm.

In this model, whether or not performance improves will depend on whether or not the 'maximum improvement capacity' exceeds the 'decrease in quality of LTCM care'. By choosing a parameter value of 30 for 'maximum improvement capacity' the model ensures that there will always be the capacity to improve. That is, the improvement capacity is greater than the rate at which quality decreases. At this point in the model development, the improvement capacity is an exogenous variable, but as the model develops this, along with some of the other exogenous variables, is incorporated into the model structure, thus becoming a part of the dynamics of implementation rather than an externally set parameter. Three runs of this model are shown below in figure 6-8, to illustrate the different levels of performance under a range of improvement capacities: 


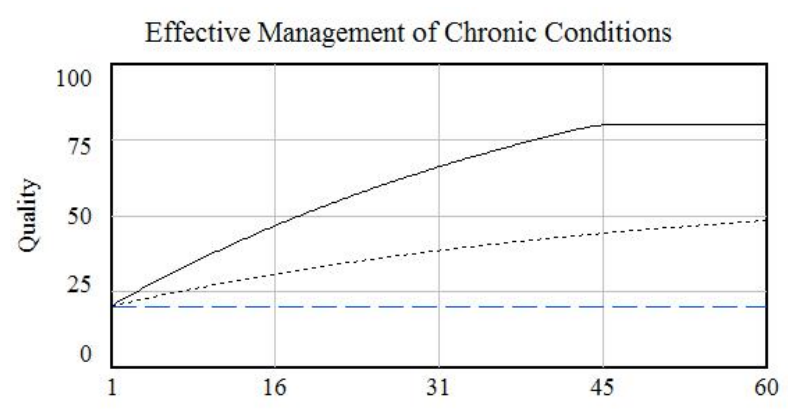

Effective Management of Chronic Conditions : full capacity

Effective Management of Chronic Conditions : some capacity

Effective Management of Chronic Conditions : no capacity

Figure 6-8 Effective management of chronic conditions: impact of capacity

In the 'Full Capacity' run, which assumes that the 'maximum improvement capacity' is 30 , the performance goal, which equates to 80 per cent of best practice, is reached in about three and half years. If capacity is reduced so that 'maximum improvement capacity' is only 15 , the performance goal is not reached, rising to 50 per cent of best practice at the end of the simulation. If the practice has no capacity to improve, that is, 'maximum improvement effort baseline' is set to zero, performance does not improve, staying at the level of the baseline performance, which is set at 20 per cent of best practice.

This initial model therefore incorporates limits to improvement capacity and acknowledges that unless continuous effort is made to improve care for people with long-term conditions, then performance will fall to a baseline level that is comparable to the accepted level within the practice's peer group.

This section has described the core goal-seeking structure around which the key ideas of the health experts have been built. The next section begins the process of integrating the ideas of the health experts into this structure. The key ideas will be built into the model, one step at a time, creating an increasingly complex model with a wide range of dynamics. The full model will be used in chapter 7 to explore the implications of the overall theory. In this section, each addition to the model will be described and the dynamics associated with it explained, while keeping all other variables constant. Thus, it 
is hoped that the impact of each core theme within the theory can be understood more clearly.

The build-up of the full model begins with the concept of clinical engagement, which was central to the expert theory of implementation.

\subsection{Clinical Engagement}

The previous section described how the structure of goal attainment has been modelled. The theory developed in the previous chapter however highlights that while there maybe an officially stated goal, the engagement with that goal may not be 100 per cent, and as a consequence efforts to achieve that stated goal are limited. This section describes how clinical engagement has been incorporated into the model and how it affects the attainment of the goal.

\subsubsection{Stated and Implicit Goals}

To reflect the impact of clinical engagement on the pursuit of improvement goals, an additional concept has been incorporated into the model, an implicit goal, which is a consequence of changing levels of level of clinical engagement. In this model the 'goal for quality of LTCM' is no longer the only driver of the 'desired improvement in quality'. Instead it is now modified by an implicit goal, the goal that the primary care team are really striving for. Thus the 'goal for quality of LTCM' shown in the goal-seeking structure has been replaced by the variable 'implicit goal for quality of LTCM'. The less support the clinical team have for the stated goal of the programme, the more support they will have for an implicit goal, which better reflects what they think is desirable and/or feasible. The model structure for this implicit goal is shown in figure 6-9. 


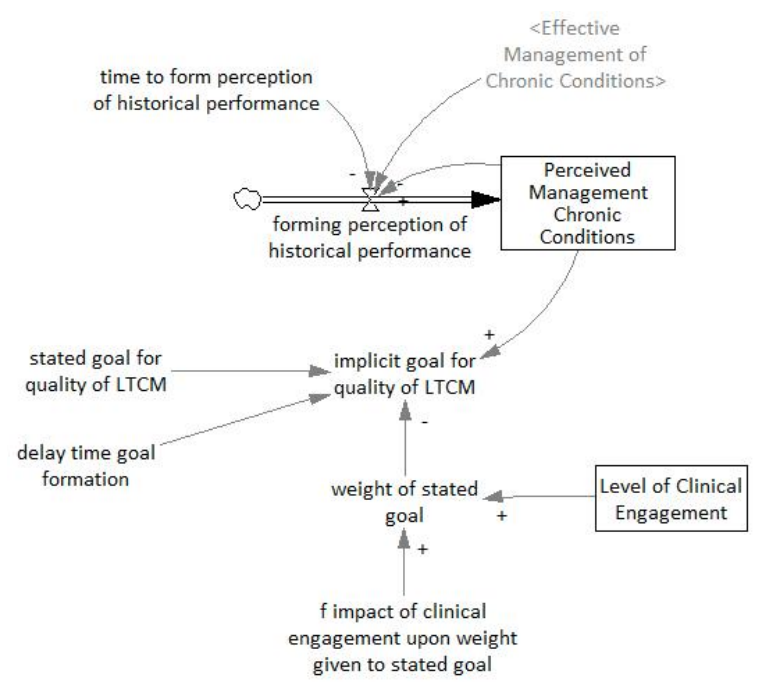

Figure 6-9 Clinical engagement and the development of the implicit goals

Perception of what is possible is influenced by current practice and how that has changed over time. That is, the clinicians' perception of historical patterns of performance will influence what they believe is possible in the future, with actual performance becoming, over time, the traditional or historic performance of tomorrow. This is based on the formulation initially developed by Forrester (1975a) in his work on planning and goal creation. In this structure the implicit goal is affected by three variables; i) the stated goal for the improvement initiative, ii) the historical performance that the practice has been able to achieve and iii) the weight given to the stated improvement goal. Thus, when actual performance persists below historical performance, the perception of traditional performance declines accordingly, and a downward spiral of practice occurs contributing to a lowering of what is perceived as possible and thus a lowering of goals. If, on the other hand, actual performance is higher than historical performance it will continue to increase, limited only by the improvement capacity. In this model, whether the implicit goal, that actually drives change, is driven more by historical performance or by the stated goal is a function of clinical engagement. The level of clinical engagement determines the weight given to the stated goal.

The consequence of this relationship, in the model, is that even where there is enough improvement capacity, the explicit goal may not be achieved because clinicians do not 
believe it is either worthwhile and/or possible. Figure 6-10 shows three scenarios, each of which maintains 'maximum capacity to improve' at 30, the figure used in the initial goal-seeking model. The first scenario is the baseline run in which there is 100 per cent engagement. As a consequence the stated goal is the driver of performance and as a result the simulation run is identical to the 'full capacity' run shown in figure 6-7. The second run lowers engagement to 50 per cent, so that performance is driven by a balance of the stated goal and historical performance. The third scenario assumes no engagement with the stated goal, in which case performance is driven by historical performance.

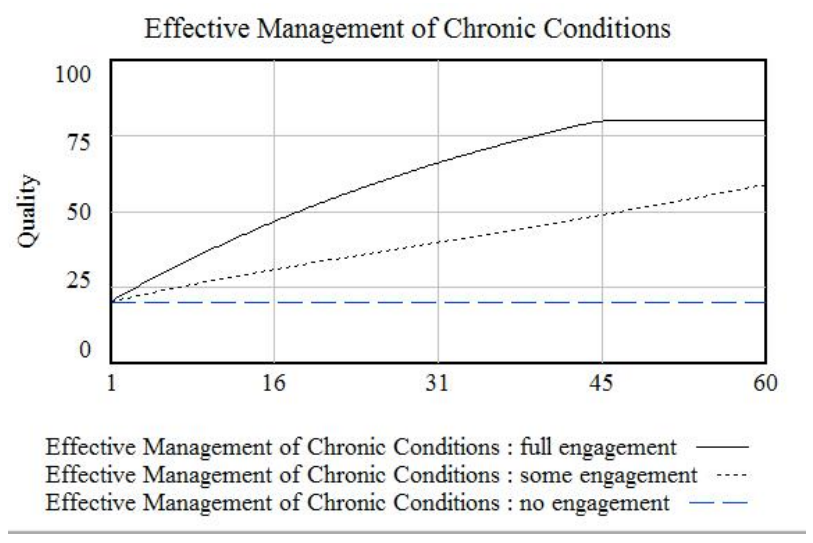

Figure 6-10 Effective management of chronic conditions: impact of clinical engagement

Central to this output is the relationship between the level of clinical engagement and the weight given to the stated goal. In this formulation the 'weight of stated goal' is a function of 'level of clinical engagement' and ' $\mathrm{f}$ (impact of clinical engagement upon weight given to stated goal)'. The ' $\mathrm{f}$ put at the beginning of the model variable description indicates that this is a function describing the relationships between two variables. This is modelled by setting up a Lookup function in the Vensim modelling software used for the research. This function is shown in figure 6-11. 


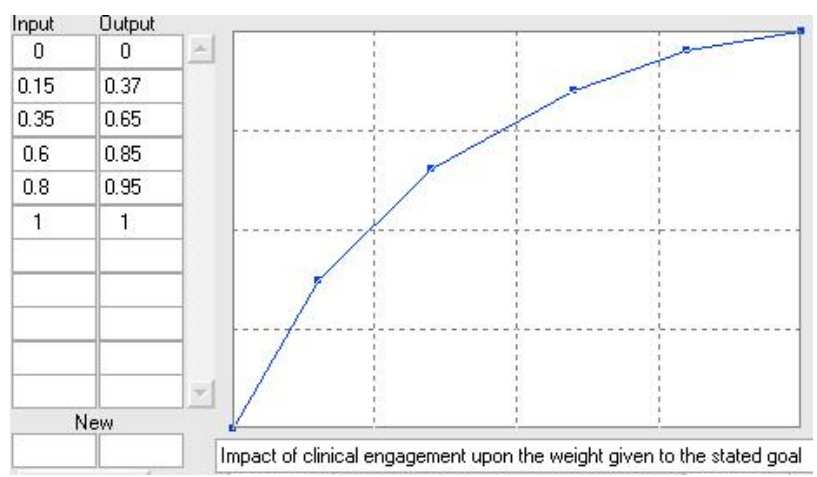

Figure 6-11 Impact of clinical engagement upon the weight given to the stated goal

The function table shown above describes the causal relationship between the level of clinical engagement and the weight given to the stated goal. The numbers on the lefthand side of the graph show the input numbers (level of clinical engagement) and the output numbers (impact upon the weight given to the stated goal). As the graph shows, if the level of clinical engagement is zero then the weight given to the stated goal is also zero, that is, the stated goal is not accepted by the clinical team. As clinical engagement rises so does the weight given to the stated goal until a stage is reached where, with 100 per cent engagement (level of clinical engagement $=1$ ), the clinical team give their full support to the stated goal.

In dynamic models these function tables play an important role, in that they translate qualitative statements such as, "The result of clinical engagement is that front line staff understand and support ... [the organisation's]... goals" (Beasley, 2006), into quantitative and testable propositions. This function table specifically describes the relationship between clinical engagement and support for the stated goal. If clinical engagement is 15 per cent then the weight given to the stated goal is 37 per cent, with major emphasis instead being put upon historical performance. If clinical engagement is 60 per cent the weight given to the stated goal is 85 per cent. The advantage of these tables is that the assumptions made about the links between, in this case clinical engagement and support for the stated goal, are explicit, testable and, as part of the simulation, able to be changed. 
The curve used in this table, as discussed earlier, is a common pattern used when there are goals towards which the system is striving. In these cases, the relationship is rarely linear, and often follows a pattern where the incremental increase gets smaller as performance gets closer to the goal being sought, in this case support for the stated goal. Sensitivity tests to assess the validity of these causal relationships are discussed in chapter 7, where the complete model is used to undertake a series of policy experiments.

This function reflects whether or not the clinicians believe that the goal is either desirable or achievable. In the expert theory however, these two concepts of desirability and achievability are treated separately. Clinicians may believe that the goal is desirable as it is something they have been involved in developing and/or because it is consistent with the accepted norms of clinical practice. However, they may also believe that it is not achievable. That is, they support the aspiration inherent within the goal, but do not believe it is achievable within the resources available. The model so far reflects whether or not clinicians believe the goal is worthwhile. Whether or not they believe it is achievable is an additional element that is incorporated into the model in the following section.

6.6.2 A Great Idea But Not In That Timeframe

Barlas and Yasarcan (2008) point out that support for a stated goal is, in part, a function of the time horizon involved. That is, the weighting given to a stated goal is also influenced by the time needed to achieve it. A desirable goal may be considered feasible if there are three years to achieve it. The same goal, however, may be considered unachievable if it has to be achieved in three months. In the model (figure 6.12) this is incorporated with the variable, 'remaining time and work ratio' which is an estimate of how many months would be needed to close the gap between current perceived performance, 'Perceived Management of Chronic Conditions' and the stated goal, 'stated goal for quality of LTCM' relative to the time remaining to achieve the goal, 'Implementation Timeframe'. Note that the gap is not between actual performance and the goal, but between perceived performance and the goal. This is because in a dynamic environment the information feedback about actual performance has delays built into it so that perceived performance lags behind actual performance by a feedback delay. The 
details of this delay and how it is modelled will be described in section 6.8 below on the quality of service delivery.

The 'remaining time and work ratio' affects the clinical team's 'self-efficacy', that is, their belief the stated performance goal can be achieved within the time available (figure 6.12). Self-efficacy is a major factor affecting the 'effort to improve care', which now becomes a function of the resources providing the improvement capacity, 'maximum improvement capacity', 'level of clinical engagement' and self-efficacy'.

Effort to improve care $=($ maximum improvement capacity/12 $) *$ level of clinical engagement * self-efficacy

Barlas and Yasarcan (2008) also add another, subtler, component to this idea. In their formulation people, as well as having some long-term horizon within which they assess the feasibility of what they are being asked to do, they also have a short-term horizon. If they believe that the goal can be achieved within this short-term horizon then, regardless of the actual achievement date they will continue to pursue the goal. It is as though they say, "I'm almost there so let's keep going". The structure for incorporating timeframe into the model is shown in figure 6-12. As I will show later, incorporating timescale into the model provides a basis upon which to incorporate resource demands, which is another central idea within the theory.

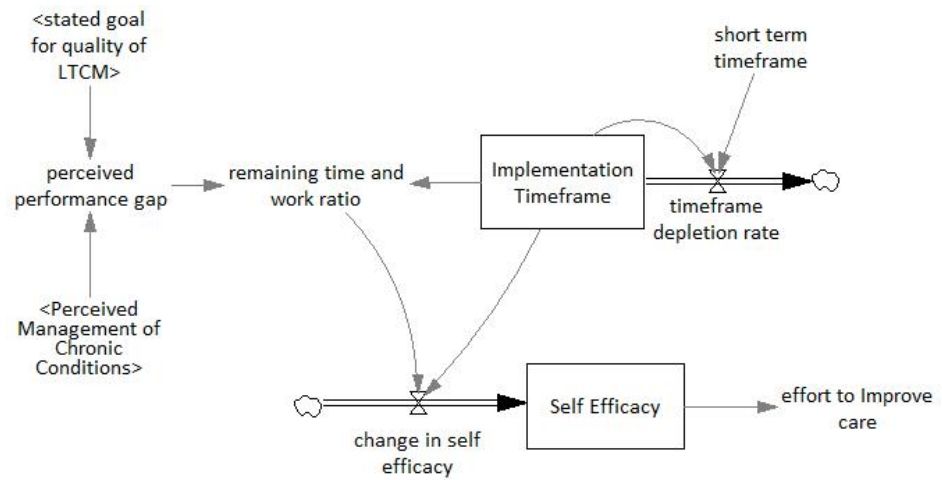

Figure 6-12 Timeframes and self-efficacy 
The impact of a changing time horizon upon the 'Effective Management of Chronic Conditions' is shown in figure 6-13. The following runs assume 100 per cent clinical engagement and a 'maximum improvement capacity' of 30 . That is, everything is in place to support and drive performance towards best practice. All that is changed is the time available to reach the goal. Furthermore, in keeping with the simulation runs shown above, the 'stated goal for quality LTCM' remains at 80 . The following runs show the impact, on both the 'Effective Management of Chronic Conditions' and on 'self-efficacy', of a time horizon of 36 months, 24 months and 18 months within which to achieve the stated goal.
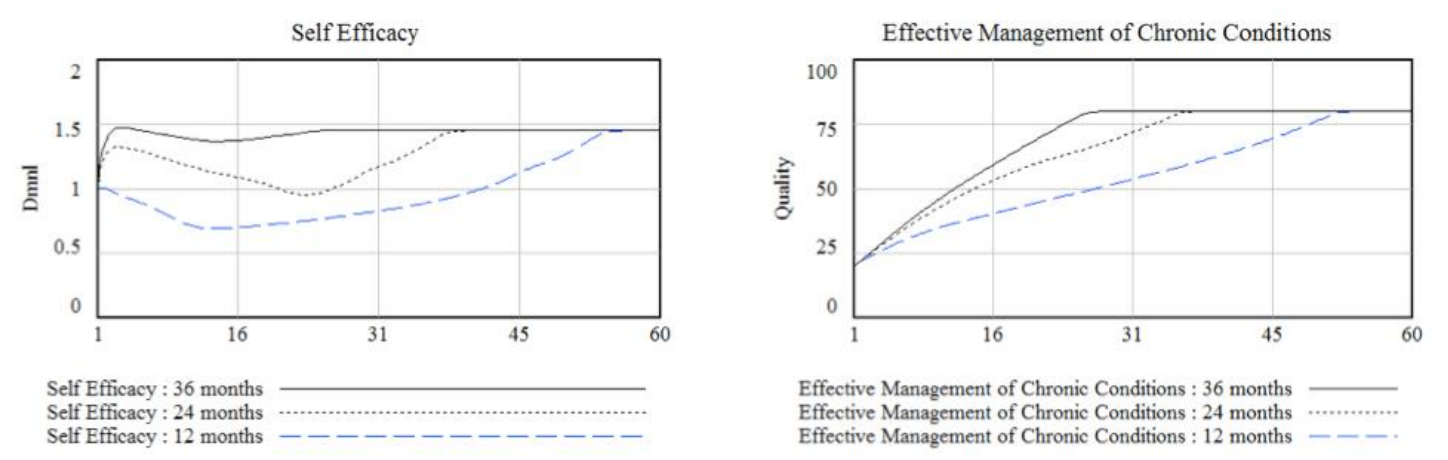

Figure 6-13 Impact of self-efficacy upon the effective management of chronic conditions

With a time horizon of 36 months, the model improves upon the last run. This is because the timescale is long enough to give the clinical team confidence that the goal is achievable given that capacity they have. Self-efficacy quickly grows (all numbers above 1 have a positive impact upon improvement, all numbers below 1 have a negative impact), extending the capacity of the practice to improve performance. While there is an initial dip in self-efficacy after month 3 , reflecting the gap between the time available and the work yet to do, their large improvement capacity and 100 per cent engagement with the goal, continues to improve performance and self-efficacy soon rises again, only levelling off once the goal has been achieved.

When the timescale is reduced to 24 months self-efficacy declines, after an initial rise as clinicians fail to develop a belief that the goal can be achieved. When the 24-month period has elapsed the timeline ceases to have an impact and performance is driven by the clinical team's continuing engagement with the goal and a large improvement 
capacity. Self-efficacy rises alongside performance, as it is no longer depressed by the pressure of a timeline, and the goal is achieved after 36 months. With a time horizon of only 12 months the previous pattern is repeated, except that the decline in self-efficacy is shorter and steeper as the team, from the onset, does not believe that the goal can be achieved within the timeline of 12 months. Once the timeline is passed the timeline pressure is removed and performance once again rises, driven by their 100 per cent engagement with the goal and their maximum improvement capacity.

Figure 6-14 'self-efficacy' feeds back to influence the 'Effective Management of Chronic Conditions'. High levels of self-efficacy increase the improvement effort, which in turn increase the 'Effective Management of Chronic Conditions'. As clinicians perceive the quality of care increasing, they are reinforced in their belief that achieving the goal is possible, thereby increasing their efforts further towards that goal. But, as with all feedback loops the reverse is also possible and that is one of the reasons for the poorer performance when the timescale is reduced. Not confident that the goal can be reached within the timeframe the efforts to improve performance are limited, resulting in a slower rate of improvement.

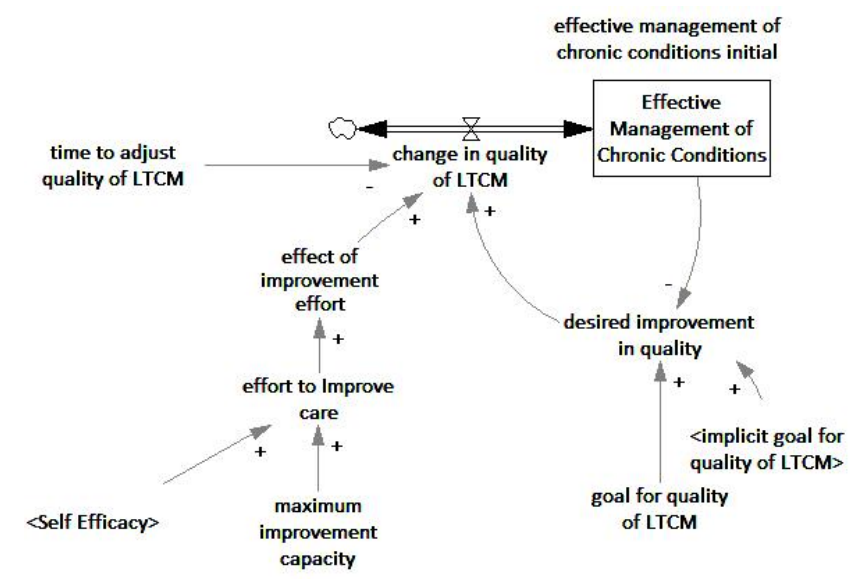

Figure 6-14 Impact of self-efficacy on the improvement effort

\subsubsection{The Complexities of Clinical Engagement}

Thus, clinical engagement is now modelled in a manner much closer to the idea put forward by the health experts. In the current formulation, it now affects not only the overall support for the stated goal (R1 in the CLD figure 6-2) but also whether or not 
the practice team believe it can be achieved within the timeframe (R6 in the CLD figure 6-2). The modelling has, therefore, elicited a more complex understanding of clinical engagement and its importance in implementation. Not only does clinical engagement, in Beasley's words, (Beasley, 2006) result in 'understanding and support' for the goals being set but it is also an important precursor of the improvement effort needed to achieve them. As the model is showing, it is quite possible to understand and support the goals and yet not undertake the work required to achieve them. While related, they have distinctly different drivers, both of which are subsumed in the concept of clinical engagement. However, as the expert theory posits, clinical engagement is only half the engagement story. Without the engagement of patients, improved quality of LTCM will always be limited. The following section describes how patient engagement has been incorporated into the model.

\subsection{Patient Engagement}

\subsubsection{Self-care and Symptomatic Patients}

The importance of patient engagement in the theory of implementation is that engaged patients develop a set of self-management skills that have two distinct benefits. The first of these is that, over time, their use of clinical services will decline and thereby place less demand on scarce clinical resources. This is feedback loop B1 discussed in chapter 5 (figure 6-2). It should be noted this is not true for an individual in that, over time, they will age and their condition will deteriorate, thus requiring increasing use of health services. However, the model does not differentiate between individuals, focusing instead on populations. For populations, the model assumes that at any point in time, patients with self-management skills will make less use of health resources than those who do not. Furthermore, over time their use of health resources will rise at a slower rate than those who do not have such skills and rely, instead, on health experts to manage their symptoms.

The second is that an engaged patient will involve themselves more in the programme of care and have higher levels of adherence to programmes and protocols and thereby improved health outcomes. This is feedback loop R4 (figure 6-2). Without patient 
engagement, care is self-limiting in that no matter how efficacious the treatment, unless patients adhere to the treatment protocols, the improvement in their conditions will be limited. Adherence to the treatment protocols may simply require taking appropriate medication in the appropriate way for the appropriate time period. For patients with long-term conditions, however, there is often a requirement for significant lifestyle changes, without which, the programme of care prescribed by the clinical team will have only limited impact.

Patient engagement has been incorporated into the model by separating the long-term condition patient population into those who interact with the health services on a symptomatic basis and those who are actively involved in self-management. The behaviour characteristics of 'symptomatic' patients are that they tend to go to see their doctor only when their symptoms reach a stage where they require clinical intervention. They are motivated not so much by a desire for 'good health' but by a current problem, manifesting itself as a symptom such as breathlessness, swollen ankles, or pain. When their symptoms ease, they not only stop seeing the clinician but often stop the medication and/or other aspects of their treatment programme. This is a common problem for clinicians dealing with long-term conditions. A patient may, on any particular day, be feeling quite well, despite that fact that their condition is deteriorating.

This lack of a direct and immediate connection between the symptoms and how well someone is at any point in time is a major challenge in engaging patients to take an active role in managing their condition. Self-management patients on the other hand have a level of knowledge, skills and confidence, described as 'patients health literacy' in the CLD (figure 6-2), that enable them to take a more active role in managing their condition. They are aware that to keep their condition under control they not only need to take their appropriate medication but also have to undertake lifestyle changes, often requiring changes in their diet and increased levels of exercise.

Getting patients to this point however does not come easily and, in the context of the model, it requires the application of clinical resources, which in the CLD, in figure 6-2, was described as 'effort to improve care'. That is, additional resources applied to developing self-management skills in their patients. Thus, to get the benefits of improved health 
outcomes and reduced use of health resources over time, the clinical practice has to invest more resource up front.

In this model, the symptomatic LTC patients engage in 'Symptomatic LTC Patient Visits'. Some of these, through the efforts of the primary care practice, become selfcare patients, 'symptomatic LTC patients engaging in self-care', thus, building up the number of 'Self-care Patient Visits' (figure 6-15).

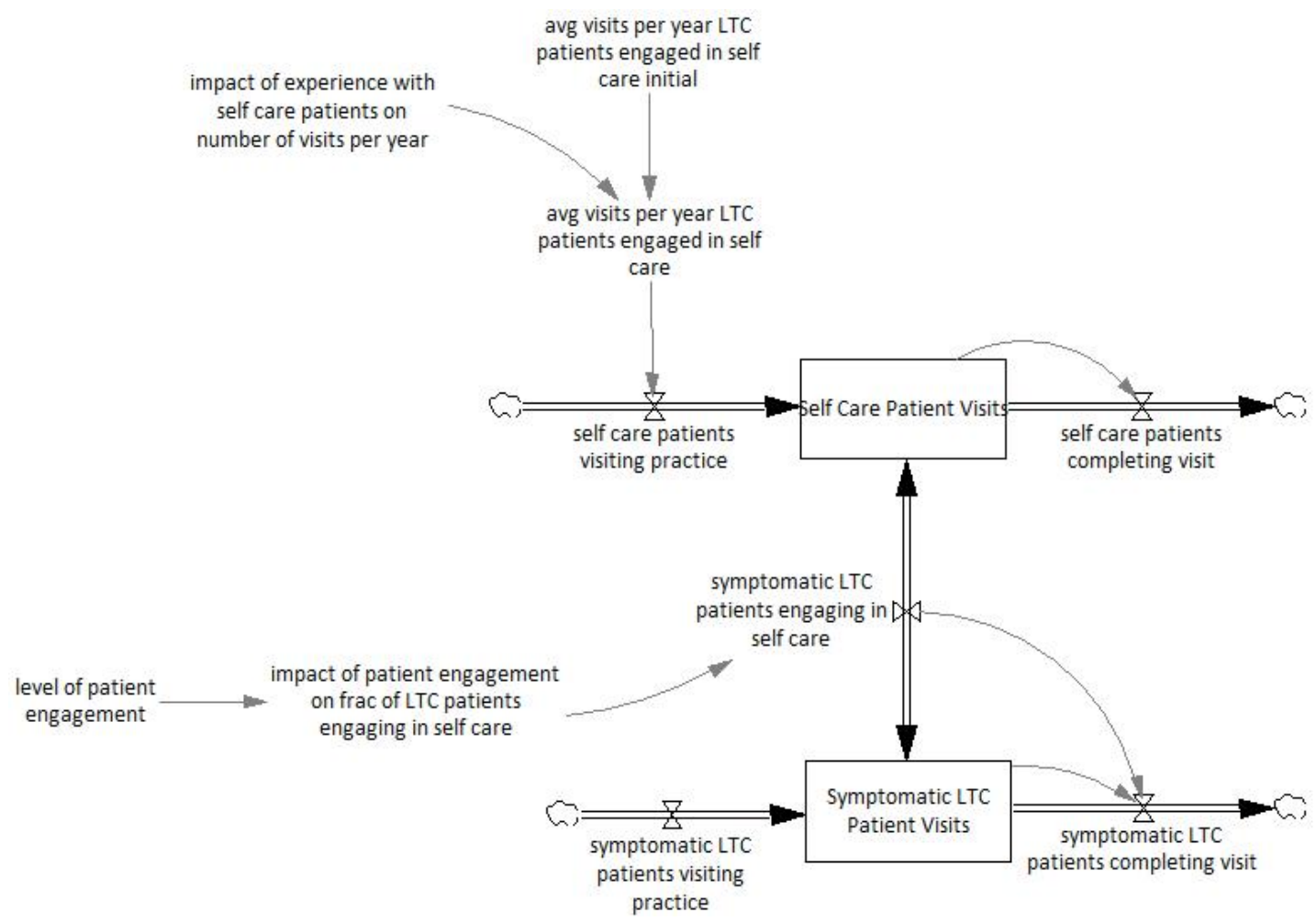

Figure 6-15 Patient engagement and its impact upon self care

The key parameters in this model are:

\begin{tabular}{lll}
\hline Model Variable & Unit of Measure & Parameter \\
\hline avg visits per year symptomatic LTC patients initial & 1/Month & 12 \\
avg visits per year LTC patients engaged in self-care initial & 1/Month & 5 \\
level of patient engagement & Dmnl & .35 \\
\hline
\end{tabular}

Table 6-4 Key Parameters for Patient Engagement 
These parameters reflect the situation whereby symptomatic patients make more visits to their doctors than do patients engaging in self-management. While the evidence is not conclusive, there is support in the literature for the idea that patients who engage in self-care have reduced health care resource use (Singh, 2005). Furthermore, research indicates that a patient's health status is a major factor in the use of primary care services (Cumming, Stillman, Liang, Poland, \& Hannis, 2010) and that self-care programmes show improvements on a number of patient outcomes (Nolte \& McKee, 2008). This model adopts the perspective that helping patients better manage their symptoms increases their health status, and thereby has an impact upon healthcare utilisation.

A level of patient engagement of 0.35 was used for the baseline so as to be able to explore the implications of both lifting and decreasing this level to reflect different population mixes. Lower decile populations tend to have fewer resources, less knowledge and thereby lower levels of self-care amongst those with long-term conditions. Conversely, higher decile populations often have abundant resources, greater knowledge and thereby take a more active role in the management of their condition.

6.7.2 Efforts to Increase Levels of Patient Engagement

There are two key aspects to the way the model determines how many patients engage in self-management. The first is through the direct efforts of the practice to engage patients. The greater the effort made by the practice to engage patients, the greater the level of patients engagement, and thereby the numbers of patients who flow from the stock of 'Symptomatic LTC patients visits' to the stock of 'Self-care Patient Visits'. The relationship between patient engagement and the numbers of patients engaging in selfcare is shown in figure 6-16. 


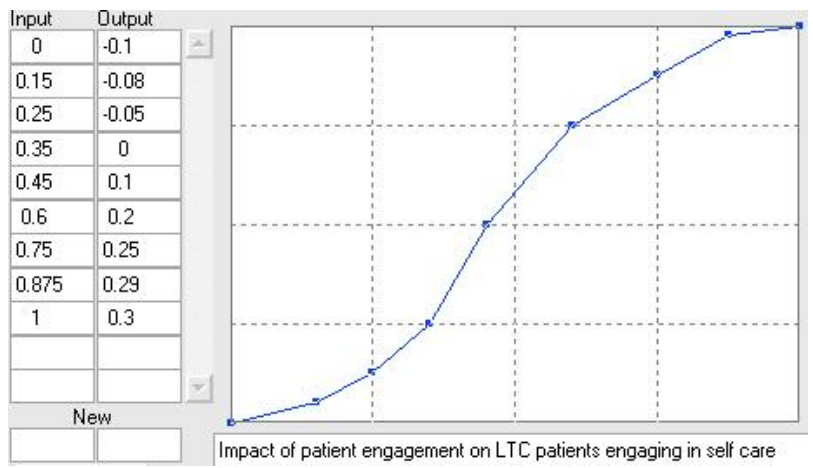

Figure 6-16 Impact of patient engagement on self care

When patient engagement is at 35 per cent ( 0.35 in the table), which is the initial level set, there is no increase in the numbers of patients taking up self-care. As a consequence the percentage of self-care and symptomatic patients remains constant (output $=0$ ). In the simulation this translates into the number of patients flowing between the symptomatic and self-care stocks being zero. If the level of patient engagement rises above that then there is an increase in the flow from the symptomatic group to the selfcare group. With 100 per cent engagement ( 1 in the table) this flow increases the initial number of patients engaging in self-care by 30 per cent. That is, the model takes the initial number of self-care patient visits, which is the initial number of self-care patients multiplied by their average number of visits and increases this sum by 30 per cent. The formulation for this is:

symptomatic LTC patients engaging in self-care $=\left(\left(\right.\right.$ self care patients initial $*_{\text {impact }}$ of patient engagement on frac of LTC patients engaging in self care)*avg visits per year LTC patients engaged in self care)/12

If patient engagement drops below 35 per cent then the patients will flow the other way, that is, a number of those currently engaged in self-care will cease self-care and enter the stock of the symptomatic patients.

The key driver of patient engagement is the amount of time the primary care practice engages in patient engagement activities. In the CLD (figure 6-2) this was balancing the 'effort to improve care' between the need to directly work on the 'effective management of chronic conditions' and the need to make an 'effort to engage patient'. 


\subsubsection{The Learning Curve}

The second way in which the model determines the number of patients taking up selfcare is by affecting the average visits per year of the LTC patients engaged in self-care: 'avg visits per year LTC patients engaged in self-care'. This is brought about by the effect of experience and the learning that comes with it (figure 6-17).

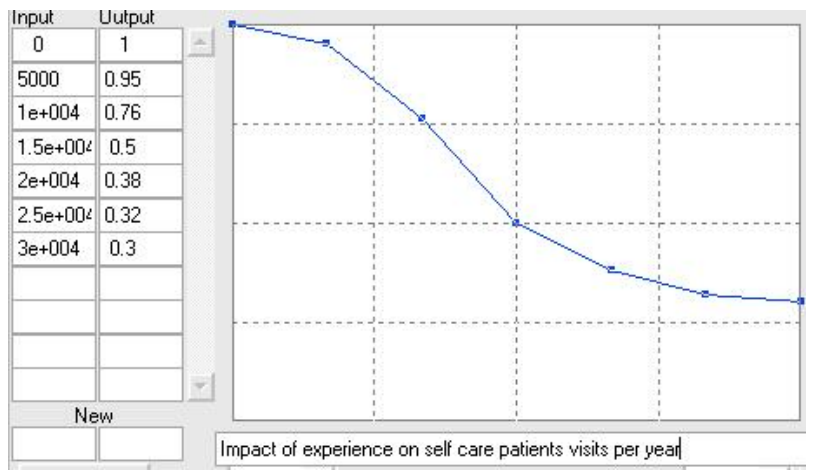

Figure 6-17 Impact of experience on visits by self care patients

As the practice gains more experience in working with and developing self-management skills with patients who have long-term conditions, so the number of visits by those patients declines. With no additional experience ( 0 in the table), the current number of visits by self-care patients remains the same ( 1 in the table). By the time the practice has had experience with 5,000 patients, the visit rate has declined to 95 per cent of the initial number of visits set. By the time the practice has had experience with 15,000 (1.5e+004) self-care patients, it reduces to 50 per cent of the baseline, and reduces to 30 per cent of the initial number when the practice has had experience working with 30,000 (3e+004) self-care patients. What the model is replicating is the increasing effectiveness of interventions to improve self-care skills in patients as the clinical team become more experienced.

This reduction in the number of visits per year made by self-care patients also reflects the fact that as a practice begins to work in this way it will be working predominantly with 'new' self-care patients who need additional time to develop and support their new self-care practice. Over time, as an increasing percentage of their enrolled population undertakes increasing levels of self-care, a greater percentage of that group will be 
'experienced' self-care patients increasingly able to manage their own condition and less dependent on a visit to the primary care practice to resolve symptom management problems. As a cohort therefore, their average number of visits will decline as an increasing number, over time, shift from a pattern of symptomatic care to self-care.

However, to get this experience, the practice has to dedicate more time to patient engagement activities. In the following scenario the number of hours involved in patient engagement activities, per clinician, has been increased from the baseline value of two hours per week to five. The consequence of these increased efforts is seen in the impact upon the quality of long-term condition management as shown below in figure 6-18.

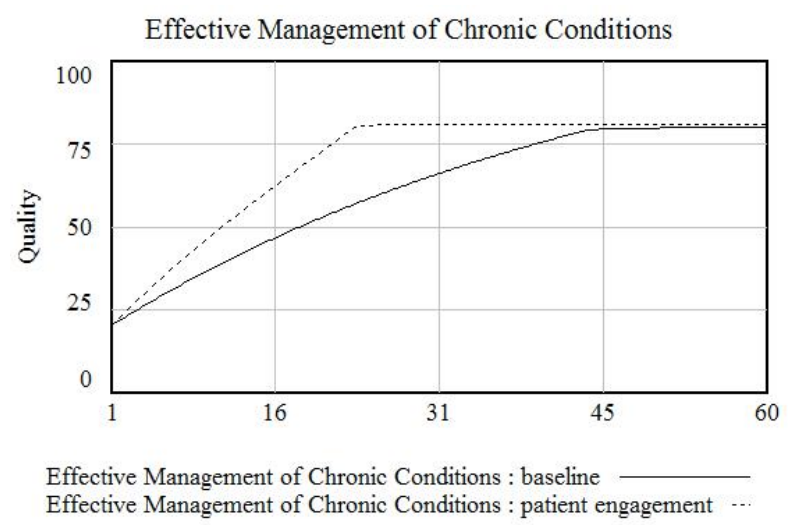

Figure 6-18 Impact of engaged patients on the effective management of chronic conditions

As the graph shows, when patients are engaged in the process of care, the ability to improve increases considerably, reflecting research that shows self-care programmes do have an impact upon clinical outcomes (Bower et al., 2012; Nolte \& McKee, 2008). As pointed out above, and strongly stated in the theory ( $\mathrm{R} 4$ in figure 6.2), patient engagement is central to improved care for people with long-term conditions. Without it progress will always be limited.

It should be noted however, that the model at this point is only highlighting the impact of single variables, in this case 'patient engagement'. The runs in this section keep all other key variables constant, for example, 'level of clinical engagement' stays at 1 and improvement capacity stays at the maximum of 30 . The interactions between each of these are explored in the policy experiments described in chapter 7. 


\subsection{Quality of Service Delivery}

In the model to this point, the 'effective management of chronic conditions' is determined by the improvement capacity in the practice which has a baseline maximum capacity which is then modified by i) clinical engagement, which influences the goals that the practice team will strive for and the effort they will put into achieving those goals; and ii) patient engagement, which has a major impact upon how much improvement is possible, regardless of the effort made by the clinical team. The third key dynamic in the expert theory is the quality of service delivery. How this is incorporated into the model is described below.

6.8.1 Improvement Efforts and Process Quality

There are two key factors in the expert theory that impact upon the quality of service delivery. The first is ensuring that the services are focused on those most in need. This requires an understanding of the enrolled population and their health needs (R2 in figure 6-2). The second is the use of evidence to inform the delivery processes. That is, ensuring that the interventions used are informed by the evidence and more likely than not to have a positive impact upon the health outcomes for that population ( $R 3$ in figure 6-2). These two factors combine to impact upon the 'quality of service delivery'. The model structure for this is shown in figure 6-19.

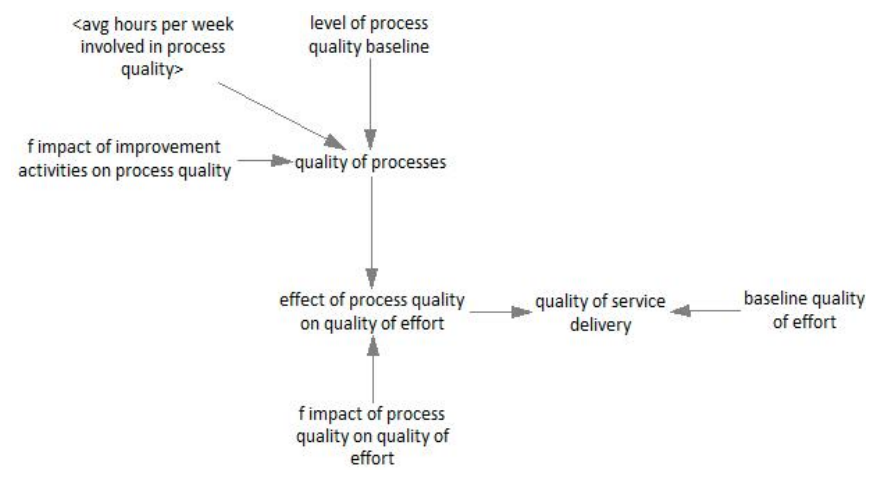

Figure 6-19 Quality of service delivery

In this structure the level of process quality is affected by the effort put into quality improvement, 'avg hours per week involved in service quality' and a function table that 
describes the impact that this activity has, 'f impact of improvement activities on process quality'. The function table is shown in (figure 6-20):

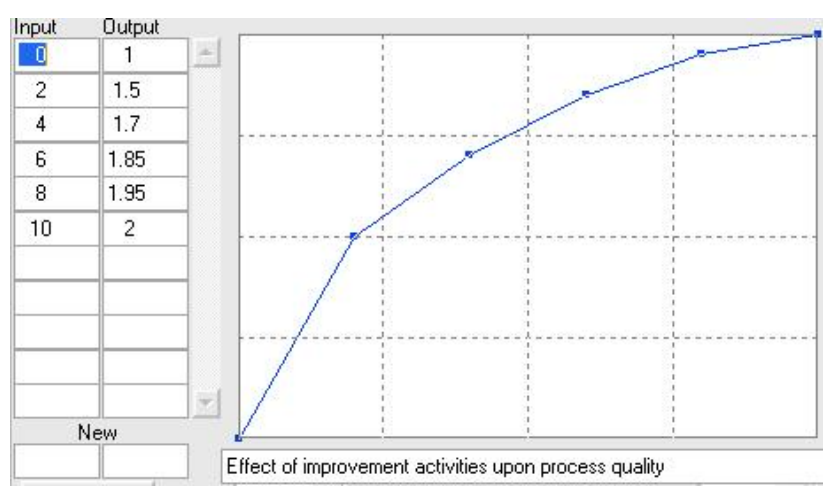

Figure 6-20 Effect of improvement activities on process quality

In this formulation, effort to improve process quality has diminishing returns, so that the impact upon quality of each incremental increase in effort decreases over time. The model baseline assumes that there is no quality improvement effort underway, so that the hours put into process improvement equals zero. As shown in the function table, this means that the baseline level of process quality remains constant. When 10 hours per week are invested into process improvement, the level of process quality is doubled, that is increased by 100 per cent.

The impact that any increase in process quality has on the quality of effort to improve the management of long-term conditions is also specified in a function table (figure 621).

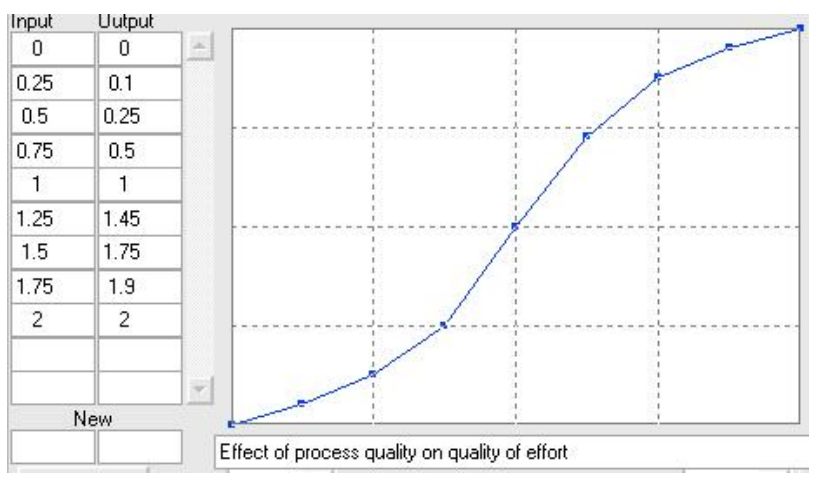

Figure 6-21 Effect of process quality on quality of effort 
In this case, initial improvements in process quality have an increasing impact upon the quality of effort. So, for example, if process quality is increased by 25 per cent, (goes from 1 to 1.25) the quality of effort increases by 45 per cent (from 1 to 1.45). A further increase of 25 per cent (rising from 1.25 to 1.5) only results in a 30 per cent increase in the quality of effort (1.45 to 1.75 ), with subsequent 25 per cent increases only delivering 15 and then 10 per cent increases in quality of effort. This s-shaped curve is common in situations where initial gains are relatively easier to obtain, becoming harder as overall quality increase.

In terms of the expert theory the consequences of these two factors are that efforts to improve performance are more likely to deliver good clinical outcomes and thereby improve the overall quality of care for patients with long-term conditions.

The consequences for the 'effective management of chronic conditions', of investing time in process quality is shown below in figure 6-22.

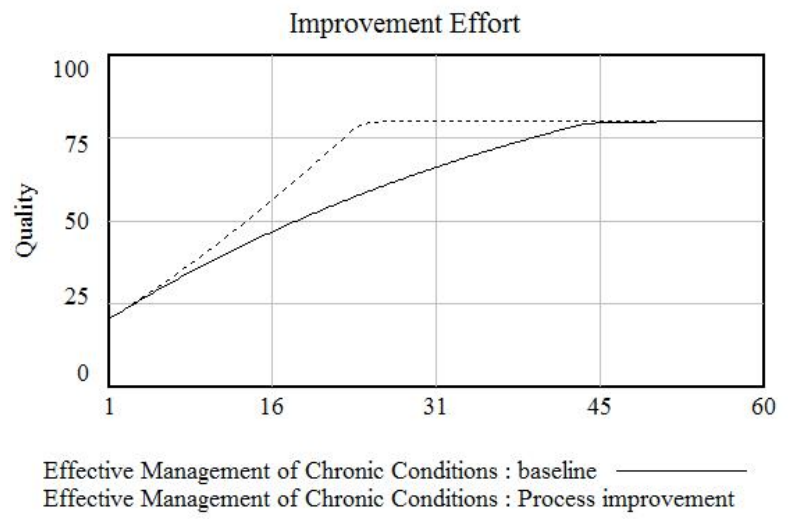

Figure 6-22 Impact of process improvement

Process improvement shows a similar impact as patient engagement, that is, both are able to increase the improvement rate allowing the target to be met within about two rather than three and a half years. 


\subsubsection{The Importance of Feedback}

One of the key ideas raised by the health experts, in the context of process quality, was the importance of feedback. It is no good collecting data if that data is not fed back to the clinicians. This idea is incorporated into the model as 'time to form perception of historical performance', which reflects the time it takes the clinical team to become aware of changes in the 'effective management of chronic conditions'. This is shown below in figure 6-23.

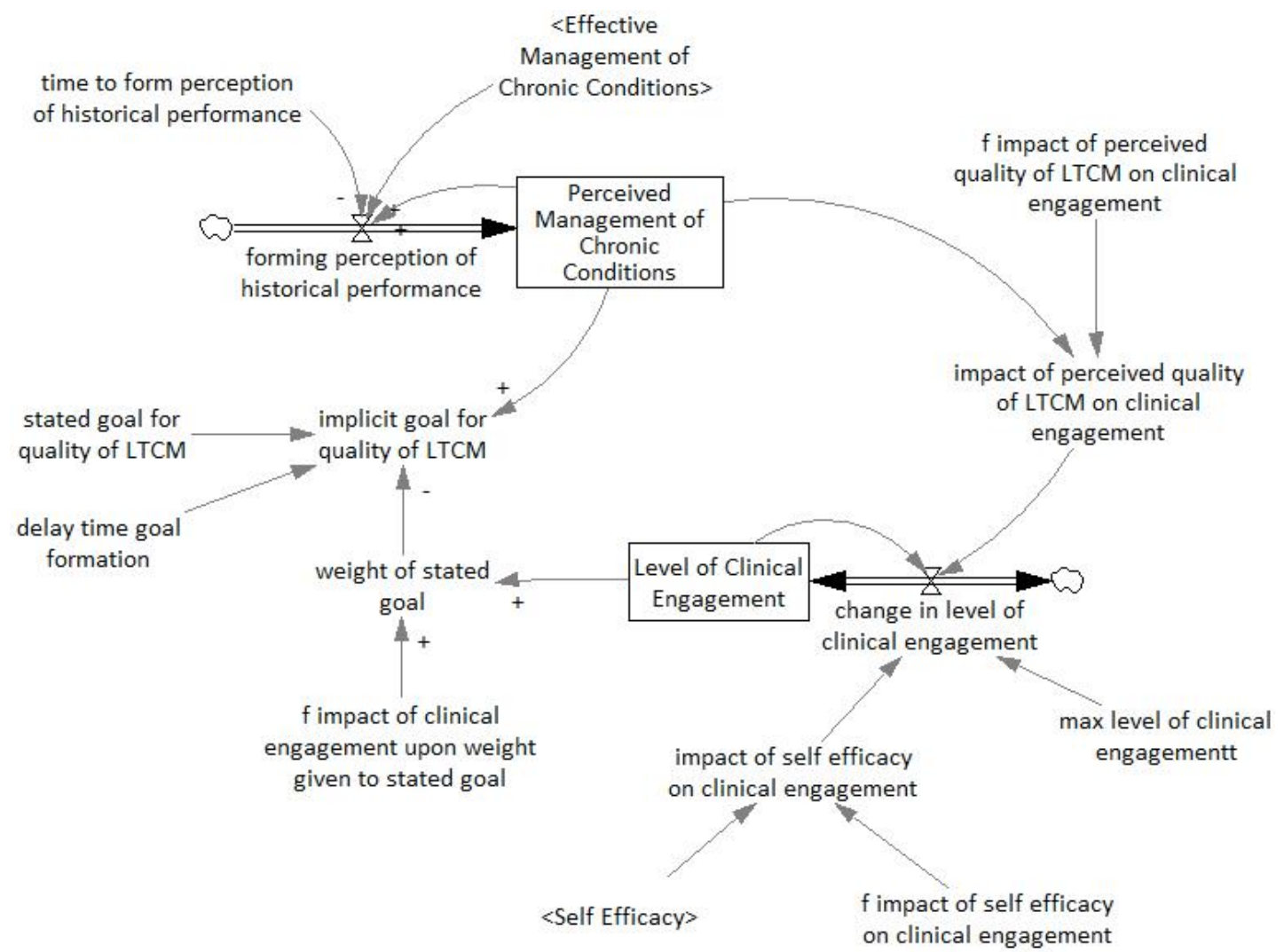

Figure 6-23 Time to perceive change in performance

To illustrate the consequence of feedback, the following graph (figure 6-24) compares the earlier run 'some engagement' in which the 'level of clinical engagement' was set at an initial value of 0.5 with the same values, except that the 'time to form perception of historical performance' is set at 60 to simulate the idea of no feedback. 


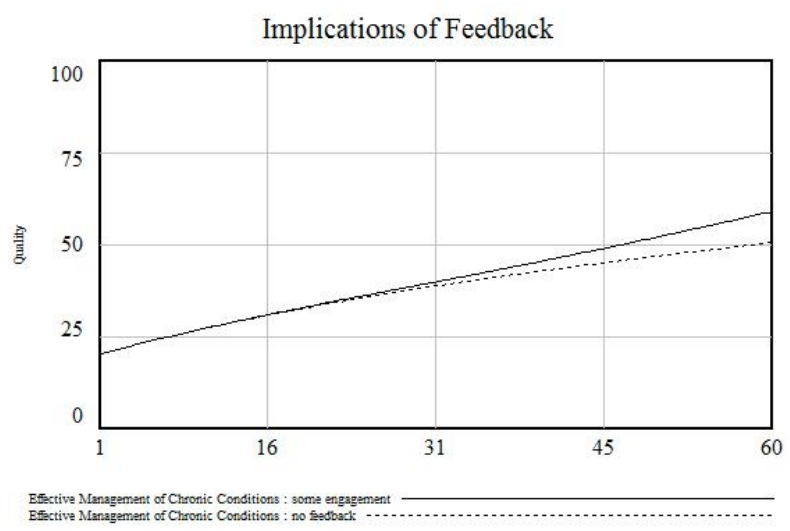

Figure 6-24 Implications of feedback

What this shows is that while process improvement does, as expected, improve the quality of LTCM, if process improvement does not incorporate improvements in data feedback so that the clinical team is aware of the consequences of their efforts it is possible to undermine the improvement effort. This is clearly shown in figure 6.24 where there is no feedback provided. While things are improving, the clinical team is unaware of this and illustrates the importance of feedback in the model. Doctors treating people with diabetes, for example, often see little change in the patients they are dealing with, because of the debilitating nature of the condition, and can become disillusioned with the care they are providing, feeling in some cases, that there is nothing they can do to improve matters.

\subsubsection{Impact Upon the Quality of Long-Term Condition Management}

With the incorporation of service quality and feedback into the model the 'effective management of chronic conditions' is now influenced by the 'desired improvement in quality', the 'effect of improvement effort' and the 'pct self-care patients'. The 'change in quality of LTCM' is formulated:

$\operatorname{MIN}($ desired improvement in quality, effect of improvement effort $)) *(1+$ pct self care patients $* 5$ )

In this formulation, the amount of change is the minimum of what is desired and what the practice is capable of delivering plus the effect of patients engaging in self-care. 
What is desired, the 'desired improvement in quality', is driven by the level of clinical engagement. What the practice is capable of, is driven by a mix of the effort they put in, 'improvement effort', the 'quality of that effort' and the success they have in increasing the percentage of their enrolled population who engage in self-care, 'pct self-care patients'. Underpinning that effort is the 'level of clinical engagement', the clinicians 'self-efficacy' and the 'quality of service delivery'.

As a consequence of the interplay between these factors if i) there is sufficient improvement effort, and ii) the quality of that effort is at a high enough level and iii) there is a sufficient percentage of self-care patients, then the rate of improvement will match the desired improvement in quality. If any of those factors are insufficient then the rate of improvement will fall below what is desired.

The model now captures the key factors in the expert theory incorporating the interplay between the three key constructs, namely, 'clinical engagement', 'patient engagement' and the 'quality of service delivery'. While this chapter has attempted to highlight the impact of these acting independently, chapter seven brings all of them together in a series of experiments to explore the implications for designing and implementing programmes to improve care for people with long-term conditions within a primary care practice. 


\subsection{Replication of Simulation Runs}

The following table provides the inputs needed, in the form of Vensim .cin files, to replicate the runs shown in chapter 6 .

\begin{tabular}{lll}
\hline Figure & Simulation Run & .cin file \\
\hline 6.7 & full capacity & full capacity.cin \\
& some capacity & some capacity.cin \\
& no capacity & nopacity.cin \\
\hline 6.9 & full engagement & full engagement.cin \\
& some engagement & some engagement.cin \\
& no engagement & no engagement.cin \\
\hline 6.12 & 36 months & 36 months.cin \\
& 24 months & 24 months.cin \\
& 12 months & 12 months.cin \\
\hline 6.16 & Baseline & Baseline.cin \\
& Patient Engagement & Patient Engagement.cin \\
\hline 6.20 & Baseline & Baseline.cin \\
& Process Improvement & Process Improvement.cin \\
\hline 6.21 & Some Engagement & Some Engagement.cin \\
& No Feedback & No Feedback.cin \\
\hline
\end{tabular}

\subsection{Summary}

Simulation is a tool that is increasingly being used to develop management theories. The advantages of using simulation to develop such theories, in the context of implementation is that they i) enforce a level of internal consistency; ii) allow researchers to explore the multi-dimensional nature of implementation and iii) provide a 'laboratory' within which to conduct experiments that explore the implications of the theory that has been developed.

This chapter provided a description of the model building process. Beginning with the goal-seeking core of the model, the chapter described how each of the key themes captured in the CLD, was incorporated in the development of the SD simulation model. The chapter also described the scope of the model, focusing on the dynamics of implementation within a single primary care practice. 


\section{Model Experiments \\ What Can the Theory Tell Us?}

\subsection{Introduction}

This chapter discusses the key results that have emerged from building the simulation model and from conducting model experiments.

The purpose of building a simulation model, chapter 6 , is to extend and clarify the theory (Jaccard \& Jacoby, 2010) developed in chapter 5. Chapter 5 developed a theory that describes a complex pattern of interactions important in the implementation of new heath care practices in primary care. In developing the simulation model, the aim is to provide a mechanism for conducting 'virtual experiments' within which the propositions, arising from the theory can be tested and refined.

Building a simulation model however is also a learning process, involving many trialand-error steps, often referred to as iterations, in the pursuit of a model that can faithfully represent the key factors in the qualitative theory. During this process new variables are often required to 'fill-in' the logic, and new insights emerge as the model is 'iterated'. This chapter therefore, will describe the results, not just as consequences, or 'end-products' of the simulation model, but also as consequences of the model building process itself. My aim is to describe the model-building process as a key part of the ongoing development of the theory and not to position the simulation as a static 'tool' that allows one to simply test a theory, that has already been fully developed.

Prior to describing this process however, it is important to validate the model, that is explore its 'soundness and usefulness' (Forrester \& Senge, 1980, p. 210).

\subsection{Model Validation}

As pointed out by Groesser and Schwaninger (2012, p. 157), model validity is a property that the model has of 'adequately reflecting the system being modelled, contingent on the model's purpose. Thus the assessment of the validity of any model has to take into 
account the purpose for which it was developed. In this case, the model was developed to help in the development of a theory of implementation, focusing on the implementation, by primary care practices in New Zealand, of new health practices, for people with chronic conditions. While validation tests aim to establish 'trust and confidence in the model' (Forrester \& Senge, 1980) one has to accept that it is impossible to develop absolute validity, or confidence (Sterman, 2000). Furthermore, while validation is a process of comparing the model with the 'empirical reality' (Maani \& Cavana, 2000, p. 69);

"It is important to realize that the word 'empirical' means 'derived from or guided by experience or experiment' (Random House Unabridged Dictionary of the English Language). Hence, empirical information for testing a model includes information in many forms other than numerical statistics" (Forrester \& Senge, 1980, p. 210)

Forrester (1992) goes further in his discussion about data used in SD modelling by describing the 'rich sources of information' that go beyond numerical data. The challenge is, therefore, to develop confidence in a model using as wide a range of information as possible.

So, while 'absolute validity' is not possible and every model will, by definition, be a simplification of the real world, what is required is a model that can provide insights into the system under study; what Jac Vennix (1996, p. 89) refers to as a requisite decision model. Furthermore, as Meadows and Robinson (1985, p. 382) point out, these insights mostly affect the 'world of ideas', making concrete, "some major old or new ways of thinking about the systems we live in", and contributing to the debate, not ending it.

There are many tests used to assess the validity of a SD model and Forrester and Senge (1980) describe 17 different tests for model validation. Most of these tests are however targeted at two key areas; model structure and model behaviour. Taking into account the purpose of building the simulation model, the validation tests, therefore, have to assess whether or not the structure and behaviour of the model is robust enough to provide support for the theory of implementation, being proposed in this research. 


\subsubsection{Validation of Model Structure}

One of the most important aspects of the model's validation was that the model's structure was developed in interaction with seven leading health experts, closely involved in the design and implementation of programmes for the care of people with chronic conditions. Their understanding of what was involved in implementing such programmes, described in detail in chapters 4 and 5 , provided the basis upon which to identify model concepts and their relationships. As described earlier, this understanding, developed from a number of interviews and feedback sessions, helped to develop and refine the model structure. Conducting a second interview to assess whether or not my interpretation of what was said was valid, or not, increased confidence that I was able to translate their words into a causal model, initially a cognitive map, while retaining the integrity of their words. Similar to the way in which Sastry (1997) used a textual analysis of a theory of organisational change to categorise key concepts in her model, I used the thematic analysis of the interview material to categorise the concepts put forward by the health experts into six themes. Feeding back, and discussing the theory that resulted in the form of a CLD also helped to confirm, not only that the development of the CLD did in fact reflect their thinking but also that, in bringing together their different ideas into a coherent model, it provided insights that were not available within individual perspectives. The involvement of the 'subject experts' (Homer et al., 2005), who are also potential users of the model, in the development and critique of the model is a procedural element (Schwaninger \& Grosser, 2008) that has helped to improve the model's validity. Conducting the discussion of the model, as described in chapter 5, using the set of criteria described by Schwaninger and Groesser, (2008, p. 451) also helped to develop confidence in the structural validity of the model.

A key aspect of the model was translating verbal statements of the 'health experts' into causal relationships. These were expressed as 'lookup' tables and discussed in chapter 6. So, for example, the link between clinical engagement and support for the goal being set was expressed as a function linking clinical engagement to the weight given to that goal. The 'lookup' table, shown in chapter 6 is reproduced below: 


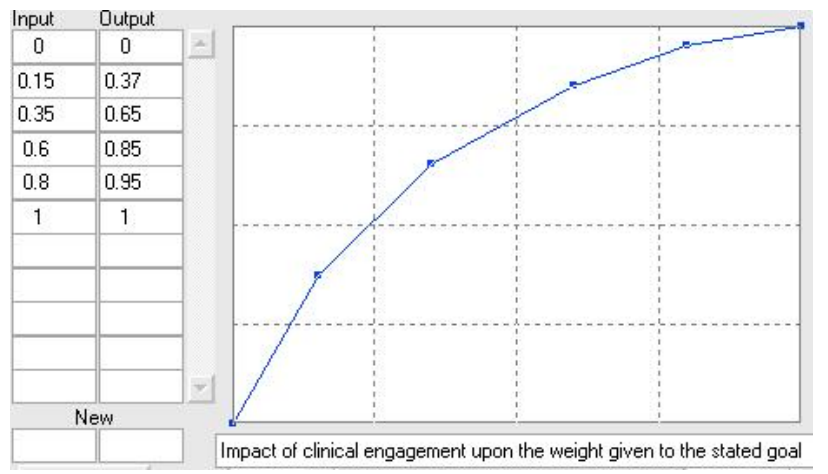

Figure 7-1 Validation testing: impact of clinical engagement on weight given to stated goal It was argued, in chapter 6 , that while there was no numerical studies to support this relationship, it did conform to a common pattern. However, it is important to conduct sensitivity analysis on the causal relationships to test the model's sensitivity to changes in this pattern. The results of the sensitivity tests for the causal relationship between clinical engagement and weight given to the stated goal are shown below in figure 7-2.
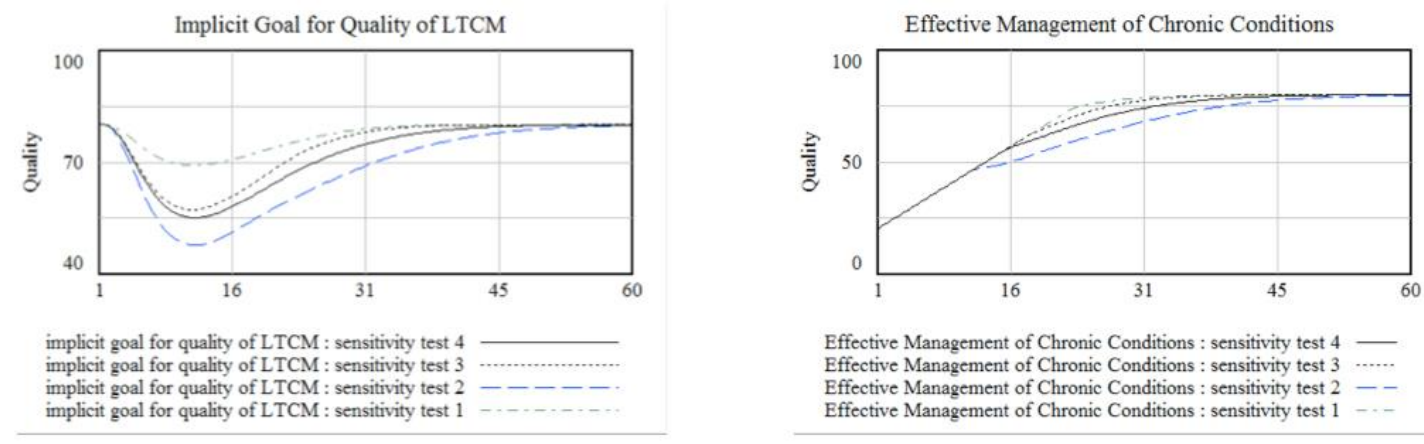

Effective Management of Chronic Conditions : sensitivity test 4 Effective Management of Chronic Conditions : sensitivity test $3 \ldots . .$.
Effective Management of Chronic Conditions : sensitivity test $2 \ldots-$ Effective Management of Chronic Conditions : sensitivity test 1

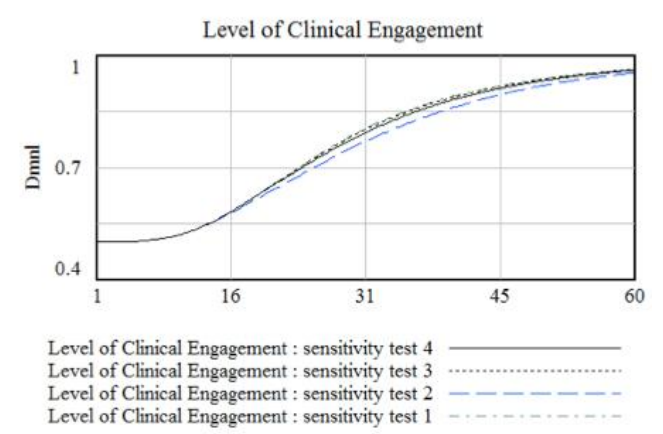

Figure 7-2 Testing the impact of clinical engagement on the weight given to the stated goal

The graphs show the impact of changing the nature of the causal relationship between clinical engagement and the weight given to the stated goal. Sensitivity test 1 is the 
default run, with the causal relationship as shown in figure 7-1. Sensitivity test 2 changes the curve so that as clinical engagement rises, the weight given to the stated goal rises at an increasing rate, rather than at a decreasing rate, as in the default run. In sensitivity run 3 the relationship conforms to a S-shaped curve and in sensitivity test 4 , the relationship is linear. In all cases, the range was from 0 to 1 . As can be seen in figure 72 the effect is to extend the impact of a low level of clinical engagement with the biggest drop being in sensitivity test 2 . This however, only has a small impact on the 'effective management of chronic conditions' and consequently an even smaller impact on the 'level of clinical engagement'. While there is a significant effect on the 'implicit goal for quality of LTCM', the pattern of change is unaltered.

Similar experiments were conducted on each of the 'lookup' functions, the results of which are shown in Appendix 3.

What emerged from these tests was that model behaviour, especially the key output variable, 'effective management of chronic conditions' was largely insensitive to changes in the causal relationships, indicating that the model is robust under a range of causal assumptions.

\subsubsection{Validation of Model Behaviour}

If the first set of tests focused on model structure, the second set focus on model behaviour. One of the most common forms of behaviour tests are 'model reproduction tests' (Schwaninger \& Grosser, 2008), which aim to test the model against historical time-series data. The problem here is that such data does not exist. The model, presented here, is a 'theory', the individual parts of which were developed on the basis of interviews and tested against the available literature, as discussed in chapter 5 . While evidence can be found for discrete parts, such as the effect of patient engagement on improved clinical outcomes, there is no research that explores the system of causality described in this model. What has to be tested instead is whether or not the model has 'plausible outputs' (Homer, 1996).

One such test of 'plausibility' was the responses of the experts when presented with model outputs, and the conclusions that resulted from those outputs. At a minimum the 
model passed the 'face validity' test in that the results were not dismissed as implausible. Instead they engaged the experts in reflecting on their own issues, with four of the five who received the feedback, picking on aspects of model behaviour that reflected and provided insights into issues they were facing.

A second behaviour test that has been applied is the 'extreme conditions test' in which key input variables were pushed to extreme values to test whether or not the model still behaved 'sensibly' under those conditions. The following table (table 7.1) shows the input variables, including the 'baseline' value and the extreme tests that were applied in the test. In all cases their effect was assessed on the key output variable 'effective management of chronic conditions', which continued to behave in plausible and understandable ways.

\begin{tabular}{llll}
\hline Input variable & Baseline Value & Low Extreme Test & High Extreme Test \\
\hline level of client engagement initial & 0.5 & 0 & 1 \\
maximum improvement capacity & 30 & 1 & 100 \\
number of GPs initial & 6 & 1 & 10 \\
implementation timeframe initial & 36 & 0 & 60 \\
$\begin{array}{l}\text { pct LTC patients engaged in self-care } \\
\text { initial }\end{array}$ & 0.1 & 0 & 1 \\
$\begin{array}{l}\text { avg hours per week involved in } \\
\text { process quality }\end{array}$ & 0 & 0 & 10 \\
stated goal for quality of LTCM & 80 & 1 & 100 \\
time to form perception of historical & 3 & 0 & 60 \\
performance & 1 & 1 & 2 \\
level of process quality baseline & 6 & & 24 \\
time to adjust quality of LTCM & & 0 & \\
\hline
\end{tabular}

Table 7-1 Parameters for the extreme conditions tests

As with any model, validation tests are no substitute for utility, and the real test is whether or not any policies implemented in the real world produce the results predicted by the model (Vennix, 1996). At this point validation tests have indicated that the model has a degree of robustness, and that it passes face validity tests with those involved in helping with its development. Whether or not the policy implications that result from 
the model have any usefulness, is the subject of the rest of this chapter. The following sections discuss the design of simulation experiments, how the experiments have been structured and the results from a series of experiments.

\subsection{Designing Simulation Experiments}

While simulations allow 'experiments' to be run to test and refine theories, they have a number of differences from laboratory experiments, both in their intent and in the way they are designed. The following table (table 7-2) adapted from Jaccard, \& Jacoby (2010) describes some these differences.

\section{The Difference Between Simulations and Laboratory Experiments}

Laboratory Experiments

'Tight' investigator control over presentation of 'stimuli', and the sequence of events

Attempts to eliminate nonfocal factors and/or hold them constant

Concentrates on a limited number of independent and dependent variables

To retain experimenter control laboratory experiments 'tie' and 'untie' variables in ways that may divorce them from everyday reality

Suited for evaluating the inputs and outcomes of a process

\section{Simulation Experiments}

Events, their sequence and their consequences emerge out of the model variables (participants) and their interactions

Permits nonfocal factors to vary freely

Includes a great number of variables, particularly potential causes of the variables of interest

Strive to keep variables 'tied' in a manner that is consonant with the way in which they are associated with the everyday world

Greater emphasis is placed on the process itself

Table 7-2 difference between simulation and laboratory experiments

Simulation experiments are designed to work with the complexity of the situation, allowing that complexity to evolve and deliver, often surprising, results. The differences outlined in the above table highlight that simulations have a useful place in social research where the system being researched is both complex and dynamic, where establishing strict experimenter control serves only to disguise or constrain the very complexity one is trying to understand. While simulations give up much of the control desired by those conducting laboratory experiments they do provide a mechanism to "...confront us with the implications of what we think we know" (Pagels, 1988), and in doing so challenge and test the robustness of our thinking. The very act of developing a 
simulation can be seen as an aid to developing theory (Jaccard and Jacoby, 2010), forcing a degree of rigour that gives the theory the 'potential to be stronger - in terms of both robustness and reach' (Schwaninger and Grösser, 2008).

In designing the simulation experiments my interest is to explore two key areas. The first is the impact of context and the question of why implementation works in some contexts and not in others (Hoddinott et al., 2010). The key contextual factors that the theory described and which are explored in the simulation are: i) the workload of the practice; ii) the level of knowledge, skills and confidence amongst the enrolled population; and iii) the level of knowledge, skills and confidence of the practice team. The second is the internal processes of the practice itself, especially in terms of i) where it balances its time between delivering direct patient care and efforts to engage the patient in the care process; ii) its ability to change; and iii) the speed and quality of the feedback processes.

Researchers tend to agree however that implementation involves a dynamic interplay between 'multilevel phenomenon' that are influenced by and influence each other, in a complex pattern of interaction that changes over time (Greenhalgh et al., 2005; Klein \& Sorra, 1996). Faced with this complexity many researchers shy away from the 'crocodile swamp' (Schon, 1983) while others, acknowledging the complexity, focus on key factors within it, for example, clinical engagement (Ham, 2003), or decision support tools (Garg et al., 2005).

Because models of implementation are complex and dynamic, they often produce nonintuitive results. Without formal tools it is difficult to infer behaviour over time from verbal explanations. Yet it is such complex dynamics - involved in explaining patterns of implementation - that are of most interest to those researching and implementing new patterns of health service delivery.

\subsection{Structure for the Simulation Experiments}

The experiments discussed below begin with a simulation run that uses initial settings to represent a primary care practice operating in a 'no change' situation. That is, a practice responding to the immediate needs of its enrolled population and having only a limited 
involvement in the proactive management of long-term conditions. These initial conditions are then modified to examine more complex scenarios in which the context and internal processes are modified. For every scenario, I test a number of model parameters by adjusting key model variables and applying them to three different types of primary care practice. The following sections describe these experiments and the insights they provide for refining and testing the theory developed in chapter five.

The results of these experiments indicate that the theory described in chapter five does highlight important aspects of implementation dynamics and that a successful implementation of new healthcare practise within primary care requires not just the identification of key factors, such as clinical and patient engagement, but an understanding of the complex interplay between these factors. Furthermore, the simulation results indicate that implementation will always be a 'local affair', in which the unique characteristics of each primary care practice need to be taken into account.

The model experiments discussed below show how each of the key theoretical constructs, clinical engagement, patient engagement and quality of service delivery can be developed, and undermined, often in surprising and counterintuitive ways.

\subsection{Exploring the Behaviour of Different Primary Care Practices}

To explore these constructs, the experiments are built around three practice settings, which are designed to approximate a range of primary care practices. These practice settings are based on their resource levels and the level of health literacy within their enrolled population and are described below.

Practice A portrays a reasonably busy practice with some improvement capacity that has set itself a challenging target for improving the care for people with long-term conditions. It has a GP/patient ratio of one GP to 1,667 patients, which is about average for New Zealand and is representative of a practice working in the 'middle ground' in terms of patient resources and requirements. The New Zealand Ministry of Health uses a ratio of one GP to 1,400 patients as equivalent to 1 full-time equivalent (FTE), (Fretter and Pande, 2006). As this model also incorporates clinical support staff into the resource mix, the ratio of 1:1,667 can be considered close to the Ministry of 
Health baseline. Resource availability is calculated in the model by multiplying the number of GPs by available hours per week enhanced by the availability of clinical support. Clinical support is expressed as a ratio of support staff to GPs, which in the baseline is set to 0.5 , that is, 0.5 FTE clinical support staff for every GP in the practice. Therefore, in a practice of six GPs, with a support staff ratio of 0.5 per GP there are a total of nine FTE staff in the practice; six GPs and three clinical support staff.

This practice also serves a population of $10,000,70$ per cent of whom have long-term conditions. Of this population group 20 per cent are engaged in self-care behaviours. It is also a practice with some experience in implementing programmes for the care of people with chronic conditions (Effective Management of Chronic Conditions initial $=$ 20). In addition to normal workload the practice is targeting an average of 2 hours per GP per week on patient engagement activities. To invest this time in improving the model of care and to meet patient demand, the practice has to operate at 108 per cent of capacity, which equates to the practice team working an average of 43 hours a week, with 100 per cent capacity being based on a forty-hour week. Much of its workload is taken up responding to acute care with limited spare capacity to take on additional work. In modelling this improvement initiative, the model settings assume that it will take 12 months to get all staff working an average of two hours per week on these activities and, as a consequence 12 months for the full benefits to be realised.

While the practice may be very effective within the traditional acute paradigm, it has only limited experience operating outside the norm of the 10-minute consultation initiated by the patient. It does not have the systems and practices in place to monitor populations, or experience in utilising multi-disciplinary teams or taking a more active role in managing a patient's interactions with the practice. In the baseline runs the patient population is locked into a similar paradigm, with only 20 per cent of the enrolled population engaged in any sort of self-care; visits to the doctor being determined by symptom severity with little regard given to self-responsibility in either preventing or managing their condition which they see as the responsibility of doctors.

This is a common starting point for many primary care practices involved in trying to implement programmes for the care of people with chronic conditions. While they maybe highly skilled and experienced in standard medical care, they have only limited 
experience and resources to implement the new care practices and systems required to deal with chronic conditions. Even though the clinicians are often very supportive of the idea, patients are generally passive receivers of care provided by the doctor.

The key parameters for Practice A are:

\begin{tabular}{lll}
\hline Model Variable & Unit of Measure & Parameter \\
\hline Number of GPs & GPs & 6 \\
Number of enrolled patients & Patients & 10,000 \\
Initial average number of support staff per GP & Staff & 0.5 \\
Per cent enrolled population with long-term conditions & Dmnl & 0.7 \\
Per cent LTC patients engaged in self-care initial & Dmnl & 0.2 \\
$\begin{array}{l}\text { Average hours per week involved in patient engagement } \\
\text { activities target }\end{array}$ & Month & 2 \\
$\begin{array}{l}\text { Time for patient engagement activities to be fully } \\
\text { implemented }\end{array}$ & Month & 12 \\
\hline Table 7-3 Key parameters for practice A & &
\end{tabular}

Practice $\mathbf{B}$ is a well-resourced practice with eight GPs serving an enrolled population of 10,000, giving it a GP/patient ratio of 1:1,250. While it has the same number of patients as Practice A, 30 per cent of its patient population are engaged in self-care activities, indicating that not only do they have more experience in managing long-term conditions but also that their patient population has a greater level of knowledge and skills than those enrolled in Practice A. Like Practice A, this practice has 0.5 FTE clinical support staff for every GP in the practice. As a consequence, increasing the time spent on patient engagement activities by 2 hours only brings it up to 80 per cent of operating capacity.

These conditions are representative of a practice operating in a well-off area where the population is relatively healthy and well educated. Furthermore, such areas attract clinicians' and, as a consequence, the GP to patient ratio is relatively low and they have spare capacity to invest in improvement activities. All other parameters are identical to Practice A.

The key parameters for Practice B are: 


\begin{tabular}{lll}
\hline Model Variable & Unit of Measure & Parameter \\
\hline Number of GPs & GPs & 8 \\
Number of enrolled patients & Patients & 10,000 \\
Initial average number of support staff per GP & Staff & 0.5 \\
Per cent enrolled population with long-term conditions & Dmnl & 0.7 \\
Per cent LTC patients engaged in self-care initial & Dmnl & 0.3 \\
$\begin{array}{l}\text { Average hours per week involved in patient engagement } \\
\text { activities target }\end{array}$ & Month & 2 \\
$\begin{array}{l}\text { Time for patient engagement activities to be fully } \\
\text { implemented }\end{array}$ & Month & 12 \\
\hline Table 7-4 Key parameters for practice B & &
\end{tabular}

In contrast, Practice $\mathbf{C}$ is a poorly resourced practice with five GPs serving a patient population of 10,000, giving it a GP/patient ratio of 1:2,000. Furthermore, only 10 per cent of its patient population is engaged in self-care activities. To invest two hours per week on patient activities the practice has to operate at 130 per cent of capacity, equating to an average of 52 hours per week for everyone working in the practice. In addition, with only 10 per cent of its enrolled population engaged in self-care activities the level of knowledge and skills of their patients are less than the enrolled populations in practices $\mathrm{A}$ and $\mathrm{B}$.

This is typical of primary care practice operating in the poorer suburbs of Auckland, where patient ratios are high, GP turnover is high, and it is difficult attracting new clinicians into the area. 
The key parameters for Practice $\mathrm{C}$ are:

\begin{tabular}{lll}
\hline Model Variable & Unit of Measure & Parameter \\
\hline Number of GPs & GPs & 5 \\
Number of enrolled patients & Patients & 10,000 \\
Initial average number of support staff per GP & Staff & 0.5 \\
Per cent enrolled population with long-term conditions & Dmnl & 0.7 \\
Per cent LTC patients engaged in self-care initial & Dmnl & 0.1 \\
$\begin{array}{l}\text { Average hours per week involved in patient engagement } \\
\text { activities target }\end{array}$ & Month & 2 \\
$\begin{array}{l}\text { Time for patient engagement activities to be fully } \\
\text { implemented }\end{array}$ & Month & 12 \\
\hline Table 7-5 Key parameters for practice C & &
\end{tabular}

The above description describes a range of primary care practices with varying levels of resource and different enrolled populations. Their purpose is to reflect the different contexts within which many primary care practices operate and to provide a range of contexts within which to explore the impact of different interventions.

Before undertaking the experiments however the base case is set up to run in a 'status quo' setting. 


\subsection{Establishing the Base Case}

Prior to conducting the simulation experiments the model is set up to replicate a 'no change' situation. Key parameters for this run are:

\begin{tabular}{lll}
\hline Model Variable & Unit of Measure & Parameter \\
\hline Effective management of chronic conditions initial & Quality & 20 \\
Stated goal for quality of LTCM & Quality & $21^{6}$ \\
Level of clinical engagement initial & Dmnl & 0.5 \\
Maximum improvement capacity & Effort & 30 \\
Implementation timeframe & Month & 36 \\
Number of GPs initial & GPs & 6 \\
Initial average number of support staff per GP & Staff & 0.5 \\
Number of patients initial & Patients & 10,000 \\
Per cent LTC patients engaged in self-care initial & Dmnl & 0.2 \\
Per cent enrolled population with LTCs & Dmnl & 0.7 \\
Average hours per week involved in patient engagement & Month & 2 \\
activities target & & 1 \\
Level of process quality baseline & Dmnl &
\end{tabular}

Table 7-6 Key parameters for the base case

In this case, the practice has no improvement goal, and there is 100 per cent 'engagement' with the status quo. Resources are the same as in Practice A, that is, six GPs serving a population of 10,000 . The clinical team is happy with and committed to the current direction and have no desire to change. The purpose of establishing this status quo run is that it gives a 'baseline' against which the simulation experiments can be compared.

As shown in figure 7-7, the 'Effective Management of Chronic Conditions' is maintained at 20, with no change in the level of clinical engagement, resource demand and no change in the percentage of the enrolled patient population engaging in self-care.

\footnotetext{
${ }^{6}$ The figure of 21 has been used to avoid a 'division by 0' error in the model. As change is driven by the gap between the 'effective management of chronic conditions initial' and the 'stated goal for quality of LTCM' having them at exactly the same starting point does cause a division by 0 error'.
} 

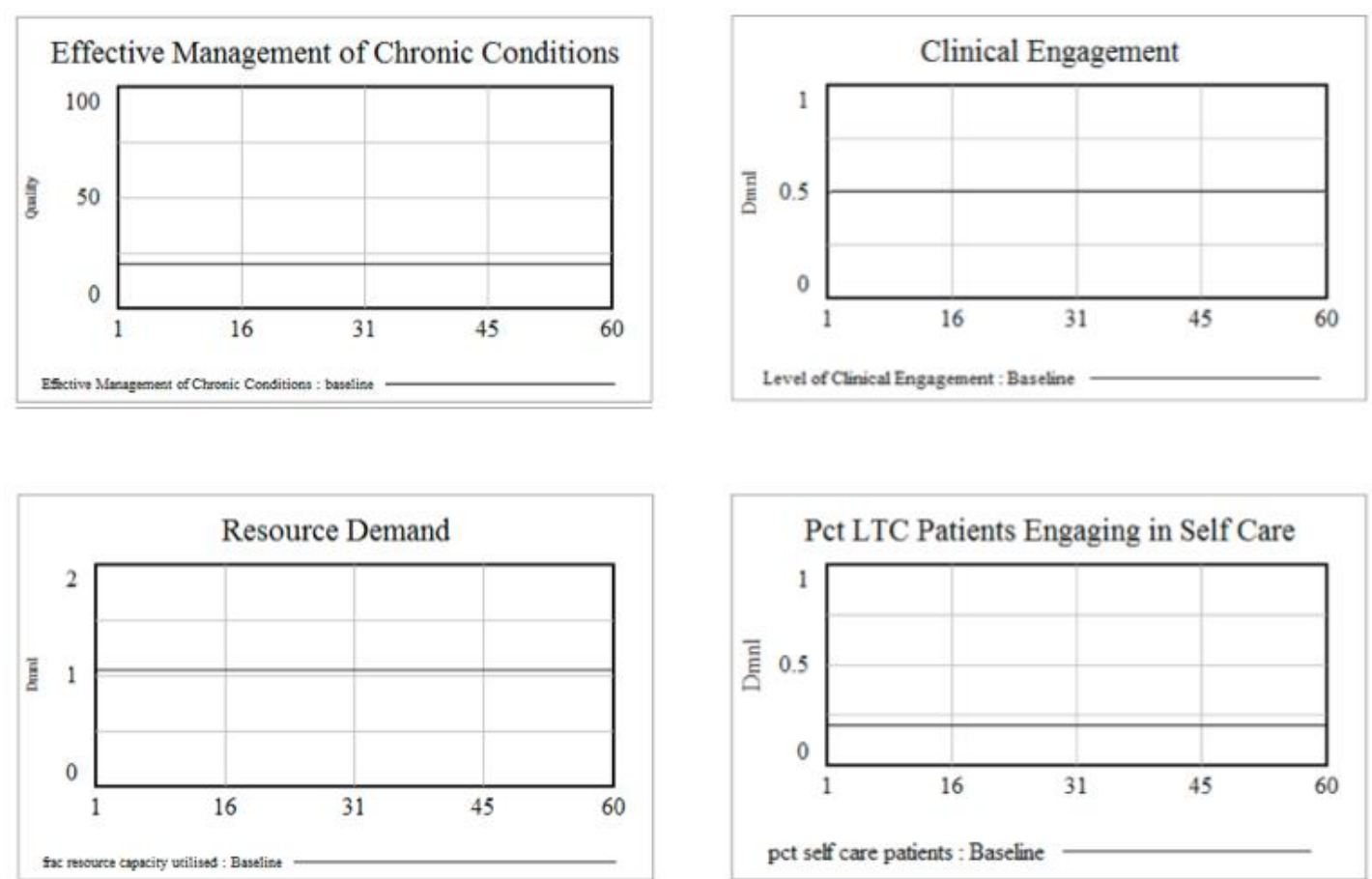

Table 7-7 Base case scenario

This is essentially a 'business as usual' scenario. It is simplistic in that it assumes no change in the volume or type of patient, but it does provide a useful basis for comparison with future simulation experiments. As the graphs show, this is a practice performing at around 20 per cent of best practice, which, as discussed in chapter 6 is considered to be about 'average', with only 20 per cent of their LTC patients achieving good control of their condition. They are also operating at around 100 per cent of capacity; resource demand equals 1 , which in this model equates to an average workload across the practice of 40 hours per week. In addition, 20 per cent of their LTC patients are engaging in self-care activities.

The next section introduces the simulation experiments, which alter key variables and explores their impact in each of the three practices.

\subsection{Running the Simulation Experiments}

This section describes a number of simulation experiments which are intended to explore the dynamics involved in the interplay between the three main constructs; 
clinical engagement, patient engagement and quality of service delivery. Each experiment is conducted within the three different primary care practices described above.

\subsection{Improving the Quality of Care by Setting Higher Goals}

The first set of experiments explores the behaviour of the three practices when faced with the task of improving the quality of care for people with long-term conditions. As has been the case throughout this research, I am assuming that the primary care team want to improve and that there are no technology barriers to doing so. As noted above my interest is in exploring the behavioural issues, rather than technical ones so the model assumes no technical barriers to improvement in any of the practices.

The first experiment simply changes the goal that the practices are trying to achieve, to explore how the level of goal set affects performance. In each case the same baseline conditions exist and the experiments involves changing the goals. The baseline conditions for each practice are:

\begin{tabular}{lll}
\hline Model Variable & Unit of Measure & Parameter \\
\hline Effective management of chronic conditions initial & Quality & 20 \\
\hline Stated goal for quality of LTCM & Quality & varies in each \\
& & 0.5 \\
Level of clinical engagement initial & Dmnl \\
Maximum improvement capacity & Effort & 30 \\
Implementation timeframe & Month & 36 \\
Number of GPs initial & GPs & varies between \\
& & practices \\
\hline Initial average number of support staff per GP & Staff & 0.5 \\
Number of patients initial & Patients & 10,000 \\
Per cent LTC patients engaged in self-care initial & Dmnl & varies between \\
& & practices \\
\hline Per cent enrolled population with LTCs & Dmnl & 0.7 \\
Average hours per week involved in patient engagement & Month & 2 \\
activities target & & Dmnl
\end{tabular}

Table 7-8 Key parameters for higher goals experiment 
Unlike the status quo run, there is now a gap between the current quality of care and the goal. In addition, clinical engagement is only 50 per cent indicating less than full support for the goals they are trying to achieve. In this experiment the specific changes are to set different levels of goal for each of the practices to explore how different goals affect different practices. The goals set are expressed as a percentage of best practice, specifically, 60, 80 and 100 per cent. All are significantly above the current levels of quality, which is set at 20 per cent. Each practice has been given 36 months to achieve the goal. The following graphs show the results for 'Effective Management of Chronic Conditions' after five years for each of the three practices.

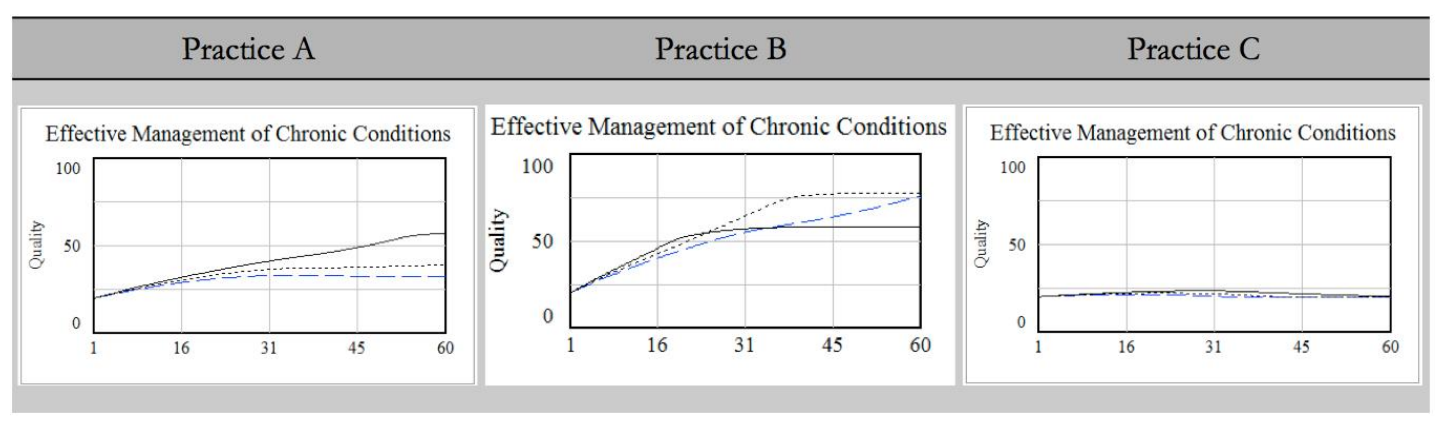

Figure 7-3 Policy experiment: adjusting goals

Legend:

$\begin{array}{lll} & = & 60 \text { per cent of best practice } \\ \ldots \ldots \ldots \ldots \ldots & = & 80 \text { per cent of best practice } \\ -\cdots---- & = & 100 \text { per cent of best practice }\end{array}$

The first thing that these results show is that the better resourced the practice is the better they perform. As can be seen from the graphs the well-resourced practice (Practice B) gets very close to achieving its goal, under the 60 and 80 per cent scenarios, while Practice A lifts its performance to 50 per cent of best practice, under the 60 per cent scenario, while the poorly-resourced practice changes very little under any of the scenarios. This is not surprising given that the model assumes all primary care practices want to improve and that there is no difference in the knowledge and skills of the individuals within the practice teams. As a consequence it is their differing level of resources that makes the difference. 
More interestingly however, this experiment also indicates that setting higher goals does not necessarily translate into better performance. In fact, for Practice C, lifting the goals had little discernable impact on performance, which lifted slightly over the first three years and then dropped, never moving far away for the initial settings. This is to be expected given that the practice not only has less resources but also has a less literate patient population. In contrast, Practice B, with extensive resources and a well-informed and literate population, improved significantly, although the shift from the 80 per cent goal to the 100 per cent goal was a 'bridge too far', resulting in a lowering of performance. Practice A, while improving significantly when the goal was set at 60 per cent of best practice, performed less well when the goals were lifted to 80 and 100 per cent. For Practices A and B, setting improvement goals was enough to drive performance improvement. A clear goal and a desire to achieve it was enough to lift performance. However, in both cases, continuing to set even higher goals did not result in continuing performance improvements. A point was reached where higher goals led to lower performance.

7.8.1 Striving for 'Best Practice' Is Not Always the Best Option

These simulation runs highlight the importance of resource constraints. No matter how engaged clinicians are with the idea of improving care for people with chronic conditions, their efforts will fall away as the pressure of normal acute care takes up whatever spare resource there is. As resource constraints become increasingly evident, then efforts to implement the new practices fall away. This has some significant consequences for practice and theory.

A common response to performance that is lower than desired is for the policy setting organisations to establish 'best practice' targets. In 2007 the National Health Committee produced a comprehensive document on what was required to improve care for people with LTCs (National Advisory Committee on Health and Disability, 2007). To address the changes required in primary care their opening remarks were that they believed that "...chronic care models will be assisted in New Zealand through fully implementing the vision of the Primary Health Care Strategy."[my emphasis]. There seems to be a common belief that context doesn't matter and that success will only come if the 'best' is implemented. Like many such policy documents the focus is often on implementing all 
aspects of the policy to the fullest extent. It is assumed that implementing 'best practice' will deliver better performance.

However, this simulation experiment highlights that bigger targets produce bigger resource demands and as the following outputs show, that can lead to performance being lower than that achieved by setting lower targets (figure 7.4). The following graphs explore the results for Practice A, under the 80 and 100 per cent scenarios.

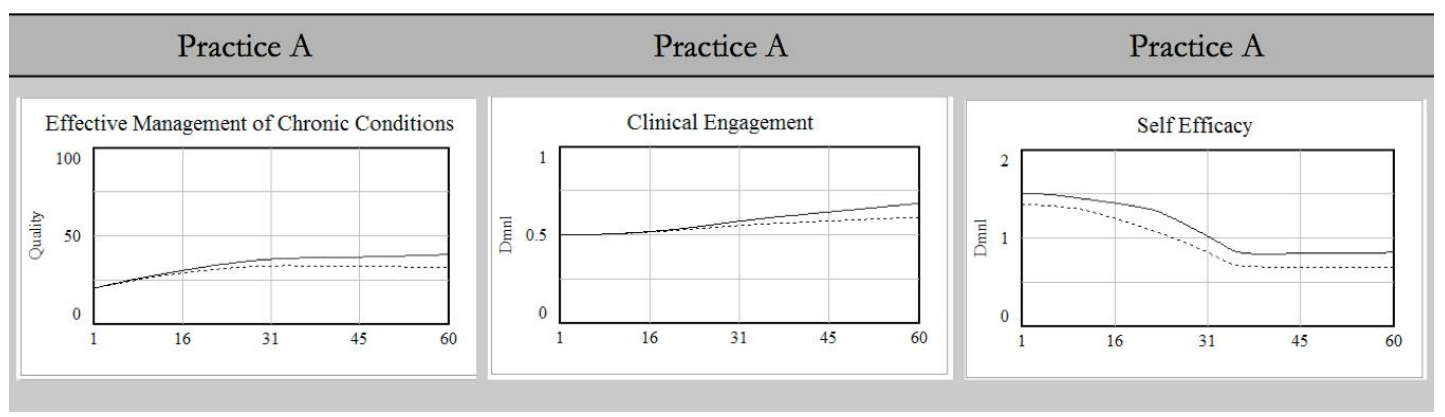

Figure 7-4 Policy experiment: striving for best practice

Legend:

$\begin{array}{ll} & =80 \text { per cent of best practice } \\ \ldots \ldots \ldots \ldots & =100 \text { per cent of best practice }\end{array}$

The behaviour of the model in these two scenarios show a gradual increase in the effective management of long-term conditions, levelling off in the case of the 100 per cent scenario after two years, underpinned by a rising level of engagement amongst clinicians. While performance does improve, neither the 80 per cent, nor 100 per cent targets are reached within the five years of the simulation, and after 12 months the 'Effective Management of Chronic Conditions' is lower when the goal is set at 100 per cent.

However, beginning with a degree of self belief, confidence declines and within two and half years self-efficacy has, in both scenarios, dropped below ' 1 ' indicating that the practice team no longer think that achieving the goal is possible. As described in chapter 6, any self-efficacy score greater that 1 reflects a positive belief that the change can be implemented successfully. Despite the belief that the goal is not achievable within the 
timeframe, clinicians' still believe long-term condition management is something worthwhile, and engagement continues to rise, albeit at a slowing rate. However, the declining level of self-efficacy reflects a gradual lowering of their belief that the goal is achievable. Factors affecting this are the fraction of resource capacity being 109 per cent, indicating a clinical team working just under 44 hours per week, and the gap between the stated goal and perceived effectiveness of chronic condition management, within an increasingly tight implementation timeframe. This figure is calculated by the model as a ratio of i) total workload time, based on number and type of patients; and ii) total time available, based on the number of GPs and the ratio of support staff to GPs. As the target deadline gets closer, it becomes increasingly clear that the practice will not achieve the goal. This is reflected in the remaining time to work ratio dropping at an increasing rate, undermining self-efficacy and subsequently the efforts put into improvement. So even though the clinicians believe it is worthwhile, as reflected in the continuing rise in clinical engagement, they do not believe it is achievable within the resource and time constraints they are facing. They run out of commitment and resource to spread the implementation further.

This result reflects a recent two-year study of primary care practices involved in implementing Patient-Centred Medical Homes (PCMH) in the United States (Crabtree et al., 2010). This research showed that despite successfully implementing many factors in the PCMH, there was limited improvement in patient outcomes and in some cases patient outcomes declined (Jaén, et al., 2010). A major reason put forward by the research team for these results was that success in implementation was heavily dependent on 'baseline conditions' and what they referred to as the 'adaptive reserve' of the practice. That is, its ability to learn and adapt to changing demands. As a consequence they emphasise the need for the implementation of new models of care to be a 'locally driven effort' (Nutting et al., 2010). The simulation model adds another element to that perspective, indicating that the goals being sought need to be matched to the capability of the practice to respond. This has significant implications for a country like New Zealand, where policy, and many of the practice targets, are set nationally and regionally. 


\subsubsection{The Impact of Resources}

When the same experiment is run with Practice B the same pattern occurs, albeit with higher levels of overall performance. A context of very tight resource constraints, such as Practice A, can place significant restrictions on what is possible and simply setting higher targets can in fact decrease performance. If the context is different however, 'going for broke' and setting high targets can have significant positive impacts. The following outputs (figure 7.5) show the performance for Practice A and Practice B when the target is set at 100 .

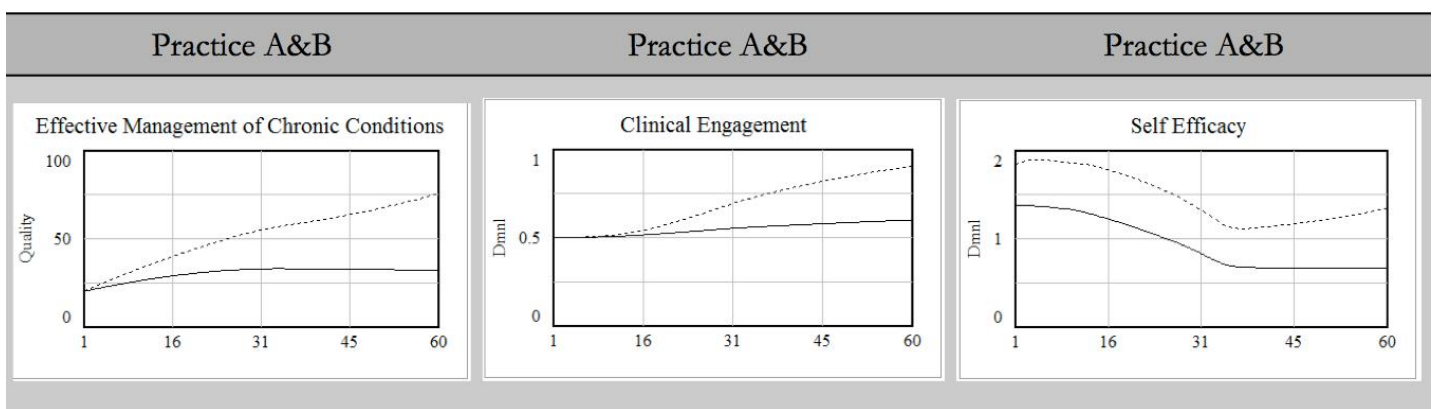

Figure 7-5 Policy experiment: impact of resources

Legend:

\begin{tabular}{lll} 
& $=$ & Practice A \\
\hdashline------ & $=$ & Practice $\mathrm{B}$
\end{tabular}

In this run, Effective Management of Chronic Conditions' rises rapidly within Practice $\mathrm{B}$ and although the 'best practice target' is not reached within the five years of the simulation the practice is well on the way and continuing to improve. Furthermore, this has been achieved by a highly engaged clinical team, who have confidence in their ability to improve. While self-efficacy declines, as the initial deadline of 36 months looms closer, once the deadline has past, their self-efficacy rises, reflecting their ability to improve, rather than an ability to reach a given target within a given deadline. 


\subsubsection{The Interaction Between Goal Setting \& Resource Constraints}

What the last experiment shows is what is commonly seen in practice: clinicians engage with the idea of developing new practices for people with chronic conditions and willingly put effort into helping implement it. As a consequence of this engagement, the effective management of chronic conditions improves, dependent upon the level of resources that can be applied to the task. In Practice B, the resource levels gave the team a great deal of confidence, and that is reflected in the improvement efforts delivering significantly improved levels of care. However, the rate of progress is much slower in Practice A. Despite continuing support for the idea, improvement in quality is severely limited. This often happens when the improvement efforts become resource constrained. While improvement in the quality of care bolsters the ongoing engagement of clinicians in the idea, the extensive effort required to implement it limits progress. The positive feedback loop generated by clinical engagement and improving performance is eventually overridden by a negative feedback loop in which continued efforts use up scarce resources, that as it becomes utilised, lowers the clinician's sense of self-efficacy which then undermines their efforts. So, despite agreeing that the idea is good, efforts to implement it are limited by the level of available resource.

The causal connections between these graphs is shown in figure 7-6, in which the graphs are placed on the causal theory, first described in chapter 5 .

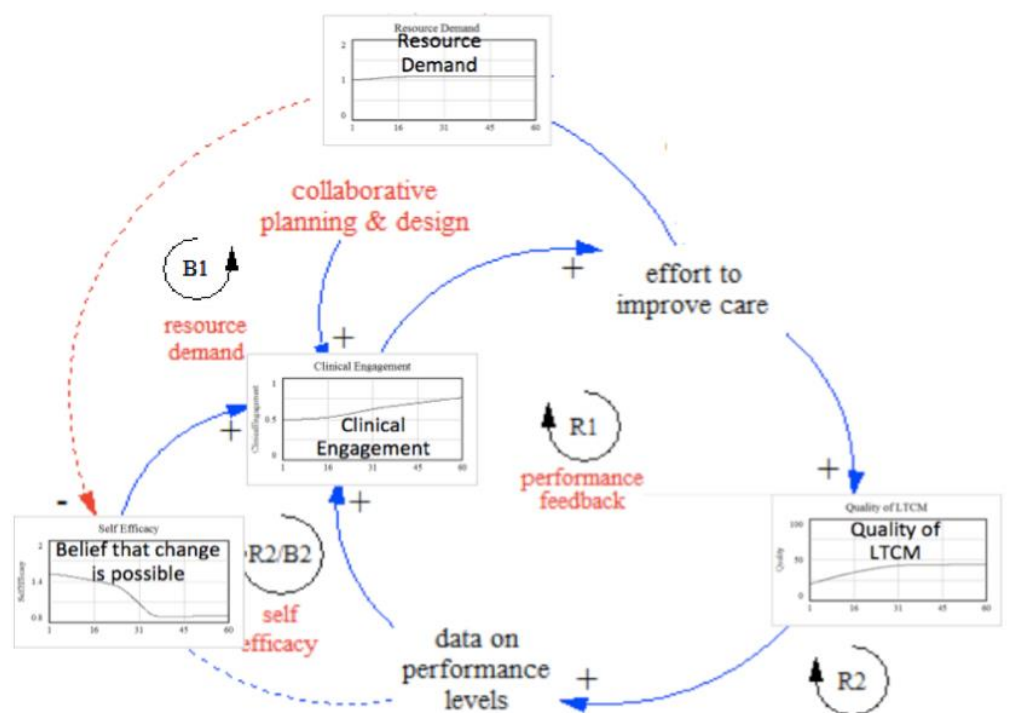

Figure 7-6 CLD and simulated behaviour 
Superimposing the quantitative simulation model over the qualitative theory aims to provide a better sense of how these simulation experiments relate to the theory described in chapter 5 .

Clinical engagement drives the effort to improve and the feedback on performance supports an ongoing rise in that engagement (R1). However the effort required to improve performance increases resource demand, which in turn undermines selfefficacy (B1). Depending on that level of self-efficacy the feedback on performance levels can serve to increase or decrease clinical engagement and therefore performance over time (R2/B2).

\subsubsection{Matching Goals to Performance}

To test this idea further, the following experiment adjusts the goals over time so that the gap between current performance and the target goal is not so great as to undermine clinicians' self-efficacy. In this scenario, based on Practice A, the goal was gradually increased over time. At the beginning of the simulation the goal was set at 40 , rising to 50 after 18 months and to 60 after four years. The results are shown below in figure 7-7. While improvements can be seen in both the Effective Management of Chronic Conditions' and in 'Clinical Engagement' the most significant shift is in Self-efficacy'. While there are drops, firstly, during the third year when the target deadline approaches and the practice team is still short of its target and again in year four when the target is increased further. However, these declines are not great and the ongoing increase in performance serves to support the belief in the practice that improvement is possible.

This scenario reflects an approach that is based on using current performance and improvement capability as the basis for goal setting rather than an arbitrary goal of 'best practice' and emphasises the point made earlier, that implementation really has to be a local affair and that there are many different implementation pathways. 


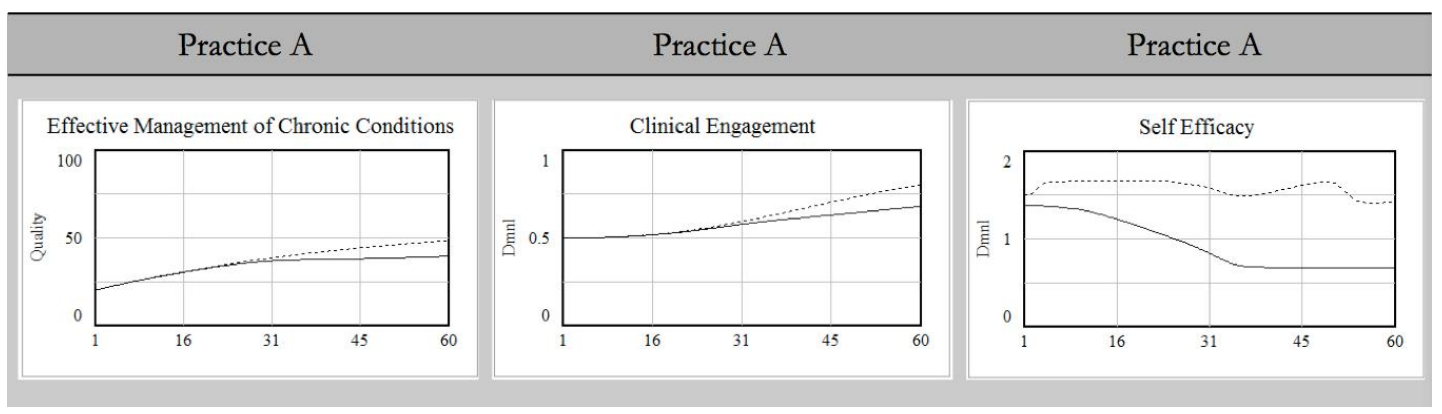

Figure 7-7 Policy experiment: matching goals to performance

Legend:

$\begin{array}{lll} & = & \text { Practice A } \\ ------- & = & \text { Practice B. }\end{array}$

7.8.5 From Best Practice to 'Local Improvements'

The implications arising from these simulation experiments are that 'best practice' in one setting may not necessarily be 'best practice' in another (R. Stacey, 2006) and that trying to impose it, without taking into account the baseline conditions of the practice will only result in already stressed and resource constrained practices failing to meet the target and possibly reducing overall performance. This point has also been identified by others (Hovmand \& Gillespie, 2006). Implementing best practice does not always improve performance, and the context within which best practice is being implemented needs to be looked at closely. In the examples just discussed, implementing best practice in a practice that is already busy, with limited resources for improvement, can result in lower levels of performance. Setting unrealistic targets can in fact undermine performance.

The second implication is that terms like clinical engagement, which have been central to the literature on the implementation of new health practices, are much more complex than they have been portrayed. Engagement has, at least, to be separated into engagement with the idea and the belief that the idea is in fact implementable. Support for best practice does not mean that, in any specific context, clinicians believe that it can be implemented successfully and this belief is a significant driver of performance. 
What these initial experiments are showing is that actual performance improvements are driven by a subtle mix of the goal being set, the support for that goal, the belief that it can in fact be implemented and the resources available to be applied to the task.

Having highlighted the complexity underpinning the construct of clinical engagement the next set of experiments explores the impact of differing levels of clinical engagement.

\subsection{The Impact of Clinical Engagement}

The previous experiment modified the goals being sought and clinical engagement, beginning at an initial value of 0.5 , slowly rose throughout the period of the simulation. That experiment emphasised again that clinical engagement has at least two key factors that research needs to take account of, namely, engagement with the idea as something that is worthwhile doing, and belief that the goal is in fact achievable within the timeframe. In this experiment, it is the engagement with the idea as being worthwhile that is varied. That is, the experiments begin with a scenario in which there is only a limited level of clinical engagement and finish with a scenario in which there is complete support for the stated goal. As a consequence it is the goal that is the driver of effort, past and current performance having no effect on engagement and/or effort. The baseline scenario conditions for this experiment are identical to the previous experiment, except that the Level of clinical engagement initial' is now the variable that is altered in each experiment. 


\begin{tabular}{lll}
\hline Model Variable & Unit of Measure & Parameter \\
\hline Effective management of chronic conditions initial & Quality & 20 \\
Stated goal for quality of LTCM & Quality & 80 \\
\hline Level of clinical engagement initial & Dmnl & varies with each \\
experiment
\end{tabular}

Table 7-9 Key parameters: clinical engagement

\subsubsection{Changing the Initial Conditions for Clinical Engagement}

The goal set for each practice in this experiment is 80 per cent of best practice and the level of clinical engagement is varied. In scenario 1, the level of clinical engagement is set at 0.5 , rising to 0.75 in scenario 2 and to 1.0 in scenario 3 . The results are show in figure 7-8. 


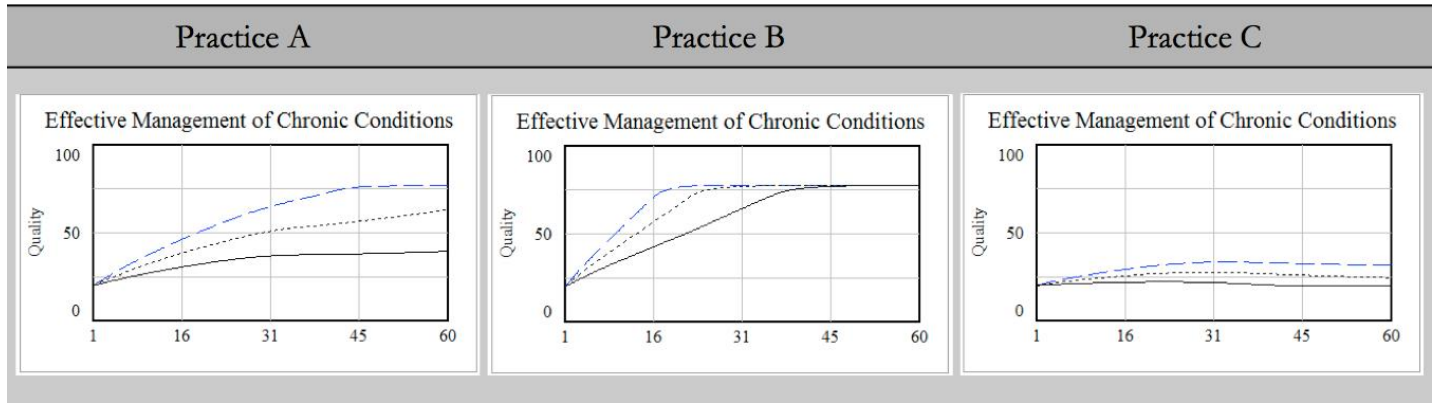

Figure 7-8 Policy experiment: changing initial levels of clinical engagement

Legend:

\begin{tabular}{|c|c|c|}
\hline & & Clinical Engagement Initial $=0.5$ \\
\hline 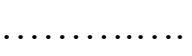 & & agement Initial $=0.75$ \\
\hline & & Clinical Engagement Initial $=1.0$ \\
\hline
\end{tabular}

In this experiment, the results are more consistent than in the first experiment: as clinical engagement goes up, so does performance. The well-resourced practice performs better than the others, but even Practice C improves, when clinical engagement rise to 75 and 100 per cent. This is not surprising, as an initial value of 0.5 for clinical engagement means that the stated goal is given a weighting of 0.5 , the other 50 per cent being driven by their perception of actual performance. So, with clinical engagement set at 0.5 the effort to improve is moderated by their perception of what they consider the practice is capable of doing, as reflected in actual performance. When clinical engagement is set to 1 then it is the goal, rather than perceptions of performance capability that drive effort.

With clinical engagement set at an initial value of 0.5 , the level of engagement over time is driven by a $50 / 50$ balance between the stated goal and the perception of current performance. With the level of clinical engagement set at an initial value of 0.75 then the balance is shifted in favour of the stated goal with their perception of current performance only having a 25 per cent influence. When the level is set at 1 clinical engagement is unmoved by actual performance. The stated goal is the driver of engagement and no matter what happens to performance a belief in the goal remains unchanged. 


\subsubsection{Like the Idea But Is It Possible?}

However, in this model, efforts to improve are not just driven by clinical engagement.

They are also driven by self-efficacy, a belief that the goal, however laudable, can also be implemented with the resources available and within the timeframe. This impact of this is shown in the graphs below, which show the performance in terms of Effective Management of Chronic Conditions', 'Clinical Engagement' and Self-efficacy' for each practice when the goal is set at 80 per cent of best practice and clinical engagement is initialised at 0.75 .

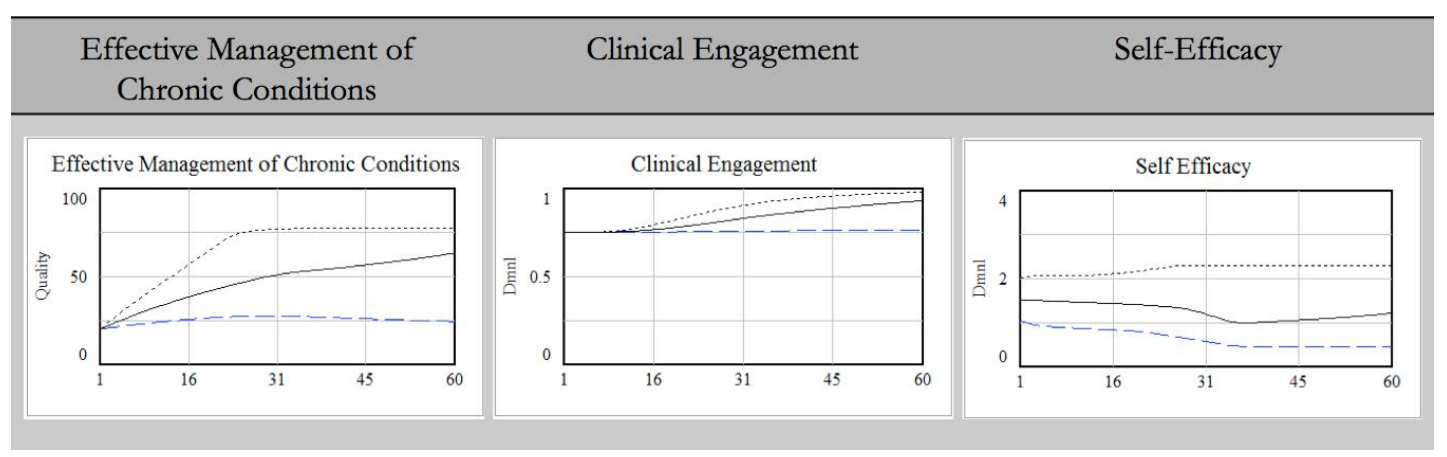

Figure 7-9 Policy experiment: clinical engagement and self-efficacy

Legend:

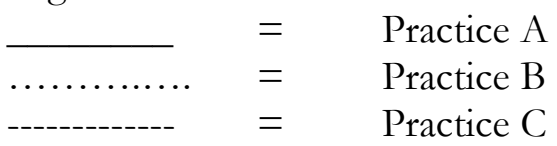

Practice B achieves the goal within two years and clinical engagement continues to rise over the five years of the simulation. In addition, they begin the change process supremely confident that they can achieve the goal, a confidence that does not diminish. So, with a well-resourced practice, clinicians who support the goal and are confident that they can achieve it the results are outstanding. For Practice A, while performance does improve it can be seen that 'under the surface' there is a tension between a strong and growing commitment to what the practice is trying to achieve and a decreasing belief that the goal can in fact be achieved. The tension between these two dynamics impacts overall performance improvement. For Practice C, their lack of resources, once 
again, limits any chance of significant improvement. The clinicians within Practice $\mathrm{C}$ support the idea but know that they can never achieve it.

What happens however, if clinical engagement is much lower? The following experiment lowers the initial value of clinical engagement to 0.2 . In this scenario only 20 per cent is weighted to the stated goal with 80 per cent being weighted towards their perception of actual performance. In this scenario the driver of effort is largely knowledge of what has been achieved in the past, which as described above is a situation in which Effective Management of Chronic Conditions' has remained 20 per cent of best practice. In this scenario, the goal is, as above, 80 per cent of best practice, but each practice begins with a much lower level of commitment to that goal. The following graphs (figure 7-10) show the impact upon Effective Management of Chronic Conditions', 'Clinical Engagement' and Self-efficacy' for each practice:

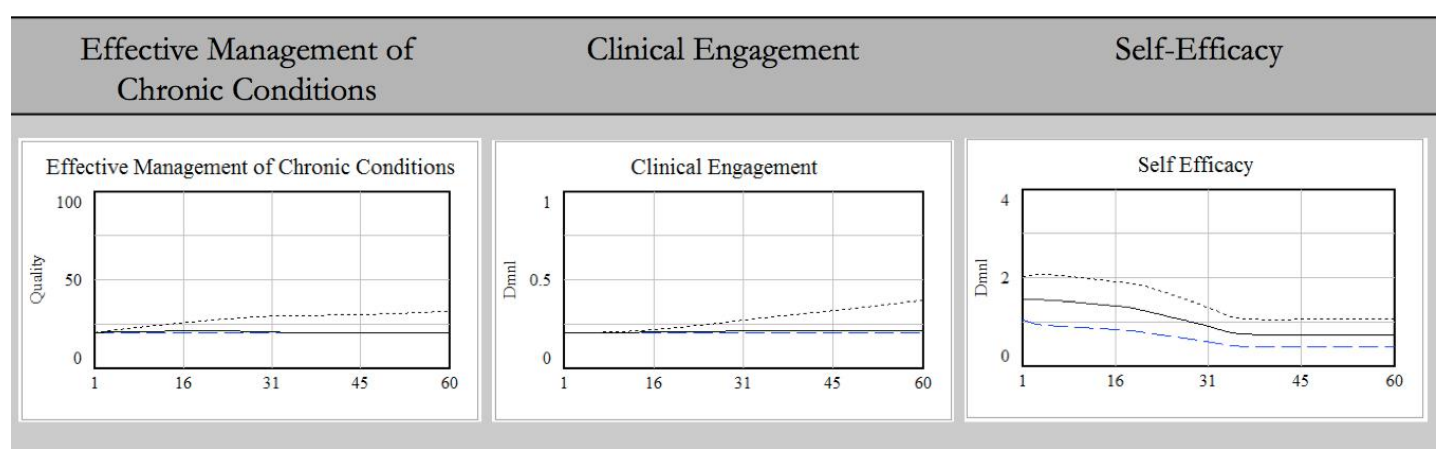

Figure 7-10 Policy experiment: lower levels of clinical engagement

Legend:



As the graphs show unless clinicians are engaged with and committed to the goal, little can be achieved. In the case of Practice A and Practice C, performance in fact drops. While Practice B does improve, driven by their large resource base, the improvement is marginal. Thus clinical engagement works in different ways in different practice contexts. If clinical engagement is high, those practices that have the resources to improve will do 
so and the performance gap between the best performing and least performing increases considerably over time. However, if clinical engagement is low nobody, even the most highly-resourced practice, is likely to improve much, and even after five years the difference between the best and worst performing practice is not great.

Clinical Engagement, in this model, seems therefore to be a 'necessary but not sufficient' component of successful implementation. If there is little clinical engagement with the improvement goal then, regardless of the resource levels, improvement will be severely limited. As the high levels of self-efficacy for Practices A and B, shown above, indicate, they may believe that the improvement is possible, but it is not one they support, once again highlighting the two important factors underpinning the concept.

So, while the simulation is consistent with the idea that clinical engagement is important to performance improvement, resource constraints still provide the 'trump card'. Even with a team of highly engaged clinicians, improvement will always be constrained by the resources available.

So, is it possible in this model for poorly resourced practices to make significant gains in terms of the Effective Management of Chronic Conditions'? The next experiment explores the impact of investing increased hours in patient engagement activities.

\subsection{Options for a Resource Constrained Practice- Patient Engagement}

One of the key insights that the model is highlighting are the different challenges being faced by a resource-constrained practice operating in a high needs area. In this context, practices have to deal with higher patient/GP ratios and lower levels of selfmanagement skills in the patient population. What the model shows is that, in highly resource-constrained practices (Practice $\mathrm{C}$ in this model) interventions that require additional work on the part of the practice team have little if any positive impact upon the 'Effective Management of Chronic Conditions'. In fact exhortations to 'do more' often lead to lower levels of performance. 
7.10.1 Investing in Patient Engagement Activities

Before exploring specific strategies for Practice C, the following experiment shows the impact of changing the amount of time spent on patient engagement activities across all three practices. The parameters used in this experiment are shown below in table 7-10.

\begin{tabular}{lll}
\hline Model Variable & Unit of Measure & Parameter \\
\hline Effective management of chronic conditions initial & Quality & 20 \\
Stated goal for quality of LTCM & Quality & 80 \\
Level of clinical engagement initial & Dmnl & 0.5 \\
Maximum improvement capacity & Effort & 30 \\
Implementation timeframe & Month & 36 \\
Number of GPs initial & GPs & varies between \\
& & practices \\
Initial average number of support staff per GP & Staff & 0.5 \\
Number of patients initial & Patients & 10,000 \\
Per cent LTC patients engaged in self-care initial & Dmnl & varies between \\
& & practices \\
\hline Per cent enrolled population with LTCs & Dmnl & 0.7 \\
Average hours per week involved in patient engagement & Month & varies within \\
activities target & & Dmnl \\
Level of process quality baseline & & 1 \\
\hline Table $7-10$ Key pariment
\end{tabular}

Table 7-10 Key parameters: resource constrained practice 
The following graphs (figure 7-11) show the impact across all three practices of investing varying amount of time in patient engagement.

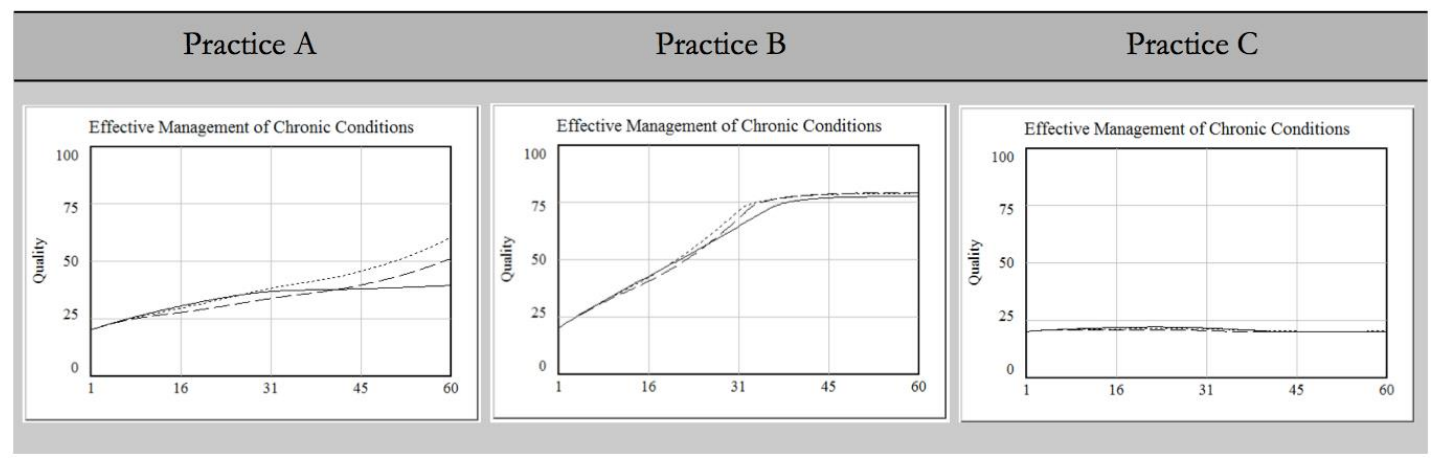

Figure 7-11 Policy experiment: changing levels of patient engagement activities

Legend:

$\begin{array}{ll} & \text { Time on patient engagement activities }=2 \text { hours per week } \\ \ldots \ldots \ldots \ldots \ldots & \text { Time on patient engagement activities }=5 \text { hours per week } \\ -\cdots--\ldots-. & \text { Time on patient engagement activities }=10 \text { hours per week }\end{array}$

While Practices A and B show considerable benefit from investing in patient engagement activities, Practice $C$ is unable to lift performance above its baseline level. To explore the drivers of this, the following graphs show the per cent of patient engaged in self-care activities and the resource demand for Practice $C$ when the hours spent on improvement activities shifts from two hours per week in scenario 1, to 5 hours per week in scenario 2 and 10 hours per week in scenario 3. The results, shown in figure 7-12, highlight the difficulty of making changes in such a resource-constrained environment. 


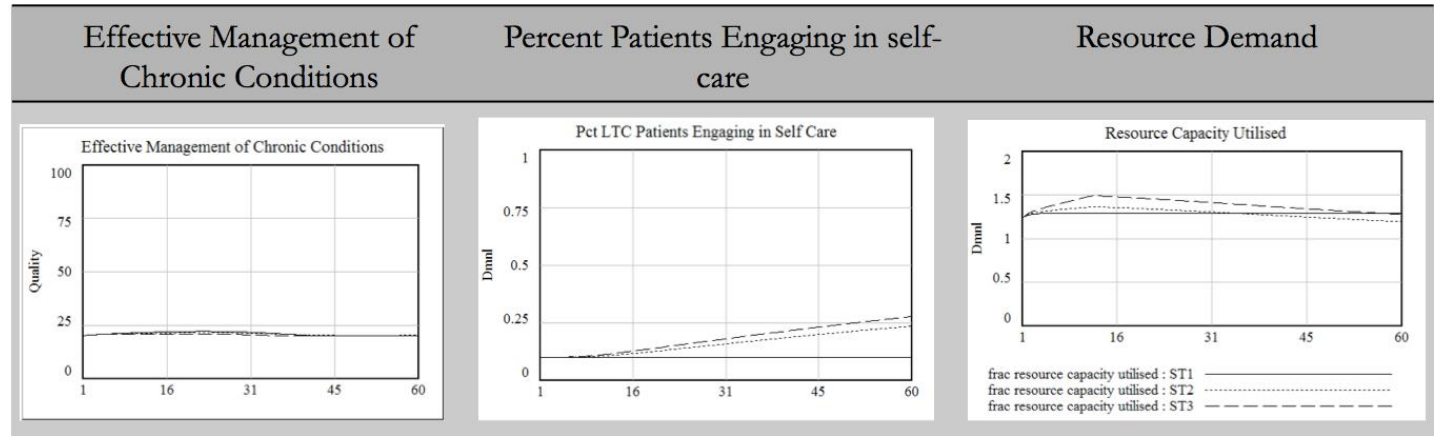

Figure 7-12 Policy experiment: impact of changing investment in patient engagement activities in practice $\mathrm{C}$

Legend:

$\begin{array}{lll} & = & \text { Time on patient engagement activities }=2 \text { hours per week } \\ \ldots \ldots \ldots \ldots . . & = & \text { Time on patient engagement activities }=5 \text { hours per week } \\ -\cdots------ & = & \text { Time on patient engagement activities }=10 \text { hours per week }\end{array}$

Throughout the five years of the simulation, there is little change in the 'Quality of LTCM', although the graphs for the 5 hours and 10 hours per week scenario show a minimal rise after about three and a half years. While the difference is not great, the trend is towards increasing improvement in both the 5 hours and the 10 hours scenarios. The key driver of this is that the time spent improving patient engagement results in an increasing percentage of patients engaging in self-care. Scenario 1, with two hours per week invested in patient engagement activities, has no impact upon the percentage of patients engaging in self-care, however, when the number of hours per week is lifted to five the per cent rises to 28 per cent after five years and when the number of hours per week is lifted to 10 the percentage after five years rises to 37 per cent. This has an impact on resource demand. For the first three years the resource demand, driven by the extra hours put into patient engagement activities rises, only falling below its starting position around month 36. Resource demand, through its impact upon self-efficacy, as has been shown earlier has a significant impact upon performance. The change in resource demand also highlights the delays involved in changing patient behaviour. The time has to be spent and the extra hours absorbed for a considerable period before the benefits of improved patient engagement begin to show. 
So, given the rise in the percentage of patients engaging in self-care, why is the

'Effective Management of Chronic Conditions' so slow to rise? The reason can be seen in the following graphs, which show the impact upon 'Clinical Engagement' and 'Selfefficacy' in this experiment.

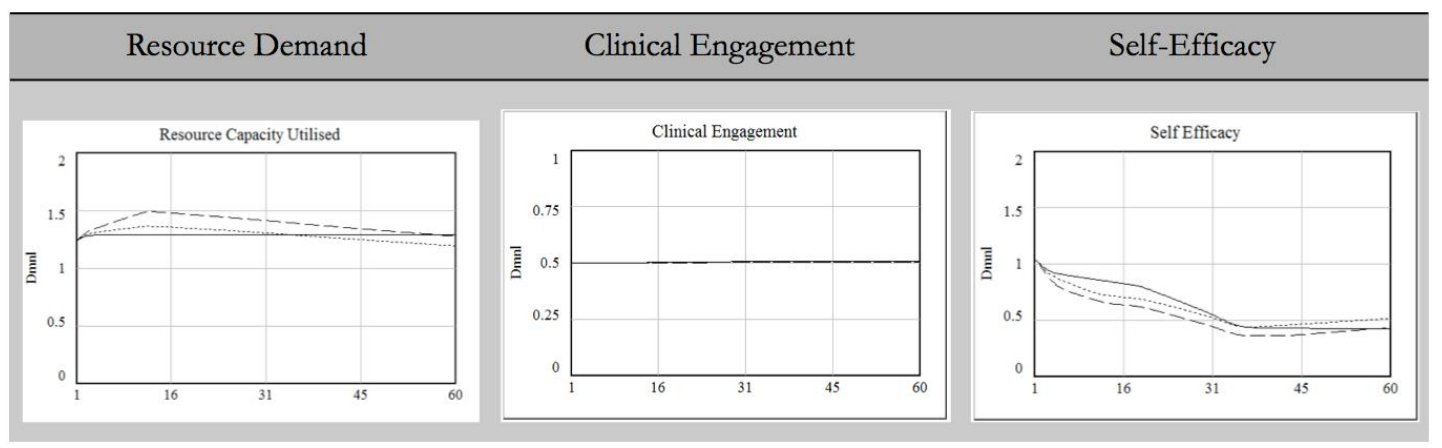

Figure 7-13 Policy experiment: impact of high resource demands in practice $\mathrm{C}$

Legend:

\begin{tabular}{|c|c|c|}
\hline & $\begin{array}{l}= \\
=\end{array}$ & $\begin{array}{l}\text { Time on patient engagement activities }=2 \text { hours per week } \\
\text { Time on patient engagement activities }=5 \text { hours per week }\end{array}$ \\
\hline 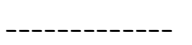 & & Time on patient engagement activities $=10$ hours per week \\
\hline
\end{tabular}

In this model 'Clinical Engagement' is a function of the stated goal and the perception of current performance. So, as is the case in this scenario, when 'Clinical Engagement' is initially set at 50 per cent, half of the efforts to improve are driven by a desire to close the gap between current performance and the stated goal, and the other half are driven by the perception of current performance, which is seen as the indicator of what is possible. In this scenario, the consequence is that the gap between the current and future state is so large, requiring a significant increase in resources that self-efficacy declines rapidly over the first three years, acting as a constraint upon performance improvement, and the perception of that very slow and minimal improvement only serves to reinforce the belief that change is not possible. A resource demand of over 1 indicates that the only way the work can get done is through work over and above the normal working week. This impacts self-efficacy and as a consequence 'Clinical Engagement', and thereby the 'Effective Management of Chronic Conditions' does not rise. 


\subsubsection{Being Realistic About What is Possible}

In the first set of experiments it was shown that goal setting itself can act as a brake upon performance and that it was possible to improve results by taking account of the local context and slowly increasing targets as performance itself improved. In the following example, the key parameters are identical to scenario 2 , in which the practice increases the amount of time spent on patient engagement activities to five hours per week, except that the goals are increased slowly over time. At the start of the experiment, the goal is set at 40, increasing to 50 after two years and then to 60 after three and a half years. The results are shown below in figure 7-14.

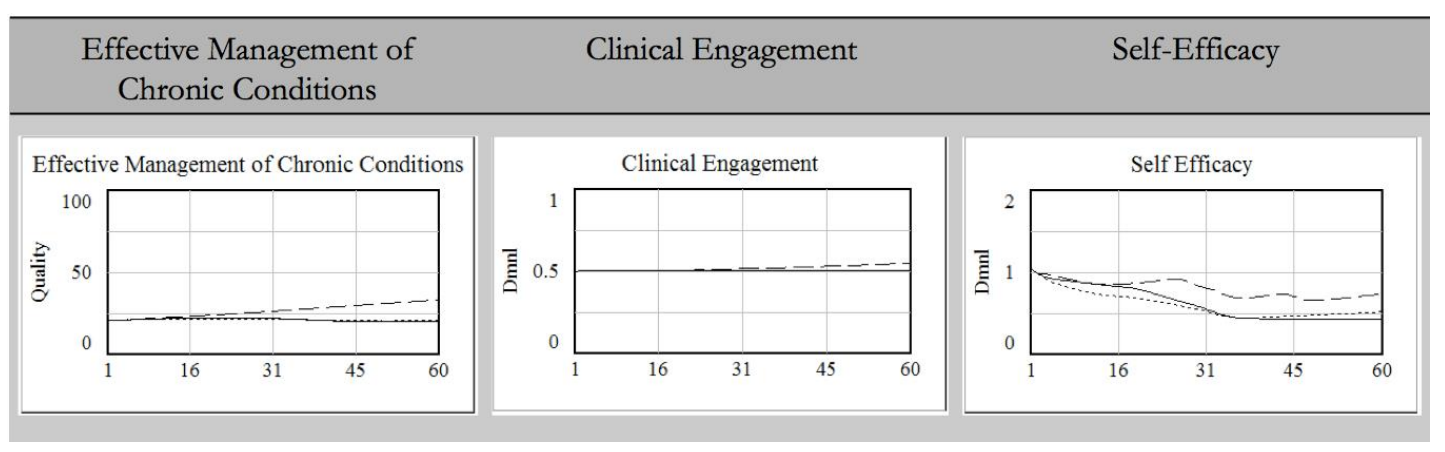

Figure 7-14 Policy experiment: setting targets based on local capabilities in practice C

Legend:

Time on patient engagement activities $=2$ hours per week

…...... Time on patient engagement activities $=5$ hours per week

Time on patient engagement activities $=5$ hours per week with the goals slowly rising to 60

The results show a considerable improvement in performance and a significant rise in clinical engagement over time. Beginning with a goal of only 40 means that the gap between the current state and the goal is not so great as to drive self-efficacy downward. In fact, the relatively lower improvement target creates great confidence in the practice and they start with a much higher level of self-efficacy. It drops when the target is increased to 50, and again when the target is increased to 60 , however it rises again as performance improves. Because self-efficacy does not drop as significantly as in the other scenarios effort is maintained and quality improves. With self-efficacy remaining 
higher and performance improving 'Clinical Engagement' gets a double push - they see improvements in care and believe that the targets are achievable - and after two years begins to rise, increasing more rapidly over time.

What this experiment shows, is that despite the enormous impact of resource levels, a carefully planned implementation programme, that acknowledges the local context can deliver improvements in performance. One aspect driving this is that Patient Engagement' while taking a long time to deliver benefits does so at an increasing rate over time. It builds momentum, by not only decreasing resource demand as the benefits of self-care translate into lower usage of health resources, but also directly contributing to the quality of care experienced by the patient.

\subsection{Improving Service Delivery}

So far the experiments have focused on the goal being sought, clinical engagement and patient engagement. Central to the theory however is the concept, 'Quality of Service Delivery.' Underpinning this was the idea that improving a practice's understanding of the enrolled population and how specific processes impact upon clinical outcomes can improve the 'quality of effort'. In the model, this is operationalised as a multiplier of the impact of effort upon the improvement effort, which drives the 'change in quality of LTCM'. This is shown below in figure 7-15, an extract from the model.

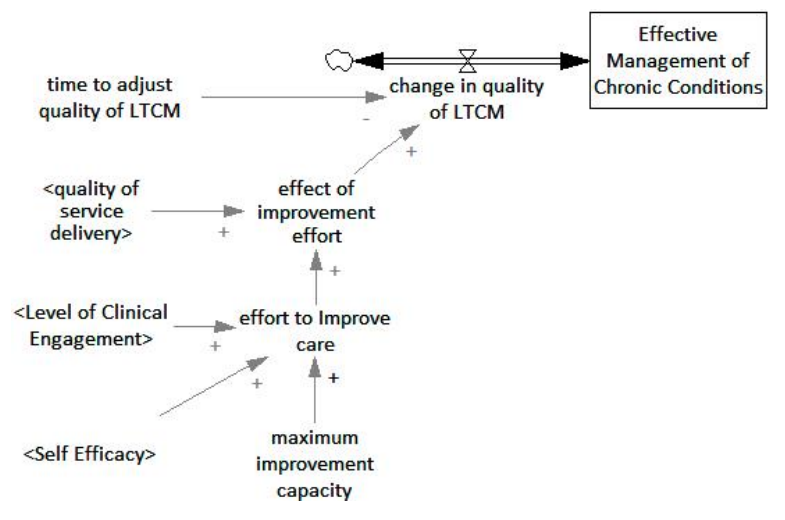

Figure 7-15 Model structure: impact of service delivery 
So, while the 'Level of Clinical Engagement' and 'self-efficacy' drive the improvement effort, how much impact that effort will have on the 'change in quality of LTCM' will be influenced by the 'quality of service delivery'. The next set of experiments explores the impact of changing the quality of effort, which in the model is driven by investing time in improving service quality improvement. In this context, while some time may be involved in exploring process improvement initiatives, the main activity is the use of information technology, during the patient consults, technology that captures and stores patient information in a way that can be used to provide information on the health of the enrolled population and the impact of practice initiatives. The use of such 'decision support' technology has been a major part of improvement efforts in primary care (Garg et al., 2005). It should be noted that the model takes no account of the financial investments required to implement such systems. It does assume however, that using such systems does take time to implement and add to the overall workload. The results of this experiment are shown below in figure 7-16.

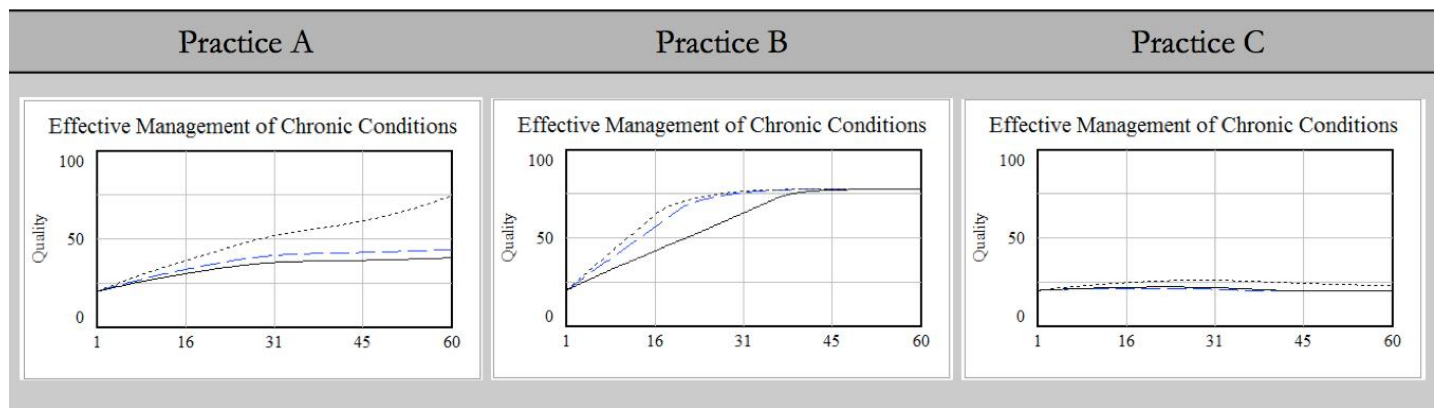

Figure 7-16 Policy experiment: impact of process improvement activities

Legend:

Time on service quality $=0$ hours per week Time on service quality $=5$ hours per week Time on service quality $=10$ hours per week

Once again, the impact of resources is evident, with the better-resourced practices benefiting more from the investment in process improvement. Practice A, while it does not achieve the 80 per cent target, with an investment of five hours per week, reaches just over 50 per cent after five years. Practice B, easily achieves its target under all scenarios. Practice $\mathrm{C}$ on the other hand receives no discernable benefit from the investment. As with the goal setting scenario, trying too hard can have detrimental 
effects and in all practices the best performance comes from investing five hours per week. Lifting that investment to 10 hours per week only serves to substantially increase resource demand and thereby negatively affecting self-efficacy, the result of which is seen in lower levels of quality across all three practices; lower than that gained by investing five hours per week.

Regardless of resource levels, the experiments are showing that it is always important to balance the efforts required to change with the impact those efforts have on resource demand. While investing in improvement initiatives have a positive impact upon performance, too much time invested only serves to trigger the self-efficacy negative feedback loop, in which support for the idea and the positive effects of the intervention are undermined by a decreasing belief that the goals can be achieved within the resource and time constraints.

\subsection{Summary}

The purpose of building the simulation model was to extend and clarify the theory, initially captured in the CLD described in chapter 5 . This chapter described the validation process undertaken to build confidence in the model, as well as the challenges of building such models where empirical data is scarce. While accepting the impossibility of achieving absolute validity, the chapter described how the structure of the model was tested with the interviewees throughout the process. The causal relationships were tested by the use of sensitivity analysis and one example shown. The behaviour of the model was also tested with the health experts interviewed and where possible with the available literature. Finally 'extreme condition' tests were run to test the plausibility of the model outputs where key inputs were set at extreme levels.

The chapter then described the design of the simulation experiments, in particular the use of three different 'archetypal' primary care practices operating in three different contexts. Following the base case, a number of simulation experiments were undertaken to explore the impact of changes in i) goal setting, based around percentages of best practice; ii) clinical engagement; iii) patient engagement; and iv) service delivery. These 
experiments provided insights into the practical realities of implementing new health practices in a primary care practice.

The simulation model aimed to capture the dynamic consequences of a range of concepts, identified by health experts, interacting over time in the context of three 'typical' primary care practices operating in New Zealand. It has been used to run a series of policy experiments that have shown the consequences of different resources and different practices and, above all, highlighted that any strategy has to take into account the local context, especially the resource levels within the practice and the extent to which the population served by the practice has adopted self-care practices.

What can be concluded from these experiments, and the qualitative theory that it was based on, is discussed in chapter 8 . 


\section{Conclusions}

\section{Learning and Limitations}

\subsection{Discussion}

A central idea in this research has been that implementation, by its very nature, is messy, with many factors interacting with each other in surprising and often counterintuitive ways to affect how the implementation pathway unfolds. Furthermore, this research has taken up Donald Schön's challenge to leave the high ground of 'technical rigor' and enter the 'swampy lowland' where the implementation is much more complex and messy (Schon, 1983, p. 42). In doing so, this research has attempted to find a way of living in the swamp by using qualitative and quantitative systems modelling methods that, while they may not have the level of precision and control of other experimental and survey methods, have provided a glimpse into the characteristics of the swamp and some of its inhabitants. To continue the ecological metaphor, this research has explored how well-known characteristics of the swamp, such as goal-setting, clinical engagement, patient engagement and service quality interact, influencing how well those primary care practices trying to live in the swamp survive and prosper, or stagnate and die.

The research shows, almost above all else, that it is possible to explore the complexities of implementation and reveal something of how it works without overly simplifying and controlling the situation under study. The research has also shown that while there are key factors, such as clinical engagement, that are important to the success of implementing new health initiatives, the differing context within which primary care practices find themselves, means that successful implementation will always “...be a locally driven effort" (Nutting et al., 2010, p. S45).

The significance of these results is that those trying to improve primary care, and those trying to develop national and regional performance targets, need to be much more cognizant of context.

The theory of implementation described in chapter 5 shows that it is possible to describe, in a coherent manner, variables involved in implementation, along with their 
patterns of interaction. The simulation results, described in chapter 7 , explored those patterns of interaction in detail and highlighted that many of the factors identified, in the literature, as being important are better described as 'necessary but not sufficient'. Furthermore, the experiments, comparing results within and between different practices, helped to clarify what influences their ability to support successful implementation.

\subsection{Implications for Theory}

This research attempted to answer three key questions:

- What is the system of causality underpinning the key factors known to be important in the implementation of new models of chronic health care management?

- How does context influence this system?

- Can the answers to the first two questions provide a model of implementation that informs both theory and practice?

So what answers does this research provide?

\subsubsection{Developing a System of Causality}

Building on the ideas of the seven health experts, this research developed a theory of implementation, described in chapter five, that has many of the key factors already known to be important in implementing new health initiatives. Much has been written about the importance of clinical engagement (Beasley, 2006; Ruston \& Tavabie, 2010) and the central role it plays (Ham, 2003). The same can be said for patient engagement (Jordan et al., 2008), which is supported by an extensive literature on how to achieve better self-care (Bower et al., 2012; Jordan \& Osborne, 2007; Vickery, Golaszewski, Wright, \& Kalmer, 1988), and how to deliver high quality healthcare systems (Si et al., 2005). Although less has been said about two other factors that emerged as important in this research - goal setting and self-efficacy - they are not completely new to this area of research (Bandura \& Cervone, 1983; Lee \& Ko, 2009). What is new, is the connecting of these factors into a coherent system of causality that helps explain why they are important and what causes them to grow and decay under different contexts. Using the literature to both enhance and 'fill-in-the-gaps' in the ideas of the health experts, this 
research has developed a system of causality that combines known factors into an explicit and testable theory of implementation. Furthermore, the literature allowed me to identify some areas, for example the negative impact of trying to implement 'best practice', where empirical research has demonstrated the importance of some of these causal relationships.

This research, therefore, contributes to the growing literature on issues of implementation in healthcare (Damschroder et al., 2009; Proctor et al., 2009), by putting known factors into a causal system that is sensitive to context. Furthermore, it posits 'micro-theories' (Schwaninger \& Grosser, 2008) that explain how these key factors develop over time. For example, the theory describes how clinical engagement is developed and how it can be undermined. It can be given a 'kick-start' by ensuring clinicians are involved early on in the planning and design of the implementation programme. It is more likely to be maintained when clinicians get feedback that provides them with information about the impact of the initiative on their patients and enrolled population. However, clinical engagement will be undermined if the improvement goals set go beyond what clinicians believe is achievable within the resource constraints and timeframe. Each of these theoretical propositions finds support in the literature. So, while the simulation model, developed from the theory, takes qualitative statements such as "clinical engagement is central to the improvement effort", and posits a quantitative level of impact it does so in a way that is empirically testable and in doing so, provides the opportunity for the theory to be tested and refined.

\subsubsection{The Impact of Context}

The theory, and the simulation model supporting it have shown how context has a significant impact upon, for example, clinical engagement and the overall quality of healthcare provided. In this research, the key contextual factors are the GP/patient ratios, which indicate the overall workload of the practice and the percentage of the enrolled population engaged in self-care, which is indicative of the level of health literacy in that population. The experiments described in chapter 7 show, for example, that while setting stretch goals can help drive performance improvements in reasonably and well-resourced practices (Practices A and B), they only serve to undermine self- 
efficacy in a poorly resourced practice resulting in stagnant or lower levels of performance. Even in Practices 'A' and 'B', if the stretch is too far, self-efficacy is undermined and performance drops. This is consistent with the goal-setting literature (Locke \& Latham, 2002) that describes the relationship between goals, effort and performance. It is also consistent with the literature in highlighting that goals that go beyond what the participants believe is possible will undermine performance. Locke, Latham and Erez, (1988), commenting on earlier work by Erez and Zidon (Erez \& Zidon, 1984) note the finding that, “.... when commitment drops markedly in response to increasingly difficult goals, performance drops accordingly" (Locke et al., 1988, p. 23).

This incorporation of context into the theory responds to growing acknowledgement that implementation is always context dependent and while some factors have universal application, how they play out and affect the success of the implementation will always be influenced by the context within which they exist.

By describing a system of causality that underpins the implementation of new health initiatives and showing how this system is affected by the context within which it operates, this research can help inform our theories of implementation. As discussed in chapter 2, most theories of implementation are essentially factor theories (Yin, 2003), describing those factors considered to be important in delivering improved performance within an implementation programme. Furthermore, those factors affecting implementation are, in most cases studied, 'in an isolated fashion' (Durlak \& DuPre, 2008), working within a paradigm that, “....assembles a list of independent variables and determines those that are most highly correlated with the dependent variable" (Yin, 2003, p. 14). Recent research has confirmed that implementation is a complex process, involving many interacting factors (Durlak \& DuPre, 2008; Greenhalgh et al., 2005), and that it is important to consider the initiative's interaction with context, when implementing innovations in the messy reality of real world settings. Because of this it is important to consider the complex system within which interventions occur (Hoddinott et al., 2010).

The SD literature, embedded as it is within ideas of complex patterns of causality, has a number of examples describing the dynamics of innovation and implementation (Hovmand \& Gillespie, 2006; Repenning, 2002; K. Taylor \& Dangerfield, 2005). This 
research adds to that growing body of literature by focusing on a specific implementation challenge, new health initiatives designed to improve care for people with chronic conditions, within the specific context of primary care.

\subsection{Implications For Practice}

This research has a number of implications for policy and practice in primary healthcare. It cautions against the blanket imposition of 'national targets' in favour of local targets based on the capability of the practice. It favours incremental improvement steps, rather that large shifts in performance expectations. It also emphasises the need to support poorly resourced practices in their attempts to shift the focus of care, as without it little change is likely to occur.

\subsubsection{Design Propositions}

One of the significant things that this research does provide is a set of propositions that can be used to guide the design of new initiatives. These include:

i. Understand what the clinical team believe they are capable of before establishing performance targets.

ii. Involve clinicians, from the outset, in the planning and design of the new initiative.

iii. Establish feedback loops that keep the clinical teams informed about how their efforts are impacting the health of the population they serve.

iv. Establish performance targets that are based on current performance and capability, rather than some external national or regional goal, or concept of best practice. If, for example, HbA1c, a measure of blood glucose levels and is an indicator of how well the patient has their diabetes under control, is an important clinical outcome, rather than setting an arbitrary target such as '50 per cent of patients should have HbA1c levels $\leq$ 9', set a target that is based on current performance, within a time period. For example, 'increase the number of diabetic patients with HbA1c $\leq 9$ by 10 per cent per year for the next three years. 
v. Give patient engagement a high priority, early on in the programme. The benefits take a while in coming but can help reduce resource demand and thereby free up resource for further improvement efforts.

vi. Identify the GP/patient ratios, within the practice, in relation to local, regional and national averages, as well as the health literacy of the population being served, as they will heavily influence the size and speed of any performance improvement.

\subsection{Limitations of the Research}

There are two significant limitations to this research. The first is that the theory, described in chapter 5 , was developed from ideas presented by seven individuals. While these were seven senior and experienced individuals with extensive knowledge, incorporating national and regional as well as clinical, policy and managerial perspectives, it is possible that their combined perspectives are lacking in some factor that is of crucial importance to the theory that emerged. However, a review of the literature and feedback from the experts interviewed, indicates that improvements in the theory are more likely to come from adding further detail, for example, the impact of technology on the feedback mechanisms to clinicians, rather than any completely new factor not already incorporated into the theory. The second limitation is that the lack of empirical data in the implementation research means that the size and direction of impact of the causal connections may be wrong in some seriously important ways. While attempts have been made to find empirical or theoretical support for the causal connections in the model, it is possible that there are errors in there that would be significant to substantially alter the propositions that flow from it. The research has tried to minimise this by running a series of sensitivity tests, as discussed in chapter 6 , but the fact remains that, to-date, the lack of empirical data is a significant limitation. Furthermore, the model does point to very specific areas where data could be collected to provide further testing of the theory.

\subsection{Future Research}

As is often the case it is the limitations of any research that offer up the opportunities for further study. Because of its explicit, and thereby testable, description of the 
dynamics of implementation, the theory described in chapter 5 is able to be tested with a broader group of health professionals. In doing so the theory could be expanded with the addition of new variables and causal connections. It could also be 'filled in' by disaggregating key variables. It would also be interesting to conduct some empirical studies that helped refine the causal connections. Are the size and shape of the causal connections appropriate? How much do they vary in different contexts and what determines that variation? So, while the simulation has made some distinctions between well and poorly resourced practices, it would be interesting to conduct some empirical research on the impact of resource capacity and ability to implement new health initiatives.

Another area of future research focuses less on content and more on process. This research has attempted to describe a process for extracting information from domain experts, through a series of steps that refine that information and, through the development of a simulation, explore the consequences of their perspectives. This is about the process of conceptualising models, deciding what they should include, and what should be disaggregated. This is of considerable interest to researchers, using modelling techniques, and has been the subject of numerous papers (Eden et al., 2009; Kim \& Andersen, 2012; Luna-Reyes \& Andersen, 2003), as the choices made can have a significant impact upon the scope, structure and behaviour of the model. This research uses cognitive mapping as the primary organising mechanism for the qualitative data. Future research may explore how different coding techniques (Kim \& Andersen, 2012; Sastry, 1997) could help improve the process for translating the rich descriptions people provide into the more formal structures of CLDs and Stock-Flow models. While this research has endeavoured to describe such a process it is clear that much has still to be learnt about how to minimise the distortion that will inevitably occur in any translation process.

Conducting research that tries to capture the complex realities of implementing new health innovations to tackle the growing burden of chronic disease is fraught with challenges. It is hoped that the research described in this thesis provides some useful and informative insights into that process. Furthermore, it is hoped that the conclusions it has reached helps to inform clinicians, managers and policy makers who are trying to 
improve health services for the growing number of people who are having to cope, on a daily basis, with multiple and complex chronic conditions. 


\section{References}

Abdel-Hamid, T. K. (2002). Modeling the dynamics of human energy regulation and its implications for obesity treatment. System Dynamics Review, 18(4), 431-471. doi: $10.1002 /$ sdr. 240

Achterberg, T. v., Schoonhoven, L., \& Grol, R. (2008). Nursing implementation science: How evidence-based nursing requires evidence-based implementation. Journal of Nursing Scholarship, 40(4), 9.

Ackoff, R., L. (1981). Creating the corporate future: Plan or be planned for. New York: John Wiley \& Sons.

Aharoni, Y., Tihanyi, L., \& Connelly, B. L. (2011). Managerial decision-making in international business: A forty-five-year retrospective. Journal of World Business, 46(2), 135-142. doi: 10.1016/j.jwb.2010.05.001

Akkermans, H., \& Romme, G. (2003). System dynamics at the design-science interface: Past, present and future. Paper presented at the International System Dynamis Conference.

Alexander, C. (1964). Notes on the synthesis of form. London: Harvard University Press.

Anantatmula, V., \& Kanungo, S. (2006). Structuring the underlying relations among the knowledge management outcomes. Journal of Knowledge Management, 10(4), 25-42. doi: $10.1108 / 13673270610679345$

Anderson, G. F. (2003). Physician, public, and policymaker perspectives on chronic conditions. Archives of Internal Medicine, 163, 6.

Argyris, C. (1993). Knowledge for action: A guide to overcoming barriers to organizational change. San Francisco: Jossey-Bass.

Argyris, C. (1996). Actionable knowledge: Design causality in the service of consequential theory. The Journal of Applied Behavioural Science, 32(4), 390-406.

Argyris, C. (2004). Reasons and rationalizations: The limits to organizational knowledge. Oxford: Oxford University Press.

Argyris, C., Putnam, R., \& McLain Smith, D. (1985). Action science. London: Jossey-Bass Publishers.

Argyris, C., \& Schon, D. A. (1974). Theory in practice: Increasing professional effectiveness. San Francisco: Jossey-Bass Publishers.

Axelrod, R. (2003). Advancing the art of simulation in the social sciences. Japanese Journal for Management Information System, 12(3), 1-2.

Bakken, B. E. (2008). On improving dynamic decision-making: Implications from multiple-process cognitive theory. Systems Research and Behavioural Science, 25, 493501.

Bandura, A., \& Cervone, D. (1983). Self-evaluative and self-efficacy mechanisms governing the motivational Eeffects of goal systems. Journal of Personality and Social Psychology, 45(5), 12. 
Barlas, Y., \& Yasarcan, H. (2008). A comprehensive model of goal dynamics in organizations: Setting, evaluation and revision. In H. Qudrat-Ullah, J. M. Spector \& P. I. Davidsen (Eds.), Complex Decision Making: Theory and Practice. NECSI Cambridge/Massachusetts: Springer.

Barley, S. R., \& Kunda, G. (1992). Design and devotion: Surges of rational and normative ideologies of contol in managerial discourse. Administrative Science Quarterly, 37, 37.

Barnett, P., \& Barnett, R. (2005). Reform and change in health service provision. In K. Dew \& P. Davis (Eds.), HYealth and Society in Aotearoa New Zealand. Auckland: Oxford University Press.

Bayer, S., Barlow, J., \& Curry, R. (2007). Assessing the impact of a care innovation: telecare. System Dynamics Review, 23(Spring), 61-80.

Beasley, C. (2006). Reflections. Nursing Standard, 20(50), 1.

Beer, S. (1994). Brain of the Firm, 2nd Edition. Chichester: John Wiley and Sons.

Bertalanffy, L. v. (1969). General systems theory: Foundations, development, applications. New York: George Braziller.

Bindman, A. B., Forrest, C. B., Britt, H., Crampton, P., \& Majeed, A. (2007). Diagnostic scope of and exposure to primary care physicians in Australia, New Zealand and The United States: Cross sectional analysis of results from three national surveys. [on-line]. British Medical Journal, BMJ, doi:10.1136/ bmj.39203.658970.55 (published 15 May 2007).

Bodenheimer, T., Lorig, K., Holman, H., \& Grumbach, K. (2002). Patient self management of chronic disease in primary care. Journal of the American Medical Association, 288(19), 2469-2475.

Bodenheimer, T., Wagner, E. H., \& Grumbach, K. (2002a). Improving primary care for patients with chronic illness. Journal of the American Medical Association, 288(14), 1775-1779.

Bodenheimer, T., Wagner, E. H., \& Grumbach, K. (2002b). Improving primary care for patients with chronic illness - part 2. Journal of the American Medical Association, 288(15), 1909 - 1914.

Bossel, H. (2007). Systems and models: Complexity, dynamics, evolution, sustainability. Norderstedt, Germany: Books on Demand GmbH.

Bower, P., Kennedy, A., Reeves, D., Rogers, A., Blakeman, T., Chew-Graham, C., . . . Thompson, D. (2012). A cluster randomised controlled trial of the clinical and cost-effectiveness of a 'whole systems' model of self-management support for the management of long- term conditions in primary care: trial protocol. [Randomized Controlled Trial

Research Support, Non-U.S. Gov't]. Implement Sci, 7, 7. doi: 10.1186/1748-5908-7-7

Bowns, I. R., \& McNulty, T. (1999). Re-engineering Leicester Royal Infirmary: School of Health and Related Research, University of Sheffield.

Bradley, E. H., Tashonna, W. R., Baker, D., Schlesinger, M., Inouye, S., K, Barth, M. C., ... Koren, M. J. (2004). Translating research into practice: Speeding the adoption of innovative care programs. 
Cavana, R. Y., \& Clifford, L. V. (2006). Demonstrating the utility of system dynamics for public policy analysis in New Zealand: The case of excise tax policy on tobacco. System Dynamics Review, 22(4), 321-348.

Cavana, R. Y., Davies, P. K., Robson, R. M., \& Wilson, K. J. (1999). Drivers of quality in health services: Different worldviews of clinicians and policy managers revealed. System Dynamics Review, 15(3), 10.

Cavana, R. Y., Delahaye, B. L., \& Sekaran, U. (2001). Applied business research: Qualitative and quantitative methods. Milton, Queensland 4064: John Wiley \& Sons Ltd.

Cavana, R. Y., \& Mares, E. D. (2004). Integrating critical thinking and systems thinking: from premises to causal loops. System Dynamics Review, 20(3), 13.

Cavana, R. Y., \& Tobias, M. (2009). Integrative system dynamics: Analysis of policy options for tobacco control in New Zealand. Systems Research and Behavioral Science, 25(5), 675-694. doi: 10.1002/sres.934

Checkland, P. (1993). Systems Thinking, Systems Practice. Chichester: John Wiley and Sons.

Checkland, P. (2000). Soft systems methodology: A thirty year retrospective. Systems Research and Behavioural Science, 17, S11-S58.

Checkland, P., \& Holwell, S. (1998). Information, systems and information systems: Making sense of the field. Chichester: John Wiley and Sons.

Coleman, A., Checkland, K., Harrison, S., \& Hiroeh, U. (2010). Local histories and local sensemaking: A case of policy implementation in the English National Health Service. Policy and Politics, 38(2), 19.

Coleman, K., Austin, B. T., Brach, C., \& Wagner, E. H. (2009). Evidence On The Chronic Care Model In The NewMillennium. Health Affairs, 28(1), 11.

Coleman, M. T., \& Newton, K. S. (2005). Supporting self-management in patients with chronic illness. American Family Physician, 72(8), 1503-1510.

Connolly, M., Boyd, M.-A., Kenealy, T., Moffitt, A., Sheridan, N., \& Kolbe, J. (2010). Alleviating the burden of chronic conditions in New Zealand: the ABCC NZ study workbook 2010 (pp. 50): University of Auckland.

Connolly, M., Keneally, T., Barber, A. P., Carswel, P., Clinton, J., Dyall, L., . . Sheridan, N. (2011). National variability in provision of health services for major longterm conditions in New Zealand (a report from the ABCC NZ study). New Zealand Medical Journal, 124(1344), 20.

Counties Manukau District Health Board. (2006). District strategic plan 2006-2011: Counties Manukau District Health Board.

Coyle, G. R., \& Alexander, M. D. W. (1997). Two approaches to qualitative modellling of a nation's drugs trade. System Dynamics Review, 13(3), 18.

Creswell, J. W. (2009). Research design: Qualitative, quanititative and mixed methods approaches (3 ed.). Thousand Oaks, California: Sage Publications Inc.

Cumming, J., Stillman, S., Liang, Y., Poland, M., \& Hannis, G. (2010). The determinants of GP visits in New Zealand. [Research Support, Non-U.S. Gov't]. Aust N Z J Public Health, 34(5), 451-457. doi: 10.1111/j.1753-6405.2010.00589.x

Cyert, R. M., \& March, J. G. (1963). A behavioral theory of the firm. Englewood Cliffs, NJ: Prentice Hall. 
Damschroder, L. J., Aron, D. C., Keith, R. E., Kirsh, S. R., Alexander, J. A., \& Lowery, J. C. (2009). Fostering implementation of health services research findings into practice: a consolidated framework for advancing implementation science. Implementation Science, 4(50), 15. doi: 10.1186/1748-5908-4-50

Dangerfield, B. C., Fang, Y., \& Roberts, C. A. (2001). Model-based scenarios for the epidemiology of HIV/AIDS: The consequences of highly active antiretroviral therapy. System Dynamics Review, 17(2), 119-150.

Dangerfield, B. C., \& Roberts, C. (1999). Optimisation as a statistical estimation tool: An example in estimating the AIDS treatment-free incubation period distribution. System Dynamics Review, 15(3), 19.

Dennis, S. M., Zwar, N., Griffiths, R., Roland, M., Hasan, I., Powell Davies, G., \& Haris, M. (2008). Chronic disease management in primary care: from evidence to policy. Medical Journal of Australia, 188(8), 4.

Dent, E. B. (2003). The interactional model: An alternative to the direct cause and effect construct for mutually causal organizational phenomena. Foundations of Science, 8, 21.

Dewett, T., Whittier, N. C., \& Williams, D. S. (2007). Internal diffusion: The conceptualizing innovation implementation. Competitive Review: An International Business Journal, 17(1/2), 18.

Downs, G. W., \& Mohr, L. B. (1976). Conceptual issues in the study of innovation. Administrative Science Quarterly, 21, 16.

Durlak, J. A., \& DuPre, E. P. (2008). Implementation matters: A review of research on the influence of implementation on program outcomes and the factors affecting implementation. American Journal of Community Psychology, 41, 24.

Eden, C. (1988). Cognitive mapping. European Journal of Operational Research, 36, 1-13.

Eden, C. (1992). On the nature of cognitive maps. Journal of Management Studies, 29(3), 261-265.

Eden, C. (1994). Cognitive mapping and problem structuring for system dynamics model building. System Dynamics Review, 10(2-3), 257-276.

Eden, C., Ackermann, F., Bryson, J. M., Richardson, G. P., Andersen, D. F., \& Finn, C. B. (2009). Integrating modes of policy analysis and strategic management practice: Requisite elements and dilemmas. Journal of the Operational Research Society, $60,12$.

Eden, C., Ackermann, F., \& Cropper, S. (1992). The analysis of cause maps. Journal of Management Studies, 29(3 May).

Ellison-Loschmann, King, R., \& Pearce, N. (2004). Regional variations in asthma hospitalisations among Maori and non-Maori. The New Zealand Journal, 117(1188), 11.

Elwyn, G., Laitner, S., Coulter, A., Walker, E., Watson, P., \& Thomson, R. (2010). Implementing shared decision making in the NHS. British Medical Journal, 341(10).

Emery, F. E., \& Trist, E. L. (1972). Towards a social ecology. Harmondsworth, U.K.: Penguin.

Epping-Jordan, J. E., Pruitt, S. D., Bengoa, R., \& Wagner, E. H. (2004). Improving the quality of health care for chronic conditions. Quality Safety Health Care, 13, 299 305. 
Erez, M., \& Zidon, I. (1984). Effect of goal acceptance on the relationship of goal difficulty to performance. Journal of Applied Psychology, 69(1), 10.

Feachem, R. G. A., Neelam, S., K, \& White, K., L. (2002). Getting more for their dollar: A comparison of the NHS with California's Kaiser Permanente. British Medical Journal, 324(7330).

Forrester, J. W. (1961). Industrial dynamics. Oregon: Productivity Press.

Forrester, J. W. (1968). Industrial dynamics - A response to Ansoff and Slevin. Management Science, 14(9), 601-618.

Forrester, J. W. (1975a). Collected papers of Jay W. Forrester. Massachusetts: Wright-Allen Press.

Forrester, J. W. (1975b). The counterintuitive behavior of social systems Collected Papers of Jay W. Forrester. Cambridge, Massachusetts: Wright-Allen Press Inc.

Forrester, J. W. (1992). Policies, decisions and information sources for modeling. European Journal of Operational Research, 59, 42-63.

Forrester, J. W. (1994). Policies, Decisions, and Information Sources for Modeling Modeling for Learning Organisations (pp. 51-84). Portlans OR: Productivity Press.

Forrester, J. W., \& Senge, P. M. (1980). Tests for building confidence in system dynamics models TIMS Studies in Management Sciences (Vol. 14, pp. 21): New York: North Holland.

Fretter, J., \& Pande, M. (2006). Forecasting GP workforce capacity: Towards an understanding of GP workforce capacity, long-term forecasting and benchmarking tools. New Zealand.

Garg, A. X., Adhikari, N. K. J., McDonald, H., Rosas-Arellano, P. M., Devereaux, P. J., Beyene, J., ... Haynes, B. R. (2005). Effects of computerized clinical decision support systems on practitioner performance and patient outcomes: A systematic review. Journal of the American Medical Association, 293(10), 16.

Garrett, N., \& Martini, M. E. (2007). The boomers are coming: A total cost of care model of the impact of population ageing on the cost of chronic conditions in the United States. Disease Management, 10(2), 51-60.

Gentles, D., Metcalf, P., Dyall, L., Scragg, R., Black, P., Schaaf, D., . . Jackson, R. (2006). Blood pressure prevalences and levels for a multicultural population in Auckland, New Zealand: Results from the

Diabetes, Heart and Health Survey 2002/2003. New Zealand Medical Journal, 119(1245), 10.

Godfrey, P. C., \& Hill, C. W. L. (1995). The problem of unobservables in strategic management esearch. Strategic Management Journal, 16, 15.

Gonzalez-Busto, B., \& Garcia, R. (1999). Waiting lists in Spanish public hospitals: A system dynamics approach. System Dynamics Review, 15(3), 24.

Gorman, D. (2010). The utility of idealised patient journeys as a centre-piece of health workforce planning. Asia Pacific Journal of Health Management, 5(2), 6.

Graham, A. K. (1980). Parameter estimation in system dynamics modeling. In J. Randers (Ed.), Elements of the System Dynamics Method. Cambride, Massachusetts: Productivity Press. 
Greenhalgh, T., Robert, G., Bate, P., MacFarlane, F., \& Kyriakidou, O. (2005). Diffusion of innovations in health service organisation: A systematic review of the literature. Massachusetts: Blackwell Publishing Ltd.

Greenhalgh, T., Robert, G., MacFarlane, F., Bate, P., \& Kyriakidou, O. (2004). Diffusion of innovations in service organisations: Systematic review and recommendations. Millbank Quarterly, 82(4), 581-629.

Groesser, S. N., \& Schwaninger, M. (2012). Contributions to model validation: Hierarchy, process, and cessation. System Dynamics Review, 28(2), 157-181. doi: 10.1002/sdr.1466

Grol, R., \& Grimshaw, J. M. (2003). From best evidence to best practice: Effective implementation of change in patients' care. The Lancet, 362(October 11), 7.

Groves, T., \& Wagner, E., H. (2005). High quality care for people with chronic diseases: What patients with chronic conditions really need. [Editorial]. British Medical Journal, 330(19 March), 609-610.

Gustafson, D., H, Sainfort, F., Eichler, M., Adams, L., Bisognano, M., \& Steudel, H. (2003). Developing and testing a model to predict outcomes of organisational change. Health Services Research.

Ham, C. (2003). Improving the performance of health services: The role of clinical leadership. Lancet, 361, 3.

Harrison, R. J., \& Carroll, G. R. (2006). Culture and demography in organizations. Princeton, New Jersey: Princeton University Press.

Harrison, R. J., Carroll, G. R., \& Carley, K. M. (2007). Simulation modeling in organizational and management research. Academy of Management Review, 32(4), 17.

Health \& Disability Commissioner. (2007). Capital and Coast District Health Board; A report by the Health and Disability Commissioner (Case 05HDC11908). Health \& Disability Commisssion.

Hirsch, G. B., \& Homer, J. (2004a). Modeling the dynamics of health care services for improved chronic illness management. Paper presented at the 22nd International Conference of the System Dynamics Society Oxford, England.

Hirsch, G. B., \& Homer, J. (2004b). Modeling the Dynamics of Healthcare Services. Paper presented at the International Systems Dynamics Conference, Oxford.

Hirsch, G. B., Homer, J., McDonnell, G., \& Milstein, B. (2005). Achieving health care reform in the United States: Toward a whole-system understanding. Paper presented at the International System Dynamics Conference, New York.

Hirsch, G. B., \& Immediato, C. S. (1999). Microworlds and generic structures as resources for integrating care and improving health. System Dynamics Review, 15(3), 315-330.

Hirsch, G. B., Levine, R. L., \& Miller, R. L. (2007). Using system dynamics modeling to understand the impact of social change initiatives. American Journal of Community Psychology, 39(3-4), 239-253.

Hockenberry, M., Walden, M., \& Brown, T. (2007). Creating an evidence-based practice environment. Journal of Nursing Care Quality, 22(3), 10.

Hoddinott, P., Britten, J., \& Pill, R. (2010). Why do interventions work in some places and not others: A breastfeeding support group trial. Social Science and Medicine, 70, 9 . 
Hohmann, A. A., \& Shear, K. M. (2002). Community-based intervention research: Coping with the "noise" of real life in study design. American Journal of Psychiatry, $159,7$.

Holland, J. H. (1995). Hidden Order: How Adaption Builds Complexity. Canada: Perseus Books.

Holton, E. F., \& Lowe, J. S. (2007). Toward a general research process for using Dubin's theory building model. Human Resource Development Review, 6(3), 24.

Homer, J. (1993). A system dynamics model of national cocaine prevalence. System Dynamics Review, 9(1), 49-78.

Homer, J. (1996). Why we iterate: Scientific modelling in theory and practice. System Dynamics Review, 12(1), 1-19.

Homer, J., Hirsch, G. B., \& Milstein, B. (2007). Chronic illness in a complex health economy: The perils and promises of downstream and upstream reforms. System Dynamics Review.

Homer, J., Hirsch, G. B., Minniti, M., \& Pierson, M. (2004a). Models for collaboration: How system dynamics helped a community organize cost-effective care for chronic illness. Paper presented at the International Systems Dyamics Conference, Oxford.

Homer, J., Hirsch, G. B., Minniti, M., \& Pierson, M. (2004b). Models for collaboration: How system dynamics helped a community organize cost-effective care for chronic illness. System Dynamics Review, 20(3), 199-222. doi: 10.1002/sdr.295

Homer, J., Jones, A., Seville, D., Essien, J., Milstein, B., \& Murphy, D. (2005). The $C D C^{\prime}$ 's diabetes systems modeling project: Developing a new tool for chronic disease prevention and control. Paper presented at the International Systems Dynamics Conference, New York.

Homer, J., \& Milstein, B. (2004). Optimal decision making in a dynamic model of community bealth. Paper presented at the Hawaii International Conference on System Science, Big Island, Hawaii.

Homer, J., Milstein, B., Dietz, W., Buchner, D., \& Majestic, E. (2006). Obesity population dynamics: Exploring historical growth and plausible futures in the U.S. Paper presented at the 24th International System Dynamics Conference, Nijmegan.

Homer, J., Milstein, B., Wile, K., Pratibhu, P., Farris, R., \& Orenstein, D. (2008). Modeling the local dynamics of cardiovascular health: Risk factors, context and capacity. Preventing Chronic Disease, 5(2), 1-6.

Homer, J., Ritchie-Dunham, J. L., Rabbino, H., Puente, L. M., Jorgensen, J., \& Hendricks, K. (2000). Toward a dynamic theory of antibiotic resistance. System Dynamics Review, 16(4), 33.

Hovmand, P. S., Ford, D. N., Flom, I., \& Kyriakakis, S. (2009). Victims arrested for domestic violence: Unintended consequences of arrest policies. System Dynamics Review, 25, 21.

Hovmand, P. S., \& Gillespie, D. F. (2006). Dynamics of innovation implementation in social service organizations. Paper presented at the International System Dynamics Conference, Nijmegen.

Hovmand, P. S., \& Gillespie, D. F. (2008). Implementation of evidence-based practice and organizational performance. Journal of Behavioral Health Services and Research, 37(1), 17. 
Hroscikoski, M. C., Solberg, L. I., M, S.-H. J., Harper, P. G., McGrail, M. P., \& Crabtree, B. F. (2006). Challenges of change: A qualitative study of chronic care model implementation. Annals of Family Medicine, 4(4).

Hunt, K., Townsend, A., \& Wyke, S. (2003). Managing multiple morbidity in mid-life: A qualitative stidy if attitudes to drug use. British Medical Journal, 327(7419).

Huz, S., Anderson, D., F, Richardson, G., P, \& Boothroyd, R. (1997). A framework for evaluating systems thinking interventions: An experimental approach to mental health system change. System Dynamics Review, 13(2), 149-169.

Ilies, R., \& Judge, T. A. (2005). Goal regulation across time: The effects of feedback and affect. Journal of Applied Psychology, 90(3).

Institute of Medicine. (2001). Crossing the quality chasm: A new health system for the 21 st Century. Washinton, D.C.: National Academy Press.

Jaccard, J., \& Jacoby, J. (2010). Theory construction and model-building skills: A practical guide for social scientists. New York, London: The Guilford Press.

Jaine, D. R. (2009). Report on New Zealand Cost-of-Illness Studies. Wellington.

Jaques, E. (1962). Measurement of responsibility (Jaques, E. 1962. Measurement of Responsibility. Tavistock Publica- tions, London, U.K. ed.). London, U.K.: Tavistock Publications.

Jolly, K., Bradley, F., Sharp, S., Smith, H., Thompson, S., Kinmonth, A.-L., \& Mant, D. (1999). Randomised controlled trial of follow up care in general practice of patients with myocardial infarction and angina: Final results of the Southampton heart integrated care project (SHIP). bmj, 318, 706-711.

Joosten, E. A., DeFuentes-Merillas, L., de Weert, G. H., Sensky, T., van der Staak, C. P., \& de Jong, C. A. (2008). Systematic review of the effects of shared decisionmaking on patient satisfaction, treatment adherence and health status. [Research Support, Non-U.S. Gov't

Review]. Psychother Psychosom, 77(4), 219-226. doi: 10.1159/000126073

Jordan, J. E., Briggs, A., M, Brand, C., A, \& Osborne, R., H. (2008). Enhancing patient engagement in chronic disease self-management support initiatives in Australia: The need for an integrated approach. Medical Journal of Australia, 189(10), 5.

Jordan, J. E., \& Osborne, R., H. (2007). Chronic disease self management education programmes: Challenges ahead. Medical Journal of Australia, 186(2), 4.

Joseph, J. (2004). The practice of design-based research: uncovering the interplay between design, research, and the real-world context. Educational Psychologist, 39(4), 9.

Kane, R. L., Priester, R., \& Totten, A. M. (2005). Meeting the challenge of chronic illness. Maryland: The Johns Hopkins University press.

Kanter, R. M. (1984). The change masters: Corporate entrepeneurs at work. London: Unwin.

Kenealy, T., Rees, D., Sheridan, N., Moffitt, A., Tibby, S., \& Homer, J. (2012). Whole of system approach to the design and funding of CVD interventions in Counties Manukau. Australian \& New Zealand Journal of Public Health, 36(2).

Kernick, D. (2002). The demise of linearity in managing health services: A call for post normal health care. Journal of Health Services Research, 7(2), 121-124. 
Kim, H., \& Andersen, D. F. (2012). Building confidence in causal maps generated from purposive text data: Mapping transcripts of the Federal Reserve. System Dynamics Review, 28(4), 311-328. doi: 10.1002/sdr.1480

Klein, K. J., \& Knight, A. P. (2005). Innovation implementation: Overcoming the challenge. Current Directions in Psychological Science, 14, 4.

Klein, K. J., \& Sorra, J. S. (1996). The challenge of innovation implementation. Academy of Management Review, 21(4), 27.

Lane, D. C., Monefeldt, C., \& Rosenhead, J. (2000). Looking in the wrong place for healthcare improvements: A system dynamics study of an accident and emergency department. Journal of the Operational Research Society, 51, 518-531.

Lee, T., Wha, \& Ko, Y. K. (2009). Effects of self-efficacy, affectivity and collective efficacy on nursing performance of hospital nurses. Journal of Advanced Nursing, 66(4), 10.

Lewin, K. (1997). Resolving social conflicts \& field theory in social science. Washington: American Psychological Association.

Locke, E. A., \& Latham, G. P. (2002). Building a practically useful theory of goal setting and task motivation. American Psychologist, 57(9), 13.

Locke, E. A., Latham, G. P., \& Erez, M. (1988). The determinants of goal commitment. Academy of Management Review.

Luna-Reyes, L., F, \& Andersen, D. L. (2003). Collecting and analyzing qualitative data for system dynamics: Methods and models. System Dynamics Review, 19(4), 26.

Maani, K. E., \& Cavana, R. Y. (2000). Systems thinking and modelling. Auckland: Pearson.

Macy, J. (1991). Mutual causality in Buddhism and General Systems Theory: The dharma of natural systems. New York: State University of New York Press.

Marmot, M., \& Wilkinson, R. D. (2006). Social determinants of health. Oxford: Oxford University Press.

Martin, C., \& Sturmberg, J. (2008). Complex adaptive chronic care. Journal of Evaluation in Clinical Practice, 15, 7.

McCubbin, M., \& Cohen, D. (1999). A systemic and value-based approach to strategic reform of the mental health system. Health Care Analysis, 7, 57-77.

McGlynn, E. A., Asch, S. M., Adams, J., \& Keesey, J. (2003). The quality of health care delivered to adults in the United States. The New England Journal of Medicine, 348(26), 14.

Meadows, D. (1980). The unavoidable a priori. In J. Randers (Ed.), Elements of the System Dynamics Method. Cambridge: Productivity Press.

Meadows, D. H., \& Robinson, J. M. (1985). The electronic oracle: Computer models and social decisions. Chichester: John Wiley and Sons.

Milstein, B. (2008). Hygeia's constellation navigating health futures in a dynamic and democratic world Centers for Disease Control

Milstein, B., Jones, A., Homer, J. B., Murphy, D., Essien, J., \& Seville, D. (2007). Charting plausible futures for diabetes prevalence in the United States: A role for system dynamics simulation modeling. Preventing Chronic Disease, 4(3), 1-8.

Ministry of Health. (2007). Statement of intent 2007-2010. Ministry of Health. 
Ministry of Health. (2011). Better, sooner, more convenient health care in the community. Wellington: Ministry of Health.

Mooy, J. M., \& Gunning-Schepers, L. J. (2001). Computer assisted health impact assessment for intersectoral health policy. Health Policy, 57, 169-177.

Morecroft, J. D. W. (2007). Strategic modelling and business dynamics: A feedback systems approach. Chichester: John Wiley and Sons.

Morgan, D. L. (2007). Paradigms lost and pragmatism regained methodological implications of combining qualitative and quantitative methods. Journal of Mixed Methods Research, 1(1), 30.

National Advisory Committe on Health and Disability. (2007). Meeting the needs of people with chronic conditions. Wellington.

National Health Committee. (2007). MEETING THE NEEDS OF PEOPLE WITH CHRONIC CONDITIONS. Wellington.

New Zealand Guidelines Group. (2011). RapidE: Chronic care a systematic review of the literature on health behaviour change in chronic care. Wellington.

Nolte, E., \& McKee, M. (Eds.). (2008). Caring for people with chronic conditions. Berkshire, England: Open University.

Nuno, R., Coleman, K., Bengoa, R., \& Sauto, R. (2012). Integrated care for chronic conditions: the contribution of the ICCC Framework. [Review]. Health Policy, 105(1), 55-64. doi: 10.1016/j.healthpol.2011.10.006

Nurse, J., \& Edmondson-Jones, P. (2007). A framework for the delivery of public health: An ecological approach. Journal of Epidemiology and Public Health, 61, 555558.

Nutting, P. A., Crabtree, B. F., Miller, W. L., Stewart, E. E., Stange, K. C., \& Jaen, C. R. (2010). Journey to the patient-centered medical home: A qualitative analysis of the experiences of practices in the national demonstration project. Annals of Family Medicine, 8(Supplement 1), 12.

Pagels, H. R. (1988). The dreams of reason: The computer and the rise of the sciences of complexity. New York: Simon \& Schuster.

Park, B.-W., \& Ahn, J.-H. (2010). Policy analysis for online game addiction problems. System Dynamics Review, 26(2), 117-138. doi: 10.1002/sdr.436

Patterson, C. H. (1986). Theories of counseling and psychotherapy (4th ed.). New york: Harper and Row.

Porter, R. (1997). The greatest benefit to mankind: A medical bistory of humanity. New York: W.W. Norton and Company.

Proctor, E. K., Landsverk, J., Aarons, G., Chambers, D., Glisson, C., \& Mittman, B. (2009). Implementation research in mental health services: An emerging science with conceptual, methodological, and training challenges. [Research Support, N.I.H., Extramural]. Adm Policy Ment Health, 36(1), 24-34. doi: 10.1007/s10488008-0197-4

Rea, H., Kenealy, T., Wellingham, J., Moffitt, A., Sinclair, G., McAuley, S., . . A Arcus, K. (2007). Chronic care management evolves towards integrated care in Counties Manukau, New Zealand. New Zealand Medical Journal, 120(1252). 
Rees, D., Naden, D. R., \& Field, D. A. (2008). Modelling the demand for renal replacement therapies: Challenging assumptions and influencing policy. Paper presented at the International System Dynamics Conference, Athens.

Rees, D., \& Orr-Walker, D. B. (2006). System dynamics modelling as a tool in healthcare planning. Paper presented at the International System Dynamics Conference, Njeimegen.

Repenning, N. P. (2002). A simulation-based approach to understanding the dynamics of innovation implementation. Organization Science, 13(2), 19.

Richardson, G. P. (1991). Feedback thought in social science and systems theory. Philadelphia: University of Pennsylvania Press.

Ritchie-Dunham, J. L., \& Galvan, J. F. M. (1999). Evaluating epidemic intervention policies with systems thinking: A case study of dengue fever in Mexico. System Dynamics Review, 15(2), 20.

Robert Wood Johnson Foundation. (2004). Chronic conditions: Making the case for ongoing care (September 2004 Update): Robert Woods Johnson Foundation.

Romme, G. (2003). Making a difference: Organization as design. Organization Science, 14(5), 16.

Rosenhead, J., \& Mingers, J. (Eds.). (2001). Rational analysis for a problematic world revisited: Problem structuring methods for complexity, uncertainty and conflict. Chichester: John Wiley and Sons.

Rosenthal, M. P., Butterfoss, F. D., Doctor, L. J., Gilmore, L. A., Krieger, J. W., Meurer, J. R., \& Vega, I. (2006). The coalition process at work: Building care coordination models to control chronic disease. Health Promotion Practice, 7(2), $117 \mathrm{~s}-126 \mathrm{~s}$.

Royston, G., Dost, A., Townshend, J., \& Turner, H. (1999). Using system dynamics to help develop and implement policies and programmes in health care in England. System Dynamics Review, 15(3), 21.

Ruston, A., \& Tavabie, A. (2010). Fostering clinical engagement and medical leadership and aligning cultural values: An evaluation of a general practice specialty trainee integrated training placement in a primary care trust. Quality in Primary Care, 18, 6.

Sarriot, E. G., Winch, P. J., Ryan, L. J., Bowie, J., Kouletio, R., Swedburg, E., . . Pacque, M. C. (2004). A methodological approach and framework for sustainability assessment in NGO-implemented primary health care programs. International Journal of Health Planning and Management, 19, 23-41.

Sarriot, E. G., Winch, P. J., Ryan, L. J., Edison, J., Bowie, J., Swedburg, E., \& Welch, R. (2004). Qualitative research to make practical sense of sustainability in primary health care projects implemented by non-governmental organisations. International Journal of Health Planning and Management, 19, 3-22.

Sastry, A. M. (1997). Problems and paradoxes in a model of punctuated organizational change. Administrative Science Quarterly, 42, 39.

Schoen, C., Osborn, R., Squires, D., Doty, M. M., Pierson, R., \& Applebaum, S. (2011). New 2011 survey of patients with complex care needs in eleven countries finds that care Is often poorly coordinated. Health Affairs. doi: 10.1377/hlthaff.2011.0923 
Schon, D. A. (1983). The reflective practitioner: How professionals think in action. USA: Basic Books.

Schwaninger, M., \& Grosser, S. (2008). System dynamics as model-based theory making. Systems Research and Behavioural Science, 25, 447-465.

Scott, I. A. (2008). Chronic disease management: A primer for physicians. [MetaAnalysis

Review]. Intern Med J, 38(6), 427-437. doi: 10.1111/j.1445-5994.2007.01524.x

Senge, P. M. (1990). The fifth discipline: The art and practice of the learning organization. New York: Doubleday Currency.

Si, D., Bailie, R., Connors, C., Dowden, M., Stewart, A., Robinson, G., . . . Weeramanthri, T. (2005). Assessing health centre systems for guiding improvement in diabetes care. BMC Health Services Research, 5(56), 1-9.

Sibbald, B., McDonald, R., \& Roland, M. (2007). Shifting care from hospitals to the community: A review of the evidence on quality and efficiency. Journal of Health Services Research and Policy, 12(2), 110-117. doi: $\mathrm{q}$

Sibthorpe, B. M., Glasgow, N. J., \& Wells, R. W. (2005). Questioning the sustainability of primary health care innovation. MJA, 183(10), S52-S53.

Simon, H. A. (1996). The sciences of the artificial (3rd ed.). Massachusetts The MIT Press.

Singh, D. (2005). Transforming chronic care: Evidence about improving care for people with long-term conditions: HSMC. Birmingham: HSMC, University of Birmingham \& NHS Institute for Innovation and Improvement.

Singh, D. (2008). How can chronic disease management programmes operate across care settings and providers? Copenhagen.

Singh, D., \& Ham, C. (2008). Improving care for people with long term conditions: A review of UK and international frameworks: University of Birmingham HSMC \& NHS Institute for Innovation and Improvement.

Smith, G., Wolstenhome, E., \& Repper, D. (2005). Initial experiences of introducing system dynamics through a mental health project in North West England. Paper presented at the International System Dynamics Conference, New York.

Stacey, D., Bennett, C. L., Barry, M. J., Col, N. F., Eden, K. B., Holmes-Rovner, M., . . Thomson, R. (2012). Decision aids for people facing health treatment or screening decisions (Review). Cochrane Library(5).

Stacey, R. (2006). Ways of thinking about public sector governance. In R. Stacey \& D. Griffin (Eds.), Complexity and the Experience of Managing Public Sector Organisations. Oxon: Routledge.

Sterman, J. D. (1989). Modeling managerial behavior: Misperceptions of feedback in a dynamic decision making experiment. Management Science, 35(3), 20.

Sterman, J. D. (1994). Learning In and about complex systems. Reflections, 1(3), 24-51.

Sterman, J. D. (2000). Business dynamics: systems thinking and modeling for a complex world. Boston: The McGraw Hill Companies.

Syme, L. S. (2004). Social determinants of health: Community as an empowered partner. Preventing Chronic Disease, 1(1), 1 - 5.

Taylor, F. W. (1911). The principles of scientific management. New York: Harper. 
Taylor, K., \& Dangerfield, B. C. (2005). Modelling the feedback effects of reconfiguring health services. Journal of the Operational Research Society, 56, 659-675.

Taylor, K., Dangerfield, B. C., \& Le Grand, J. (2005). Simulation analysis of the consequences of shifting the balance of health care: A system dynamics approach. Journal of Health Services Research and Policy, 10(4), 196-202.

Thompson, D., Edelsberg, J., Colditz, G. A., Bird, A. P., \& Oster, G. (1999). Lifetime health and economic consequences of obesity. Archives of Internal Medicine, 159(Oct 11), 2177-2183.

Thompson, K. M., \& Tebbens, R. J. D. (2008). Using system dynamics to develop policies that matter: Global management of poliomyelitis and beyond. System Dynamics Review, 24(4), 433-449. doi: 10.1002/sdr.419

Tobias, M. I., Cavana, R. Y., \& Bloomfield, A. (2010). Application of a system dynamics model to inform investment in smoking cessation services in New Zealand. Am J Public Health, 100(7), 1274-1281. doi: 10.2105/AJPH.2009.171165

Turner, K. M. T., Nicholson, J. M., \& Sanders, M. R. (2011). The role of practitioner self-efficacy, training, program and workplace factors on the implementation of an evidence-based parenting intervention in primary care. Journal of Primary Prevention, 32(2), 18.

van Ackere, A., \& Smith, P. C. (1999a). A dynamic model of National Health Service waiting lists. System Dynamics Group.

van Ackere, A., \& Smith, P. C. (1999b). Towards a macro model of National Health Service waiting lists. System Dynamics Review, 15(3), 225-252.

Van Den Belt, M. (2004). Mediated modeling: A system dynamics approach to environmental consensus building. London: Island Press.

Vennix, J. A. M. (1996). Group model building: Facilitating team learning using system dynamics. Chichester: John Wiley and Sons.

Vickery, D. M., Golaszewski, T. J., Wright, E. C., \& Kalmer, H. (1988). The effect of self-care interventions on the use of medical service within a medicare population. Medical Care, 26(6), 10.

Vogeli, C., Shields, A. E., Lee, T. A., Gibson, T. B., Marder, W. D., Weiss, K. B., \& Blumenthal, D. (2007). Multiple chronic conditions: prevalence, health consequences, and implications for quality, care management, and costs. [Review]. J Gen Intern Med, 22 Suppl 3, 391-395. doi: 10.1007/s11606-007-0322-1

Wagner, E. H., Austin, B., Davis, C., Hindmarsh, M., Schaefer, J., \& Bonomi, A. (2001). Improving chronic illness care: Translating evidence into action. Health Affairs, 20(6), 64-78.

Wagner, E. H., Austin, B. T., \& von Korff, M. (1996). Organizing care for patients with chronic illness. The Millbank Quarterly, 74(4), 34.

Wagner, E. H., Davis, C., Schaefer, J., von Korff, M., \& Austin, B. (1999). A survey of leading chronic disease management programs: Are they consistent with the literature? Managed Care Quarterly, 7(3), 56-66.

Watzlawick, P. (Ed.). (1984). The invented reality: How do we know what we believe we know (contributions to constructivism). New York: W. W. Norton \& Company. 
Wellingham, J., Tracey, J., Rea, H., \& Gribben, B. (2003). The development and implementation of the chronic care management programme in Counties Manukau. New Zealand Medical Journal, 116(1169).

Wells, S., \& Jackson, R. (2011). Unwarranted variation in healthcare organisation and practice for long-term conditions. New Zealand Medical Journal, 124(1344), 4.

Wolstenhome, E. (1990). System enquiry: A system dynamics approach. Chichester: John Wiley and Sons Ltd.

Wolstenhome, E. (1999). A patient flow perspective of U.K. Health Services: Exploring the case for new "intermediate care" initiatives. System Dynamics Review, 15(3), 19.

Wolstenhome, E., McKelvie, D., Monk, D., Todd, D., \& Brady, C., Dr. (2008). Emerging opportunities for system dynamics in UK health and social care - The market-pull for systemic thinking. Paper presented at the International Systems Dynamics Conference, Athens.

Wolstenhome, E., Monk, D., McKelvie, D., \& Arnold, S. (2007). Coping but not coping in health and social care: masking the reality of running organisations beyond safe design capacity. System Dynamics Review, 23(4), 371-389.

World Health Organisation. (2005). Preventing chronic disease: A vital investment.

Yin, R. K. (2003). Applications of case study research, second edition. New Delhi: Sage Publications.

Ziglio, E., Hagard, S., \& Griffiths, J. (2000). Health promotion development in Europe: Achievements and challenges. Health Promotion International, 15(2), 143-154.

Zwar, N., Harris, M., Griffiths, R., Roland, M., Dennis, S., Davies, G. P., \& Hasan, I. (2006). A systematic review of chronic disease management: Australian Primary Health Care Research Institute. 
Appendix 1: Cognitive Maps 
Interviewee \#1: Primary Care Clinician and Clinical Advisor Within the Ministry of Health

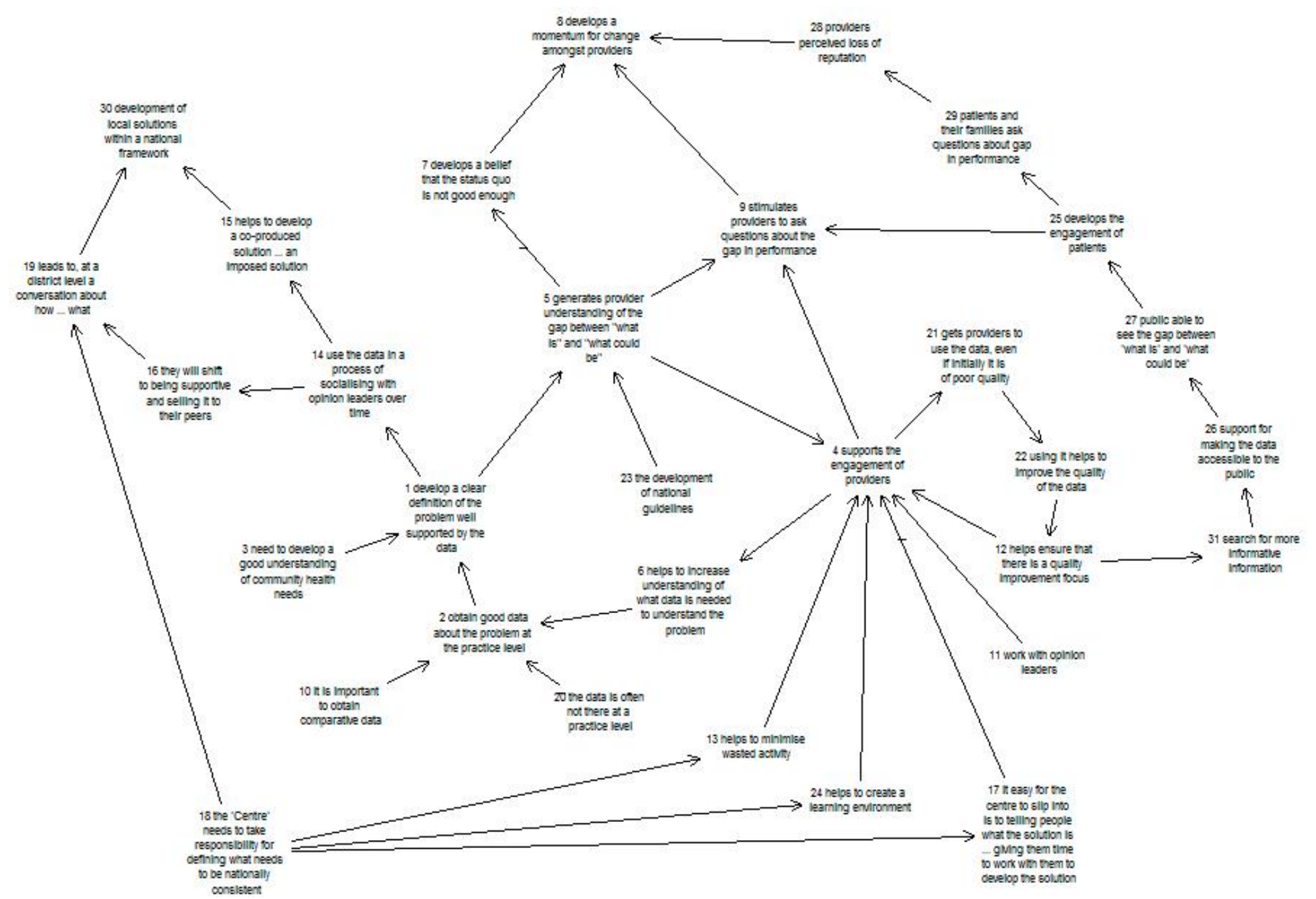

Appendix 1: Individual Cognitive Maps 
Interviewee \#2: Primary Care Clinician, Regional Planner and Manager of Primary Health Services Within a DHB

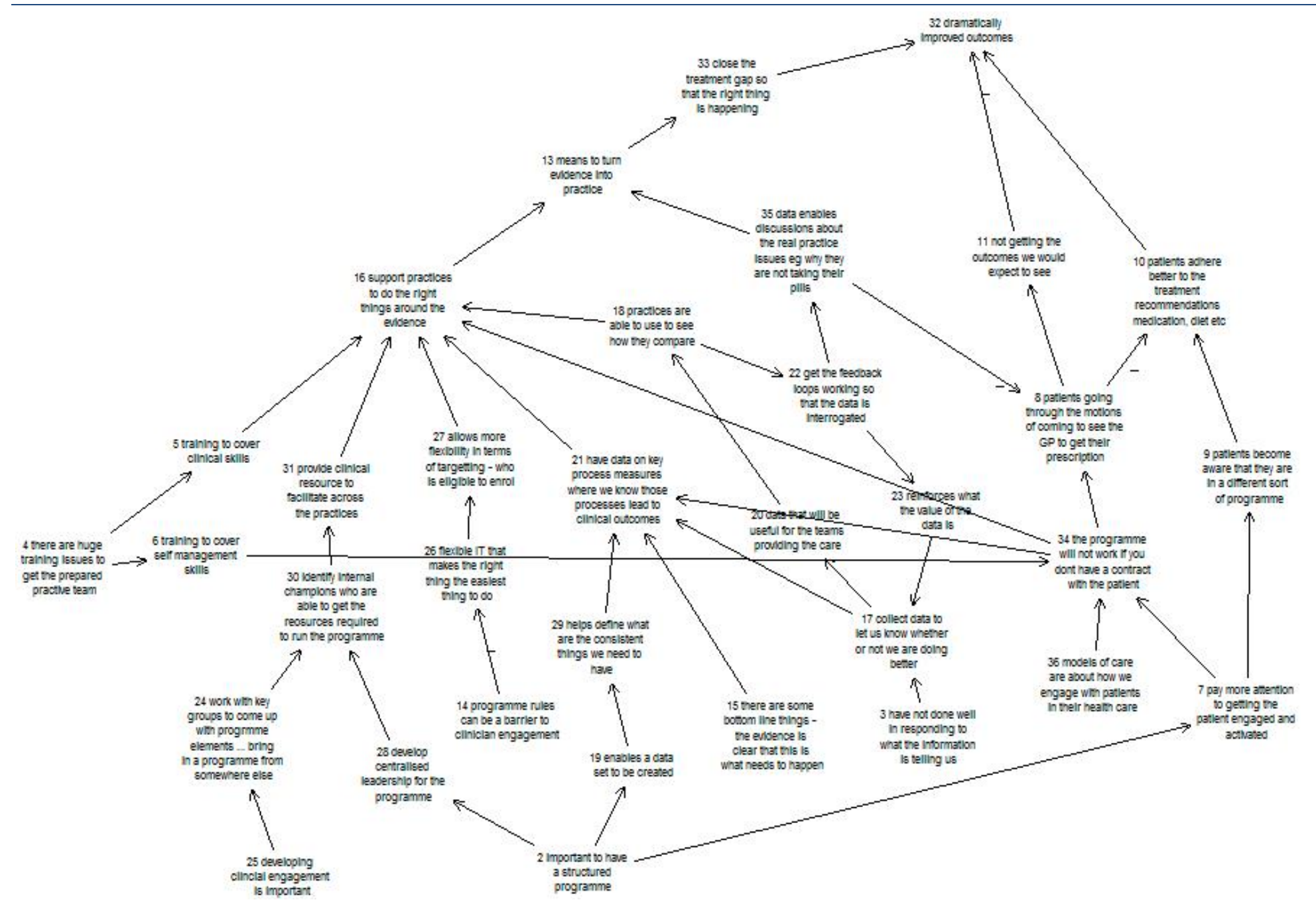

Appendix 1: Individual Cognitive Maps 
Interviewee \#3: Senior Planner Within the Ministry of Health

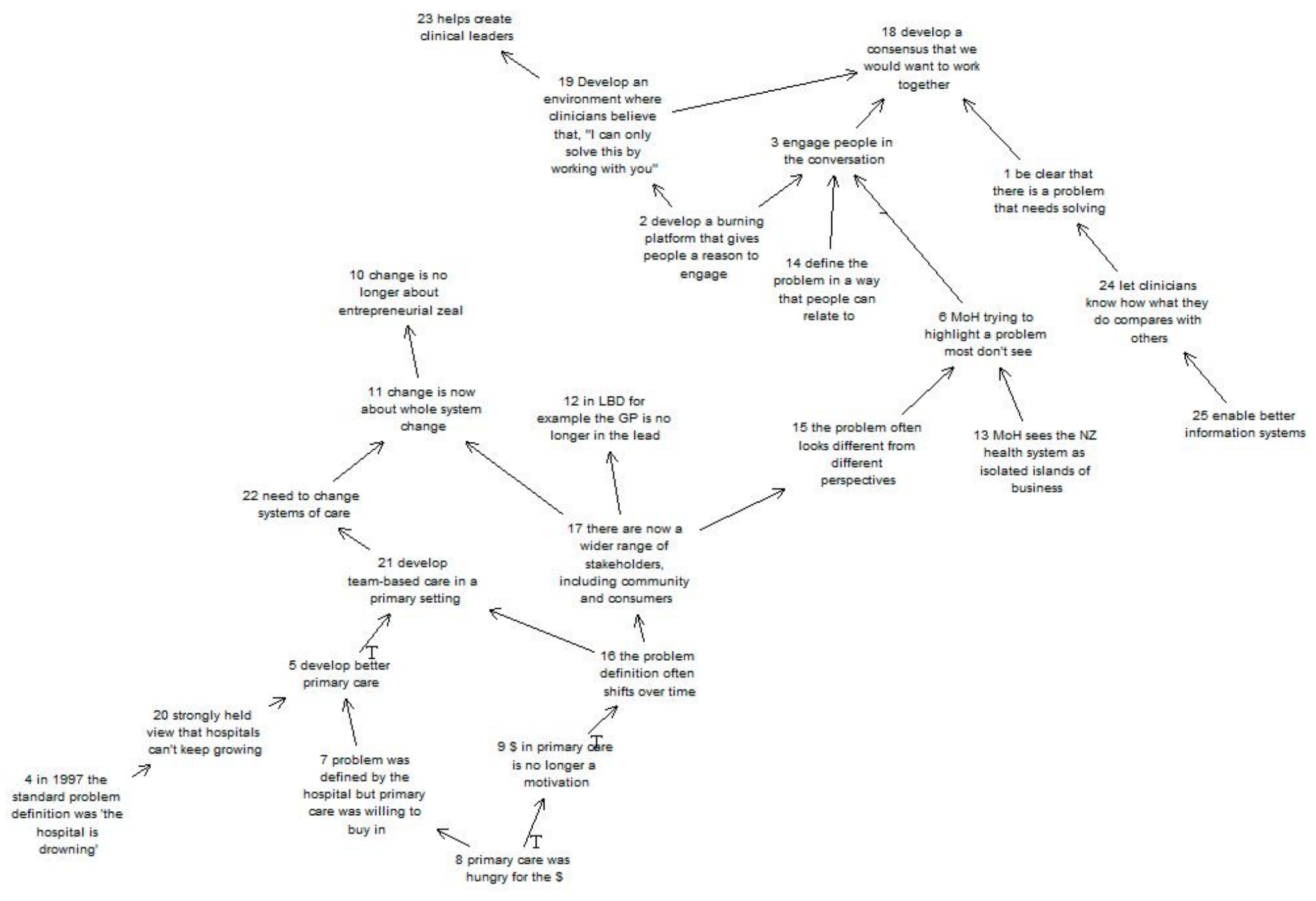

Appendix 1: Individual Cognitive Maps 


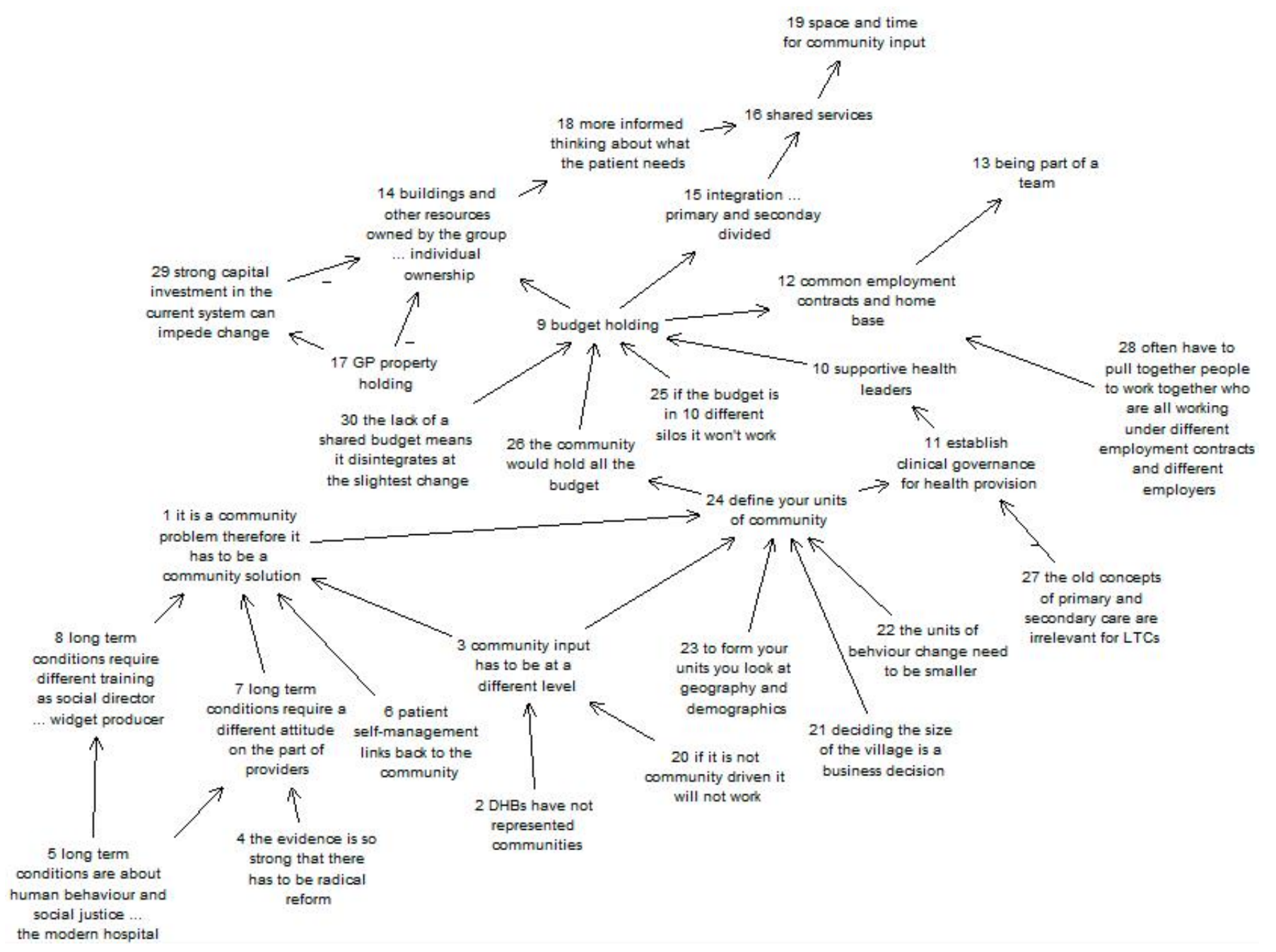


Interviewee \#5: Senior Planner Within a DHB

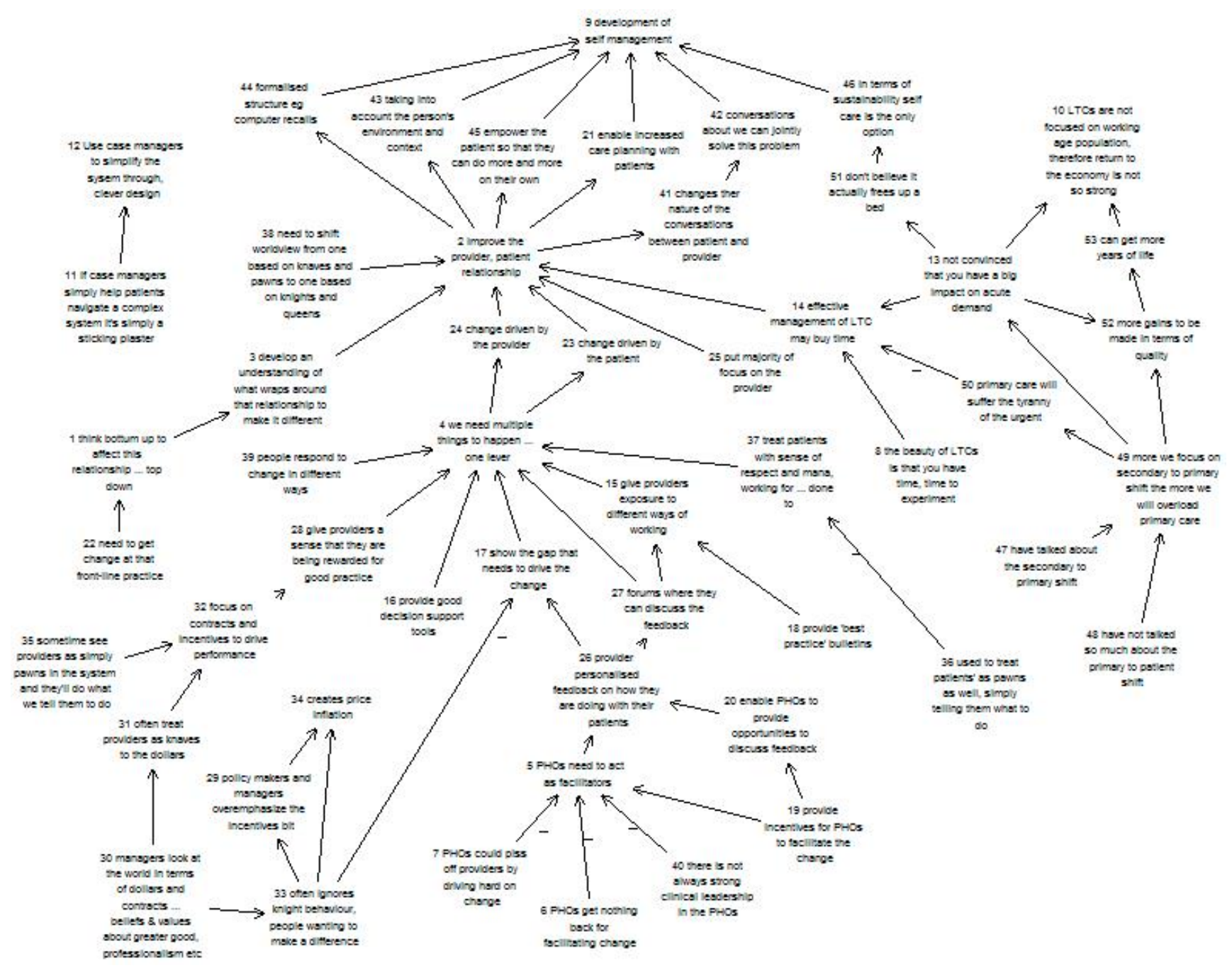


Interviewee \#6: Senior Planner Within a DHB

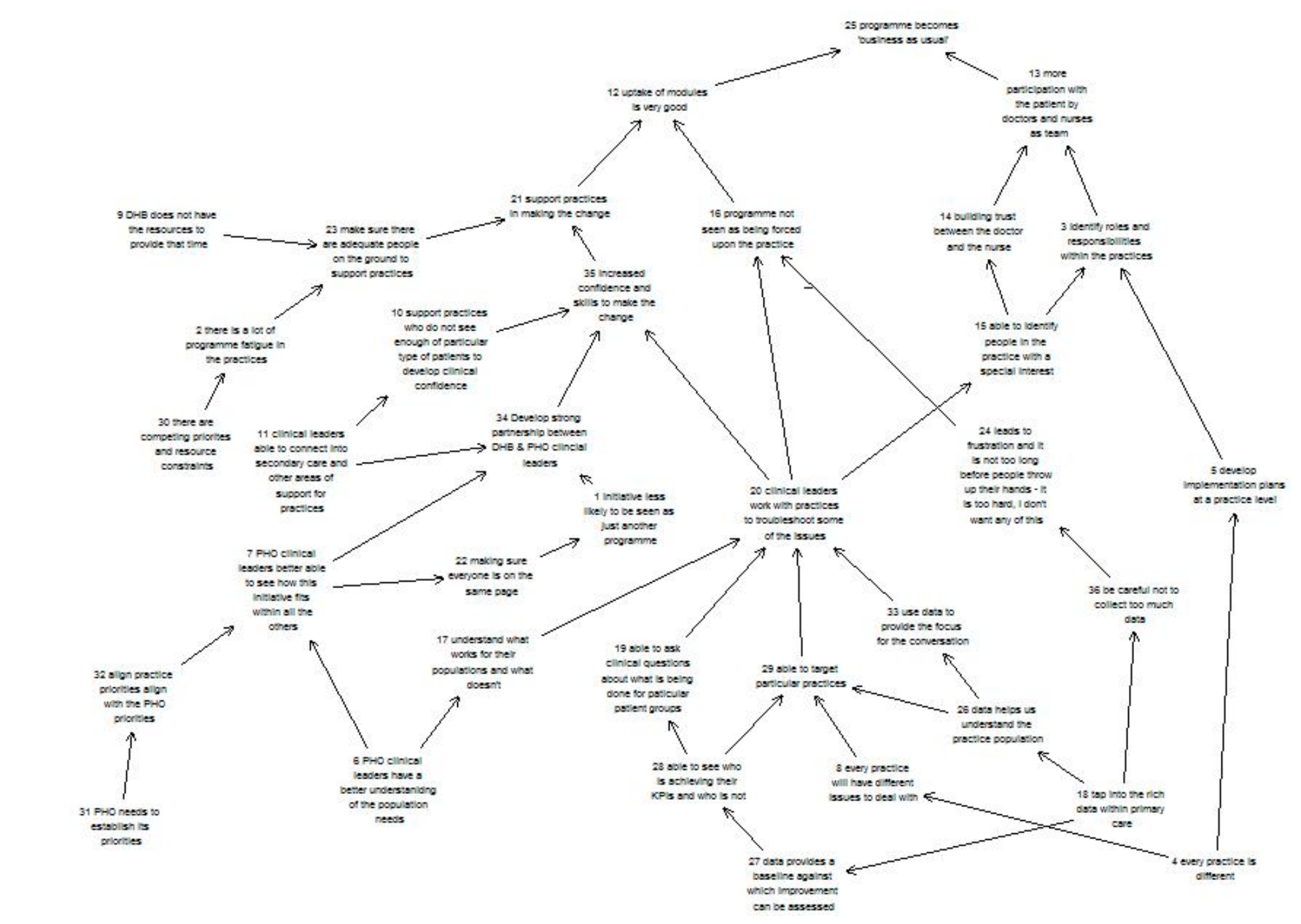

Appendix 1: Individual Cognitive Maps 
Interviewee \#7: Primary Care Clinician and Clinical Advisor Within the Ministry of Health

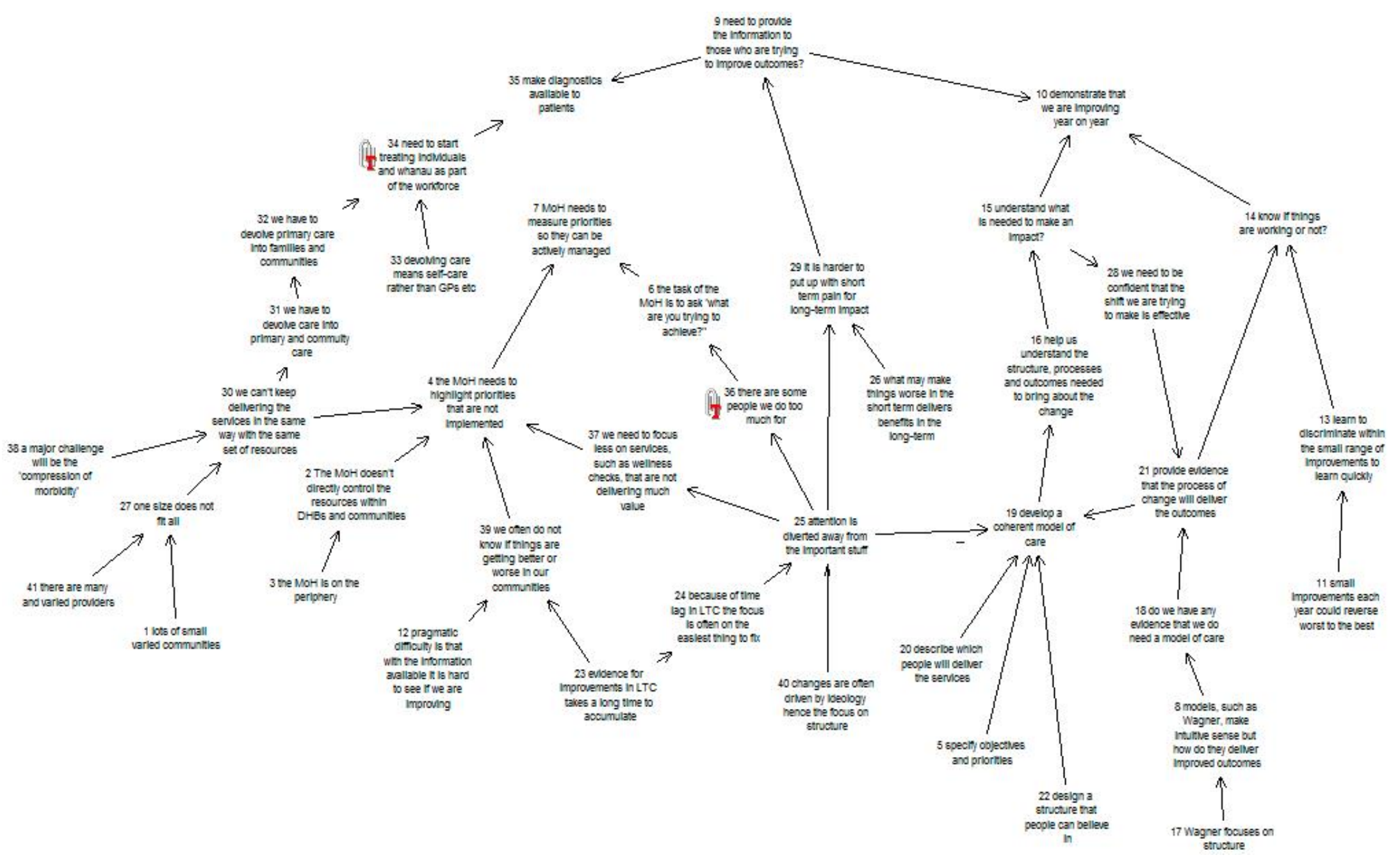


Appendix 2: Material Used to Feedback Model to Health Experts Interviewed 


\section{The Dynamics of} Implementing New Health Practices in Primary Health Care

\author{
David Rees \\ Victoria Management School
}

September 2012

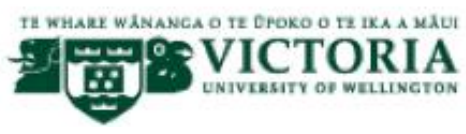

\section{A Practice Model of Implementation}

The following pages describe the initial interviews, the themes that resulted from them and depicts, in visual form, the key elements considered to be important in the successful implementation of new health practices in primary care.

The focus of the model is on the dynamics within the primary care practice, and aims to capture the key aspects of the practice that need to be

managed when trying to implement new ways of delivering care for people with long-term conditions.

The model is based on information obtained from a series of interviews with health professionals and the relevant literature.

Although many of the factors shown in the model are already well known, the difference is that it explores the relationships between them, acknowledging that success will come not from the individual factors themselves, but from finding a balance amongst a range of factors, that interact with each other over time. 


\section{Issues Involved in Implementation}

During 2009/10 I interviewed a number of health experts about the issues they considered to be important in implementing new health practices in primary care. The specific focus was on health care for people with long-term conditions.

Each person interviewed had a report produced which summarised the ideas raised during the interview. A second interview was then organised to check that the report did in fact capture the key ideas discussed.

Following the interviews an analysis was done to explore the themes that cut across all the people interviewed. The key themes that emerged from this process were:

- Performance feedback

- Engagement

- Provider Performance

- System Change

- Clinical Leadership

- Models of Care
The model focuses on the themes that relate directly to the primary care practice rather than the broader policy and structural issues within the sector. As a consequence the theme of 'System Change' was not included in the implementation model.

This is not to say that it is unimportant but reflects a desire to focus on the elements that are able to be controlled, or at least influenced, by the primary care practice charged with implementing the new care models.

In contrast the model does explore the other themes in detail, so that, for example, 'engagement' teases out the sub-themes that emerged during the interviews i.e. clinical engagement, patient engagement and community engagement.

The links between the original themes and the details within each that provided the key model variables is shown on the following page. 


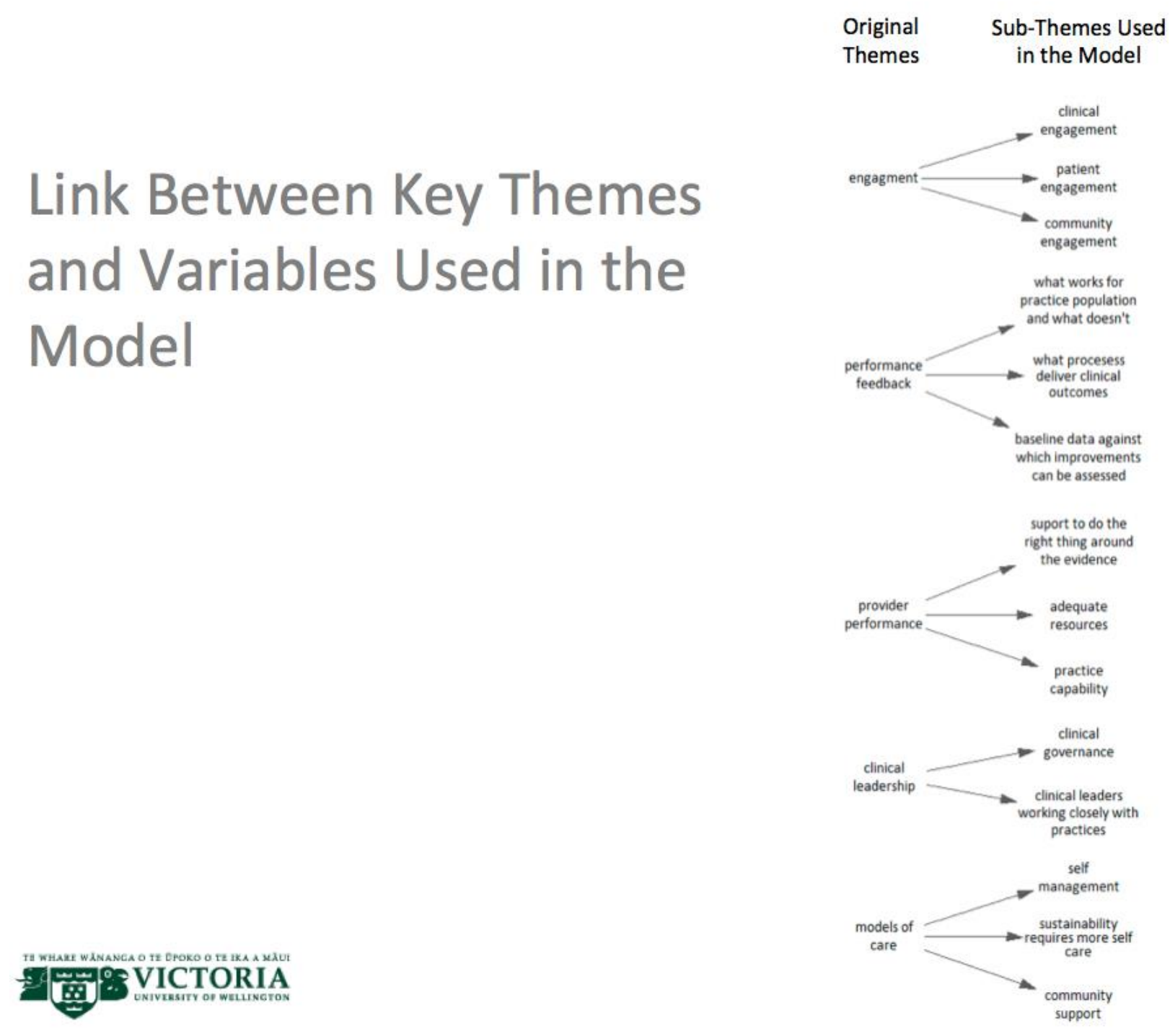




\section{Clinical Engagement}

A key element in the model is that of clinical engagement. Regardless of any other factors, unless clinicians are engaged they will not make the effort necessary to deliver more effective care.

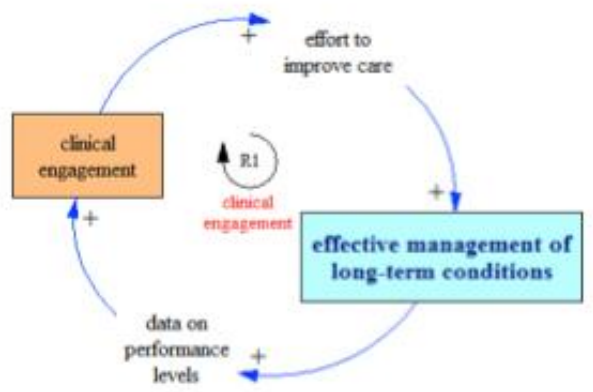

In addition, if there is not feedback to clinicians on the effect of their actions, the engagement with the proposed changes will be difficult to maintain.

The argument here is a simple one; some level of engagement must be developed if changes are to occur, and feedback has to be provided if that engagement is to be maintained.

Other aspects of the model will describe factors that can support and inhibit the development of this engagement. At this point all the model is pointing to is the central importance of engagement itself. It is a central driver of the changes needed to improve care. 


\section{Quality of Effort}

While clinical engagement is a key driver of change, a lot of the effort that results can be wasted unless the Practice has a good understanding of the needs of their enrolled population and is able to target services towards them.

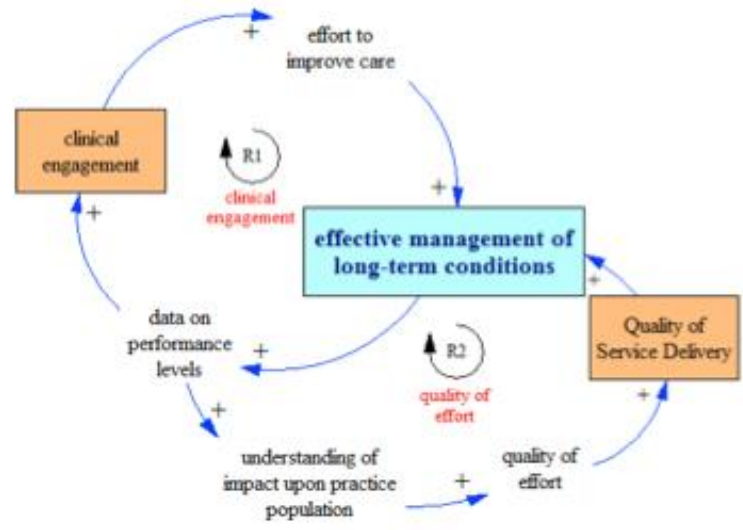

This means that the Practice has to be proactive, reaching out to those in their enrolled population who have the greatest needs. Unless this occurs their poor health will reflect in increasing usage of health services over time, making it harder and harder for the Practice to divert resources towards long-term care.

I've referred to this dynamic as 'quality of effort' to reflect that fact that it is about ensuring the effort made by the practice team is focused on areas that can deliver improvements in care. 


\section{Quality of Processes}

Ensuring that the practices of care are in fact evidence-informed and are likely to make a difference is also important in ensuring that the effort made by the practice team does in fact lead to improved clinical outcomes.

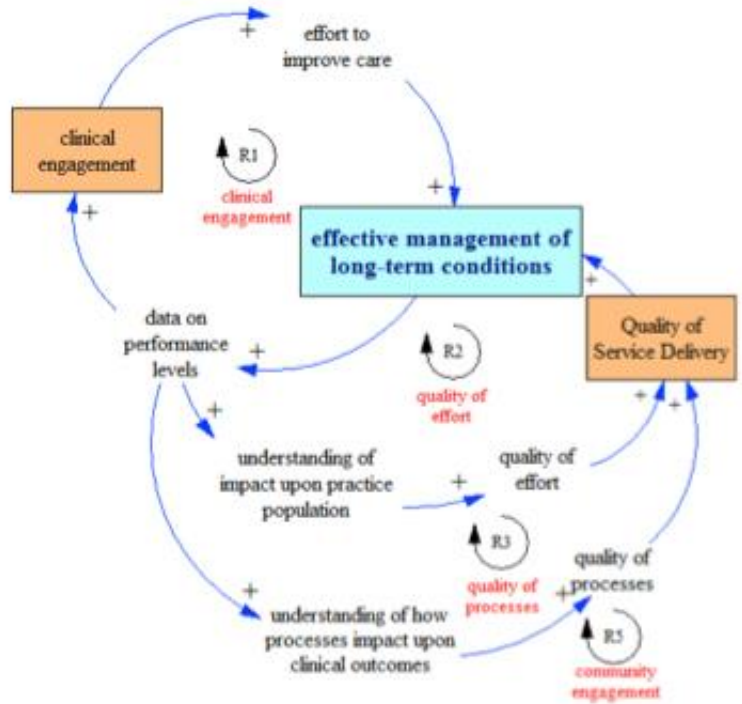

The focus here is on ensuring that the Practice is aware of the link between their own treatment process and clinical outcomes. This may, for example, refer to reducing inappropriate prescribing or 'behavioural interventions' designed to engage the patient into making lifestyle changes.

'Quality of processes' and 'quality of effort' focus on i) ensuring the Practice is targeting services

towards those most in need and ii) uses evidenceinformed treatment processes that are more

likely to deliver positive clinical outcomes.

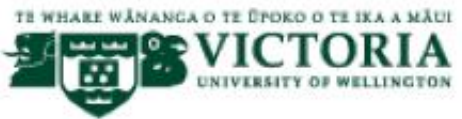




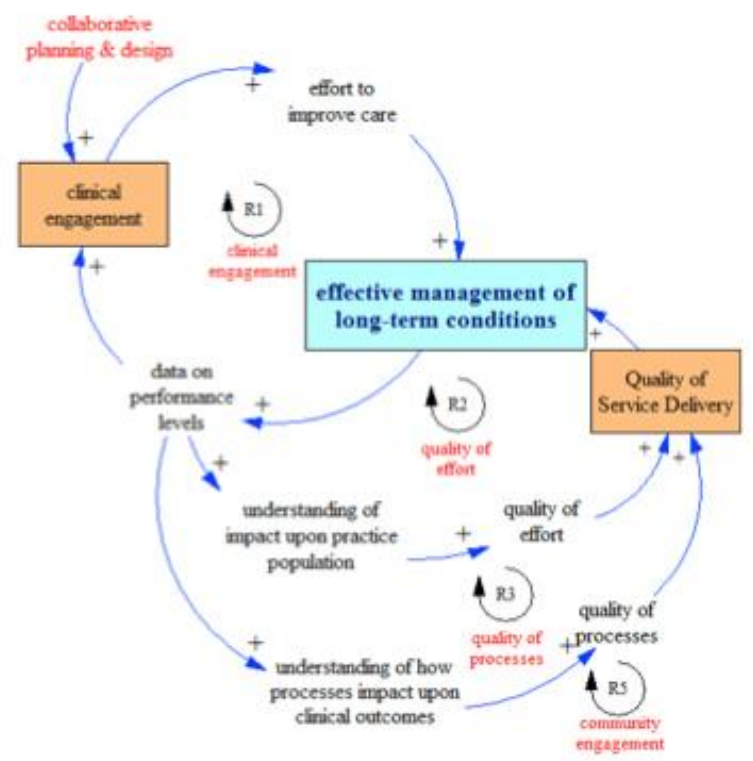

\section{Collaborative Planning \& Design}

The first dynamic highlighted the importance of clinical engagement and while it is fair to assume that the idea of improving care is shared by most clinicians, the means of implementing is often a source of great debate. As a consequence, involving the clinical team in planning and designing what specific changes will be

implemented, and how they will be implemented, is a key means of developing clinical support for the changes.

This involvement is important, not only to develop an initial level of engagement, but to maintain it over time. Unless the clinical team is involved, on an ongoing basis, their engagement and support for the changes may not be maintained.

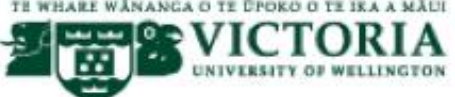




\section{Patient Engagement}

A characteristic of long-term conditions is that their treatment almost always requires active involvement of the patient themselves. Whether in terms of lifestyle changes or adherence to a treatment programme, unless the patient is engaged in the process the potential for improvement will be limited. In terms of longterm conditions, clinical interventions will always be limited by the extent to which the patient understands them and, is able and willing to adopt them.

In most cases, long-term conditions involve a treatment focused on 'care' rather than 'cure' and the outcomes evolve over months and years. This is in marked contrast to 'acute' conditions, the treatment of which generally takes place over minutes, hours and sometimes weeks. With longterm conditions the relationships with the patient is crucial and for real improvement to be made and maintained over the long term, the patient

has to be engaged as an 'equal partner' in the therapeutic relationship. 


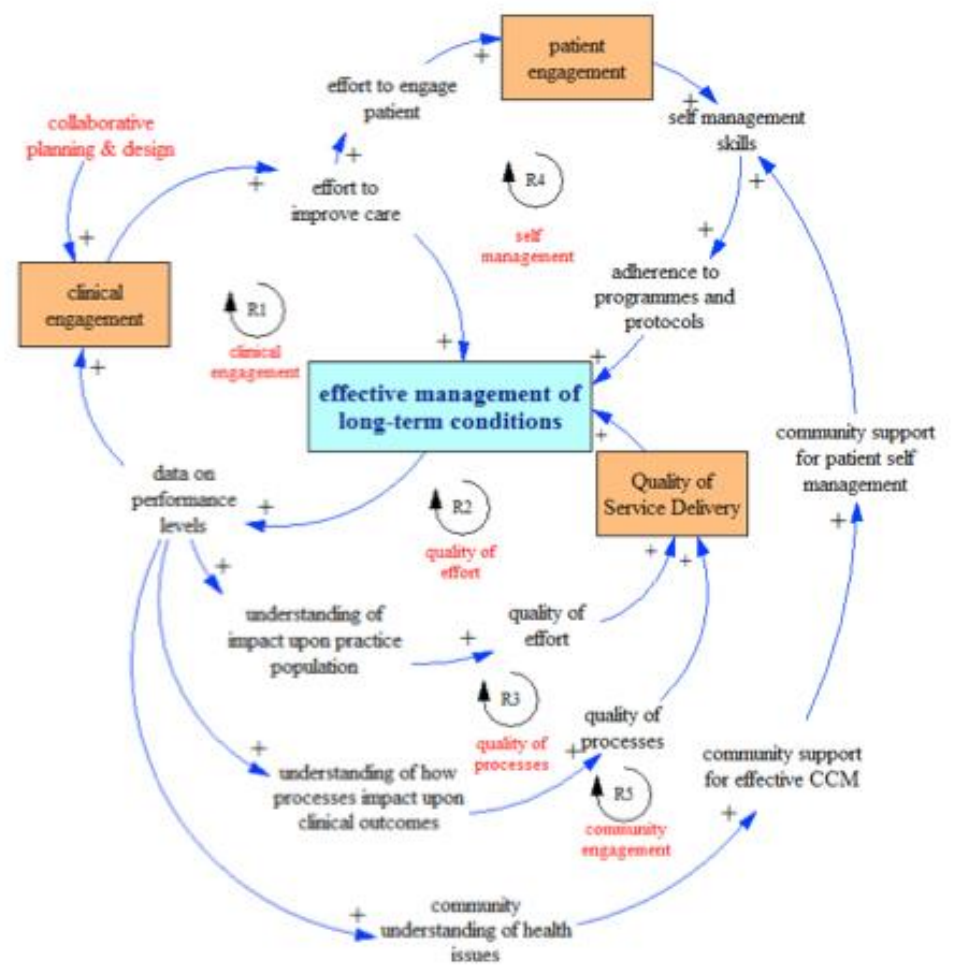

Community Engagement

Patients do not exist in isolation, but live within a broader family, whānau and community context.

When the connections with this broader community context are strong the patient is better supported and more able to engage with and adhere to the programme and necessary lifestyle changes.

While developing an engaged and supportive community is well beyond the limits of what a primary care practice can achieve they do have a role in i) helping to increase understanding in the community of relevant health issues and ii) increasing understanding in the patients family and whānau so that they can play a supportive role in the patients treatment.

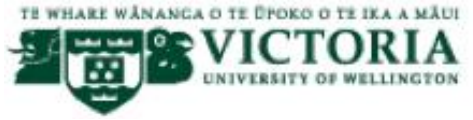

Appendix 2: Feedback Material 


\section{Resource Demand \& Self Efficacy}

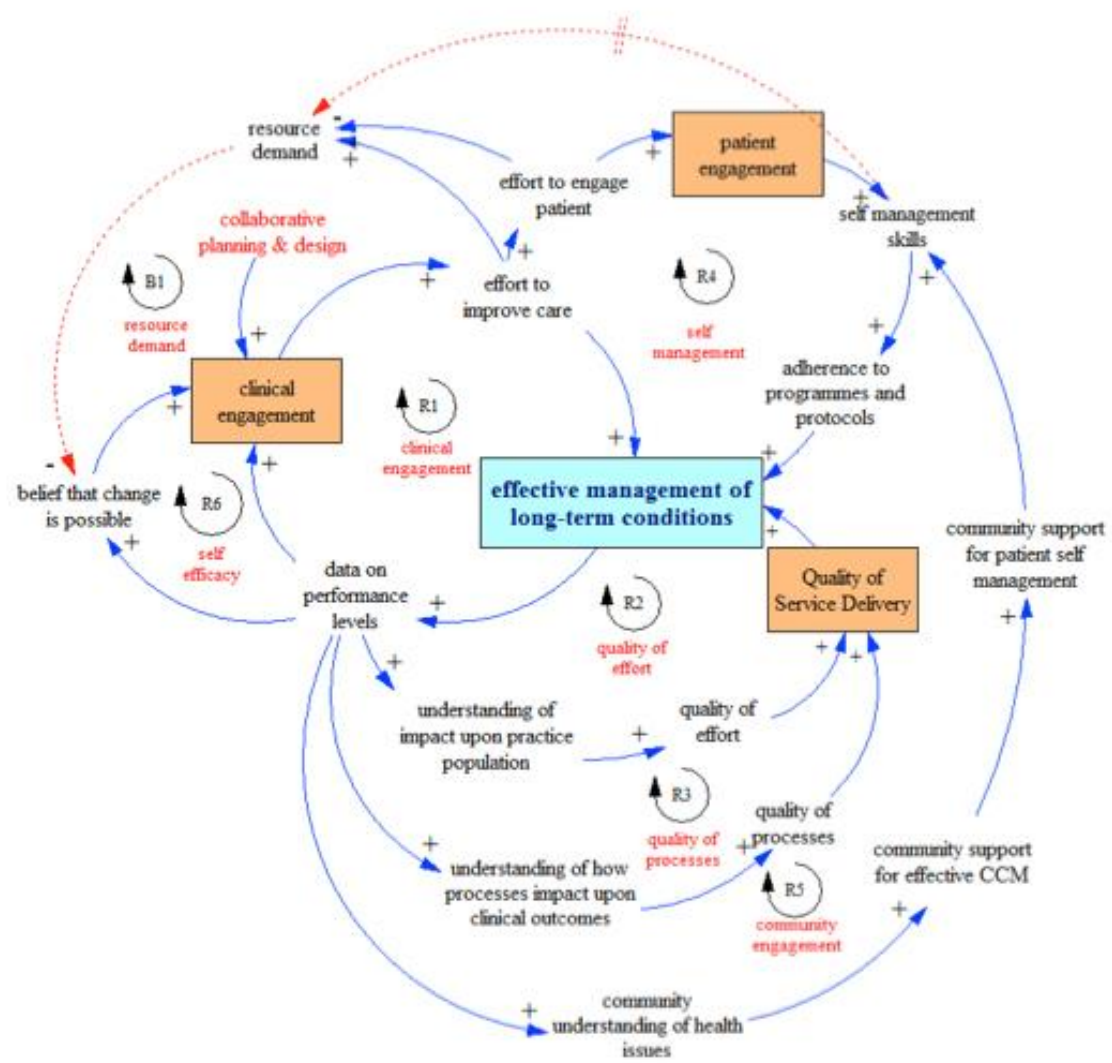

Making the changes required to improve care for people with long-term conditions not only requires effort, it also requires resources. If those resources and scarce, or not available, the belief amongst the clinical team that the change is in fact even possible - self efficacy - is diminished.

To become engaged, or to stay engaged throughout the change the clinical team have to have a belief that it can be done, that they have the resources required.

This is not to say that nothing occurs without adequate resources, as there are many examples of people simply trying harder, making do with what they have. However, even the most dedicated team will come up against resource barriers and unless they are addressed, their levels of self efficacy will drop and undermine the engagement and support they have for the changes being made.

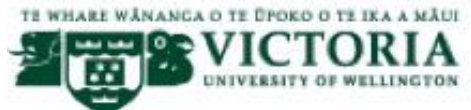




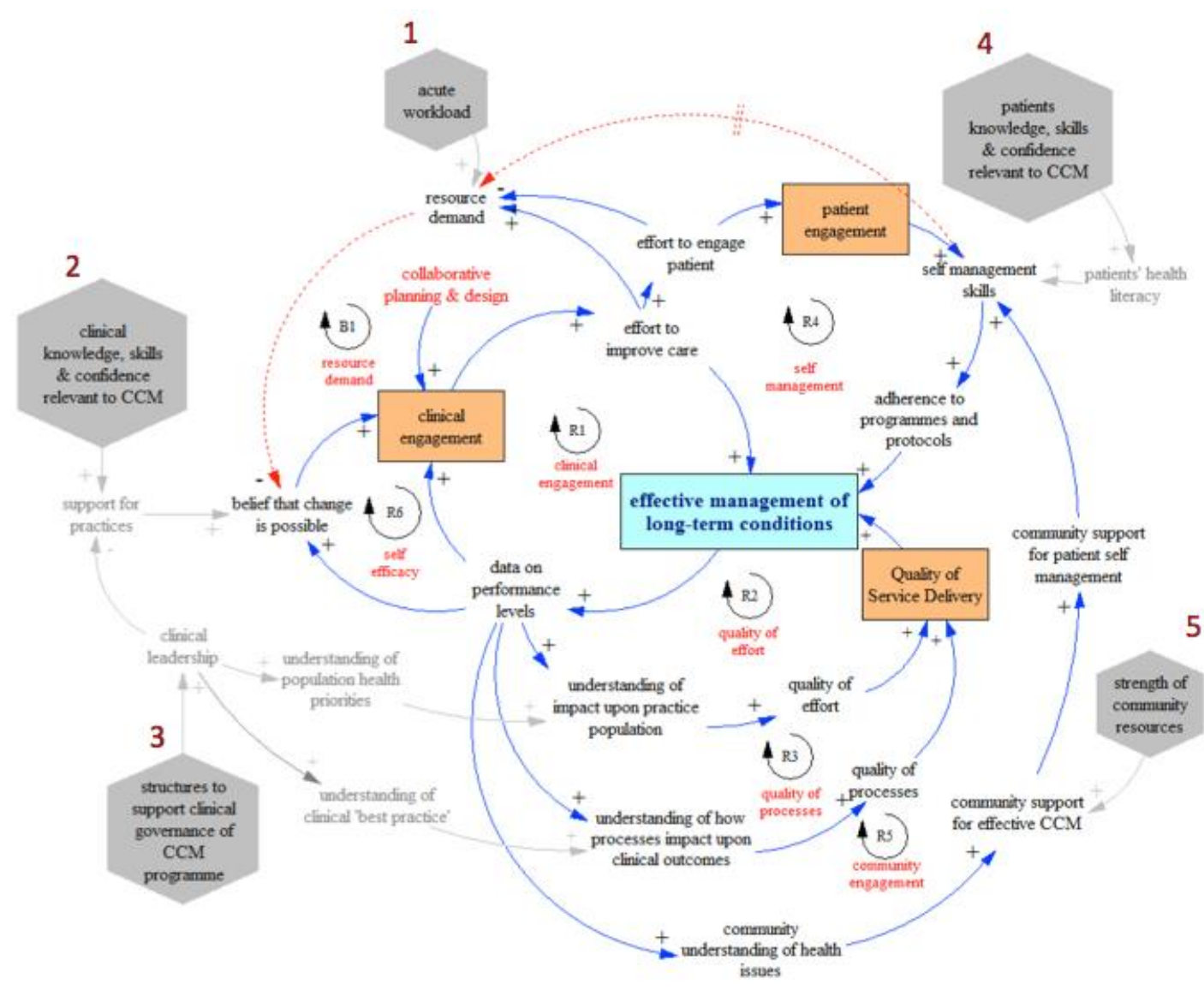

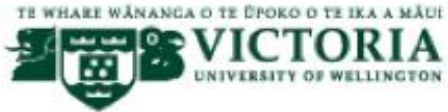

\section{Context}

The context within which the primary care practice operates will have an important impact upon how the dynamics discussed above will play out.

For example, the level of 'acute workload' (1) will have a significant impact upon the team's self-

efficacy. Resource constrained practices will find it harder to implement the changes without some form of support. Whether that be in terms of money, time, knowledge, or skills (2).

Clinical leadership (3) has an important role to play here and where it is strong the practice team

5 is better able to cope with resource constraints and is better placed to ensure that the efforts they do make are targeted in the right areas and are strongly evidence-based.

Furthermore practices in areas where patients have low levels of health literacy (4)and limited community support (5) will require greater effort on part of the team to develop the patient and community engagement needed to deliver improved outcomes.

It is these contextual elements that will influence what is the most appropriate balance of actions required to successfully implement improved care within any given practice. 


\section{Appendix 3: Sensitivity Tests}

This appendix describes the sensitivity test undertaken on the 'lookup' functions that describe key casual relationships in the model. The sensitivity analysis test four different casual relationships for each lookup function:

- S-shaped growth: in which the relationships between the variables in the model correspond to a s-shaped curve.

- Linear growth: in which the relationships between the variables are depicted as a straight line

- Decreasing growth: in which a 'convex' curve is used to depict rapid growth that tails off as the causal variable decreases.

- Increasing growth: in which a 'concave' curve is used to depict slow growth rising rapidly as the casual variables reaches it upper limit.

Each of the baseline lookup functions utilised on of these curves so the sensitivity tests compare the baseline against three alternatives. For these sensitivity test the goals was set at 80 per cent of best practice. The output graphs show the impact on the variables of concern as well as the impact upon the 'effective management of chronic conditions'.

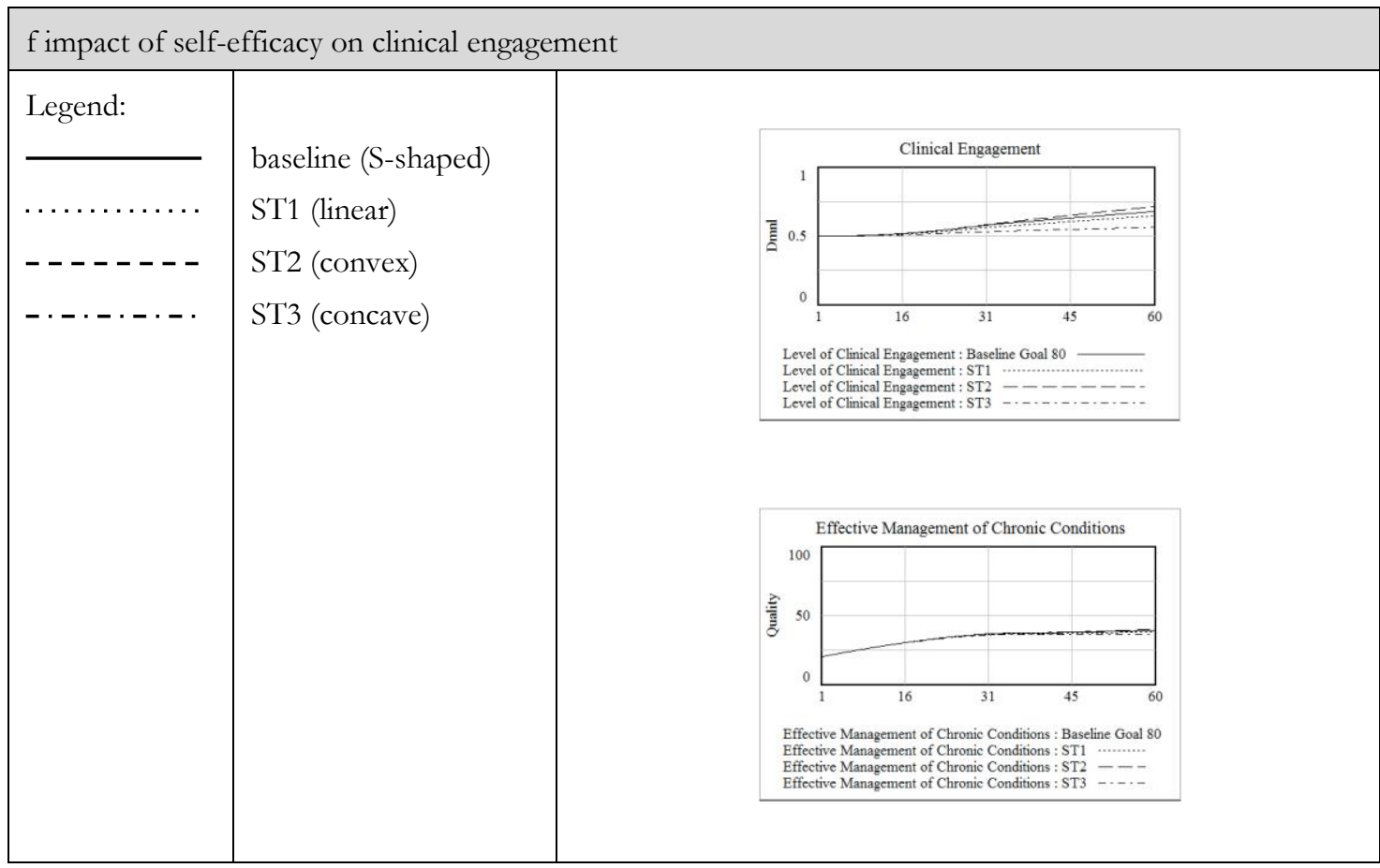




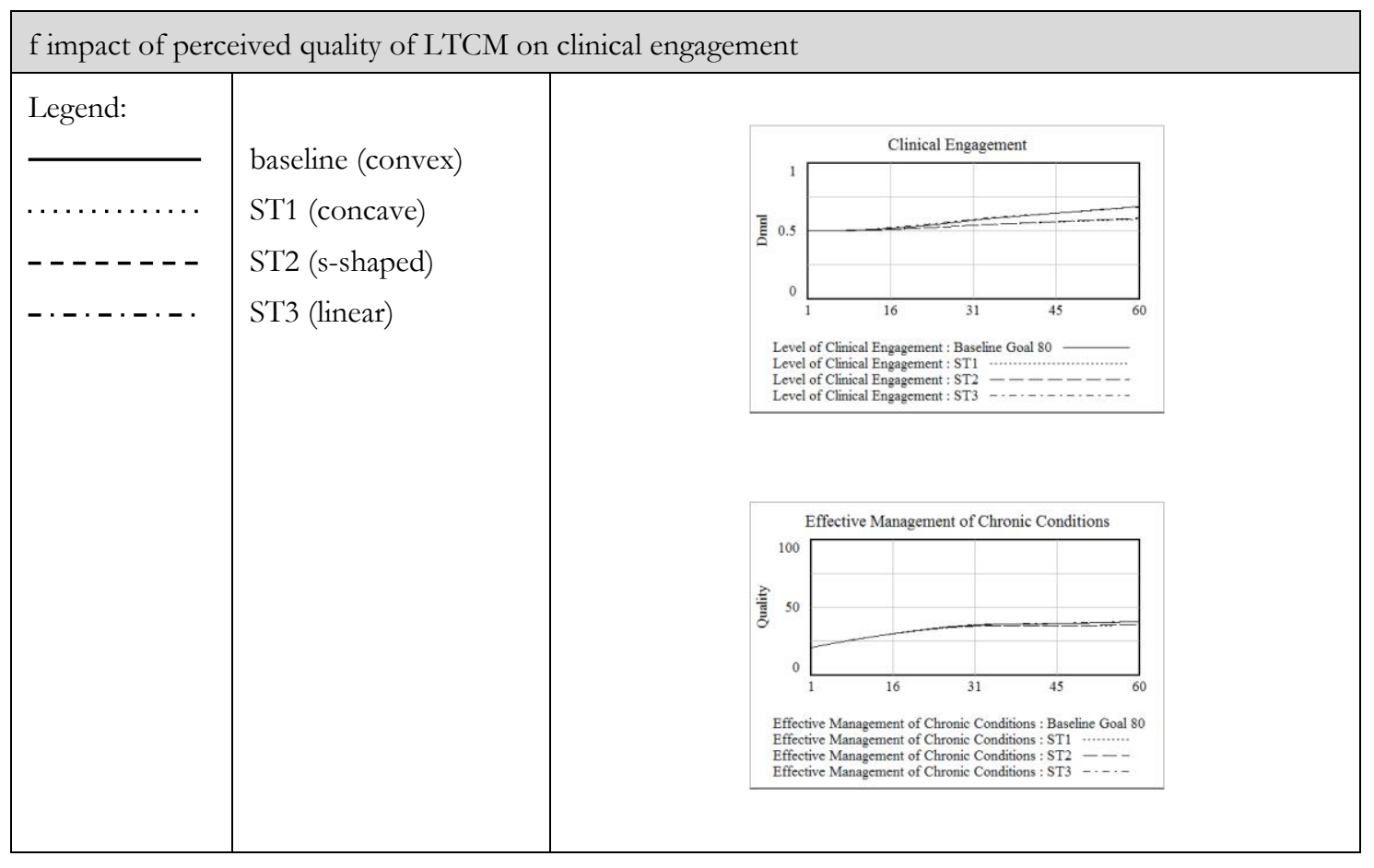

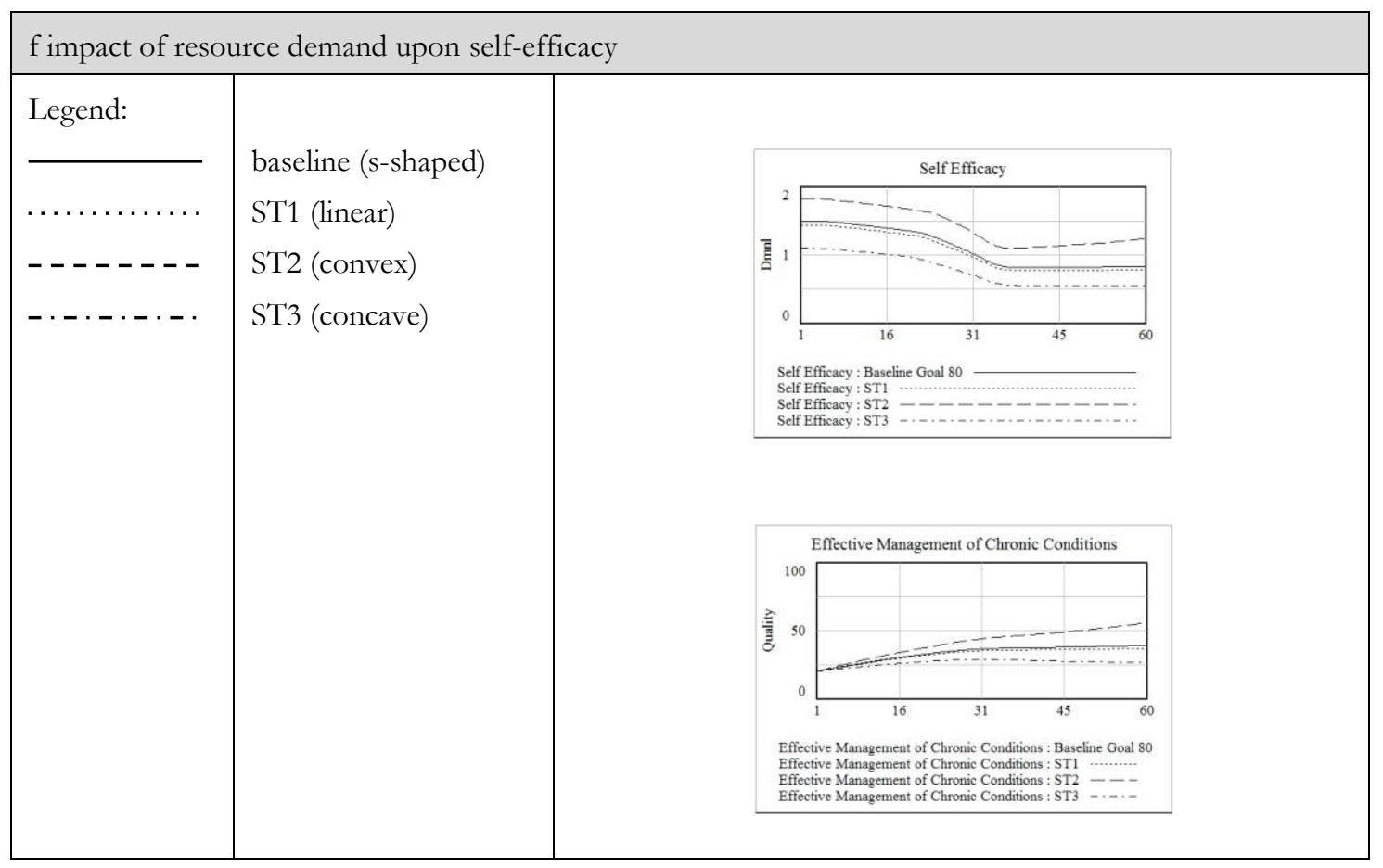




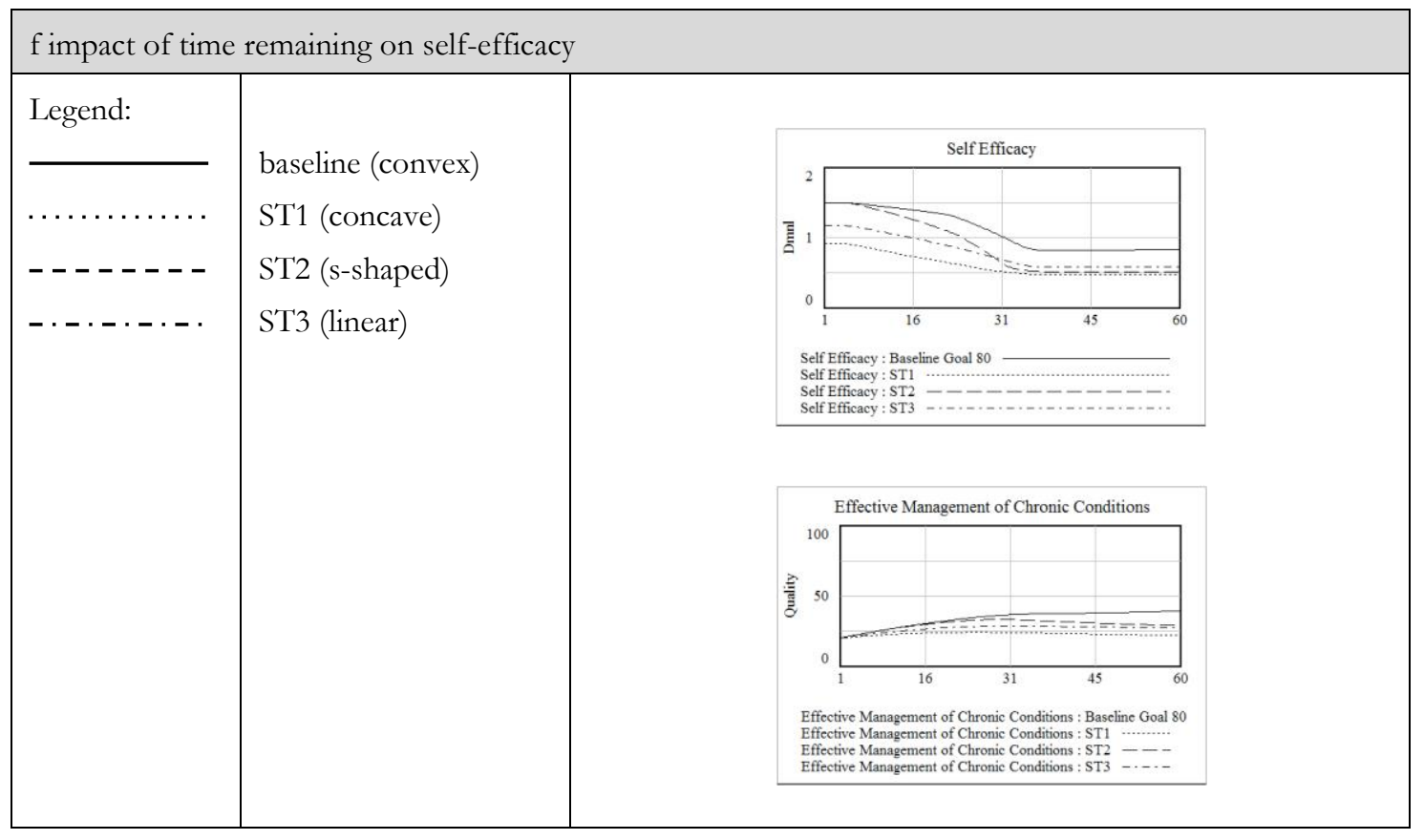

f impact of improvement activities on process quality

\begin{tabular}{|l|l|l|l|l|l|}
\hline Legend: & baseline (convex) \\
\hline$\ldots . . . . .$. & $\begin{array}{l}\text { ST1 (concave) } \\
\text { ST2 (s-shaped) } \\
\text { ST3 (linear) }\end{array}$ & $\begin{array}{l}\text { NOTE: these tests } \\
\text { were done assuming } 5 \\
\text { hours per week } \\
\text { involved in process } \\
\text { improvement activities } \\
\text { and 24 months as the } \\
\text { time for the benefit of } \\
\text { those activities to take } \\
\text { full effect. }\end{array}$ \\
\hline
\end{tabular}




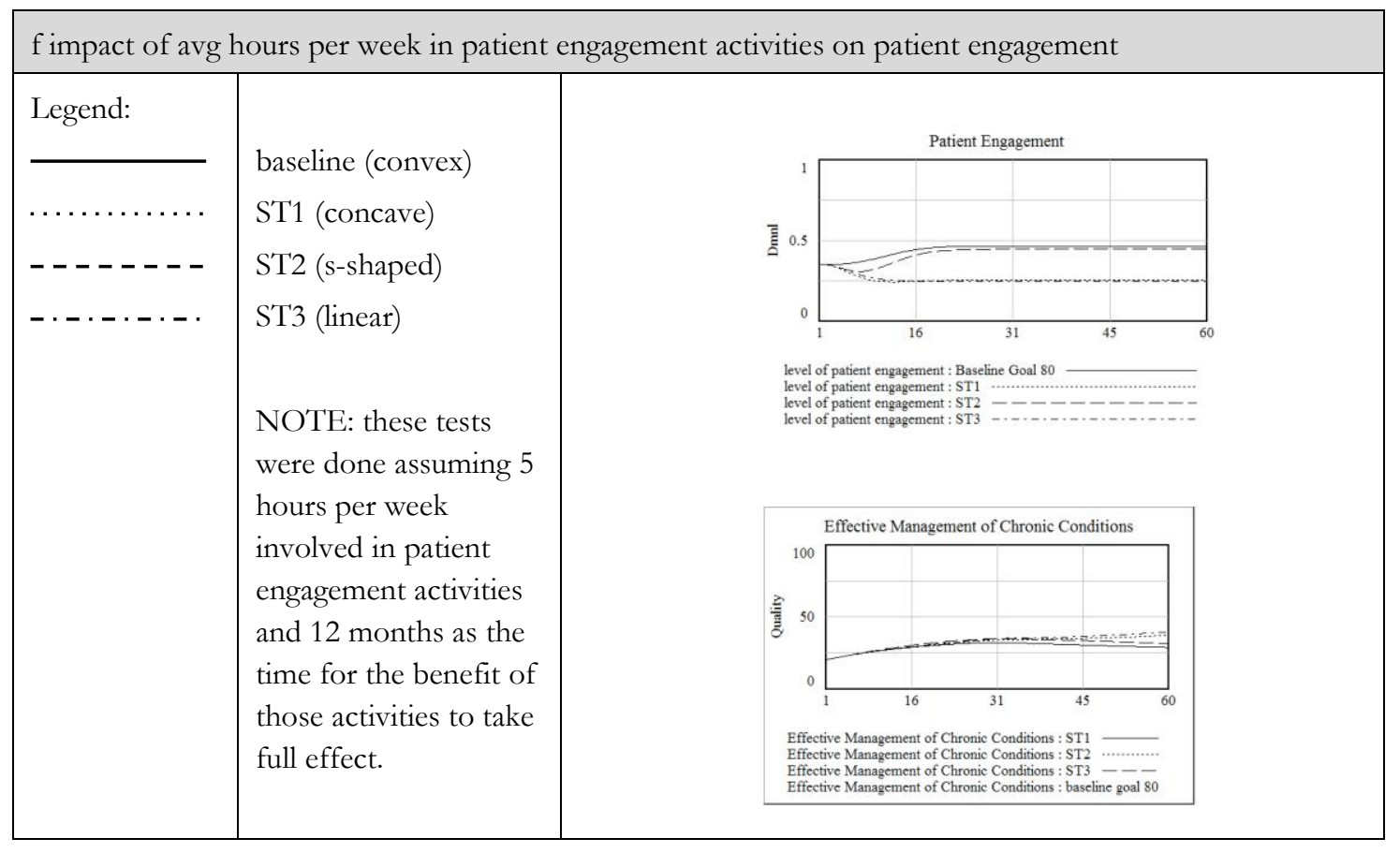

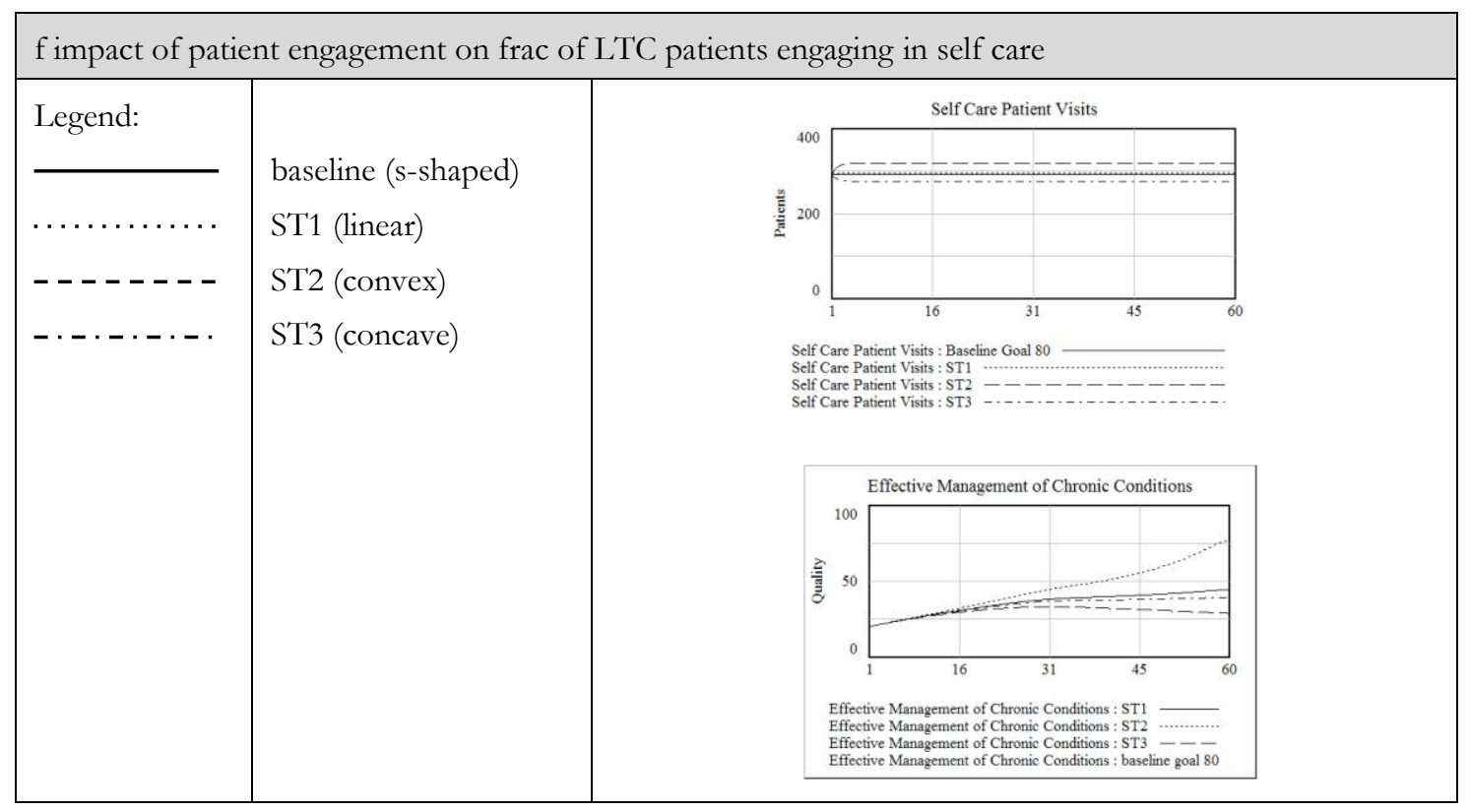




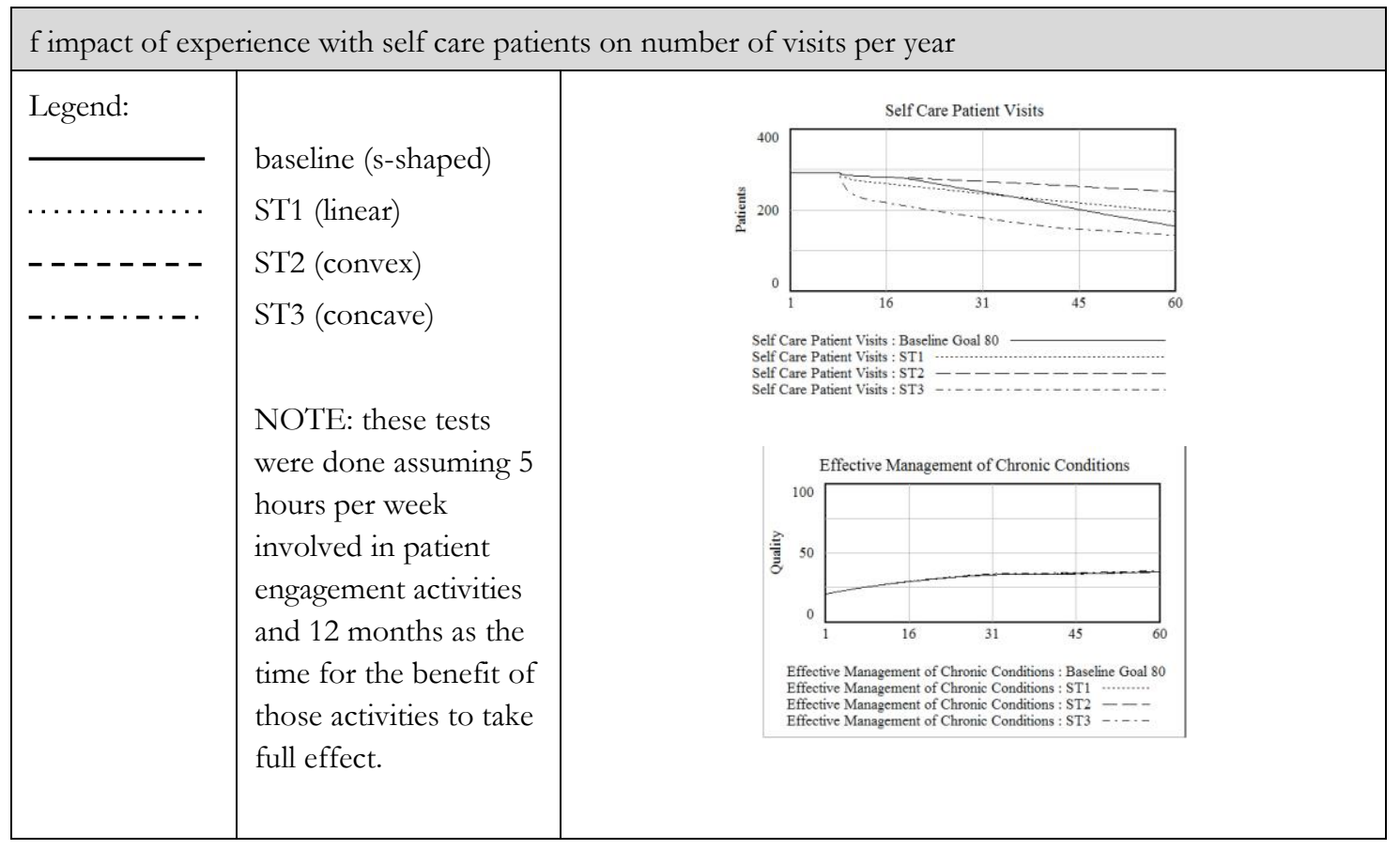

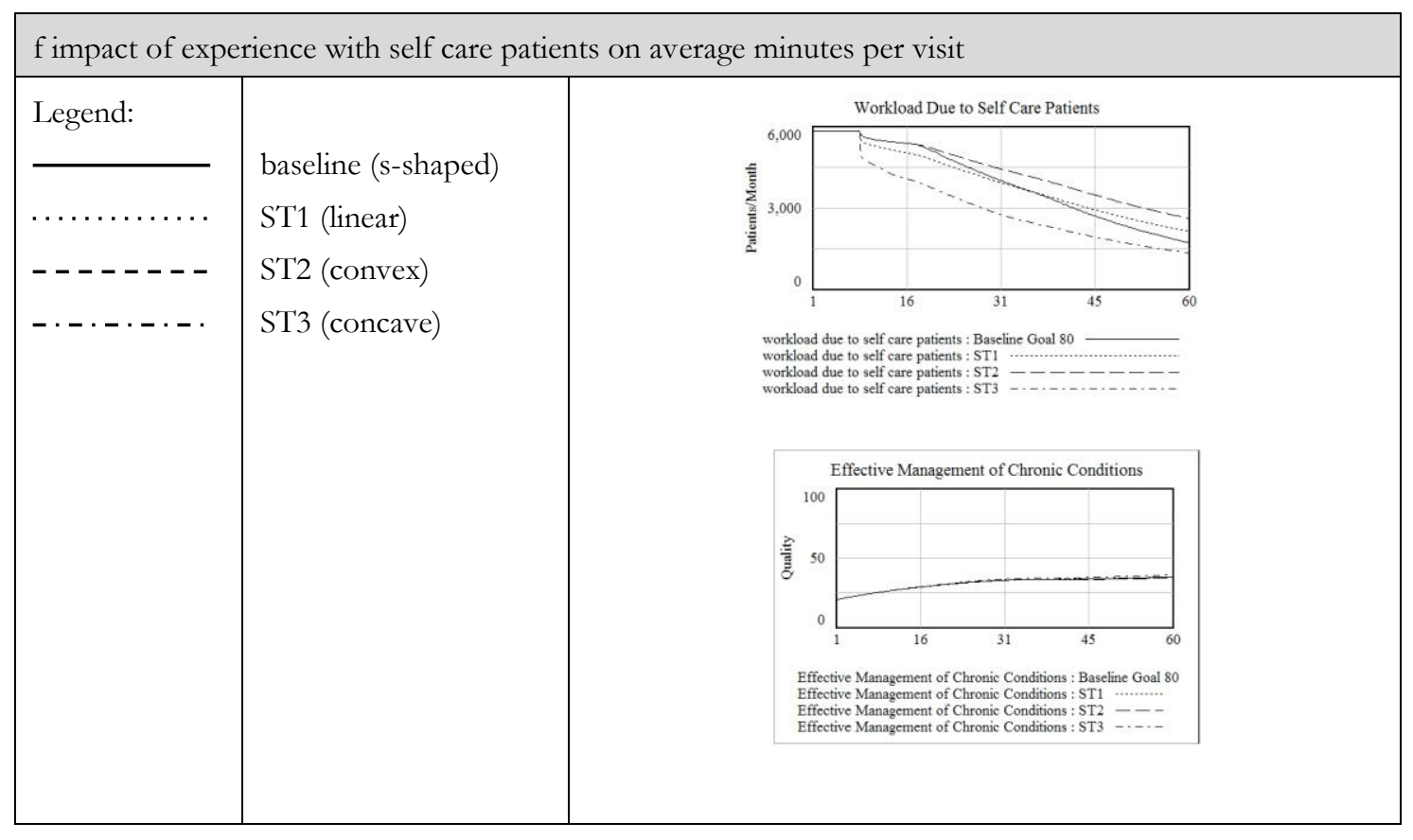


Appendix 4: Model Documentation ${ }^{7}$

\section{Documentation of Dynamics of Implementation in Primary Care_020113}

\section{Model Assessment Results}

\begin{tabular}{lc} 
Model Information & Number \\
Total Number of Variables & 143 \\
Total Number of State Variables (Level+Smooth+Delay & 15 \\
\hline Variables) & \\
Total Number of Stocks (Stocks in Level+Smooth+Delay & 21 \\
Variables) $\dagger$ & 0 \\
Total Number of Macros & \\
\hline Time Unit & Month \\
Initial Time & 1 \\
Final Time & 60 \\
Time Step & 0.25 \\
Model Is Fully Formulated & Yes
\end{tabular}
7 The model documentation was done using SDM-Doc, a SD documentation tool for Vensim. The documentation is laid out to match the model views in Vensim. Each table of
equations is preceded by a picture of the model structure in that view. The software is available from: http://tools.systemdynamics.org/documenting-and-explaining-models/ 


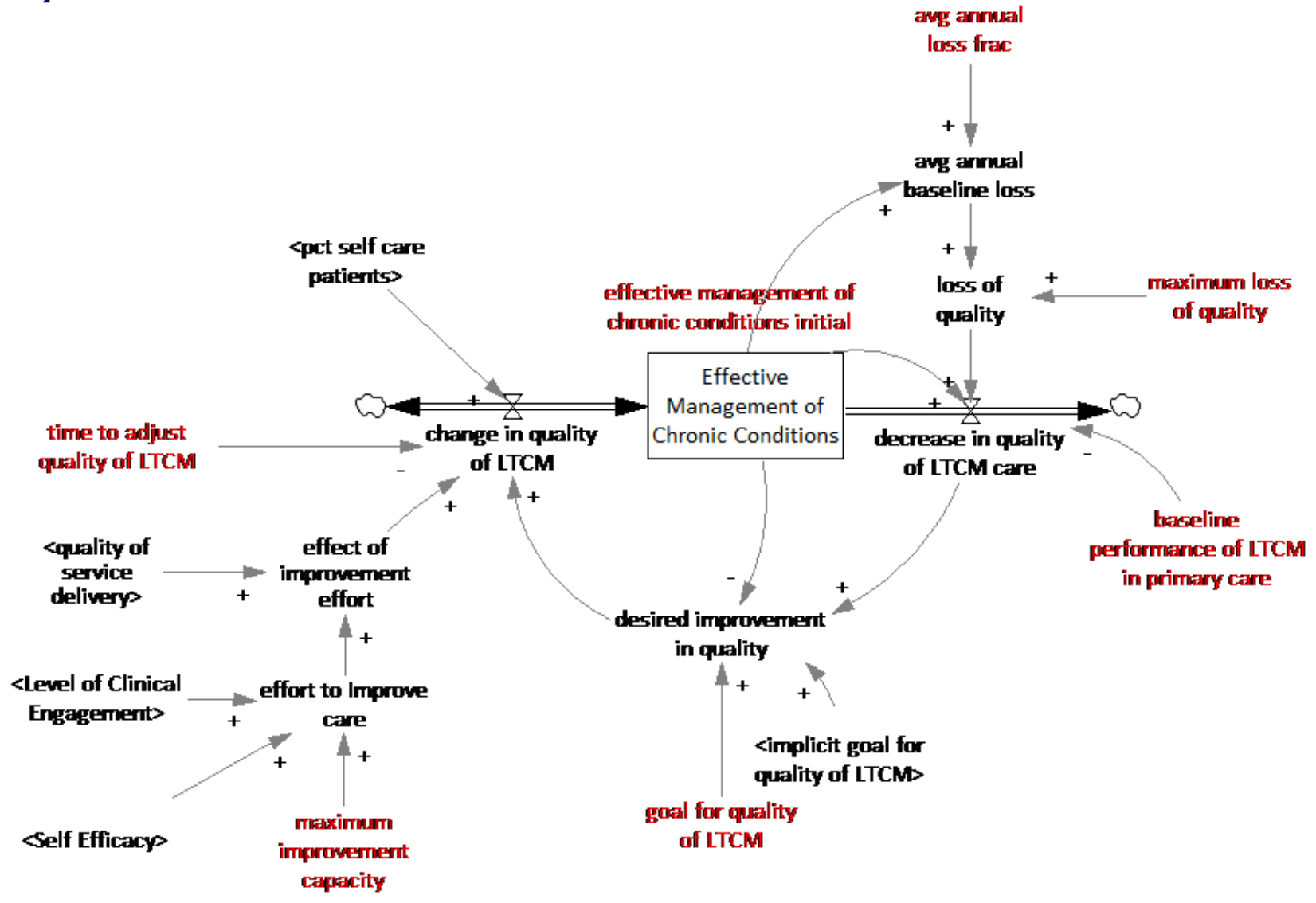




\begin{tabular}{|c|c|c|c|}
\hline Module & Group & Type & Variable Name and Description \\
\hline Default & \begin{tabular}{|c||} 
Dynamics of \\
Implementation \\
in Primary \\
Care 020113 \\
(Default)
\end{tabular} & \begin{tabular}{|c|} 
\\
$A$ \\
VAB \\
VAB
\end{tabular} & $\begin{array}{l}\text { avg annual baseline loss (Quality/Month) } \\
\text { = Effective Management of Chronic Conditions } \\
\text { Description: This is the baseline loss of qualitry, } \\
\text { and/or improve quality then this represents the quality that will be lost until it reaches the baseline level of } \\
\text { performance } \\
\text { Present in } 1 \text { view: } \\
\text { - Quality of LTCM } \\
\text { Used by: } \\
\text { - loss of quality - This is the amount of quality that will be lost each month due to the effects of 'forgetting' }\end{array}$ \\
\hline Default & \begin{tabular}{|c||} 
Dynamics of \\
Implementation \\
in Primary \\
Care 020113 \\
(Default)
\end{tabular} & $\begin{array}{c}\mathrm{C} \\
\mathrm{W} \\
\mathrm{V} / \mathrm{B}\end{array}$ & $\begin{array}{l}\text { avg annual loss frac (1/Month }[0,1,0.05]) \\
=0.25 \\
\text { Description: This is the annual loss fraction, assuming no efforts are made to build and/or maintain knowledge and } \\
\text { skills in providing care for people with long-term conditions. } \\
\text { Present in } 1 \text { view: } \\
\text { - Quality of LTCM } \\
\text { Used by: } \\
\text { - } \frac{\text { avg annual baseline loss - This is the baseline loss of qualitry, due to forgetting, and turnover. If no efforts }}{\text { are made to manage and/or improve quality then this represents the quality that will be lost until it reaches }} \\
\text { the baseline level of performance }\end{array}$ \\
\hline
\end{tabular}




\begin{tabular}{|c|c|c|c|}
\hline Default & $\mid \begin{array}{c}\text { Dynamics of } \\
\text { Implementation } \\
\text { in Primary } \\
\text { Care } 020113 \\
\text { (Default) }\end{array}$ & $\mid \begin{array}{c}C \\
\text { C } \\
\text { VAt }\end{array}$ & $\begin{array}{l}\text { baseline performance of LTCM in primary care (Quality) } \\
=20 \\
\text { Description: This is the baseline performance of surrounding primary care practices. The figure of } 20 \text { is based on } \\
\text { data from South Auckland inidicating that only 20per cent of diabetes pateints meet the clinical criteria for 'in } \\
\text { control'. } \\
\text { Present in 1 view: } \\
\text { - Quality of LTCM } \\
\text { Used by: } \\
\text { - } \frac{\text { decrease in quality of LTCM care - This is the decrease in quality per month - a function of an annual loss }}{\text { function and the baseline performance of peer practices }}\end{array}$ \\
\hline Default & $\mid \begin{array}{c}\text { Dynamics of } \\
\text { Implementation } \\
\text { in Primary } \\
\text { Care } 020113 \\
\text { (Default) }\end{array}$ & $\begin{array}{l}F, A \\
\text { WAb } \\
\text { F }\end{array}$ & 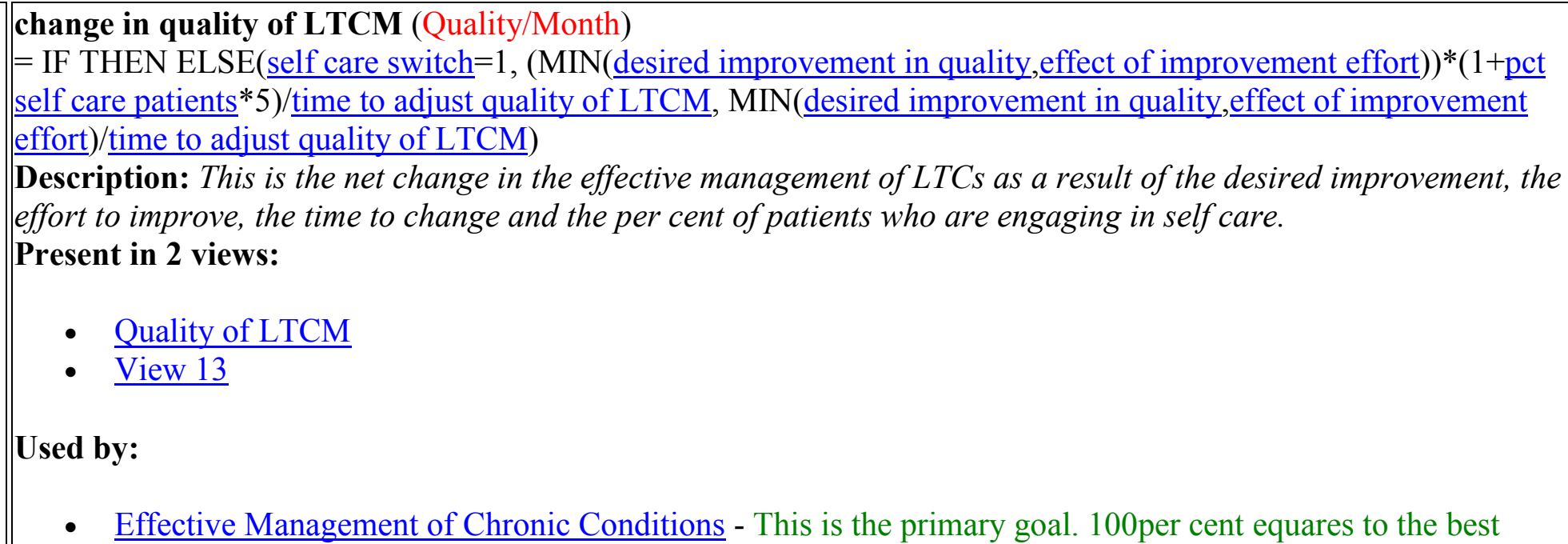 \\
\hline
\end{tabular}




\begin{tabular}{|c|c|c|c|}
\hline & & & possible quality across all patients in the practice. \\
\hline
\end{tabular}




\begin{tabular}{|c|c|c|c|}
\hline & & & $\begin{array}{l}\text { - change in quality of LTCM - This is the net change in the effective management of LTCs as a result of the } \\
\text { desired improvement, the effort to improve, the time to change and the per cent of patients who are engaging } \\
\text { in self care. }\end{array}$ \\
\hline Default & \begin{tabular}{||c||} 
Dynamics of \\
Implementation \\
in Primary \\
Care 020113 \\
(Default)
\end{tabular} & $\begin{array}{r}\text { A } \\
\text { VA }\end{array}$ & $\begin{array}{l}\text { effect of improvement effort (Quality) } \\
=\text { IF THEN ELSE(service delivery switch=1, effort to Improve care* (quality of service delivery } * 2 \text { ), effort to } \\
\text { Improve care) } \\
\text { Description: This is the effect the improvement effort will have on the change in manaement of LTCs } \\
\text { Present in } 1 \text { view: } \\
\text { - Quality of LTCM } \\
\text { Used by: } \\
\text { - change in quality of LTCM - This is the net change in the effective management of LTCs as a result of the } \\
\text { desired improvement, the effort to improve, the time to change and the per cent of patients who are engaging } \\
\text { in self care. }\end{array}$ \\
\hline
\end{tabular}




\begin{tabular}{|c|c|c|c|}
\hline & & & $\begin{array}{l}\text { - } \text { Clinical Engagement } \\
\text { Used by: } \\
\text { - } \frac{\text { desired improvement in quality - This is the desired improvement goal - a function of the target they are }}{\text { trying to reach, the time it takes them to make changes and the gap between their goal and current practice. }} \\
\text { - } \frac{\text { avg annual baseline loss - This is the baseline loss of qualitry, due to forgetting, and turnover. If no efforts }}{\text { are made to manage and/or improve quality then this represents the quality that will be lost until it reaches }} \\
\text { the baseline level of performance } \\
\text { - } \text { decrease in quality of LTCM care - This is the decrease in quality per month - a function of an annual loss } \\
\text { function and the baseline performance of peer practices } \\
\text { forming perception of historical performance - This is the change in perception of performance }\end{array}$ \\
\hline Default & \begin{tabular}{|} 
Dynamics of \\
Implementation \\
in Primary \\
Care 020113 \\
(Default)
\end{tabular} & $\begin{array}{c}\text { LI,C } \\
\text { VAt } \\
\text { VAs }\end{array}$ & $\begin{array}{l}\text { effective management of chronic conditions initial (Quality) } \\
=20 \\
\text { Description: This is the initial level of quality. The initial figure of } 20 \text { is based on data from South Auckland } \\
\text { inidicating that only 20per cent of diabetes pateints, in a chronic care programme, met the clincial guidelines for } \\
\text { being 'iin control'. } \\
\text { Present in } 2 \text { views: } \\
\text { - Quality of LTCM } \\
\text { - } \frac{\text { Clinical Engagement }}{\text { Used by: }} \\
\text { - } \frac{\text { Perceived Management of Chronic Conditions - This is the perception of effectiveness of LTC management }}{\text { peffective Management of Chronic Conditions }- \text { This is the primary goal. 100per cent equares to the best }}\end{array}$ \\
\hline
\end{tabular}




\begin{tabular}{|c|c|c|c|}
\hline Default & \begin{tabular}{|c||} 
Dynamics of \\
Implementation \\
in Primary \\
Care 020113 \\
(Default)
\end{tabular} & $\begin{array}{c}G \\
6\end{array}$ & $\begin{array}{l}\text { effort to Improve care (Effort) } \\
=\text { GAME (IF THEN ELSE(effort to improve switch }=1 \text {, (maximum improvement capacity/12)*(Level of Clinical } \\
\text { Engagement)*(Self-efficacy), maximum improvement capacity/12)) } \\
\text { Description: This is the amount of effort the primary care team is able and willing to put into improving their } \\
\text { practice } \\
\text { Present in } 2 \text { views: } \\
\text { - } \frac{\text { Quality of LTCM }}{\text { Self-efficacy }} \\
\text { Used by: } \\
\text { - } \frac{\text { effect of improvement effort }- \text { This is the effect the improvement effort will have on the change in }}{\text { manaement of LTCs }}\end{array}$ \\
\hline Default & \begin{tabular}{|c||} 
Dynamics of \\
Implementation \\
in Primary \\
Care 020113 \\
(Default)
\end{tabular} & $\begin{array}{c}\mathrm{C} \\
\text { VAB }\end{array}$ & $\begin{array}{l}\text { effort to improve switch }(\mathrm{Dmnl}[0,1,1]) \\
=1 \\
\text { Description: Scenario switch - swtiches the slef efficacy and clinical engagement sectors on/off } \\
\text { Present in } 2 \text { views: } \\
\text { - } \underline{\text { Quality of LTCM }} \\
\text { Used by: } \\
\text { - } \frac{\text { effort to Improve care - This is the amount of effort the primary care team is able and willing to put into }}{\text { improving their practice }}\end{array}$ \\
\hline Default & Dynamics of & $\mathrm{C}$ & goal for quality of LTCM (Quality $[0,100,1]$ ) \\
\hline
\end{tabular}




\begin{tabular}{|c|c|c|c|}
\hline & \begin{tabular}{|c||} 
Implementation \\
in Primary \\
Care 020113 \\
(Default)
\end{tabular} & VA⿱ & $\begin{array}{l}=80 \\
\text { Description: The goal set for quality of LTCM when the dynamics of the implicit goal being turned off } \\
\text { Present in } 1 \text { view: } \\
\text { - Quality of LTCM } \\
\text { Used by: } \\
\text { - } \frac{\text { desired improvement in quality - This is the desired improvement goal - a function of the target they are }}{\text { trying to reach, the time it takes them to make changes and the gap between their goal and current practice. }}\end{array}$ \\
\hline Default & \begin{tabular}{||c||} 
Dynamics of \\
Implementation \\
in Primary \\
Care 020113 \\
(Default)
\end{tabular} & & $\begin{array}{l}\text { goal switch (Dmnl }[0,1,1]) \\
=1 \\
\text { Description: Scenario switch - switches the implicit goal effect on/off } \\
\text { Present in } 1 \text { view: } \\
\text { - Quality of LTCM } \\
\text { Used by: } \\
\text { - } \quad \text { desired improvement in quality - This is the desired improvement goal - a function of the target they are } \\
\text { trying to reach, the time it takes them to make changes and the gap between their goal and current practice. }\end{array}$ \\
\hline Default & \begin{tabular}{||c||} 
Dynamics of \\
Implementation \\
in Primary \\
Care 020113 \\
(Default)
\end{tabular} & 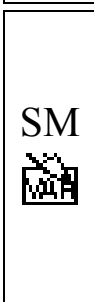 & 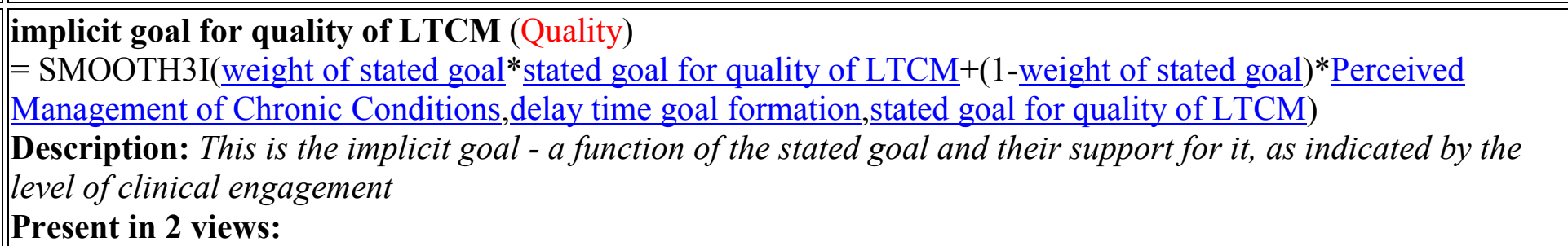 \\
\hline
\end{tabular}




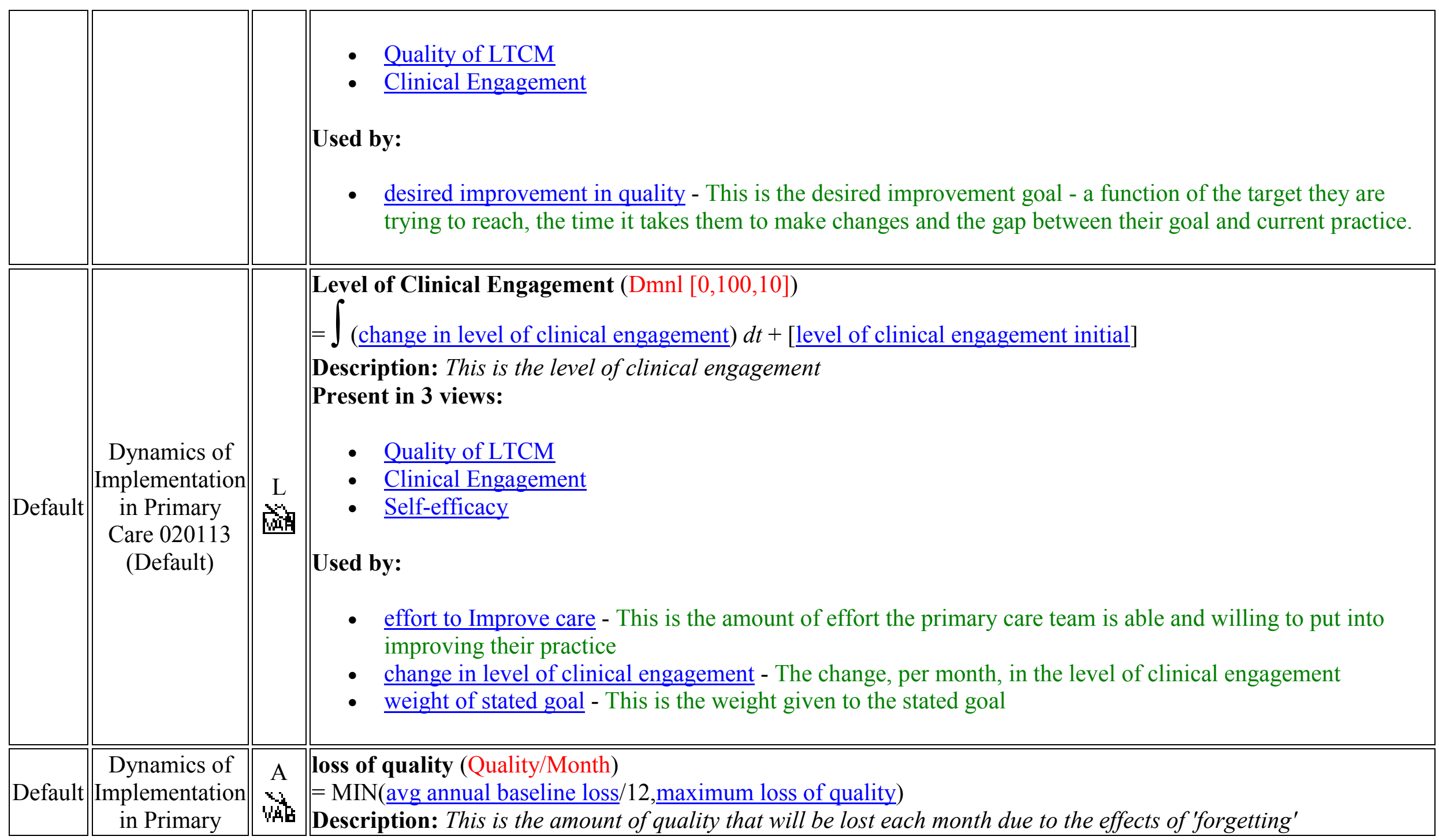




\begin{tabular}{|c|c|c|c|}
\hline & $\begin{array}{c}\text { Care } 020113 \\
\text { (Default) }\end{array}$ & & $\begin{array}{l}\text { Present in } 1 \text { view: } \\
\text { - Quality of LTCM } \\
\text { Used by: } \\
\text { - } \frac{\text { decrease in quality of LTCM care - This is the decrease in quality per month - a function of an annual loss }}{\text { function and the baseline performance of peer practices }}\end{array}$ \\
\hline Default & \begin{tabular}{|c|} 
Dynamics of \\
Implementation \\
in Primary \\
Care 020113 \\
(Default) \\
\end{tabular} & 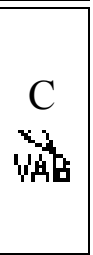 & $\begin{array}{l}\text { maximum loss of quality (Quality/Month }[0,20,1] \text { ) } \\
=10 \\
\text { Description: This is the maximum loss of units of quality per month. } \\
\text { Present in } 1 \text { view: }\end{array}$ \\
\hline
\end{tabular}




\begin{tabular}{|c|c|c|c|}
\hline & & & $\begin{array}{l}\text { - } \underline{\text { Quality of LTCM }} \\
\text { Used by: } \\
\text { - loss of quality - This is the amount of quality that will be lost each month due to the effects of 'forgetting' }\end{array}$ \\
\hline Default & \begin{tabular}{|} 
Dynamics of \\
Implementation \\
in Primary \\
Care 020113 \\
(Default)
\end{tabular} & & $\begin{array}{l}\text { pet self care patients (Dmnl) } \\
=\text { Number of Self Care Patients/(Number of Self Care Patients }+ \text { Cumulative Number of Symptomatic LTC Patients) } \\
\text { Description: Per cent of self care patients who engage in self care } \\
\text { Present in } 2 \text { views: } \\
\text { - } \frac{\text { Quality of LTCM }}{\text { Number of Patients }} \\
\text { Used by: } \\
\text { - } \frac{\text { change in quality of LTCM - This is the net change in the effective management of LTCs as a result of the }}{\text { desired improvement, the effort to improve, the time to change and the per cent of patients who are engaging }} \\
\text { in self care. }\end{array}$ \\
\hline Default & \begin{tabular}{|} 
Dynamics of \\
Implementation \\
in Primary \\
Care 020113 \\
(Default)
\end{tabular} & $\begin{array}{c}A \\
\text { WAb }\end{array}$ & $\begin{array}{l}\text { quality of service delivery (Quality/Effort) } \\
=\text { IF THEN ELSE(Time }<\text { Start time (month) for process improvement, baseline service quality, IF THEN } \\
\text { ELSE(Time }>=\text { Time (month) for process improvement to have full effect, quality of processes, } \text {, baseline service } \\
\text { quality + (quality of processes-baseline service quality)*(Time-Start time (month) for process improvement)/(Time } \\
\text { (month) for process improvement to have full effect-Start time (month) for process improvement)) }) \\
\text { Description: This is the current quality of service delivery } \\
\text { Present in } 2 \text { views: }\end{array}$ \\
\hline
\end{tabular}




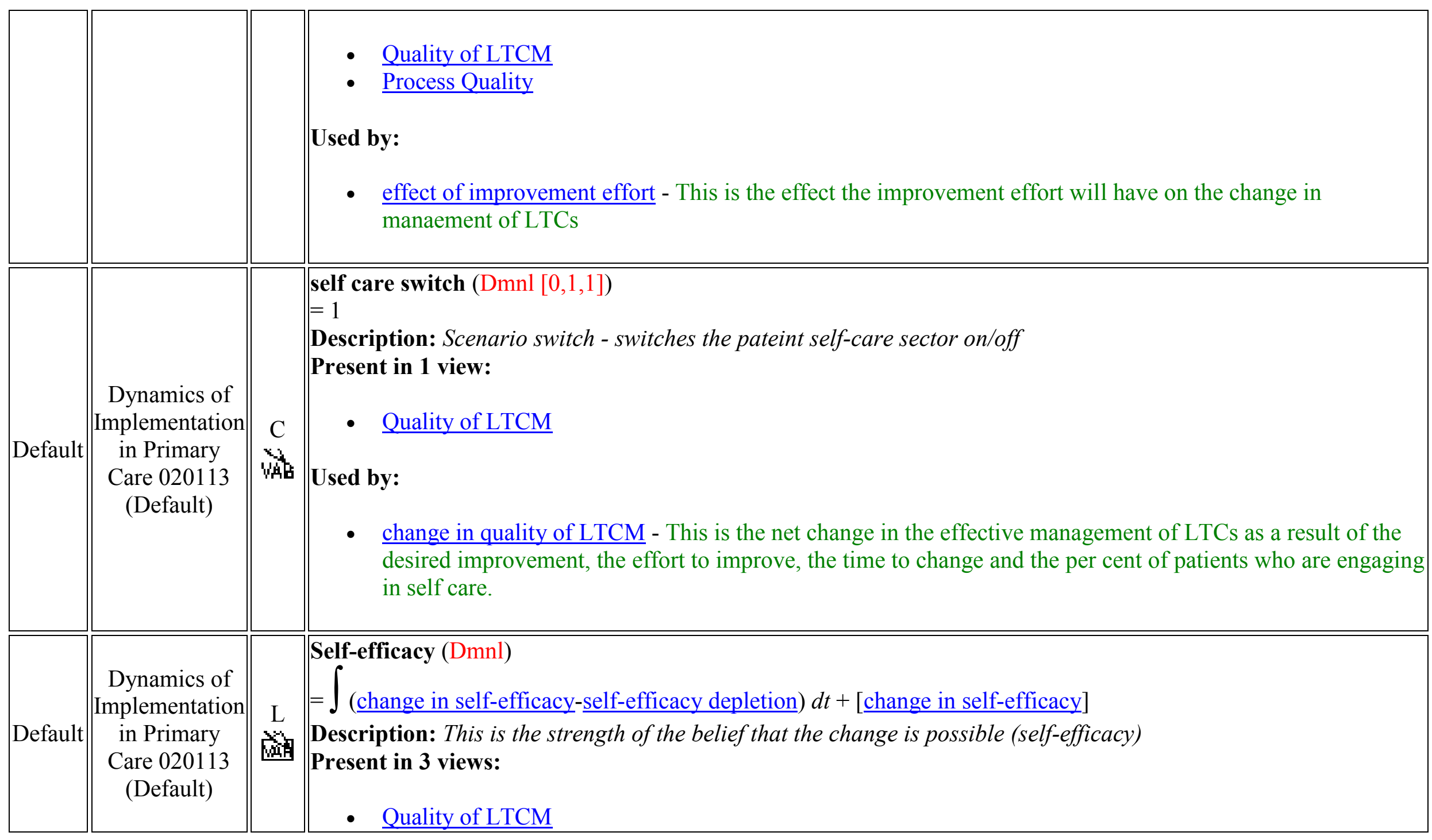




\begin{tabular}{|c|c|c|c|}
\hline & & & $\begin{array}{l}\text { - } \text { Clinical Engagement } \\
\text { Used by: } \\
\text { - } \frac{\text { effort to Improve care - This is the amount of effort the primary care team is able and willing to put into }}{\text { improving their practice }} \\
\text { - } \frac{\text { self-efficacy depletion - The depletion of self-efficacy within the timeframe of the gaol being set }}{\text { impact of self-efficacy on clinical engagement - This is the effect of self-efficacy upon the level of clinical }} \\
\text { engagement }\end{array}$ \\
\hline Default & $\mid \begin{array}{c}\text { Dynamics of } \\
\text { Implementation } \\
\text { in Primary } \\
\text { Care } 020113 \\
\text { (Default) }\end{array}$ & $\begin{array}{r}\mathrm{C} \\
\mathrm{VA}\end{array}$ & $\begin{array}{l}\text { service delivery switch }(\mathrm{Dmnl}[0,1,1]) \\
=1 \\
\text { Description: Scneario switch - switches the process quality sector on/off } \\
\text { Present in } 1 \text { view: } \\
\text { - Quality of LTCM } \\
\text { Used by: } \\
\text { - } \frac{\text { effect of improvement effort }- \text { This is the effect the improvement effort will have on the change in }}{\text { manaement of LTCs }}\end{array}$ \\
\hline Default & \begin{tabular}{|c|} 
Dynamics of \\
Implementation \\
in Primary \\
Care 020113 \\
(Default) \\
\end{tabular} & $\begin{array}{r}\mathrm{C} \\
\mathrm{VA}\end{array}$ & $\begin{array}{l}\text { time to adjust quality of LTCM (Month }[1,24,1] \text { ) } \\
=6 \\
\text { Description: This is the time it takes the practice to make changes to the quality of its practice } \\
\text { Present in } 1 \text { view: }\end{array}$ \\
\hline
\end{tabular}




$\mid$\begin{tabular}{l|l} 
Used by: \\
$-\frac{\text { Quality of LTCM }}{\text { change in quality of LTCM - This is the net change in the effective management of LTCs as a result of the }}$ \\
in self care.
\end{tabular}




\section{Clinical Engagement}

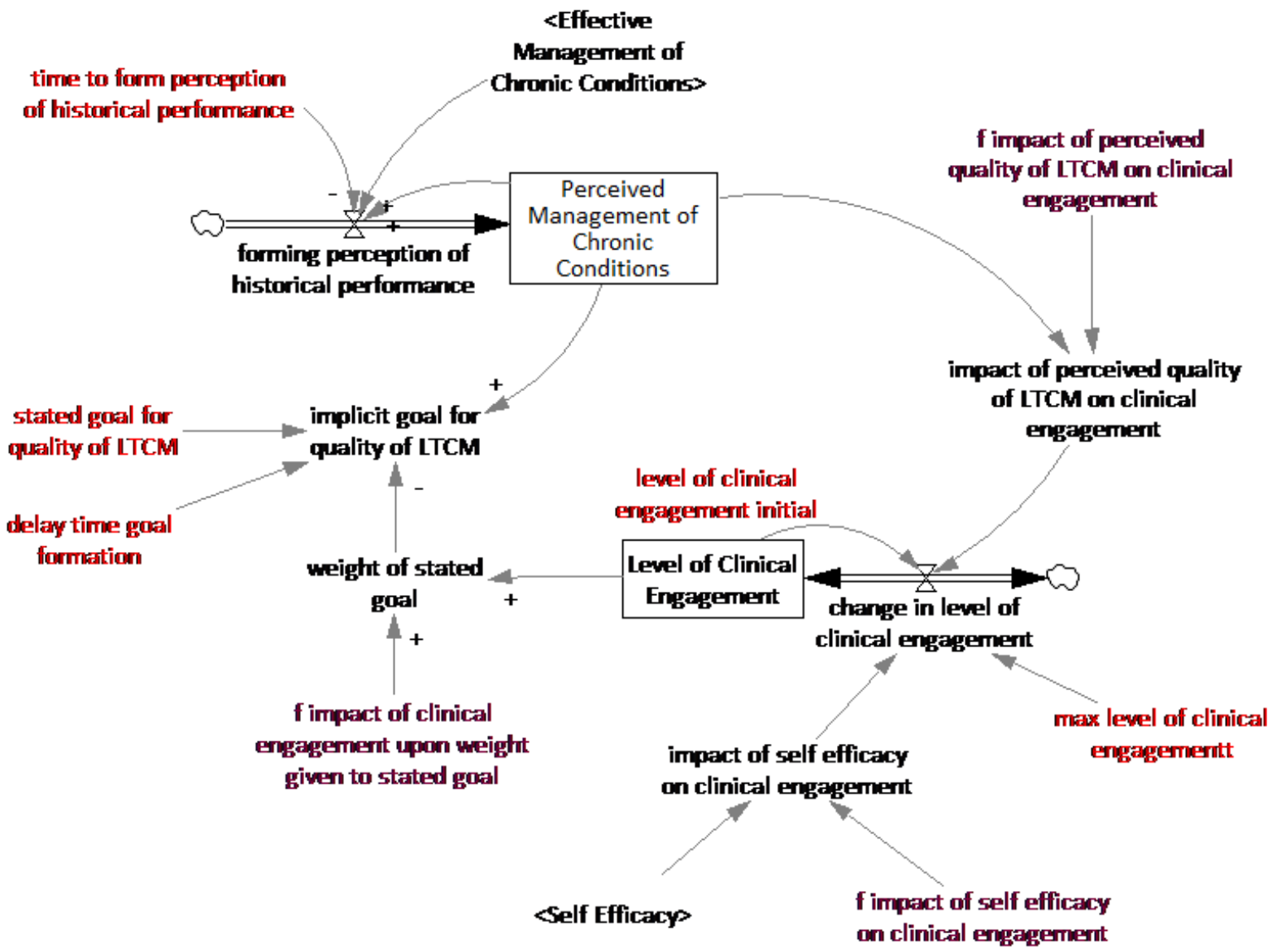




\begin{tabular}{|c|c|c|c|}
\hline Module & Group & Type & Variable Name and Description \\
\hline Default & $\begin{array}{c}\text { Dynamics of } \\
\text { Implementation } \\
\text { in Primary } \\
\text { Care } 020113 \\
\text { (Default) }\end{array}$ & \begin{tabular}{|l|} 
\\
$F, A$ \\
VAB \\
君 \\
\end{tabular} & $\begin{array}{l}\text { change in level of clinical engagement }(1 / \text { Month }) \\
=\text { IF THEN ELSE( }(\text { engagement switch }=1 \text {, (impact of perceived quality of LTCM on clinical engagement } * \text { impact of } \\
\text { self-efficacy on clinical engagement }) *(\text { max level of clinical engagementt-Level of Clinical Engagement }-0.01), 0) \\
\text { Description: The change, per month, in the level of clinical engagement } \\
\text { Present in } 1 \text { view: } \\
\text { - Clinical Engagement } \\
\text { Used by: } \\
\text { - Level of Clinical Engagement }- \text { This is the level of clinical engagement }\end{array}$ \\
\hline Default & $\begin{array}{c}\text { Dynamics of } \\
\text { Implementation } \\
\text { in Primary } \\
\text { Care } 020113 \\
\text { (Default) }\end{array}$ & & 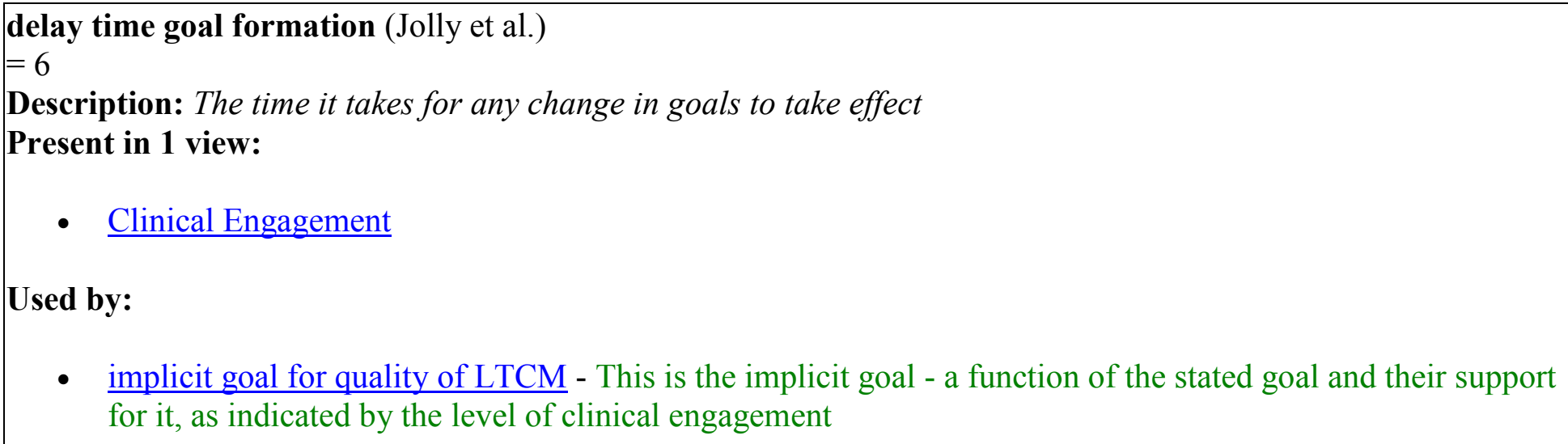 \\
\hline Default & $\begin{array}{c}\text { Dynamics of } \\
\text { Implementation } \\
\text { in Primary }\end{array}$ & $\begin{array}{c}\mathrm{L} \\
\mathrm{x} \\
\mathrm{m}\end{array}$ & $\begin{array}{l}\text { Effective Management of Chronic Conditions (Quality) } \\
=\iint^{\text {change in quality of LTCM-decrease in quality of LTCM care })} d t+\text { [effective management of chronic }\end{array}$ \\
\hline
\end{tabular}




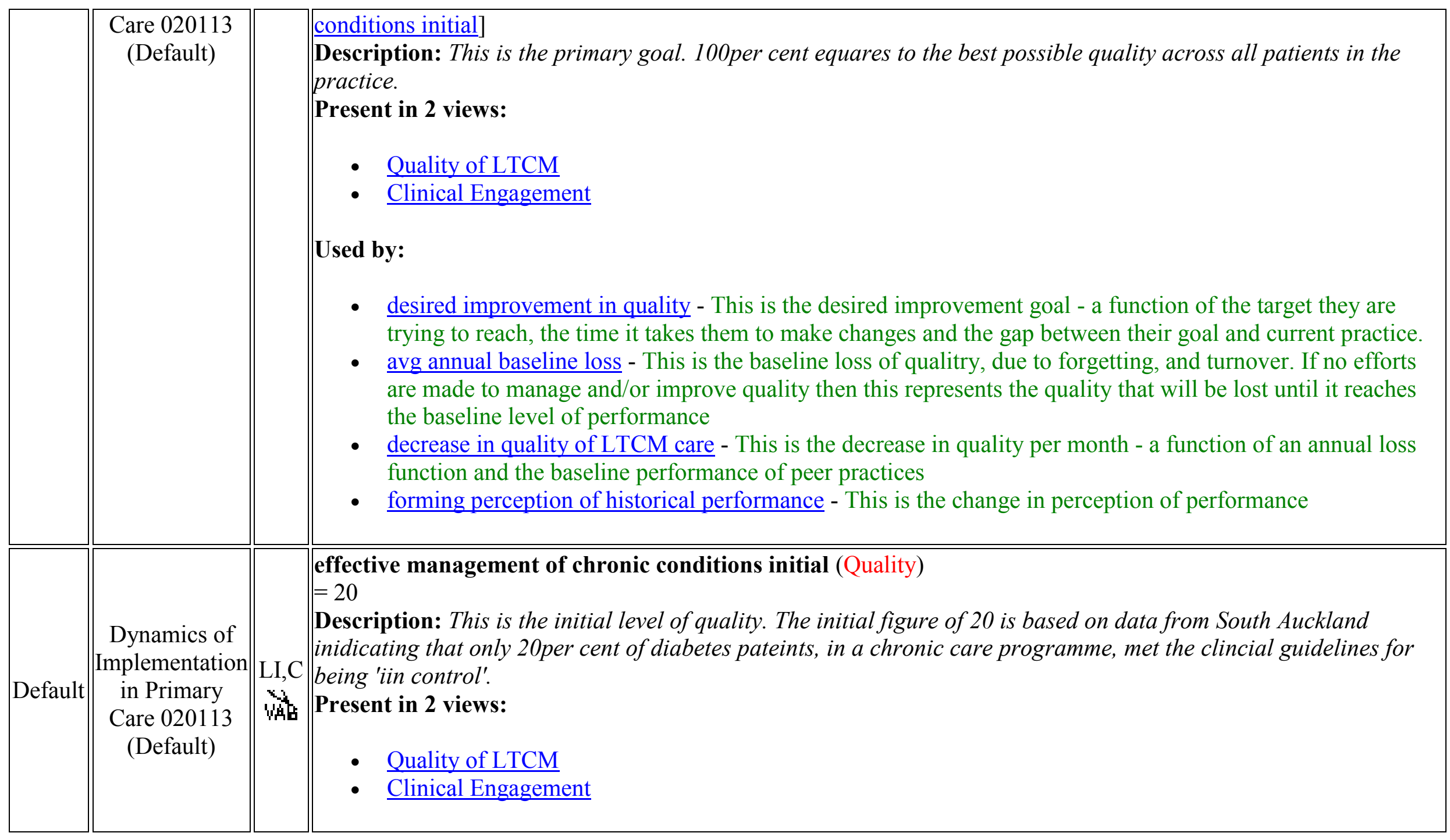




\begin{tabular}{|c|c|c|c|}
\hline & & & $\begin{array}{l}\text { Used by: } \\
\text { - } \frac{\text { Perceived Management of Chronic Conditions - This is the perception of effectiveness of LTC management }}{\text { - }} \text { poffective Management of Chronic Conditions }- \text { This is the primary goal. 100per cent equares to the best } \\
\text { possible quality across all patients in the practice. }\end{array}$ \\
\hline Default & \begin{tabular}{|l||} 
Dynamics of \\
Implementation \\
in Primary \\
Care 020113 \\
(Default)
\end{tabular} & $\begin{array}{c}\mathrm{C} \\
\text { VAB }\end{array}$ & $\begin{array}{l}\text { engagement switch (Dmnl }[0,1,1]) \\
=1 \\
\text { Description: Scenario switch - switches the impact of perceived performance on clinical engagement on/off } \\
\text { Present in } 1 \text { view: } \\
\text { - Clinical Engagement } \\
\text { Used by: } \\
\text { - change in level of clinical engagement - The change, per month, in the level of clinical engagement }\end{array}$ \\
\hline
\end{tabular}




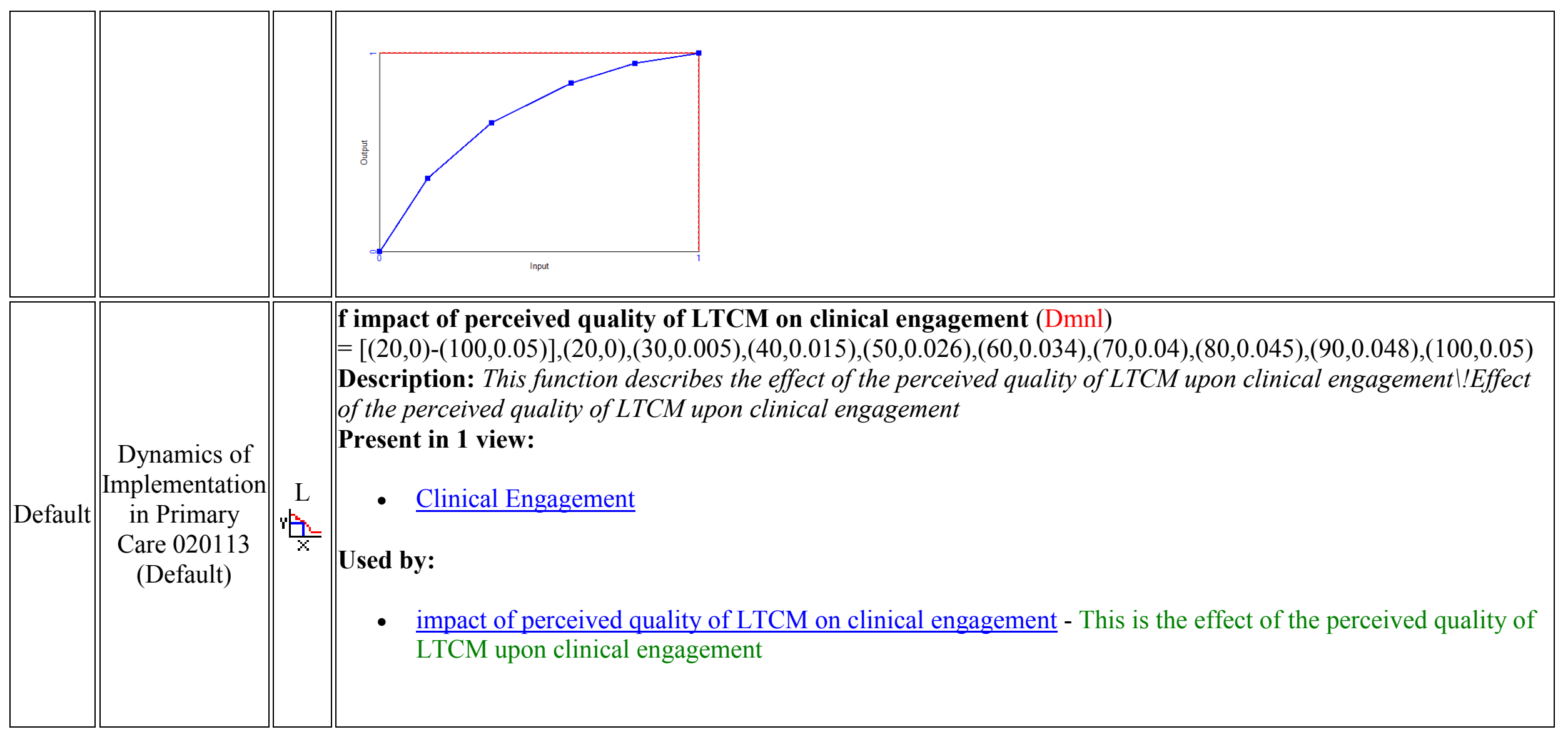




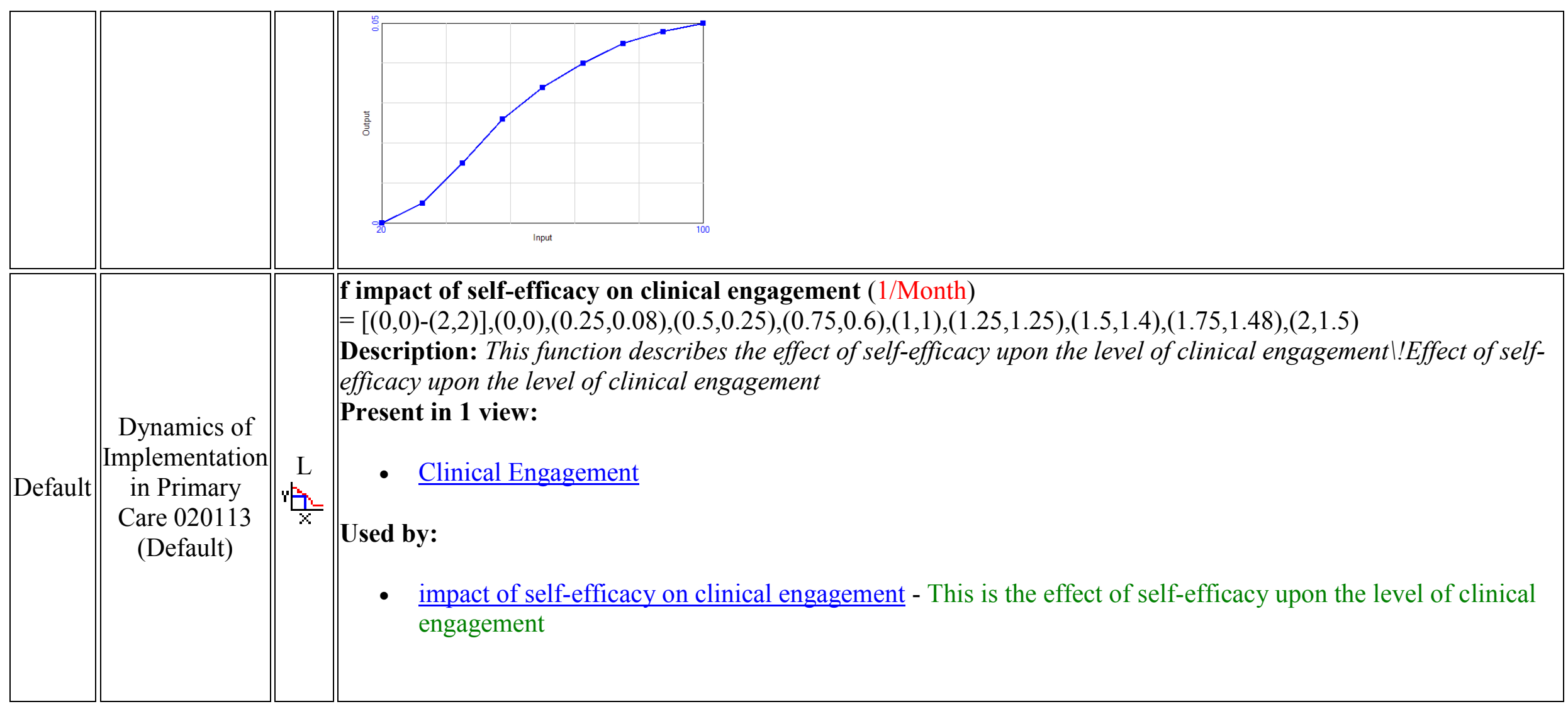




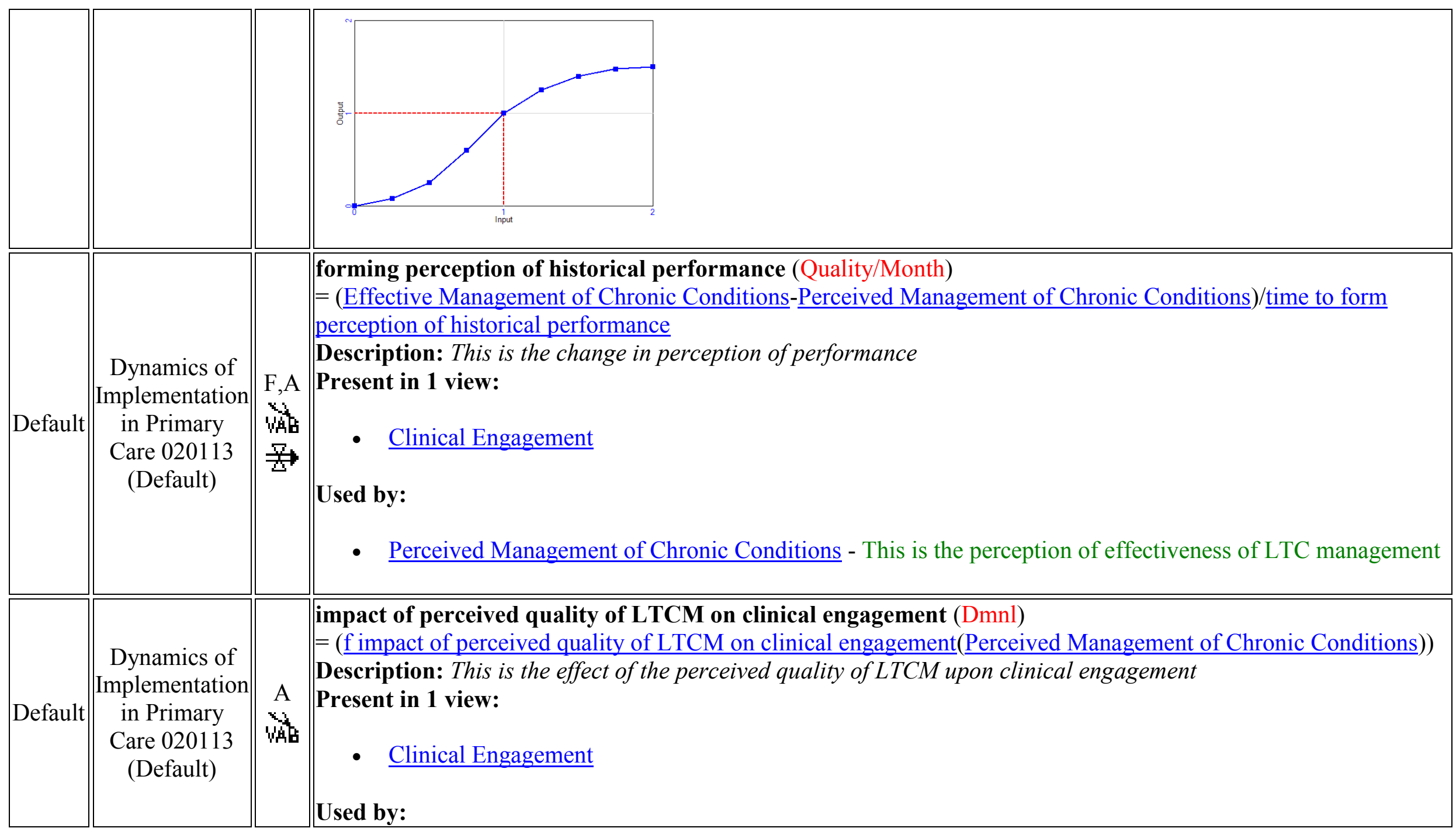




\begin{tabular}{|c|c|c|c|}
\hline & & & - change in level of clinical engagement - The change, per month, in the level of clinical engagement \\
\hline Default & $\mid \begin{array}{c}\text { Dynamics of } \\
\text { Implementation } \\
\text { in Primary } \\
\text { Care } 020113 \\
\text { (Default) }\end{array}$ & $\begin{array}{c}\text { A } \\
\text { VAt }\end{array}$ & $\begin{array}{l}\text { impact of self-efficacy on clinical engagement (1/Month) } \\
=\text { f impact of self-efficacy on clinical engagement(Self-efficacy) } \\
\text { Description: This is the effect of self-efficacy upon the level of clinical engagement } \\
\text { Present in } 1 \text { view: } \\
\text { - Clinical Engagement } \\
\text { Used by: } \\
\text { - change in level of clinical engagement - The change, per month, in the level of clinical engagement }\end{array}$ \\
\hline Default & $\mid \begin{array}{c}\text { Dynamics of } \\
\text { Implementation } \\
\text { in Primary } \\
\text { Care } 020113 \\
\text { (Default) }\end{array}$ & $\begin{array}{l}\text { SM } \\
\text { 离出 }\end{array}$ & 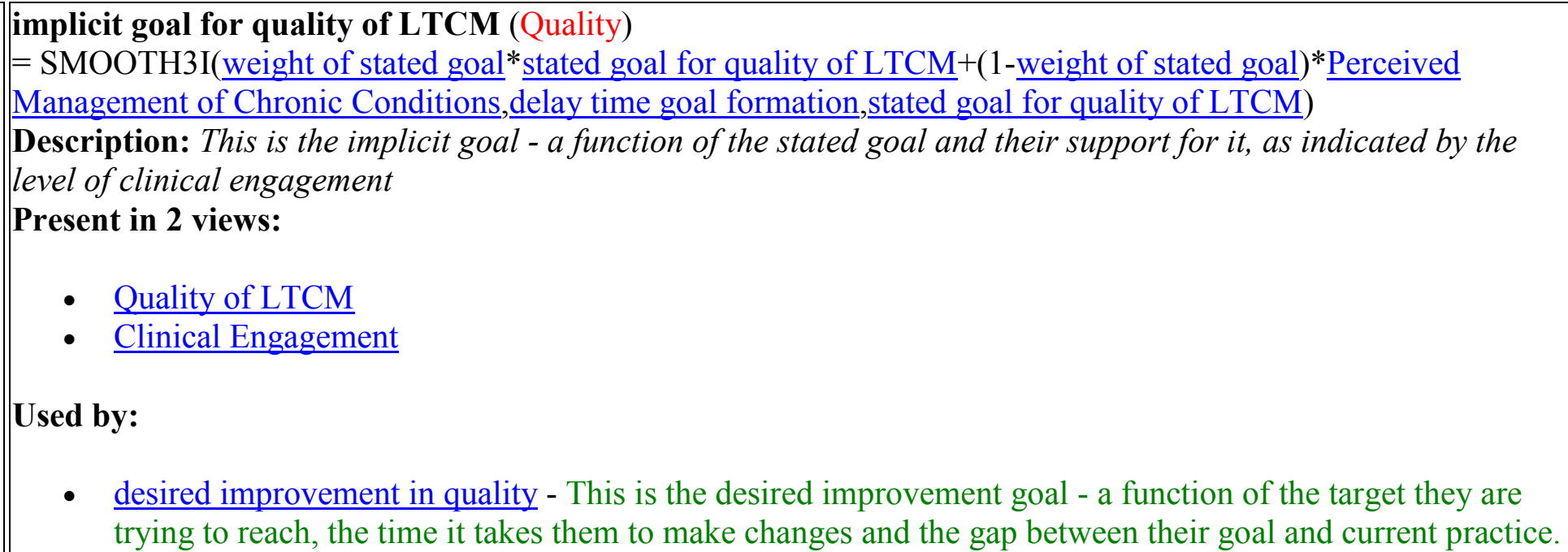 \\
\hline
\end{tabular}




\begin{tabular}{|c|c|c|c|}
\hline Default & \begin{tabular}{|c||} 
Dynamics of \\
Implementation \\
in Primary \\
Care 020113 \\
(Default)
\end{tabular} & $\begin{array}{c}\mathrm{L} \\
\mathrm{x} \\
\mathrm{M}\end{array}$ & 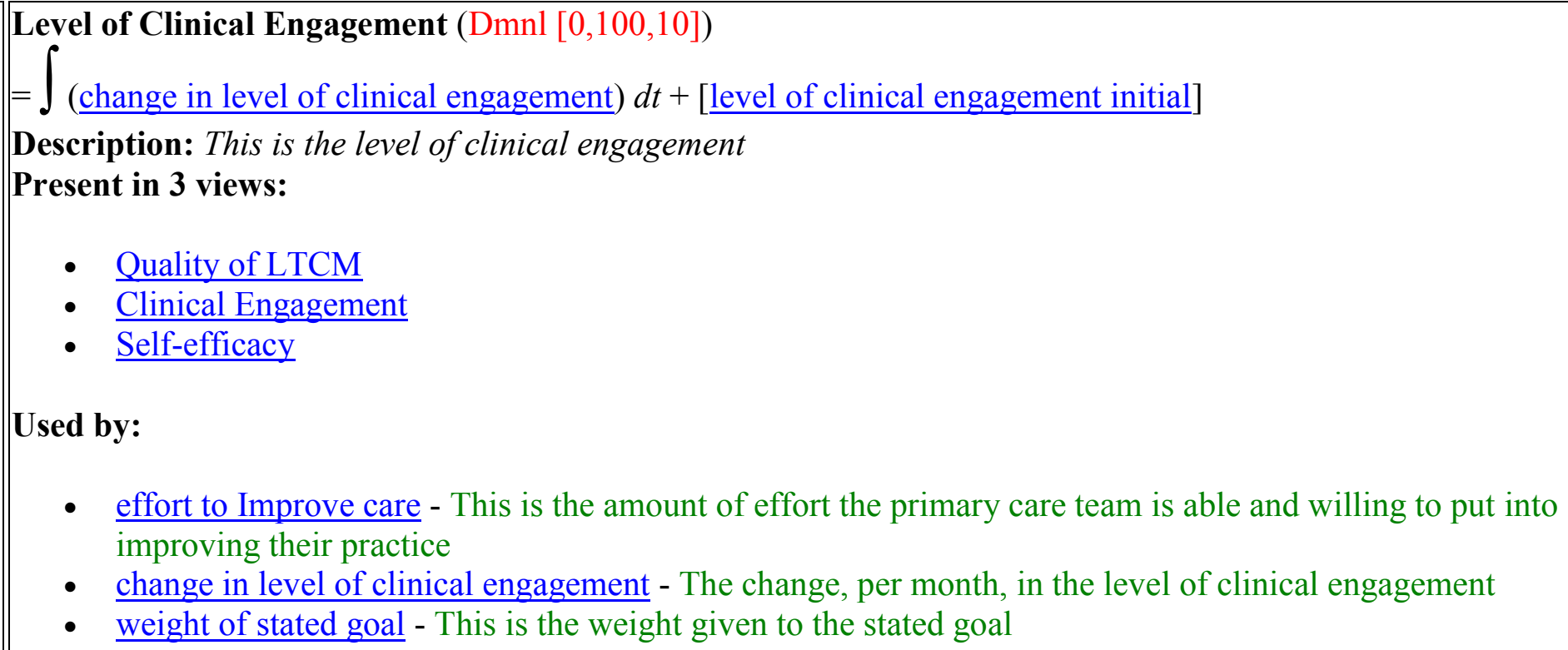 \\
\hline Default & Dynamics of & C & max level of clinical engagementt (Dmnl $[0,1,0.1])$ \\
\hline
\end{tabular}




\begin{tabular}{|c|c|c|c|}
\hline & \begin{tabular}{|c||} 
Implementation \\
in Primary \\
Care 020113 \\
(Default)
\end{tabular} & Vin & $\begin{array}{l}\text { 1 } \\
\text { Description: max possible level of clinical engagement } \\
\text { Present in } 1 \text { view: } \\
\quad \text { Clinical Engagement } \\
\text { Used by: } \\
\quad \text { change in level of clinical engagement - The change, per month, in the level of clinical engagement }\end{array}$ \\
\hline Default & \begin{tabular}{|c||} 
Dynamics of \\
Implementation \\
in Primary \\
Care 020113 \\
(Default)
\end{tabular} & $\begin{array}{c}\mathrm{L} \\
\mathrm{m}\end{array}$ & 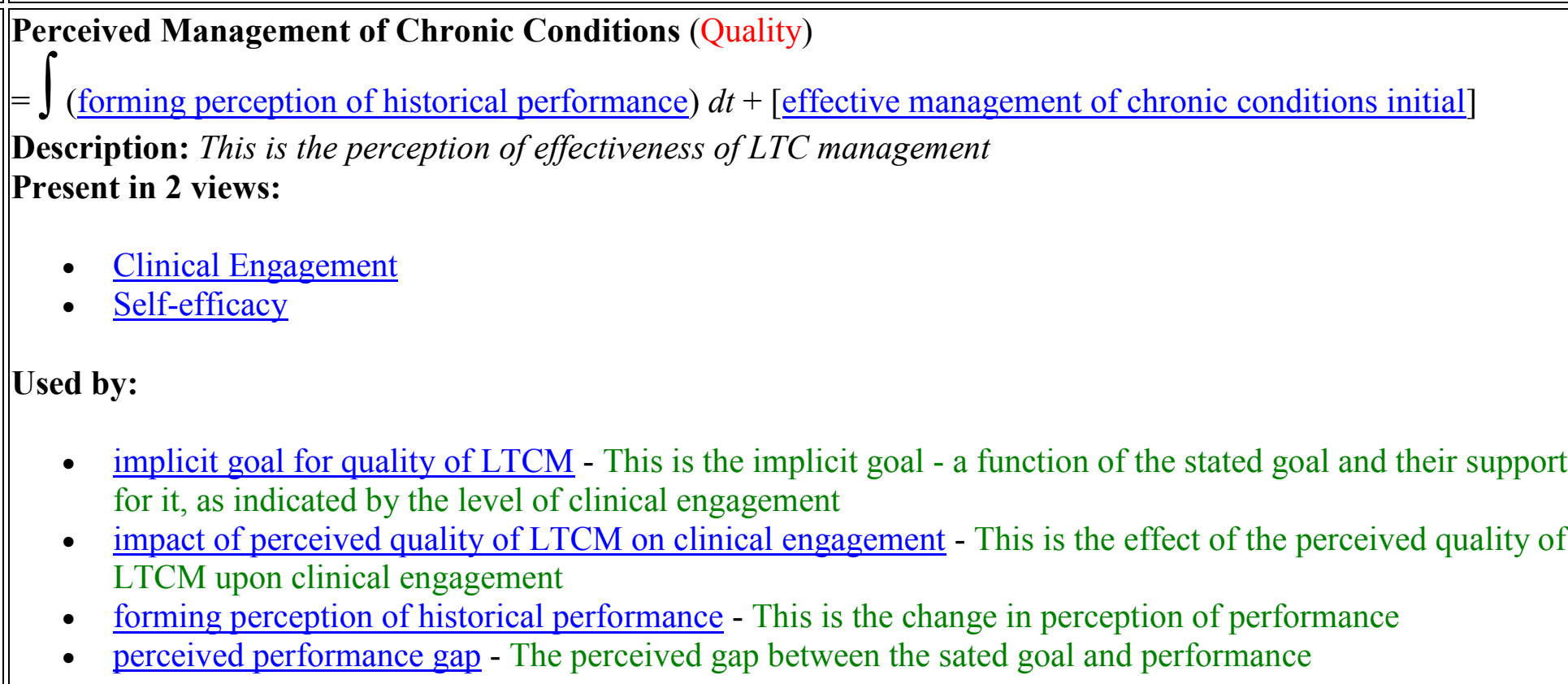 \\
\hline Default & Dynamics of & $\overline{\mathrm{L}}$ & Self-efficacy (Dmnl) \\
\hline
\end{tabular}




\begin{tabular}{|c|c|c|c|}
\hline & $\begin{array}{c}\text { Implementation } \\
\text { in Primary } \\
\text { Care } 020113 \\
\text { (Default) }\end{array}$ & 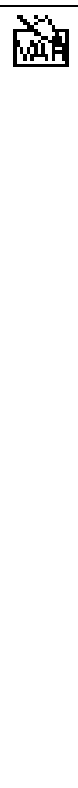 & $\begin{array}{l}\left.=\int \text { (change in self-efficacy-self-efficacy depletion }\right) d t+[\text { change in self-efficacy] } \\
\text { Description: This is the strength of the belief that the change is possible (self-efficacy) } \\
\text { Present in } 3 \text { views: } \\
\text { - } \frac{\text { Quality of LTCM }}{\text { - }} \text { Slinical Engagement } \\
\text { Used by: } \\
\text { - } \frac{\text { effort to Improve care - This is the amount of effort the primary care team is able and willing to put into }}{\text { improving their practice }} \\
\text { - } \frac{\text { self-efficacy depletion - The depletion of self-efficacy within the timeframe of the gaol being set }}{\text { impact of self-efficacy on clinical engagement }- \text { This is the effect of self-efficacy upon the level of clinical }} \\
\text { engagement }\end{array}$ \\
\hline
\end{tabular}




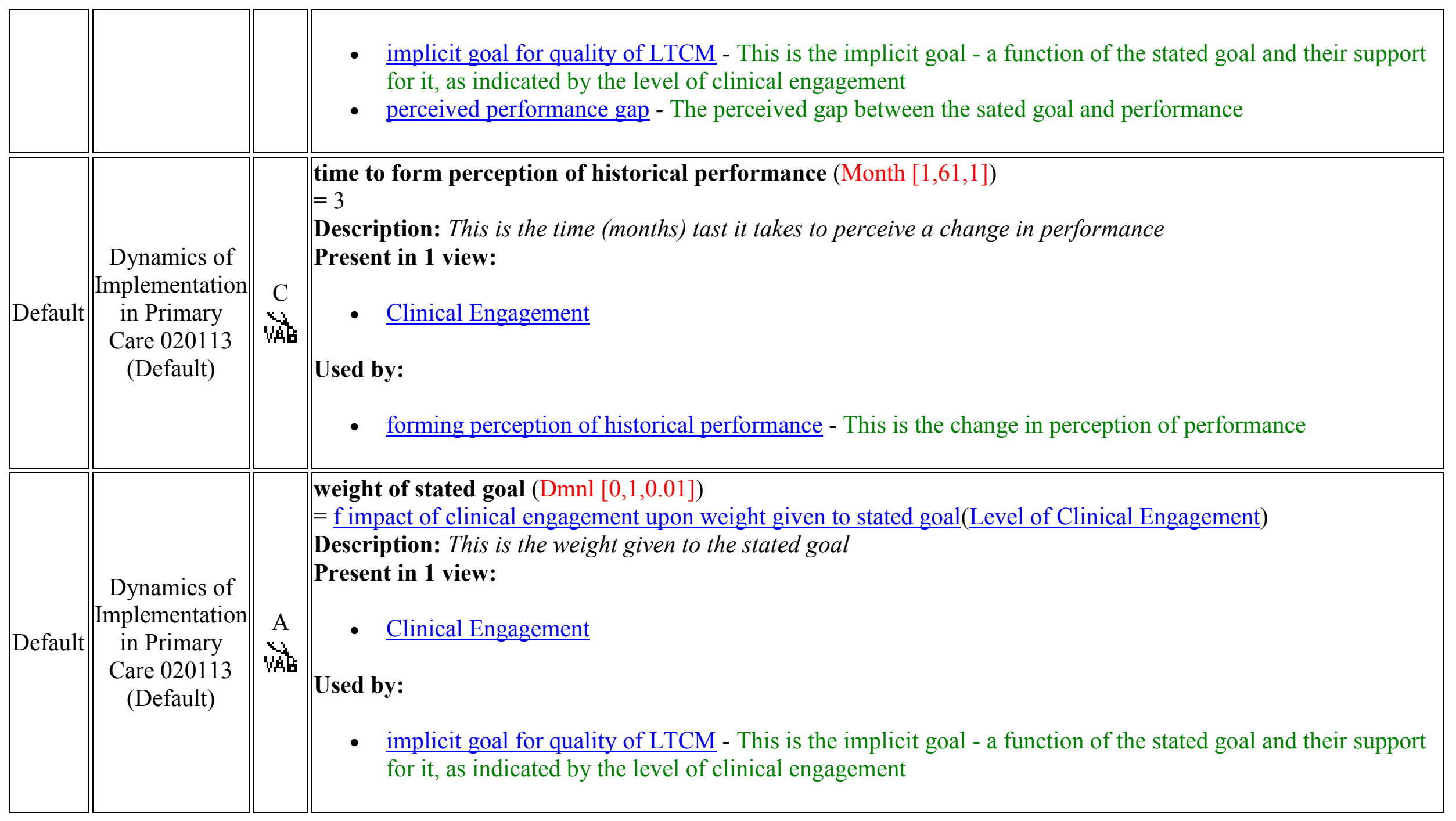


top Self-efficacy (23 variables)

Self Efficacy

HOME

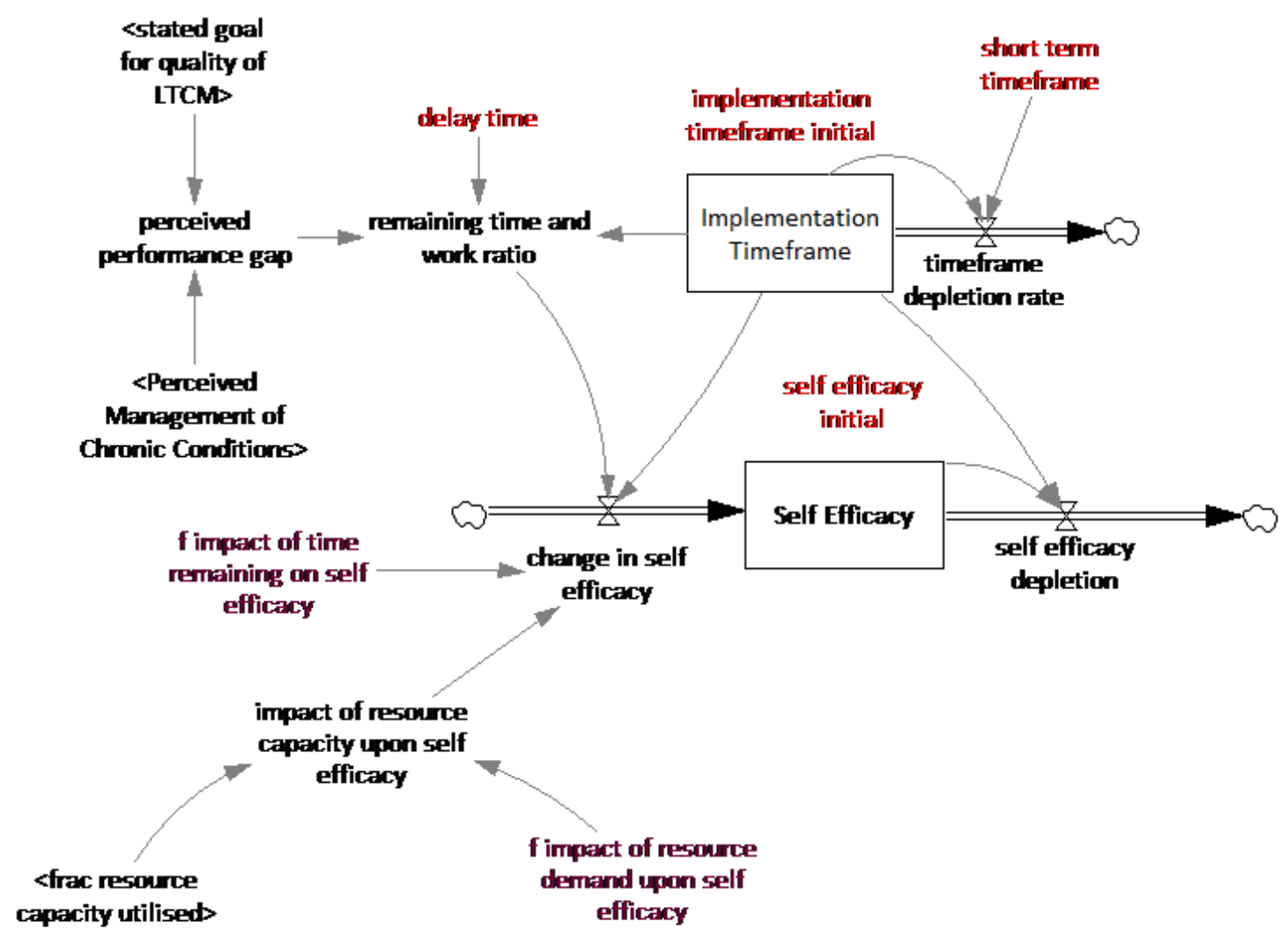

Appendix 4: Model Documentation 


\begin{tabular}{|c|c|c|c|}
\hline Module & Group & Type & Variable Name and Description \\
\hline Default & $\begin{array}{c}\text { Dynamics of } \\
\text { Implementation } \\
\text { in Primary } \\
\text { Care } 020113 \\
\text { (Default) }\end{array}$ & $\begin{array}{c}\text { LI,F,A } \\
\text { VAB } \\
\text { 荧 }\end{array}$ & 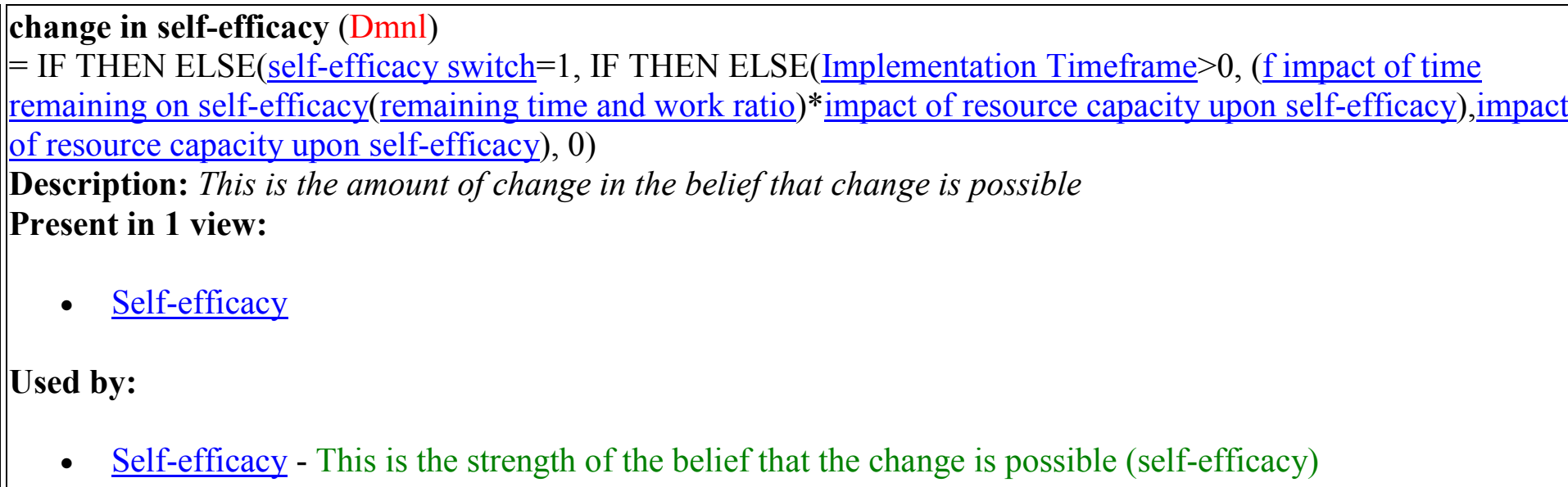 \\
\hline Default & $\begin{array}{c}\text { Dynamics of } \\
\text { Implementation } \\
\text { in Primary } \\
\text { Care } 020113 \\
\text { (Default) }\end{array}$ & $\begin{array}{c}\mathrm{C} \\
\mathrm{W} \\
\mathrm{V} / \mathrm{b}\end{array}$ & $\begin{array}{l}\text { delay time (Jolly et al.) } \\
=3 \\
\text { Description: The time, in months, before the perceived performance gap has an effect upon the remaining time } \\
\text { and work ratio } \\
\text { Present in } 1 \text { view: } \\
\text { - Self-efficacy } \\
\text { Used by: } \\
\text { - } \frac{\text { remaining time and work ratio }- \text { This is the ratio between the remaining time (months) and the amount (in }}{\text { months) of work still to do to reach the goal }}\end{array}$ \\
\hline Default & Dynamics of & $\mathrm{G}$ & effort to Improve care (Effort) \\
\hline
\end{tabular}




\begin{tabular}{|c|c|c|c|}
\hline & $\begin{array}{c}\text { Implementation } \\
\text { in Primary } \\
\text { Care 020113 } \\
\text { (Default) }\end{array}$ & 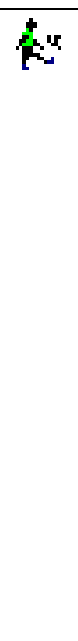 & $\begin{array}{l}\text { = GAME (IF THEN ELSE( }(\text { effort to improve switch }=1 \text {, (maximum improvement capacity/12)*(Level of Clinical } \\
\text { Engagement)*(Self-efficacy), maximum improvement capacity/12)) } \\
\text { Description: This is the amount of effort the primary care team is able and willing to put into improving their } \\
\text { practice } \\
\text { Present in } 2 \text { views: } \\
\text { - Quality of LTCM } \\
\text { - } \underline{\text { Self-efficacy }} \\
\text { Used by: } \\
\text { - } \frac{\text { effect of improvement effort - This is the effect the improvement effort will have on the change in }}{\text { manaement of LTCs }}\end{array}$ \\
\hline Default & $\mid \begin{array}{c}\text { Dynamics of } \\
\text { Implementation } \\
\text { in Primary } \\
\text { Care } 020113 \\
\text { (Default) }\end{array}$ & $\begin{array}{c}\mathrm{C} \\
\mathrm{W} / \mathrm{B}\end{array}$ & 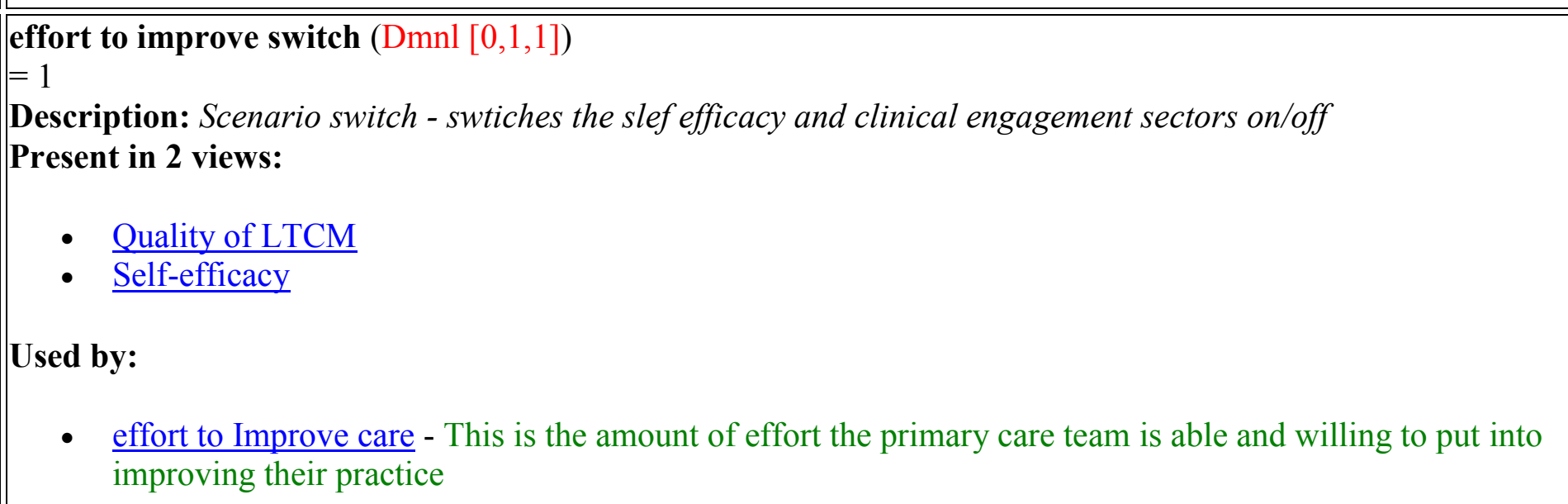 \\
\hline Default & $\begin{array}{c}\text { Dynamics of } \\
\text { Implementation }\end{array}$ & $\mathrm{L}$ & $\begin{array}{l}\text { f impact of resource demand upon self-efficacy }(\mathrm{Dmnl}) \\
=[(0.5,0)-(1.5,2)],(0.5,1.3),(0.65,1.25),(0.8,1.14),(1,0.9),(1.2,0.6),(1.35,0.43),(1.5,0.38)\end{array}$ \\
\hline
\end{tabular}




\begin{tabular}{|c|c|c|c|}
\hline & $\begin{array}{c}\text { in Primary } \\
\text { Care } 020113 \\
\text { (Default) }\end{array}$ & $\frac{{ }^{2}}{x}$ & $\begin{array}{l}\text { Description: This function describes the effect of resource demand upon self-efficacyl!Effect of resource demand } \\
\text { upon self-efficacy } \\
\text { Present in } 1 \text { view: } \\
\text { - Self-efficacy } \\
\text { Used by: } \\
\text { - impact of resource capacity upon self-efficacy - This is the effect of resource demand upon self-efficacy }\end{array}$ \\
\hline
\end{tabular}




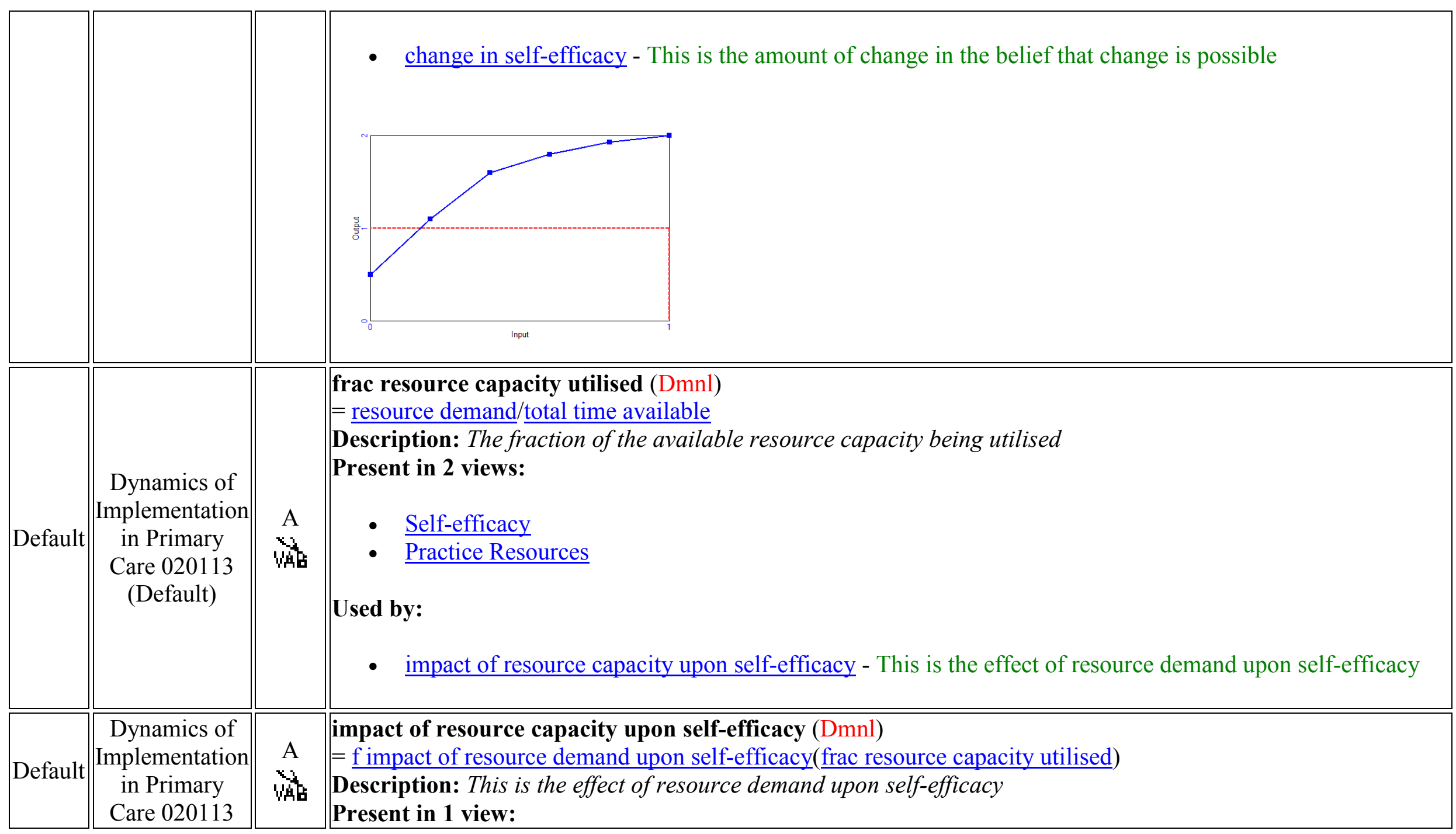




\begin{tabular}{|c|c|c|c|}
\hline & (Default) & & $\begin{array}{l}\text { - } \underline{\text { Self-efficacy }} \\
\text { Used by: } \\
\text { - change in self-efficacy - This is the amount of change in the belief that change is possible }\end{array}$ \\
\hline Default & \begin{tabular}{|} 
Dynamics of \\
Implementation \\
in Primary \\
Care 020113 \\
(Default)
\end{tabular} & 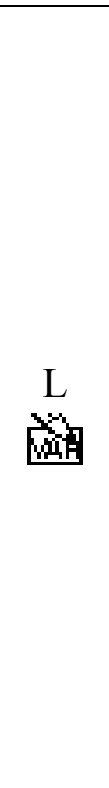 & 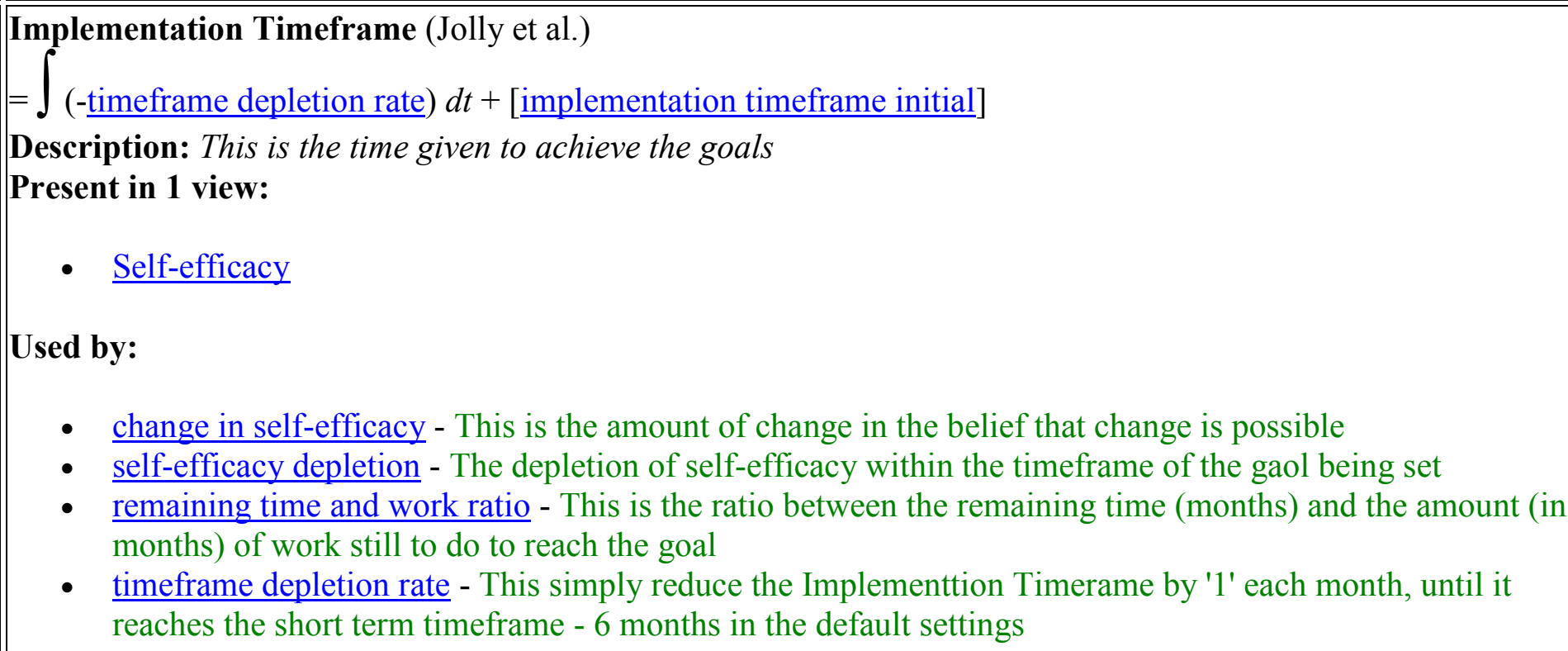 \\
\hline Default & \begin{tabular}{|c||} 
Dynamics of \\
Implementation \\
in Primary \\
Care 020113 \\
(Default)
\end{tabular} & $\begin{array}{c}\text { LI,C } \\
\text { VA⿱ }\end{array}$ & $\begin{array}{l}\text { implementation timeframe initial (Month }[0,60,1] \text { ) } \\
=36 \\
\text { Description: This is the time (months) given to achieve the goal - initial setting } \\
\text { Present in } 1 \text { view: }\end{array}$ \\
\hline
\end{tabular}




\begin{tabular}{|c|c|c|c|}
\hline & & & 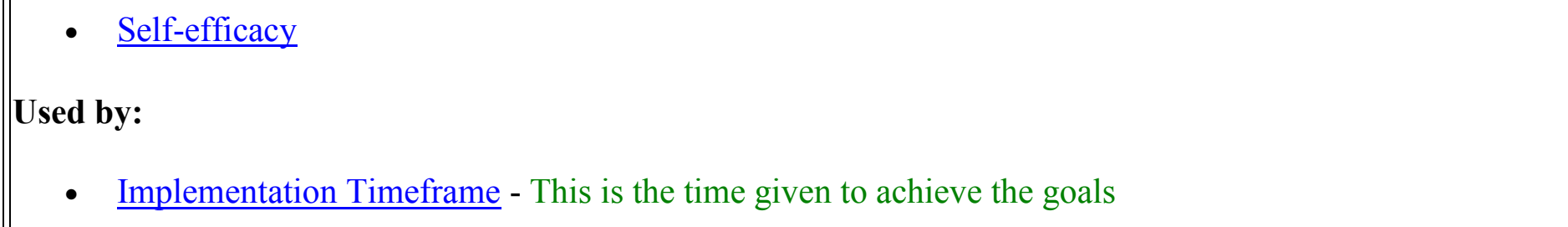 \\
\hline Default & \begin{tabular}{|} 
Dynamics of \\
Implementation \\
in Primary \\
Care 020113 \\
(Default)
\end{tabular} & $\begin{array}{c}\mathrm{L} \\
\text { 緭 }\end{array}$ & $\begin{array}{l}\text { Level of Clinical Engagement (Dmnl }[0,100,10]) \\
\left.=\int \text { (change in level of clinical engagement }\right) d t+[\text { level of clinical engagement initial }] \\
\text { Description: This is the level of clinical engagement } \\
\text { Present in } 3 \text { views: } \\
\text { - Quality of LTCM } \\
\text { - } \underline{\text { Clinical Engagement }} \\
\text { Used by: } \\
\text { - } \frac{\text { effort to Improve care - This is the amount of effort the primary care team is able and willing to put into }}{\text { improving their practice }} \\
\text { - } \frac{\text { change in level of clinical engagement - The change, per month, in the level of clinical engagement }}{\text { - weight of stated goal - This is the weight given to the stated goal }}\end{array}$ \\
\hline Default & \begin{tabular}{|l||} 
Dynamics of \\
Implementation \\
in Primary \\
Care 020113 \\
(Default)
\end{tabular} & $\begin{array}{c}\mathrm{C} \\
\mathrm{VAb}\end{array}$ & $\begin{array}{l}\text { maximum improvement capacity (Effort }[1,200,1] \text { ) } \\
=30 \\
\text { Description: This is the maximum capacity the practice has to make changes to quality. Equates to 'adaptive } \\
\text { reserve' described in NDP - patient-centred medical home papers } 2010 . \\
\text { Present in } 2 \text { views: }\end{array}$ \\
\hline
\end{tabular}




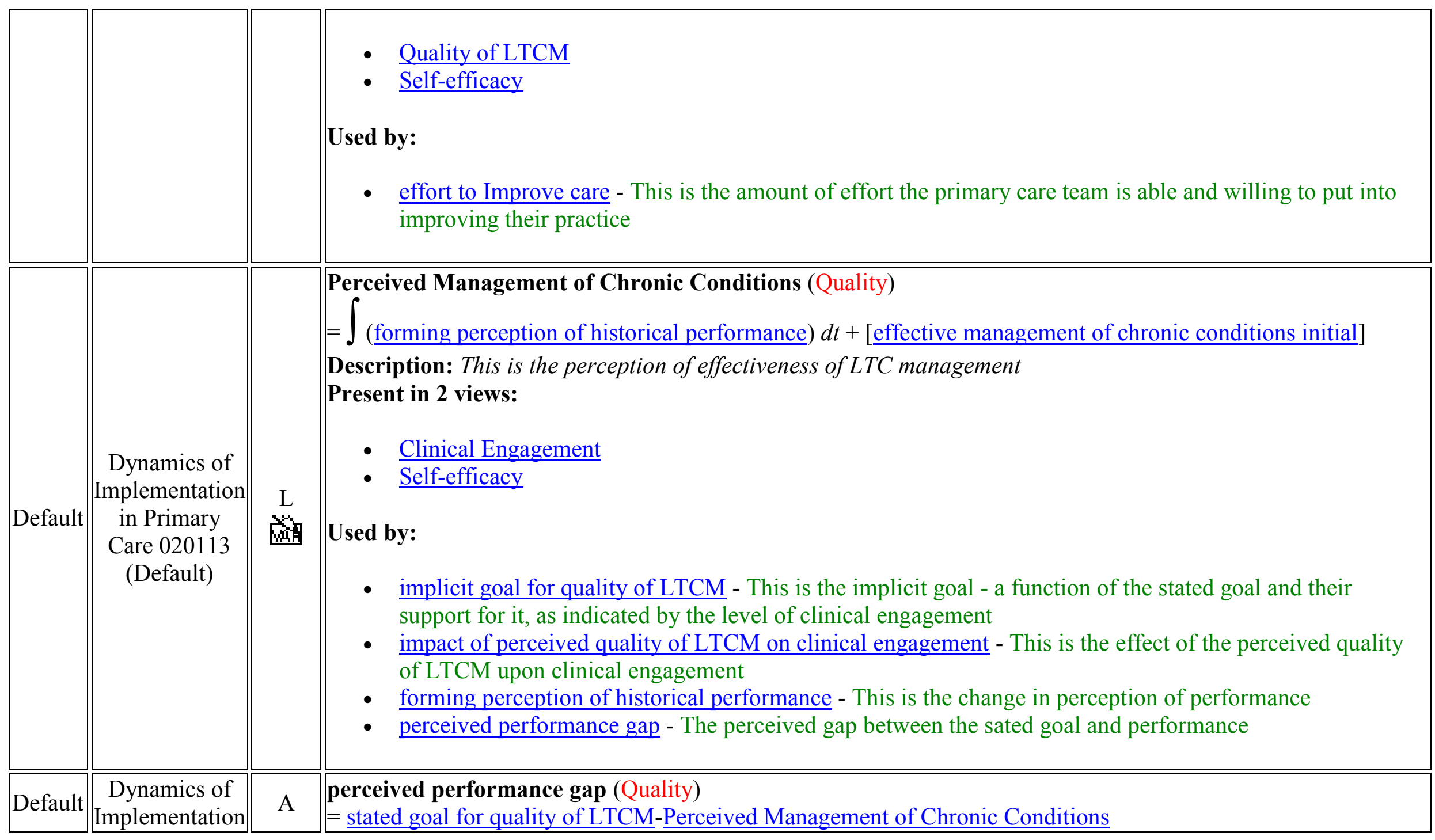




\begin{tabular}{|c|c|c|c|}
\hline & $\begin{array}{c}\text { in Primary } \\
\text { Care } 020113 \\
\text { (Default) }\end{array}$ & VAb & $\begin{array}{l}\text { Description: The perceived gap between the sated goal and performance } \\
\text { Present in } 1 \text { view: } \\
\text { - Self-efficacy } \\
\text { Used by: } \\
\text { - } \frac{\text { remaining time and work ratio - This is the ratio between the remaining time (months) and the amount (in }}{\text { months) of work still to do to reach the goal }}\end{array}$ \\
\hline Default & $\mid \begin{array}{c}\text { Dynamics of } \\
\text { Implementation } \\
\text { in Primary } \\
\text { Care } 020113 \\
\text { (Default) }\end{array}$ & $\begin{array}{l}\text { SM } \\
\text { 站 }\end{array}$ & $\begin{array}{l}\text { remaining time and work ratio (Month/Quality) } \\
\text { = SMOOTH3( }(\underline{\text { Implementation Timeframe }}+1) / \text { perceived performance gap, }, \text { delay time }) \\
\text { Description: This is the ratio between the remaining time (months) and the amount (in months) of work still to do } \\
\text { to reach the goal } \\
\text { Present in } 1 \text { view: } \\
\quad \text { - Self-efficacy } \\
\text { Used by: } \\
\quad \text { change in self-efficacy - This is the amount of change in the belief that change is possible }\end{array}$ \\
\hline Default & $\mid \begin{array}{c}\text { Dynamics of } \\
\text { Implementation } \\
\text { in Primary } \\
\text { Care } 020113 \\
\text { (Default) }\end{array}$ & $\begin{array}{c}\mathrm{L} \\
\mathrm{W}\end{array}$ & $\begin{array}{l}\text { Self-efficacy (Dmnl) } \\
\left.=\int_{\text {(change in self-efficacy-self-efficacy depletion }}\right) d t+\text { [change in self-efficacy] } \\
\text { Description: This is the strength of the belief that the change is possible (self-efficacy) } \\
\text { Present in } 3 \text { views: } \\
\quad \text { Quality of LTCM }\end{array}$ \\
\hline
\end{tabular}




\begin{tabular}{|c|c|c|c|}
\hline & & & $\begin{array}{l}\text { - } \text { Clinical Engagement } \\
\text { Used by: } \\
\text { - } \frac{\text { effort to Improve care - This is the amount of effort the primary care team is able and willing to put into }}{\text { improving their practice }} \\
\text { - } \frac{\text { self-efficacy depletion - The depletion of self-efficacy within the timeframe of the gaol being set }}{\text { impact of self-efficacy on clinical engagement - This is the effect of self-efficacy upon the level of clinical }} \\
\text { engagement }\end{array}$ \\
\hline Default & \begin{tabular}{|} 
Dynamics of \\
Implementation \\
in Primary \\
Care 020113 \\
(Default)
\end{tabular} & 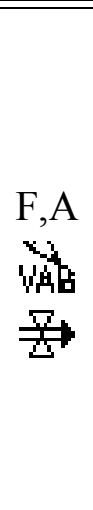 & $\begin{array}{l}\text { self-efficacy depletion (Dmnl) } \\
\text { = IF THEN ELSE(Implementation Timeframe }>0 \text {, Self-efficacy, } 0) \\
\text { Description: The depletion of self-efficacy within the timeframe of the gaol being set } \\
\text { Present in } 1 \text { view: } \\
\text { - Self-efficacy } \\
\text { Used by: } \\
\text { - Self-efficacy - This is the strength of the belief that the change is possible (self-efficacy) }\end{array}$ \\
\hline Default & \begin{tabular}{|} 
Dynamics of \\
Implementation \\
in Primary \\
Care 020113 \\
(Default)
\end{tabular} & $\begin{array}{c}\mathrm{C} \\
\mathrm{VA} \\
\mathrm{VAB}\end{array}$ & $\begin{array}{l}\text { self-efficacy initial (Dmnl) } \\
=1 \\
\text { Description: The strength of the belief that change is possible - intial setting } \\
\text { Present in } 1 \text { view: } \\
\text { - Self-efficacy }\end{array}$ \\
\hline
\end{tabular}




\begin{tabular}{|c|c|c|c|}
\hline Default & \begin{tabular}{|} 
Dynamics of \\
Implementation \\
in Primary \\
Care 020113 \\
(Default)
\end{tabular} & $\begin{array}{c}C \\
\text { VA }\end{array}$ & $\begin{array}{l}\text { self-efficacy switch (Dmnl }[0,1,1]) \\
=1 \\
\text { Description: Scenario switch - switches the impact of resource capacity on self-efficacy on/off } \\
\text { Present in } 1 \text { view: } \\
\text { - Self-efficacy } \\
\text { Used by: } \\
\quad \text { change in self-efficacy - This is the amount of change in the belief that change is possible }\end{array}$ \\
\hline Default & \begin{tabular}{|} 
Dynamics of \\
Implementation \\
in Primary \\
Care 020113 \\
(Default)
\end{tabular} & $\begin{array}{c}\mathrm{C} \\
\mathrm{W} \\
\mathrm{W}\end{array}$ & $\begin{array}{l}\text { short term timeframe (Month }[0,12,1] \text { ) } \\
=6 \\
\text { Description: The short term timeframe is the timeframe over which performance is managed. } \\
\text { Present in } 1 \text { view: } \\
\text { - Self-efficacy } \\
\text { Used by: } \\
\text { - } \frac{\text { timeframe depletion rate - This simply reduce the Implementtion Timerame by ' } 1 \text { ' each month, until it }}{\text { reaches the short term timeframe - } 6 \text { months in the default settings }}\end{array}$ \\
\hline Default & \begin{tabular}{|} 
Dynamics of \\
Implementation \\
in Primary \\
Care 020113 \\
(Default)
\end{tabular} & $\begin{array}{l}\mathrm{G} \\
\mathrm{A}^{\circ}\end{array}$ & $\begin{array}{l}\text { stated goal for quality of LTCM (Quality }[0,100,10]) \\
=\text { GAME (80) } \\
\text { Description: This is the quality goal, as set by 'external parties'. This could be the Ministry of Health (MoH) or } \\
\text { those responsible for setting and delivering service targets in thier locality e.g. District Health Boards (DHBs) or } \\
\text { Primary Health Organisaitons (PHOs). } \\
\text { Present in } 2 \text { views: }\end{array}$ \\
\hline
\end{tabular}




\begin{tabular}{|c|c|c|c|}
\hline & & & 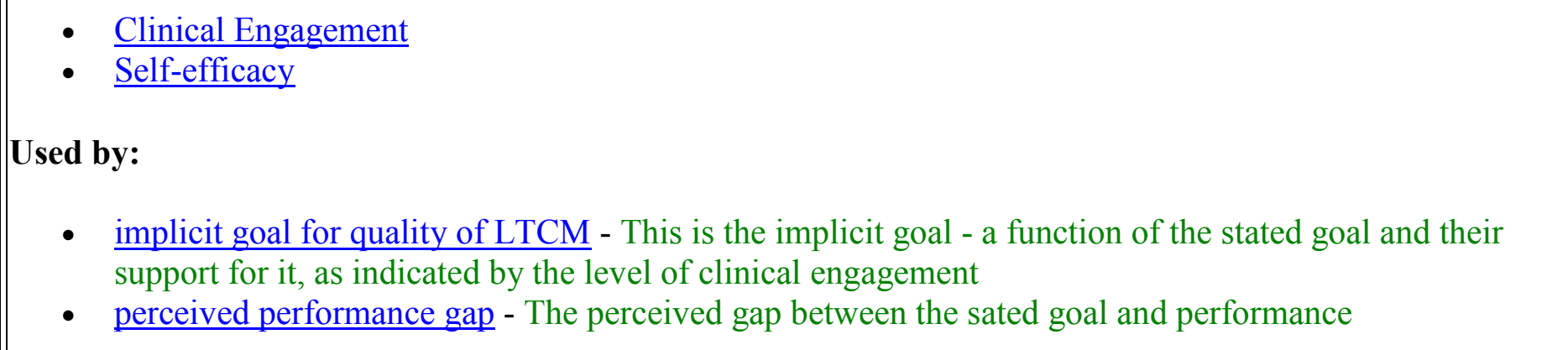 \\
\hline Default & $\begin{array}{c}\text { Dynamics of } \\
\text { Implementation } \\
\text { in Primary } \\
\text { Care } 020113 \\
\text { (Default) }\end{array}$ & $\begin{array}{l}\text { F,A } \\
\text { VAB } \\
\text { 居 }\end{array}$ & $\begin{array}{l}\text { timeframe depletion rate (Month/Month) } \\
\text { = IF THEN ELSE(Implementation Timeframe }>\text { short term timeframe, } 1,0) \\
\text { Description: This simply reduce the Implementtion Timerame by '1' each month, until it reaches the short term } \\
\text { timeframe - } 6 \text { months in the default settings } \\
\text { Present in } 1 \text { view: } \\
\text { - Self-efficacy } \\
\text { Used by: } \\
\text { - Implementation Timeframe - This is the time given to achieve the goals }\end{array}$ \\
\hline
\end{tabular}


rop Process Quality (9 variables)

\section{Process Quality}

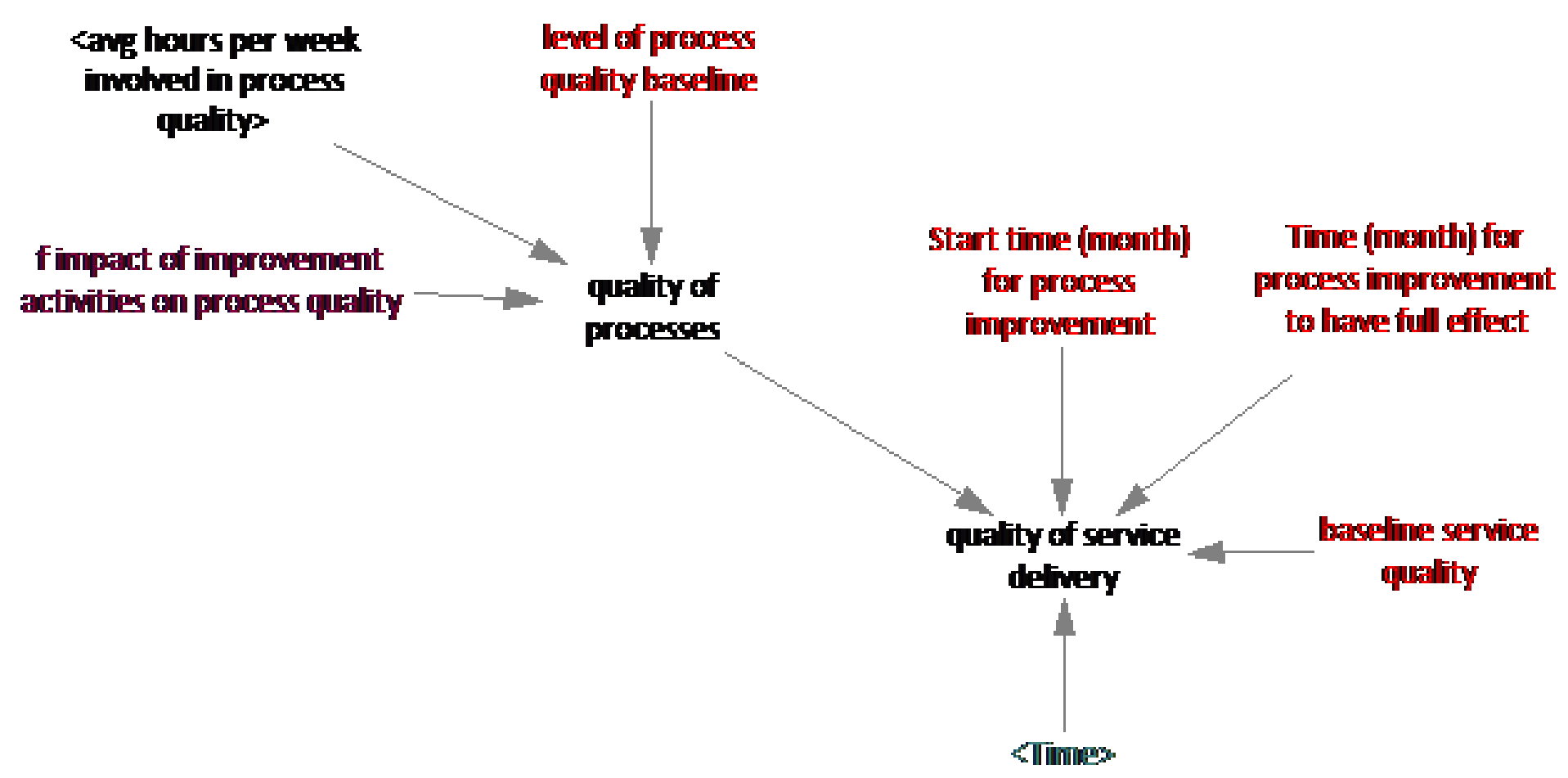




\begin{tabular}{|c|c|c|c|}
\hline Module & Group & Type & Variable Name and Description \\
\hline Default & $\mid \begin{array}{c}\text { Dynamics of } \\
\text { Implementation } \\
\text { in Primary } \\
\text { Care } 020113 \\
\text { (Default) }\end{array}$ & & $\begin{array}{l}\text { avg hours per week involved in process quality (Month }[0,10,1]) \\
=0 \\
\text { Description: The average hours per week per GP spend on process improvement activities } \\
\text { Present in } 2 \text { views: } \\
\text { - } \frac{\text { Process Quality }}{\text { Total Workload }} \\
\text { Used by: } \\
\text { - } \frac{\text { quality of processes - This is the current level of process quality }}{\text { img minutes per week involved in process improvement - Average time (minutes) per GP spent on process }}\end{array}$ \\
\hline Default & $\mid \begin{array}{c}\text { Dynamics of } \\
\text { Implementation } \\
\text { in Primary } \\
\text { Care } 020113 \\
\text { (Default) }\end{array}$ & $\begin{array}{c}C \\
\text { VA }\end{array}$ & $\begin{array}{l}\text { baseline service quality (Quality/Effort) } \\
=1 \\
\text { Description: This is the baseline quality of service delivery } \\
\text { Present in } 1 \text { view: } \\
\text { - Process Quality } \\
\text { Used by: } \\
\text { - quality of service delivery - This is the current quality of service delivery }\end{array}$ \\
\hline Default & $\mid \begin{array}{c}\text { Dynamics of } \\
\text { Implementation }\end{array}$ & 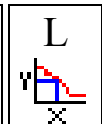 & $\begin{array}{l}\text { f impact of improvement activities on process quality (Dmnl) } \\
=[(0,1)-(10,2)],(0,1),(2,1.5),(4,1.7),(6,1.85),(8,1.95),(10,2)\end{array}$ \\
\hline
\end{tabular}




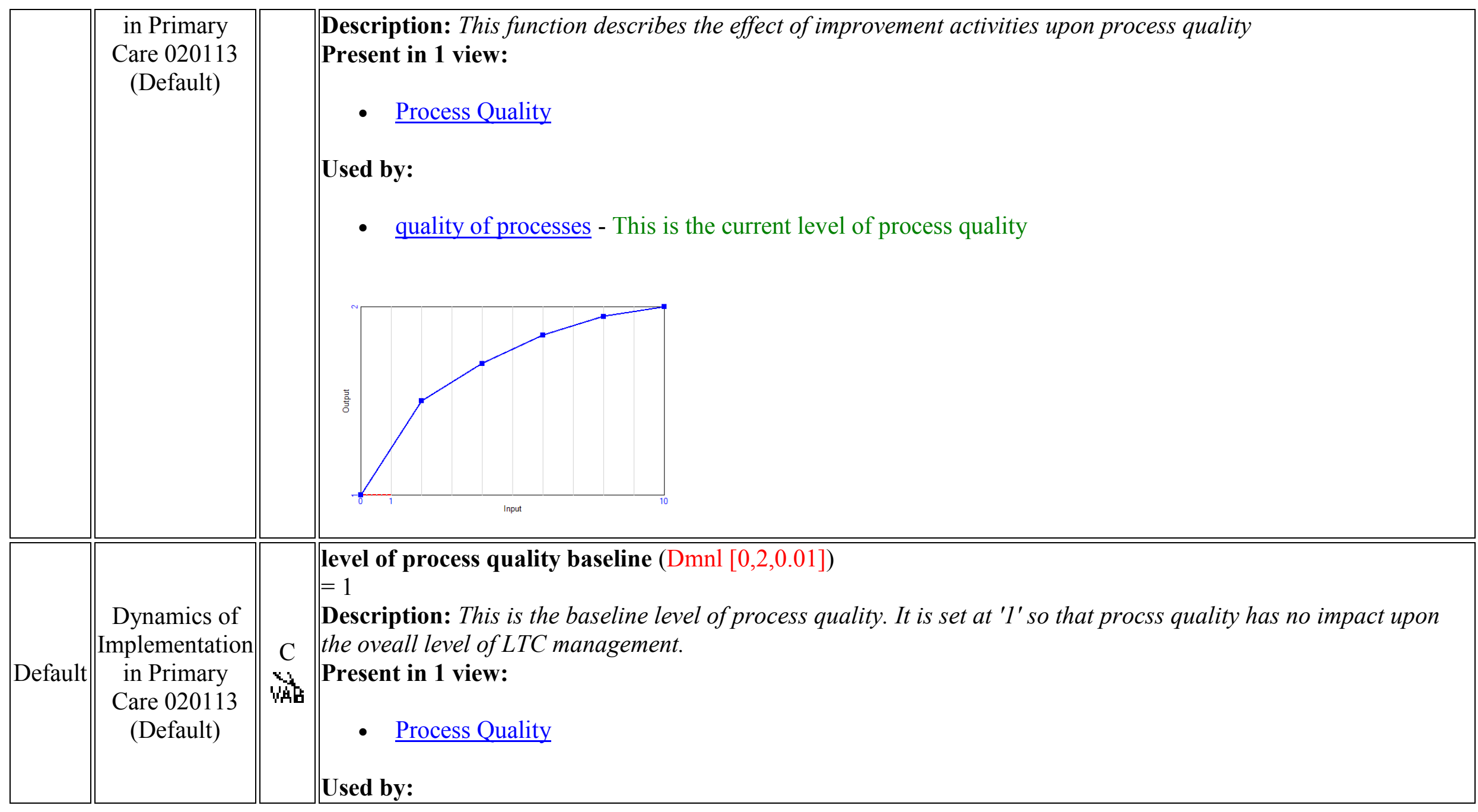




\begin{tabular}{|c|c|c|c|}
\hline & & & y of processes - This is the current level of process quality \\
\hline Default & \begin{tabular}{|c||} 
Dynamics of \\
Implementation \\
in Primary \\
Care 020113 \\
(Default)
\end{tabular} & $\begin{array}{c}A \\
\text { VA }\end{array}$ & $\begin{array}{l}\text { quality of processes (Dmnl) } \\
=\text { f impact of improvement activities on process quality (avg hours per week involved in process quality)*level of } \\
\text { process quality baseline } \\
\text { Description: This is the current level of process quality } \\
\text { Present in 1 view: } \\
\text { - Process Quality } \\
\text { Used by: } \\
\text { - quality of service delivery - This is the current quality of service delivery }\end{array}$ \\
\hline Default & \begin{tabular}{|c||} 
Dynamics of \\
Implementation \\
in Primary \\
Care 020113 \\
(Default)
\end{tabular} & $\begin{array}{c}A \\
\text { VA }\end{array}$ & $\begin{array}{l}\text { quality of service delivery (Quality/Effort) } \\
=\text { IF THEN ELSE(Time }<\text { Start time (month) for process improvement, baseline service quality, IF THEN } \\
\text { ELSE(Time }>=\text { Time (month) for process improvement to have full effect, quality of processes, baseline service } \\
\text { quality }+ \text { (quality of processes-baseline service quality)*(Time-Start time (month) for process improvement)/(Time } \\
\text { (month) for process improvement to have full effect-Start time (month) for process improvement)) }) \\
\text { Description: This is the current quality of service delivery } \\
\text { Present in } 2 \text { views: } \\
\text { - Quality of LTCM } \\
\text { - } \underline{\text { Process Quality }} \\
\text { Used by: } \\
\text { - effect of improvement effort - This is the effect the improvement effort will have on the change in }\end{array}$ \\
\hline
\end{tabular}




\begin{tabular}{|c|c|c|c|}
\hline & & & manaement of LTCs \\
\hline Default & \begin{tabular}{||c||} 
Dynamics of \\
Implementation \\
in Primary \\
Care 020113 \\
(Default)
\end{tabular} & $\begin{array}{c}\mathrm{C} \\
\mathrm{W} \\
\mathrm{VAB}\end{array}$ & $\begin{array}{l}\text { Time (Jolly et al.) for process improvement to have full effect (Month }[1,121,1] \text { ) } \\
=1 \\
\text { Description: This is the time at which the impact of the process improvement activity takes full efffect } \\
\text { Present in } 1 \text { view: } \\
\text { - Process Quality } \\
\text { Used by: } \\
\text { - quality of service delivery - This is the current quality of service delivery }\end{array}$ \\
\hline
\end{tabular}




\section{op Number of Patients (21 variables)}

Patient Numbers

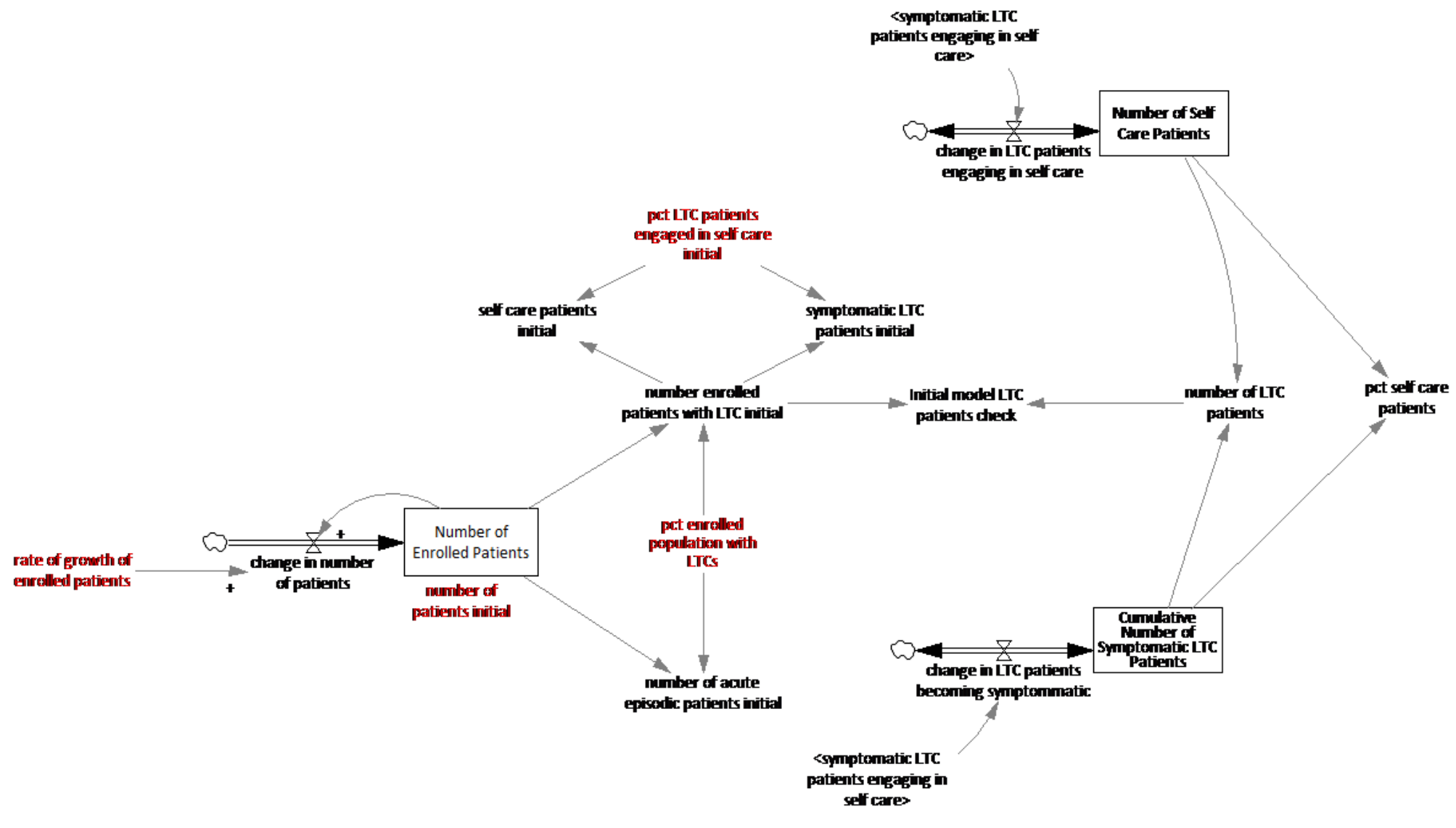




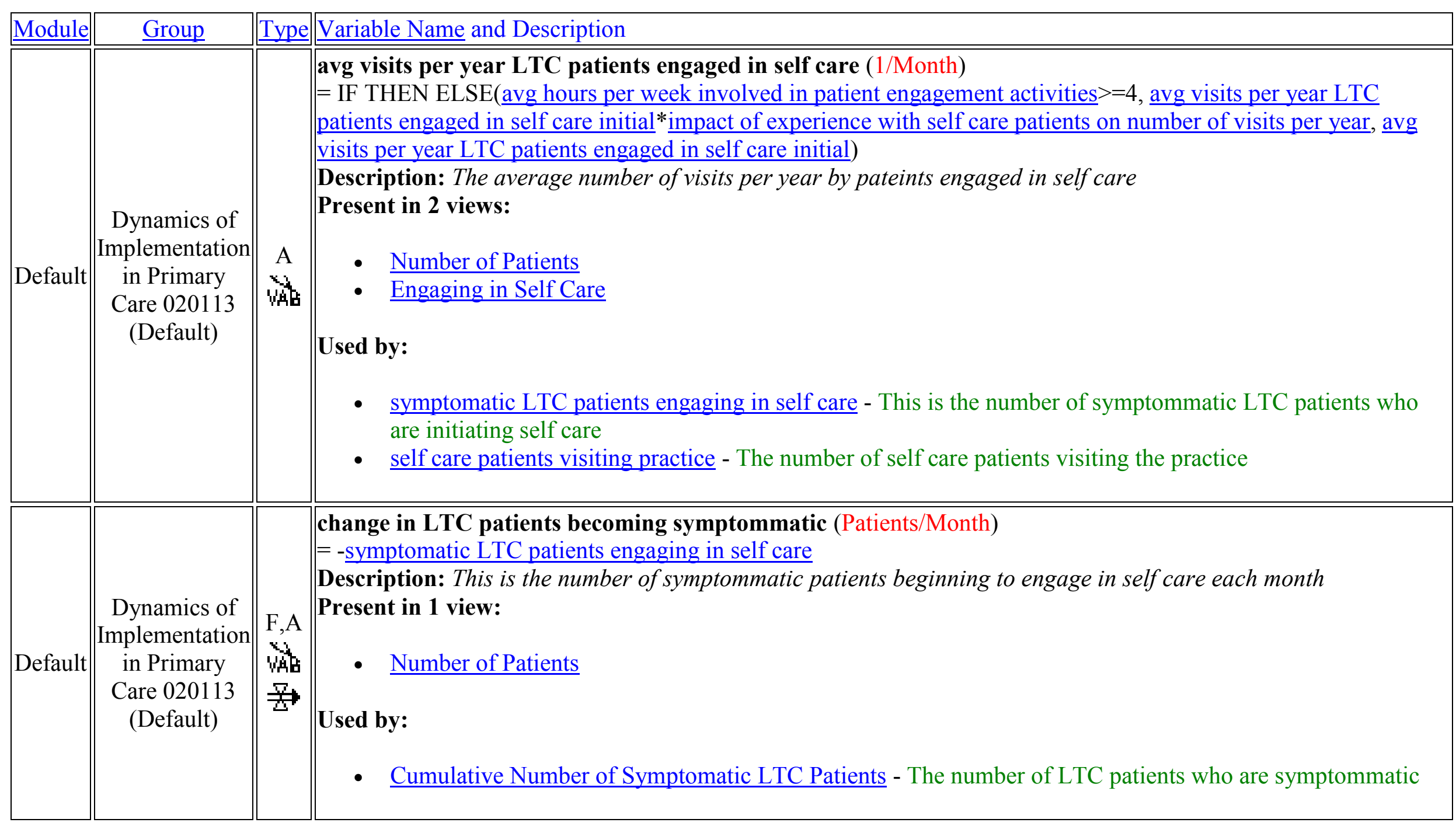




\begin{tabular}{|c|c|c|c|}
\hline Default & \begin{tabular}{|c} 
Dynamics of \\
Implementation \\
in Primary \\
Care 020113 \\
(Default)
\end{tabular} & 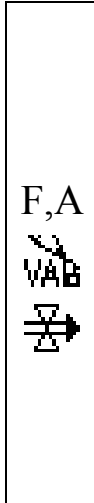 & $\begin{array}{l}\text { change in LTC patients engaging in self care (Patients/Month) } \\
=\text { symptomatic LTC patients engaging in self care } \\
\text { Description: This is the number of symptommatic LTC patients beginning to engage in self care each month. } \\
\text { Present in } 1 \text { view: } \\
\text { - } \quad \text { Number of Patients } \\
\text { Used by: } \\
\text { - Number of Self Care Patients - The number of LTC patients engaging in self care }\end{array}$ \\
\hline Default & \begin{tabular}{|c} 
Dynamics of \\
Implementation \\
in Primary \\
Care 020113 \\
(Default)
\end{tabular} & 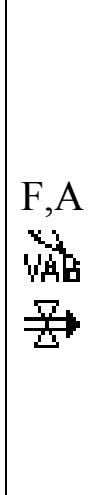 & $\begin{array}{l}\text { change in number of patients (Patients/Month) } \\
=\text { Number of Enrolled Patients* }{ }^{*} \text { ate of growth of enrolled patients/12 } \\
\text { Description: This the change in the number of patients } \\
\text { Present in } 1 \text { view: } \\
\text { - } \quad \text { Number of Patients } \\
\text { Used by: } \\
\text { - Number of Enrolled Patients }- \text { This is the number of patients }\end{array}$ \\
\hline Default & \begin{tabular}{||c} 
Dynamics of \\
Implementation \\
in Primary \\
Care 020113 \\
(Default)
\end{tabular} & $\begin{array}{c}\mathrm{L} \\
\mathrm{W} \\
\end{array}$ & 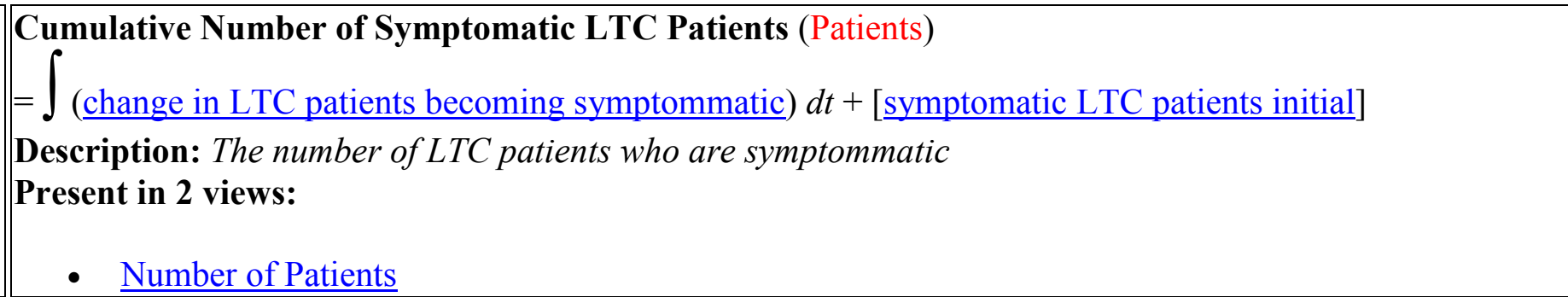 \\
\hline
\end{tabular}




\begin{tabular}{|c|c|c|c|}
\hline & & & $\begin{array}{l}\text { - } \text { Engaging in Self Care } \\
\text { Used by: } \\
\text { - symptomatic LTC patients visiting practice - The number of symptomatic patients visiting the practice } \\
\text { - } \text { pct self care patients - Per cent of self care patients who engage in self care } \\
\text { - }\end{array}$ \\
\hline Default & \begin{tabular}{||c||} 
Dynamics of \\
Implementation \\
in Primary \\
Care 020113 \\
(Default)
\end{tabular} & $\begin{array}{c}\text { A } \\
\text { VA } \\
\text { VA⿱ }\end{array}$ & 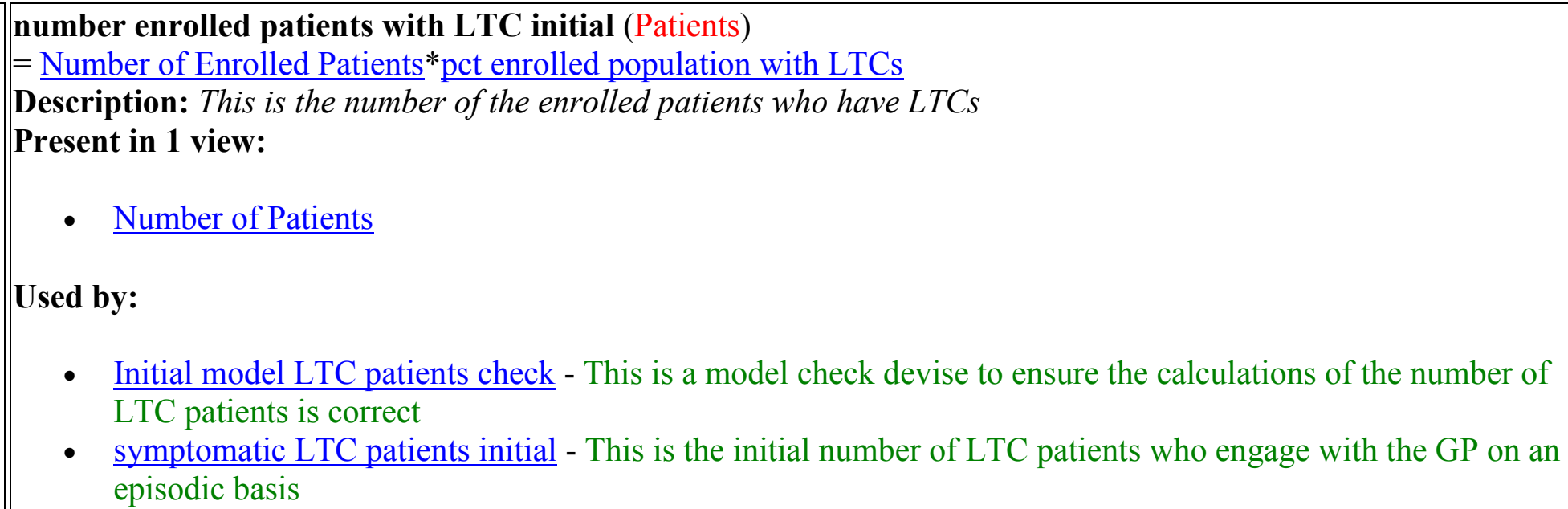 \\
\hline
\end{tabular}




\begin{tabular}{|c|c|c|c|}
\hline & & & - self care patients initial - This is the initial number of LTC patients who are engaging in self care \\
\hline Default & \begin{tabular}{|c||} 
Dynamics of \\
Implementation \\
in Primary \\
Care 020113 \\
(Default)
\end{tabular} & $\begin{array}{c}\mathrm{L} \\
\text { 䋸 }\end{array}$ & 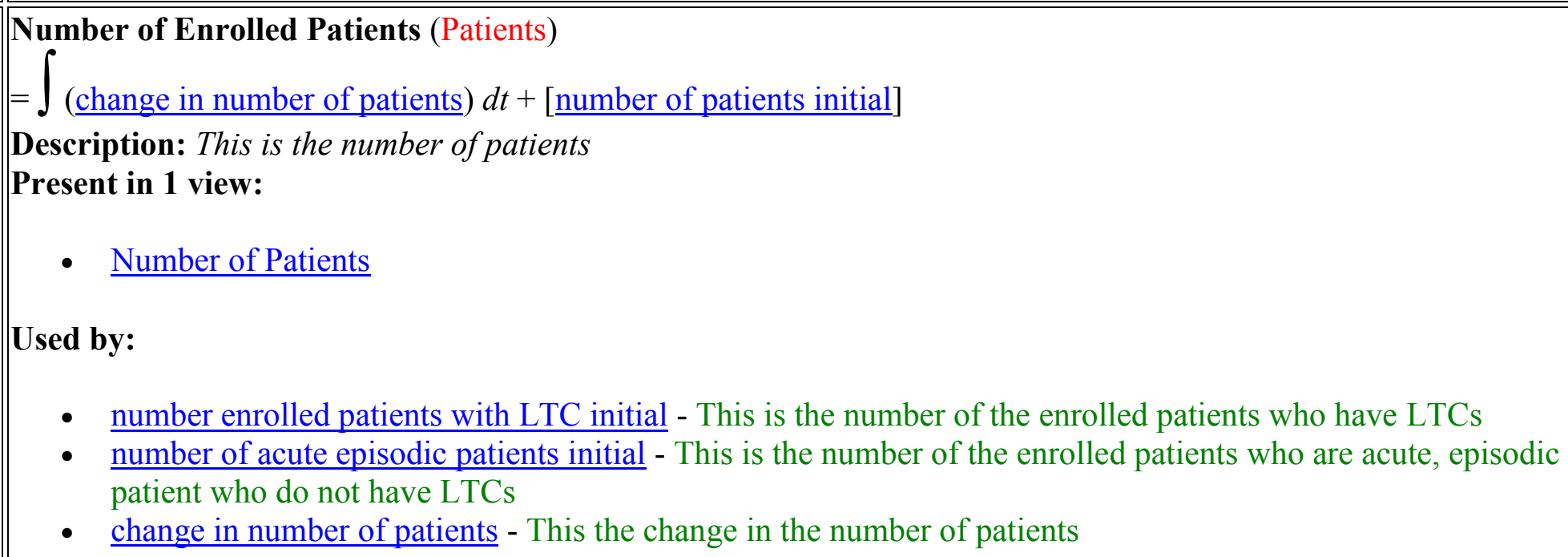 \\
\hline
\end{tabular}




\begin{tabular}{|c|c|c|c|}
\hline Default & \begin{tabular}{||} 
Dynamics of \\
Implementation \\
in Primary \\
Care 020113 \\
(Default)
\end{tabular} & $\begin{array}{c}\text { A } \\
\text { VA⿱⺊口}\end{array}$ & $\begin{array}{l}\text { number of LTC patients (Patients) } \\
=\text { Number of Self Care Patients }+ \text { Cumulative Number of Symptomatic LTC Patients } \\
\text { Description: The number of LTC patients } \\
\text { Present in } 1 \text { view: } \\
\text { - Number of Patients } \\
\text { Used by: } \\
\text { - } \quad \underline{\text { Initial model LTC patients check }} \text { - This is a model check devise to ensure the calculations of the number of } \\
\quad \text { LTC patients is correct }\end{array}$ \\
\hline Default & \begin{tabular}{||c||} 
Dynamics of \\
Implementation \\
in Primary \\
Care 020113 \\
(Default)
\end{tabular} & $\begin{array}{l}\text { LI,C } \\
\text { Wab }\end{array}$ & $\begin{array}{l}\text { number of patients initial (Patients) } \\
=10000 \\
\text { Description: This is the number of patients initial } \\
\text { Present in } 1 \text { view: } \\
\text { - Number of Patients } \\
\text { Used by: } \\
\text { - Number of Enrolled Patients - This is the number of patients }\end{array}$ \\
\hline Default & \begin{tabular}{||c||} 
Dynamics of \\
Implementation \\
in Primary \\
Care 020113 \\
(Default)
\end{tabular} & 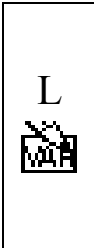 & $\begin{array}{l}\text { Number of Self Care Patients (Patients) } \\
=\int_{\text {(change in LTC patients engaging in self care })} d t+[\text { self care patients initial }] \\
\text { Description: The number of LTC patients engaging in self care } \\
\text { Present in } 1 \text { view: }\end{array}$ \\
\hline
\end{tabular}




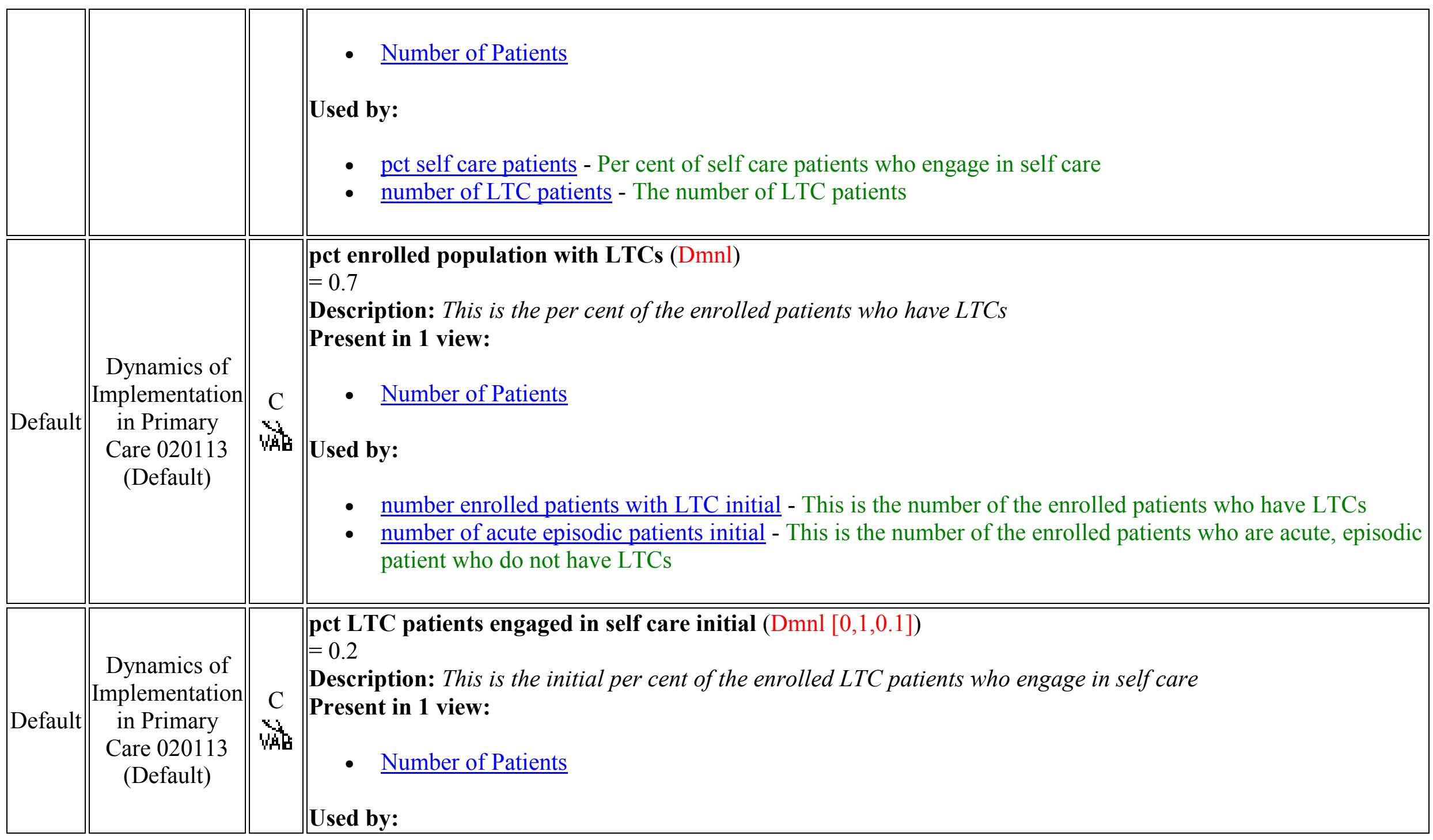




\begin{tabular}{|c|c|c|c|}
\hline & & & 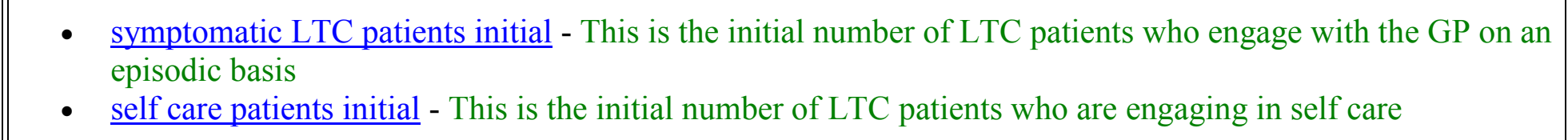 \\
\hline Default & \begin{tabular}{||c} 
Dynamics of \\
Implementation \\
in Primary \\
Care 020113 \\
(Default)
\end{tabular} & $\begin{array}{c}\text { A } \\
\text { WA }\end{array}$ & $\begin{array}{l}\text { pet self care patients }(\mathrm{Dmnl}) \\
=\text { Number of Self Care Patients/(Number of Self Care Patients }+ \text { Cumulative Number of Symptomatic LTC Patients }) \\
\text { Description: Per cent of self care patients who engage in self care } \\
\text { Present in } 2 \text { views: } \\
\text { - } \frac{\text { Quality of LTCM }}{\text { Number of Patients }} \\
\text { Used by: } \\
\text { - } \frac{\text { change in quality of LTCM - This is the net change in the effective management of LTCs as a result of the }}{\text { desired improvement, the effort to improve, the time to change and the per cent of patients who are engaging }} \\
\text { in self care. }\end{array}$ \\
\hline
\end{tabular}




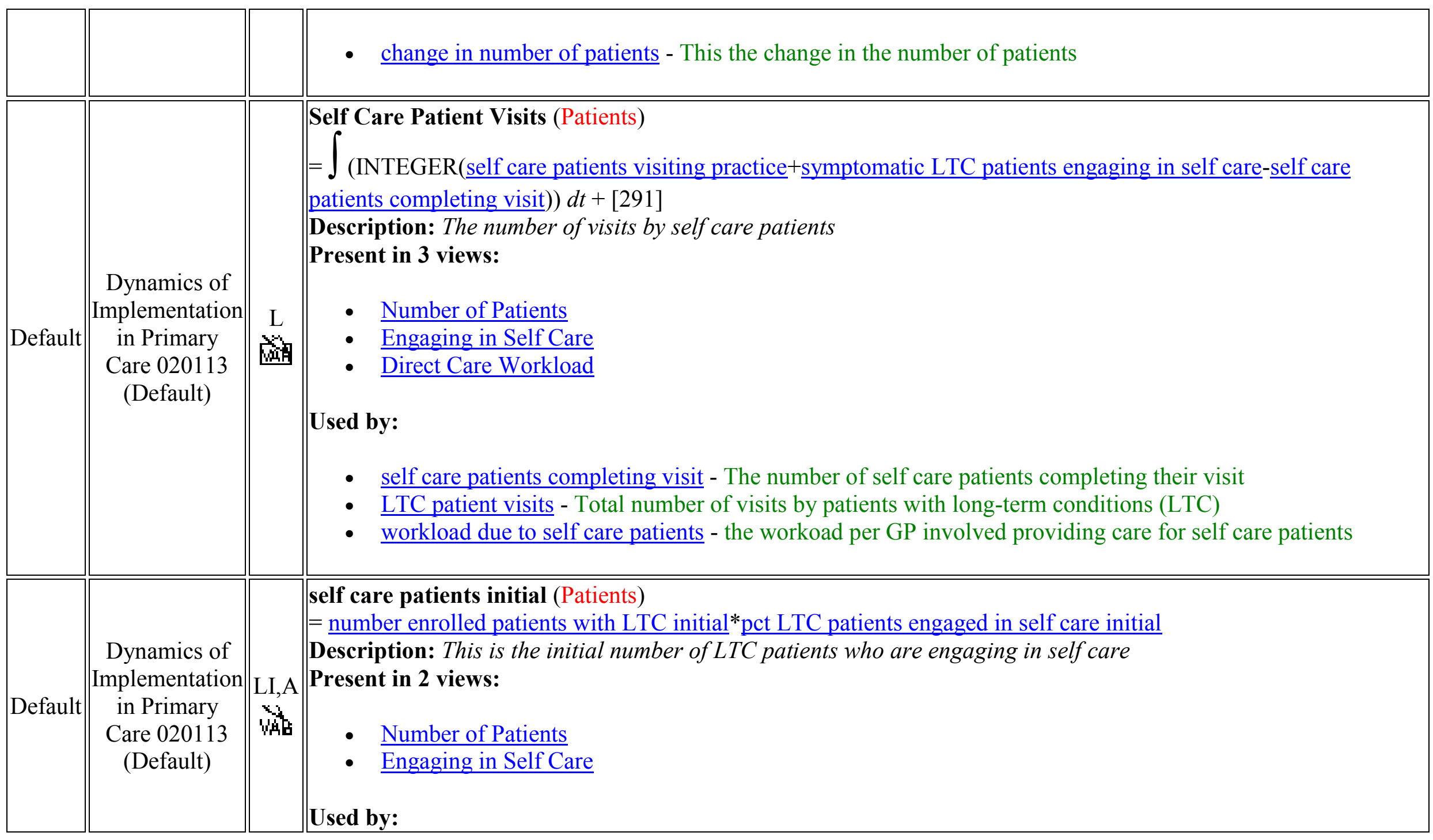




\begin{tabular}{|c|c|c|c|}
\hline & & & $\begin{array}{l}\text { - } \text { symptomatic LTC patients engaging in self care - This is the number of symptommatic LTC patients who } \\
\text { are initiating self care } \\
\text { - } \text { self care patients seeking care per month initial - The number of self care patients seeking care per month } \\
\text { - }\end{array}$ \\
\hline Default & \begin{tabular}{|c} 
Dynamics of \\
Implementation \\
in Primary \\
Care 020113 \\
(Default)
\end{tabular} & $\begin{array}{l}\text { F,A } \\
\text { Wha } \\
\text { 差 }\end{array}$ & 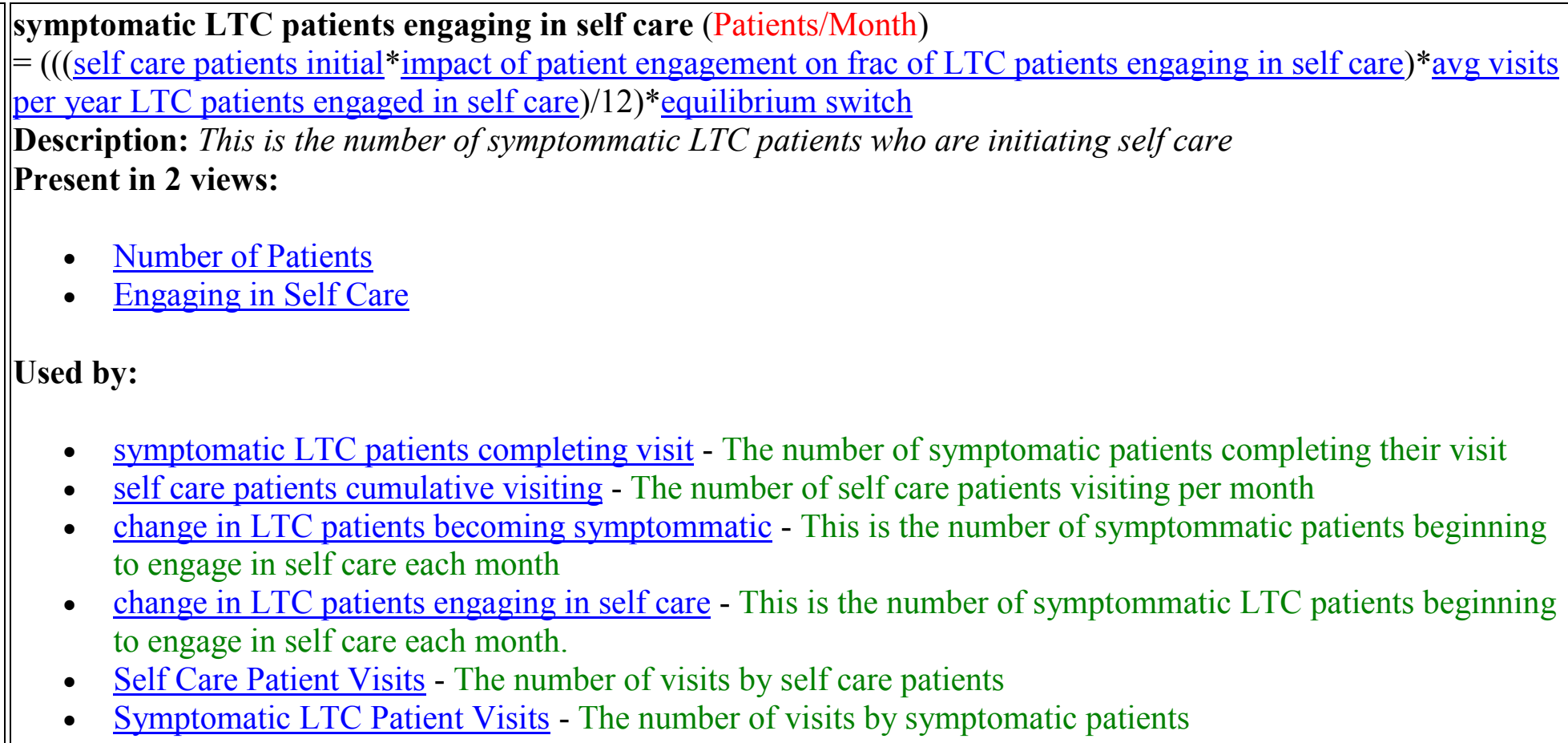 \\
\hline Default & $\begin{array}{c}\text { Dynamics of } \\
\text { Implementation } \\
\text { in Primary }\end{array}$ & $\begin{array}{l}\text { LI,A } \\
\text { vas } \\
\text { Vats }\end{array}$ & $\begin{array}{l}\text { symptomatic LTC patients initial (Patients) } \\
=\text { number enrolled patients with LTC initial*(1-pct LTC patients engaged in self care initial) } \\
\text { Description: This is the initial number of LTC patients who engage with the GP on an episodic basis }\end{array}$ \\
\hline
\end{tabular}




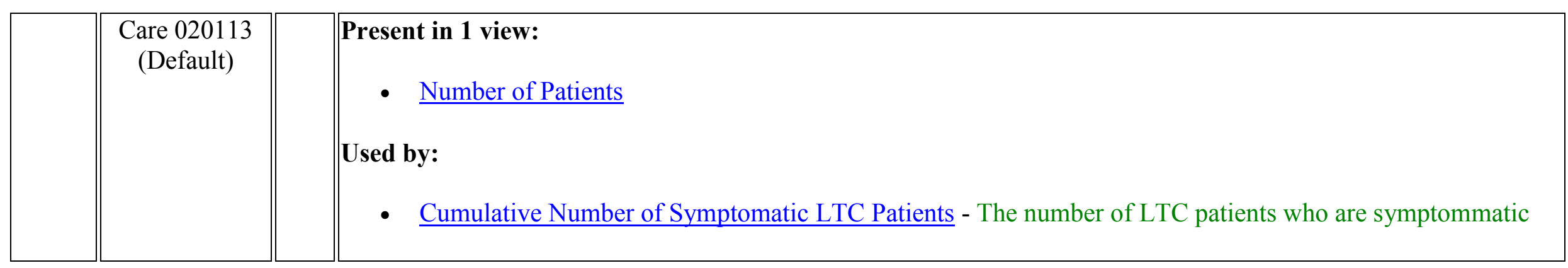




\section{тор Engaging in Self Care (30 variables)}

Self Care Patients

fimpact of experience with self

HOME

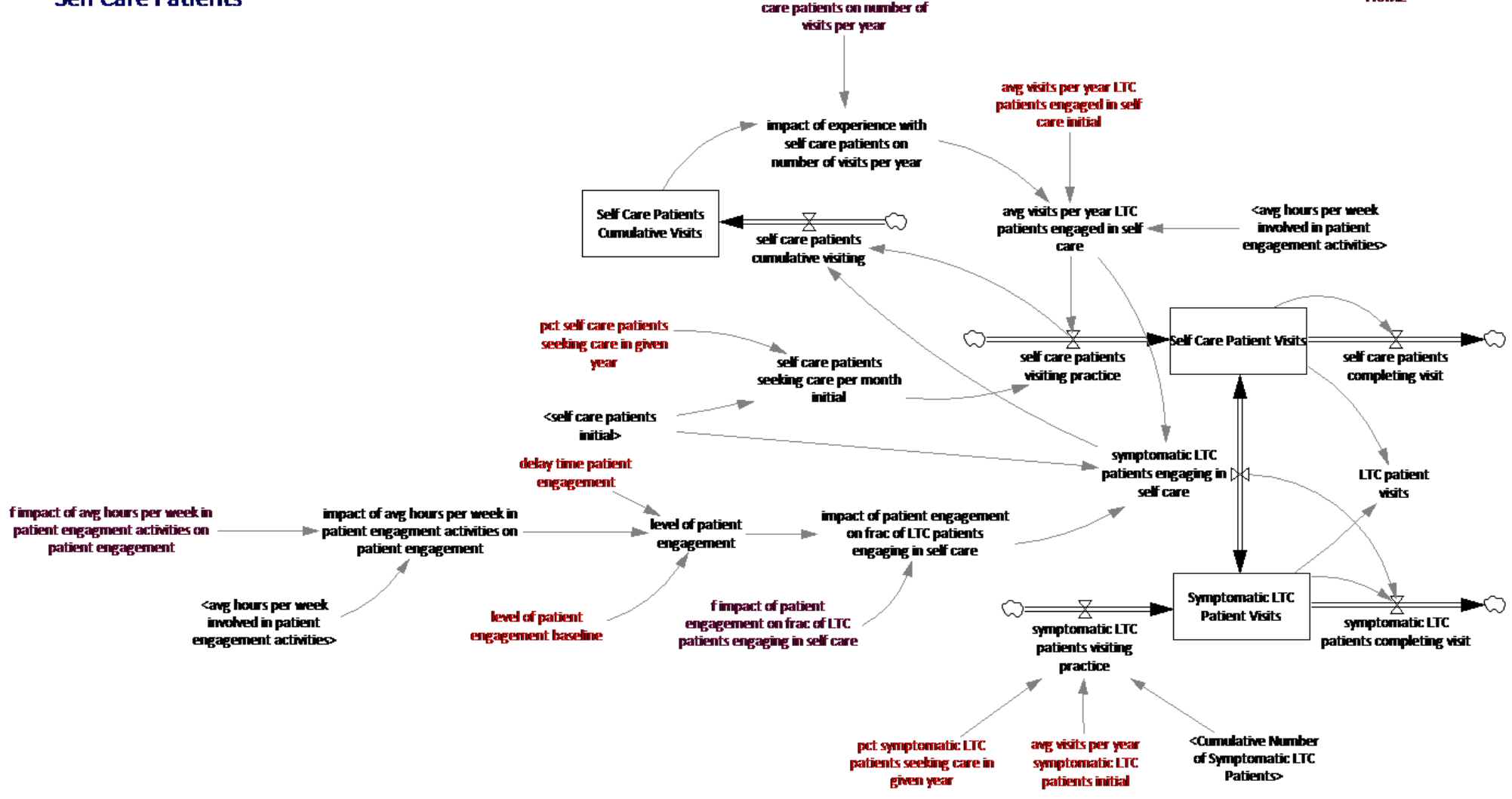




\begin{tabular}{|c|c|c|c|}
\hline Module & Group & Type & Variable Name and Description \\
\hline Default & \begin{tabular}{|c} 
Dynamics of \\
Implementation \\
in Primary \\
Care 020113 \\
(Default)
\end{tabular} & $\begin{array}{c}\text { A } \\
\text { Vat } \\
\text { Vat }\end{array}$ & 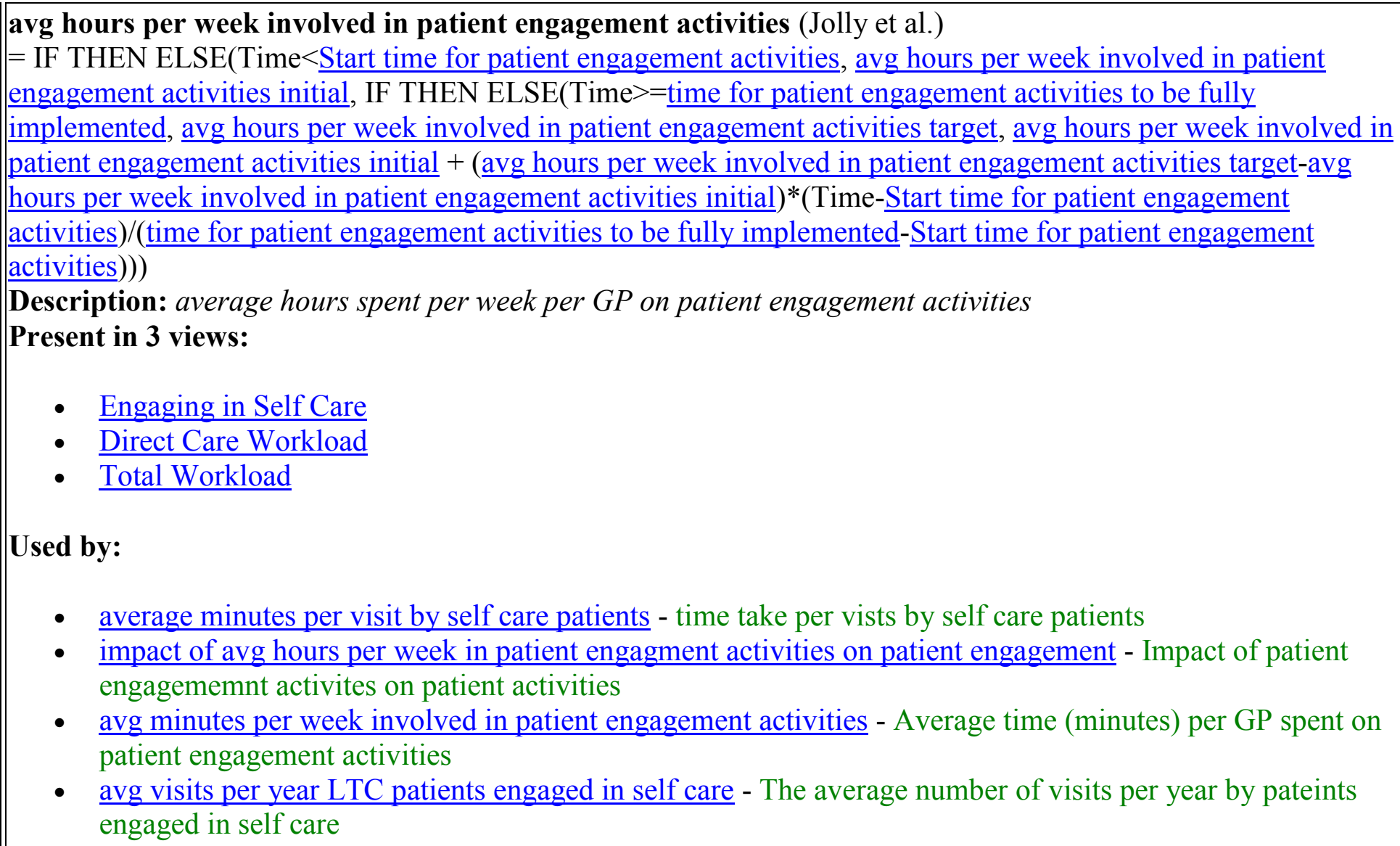 \\
\hline Default & \begin{tabular}{|c|} 
Dynamics of \\
Implementation \\
in Primary \\
\end{tabular} & $\begin{array}{c}\text { A } \\
\text { V } \\
\text { VAb }\end{array}$ & $\begin{array}{l}\text { avg visits per year LTC patients engaged in self care (1/Month) } \\
=\text { IF THEN ELSE(avg hours per week involved in patient engagement activities }>=4 \text {, avg visits per year LTC } \\
\text { patients engaged in self care initial } \\
\end{array}$ \\
\hline
\end{tabular}




\begin{tabular}{|c|c|c|c|}
\hline & $\begin{array}{l}\text { Care } 020113 \\
\text { (Default) }\end{array}$ & & $\begin{array}{l}\text { visits per year LTC patients engaged in self care initial) } \\
\text { Description: The average number of visits per year by pateints engaged in self care } \\
\text { Present in } 2 \text { views: } \\
\text { - } \text { Number of Patients } \\
\text { Used by: } \\
\text { - } \\
\text { symptomating in Self Care } \\
\text { are initiating self care } \\
\text { self care patients visiting practice - The number of self care patients visiting the practice }\end{array}$ \\
\hline Default & \begin{tabular}{|c||} 
Dynamics of \\
Implementation \\
in Primary \\
Care 020113 \\
(Default)
\end{tabular} & $\begin{array}{c}\mathrm{C} \\
\mathrm{V} \\
\mathrm{V} A \mathrm{~B}\end{array}$ & $\begin{array}{l}\text { avg visits per year LTC patients engaged in self care initial (1/Month) } \\
=5 \\
\text { Description: The average visits per year by patients engaged in self care - initia value } \\
\text { Present in } 1 \text { view: } \\
\text { - } \quad \text { Engaging in Self Care } \\
\text { Used by: } \\
\text { - } \quad \text { avg visits per year LTC patients engaged in self care - The average number of visits per year by pateints } \\
\text { engaged in self care }\end{array}$ \\
\hline Default & \begin{tabular}{|c||} 
Dynamics of \\
Implementation \\
in Primary \\
Care 020113
\end{tabular} & $\begin{array}{c}\mathrm{C} \\
\mathrm{V} \\
V_{A B}\end{array}$ & $\begin{array}{l}\text { avg visits per year symptomatic LTC patients initial (1/Month) } \\
=12 \\
\text { Description: The average visits per year by symptomatic patients - initial value } \\
\text { Present in } 1 \text { view: }\end{array}$ \\
\hline
\end{tabular}




\begin{tabular}{|c|c|c|c|}
\hline & (Default) & & $\begin{array}{l}\text { - Engaging in Self Care } \\
\text { Used by: } \\
\text { - } \quad \text { symptomatic LTC patients visiting practice - The number of symptomatic patients visiting the practice }\end{array}$ \\
\hline Default & \begin{tabular}{|} 
Dynamics of \\
Implementation \\
in Primary \\
Care 020113 \\
(Default)
\end{tabular} & $\begin{array}{c}\mathrm{L} \\
\mathrm{x} \\
\mathrm{m}\end{array}$ & 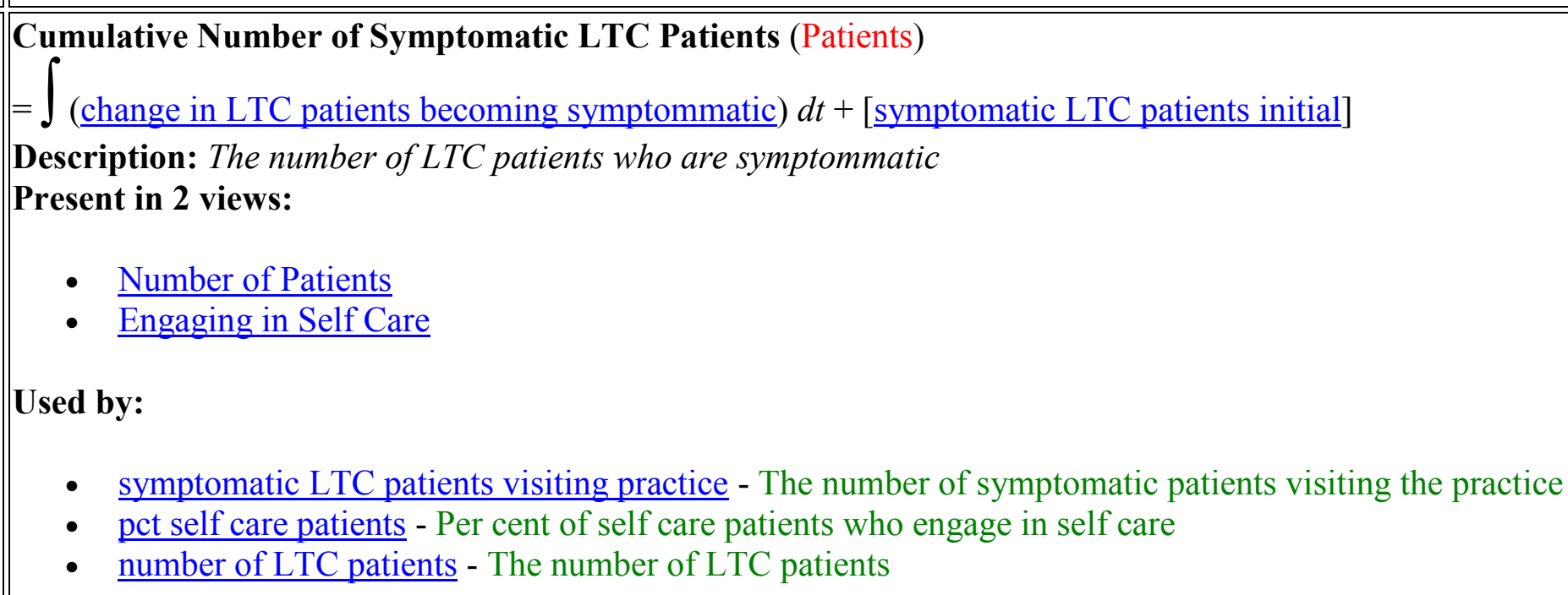 \\
\hline Default & \begin{tabular}{|} 
Dynamics of \\
Implementation \\
in Primary \\
Care 020113 \\
(Default)
\end{tabular} & $\begin{array}{c}\mathrm{C} \\
\text { Wab }\end{array}$ & $\begin{array}{l}\text { delay time patient engagement (Jolly et al.) } \\
=6 \\
\text { Description: Te delay in patient engagement activities resulting in chagnes in patient engagement } \\
\text { Present in } 1 \text { view: } \\
\quad \text { Engaging in Self Care }\end{array}$ \\
\hline
\end{tabular}




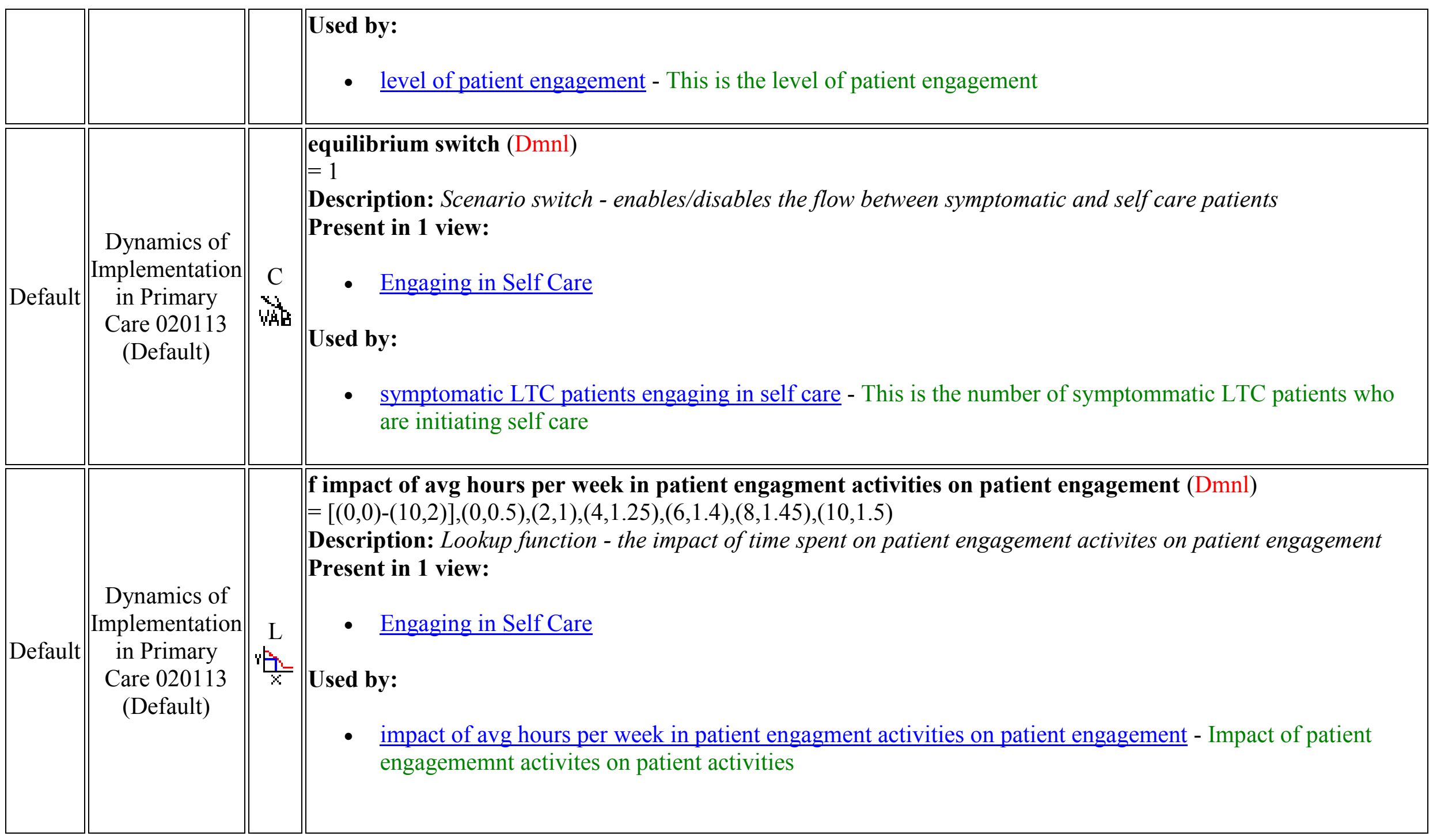




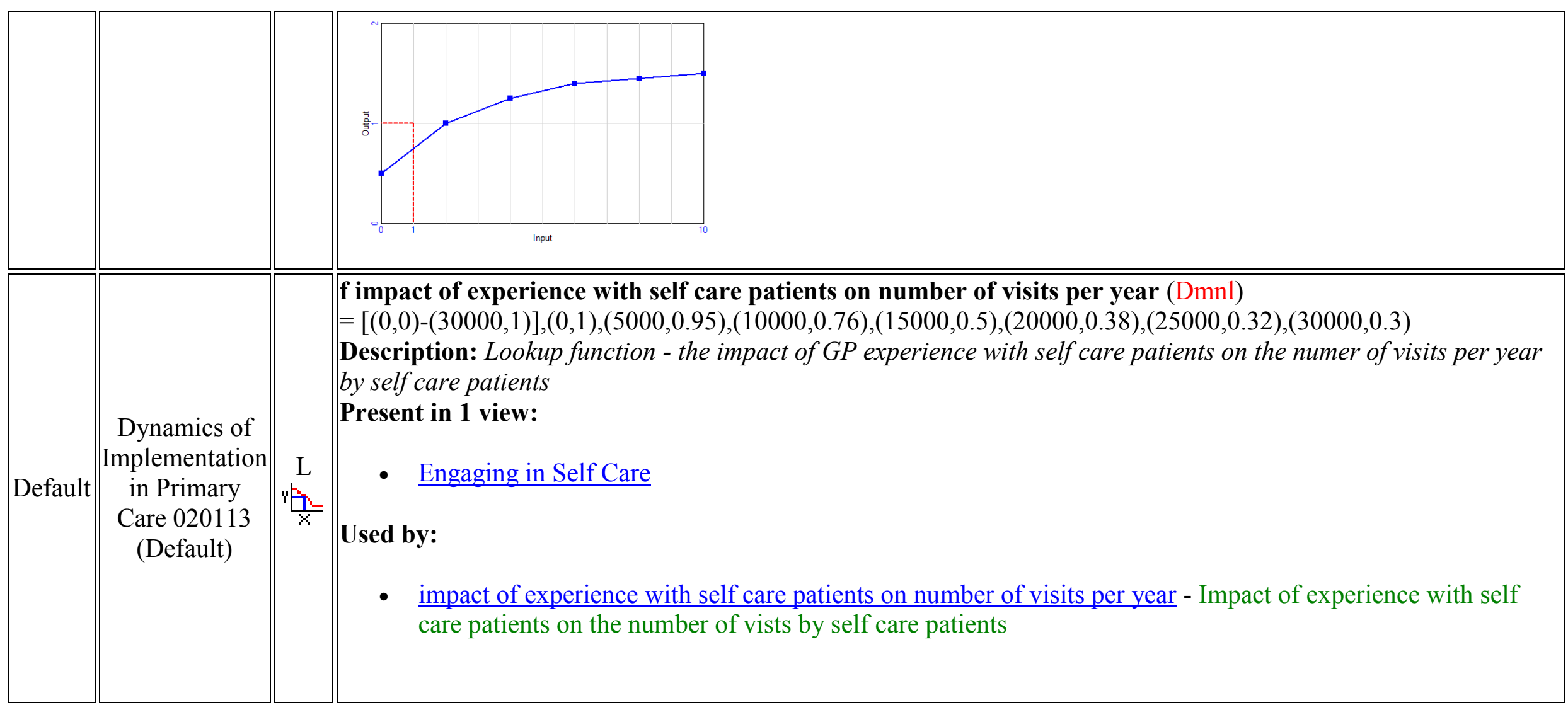




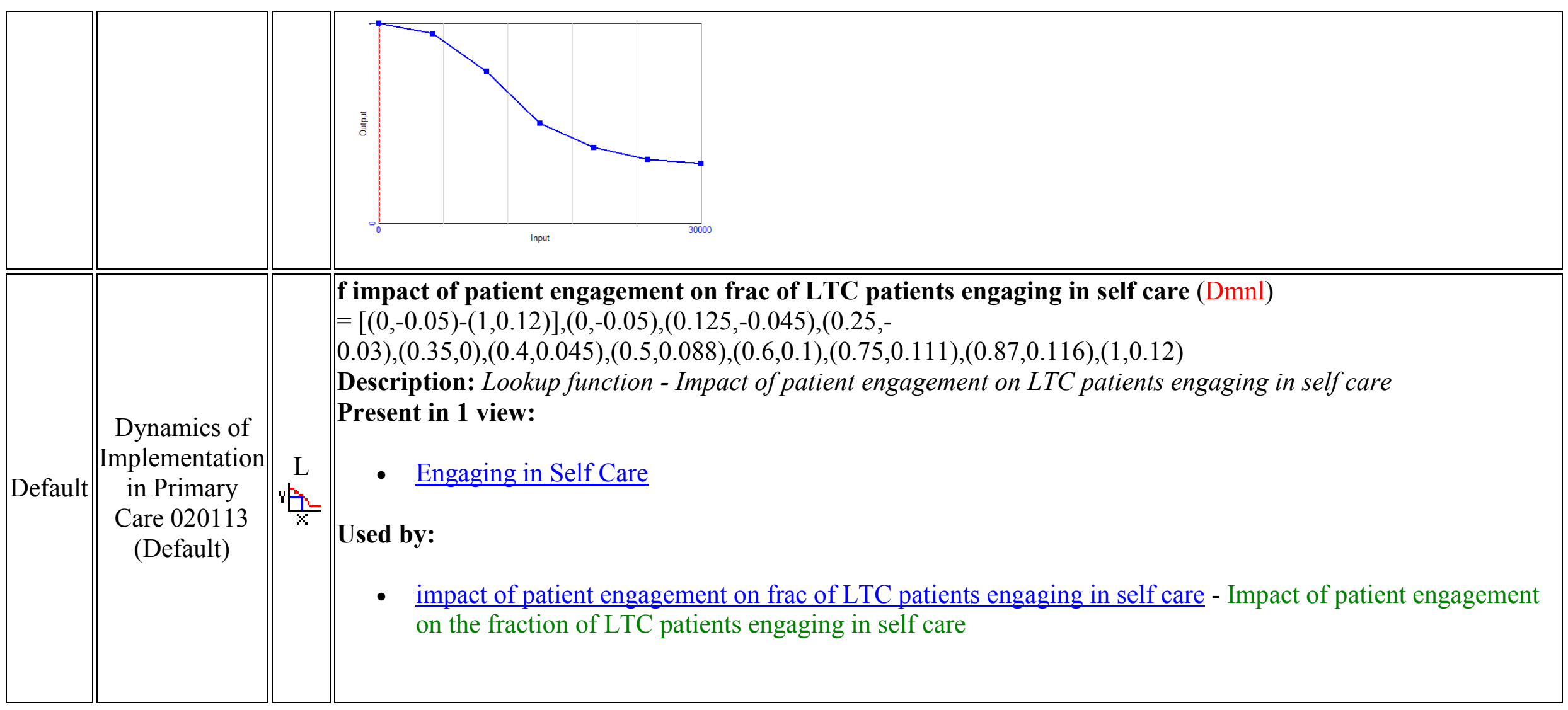




\begin{tabular}{|c|c|c|c|}
\hline & & & 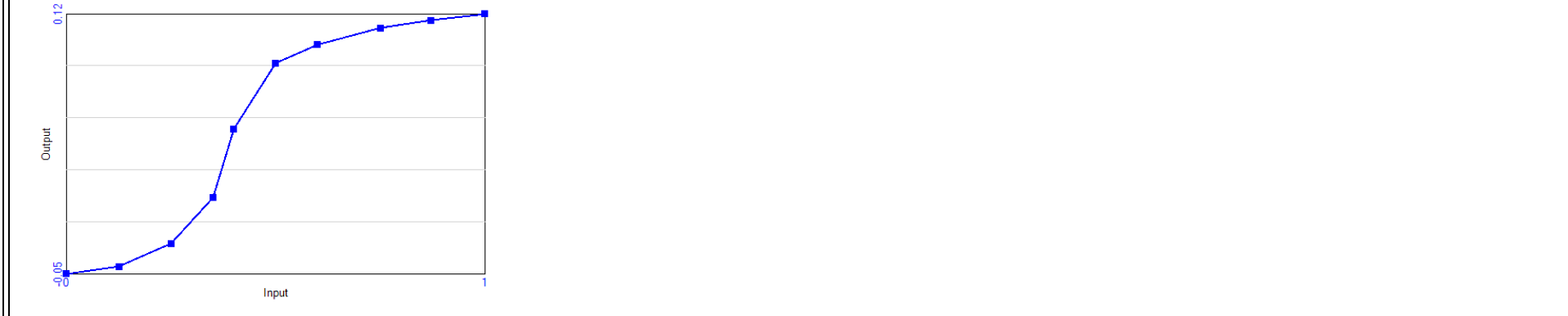 \\
\hline Default & $\begin{array}{c}\text { Dynamics of } \\
\text { Implementation } \\
\text { in Primary } \\
\text { Care } 020113 \\
\text { (Default) }\end{array}$ & $\begin{array}{c}\text { A } \\
\text { Whb }\end{array}$ & $\begin{array}{l}\text { impact of avg hours per week in patient engagment activities on patient engagement (Dmnl) } \\
=\underline{\text { f impact of avg hours per week in patient engagment activities on patient engagement }(\text { avg hours per week }} \\
\text { involved in patient engagement activities) } \\
\text { Description: Impact of patient engagememnt activites on patient activities } \\
\text { Present in } 1 \text { view: } \\
\text { - Engaging in Self Care } \\
\text { Used by: } \\
\quad \text { level of patient engagement - This is the level of patient engagement }\end{array}$ \\
\hline Default & \begin{tabular}{|} 
Dynamics of \\
Implementation \\
in Primary \\
Care 020113 \\
(Default)
\end{tabular} & $\begin{array}{c}\mathrm{A} \\
\mathrm{W} \\
\mathrm{W}\end{array}$ & $\begin{array}{l}\text { impact of experience with self care patients on number of visits per year (Dmnl) } \\
=\text { f impact of experience with self care patients on number of visits per year (Self Care Patients Cumulative Visits) } \\
\text { Description: Impact of experience with self care patients on the number of vists by self care patients } \\
\text { Present in } 1 \text { view: } \\
\quad \text { Engaging in Self Care } \\
\text { Used by: }\end{array}$ \\
\hline
\end{tabular}




\begin{tabular}{|c|c|c|c|}
\hline & & & $\begin{array}{l}\text { - avg visits per year LTC patients engaged in self care - The average number of visits per year by pateints } \\
\text { engaged in self care }\end{array}$ \\
\hline
\end{tabular}




\begin{tabular}{|c|c|c|c|}
\hline Default & \begin{tabular}{|l} 
Dynamics of \\
Implementation \\
in Primary \\
Care 020113 \\
(Default)
\end{tabular} & $\begin{array}{r}\mathrm{C} \\
\mathrm{W}\end{array}$ & $\begin{array}{l}\text { level of patient engagement baseline (Dmnl }[0,1,0.1]) \\
=0.35 \\
\text { Description: The level of patient engagement - initial value } \\
\text { Present in } 1 \text { view: } \\
\text { - Engaging in Self Care } \\
\text { Used by: } \\
\text { - level of patient engagement - This is the level of patient engagement }\end{array}$ \\
\hline Default & \begin{tabular}{|l} 
Dynamics of \\
Implementation \\
in Primary \\
Care 020113 \\
(Default)
\end{tabular} & $\begin{array}{c}\text { A } \\
\text { W }\end{array}$ & $\begin{array}{l}\text { LTC patient visits (Patients) } \\
=\text { Symptomatic LTC Patient Visits }+ \text { Self Care Patient Visits } \\
\text { Description: Total number of visits by patients with long-term conditions }(\text { LTC) } \\
\text { Present in } 1 \text { view: } \\
\quad \text { - Engaging in Self Care }\end{array}$ \\
\hline
\end{tabular}




\begin{tabular}{|c|c|c|c|}
\hline Default & \begin{tabular}{|c} 
Dynamics of \\
Implementation \\
in Primary \\
Care 020113 \\
(Default)
\end{tabular} & $\begin{array}{c}\mathrm{C} \\
\mathrm{VA} \\
\mathrm{VAB}\end{array}$ & $\begin{array}{l}\text { pct symptomatic LTC patients seeking care in given year (Dmnl) } \\
=0.7 \\
\text { Description: The per cent of symptomatic patients who seek care in a given year } \\
\text { Present in } 1 \text { view: } \\
\text { - Engaging in Self Care } \\
\text { Used by: } \\
\quad \text { symptomatic LTC patients visiting practice - The number of symptomatic patients visiting the practice }\end{array}$ \\
\hline Default & \begin{tabular}{|c} 
Dynamics of \\
Implementation \\
in Primary \\
Care 020113 \\
(Default)
\end{tabular} & $\begin{array}{c}\mathrm{L} \\
\text { 繮 }\end{array}$ & 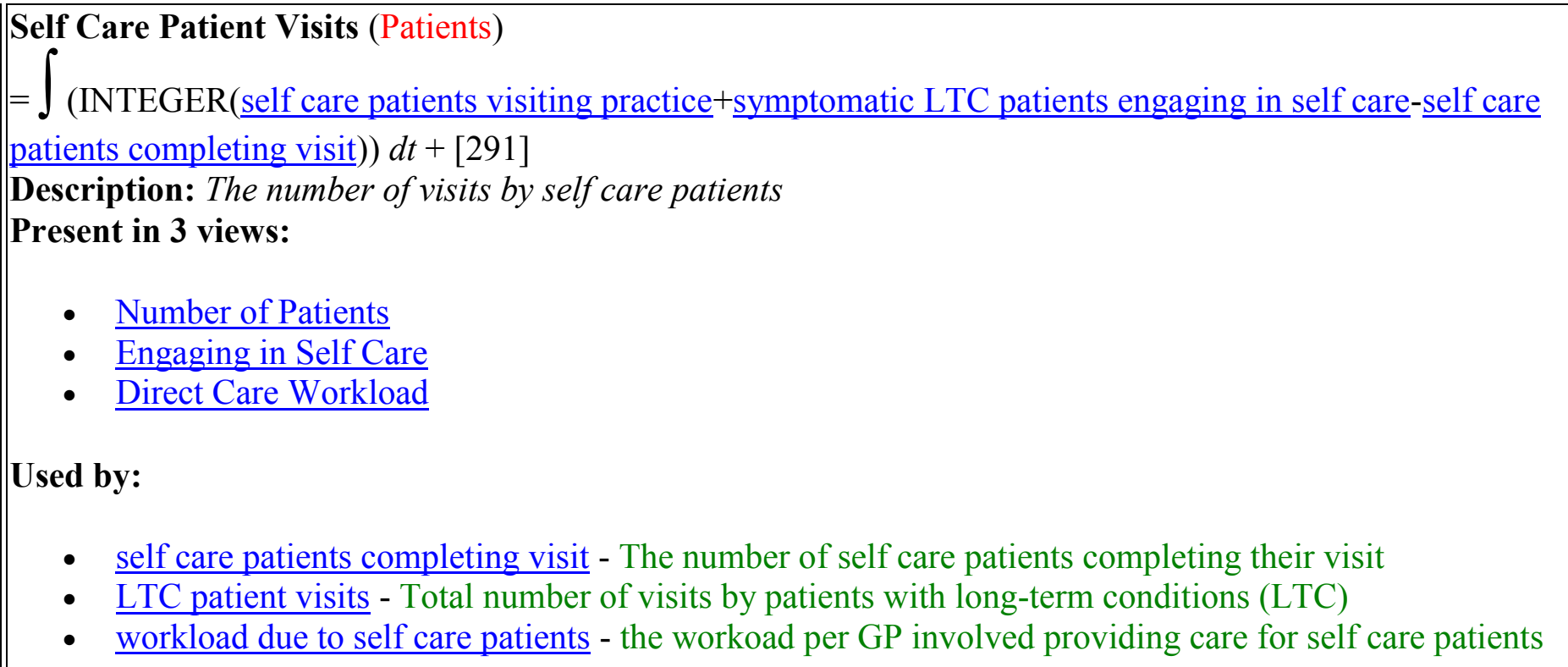 \\
\hline Default & Dynamics of & $\mathrm{F}, \mathrm{A}$ & self care patients completing visit (Patients/Month) \\
\hline
\end{tabular}




\begin{tabular}{|c|c|c|c|}
\hline & \begin{tabular}{|c|} 
Implementation \\
in Primary \\
Care 020113 \\
(Default)
\end{tabular} & $\begin{array}{l}\text { Vab } \\
\text { 吾 }\end{array}$ & $\begin{array}{l}=\text { Self Care Patient Visits } \\
\text { Description: The number of self care patients completing their visit } \\
\text { Present in } 1 \text { view: } \\
\text { - } \quad \text { Engaging in Self Care } \\
\text { Used by: } \\
\text { - Self Care Patient Visits - The number of visits by self care patients }\end{array}$ \\
\hline Default & \begin{tabular}{|c||} 
Dynamics of \\
Implementation \\
in Primary \\
Care 020113 \\
(Default)
\end{tabular} & $\begin{array}{l}F, A \\
\text { VAb } \\
\text { F }\end{array}$ & $\begin{array}{l}\text { self care patients cumulative visiting (Patients/Month) } \\
=\text { self care patients visiting practice+ }+ \text { symptomatic LTC patients engaging in self care } \\
\text { Description: The number of self care patients visiting per month } \\
\text { Present in } 1 \text { view: } \\
\text { - Engaging in Self Care } \\
\text { Used by: } \\
\quad \text { Self Care Patients Cumulative Visits - the cumulative number of self care patients visiting their GP }\end{array}$ \\
\hline Default & \begin{tabular}{|c||} 
Dynamics of \\
Implementation \\
in Primary \\
Care 020113 \\
(Default)
\end{tabular} & $\begin{array}{c}\mathrm{L} \\
\text { 离出 }\end{array}$ & $\begin{array}{l}\text { Self Care Patients Cumulative Visits (Patients) } \\
=\int \text { (self care patients cumulative visiting) } d t+[350] \\
\text { Description: } \text { the cumulative number of self care patients visiting their GP } \\
\text { Present in } 2 \text { views: } \\
\quad \text { Engaging in Self Care }\end{array}$ \\
\hline
\end{tabular}




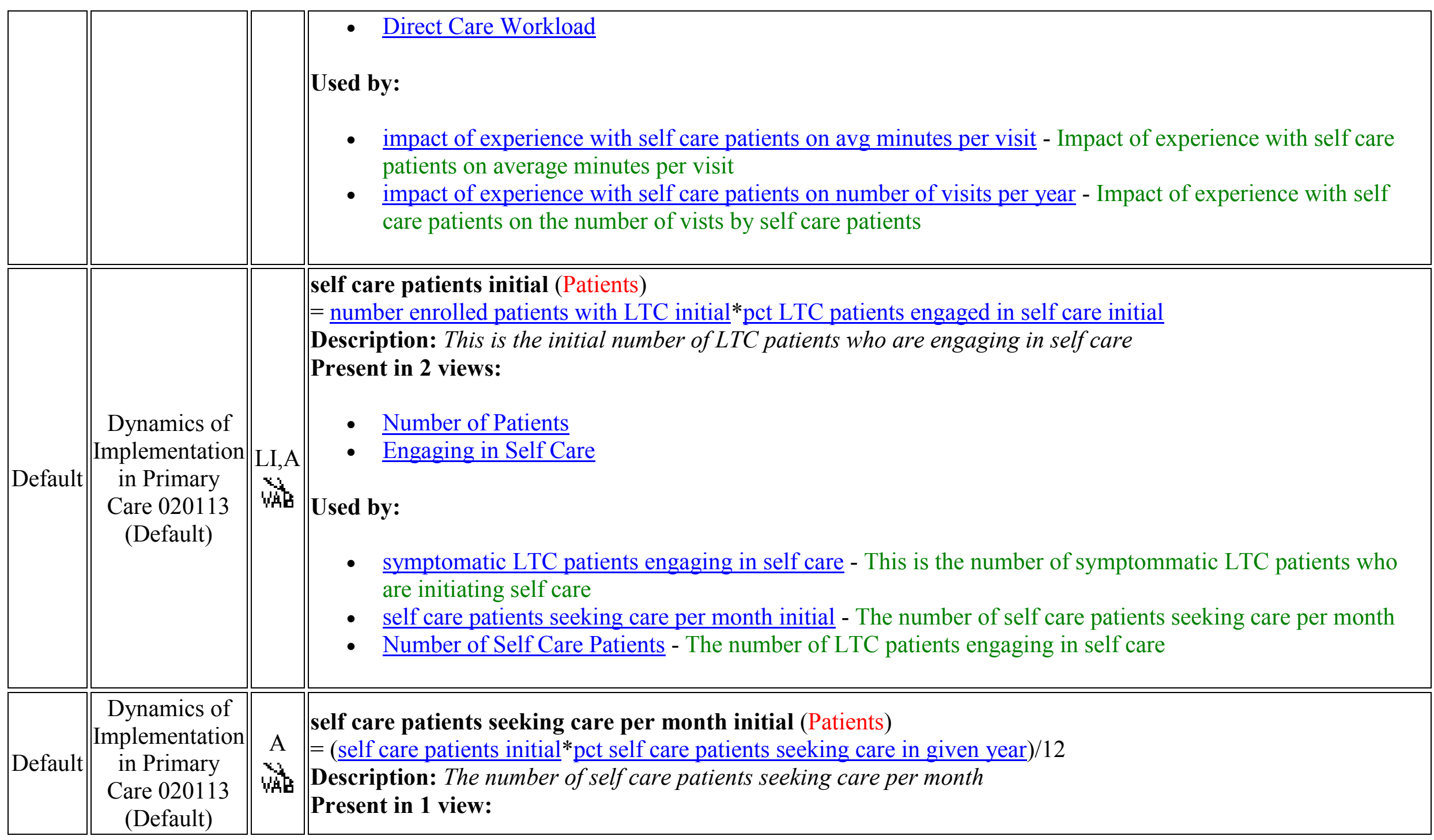




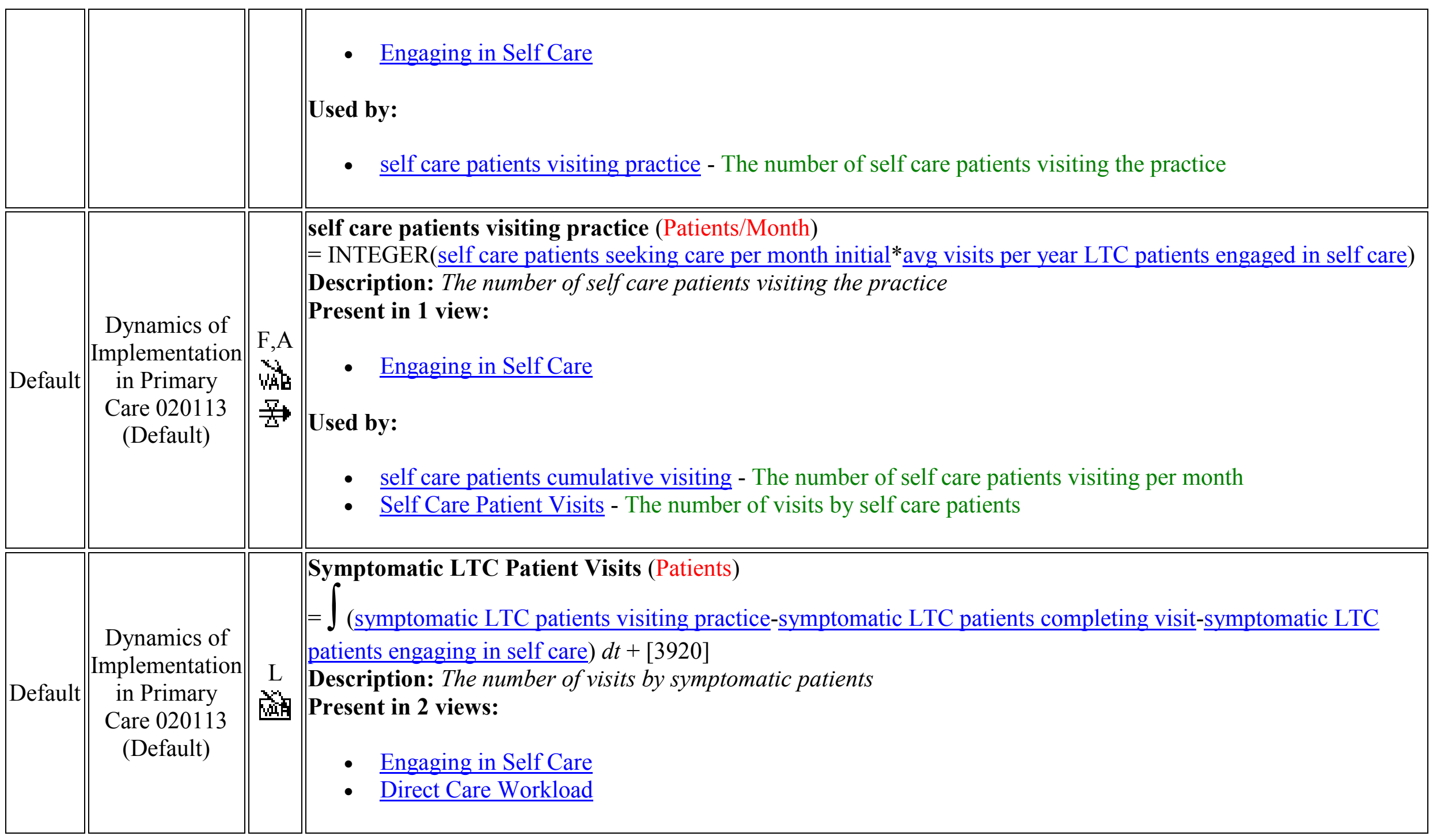




\begin{tabular}{|c|c|c|c|}
\hline & & & $\begin{array}{l}\text { Used by: } \\
\text { - } \text { symptomatic LTC patients completing visit - The number of symptomatic patients completing their visit } \\
\text { - } \frac{\text { LTC patient visits - Total number of visits by patients with long-term conditions (LTC) }}{\text { workload due to symptomatic LTC patients - the total lworkload, per GP, providing care to symptomatic }} \\
\text { patients }\end{array}$ \\
\hline Default & \begin{tabular}{|l||} 
Dynamics of \\
Implementation \\
in Primary \\
Care 020113 \\
(Default)
\end{tabular} & $\begin{array}{l}\text { F,A } \\
\text { VAB } \\
\text { 劳 }\end{array}$ & $\begin{array}{l}\text { Symptomatic LTC patients completing visit (Patients/Month) } \\
=\text { (Symptomatic LTC Patient Visits-symptomatic LTC patients engaging in self care) } \\
\text { Description: The number of symptomatic patients completing their visit } \\
\text { Present in } 1 \text { view: } \\
\text { - Engaging in Self Care } \\
\text { Used by: } \\
\text { - Symptomatic LTC Patient Visits - The number of visits by symptomatic patients }\end{array}$ \\
\hline
\end{tabular}




\begin{tabular}{|c|c|c|c|}
\hline & & & 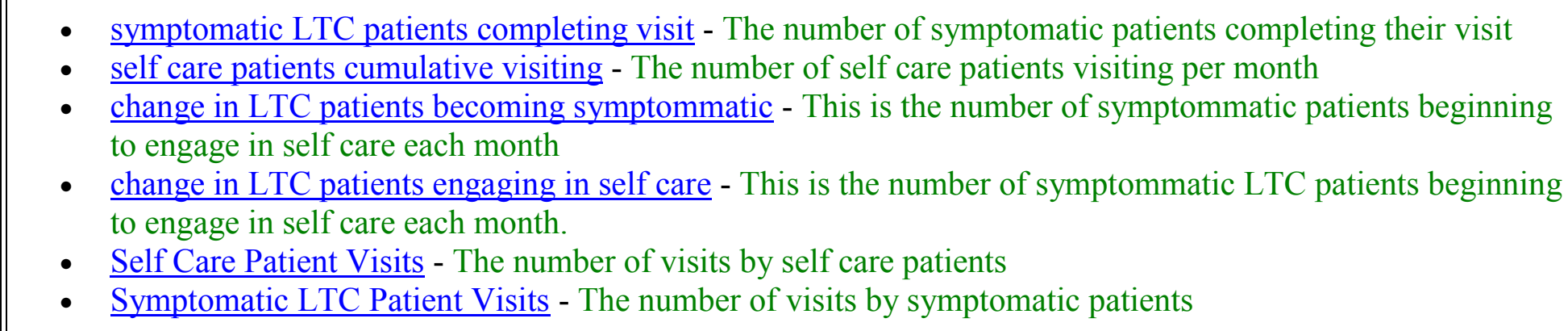 \\
\hline Default & \begin{tabular}{|c|} 
Dynamics of \\
Implementation \\
in Primary \\
Care 020113 \\
(Default)
\end{tabular} & $\begin{array}{l}F, A \\
\text { WAB } \\
\text { 吾 }\end{array}$ & $\begin{array}{l}\text { symptomatic LTC patients visiting practice (Patients/Month) } \\
=((\text { Cumulative Number of Symptomatic LTC Patients*pct symptomatic LTC patients seeking care in given } \\
\left.\text { year })^{*} \text { avg visits per year symptomatic LTC patients initial }\right) / 12 \\
\text { Description: The number of symptomatic patients visiting the practice } \\
\text { Present in } 1 \text { view: } \\
\text { - Engaging in Self Care } \\
\text { Used by: } \\
\text { - Symptomatic LTC Patient Visits }- \text { The number of visits by symptomatic patients }\end{array}$ \\
\hline
\end{tabular}




\section{rop Direct Care Workload (18 variables)}

Direct Care Workload

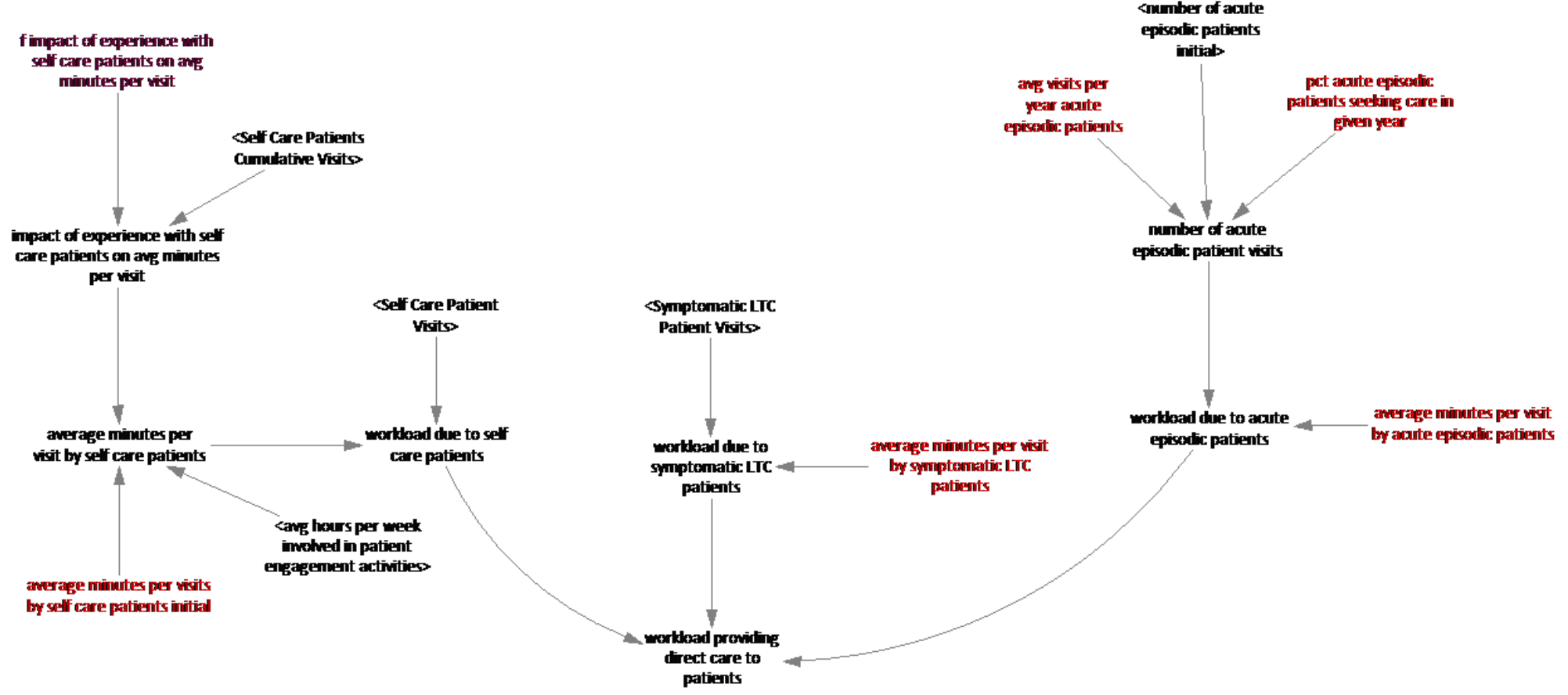




\begin{tabular}{|c|c|c|c|}
\hline Module & Group & Type & Variable Name and Description \\
\hline Default & \begin{tabular}{||c||} 
Dynamics of \\
Implementation \\
in Primary \\
Care 020113 \\
(Default)
\end{tabular} & $\begin{array}{c}\mathrm{C} \\
\mathrm{W} \\
\mathrm{W}\end{array}$ & $\begin{array}{l}\text { average minutes per visit by acute episodic patients (1/Month) } \\
=10 \\
\text { Description: The average time spent per visit by acute patients } \\
\text { Present in } 1 \text { view: } \\
\text { - Direct Care Workload } \\
\text { Used by: } \\
\text { - workload due to acute episodic patients - The workload per GP in provding care for acute patients }\end{array}$ \\
\hline Default & \begin{tabular}{||c||} 
Dynamics of \\
Implementation \\
in Primary \\
Care 020113 \\
(Default)
\end{tabular} & $\begin{array}{c}\text { A } \\
\text { VAb }\end{array}$ & 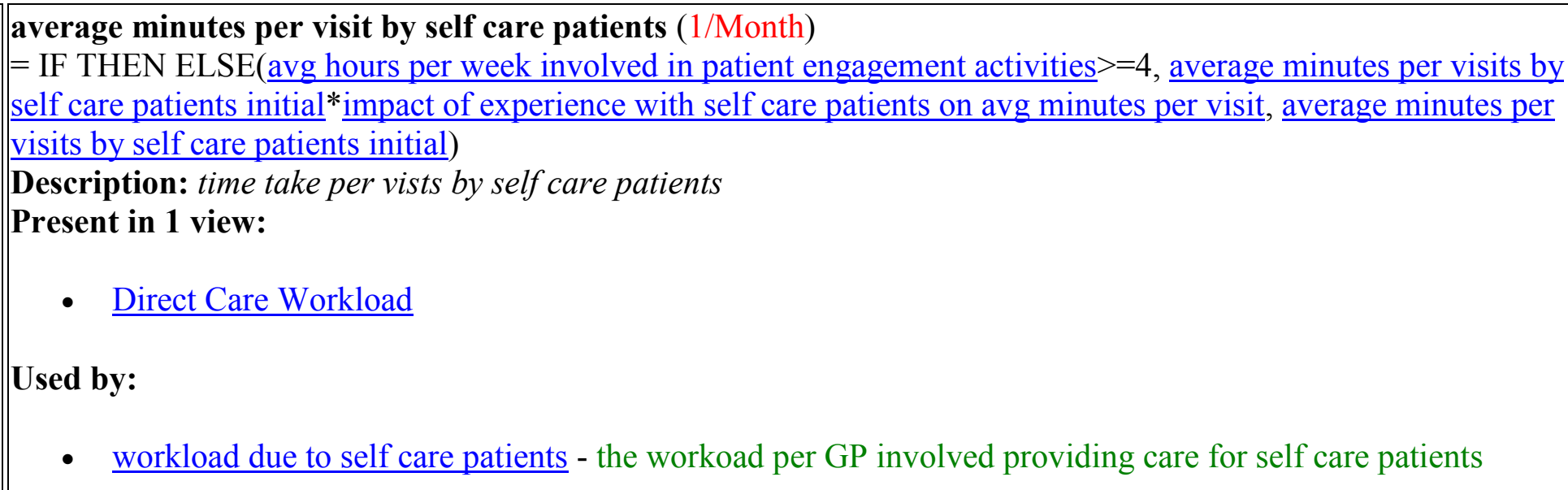 \\
\hline Default & \begin{tabular}{|c|} 
Dynamics of \\
Implementation \\
in Primary
\end{tabular} & 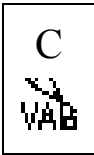 & $\begin{array}{l}\text { average minutes per visit by symptomatic LTC patients (1/Month) } \\
=12 \\
\text { Description: time taken per vists by symptomatic patients }\end{array}$ \\
\hline
\end{tabular}




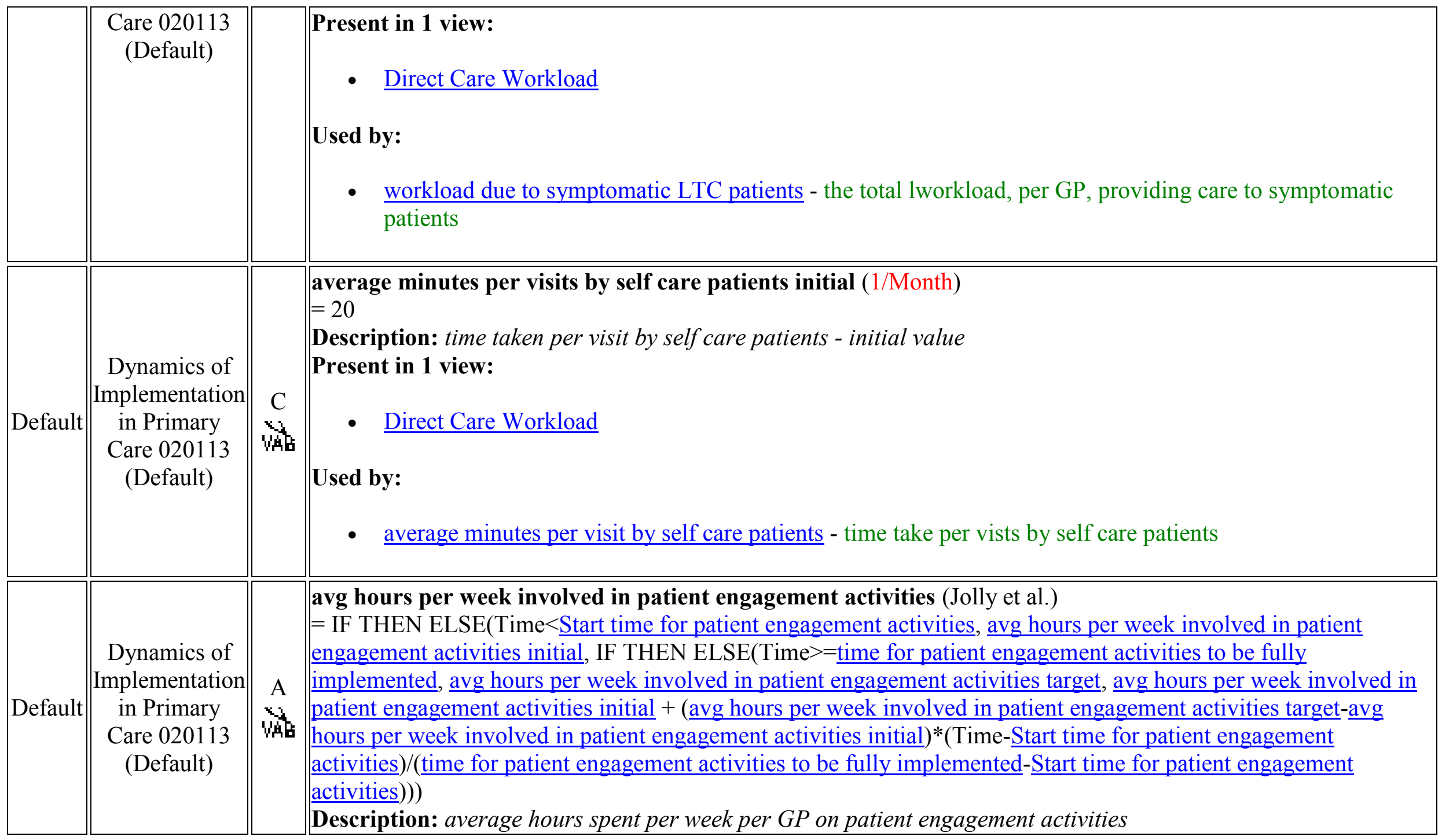




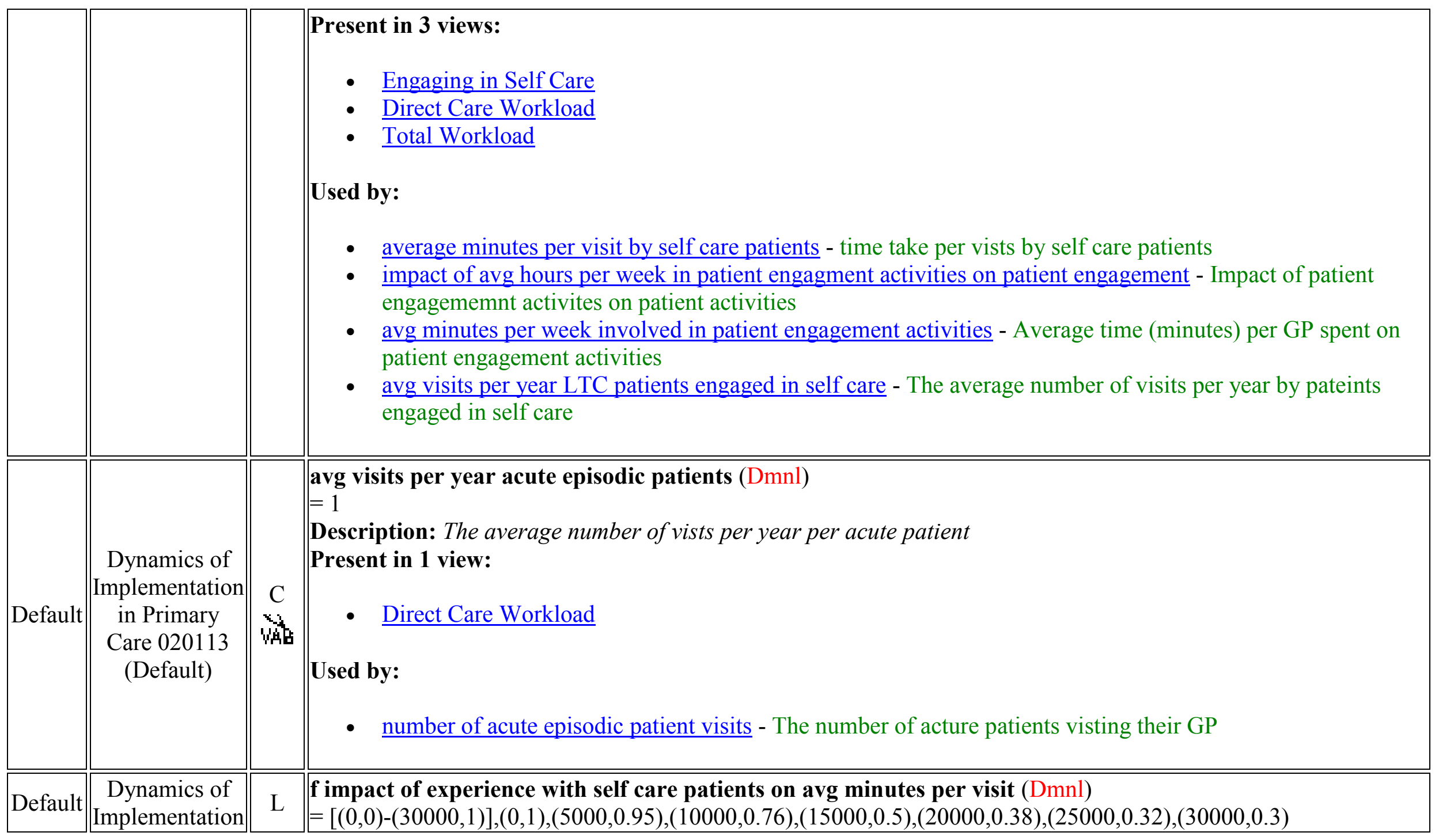




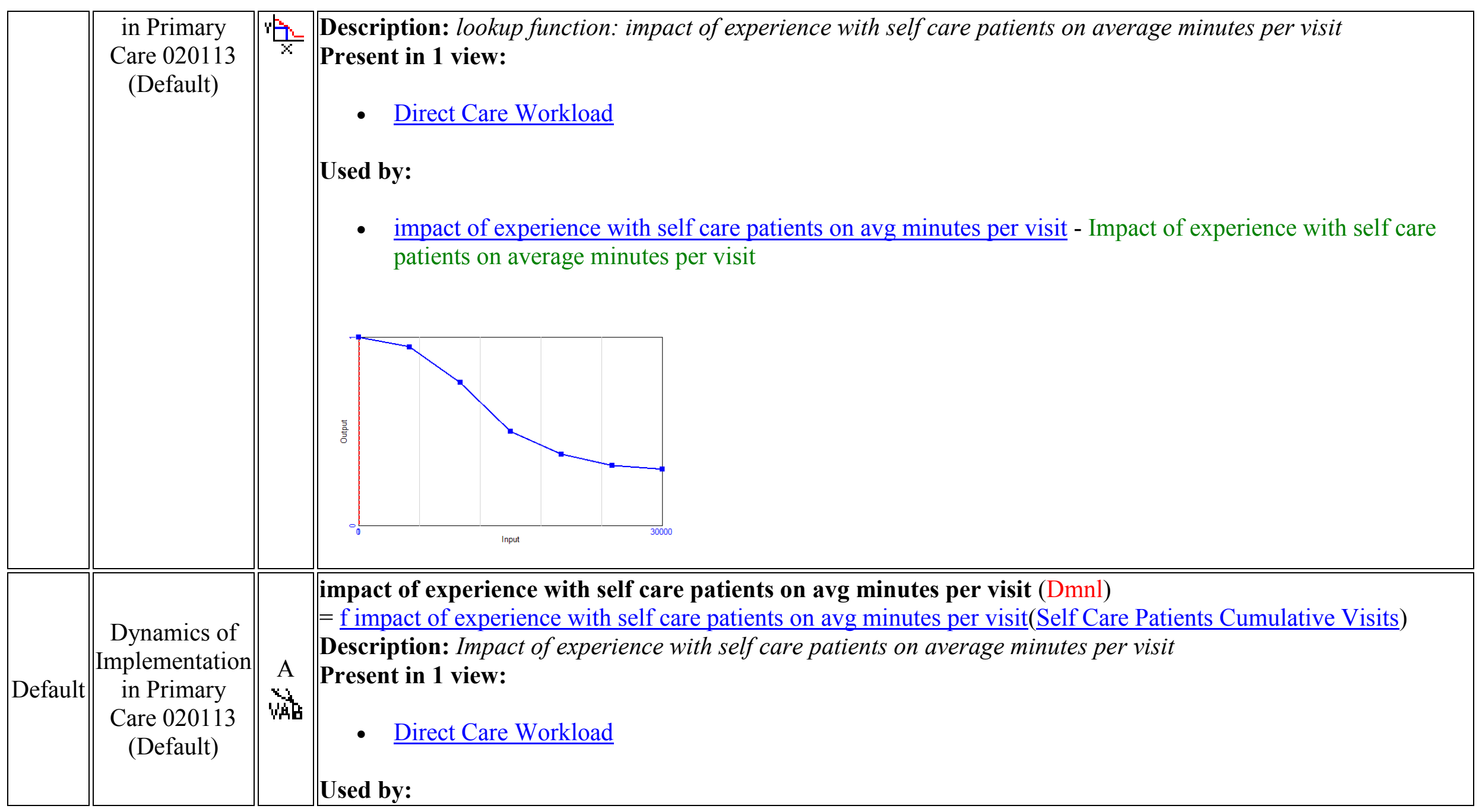




\begin{tabular}{|c|c|c|c|}
\hline & & & - average minutes per visit by self care patients - time take per vists by self care patients \\
\hline Default & $\mid \begin{array}{c}\text { Dynamics of } \\
\text { Implementation } \\
\text { in Primary } \\
\text { Care } 020113 \\
\text { (Default) }\end{array}$ & $\begin{array}{c}A \\
\text { VAt }\end{array}$ & $\begin{array}{l}\text { number of acute episodic patient visits (Patients) } \\
=\left(\text { number of acute episodic patients initial* }{ }^{*} \text { pct acute episodic patients seeking care in given year) }{ }^{*} \text { avg visits per }\right. \\
\text { year acute episodic patients } 12 \\
\text { Description: The number of acture patients visting their GP } \\
\text { Present in } 1 \text { view: } \\
\quad \text { Direct Care Workload } \\
\text { Used by: } \\
\quad \text { workload due to acute episodic patients - The workload per GP in provding care for acute patients }\end{array}$ \\
\hline Default & \begin{tabular}{|c|} 
Dynamics of \\
Implementation \\
in Primary \\
Care 020113 \\
(Default)
\end{tabular} & $\begin{array}{c}\text { A } \\
\text { VAt } \\
\text { VA }\end{array}$ & $\begin{array}{l}\text { number of acute episodic patients initial (Patients) } \\
=\text { Number of Enrolled Patients*(1-pct enrolled population with LTCs) } \\
\text { Description: This is the number of the enrolled patients who are acute, episodic patient who do not have LTCs } \\
\text { Present in } 2 \text { views: } \\
\text { - } \underline{\text { Number of Patients }} \\
\text { - } \underline{\text { Direct Care Workload }} \\
\text { - number of acute episodic patient visits - The number of acture patients visting their GP }\end{array}$ \\
\hline Default & Dynamics of & $\mathrm{C}$ & pct acute episodic patients seeking care in given year (Dmnl) \\
\hline
\end{tabular}




\begin{tabular}{|c|c|c|c|}
\hline & $\begin{array}{c}\text { Implementation } \\
\text { in Primary } \\
\text { Care } 020113 \\
\text { (Default) }\end{array}$ & 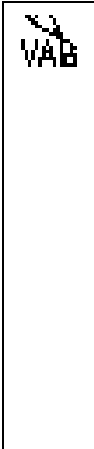 & $\begin{array}{l}=0.2 \\
\text { Description: The per cent of acute patients that seek care in any given year } \\
\text { Present in } 1 \text { view: } \\
\text { - Direct Care Workload } \\
\text { Used by: } \\
\text { - number of acute episodic patient visits - The number of acture patients visting their GP }\end{array}$ \\
\hline Default & \begin{tabular}{||c||} 
Dynamics of \\
Implementation \\
in Primary \\
Care 020113 \\
(Default)
\end{tabular} & $\begin{array}{c}\mathrm{L} \\
\mathrm{x} \\
\mathrm{M}\end{array}$ & 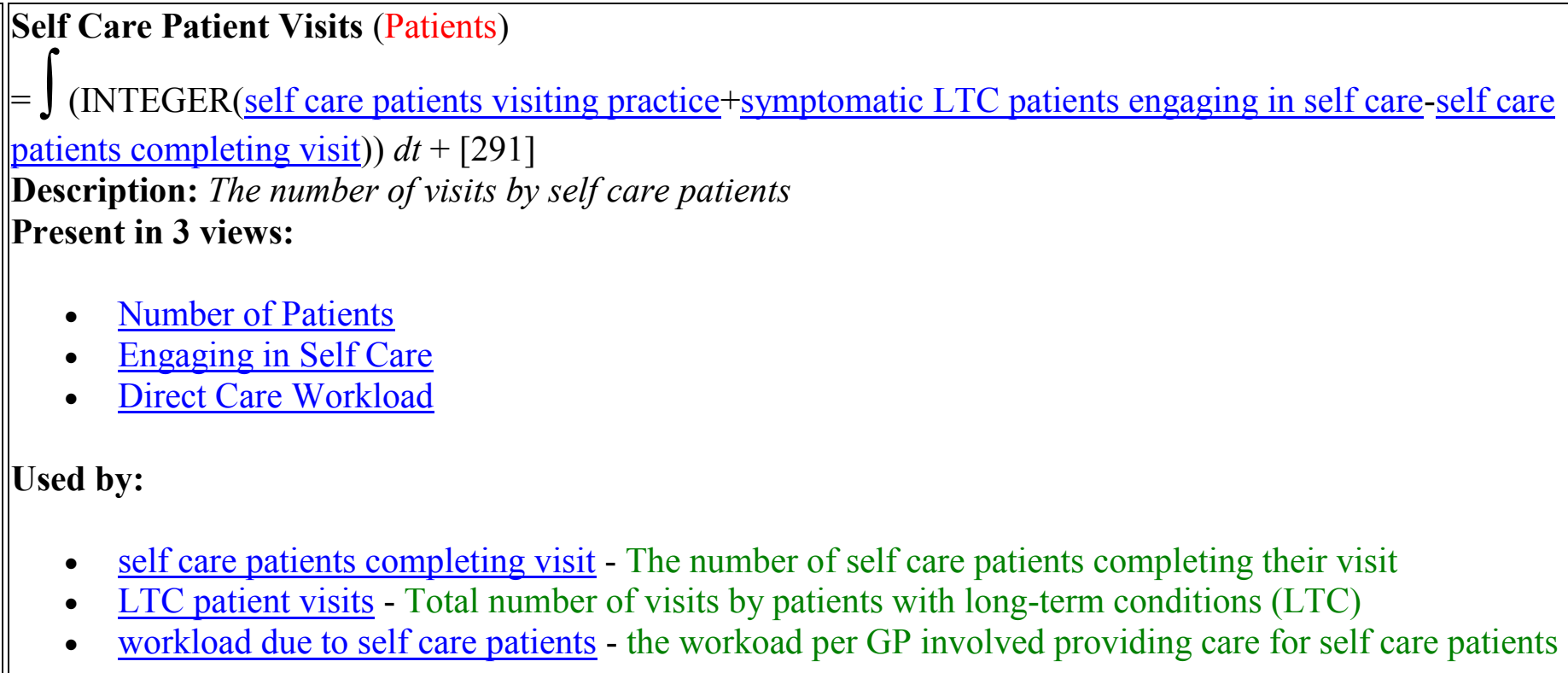 \\
\hline Default & \begin{tabular}{|c||} 
Dynamics of \\
Implementation
\end{tabular} & $\mathrm{L}$ & Self Care Patients Cumulative Visits (Patients) \\
\hline
\end{tabular}




\begin{tabular}{|c|c|c|c|}
\hline & $\begin{array}{c}\text { in Primary } \\
\text { Care } 020113 \\
\text { (Default) }\end{array}$ & 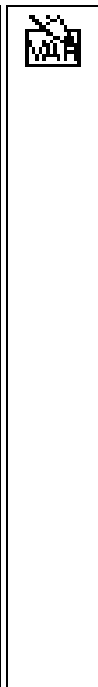 & 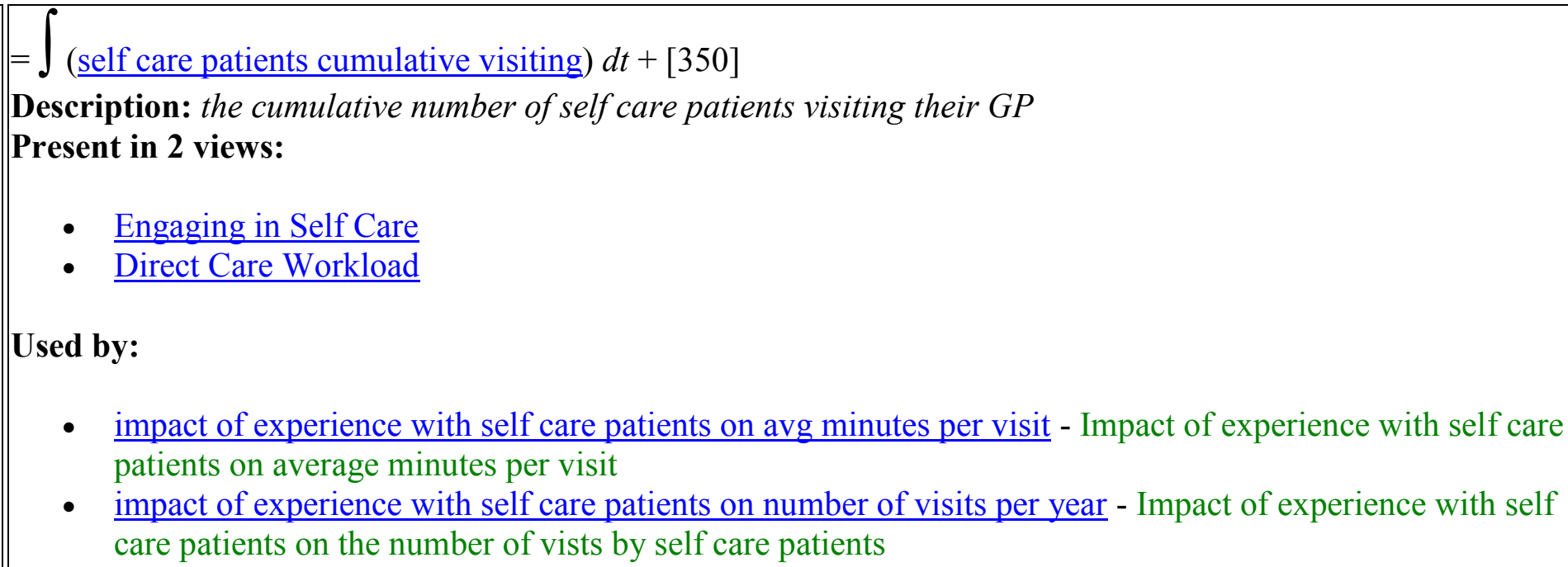 \\
\hline Default & \begin{tabular}{||c||} 
Dynamics of \\
Implementation \\
in Primary \\
Care 020113 \\
(Default)
\end{tabular} & $\begin{array}{c}\mathrm{L} \\
\text { 学自 }\end{array}$ & $\begin{array}{l}\text { Symptomatic LTC Patient Visits (Patients) } \\
=\int_{\text {(symptomatic LTC patients visiting practice-symptomatic LTC patients completing visit-symptomatic LTC }} \\
\text { patients engaging in self care) } d t+[3920] \\
\text { Description: The number of visits by symptomatic patients } \\
\text { Present in } 2 \text { views: } \\
\text { - Engaging in Self Care } \\
\text { - } \underline{\text { Direct Care Workload }} \\
\text { Used by: } \\
\text { - } \text { symptomatic LTC patients completing visit - The number of symptomatic patients completing their visit } \\
\text { - LTC patient visits - Total number of visits by patients with long-term conditions (LTC) }\end{array}$ \\
\hline
\end{tabular}




\begin{tabular}{|c|c|c|c|}
\hline & & & $\begin{array}{l}\text { - workload due to symptomatic LTC patients - the total lworkload, per GP, providing care to symptomatic } \\
\text { patients }\end{array}$ \\
\hline Default & \begin{tabular}{|} 
Dynamics of \\
Implementation \\
in Primary \\
Care 020113 \\
(Default)
\end{tabular} & $\begin{array}{r}A \\
\text { VAb }\end{array}$ & $\begin{array}{l}\text { workload due to acute episodic patients (Patients/Month) } \\
=\underline{\text { number of acute episodic patient visits* }} \text { *average minutes per visit by acute episodic patients } \\
\text { Description: The workload per GP in provding care for acute patients } \\
\text { Present in } 1 \text { view: } \\
\text { - Direct Care Workload } \\
\text { Used by: } \\
\text { - workload providing direct care to patients - The total workload per GP in provding direct care to patients }\end{array}$ \\
\hline Default & $\begin{array}{c}\text { Dynamics of } \\
\text { Implementation } \\
\text { in Primary } \\
\text { Care } 020113 \\
\text { (Default) }\end{array}$ & $\begin{array}{l}\text { A } \\
\text { VAb }\end{array}$ & $\begin{array}{l}\text { workload due to self care patients (Patients/Month) } \\
=\text { Self Care Patient Visits* average minutes per visit by self care patients } \\
\text { Description: the workoad per GP involved providing care for self care patients } \\
\text { Present in } 1 \text { view: } \\
\text { - Direct Care Workload } \\
\text { Used by: } \\
\text { - workload providing direct care to patients - The total workload per GP in provding direct care to patients }\end{array}$ \\
\hline Default & $\begin{array}{c}\text { Dynamics of } \\
\text { Implementation } \\
\text { in Primary } \\
\end{array}$ & $\begin{array}{c}A \\
\text { VAb }\end{array}$ & $\begin{array}{l}\text { workload due to symptomatic LTC patients (Patients/Month) } \\
\text { = Symptomatic LTC Patient Visits**average minutes per visit by symptomatic LTC patients } \\
\text { Description: the total lworkload, per GP, providing care to symptomatic patients }\end{array}$ \\
\hline
\end{tabular}




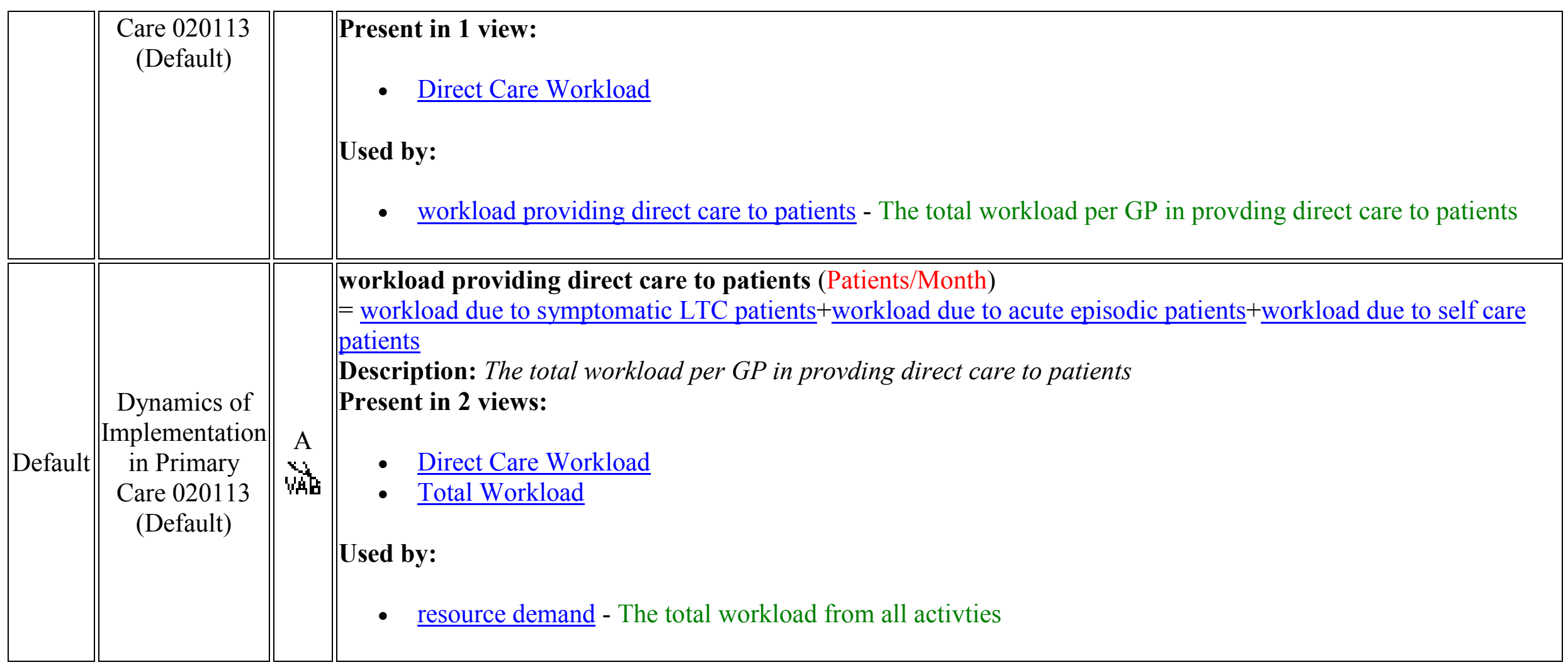




\section{oㅜ Total Workload (18 variables)}

Total Workload

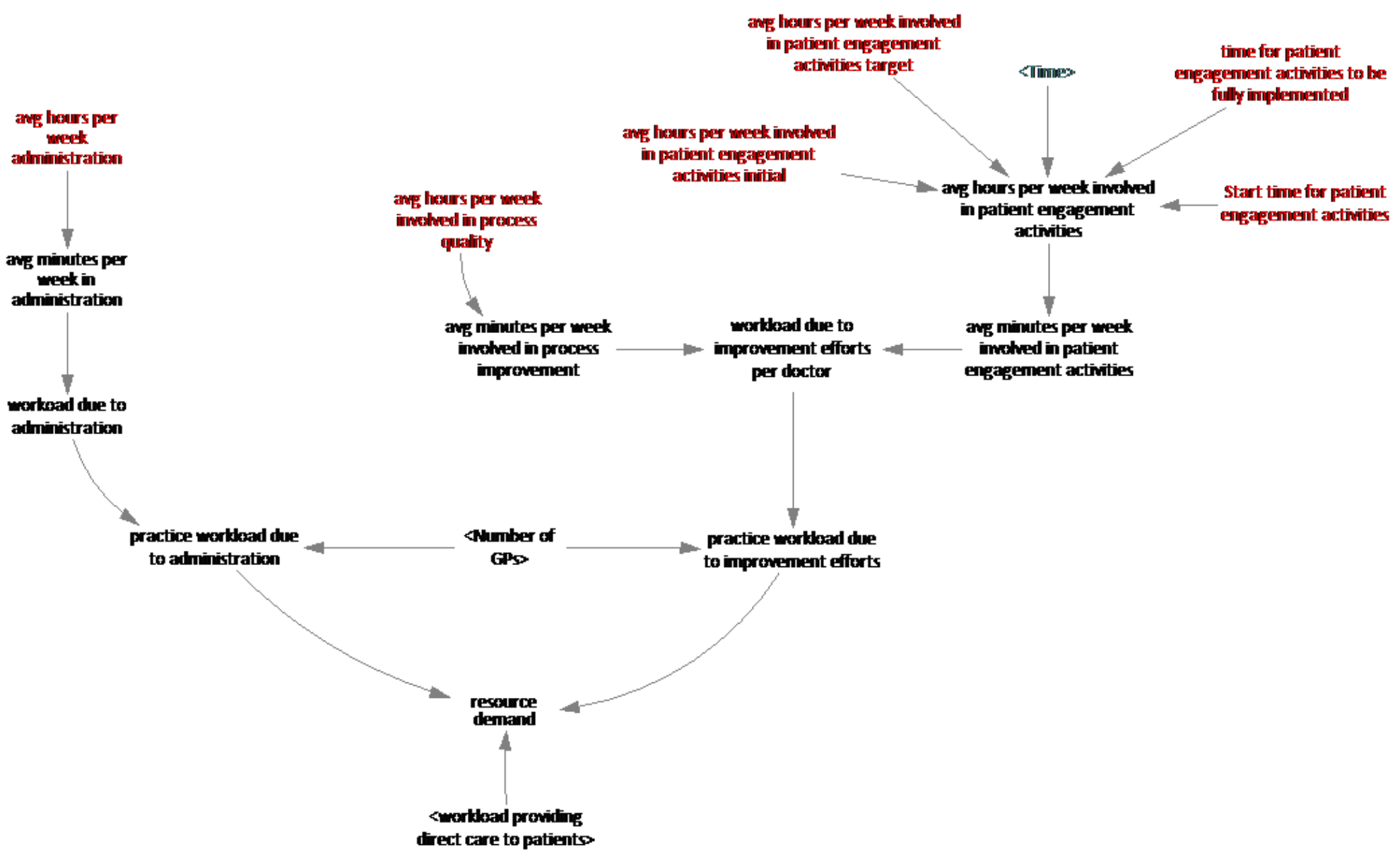




\begin{tabular}{|c|c|c|c|}
\hline Module & Group & Type & Variable Name and Description \\
\hline Default & \begin{tabular}{||c} 
Dynamics of \\
Implementation \\
in Primary \\
Care 020113 \\
(Default)
\end{tabular} & & $\begin{array}{l}\text { avg hours per week administration (Jolly et al.) } \\
=4 \\
\text { Description: The average number of hours per week per GP spend on adminstration activities } \\
\text { Present in } 1 \text { view: } \\
\text { - } \quad \text { Total Workload } \\
\text { Used by: } \\
\quad \text { avg minutes per week in administration }- \text { The average number of minutes per week per GP spent on } \\
\text { engagement activities }\end{array}$ \\
\hline Default & \begin{tabular}{||c||} 
Dynamics of \\
Implementation \\
in Primary \\
Care 020113 \\
(Default)
\end{tabular} & $\begin{array}{c}\text { A } \\
\text { Vis } \\
\text { Vhe }\end{array}$ & 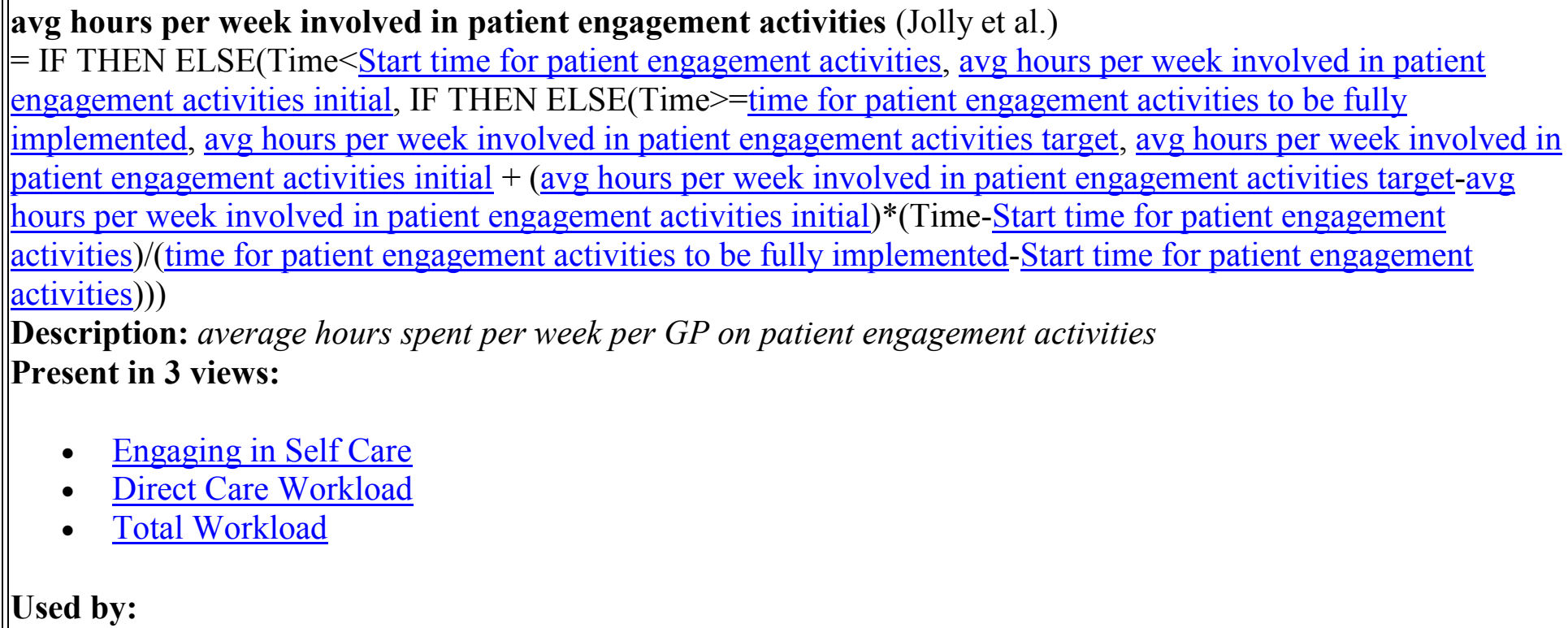 \\
\hline
\end{tabular}




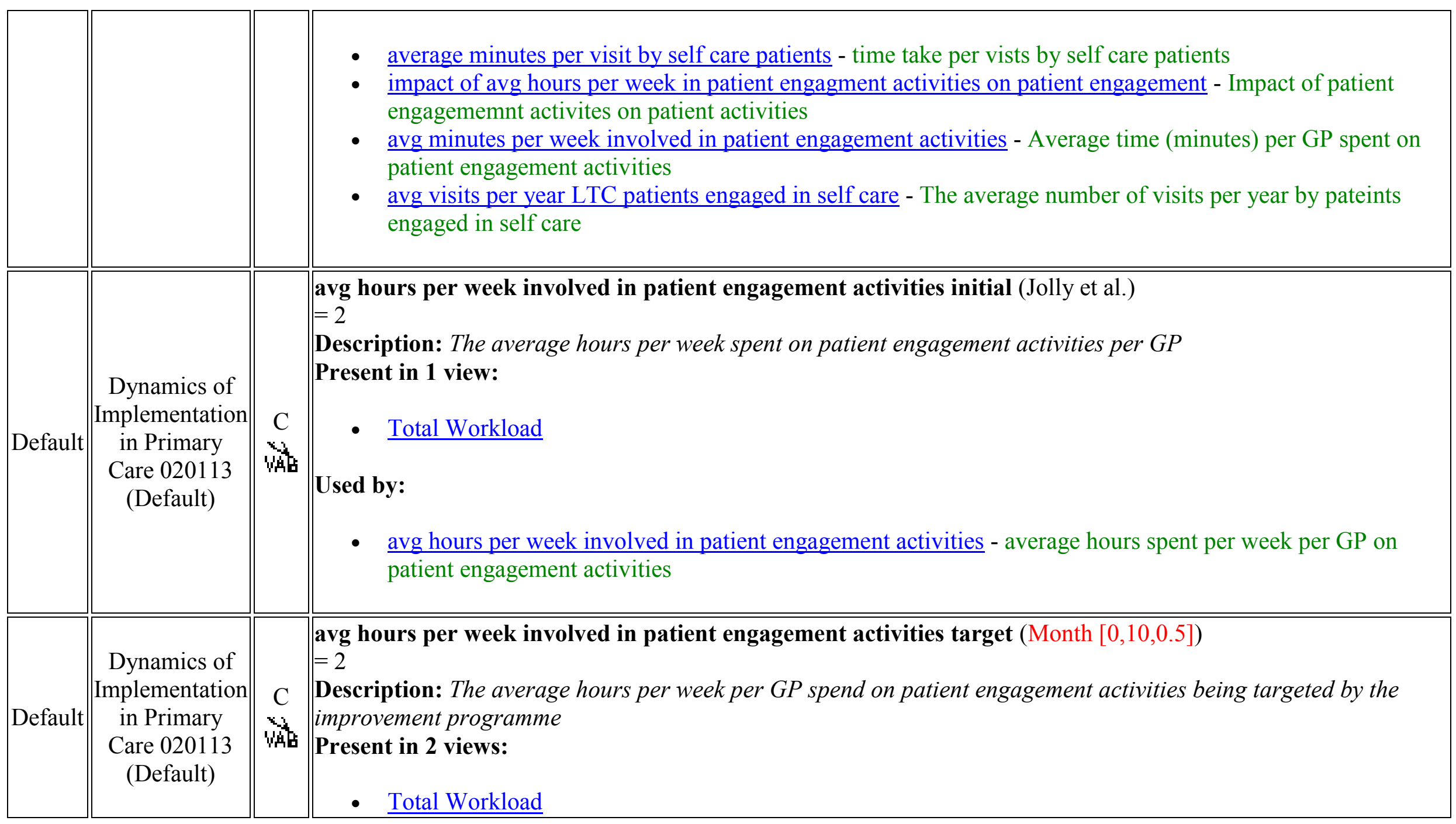




\begin{tabular}{|c|c|c|c|}
\hline & & & $\begin{array}{l}\text { - Control Panel \& Summary Graphs } \\
\text { Used by: } \\
\text { - } \frac{\text { avg hours per week involved in patient engagement activities - average hours spent per week per GP on }}{\text { patient engagement activities }}\end{array}$ \\
\hline Default & \begin{tabular}{||c||} 
Dynamics of \\
Implementation \\
in Primary \\
Care 020113 \\
(Default)
\end{tabular} & $\begin{array}{c}\mathrm{C} \\
\mathrm{YA}\end{array}$ & $\begin{array}{l}\text { avg hours per week involved in process quality (Month }[0,10,1] \text { ) } \\
=0 \\
\text { Description: The average hours per week per GP spend on process improvement activities } \\
\text { Present in } 2 \text { views: } \\
\text { - } \frac{\text { Process Quality }}{\text { Total Workload }} \\
\text { Used by: } \\
\text { - } \frac{\text { quality of processes }- \text { This is the current level of process quality }}{\text { improvement activities }}\end{array}$ \\
\hline Default & \begin{tabular}{||c||} 
Dynamics of \\
Implementation \\
in Primary \\
Care 020113 \\
(Default)
\end{tabular} & $\begin{array}{c}A \\
\text { Whb }\end{array}$ & $\begin{array}{l}\text { avg minutes per week in administration (Jolly et al.) } \\
=\text { avg hours per week administration* } 60 \\
\text { Description: The average number of minutes per week per GP spent on engagement activities } \\
\text { Present in } 1 \text { view: } \\
\quad \text { - Total Workload } \\
\text { Used by: }\end{array}$ \\
\hline
\end{tabular}




\begin{tabular}{|c|c|c|c|}
\hline & & & - workoad due to administration - This is the workload due to administration \\
\hline Default & \begin{tabular}{||c} 
Dynamics of \\
Implementation \\
in Primary \\
Care 020113 \\
(Default)
\end{tabular} & $\begin{array}{c}A \\
\text { VA }\end{array}$ & $\begin{array}{l}\text { avg minutes per week involved in patient engagement activities (Jolly et al.) } \\
=\text { avg hours per week involved in patient engagement activities* } 60 \\
\text { Description: Average time (minutes) per GP spent on patient engagement activities } \\
\text { Present in } 1 \text { view: } \\
\text { - Total Workload } \\
\text { Used by: } \\
\text { - } \frac{\text { workload due to improvement efforts per doctor }- \text { This is the additional workoad per GP due to }}{\text { improvement efforts }}\end{array}$ \\
\hline Default & \begin{tabular}{||c} 
Dynamics of \\
Implementation \\
in Primary \\
Care 020113 \\
(Default)
\end{tabular} & $\begin{array}{c}A \\
\text { Whb }\end{array}$ & $\begin{array}{l}\text { avg minutes per week involved in process improvement (Jolly et al.) } \\
=\text { avg hours per week involved in process quality*60 } \\
\text { Description: Average time (minutes) per GP spent on process improvement activities } \\
\text { Present in } 1 \text { view: } \\
\text { - } \quad \text { Total Workload } \\
\text { Used by: } \\
\text { - } \quad \frac{\text { workload due to improvement efforts per doctor }- \text { This is the additional workoad per GP due to }}{\text { improvement efforts }}\end{array}$ \\
\hline Default & Dynamics of & $\mathrm{L}$ & Number of GPs (GPs) \\
\hline
\end{tabular}




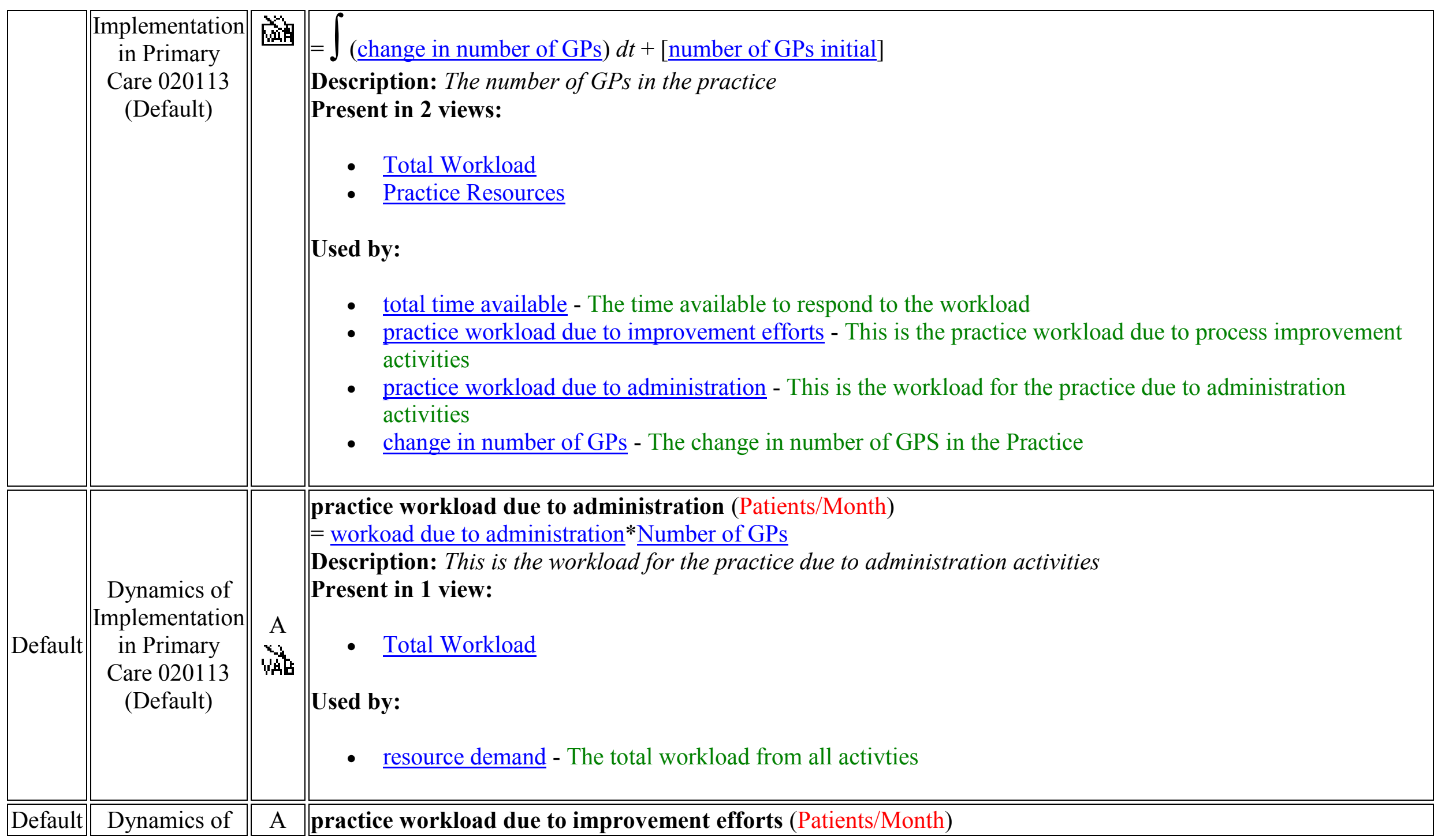




\begin{tabular}{|c|c|c|c|}
\hline & $\begin{array}{l}\text { Implementation } \\
\text { in Primary } \\
\text { Care } 020113 \\
\text { (Default) }\end{array}$ & VAB & $\begin{array}{l}\text { = workload due to improvement efforts per doctor* Number of GPs } \\
\text { Description: This is the practice workload due to process improvement activities } \\
\text { Present in } 1 \text { view: } \\
\text { - Total Workload } \\
\text { Used by: } \\
\text { - resource demand - The total workload from all activties }\end{array}$ \\
\hline Default & \begin{tabular}{||c||} 
Dynamics of \\
Implementation \\
in Primary \\
Care 020113 \\
(Default)
\end{tabular} & 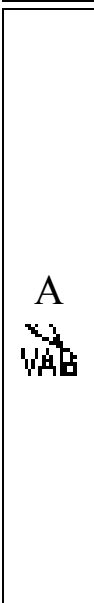 & $\begin{array}{l}\text { resource demand (Patients/Month) } \\
=\text { practice workload due to administration }+ \text { practice workload due to improvement efforts }+ \text { workload providing } \\
\text { direct care to patients } \\
\text { Description: The total workload from all activties } \\
\text { Present in } 2 \text { views: } \\
\text { - Total Workload } \\
\text { - } \underline{\text { Practice Resources }} \\
\text { Used by: } \\
\text { - frac resource capacity utilised - The fraction of the available resource capacity being utilised }\end{array}$ \\
\hline Default & \begin{tabular}{||c||} 
Dynamics of \\
Implementation \\
in Primary \\
Care 020113 \\
(Default)
\end{tabular} & $\begin{array}{c}\mathrm{C} \\
\mathrm{W} \\
\mathrm{W}\end{array}$ & $\begin{array}{l}\text { Start time for patient engagement activities (Month }[0,60,1] \text { ) } \\
=1 \\
\text { Description: This is the month in which increased pateint engagement activities are started } \\
\text { Present in } 1 \text { view: }\end{array}$ \\
\hline
\end{tabular}




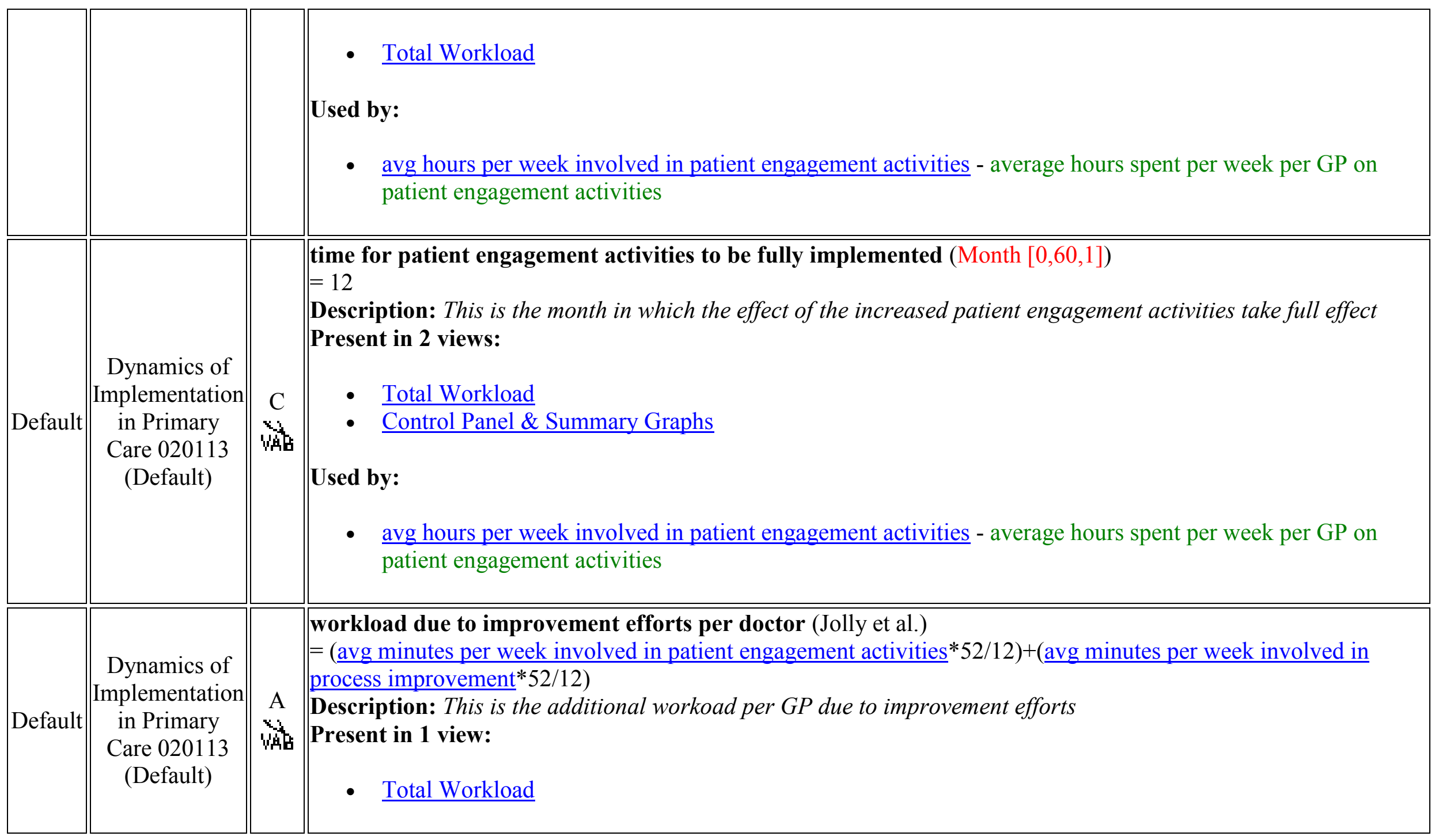




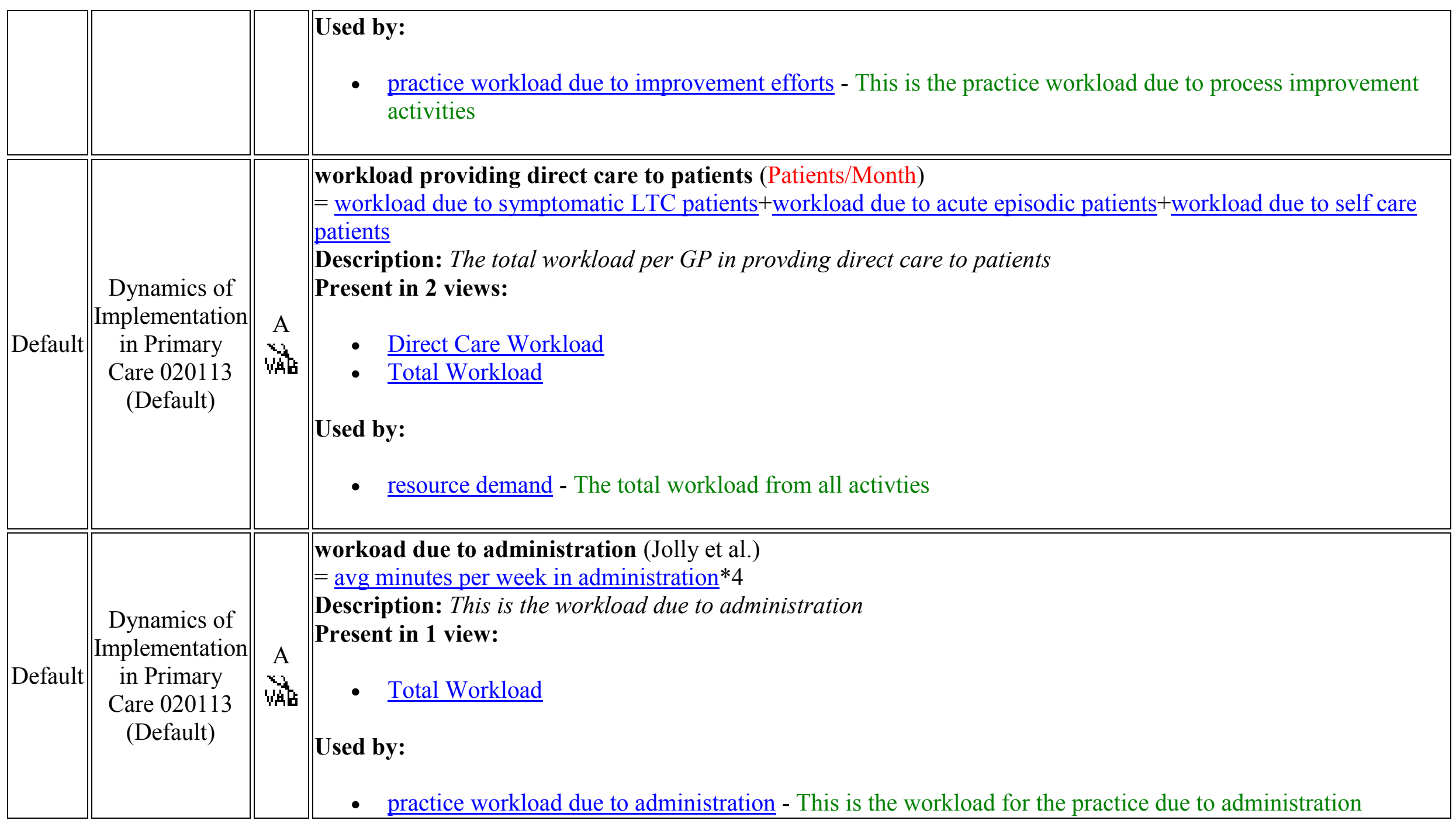




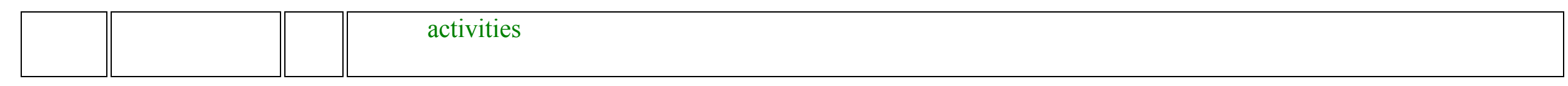




\section{тоP Practice Resources (11 variables)}

\section{Practice Resources}

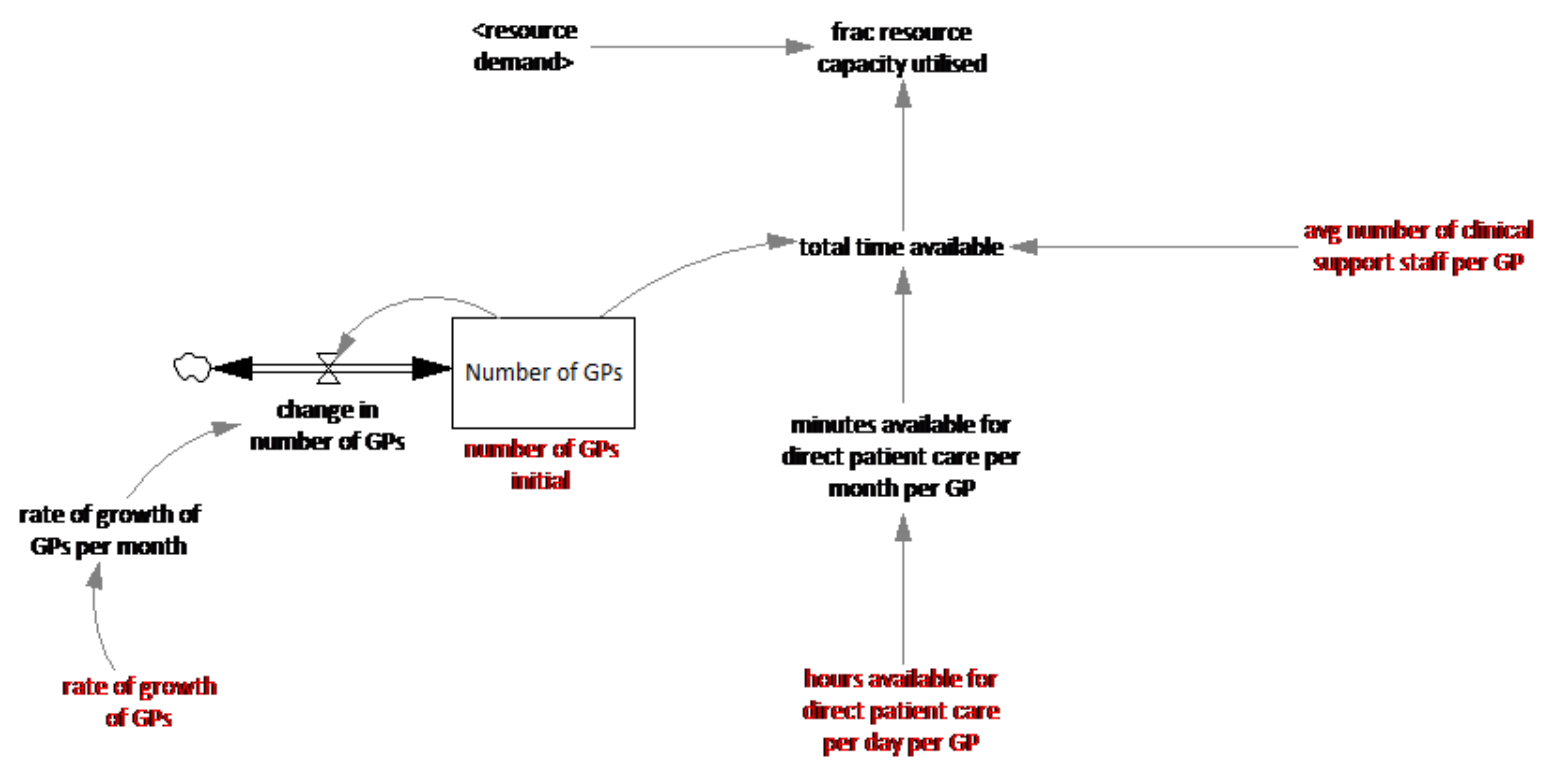




\begin{tabular}{|c|c|c|c|}
\hline Module & Group & Type & Variable Name and Description \\
\hline Default & \begin{tabular}{|c||} 
Dynamics of \\
Implementation \\
in Primary \\
Care 020113 \\
(Default)
\end{tabular} & $\begin{array}{c}C \\
\mathrm{C} \\
\mathrm{VAB}\end{array}$ & $\begin{array}{l}\text { avg number of clinical support staff per GP (Staff) } \\
=0.5 \\
\text { Description: The number of clinical support staff per GP - initial value } \\
\text { Present in } 1 \text { view: } \\
\quad \text { Practice Resources } \\
\text { Used by: } \\
\text { - total time available - The time available to respond to the workload }\end{array}$ \\
\hline Default & \begin{tabular}{|c||} 
Dynamics of \\
Implementation \\
in Primary \\
Care 020113 \\
(Default)
\end{tabular} & $\begin{array}{l}\mathrm{F}, \mathrm{A} \\
\mathrm{W}_{\mathrm{B}} \\
\mathrm{W} \\
\mathrm{F}\end{array}$ & $\begin{array}{l}\text { change in number of GPs (GPs/Month) } \\
=\text { Number of GPs* }{ }^{*} \text { rate of growth of GPs per month } \\
\text { Description: The change in number of GPS in the Practice } \\
\text { Present in } 1 \text { view: } \\
\text { - Practice Resources } \\
\text { Used by: } \\
\text { - Number of GPs - The number of GPs in the practice }\end{array}$ \\
\hline Default & \begin{tabular}{|c|} 
Dynamics of \\
Implementation \\
in Primary \\
Care 020113 \\
(Default) \\
\end{tabular} & \begin{tabular}{|c} 
A \\
Wab \\
Vab \\
\end{tabular} & $\begin{array}{l}\text { frac resource capacity utilised }(\mathrm{Dmnl}) \\
=\text { resource demand/total time available } \\
\text { Description: The fraction of the available resource capacity being utilised } \\
\text { Present in } 2 \text { views: }\end{array}$ \\
\hline
\end{tabular}




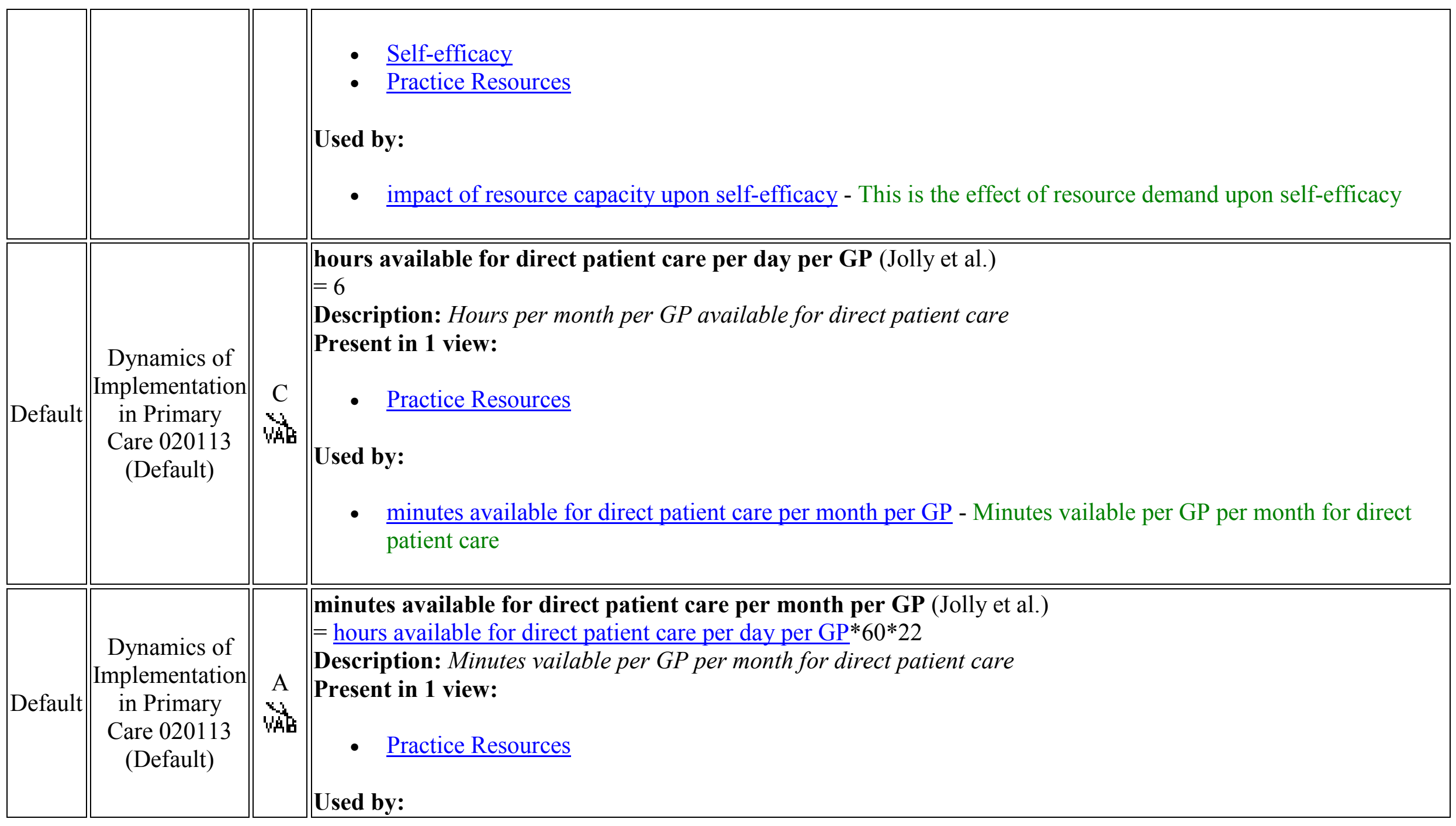




\begin{tabular}{|c|c|c|c|}
\hline Default & \begin{tabular}{|c|} 
Dynamics of \\
Implementation \\
in Primary \\
Care 020113 \\
(Default)
\end{tabular} & $\begin{array}{c}\mathrm{L} \\
\mathrm{m} \\
\mathrm{m}\end{array}$ & 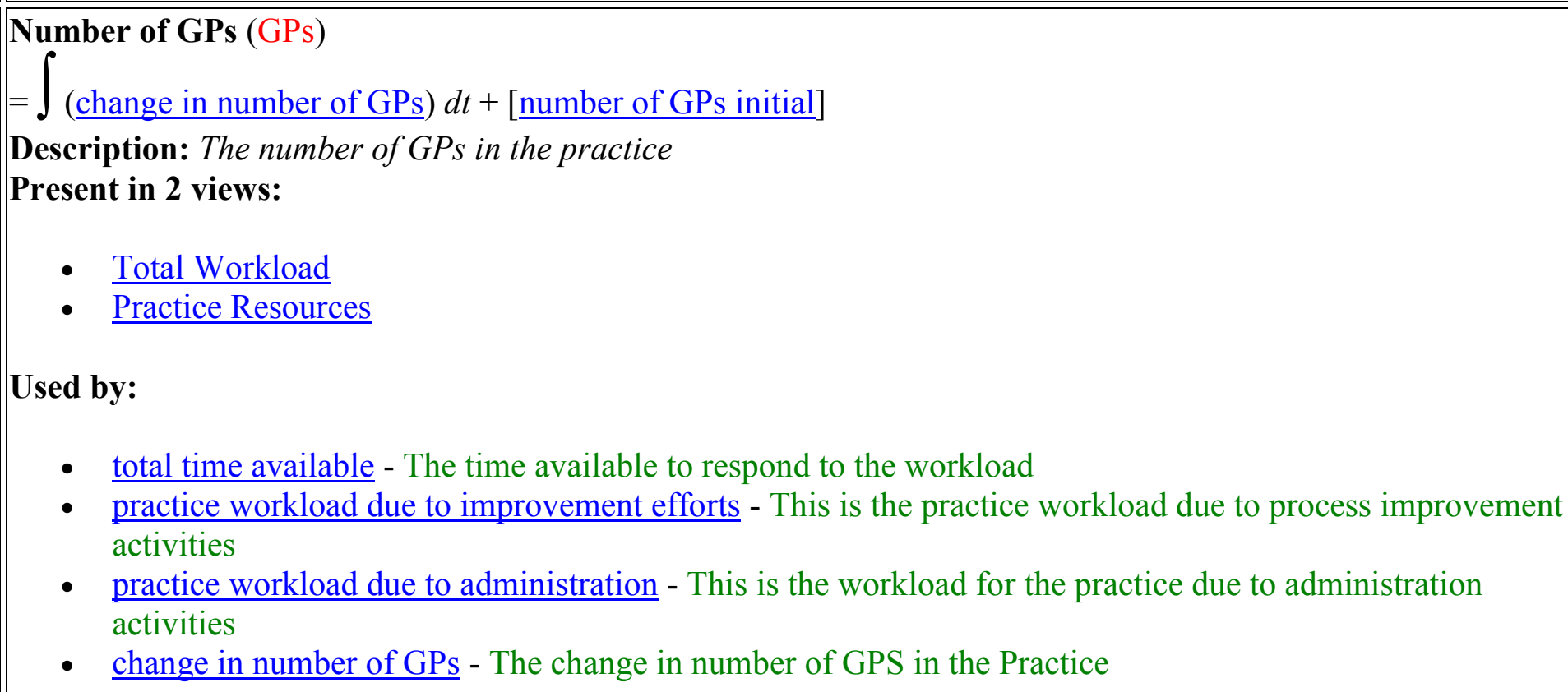 \\
\hline Default & \begin{tabular}{|c} 
Dynamics of \\
Implementation \\
in Primary \\
Care 020113 \\
(Default)
\end{tabular} & \begin{tabular}{|c|} 
LI,C \\
VAz \\
VAz
\end{tabular} & $\begin{array}{l}\text { number of GPs initial (GPs }[1,10,1]) \\
=6 \\
\text { Description: The initial number of GPs in the Practice } \\
\text { Present in } 1 \text { view: } \\
\quad \text { Practice Resources } \\
\text { Used by: }\end{array}$ \\
\hline
\end{tabular}




\begin{tabular}{|c|c|c|c|}
\hline & & & - Number of GPs - The number of GPs in the practice \\
\hline Default & $\begin{array}{c}\text { Dynamics of } \\
\text { Implementation } \\
\text { in Primary } \\
\text { Care } 020113 \\
\text { (Default) }\end{array}$ & $\begin{array}{r}C \\
\text { C } \\
\text { Vab }\end{array}$ & $\begin{array}{l}\text { rate of growth of GPs (1/Month }[0,0.02,0.001]) \\
=0 \\
\text { Description: This is the estimated growth in the number of GFPs. } \\
\text { Present in } 1 \text { view: } \\
\text { - Practice Resources } \\
\text { Used by: } \\
\text { - } \underline{\text { rate of growth of GPs per month - This is the growth rate of GPs }}\end{array}$ \\
\hline Default & $\begin{array}{c}\text { Dynamics of } \\
\text { Implementation } \\
\text { in Primary } \\
\text { Care } 020113 \\
\text { (Default) }\end{array}$ & $\begin{array}{c}\text { A } \\
\text { Wat }\end{array}$ & $\begin{array}{l}\text { rate of growth of GPs per month (1/Month) } \\
=\text { rate of growth of GPS } / 12 \\
\text { Description: This is the growth rate of GPS } \\
\text { Present in } 1 \text { view: } \\
\text { - Practice Resources } \\
\text { Used by: } \\
\text { - change in number of GPs - The change in number of GPS in the Practice }\end{array}$ \\
\hline Default & $\begin{array}{c}\text { Dynamics of } \\
\text { Implementation } \\
\text { in Primary }\end{array}$ & $\begin{array}{c}\text { A } \\
\text { VAtr }\end{array}$ & $\begin{array}{l}\text { resource demand (Patients/Month) } \\
=\text { practice workload due to administration } \\
\text { direct care to patients }\end{array}$ \\
\hline
\end{tabular}




\begin{tabular}{|c|c|c|c|}
\hline & $\begin{array}{l}\text { Care } 020113 \\
\text { (Default) }\end{array}$ & & $\begin{array}{l}\text { Description: The total workload from all activties } \\
\text { Present in } 2 \text { views: } \\
\text { - } \text { Total Workload } \\
\text { Used by: } \\
\text { - } \text { frac resource capacity utilised - The fraction of the available resource capacity being utilised }\end{array}$ \\
\hline Default & $\mid \begin{array}{c}\text { Dynamics of } \\
\text { Implementation } \\
\text { in Primary } \\
\text { Care } 020113 \\
\text { (Default) }\end{array}$ & $\begin{array}{c}\text { A } \\
\text { VAb }\end{array}$ & $\begin{array}{l}\text { total time available (Jolly et al.) } \\
=\text { Number of GPs } * \text { minutes available for direct patient care per month per GP* } * 1+\text { avg number of clinical support } \\
\text { staff per GP/2) } \\
\text { Description: The time available to respond to the workload } \\
\text { Present in } 1 \text { view: } \\
\text { - } \underline{\text { Practice Resources }} \\
\text { Used by: } \\
\text { - frac resource capacity utilised }- \text { The fraction of the available resource capacity being utilised }\end{array}$ \\
\hline Module & Group & Type & Variable Name and Description \\
\hline Module & Group & Type & Variable Name and Description \\
\hline Default & $\begin{array}{l}\text { Dynamics of } \\
\text { Implementation } \\
\text { in Primary Care } \\
020113\end{array}$ & $\begin{array}{c}C \\
\text { VAB }\end{array}$ & $\begin{array}{l}\text { avg hours per week involved in patient engagement activities target (Month }[0,10,0.5] \text { ) } \\
=2 \\
\text { Description: The average hours per week per GP spend on patient engagement activities being targeted by the } \\
\text { improvement programme }\end{array}$ \\
\hline
\end{tabular}


(Default) Present in 2 views:

- Total Workload

- $\underline{\text { Control Panel \& Summary Graphs }}$

\section{Used by:}

- $\quad$ avg hours per week involved in patient engagement activities - average hours spent per week per GP on patient engagement activities

\section{time for patient engagement activities to be fully implemented (Month $[0,60,1]$ )}

$=12$

Description: This is the month in which the effect of the increased patient engagement activities take full effect Present in 2 views:

Dynamics of Implementation

Default in Primary Care

020113

(Default)

- Total Workload

- $\quad$ Control Panel \& Summary Graphs

\section{Used by:}

- $\quad$ avg hours per week involved in patient engagement activities - average hours spent per week per GP on patient engagement activities

Module Group Type Variable Name and Description

Dynamics of F,A change in quality of LTCM (Quality/Month)

Default Implementation in Primary Care $\Rightarrow$ self care patients $* 5$ )/time to adjust quality of LTCM, MIN(desired improvement in quality, effect of improvement

020113 effort)/time to adjust quality of LTCM) 
(Default) Description: This is the net change in the effective management of LTCs as a result of the desired improvement, the effort to improve, the time to change and the per cent of patients who are engaging in self care.

Present in 2 views:

- Quality of LTCM

- $\underline{\text { View } 13}$

\section{Used by:}

- Effective Management of Chronic Conditions - This is the primary goal. 100per cent equares to the best possible quality across all patients in the practice.

decrease in quality of LTCM care (Quality/Month)

$=$ IF THEN ELSE(Effective Management of Chronic Conditions $>$ baseline performance of LTCM in primary care, loss of quality, 0 )

Description: This is the decrease in quality per month - a function of an annual loss function and the baseline performance of peer practices

Dynamics of

Implementation

Default in Primary Care

Present in 2 views:

020113

(Default)

- Quality of LTCM

- View 13

\section{Used by:}

- desired improvement in quality - This is the desired improvement goal - a function of the target they are trying to reach, the time it takes them to make changes and the gap between their goal and current practice.

- Effective Management of Chronic Conditions - This is the primary goal. 100per cent equares to the best possible quality across all patients in the practice. 
